Gabriele Marwege

\title{
Legasthenie und Dyskalkulie in der Schule
}

Eine verfassungsrechtliche Untersuchung unter besonderer

Berücksichtigung der UN-Behindertenrechtskonvention 

Gabriele Marwege

Legasthenie und Dyskalkulie in der Schule

This work is licensed under the

Creative Commons License 3.0 "by-sa", allowing you to download, distribute and print the document in a few copies for private or educational use, given that the document stays unchanged and the creator is mentioned.

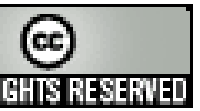


erschienen in der Reihe der Universitätsdrucke im Universitätsverlag Göttingen 2013 
Gabriele Marwege

Legasthenie und
Dyskalkulie in der Schule

Eine verfassungsrechtliche Untersuchung unter besonderer Berücksichtigung der UN-Behindertenrechtskonvention

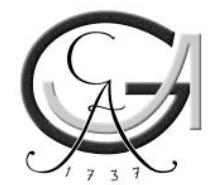

Universitätsverlag Göttingen 2013 


\title{
Bibliographische Information der Deutschen Nationalbibliothek
}

Die Deutsche Nationalbibliothek verzeichnet diese Publikation in der Deutschen Nationalbibliographie; detaillierte bibliographische Daten sind im Internet über $<$ http://dnb.ddb.de> abrufbar.

\author{
Anschrift der Autorin \\ Gabriele Marwege \\ e-mail: marwege-legasthenie@freenet.de
}

Dieses Buch ist auch als freie Onlineversion über die Homepage des Verlags sowie über den OPAC der Niedersächsischen Staats- und Universitätsbibliothek (http://www.sub.uni-goettingen.de) erreichbar und darf gelesen, heruntergeladen sowie als Privatkopie ausgedruckt werden. Es gelten die Lizenzbestimmungen der Onlineversion.

Um eine bessere Lesbarkeit für Legastheniker zu gewährleisten, wurde für diese Arbeit eine vom Standard-Format des Verlags abweichende, serifenlose Schriftart gewählt.

Satz und Layout: Annett Eichstaedt Umschlaggestaltung: Franziska Lorenz

(C) 2013 Universitätsverlag Göttingen http://univerlag.uni-goettingen.de ISBN: 978-3-86395-119-1 
Meinem Mann

und

meinen Kindern 



\section{Übersicht}

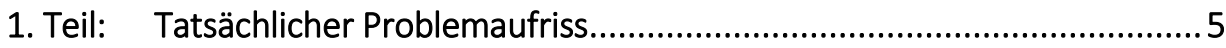

1. Kapitel: Was sind Legasthenie und Dyskalkulie? .................................... 5

2. Kapitel: Legasthenie und Dyskalkulie in der Schule................................29

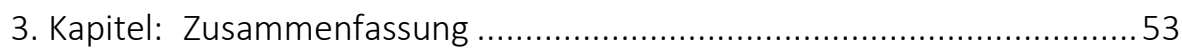

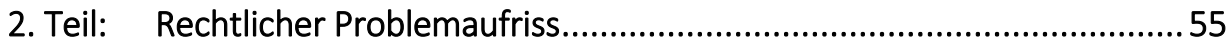

1. Kapitel: Das Sonderstatusverhältnis Schule ……...............................55

2. Kapitel: Gleichheit und Ungleichheit von Schülern und Schülerinnen

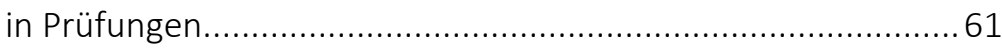

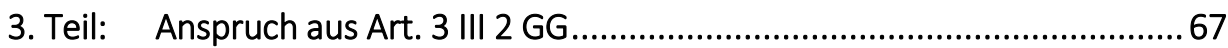

1. Kapitel: Verhältnis von Art. 3 III 2 GG und Art. 3 I GG ............................67

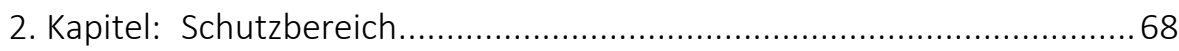

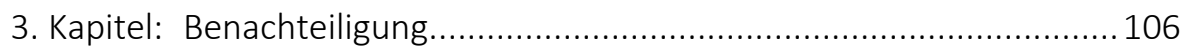

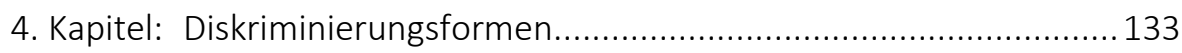

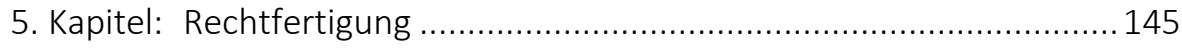

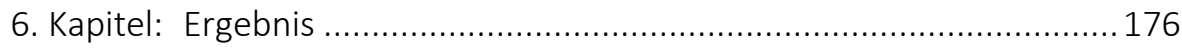

4. Teil: Anspruch aus dem Grundsatz der Chancengleichheit aus

Art. 3 I GG i.V.m. Art. 2 I und 12 I GG ............................................. 177

1. Kapitel: Konkretisierung des Grundsatzes der Chancengleichheit ........177

2. Kapitel: Grenzen des Grundsatzes der Chancengleichheit................... 178

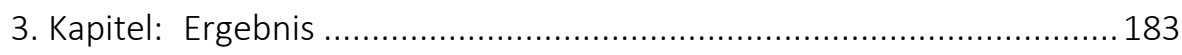

5. Teil: Nachteilsausgleich und Förderung ………..................................... 185

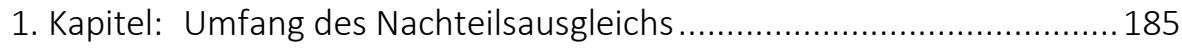

2. Kapitel: Verhältnis Nachteilsausgleich und Förderung .......................... 199

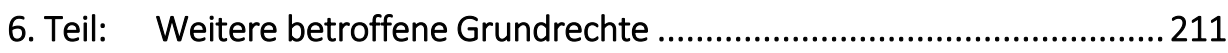

1. Kapitel: Anspruch aus Art. 2 I GG und 12 I GG ...................................211

2. Kapitel: Anspruch aus Art. 2 I und 6 || GG - Schulpflicht...................... 222

7. Teil: Anspruch aus Völker- und Europarecht ...........................................227

1. Kapitel: Anspruch aus Art. 24 BRK - Recht auf Bildung ........................227 
2. Kapitel: Weitere Völker- und Europarechtliche Regelungen .................241

8. Teil: Abwehrrecht, Leistungsrecht, Teilhaberecht ..................................245

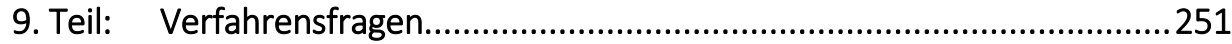

1. Kapitel: Grundentscheidung: Anerkennung von Legasthenie und

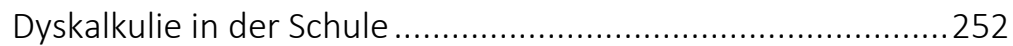

2. Kapitel: Ausgestaltung des Nachteilsausgleichs ..................................287

3. Kapitel: Verzicht der Betroffenen auf Berücksichtigung von Legasthenie und Dyskalkulie ..................................................295

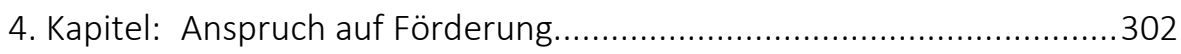

10. Teil: Datenschutz und Zeugnisbemerkung..............................................309

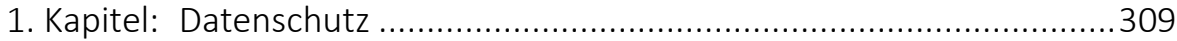

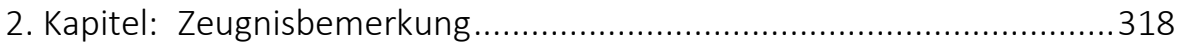

11. Teil: Amtshaftung aus $\S 839$ BGB i.V.m. Art. 34 GG ...............................333

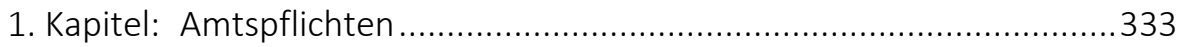

2. Kapitel: Weitere Voraussetzungen der Amtshaftung ...........................338

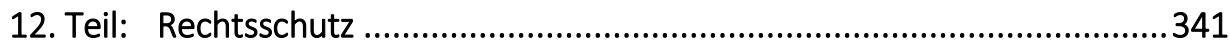

1. Kapitel: Rechtsschutz im Verfahren der Anerkennung der Behinderung und bei schulischen Prüfungen...

2. Kapitel: Rügepflichten der Eltern/des Schülers bei Nicht-Beachtung

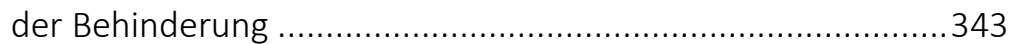

3. Kapitel: Rechtsfolgen rechtswidriger Prüfungen .................................353

13. Teil: Notwendigkeit gesetzlicher Regelungen ........................................359

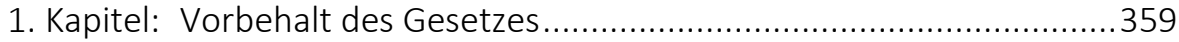

2. Kapitel: Mindestumfang gesetzlicher Regelungen ...............................362

3. Kapitel: Rechtsverordnung oder Verwaltungsvorschrift?......................364

4. Kapitel: Ansprüche bis zum Erlass gesetzlicher Regelungen .................365

14. Teil: Umgang mit Legasthenie/Dyskalkulie in der EU ................................369

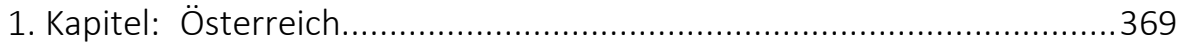

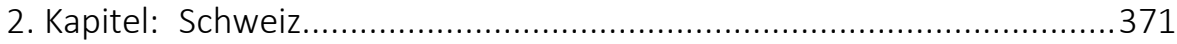

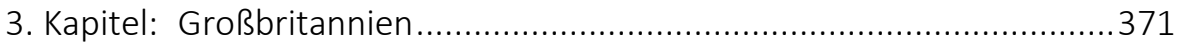

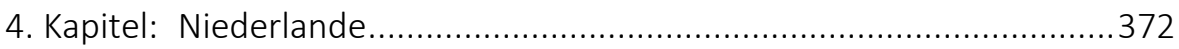

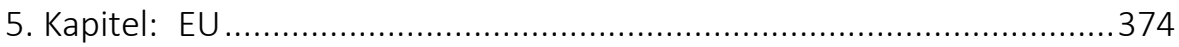

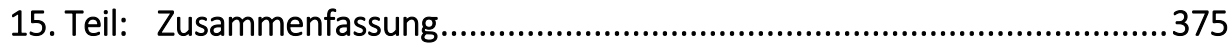




\section{Inhaltsverzeichnis}

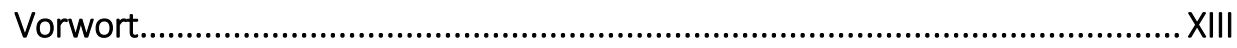

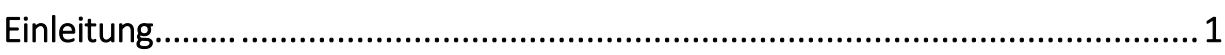

1. Teil: Tatsächlicher Problemaufriss.........................................................

1. Kapitel: Was sind Legasthenie und Dyskalkulie? .................................... 5

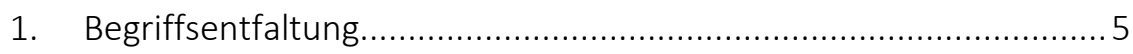

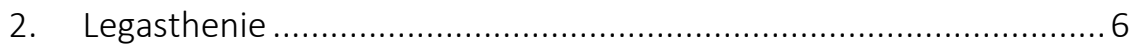

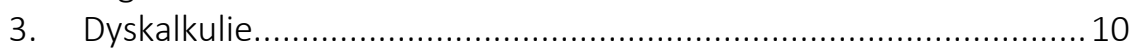

4. Kombination der Störungen .................................................... 11

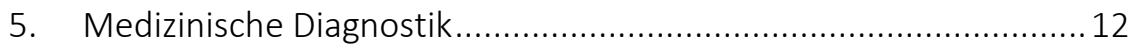

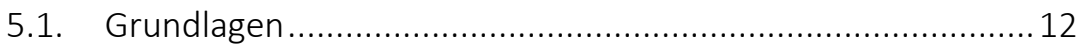

5.2. Medizinische Diagnostik nach ICD 10 und Multiaxialem

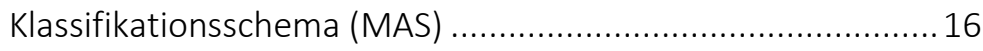

6. Pädagogische Diagnostik .......................................................... 19

7. Legasthenie versus Lese-Rechtschreib-Schwäche; Dyskalkulie versus Rechenschwäche............................................................. 20

8. Welche Folgen haben Legasthenie und/oder Dyskalkulie in

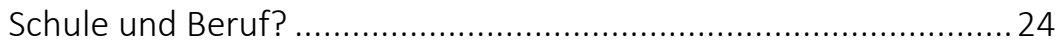

9. Welche psychischen Auswirkungen haben Legasthenie und/oder

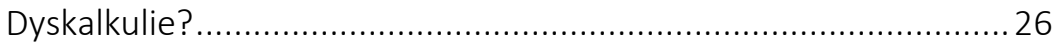

2. Kapitel: Legasthenie und Dyskalkulie in der Schule...............................29

1. Beschulung von betroffenen Schülern und Schülerinnen ...............29

2. Bewertungs- und Benotungsvorschriften......................................... 30

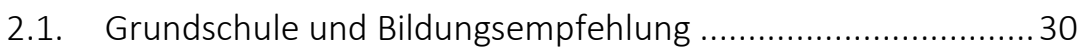

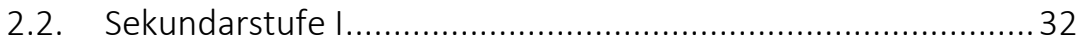

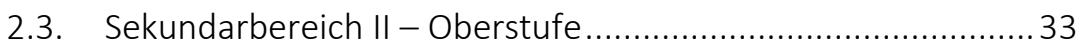

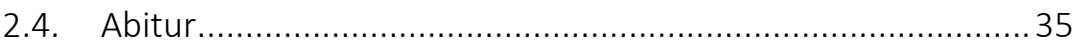

3. Die schulrechtliche Regelung zur Berücksichtigung von Legasthenie in Bayern. 
4. Grundzüge der Beschlüsse der KMK und der Landesvorschriften zur Berücksichtigung von Legasthenie und Dyskalkulie - mit

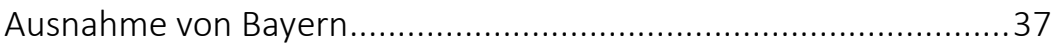

5. Berücksichtigung der Dyskalkulie in der Schule............................39

6. Förderschule statt Regelschule? ........................................40

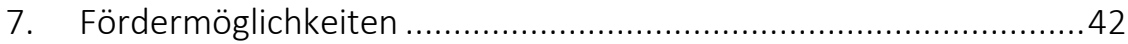

7.1. Schulische Fördermöglichkeiten ................................42

7.2. Außerschulischen Therapie und ihre Finanzierung ................45

7.2.1. Finanzierung durch die Krankenkassen nach $\S 27$ SGB V ...46

7.2.2. Finanzierung durch die Jugendämter nach $\S 35$ a SGB VIII. 48

7.2.3. Finanzierung durch die Eltern..................................49

8. Formen der Berücksichtigung in der Schule ..............................51

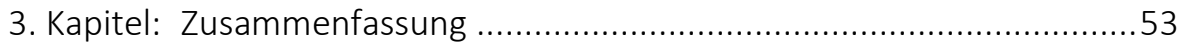

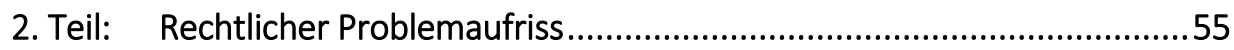

1. Kapitel: Das Sonderstatusverhältnis Schule .....................................55

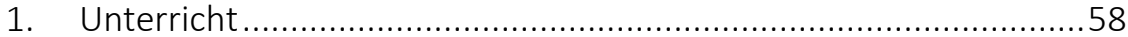

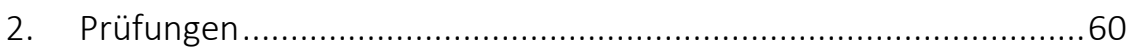

2. Kapitel: Gleichheit und Ungleichheit von Schülern und Schülerinnen in Prüfungen ............................................................61

1. Personenbezogenes Kriterium: Behinderung .............................63

2. Personenbezogenes Kriterium: Schüler und Schülerinnen mit Schwierigkeiten ..............................................................63

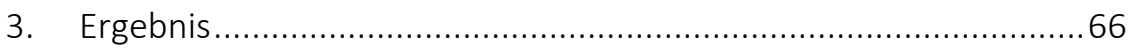

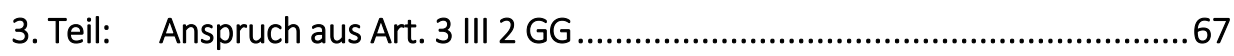

1. Kapitel: Verhältnis von Art. 3 III 2 GG und Art. 3 I GG ........................67

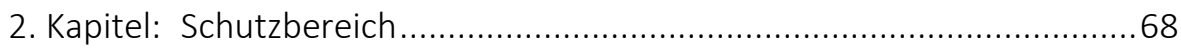

1. Der verfassungsrechtliche Begriff Behinderung ..........................68

1.1. Vorrang der Verfassung .........................................69

1.2. Wandelbarkeit des verfassungsrechtlichen Begriffs „Behinderung"

1.3. Völkerrechtsfreundliche Auslegung des Begriffs Behinderung ................................................................... 73

1.4. Grammatische Auslegung ...................................... 76

1.5. Historische Auslegung .......................................... 81

1.6. Systematische Auslegung .......................................... 82 


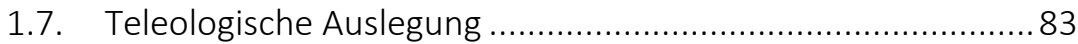

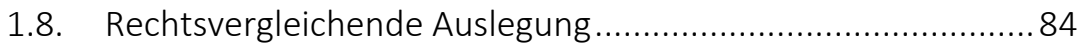

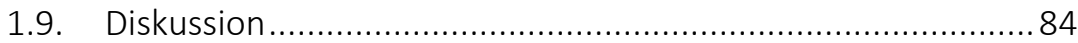

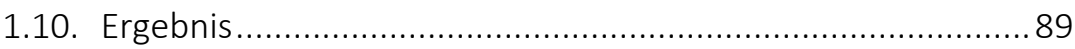

2. Qualifikation von Legasthenie/Dyskalkulie als Behinderung

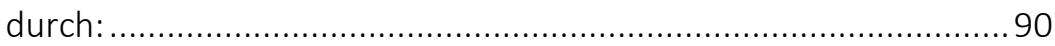

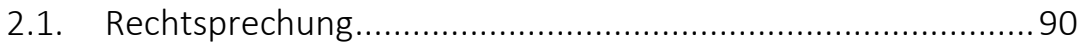

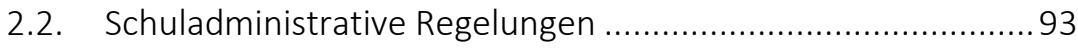

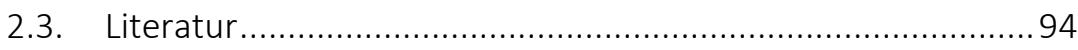

3. Der Behinderungsbegriff im Einzelnen und seine Anwendung

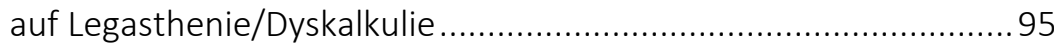

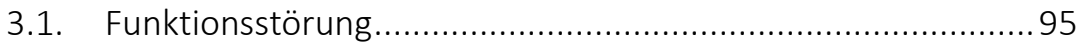

3.1.1. Medizinische Diagnostik als Grundlage ............................96

3.1.2. Jede Art der Funktionsstörung? ...................................... 97

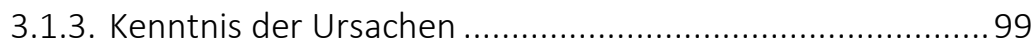

3.1.4. Defizit der medizinischen Diagnostik das Diskrepanzkriterium ............................................... 100

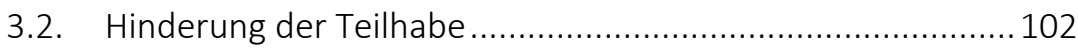

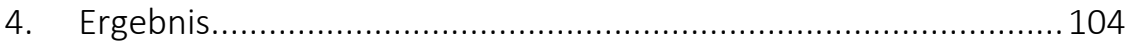

5. „Inflation“ der Behinderungen ................................................ 104

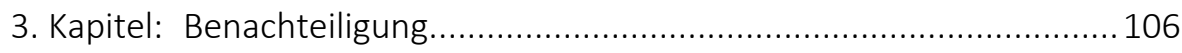

1. Völkerrechtsfreundliche Auslegung des Begriffs Benachteiligung 107

2. Objektive oder subjektive Bestimmung des Eingriffs ....................108

3. Benachteiligung durch Gleichbehandlung? ................................114

3.1. Benachteiligung durch Gleichbehandlung bezüglich äußerer Prüfungsbedingungen .......................................... 117

3.2. Benachteiligung durch Gleichbehandlung bezüglich inhaltlicher Prüfungsanforderungen ................................... 119

3.2.1. Benachteiligung durch Noten ....................................... 119

3.2.2. Benachteiligung durch Leistungsstandards .................... 122

3.3. Mittelbare Folgen als grundrechtsrelevanter Nachteil..........123

3.4. Ausschluss von Entfaltungsmöglichkeiten ............................ 124

4. Abgrenzung der Benachteiligung von der Bevorzugung nach

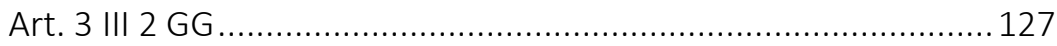

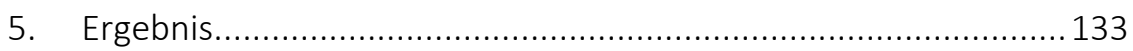




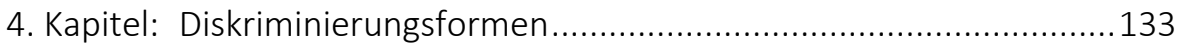

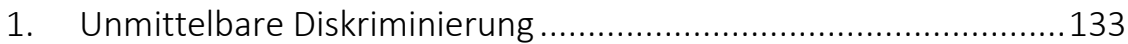

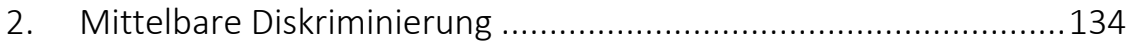

3. Diskriminierung nur bei überwiegender Betroffenheit? ..............138

4. Versagung angemessener Vorkehrungen.....................................140

5. Kausalität und/oder Konnexität...............................................143

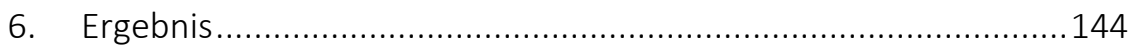

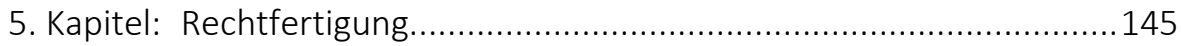

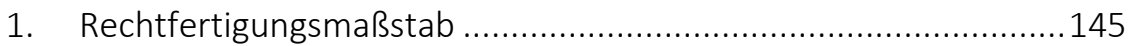

2. Gleichbehandlung bezüglich der äußeren Prüfungsbedingungen.148

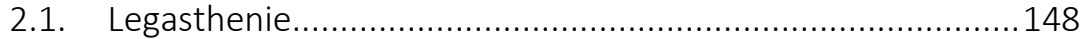

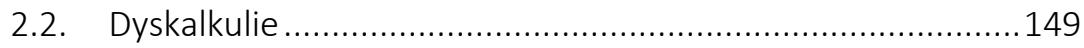

3. Gleichbehandlung bezüglich der inhaltlichen

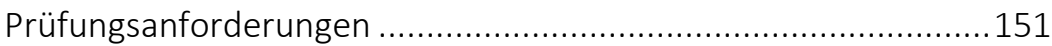

3.1. Zwingende Gründe aus der Behinderung selbst ...................152

3.2. Zwingender Grund: persönlichkeitsbedingte Eigenschaften, die die Leistungsfähigkeit prägen .......................................152

3.3. Art. 7 I GG: Sicherung von Bildungsstandards und Vergleichbarkeit der Schulabschlüsse.

3.3.1. Zulässigkeit von Standards und standardisierten

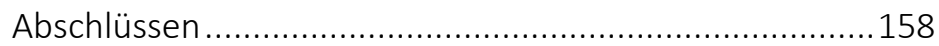

3.3.2. Abwägung zwischen Art. 7 I GG und Art. 3 III 2 GG ..........160

3.3.3. Vorrang von Art. 7 I GG bei Fehlen zwingend erforderlicher Fähigkeiten

3.3.4. Vorrang von Art. 7 I GG wegen Fehlen oder Schwächen in den Kernkompetenzen ...............................................168

3.3.4.1. Bewertung von Diktaten ..................................169

3.3.4.2. Bewertung des Faches Mathematik..................169

3.4. Grundrechte anderer Schüler ...........................................171

3.4.1. Nicht-Bewertung der Rechtschreibung und/oder des Rechnens

3.4.2. Ausschluss unqualifizierter Schüler und Schülerinnen aus einer Schulart

3.5. Pädagogische Aspekte speziell bei Legasthenie/Dyskalkulie..174 6. Kapitel: Ergebnis. 


\section{Teil: Anspruch aus dem Grundsatz der Chancengleichheit aus}

Art. 3 I GG i.V.m. Art. 2 I und 12 I GG............................................ 177

1. Kapitel: Konkretisierung des Grundsatzes der Chancengleichheit ........177

2. Kapitel: Grenzen des Grundsatzes der Chancengleichheit...................178

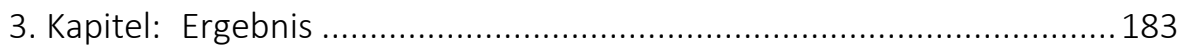

5. Teil: Nachteilsausgleich und Förderung ................................................ 185

1. Kapitel: Umfang des Nachteilsausgleichs .......................................... 185

1. Veränderungen der äußerer Bedingungen.................................186

2. Veränderungen der inhaltlichen Anforderungen ......................... 187

3. Maß und Grenze des Nachteilsausgleichs .................................. 187

3.1. Ausgleich nur bei schwerer Betroffenheit? ......................... 188

3.2. Ausgleich nur bei wesentlichen Auswirkungen?.................... 188

3.3. Ausgleich nur schulformbezogen und für begrenzte Dauer? 189

3.4. Berücksichtigung bei durchschnittlichen Noten? .................190

3.5. Ausgleich bei erfolgreichem Schulbesuch? .......................... 191

3.6. Legasthenie: zurückhaltende Bewertung der Rechtschreibung?

3.7. Legasthenie: Begrenzung des Ausgleichs auf bestimmte

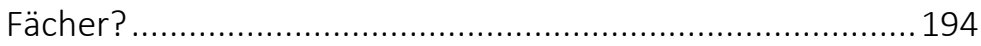

3.8. Dyskalkulie: Verzicht auf die Zulassungsnote Mathematik....197

3.9. Verhältnismäßigkeit des Nachteilsausgleichs ....................... 198

2. Kapitel: Verhältnis Nachteilsausgleich und Förderung.......................... 199

1. Vorrang des Nachteilsausgleichs vor Nicht-Bewertung von

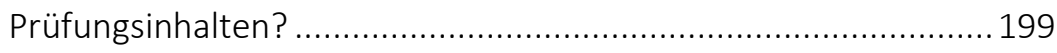

2. Bindung von Nachteilsausgleich an die Teilnahme an

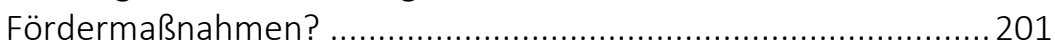

2.1. Pflicht zur Teilnahme an Förderung ...................................202

2.2. Entzug des Nachteilsausgleichs wegen fehlender

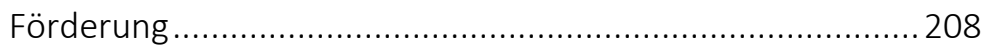

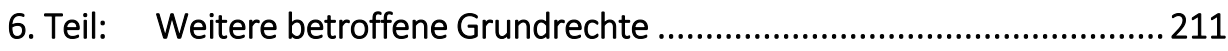

1. Kapitel: Anspruch aus Art. 2 I GG und 12 I GG ..................................211

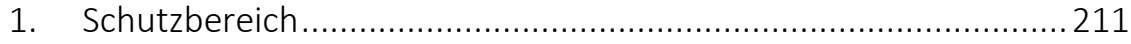

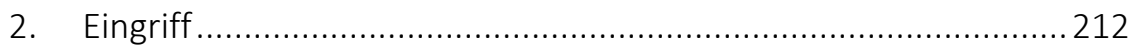

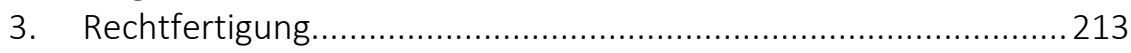

3.1. Verhältnismäßigkeit von Prüfungen ...................................2214 
3.1.1. Legitimer Zweck.....................................................214

3.1.2. Geeignetheit, Erforderlichkeit, Angemessenheit.............215

3.2. Verhältnismäßigkeit der Nicht-Berücksichtigung von Legasthenie/Dyskalkulie .....................................................219

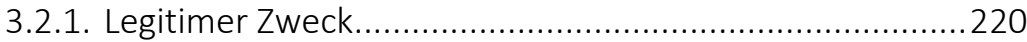

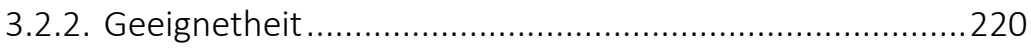

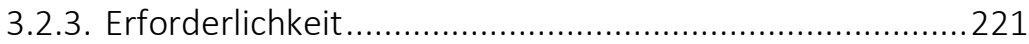

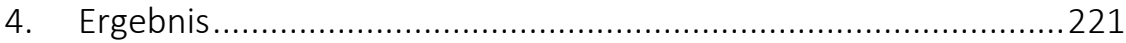

2. Kapitel: Anspruch aus Art. 2 I und 6 || GG - Schulpflicht .....................222

7. Teil: Anspruch aus Völker- und Europarecht ...........................................227

1. Kapitel: Anspruch aus Art. 24 BRK - Recht auf Bildung ........................227

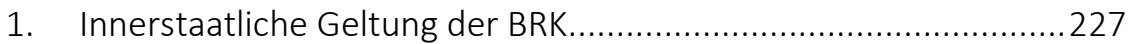

2. Unmittelbare Anwendbarkeit der BRK …..................................229

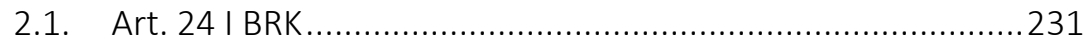

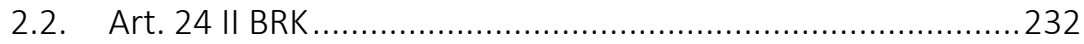

3. Völkerrechtliche Auslegung der Schulgesetze .............................233

4. Vorgaben des Art. 24 i.V.m. 5 BRK..............................................233

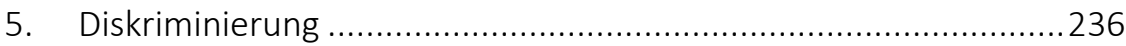

6. Rechtfertigung, Schranken, Verhältnismäßigkeit .........................237

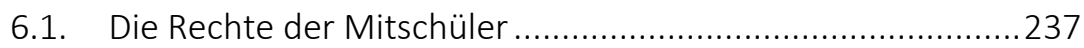

6.2. Kindeswohl des Kindes mit Behinderung .............................238

6.3. Vorbehalt der unverhältnismäßigen oder unbilligen

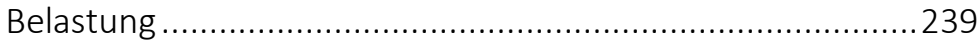

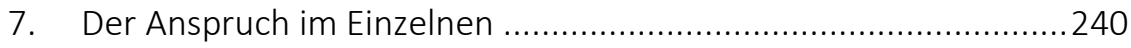

2. Kapitel: Weitere Völker- und Europarechtliche Regelungen .................241

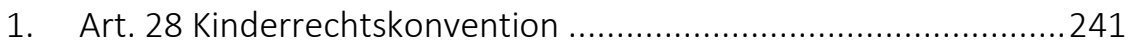

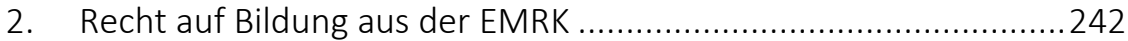

3. Rechte aus der Europäischen Grundrechtecharta.......................243

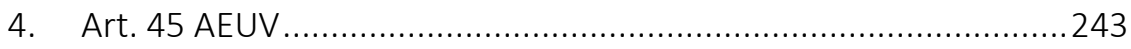

8. Teil: Abwehrrecht, Leistungsrecht, Teilhaberecht ...................................245

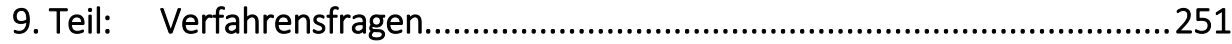

1. Kapitel: Grundentscheidung: Anerkennung von Legasthenie und

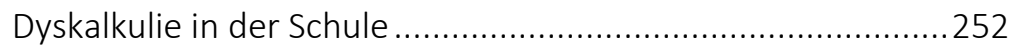

1. Verwaltungsakt: Anerkennung einer Behinderung .....................253 


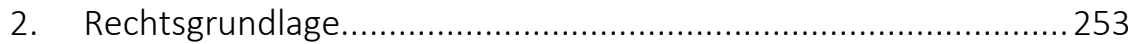

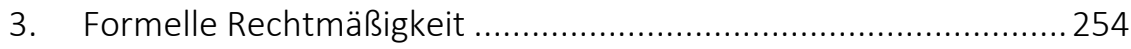

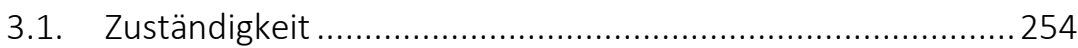

3.2. Berücksichtigung auf Antrag oder von Amts wegen? ............255

3.3. Diagnostik der Legasthenie/Dyskalkulie ..............................256

3.3.1. Relativierung der Diagnostik durch pädagogische

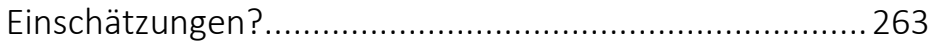

3.3.2. Inhaltliche Überprüfung der Diagnose durch die Schule? 265

3.3.3. Verhinderung von Gefälligkeitsgutachten? .....................267

3.3.4. Überprüfung der Diagnosen durch die Schulen? ............. 269

3.3.5. Gleichbehandlung aller Behinderungen .......................... 271

3.3.6. Geeignete Fachkräfte für die Diagnostik? .......................271

3.3.7. Feststellung nur durch eine von der Schule vorgegebene Fachkraft? ............................................... 274

3.4. Nachweis der Legasthenie/Dyskalkulie...............................2275

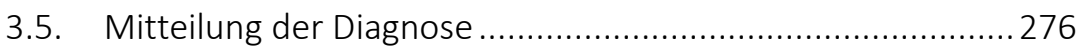

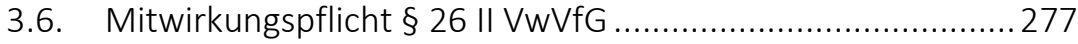

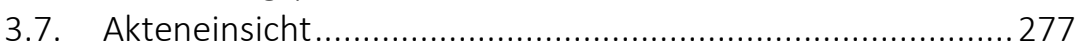

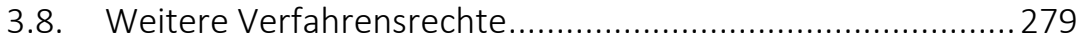

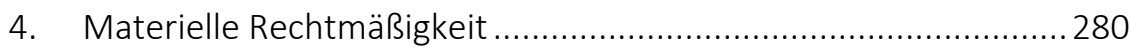

4.1. Grundentscheidung: Gebundener VA oder

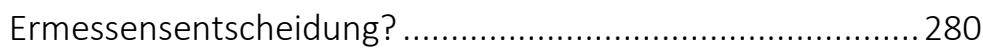

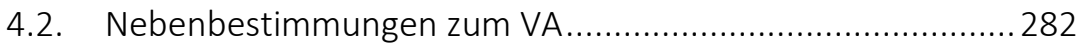

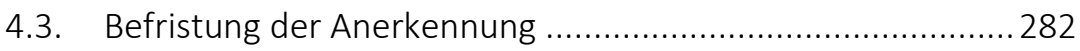

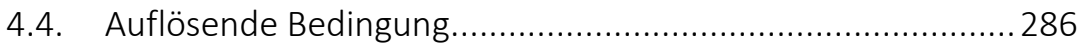

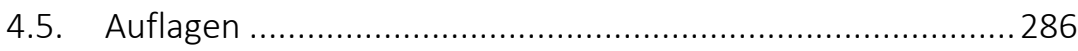

2. Kapitel: Ausgestaltung des Nachteilsausgleichs .................................287

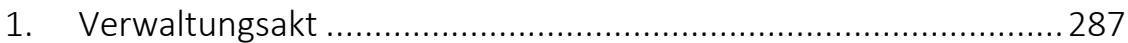

2. Gebundene Entscheidung oder Ermessensentscheidung .............287

3. Ermessen, Ermessensgrenzen, Ermessensfehler .........................289

4. Zeitpunkt der Ermessensentscheidung ....................................... 292

5. Zuständigkeit für die Festlegung ..............................................292

6. Informationspflicht gegenüber den Eltern .................................. 295

3. Kapitel: Verzicht der Betroffenen auf Berücksichtigung von Legasthenie und Dyskalkulie ..................................................295

1. Verzicht auf Berücksichtigung der Behinderung um die Zeugnisbemerkung zu vermeiden 
2. Verzicht auf Berücksichtigung der Behinderung durch NichtGeltendmachung? ...................................................... 301

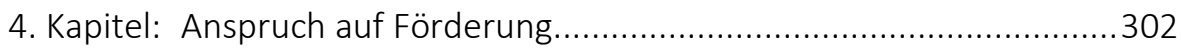

1. Anspruch auf individuelle, legasthenie-/dyskalkuliespezifische Förderung ........................................................................... 302

2. Anspruch auf spezifische Förderung aufgrund der Schulpflicht ....303

3. Zuständigkeit der Schule oder anderer Institutionen? ..................305

10. Teil: Datenschutz und Zeugnisbemerkung..............................................309

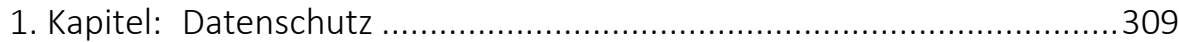

1. Datenschutz im Verfahren der Grundentscheidung und der Festlegung des Nachteilsausgleichs....

1.1. Bekanntgabe der Ergebnisse der einzelnen Achsen, insbesondere der Achsen 2 und 3 des multiaxialen Klassifikationsschema (MAS)

1.2. Schutz der Daten der Eltern.......................................... 314

1.3. Schutz der Daten der Geschwister ................................315

2. Datenschutz im schulischen Alltag .........................................315

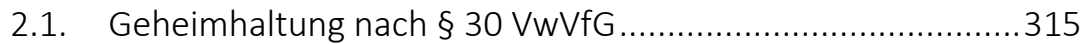

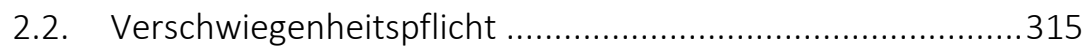

2.3. Entbindung von der Schweigepflicht ................................316

2.4. Umgang mit den Befundberichten ....................................316

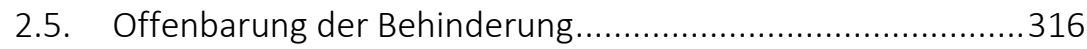

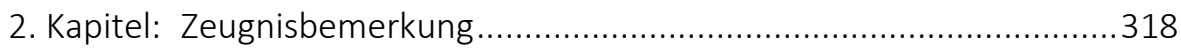

1. Unzulässigkeit bei Nachteilsausgleich .....................................319

2. Zulässigkeit zur Dokumentation der Abweichung von Leistungsstandards ............................................................320

2.1. Eingriff in Art. 3 III 2 GG ...................................... 320

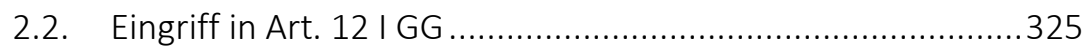

2.3. Eingriff in Art. 2 I GG durch Benennung der Legasthenie ......326

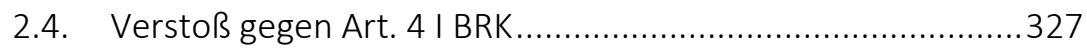

2.5. Verstoß gegen Art. 7 II BRK, Art. 3 KRK, Art. 24 II

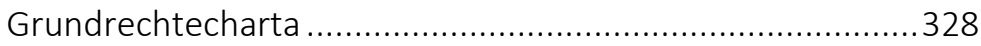

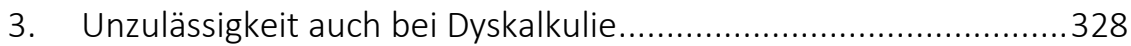

4. Besondere Auswirkungen im Bewerbungsverfahren ...................329 
11. Teil: Amtshaftung aus $\S 839$ BGB i.V.m. Art. $34 \mathrm{GG}$ 333

1. Kapitel: Amtspflichten. 333

1. Amtspflicht zur Feststellung der Legasthenie/Dyskalkulie 334

2. Amtspflicht zur legasthenie-/dyskalkuliespezifischen Förderung.. 336

3. Amtspflicht zur Berücksichtigung der Legasthenie/Dyskalkulie ....336

4. Pädagogische Freiheit der Lehrkräfte als Grenze?

5. Amtspflicht der Schulbehörden auf ordnungsgemäße Organisation

2. Kapitel: Weitere Voraussetzungen der Amtshaftung...

12. Teil: Rechtsschutz

1. Kapitel: Rechtsschutz im Verfahren der Anerkennung der Behinderung und bei schulischen Prüfungen.

2. Kapitel: Rügepflichten der Eltern/des Schülers bei Nicht-Beachtung der Behinderung

3. Kapitel: Rechtsfolgen rechtswidriger Prüfungen..................................353

13. Teil: Notwendigkeit gesetzlicher Regelungen..........................................359

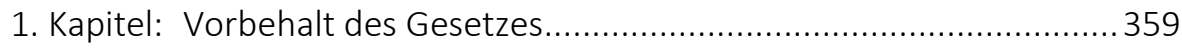

2. Kapitel: Mindestumfang gesetzlicher Regelungen ..............................362

3. Kapitel: Rechtsverordnung oder Verwaltungsvorschrift? ......................364

4. Kapitel: Ansprüche bis zum Erlass gesetzlicher Regelungen .................365

14. Teil: Umgang mit Legasthenie/Dyskalkulie in der EU...............................369

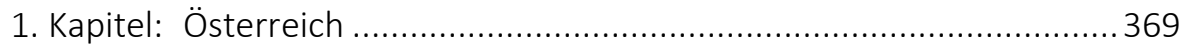

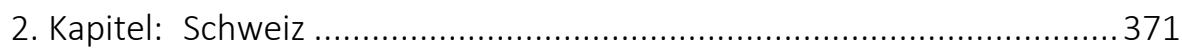

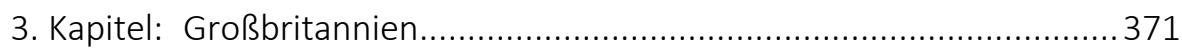

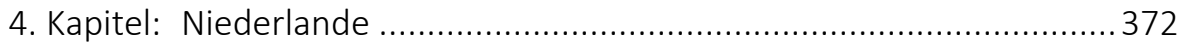

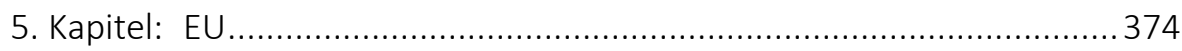

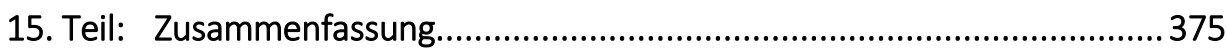


Anhang 1: Glossar medizinischer, psychologischer und pädagogischer

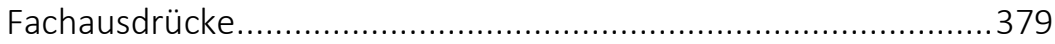

Anhang 2: Beschlüsse und Empfehlungen der Kultusministerkonferenz .......381 Anhang 3: Schulrechtliche Vorschriften der Bundesländer (außer Gesetze)..385

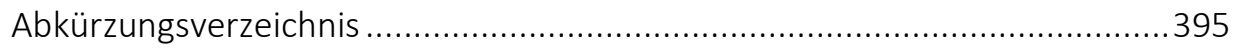

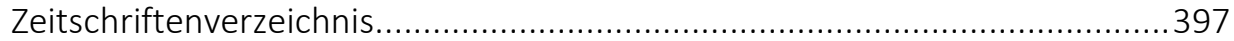

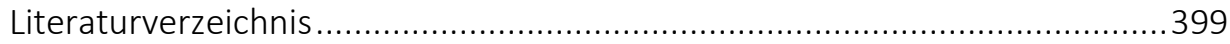




\section{Vorwort}

Die vorliegende Arbeit ist das Ergebnis einer langen Beschäftigung mit dem Thema Legasthenie. Einige Jahre lang habe ich mich in Selbsthilfegruppen und im Bundesverband Legasthenie und Dyskalkulie e.V. engagiert und dabei viel über die Legasthenie, das Leid der Kinder, den unterschiedlichen Umgang damit an Schulen und durch die Kultusministerien sowie über die Komplexität des bundesdeutschen Bildungssystems und die schwierigen sozialrechtlichen Fragen zur Finanzierung von Therapien gelernt. Besonders beschäftigt hat mich die Tatsache, dass die Frage des Umgangs mit der Legasthenie in der Schule fast ausschließlich von Pädagogen, Psychologen, Psychiatern und vielfältigen Therapeuten geführt wird. Juristen, vor allem Schulrechtler, beteiligten sich kaum an der Diskussion. Das änderte sich erst durch das Gutachten von Prof. Dr. Christine Langenfeld, die sich als erste überhaupt mit verfassungsrechtlichen Fragen zur Berücksichtigung der Legasthenie beschäftigt hat. ${ }^{1}$

Nachdem ich die ehrenamtliche Arbeit für den Selbsthilfeverband aus beruflichen Gründen beendet hatte, entschloss ich mich, die juristischen Fragen zur Berücksichtigung von Legasthenie und Dyskalkulie grundlegend aufzuarbeiten. Meine Erfahrungen mit betroffenen Kindern und aus dem Selbsthilfebereich eröffneten mir dabei einen ganz besonderen Zugang, den die Behindertenbewegung unter das Motto fasst: "Nothing about us without us." Nur mit Betroffenheit oder intensiver Erfahrung lassen sich im Diskriminierungsrecht Stolpersteine und Probleme erkennen und nachvollziehen. Allerdings war und ist mir klar, dass die rechtliche Bewertung nicht aus reiner Betroffenheit erfolgen kann und darf. Ausgangspunkte dieser Arbeit sind daher zunächst die medizinischen Grundlagen der Diagnostik, die pädagogischen Grundfragen und die sehr kontroverse Diskussion im pädagogisch-psychologisch-psychiatrischen Bereich, bevor ich die Thematik aus verfassungsrechtlicher Perspektive betrachte und aufarbeite. Einen besonderen Impuls hat die Arbeit durch die UN-Behindertenrechtskonvention erhalten, die den Bildungsbereich in einer Art und Weise beeinflusst, wie dies bei der Ratifizierung der UN-Behindertenrechtskonvention nicht vorhergesehen wurde.

Der Sonderberichterstatter für das Recht auf Bildung der UN, Vernor Muñoz, hat 2006 in seinem Bericht über das deutsche Bildungssystem ausgeführt: "Die Gleichheit der Bildungschancen und die individuelle Unterstützung für jeden einzelnen Schüler werden als Qualitätsmerkmal des Bildungswesens an-

Langenfeld, RdJB 2007, S. $211 \mathrm{ff}$ 
gesehen. Die aktuelle Tendenz im Bildungswesen geht jedoch auch dahin, die Frage der Qualität über die sozialen [und individuellen] Unterschiede zwischen den Schülern und den Mangel an Bildungschancen zu stellen. ${ }^{2}$ Er konkretisiert dies dahin: „Menschen, die ohne Behinderung leben, scheinen davon auszugehen, dass sie das Recht haben, die Bedingungen festzulegen, unter denen Menschen mit Behinderungen zu lernen haben. ${ }^{\prime 3}$ Die Bundesregierung rekurriert in ihrem Nationalen Aktionsplan zur UN-Behindertenrechtskonvention ebenfalls auf die Bedingungen und formuliert pointiert: „Behinderung ist nicht heilbar. Sie ist integraler Bestandteil der Persönlichkeit behinderter Menschen und verdient Respekt. Behindernde Strukturen und behinderndes Verhalten aber sind heilbar. ${ }^{4}$

In dieser Arbeit habe ich das Spannungsgefüge zwischen Behinderung, den Bedingungen, unter denen Schüler und Schülerinnen mit Legasthenie/Dyskalkulie zu lernen haben und behindernden Strukturen untersucht und bin vor allem der Frage nachgegangen, in welchem Umfang das Grundgesetz eine Berücksichtigung der Behinderung im Schulbereich und eine Veränderung allgemeiner Bildungsstandards für Schüler und Schülerinnen mit Legasthenie/Dyskalkulie fordert.

Besonders wichtig war mir der große schulpraktische Teil, in dem ich mich mit den Fragen beschäftige, die im Schulalltag zu beantworten sind und die in Beratungsgesprächen sowohl von Eltern als auch von Lehrern immer wieder gestellt werden.

Die Arbeit wurde im Sommersemester 2012 von der Juristischen Fakultät der Georg-August-Universität Göttingen als Dissertation angenommen. Sie ist hinsichtlich der Rechtsprechung und der landesrechtlichen Regelungen auf dem Stand von Dezember 2012, im Einzelfall Frühjahr 2013, die Literatur ist auf dem Stand von September 2011.

Ich bedanke mich bei Frau Prof. Dr. Christine Langenfeld, dass sie diese Arbeit zu einem sehr strittigen Thema betreut und mich sehr darin unterstützt hat. Außerdem bedanke ich mich bei Herrn Prof. Dr. Thomas Mann für die rasche Erstellung des Zweitgutachtens.

Ganz besonders verpflichtet bin ich Herrn Prof. Dr. Gerd Schulte-Körne, Klinik und Poliklinik für Kinder- und Jugendpsychiatrie, Psychotherapie und Psychosomatik der Ludwig-Maximilians-Universität, München, den ich bezüglich der medizinischen Grundlagen befragen konnte und der freundlicherweise die medizinischen Passagen dieser Arbeit überprüft hat.

\footnotetext{
2 Muñoz, Report Special Rapporteuer on the right to education, Rn. 66

3 Muñoz, Bildung ist ein Recht

4 Bundesregierung, Nationaler Aktionsplan zur UN-BRK, S. 21
} 
Sehr wichtig war die Zusammenarbeit mit meinem Lektor Thomas Kupka, der mir ein sehr kritischer, schwer zu überzeugender, aber immer konstruktiver und optimistischer Partner gewesen ist.

Viele rechtliche Diskussionen habe ich mit Rechtsanwältin Annette Heinisch und Rechtsanwältin Heike Bickel geführt. Sie haben diese Arbeit über die Jahre intensiv begleitet und viele der unveröffentlichten Urteile beigesteuert. Vertiefte Einblicke in das Behindertenrecht habe ich durch Prof. Dr. Theresia Degener und in das Völkerrecht durch Prof. Dr. Rudolf Geiger erhalten. Ihnen allen danke ich für den interessanten und regen Gedankenaustausch.

Sehr gefreut habe ich mich darüber, dass der Göttinger Universitätsverlag sofort meinem Anliegen entsprochen hat, diese Arbeit nicht in der an sich üblichen Schriftart "Garamond", sondern in der für Legastheniker besser lesbaren Schriftart "Calibri" zu drucken. Die Druckvorlage hat Frau Annett Eichstaedt sehr zuverlässig und zügig erarbeitet.

Die Arbeit habe ich neben meiner beruflichen Tätigkeit, meinen familiären Aufgaben und dem Schulbesuch meiner Kinder geschrieben. Daher gilt mein ganz großer Dank meinem Mann und meinen Kindern, die mir das ermöglicht, meine Arbeitsphasen akzeptiert und mich durch so manches Tief getragen haben. Ich widme deshalb diese Arbeit meinem Mann, der ein unerschütterliches Vertrauen darin hatte, dass ich diese Aufgabe meistern würde, und meinen vier Kindern, die meine Beschäftigung mit der Thematik mit starkem Gerechtigkeitssinn nicht nur begleitet, sondern für unbedingt notwendig gehalten haben.

Juni 2013

Gabriele Marwege 



\section{Einleitung}

Fehlerfrei lesen und schreiben zu können, ist Voraussetzung für die Kommunikation in technologisch hochentwickelten Ländern. ${ }^{5}$ Lesen, Schreiben und Rechnen sind Grundfertigkeiten unserer heutigen Bildungsgesellschaft und grundlegende Bereiche der Schulbildung. Es ist die primäre Aufgabe der Schule, insbesondere der Grundschule, Kindern diese Fertigkeiten beizubringen. Kinder, die in den Grundfertigkeiten Lesen, Rechtschreibung und Rechnen massive Schwierigkeiten haben oder sogar versagen, werden verspottet ${ }^{6}$, als minderbegabt, weniger intelligent, faul ${ }^{7}$, nachlässig ${ }^{8}$, unkonzentriert ${ }^{9}$ und nicht geeignet für ein Studium angesehen. Insbesondere die Fähigkeit richtig zu schreiben, wird im schulischen Bereich ${ }^{10}$ und von der Rechtsprechung ${ }^{11}$ als Indikator für Intelligenz, Sorgfalt und Studienbefähigung angesehen. Je besser die Rechtschreibleistung eines Schülers, desto besser ist die Deutschnote und desto höher ist die Chance auf eine Bildungsempfehlung für eine höhere Schule. ${ }^{12}$ Schüler mit besserer Rechtschreibung erreichen danach höhere Bildungsabschlüsse als Schüler mit schwächerer Rechtschreibung, obwohl letztere über mindestens ebenso gute intellektuelle Fähigkeiten und Begabungen verfügen mögen. ${ }^{13}$

Schwierigkeiten im Lesen, Schreiben und Rechnen können sich aus vielfältigen Gründen ergeben, etwa weil Kinder nicht ausreichend beschult werden,

5 Eggers/Fegert/Resch, Psychiatrie, S. 762; s. auch LSG Bayern, Urteil v. 23.3.2006, Az.: L 4 KR 279/09, JAmt, 2006, S. 314 ff, 315, das eine Behinderung bejaht, wenn die Teilhabe an diesen selbstverständlichen Kommunikationsformen beeinträchtigt ist.

6 Eggers/Fegert/Resch, Psychiatrie, S. 762

7 So bereits Brockhaus, in: Enzyklopädie, "Legasthenie“

8 Verlautbarung des KM Baden-Württemberg von 1979 zitiert nach Vanselow/DummerSmoch, Vernachlässigung individueller Begabungsstrukturen, S. 73

9 VG Darmstadt, Urteil v. 28. 4. 2009, Az.: 7 K 687/08.DA (3), S. 17 (nicht veröffentlicht)

10 Jägel, W.-D.; Übungs- und Prüfungsdiktate zur Rechtschreibung und Zeichensetzung, 1966, zitiert nach Vanselow/Dummer-Smoch, Vernachlässigung individueller Begabungsstrukturen, S. 69; Sieburg, Friedrich zitiert nach Vanselow/Dummer-Smoch, Vernachlässigung individueller Begabungsstrukturen, S. 69; Tulzer, Deutsch am Gymnasium, S. 102

11 VG Schleswig, Urteil v. 10.6.2009, Az.: 9 A 208/08, BeckRS 2009 38123, S. 10

12 Roos/Schöler, Schriftspracherwerb, S. 129, 132

13 Esser/Schmidt, Zschr. klin. Psychol. Psychiatr. Psychother 1993, S. 100 ff; Schulte-Körne, in: Legasthenie und Dyskalkulie 2007, Vorwort 
weil sie minderbegabt sind und ihnen deshalb die kognitiven Fähigkeiten für diese Fertigkeiten fehlen, weil sie die deutsche Sprache nicht ausreichend beherrschen oder eben weil sie Legastheniker oder Dyskalkuliker sind.

Sind Legasthenie und Dyskalkulie individuelle Einzelschicksale und ist eine dadurch beeinträchtigte schulische oder gesundheitliche Entwicklung, wie das OLG Celle urteilt, „als bedauerliche und schicksalhafte Kindheitsentwicklung des Schülers" anzusehen, die ,in erster Linie in seiner Person begründet ist und nicht auf ... Versäumnissen von Dritten beruht" ${ }^{14}$ Oder hat die Schule, wie das LSG Niedersachsen-Bremen ausführt, eine Rechtspflicht zur Berücksichtigung der Legasthenie/Dyskalkulie in der Schule und auch zur Abwendung eines persönlichen Schadens, vor allem eines Gesundheitsschadens? ${ }^{15}$ Müssen die Schulen auf Schwierigkeiten aufgrund von Legasthenie/Dyskalkulie eingehen, wie dies die Eltern der betroffenen Kinder und ihre Selbsthilfeorganisation fordern $?^{16}$ Oder handelt es sich, wie bisweilen von der Dyskalkulie behauptet, um eine Modeerscheinung ? $^{17}$ Und wenn die Schulen darauf eingehen müssen, in welchem Umfang und in welchen Verfahren müsste das geschehen?

Diesen Problemen widmet sich die vorliegende Arbeit. Sie geht der Frage nach, ob es verfassungsrechtliche oder gar völkerrechtliche Ansprüche der Schüler und Schülerinnen mit Legasthenie und Dyskalkulie gibt, die betreffenden Störungen bei der Zuweisung zu bestimmten Schularten, bei Prüfungen und bei Schulabschlussprüfungen zu berücksichtigen und, wenn dies zu bejahen ist, in welchem Umfang und in welchen Verfahren die Berücksichtigung erfolgen muss. Die Arbeit beschäftigt sich nur mit den Rechten von Schülern und Schülerinnen mit Legasthenie und/oder Dyskalkulie. Da beide Störungen ähnlich gelagert sind, werde ich im Folgenden zumeist über die Legasthenie sprechen. Legasthenie ist im Schulbereich bekannter und die Berücksichtigung umfassender geregelt, als die der Dyskalkulie. Außerdem werde ich mich vor allem auf das Abitur beziehen, weil es am umfassendsten rechtlich geregelt ist und die Berücksichtigung von Legasthenie/Dyskalkulie in diesem Bereich am umstrittensten ist. Meine Ausführungen gelten jedoch, soweit nicht ausdrücklich differenziert wird, generell für beide Störungen und sind auch auf alle Schulabschlüsse (z.B. Hauptschulabschluss, Mittlerer Schulabschluss, Abschluss an Fachoberschulen, Abschluss an berufsbildenden Schulen) übertragbar.

14 OLG Celle, Urteil v. 4.11.2008, Az.: 16 U 70/07, S. 13 (nicht veröffentlicht), aufgehoben durch Kammerbeschluss des BVerfG, Az.: 1 BvR 1711/09, v. 21.11.2012, juris

15 LSG Niedersachsen-Bremen, Urteil v. 14.1.2009, Az.: L 9 U 129/06 juris; Revision vom BSG zurückgewiesen, Urteil v. 27.4.2010; Az.: B 2 U 13/09, juris; Verfassungsbeschwerde dagegen nicht zur Entscheidung angenommen: BVerfG, Beschluss v. 8.6.2012; Az.: 1 BvR 2853/10, http://www.bverfg.de/entscheidungen/rk20120608_1bvr285310.html

16 Bundesverband Legasthenie und Dyskalkulie e.V.: http://www.bvl-legasthenie.de/schule/ schulfoerderunglegas

17 So Valtin, in: LRS und Legasthenie, S. 62 
Es ist nicht Ziel dieser rechtswissenschaftlichen Arbeit, die Schule zu reformieren und zu untersuchen, inwieweit bestehende Leistungs- und Beurteilungskriterien allgemein angemessen $\operatorname{sind}^{18}$ oder ob die bestehenden Systeme politisch und volkswirtschaftlich sinnvoll sind. ${ }^{19}$ Dies sind politische Fragestellungen und Entscheidungen. Inhalt dieser Arbeit ist es, auf der Grundlage des geltenden Rechts, insbesondere des Verfassungsrechts, zu prüfen, welche Rechte Schüler und Schülerinnen mit Legasthenie/Dyskalkulie im bestehenden System haben und ob das bestehende Schulsystem diese Rechte achtet oder gegebenenfalls verletzt.

Dazu geht die Untersuchung in folgenden Schritten vor:

Der 1. Teil gibt einen tatsächlichen Problemaufriss zu Legasthenie und Dyskalkulie. Hier geht es um die Diagnostik und deren Folgen, die Beschulung der betroffenen Schüler und Schülerinnen, die Auswirkungen von Benotungsvorschriften und um die Grundzüge der Berücksichtigung der Beeinträchtigungen durch die Kultusministerkonferenz (KMK) und die Kultusministerien. Der 2. Teil entwickelt den rechtlichen Problemaufriss zum Sonderstatusverhältnis Schule. Den Schwerpunkt der Untersuchung bildet dann der 3. Teil, in dem Art. 3 III 2 GG unter Berücksichtigung seiner völkerrechtskonformen Auslegung im Hinblick auf die UN-Behindertenrechtskonvention (BRK) geprüft wird. Anschließend wird der Grundsatz der Chancengleichheit (Teil 4) geprüft und es werden Nachteilsausgleich, Bevorzugung und Förderung voneinander abgegrenzt (Teil 5). Es folgt die Prüfung weiterer Ansprüche aus Grundrechten (Teil 6) und aus Völker- und Europarecht (Teil 7) sowie die Umsetzung des Anspruchs auf Berücksichtigung (Teil 8). Im Verfahrensteil (Teil 9) werden umfassend die konkreten Probleme geprüft, die sich in der schulischen Praxis und der diesbezüglichen Rechtsetzung bei der Berücksichtigung von Behinderungen ergeben, angefangen von Fragen der Diagnostik über die Anerkennung der Behinderung in der Schule, die Ausgestaltung von Nachteilsausgleich, den möglichen Verzicht

18 Schlee hat seine massive Kritik an dem Begriff Legasthenie in den 1970er Jahren vor allem damit begründet, dass das Legastheniekonzept nicht dazu beitragen würde, gerade die Frage der Bedeutung der Rechtschreibung für den Schulerfolg aufzuweichen. Schlee, Legasthenieforschung am Ende?, S. 160, s. auch Ruß, Legasthenie und Hochbegabung, S. $101 \mathrm{ff} ;$ umfassend zur Vernachlässigung mathematisch-naturwissenschaftlicher Begabungen im deutschen Schulsystem Vanselow/Dummer-Smoch, Vernachlässigung individueller Begabungsstrukturen

19 Zu volkswirtschaftlichen Folgen von Bildungspolitik s. die Studie der Bertelsmann-Stiftung von 2009 von Wößmann/Piopiunik, Unzureichende Bildung und die Studie des CEPS (Centre for European Policy Studies) 2010, Gros/Sagmeister, Nachkrisenzeit, http://www.ceps.be/book/ nachkrisenzeit-\%E2\%80\%93-und-was-kommt-jetzt. Der Leiter des CEPS, Daniel Gros, führte zu dieser Studie aus: „Laut Studie gibt es im deutschen Bildungssektor zu viele Schulabbrecher und zu wenige Uni-Absolventen. Das wird Deutschland in der nächsten Generation zum Land der Hilfsarbeiter machen. Verknüpft man die Akademikerquote mit den Resultaten der Pisa-Studie, liegt Warschau vor Berlin." 
der Betroffenen auf die Berücksichtigung und den Anspruch auf Förderung. Teil 10 beschäftigt sich mit Fragen des Datenschutzes und der Zulässigkeit von Zeugnisbemerkungen. Anschließend werden Fragen der Amtshaftung (Teil 11) und des Rechtsschutzes (Teil 12) geprüft. Die notwendigen Vorgaben für (künftige) gesetzliche Regelungen werden in Teil 13 erörtert. Teil 14 gibt eine kurze Übersicht über die Rechtslage in einigen europäischen Ländern und Teil 15 enthält abschließend eine knappe Zusammenfassung der Ergebnisse. 


\section{Teil: Tatsächlicher Problemaufriss}

\section{Kapitel: Was sind Legasthenie und Dyskalkulie?}

\section{Begriffsentfaltung}

Der Begriff Legasthenie setzt sich etymologisch zusammen aus dem lateinischen Wort "legere" = "lesen" und dem griechischen Wort ",asthenes" = "kraftlos", "schwach". Die Bezeichnung Legasthenie wird nur im deutschsprachigen Raum verwandt. International wird der Begriff "Dyslexie" benutzt, der auf griechisches Vokabular zurückgeht. ${ }^{20}$ Die griechische Vorsilbe "dys-“ bedeutet "un...", "miss... ", "schwierig", und hat vor allem in der Medizin die Bedeutung: von der Norm abweichend, übel, schlecht, fehlerhafter Zustand. Das Wort "lexis" bedeutet "Wort". Der Begriff "Dyslexie" bezeichnet also eine Schwäche im Erlernen des Lesens und orthografischen Schreibens bei vergleichsweise durchschnittlicher oder sogar guter Allgemeinbegabung des Kindes.

Im allgemeinen Sprachgebrauch werden die Schwierigkeiten beim Erlernen des Lesens, Schreibens und Rechtschreibens meistens kurzgefasst als "LRS" bezeichnet. Die Abkürzung steht für "Lese-Rechtschreibstörung", "Lese-Rechtschreibschwäche", "Lese-Rechtschreibschwierigkeiten“. Häufig wird LRS als Oberbegriff angesehen, unter den auch die Legasthenie zu fassen ist. In den schulrechtlichen Bestimmungen ${ }^{21}$ wird überwiegend der Begriff „,besondere Schwierigkeiten beim Erlernen des Lesens und Schreibens" benutzt. In Schleswig-Holstein und Mecklenburg-Vorpommern werden die Begriffe "Legasthe-

20 S. z.B. British Dyslexia Association: http://www.bdadyslexia.org.uk Auf allen Kontinenten gibt es Dyslexie-Vereinigungen, s. z.B. die Auflistung auf http://www.dyslexiateacher.com/resources1.html, Definition unter: www.thefreedictionary.com/Dyslexia. Auch die Überarbeitung des amerikanischen Diagnosekatalogs Diagnostic and Statistical Manual of Mental Disorders (DSM V) kehrt zur Bezeichnung "Dyslexie“ zurück, nachdem in der Version DSM IV die Bezeichnung noch „Reading Disorder“ lautete, http://www.dsm5.org/ProposedRevisions/Pages/proposedrevision.aspx?rid=84\#

21 Nachweis der schulrechtlichen Vorschriften und ihrer Fundstellen in Anhang 3: Schulrechtliche Vorschriften der Bundesländer 
Tatsächlicher Problemaufriss

nie" und "LRS“ ausdrücklich synonym verwendet ${ }^{22}$, in Bayern dagegen bezeichnen die Begriffe "Legasthenie“ und "LRS" ausdrücklich verschiedene Ausprägungen. ${ }^{23}$ In Berlin werden die Begriffe „besondere Schwierigkeiten“, „gravierende Lese- Rechtschreibstörung", "festgestellte Lese-Rechtschreibstörung ", ${ }^{24}$ und "gravierende Lese- und Rechtschreibstörung gutachterlich bestätigt" verwendet. $^{25}$

Der Begriff Dyskalkulie verbindet etymologisch das griechische "dys..." mit dem lateinischen "calculare" = "mit Rechensteinen rechnen", und bezeichnet Schwierigkeiten im Rechnen. Unter Dyskalkulie versteht man eine Störung des mathematischen Denkens bei Kindern, Jugendlichen und auch Erwachsenen, die dazu führt, dass die Betroffenen den Lernstoff des arithmetischen Grundlagenbereiches (z.B. Vergleich von Mengen, Zahlbegriff, Grundrechenarten, Dezimalsystem,) nicht oder sehr viel schwerer erlernen. ${ }^{26}$ Die Dyskalkulie wird auch bezeichnet als "Rechenstörung" und "Rechenschwäche“. Die schulischen Vorschriften sprechen von "Schwierigkeiten im Rechnen "27 oder von "Lernbeeinträchtigung im mathematischen Bereich (LimB)“. ${ }^{28}$

Die Begriffsvielfalt zeigt bereits, welche Verunsicherung schon bei der Bezeichnung der Problematik besteht und dass insoweit die Gefahr von Missverständnissen oder offenen oder versteckten Dissensen in Diskussionen groß ist. Ich benutze in dieser Arbeit die Begriffe Legasthenie und Dyskalkulie als Bezeichnung für Störungen, die die Anforderungen der medizinischen Diagnostik nach der ICD 10 erfüllen, einer Klassifikation der Weltgesundheitsorganisation, die ich in Kap. 4 dieses Teils genauer erörtern werde.

\section{Legasthenie}

Normalerweise beeinflusst die Intelligenz des Kindes vor allem zu Beginn der Grundschulzeit sowohl die Lese- als auch die Rechtschreibleistung und spielt dann mit der Zeit dafür eine zunehmend geringere Rolle. ${ }^{29}$ Je höher die Intelligenz ist, desto höher sind die Leistungen im Lesen und Rechtschreiben. ${ }^{30}$ Bei der Legasthenie klaffen demgegenüber die Intelligenz und die Leistungen im

\footnotetext{
22 SH Legasthenie-Erlass Nr. 1; MV VV-LRR Nr. 3.1.

23 BY KMBek Legasthenie Ziff. I

24 BE VO-GO § 14 VOGO Berlin

25 BE Sek I-VO § 14

26 Jacobs/Petermann, in: Lehrbuch der klinischen Kinderpsychologie, S. 209

27 BB VV-LRSR Nr. 6 I; HH Förder-Richtlinie Nr. 4; HE VO-GestSchulVerh § 37 I, NI LRR-Erlass Nr. 3.2

28 MV VV-LRR Nr. 3.2

29 Roos/Schöler, Schriftspracherwerb, S. 124, S. 125

30 Roos/Schöler, Schriftspracherwerb, S. 61, S. 90
} 
Lesen und Rechtschreiben signifikant auseinander. Die Legasthenie ist dadurch gekennzeichnet, dass bei ansonsten befriedigenden schulischen Leistungen und mindestens normaler Intelligenz das Erlernen des Lesens erschwert ist und ungewöhnlich viele Rechtschreibfehler auftreten. Bedeutsam an der Definition ist die Diskrepanz zwischen Lese-Rechtschreib-Leistungen und dem übrigen Leistungsniveau im Sinne einer Teilleistungsstörung ${ }^{31}$, worunter man die gestörte Entwicklung nur eines oder einzelner Teilfunktionssysteme des zentralen Nervensystems im Vergleich zu dessen gesamter Leistungsfähigkeit versteht. ${ }^{32}$ Das heißt, dass sich die Leistungsminderung in nur einem Teilbereich zeigt, während die Leistungen ansonsten in der normalen und erwarteten Fähigkeitsspanne liegen.

Legasthenie tritt in allen Schriftsystemen auf. ${ }^{33}$ Sie ist in ihrer Ausprägung im Wesentlichen von der Lauttreue der Orthographie der jeweiligen Sprache abhängig. ${ }^{34}$ Je konsistenter in einer Sprache Laute auch einem Buchstaben entsprechen, desto schneller wird z.B. das lautierende Lesen erlernt. Je inkonsistenter die Buchstaben-Laut-Zuordnung ist, desto komplexer ist der Prozess. Dementsprechend wird es auch schwieriger, die Laute zu verschriftlichen. ${ }^{35}$ Schüler, die eine Sprache, wie z.B. Englisch, erlernen, deren Orthographie wenig lautgetreu und daher sehr komplex ist, weisen deshalb vergleichsweise massivere Defizite im Lesen und Rechtschreiben auf als Schüler, die eine eher lautgetreue und orthographisch konsistentere Sprache, wie z.B. Finnisch, erlernen. ${ }^{36}$ Auf der Grundlage der Diagnostik nach der ICD 10 wird eine Häufigkeit von ca. 4 - $6 \%$ im deutschen Sprachraum errechnet, wobei die Häufigkeit im Grundschulalter höher ist als in späteren Altersstufen. ${ }^{37}$ Bei Legasthenie handelt es sich um eine andauernde Störung, d.h. sie bleibt bis ins Erwachsenenal-

31 Steinhausen, Psychische Störungen bei Kindern und Jugendlichen, S. 161; Warnke/SchulteKörne, in: Therapie psychischer Störungen, S. 153 chung von Aguilar, Mexikanische und deutsche Kinder mit Lese- Rechtschreibstörungen und World Dyslexia Forum 2010, mit Berichten zu den Sprachen Arabisch, Chinesisch, Englisch, Französisch, Russisch und Spanisch, http://www.worlddyslexiaforum.org

Landerl, in: Legasthenie und Dyskalkulie 2011, S. 65 f - so kann im Deutschen der Langvokal /a:/ geschrieben werden als "Wahl“, „Wal“ oder "Aal“, im Englischen kann der Vokal /i:/ geschrieben werden als "lead", ",need" oder "theme“; s. auch Schulte-Körne, Deutsches Ärzteblatt 107 (2010), S. 49 Landerl, in: Legasthenie und Dyskalkulie 2011, S. 65; Plume/Warnke, in: Schule und psychische Störungen, S. 197

37 Plume/Warnke, in: Schule und psychische Störungen, S. 197 f; Steinhausen, Psychische Störungen bei Kindern und Jugendlichen, S. 162 
Tatsächlicher Problemaufriss

ter bestehen. ${ }^{38}$ Zwar lassen sich durch optimale Förderung die Leistungen betroffener Fünftklässler im Rechtschreiben verbessern, sie bleiben aber bis ins Erwachsenenalter deutlich unterhalb des Erwartungswertes. ${ }^{39}$

Die medizinische Forschung ordnet die entsprechenden Störungen vornehmlich neuropsychologischen Defiziten im Hinblick auf linguistische Funktionen, Gedächtnisfunktionen und visuelle Fertigkeiten zu. In der linguistischen Funktion, also der lautsprachlichen Informationsverarbeitung, sind es Probleme bei der akustischen Identifikation und Unterscheidung von Phonemen, der Lautverbindung des Wortschatzes sowie der grammatikalisch-syntaktischen Struktur der Sprache. Im Bereich der Gedächtnisleistungen liegen Probleme bei der Kodierung des Gedächtnismaterials und Defekte im raschen Abrufen von Gedächtnisinformationen respektive in der Anwendung von Gedächtnisstrategien vor. Schließlich ist die visuelle Informationsverarbeitung beeinträchtigt, die zu einer Verlangsamung, einer höheren Fehlerrate und zu defizitärer orthographischer Kodierung in diesem Bereich führen. ${ }^{40}$

Insgesamt setzt sich die Legasthenie also zusammen aus einer Lesestörung und einer Rechtschreibstörung. Die Betroffenen weisen in der Regel beide Störungen auf; es gibt aber auch Betroffene, die "nur" eine Lesestörung oder "nur" eine Rechtschreibstörung haben. ${ }^{41}$

Die Lesestörung zeigt sich in Defiziten hinsichtlich der Lesegenauigkeit, des Lesetempos und des Leseverständnisses. ${ }^{42}$ Betroffene Kinder lesen meist stockend, nicht flüssig, nicht sinnhaft betonend und erheblich verlangsamt. Das Lesen bereitet größte Mühe, so dass sich die Kinder oft nicht mehr auf den Inhalt des Gelesenen konzentrieren können. Sie haben deshalb Schwierigkeiten, das Gelesene wiederzugeben oder daraus Schlüsse zu ziehen. ${ }^{43}$ Im Verlauf der Schulzeit verbessern die meisten Kinder zwar ihre Lesefähigkeit, sie lesen aber meistens nach wie vor deutlich langsamer als ihre Mitschüler. ${ }^{44}$

38 Steinhausen, Psychische Störungen bei Kindern und Jugendlichen, S. 164 f; Klicpera/Schabmann/Gasteiger-Klicpera, Legasthenie, S. 127; Schulte-Körne/Stieglitz, in: Adoleszenzpsychiatrie, S. 477; Warnke/Schulte-Körne, in: Therapie psychischer Störungen, S. 154; Plume/ Warnke, in: Schule und psychische Störungen S. 198

39 Schulte-Körne/Deimel/Jungermann/Remschmidt, Zeitschrift für Kinder- und Jugendpsychiatrie und Psychotherapie 31 (2003), 267 ff; Eggers/Fegert/Resch, Psychiatrie, S. 766; Klicpera/ Schabmann/Gasteiger-Klicpera, Legasthenie, S. 138

Steinhausen, Psychische Störungen bei Kindern und Jugendlichen, S. 162

41 Landerl/Moll/Kristina, Scientific Studies of Reading 2009, S. $359 \mathrm{ff}$

42 Schulte-Körne, Deutsches Ärzteblatt 107 (2010), S. $47 \mathrm{f}$

43 Beispiele bei Warnke/Schulte-Körne, in: Therapie psychischer Störungen, S. 153 f und Steinhausen, Psychische Störungen bei Kindern und Jugendlichen, S. 162

44 Eindringlich werden die Schwierigkeiten von (erwachsenen) Legasthenikern, Bibliotheken zu benutzen, in den Internationalen Richtlinien für das Bibliothekswesen zur Unterstützung von Legasthenikern beschrieben: Richtlinien Bibliothekswesen, Anlage 4 
Die Störung im Rechtschreiben zeigt sich meist zu Beginn der Grundschule durch Defizite in der lautgetreuen Rechtschreibung. Die korrekte Zuordnung von Buchstaben zu Lauten gelingt nicht immer (z.B. d/t), so dass die lautliche Durchgliederung des Wortes unvollständig sein kann. Zudem werden Buchstaben verwechselt (z.B. b/d), vertauscht („Artz" statt "Arzt“), ausgelassen oder hinzugefügt. ${ }^{45}$ Wenn die betroffenen Kinder im Verlauf der Grundschulzeit die lautgetreue Rechtschreibung besser beherrschen, weisen sie im Vergleich zu den Mitschülern Schwierigkeiten bei der Anwendung der orthographischen Regeln auf, machen z.B. Fehler in der Groß- und Kleinschreibung, Dehnungsfehler (,im" statt „ihm“; ,"i" statt „ie"), Fehler in der Konsonantenverdopplung (,," statt "ss") und weitere Regelfehler („schpielen" statt „spielen“, „Forsicht" statt „Vorsicht"). Darüber hinaus fällt eine Fehlerinkonstanz auf, d.h. ein und dasselbe Wort wird immer wieder unterschiedlich geschrieben. So kann auf der gleichen Heftseite ein Wort in einer Zeile richtig und in den nächsten Zeilen falsch geschrieben sein. ${ }^{46}$ Die deutsche Ergänzung zur internationalen GrundschulLese-Untersuchung (IGLU-E) hat gezeigt, dass betroffene Kinder im Laufe der Grundschulzeit in der Rechtschreibung nicht besser, sondern schlechter werden. So schreiben sie z.B. in der 2. Klasse das Wort "Matratze" in vier Formen falsch, in der 4. Klasse haben sie dann aber 12 verschiedene falsche Schreibweisen für das Wort. Je mehr Regeln sie erlernen, desto weniger können sie diese sinnvoll anwenden und desto mehr sind sie generell verunsichert und geradezu „erschlagen“. Es kommt mitunter zu einem "Rechtschreibkollaps“. ${ }^{47}$

Die Vorstellung, dass es "typische" Fehler gibt und keine Legasthenie vorliegt, wenn diese Fehler nicht oder nicht gehäuft vorliegen, ist in der Schule und auch in der Rechtsprechung nach wie vor weit verbreitet. ${ }^{48}$ In der medizinischen und psychologischen Forschung indes hat man den Ansatz, dass es ein spezifisches Fehlerprofil bei Legasthenie gibt, aufgegeben, da er sich nicht beweisen ließ. ${ }^{49}$ "Typische" Fehler, die nur ein Kind mit Legasthenie macht, gibt es $n i c h t^{50}$, vielmehr machen Kinder mit Legasthenie die gleichen Fehler wie Kinder

45 Warnke/Schulte-Körne, in: Therapie psychischer Störungen, S. 154

46 Plume/Warnke, in: Schule und psychische Störungen, S. 199

47 Löffler/Meyer-Schepers/Lischeid, in: Legasthenie und Dyskalkulie 2007, S. $273 \mathrm{ff}$

48 S. VG Darmstadt, Urteil v. 28.4.2009, Az.: 7 K 687/08.DA 3, S. 3 (nicht veröffentlicht): „Der Kl. habe 39 Rechtschreibfehler gemacht. Allerdings handele es sich bei diesen Fehlern nicht um typische Legasthenie-Fehler, sondern um Fehler, wie sie auch von zahlreichen anderen Schülern ... gemacht würden", und S. 14, wo das Gericht prüft, ob der KI. Fehler macht, die in den Leitlinien beispielhaft aufgezählt sind und zu dem Ergebnis kommt, dass der KI. vor allem "dass" und "das" falsch schreibt; anders ausdrücklich SH Legasthenie FAQ S. 18 Steinhausen, Psychische Störungen bei Kindern und Jugendlichen, S. 162 
ohne Legasthenie. ${ }^{51}$ Aus der Fehlerart allein lässt sich daher keine Diagnose stellen, vielmehr ist die Quantität der Fehler ausschlaggebend für die Diagnosestellung. Es ist typisch, dass überdurchschnittlich viele Fehler gemacht werden, so dass betroffene Kinder in Diktaten meist nicht besser als mit den Noten 5 und 6 abschneiden. ${ }^{52}$ Ebenso wenig lässt sich zwischen legastheniebedingten und nicht legastheniebedingten Fehlern unterscheiden. ${ }^{53}$

Schwierigkeiten in der Rechtschreibung wirken sich umfassend auf den schriftlichen Ausdruck aus. Dieser leidet darunter, dass das Kind bei nahezu jedem Wort darüber nachdenken muss, wie es geschrieben wird. ${ }^{54}$ Deshalb schreiben die Kinder beim freien Schreiben von Texten aus Angst vor Rechtschreibfehlern nur kurze Texte und vermeiden anspruchsvolle Wörter, bei deren Schreibung sie unsicher sind.

\section{Dyskalkulie}

Die Rechenstörung ist eine Störung im Erlernen, Verstehen und Anwenden mathematischer Grundlagenkenntnisse. ${ }^{55}$ Sie äußert sich in beständigen Minderleistungen im Lernstoff des arithmetischen Grundlagenbereiches (Mächtigkeitsverständnis, d.h. Vergleich von Mengen; kardinaler Zahlbegriff, d.h. Grundrechenarten und Dezimalsystem). ${ }^{56}$ Die betroffenen Schüler machen mit ihrer subjektiven Logik in systematisierbarer Art und Weise Fehler, die auf begrifflichen Verinnerlichungsproblemen beruhen. Das Verständnis der allgemeinen Logik der Zahlen und der mathematischen Operationen ist bei innen nicht oder nur mangelhaft entwickelt. ${ }^{57}$

Die Rechenschwierigkeiten sind sehr verschiedenartig, je nachdem welche Wahrnehmungsfähigkeiten beeinträchtigt sind: Bei der Zahlensemantik werden

51 Und sie machen 28 Fehler in einem Diktat mit etwa 45 Wörtern, Lenhard/Küspert/Becker, in: Legasthenie, S. 60

52 Plume/Warnke, in: Schule und psychische Störungen, S. 199; Warnke/Schulte-Körne, in: Therapie psychischer Störungen, S. $153 \mathrm{f}$

53 So aber die Vorstellung des VGH Kassel Beschluss v. 8.12.2011, Az.: 7 A 2621/10.Z, juris, Rn. 16 und 33, das dem Schüler dafür auch die Darlegungs- und Beweislast aufbürdet. Zu Beweisschwierigkeiten und den notwendigen Erleichterungen für Menschen mit Behinderungen Aichele/ Althoff, Kommentar zur UN-BRK, Nicht-Diskriminierung, Rn. 27

54 Roos/Schöler, Schriftspracherwerb, S. 159

55 Ausführlich: Jacobs/Petermann, in: Lehrbuch der klinischen Kinderpsychologie; Steinhausen, Psychische Störungen bei Kindern und Jugendlichen, S. 165; Fallbeispiele aus der klinischen Praxis bei Neumärker/Bzufka, in: Rechenstörungen bei Kindern, S. 82 ff; Pixner/Kaufmann, in: Legasthenie und Dyskalkulie 2011; zu den Grundlagen des Rechnens und den Anforderungen an den Erstunterricht s. Wehrmann, in: Legasthenie und Dyskalkulie 2011

56 Jacobs/Petermann, in: Lehrbuch der klinischen Kinderpsychologie, S. 207; Beispiele bei Warnke/Plume/Oehler, in: Therapie psychischer Störungen, S. 163

Wehrmann, in: Legasthenie und Dyskalkulie 2007, S. 333 
Rechenoperationen und die ihnen zugrunde liegenden Konzepte nicht ausreichend verstanden, die Größe einer Menge wird unzureichend erfasst und zu einer anderen Menge in Beziehung gesetzt, der Aufbau gegliederter Zahlenstrahl- oder Zahlenraumvorstellungen und das Überschlagen und Schätzen von Mengen und Rechenergebnissen ist erschwert. Bei der sprachlichen Zahlenverarbeitung, wie dem Erwerb der Zahlwortsequenz und der Zählfertigkeiten sowie dem Speichern von Faktenwissen (Einmaleins), treten Schwierigkeiten auf. ${ }^{58}$ Bei der Dyskalkulie handelt es sich um ein sehr stabiles Störungsbild mit chronischem Verlauf. ${ }^{59}$ Etwa 3 - 6 \% der Schüler, so wird angenommen, sind von Dyskalkulie betroffen. ${ }^{60}$ Während andere Kinder im Laufe der Grundschule dazu übergehen, arithmetische Fakten aus dem Gedächtnis abzurufen, gelingt dies Kindern mit Dyskalkulie nicht. ${ }^{61}$ Sie entwickeln daher ausgeklügelte kompensatorische Strategien, die viel Zeit kosten. ${ }^{62}$

\section{Kombination der Störungen}

Die aufgezeigten Störungen können jeweils einzeln auftreten. Dann werden sie als isolierte Lesestörung, isolierte Rechtschreibstörung und Rechenstörung bezeichnet. ${ }^{63}$ Bei der Legasthenie treten die Probleme im Bereich Lesen und Rechtschreiben in der Regel gemeinsam auf. Legasthenie und Dyskalkulie können ebenfalls gemeinsam auftreten und ein Schüler kann daher in allen Gebieten des Lesens, Schreibens, Rechtschreibens und Rechnens beeinträchtigt sein. $^{64}$

58 Vgl. Hain, Familiendynamik bei Belastungen durch umschriebene Lern- und Leistungsstörungen. S. 54; einen sehr informativen Einblick in die Denkprozesse eines Grundschulkindes mit Dyskalkulie bei den Rechenhausaufgaben gibt der Aufsatz von Hoffmann, Nachmittag eines rechenschwachen Kindes; ein Überblick über die Problemfelder findet sich bei Wieneke, Kriterienkatalog - Rechenschwäche Jacobs/Petermann, in: Lehrbuch der klinischen Kinderpsychologie, S. 210 Warnke/Plume/Oehler, in: Therapie psychischer Störungen, S. 163

61 Ansari, in: Legasthenie und Dyskalkulie 2011, S. 236

62 Pixner/Kaufmann, in: Legasthenie und Dyskalkulie 2011, S. 200, s. auch OVG Weimar, Beschluss v. 17.5.2010, Az.: 1 EO 854/10, juris, Rn. 51; Beispiele bei Wehrmann, in: Legasthenie und Dyskalkulie 2007, S. $253 \mathrm{f}$ und $257 \mathrm{f}$ 


\section{Medizinische Diagnostik}

\subsection{Grundlagen}

Medizin und Psychologie benutzen im Deutschen die Bezeichnungen LeseRechtschreibstörung (synonym Legasthenie) und Rechenstörung (synonym Dyskalkulie). ${ }^{65}$ Die Definition der Störungen ergibt sich aus der "Internationalen Klassifikation der Krankheiten und verwandter Gesundheitsprobleme" $=I C D^{66}$, in ihrer momentan aktuellen Fassung ICD 10 und der maßgeblichen deutschen Übersetzung durch das Deutsche Institut für medizinische Dokumentation und Information $\left(D_{1 M D I^{67}}\right)$. Die ICD-10 ist ein internationales Klassifikationssystem der Weltgesundheitsorganisation der Vereinten Nationen (WHO), ${ }^{68}$ mit dem ein weltweiter Standard für die Klassifikation von medizinischen Diagnosen gesetzt wird. Die ICD 10 ist nach § 295 Abs. 1 SGB V die Grundlage für die Abrechnung von Leistungen im ambulanten und stationären Bereich der gesetzlichen Krankenversicherung.

Die Definitionen für Legasthenie und Dyskalkulie lauten:

\section{F81 Umschriebene Entwicklungsstörungen schulischer Fertigkeiten}

Es handelt sich um Störungen, bei denen die normalen Muster des Fertigkeitserwerbs von frühen Entwicklungsstadien an gestört sind. Dies ist nicht einfach Folge eines Mangels an Gelegenheit zu lernen; es ist auch nicht allein als Folge einer Intelligenzminderung oder irgendeiner erworbenen Hirnschädigung oder -krankheit aufzufassen.

\section{F81.0 Lese- und Rechtschreibstörung}

Das Hauptmerkmal ist eine umschriebene und bedeutsame Beeinträchtigung in der Entwicklung der Lesefertigkeiten, die nicht allein durch das Entwicklungsalter, Visusprobleme oder unangemessene Beschulung erklärbar ist. Das Leseverständnis, die Fähigkeit, gelesene Worte wiederzuerkennen, vorzulesen und Leistungen, für welche Lesefähigkeit nötig ist,

65 Steinhausen, Psychische Störungen bei Kindern und Jugendlichen, S. 161 f, 165; Warnke/ Schulte-Körne, in: Therapie psychischer Störungen, S. 153

66 engl.: International Statistical Classification of Diseases and Relates Health Problems, http://www.who.int/classifications/icd/en

67 http://www.dimdi.de/static/de/index.html, zu den Übersetzungsrechten: http://www.dimdi.de/dynamic/de/klassi/downloadcenter/icd-10-who/\$anhangicdwho.html; in der offiziellen englischen Version der WHO, http://apps.who.int/classifications/apps/icd/icd10online, werden folgende Begriffe benutzt: Specific developmental disorders of scholastic skills; specific reading disorder; specific spelling disorder, specific disorder of arithmetical skills 
können sämtlich betroffen sein. Bei umschriebenen Lesestörungen sind Rechtschreibstörungen häufig und persistieren oft bis in die Adoleszenz, auch wenn einige Fortschritte im Lesen gemacht werden. Umschriebenen Entwicklungsstörungen des Lesens gehen Entwicklungsstörungen des Sprechens oder der Sprache voraus. Während der Schulzeit sind begleitende Störungen im emotionalen und Verhaltensbereich häufig

\section{F81.1 Isolierte Rechtschreibstörung}

Es handelt sich um eine Störung, deren Hauptmerkmal in einer umschriebenen und bedeutsamen Beeinträchtigung der Entwicklung von Rechtschreibfertigkeiten besteht, ohne Vorgeschichte einer Lesestörung. Sie ist nicht allein durch ein zu niedriges Intelligenzalter, durch Visusprobleme oder unangemessene Beschulung erklärbar. Die Fähigkeiten, mündlich zu buchstabieren und Wörter korrekt zu schreiben, sind beide betroffen.

\section{F81.2 Rechenstörung}

Diese Störung besteht in einer umschriebenen Beeinträchtigung von Rechenfertigkeiten, die nicht allein durch eine allgemeine Intelligenzminderung oder eine unangemessene Beschulung erklärbar ist. Das Defizit betrifft vor allem die Beherrschung grundlegender Rechenfertigkeiten, wie Addition, Subtraktion, Multiplikation und Division, weniger die höheren mathematischen Fertigkeiten, die für Algebra, Trigonometrie, Geometrie oder Differential- und Integralrechnung benötigt werden.

\section{F81.3 Kombinierte Störungen schulischer Fertigkeiten}

Dies ist eine schlecht definierte Restkategorie für Störungen mit deutlicher Beeinträchtigung der Rechen-, der Lese- und der Rechtschreibfähigkeiten. Die Störung ist jedoch nicht allein durch eine allgemeine Intelligenzminderung oder eine unangemessene Beschulung erklärbar. Sie soll für Störungen, verwendet werden, die die Kriterien für F81.2 und F81.0 oder F81.1 erfüllen.

Die Störungen erschweren oder beeinträchtigen das Erlernen dieser Grundfertigkeiten. Sie dürfen vor allem nicht allein auf einen Mangel an Gelegenheit zum Lernen, eine unangemessene Beschulung, eine Intelligenzminderung oder auf visuelle oder auditive Probleme zurückzuführen sein.

Die Störung wird also abgegrenzt zur Intelligenzminderung. Diese wird in Kapitel F 7 der ICD 10 definiert als verzögerte oder unvollständige Entwicklung 
der geistigen Fähigkeiten mit einem IQ von 69 und weniger. ${ }^{69}$ Sie betrifft ein völlig anderes Erscheinungsbild, um das es vorliegend aber nicht geht. ${ }^{70}$

Die medizinisch-psychologische Forschung zur Legasthenie und Dyskalkulie erfolgt vor allem in den Disziplinen Neurowissenschaften, Genetik, Kinder- und Jugendpsychiatrie und Psychologie.

Inzwischen wurde nachgewiesen, dass es umfassende Vorläuferfähigkeiten für die Kulturtechniken gibt, die in einem bestimmten Lebensalter erlernt werden müssen, um darauf aufbauend Lesen, Schreiben und Rechnen lernen zu können. Im sprachlichen Bereich, also beim Lesen und Schreiben, ist dies vor allem die Fähigkeit der sog. phonologischen Bewusstheit ${ }^{71}$, also der Fähigkeit zwischen Phonemen (Lauten), Silben, Klang und Wörtern als Teilen von Sätzen zu unterscheiden. Fehlt diese Fähigkeit oder ist sie beeinträchtigt, ist der Schriftspracherwerb deutlich erschwert. Die Fähigkeit kann nur bis zu einem bestimmten Alter wirksam gefördert werden. Im mathematischen Bereich ist ein mengen- und zahlenbezogenes Vorwissen erforderlich, also neben dem Zählen und der Zahlenkenntnis auch das Ordnen und Klassifizieren von Gegenständen nach bestimmten Merkmalen und die Mengenerfassung. ${ }^{72}$ Schwierigkeiten im basis-numerischen Bereich, also numerisch-rechnerische Fertigkeiten, die vorschulisch erworben werden, führen in der Schule zu Schwierigkeiten beim Rechnen. ${ }^{73}$

Molekular-genetische Studien liefern Hinweise dafür, dass sich zumindest auf den Chromosomen 1, 2, 6, 15, 18 Gen-Orte für Komponenten der LeseRechtschreibstörung befinden. ${ }^{74}$ Die Fähigkeit, richtig lesen und schreiben zu lernen, scheint zu einem nicht geringen Anteil genetisch determiniert zu sein. ${ }^{75}$

69 Eggers/Fegert/Resch, Psychiatrie, S. 764

70 Warnke/Wewetzer, Berufsverband der Ärzte für Kinder- und Jugendpsychiatrie und Psychotherapie Deutschland e.V. 1997, 61 ff, S. 69 f, insofern passt die Kritik von Kischel, in BeckOK GG, Art. 3, Rn. 219.1, dass die Einordnung der Legasthenie als geistige Behinderung fragwürdig ist.

71 S. Anhang 1: Glossar der Fachbegriffe

72 Umfassend dazu: Pixner/Kaufmann, in: Legasthenie und Dyskalkulie 2011

73 Pixner/Kaufmann, in: Legasthenie und Dyskalkulie 2011, S. 200; Ansari, in: Legasthenie und Dyskalkulie 2011, S. 238

74 Umfassender Überblick über den Stand der genetischen Forschung bei Scerri/Schulte-Körne, European Child and Adolescense Psychiatry 19 (2010), S. $179 \mathrm{ff}$ und Poelmans/Buitelaar/ Pauls/Franke, Molecular Psychiatry 2011, S. $365 \mathrm{ff}$

75 Schulte-Körne, Psychiatric Genetics 2008; Scerri/Schulte-Körne, European Child and Adolescense Psychiatry 19 (2010), 179 ff; Warnke/Schulte-Körne, in: Therapie psychischer Störungen, S. 154; s. auch die Geschichte der Familie Grimm mit Legasthenie-Belastung über vier Generationen: Grimm, Schicksal Legasthenie; Projekt der Universität Würzburg zur neurophysiologischen, psychometrischen und genetischen Untersuchung der Legasthenie, http://www.zv.uni-wuerzburg.de/forschungsbericht/FOBE-akt/IN-30506000/Lese- 
Es wird angenommen, dass diese Gene Hirnfunktionen regulieren, die wichtige Voraussetzungen für einen erfolgreichen Schriftspracherwerb darstellen. ${ }^{76}$ Ein weiteres medizinisches Forschungsfeld hat sich in den letzten Jahren vor allem durch die bildgebenden Verfahren wie die Positronen-Emissionen-Tomographie (PET), die Magnetresonanztomographie (MRI: Magnetic Resonance Imaging) sowie die funktionale Magnetresonanztomographie (fMRI) ergeben. Diese machen es möglich, die Funktion des Gehirns bei bestimmten Prozessen abzubilden. Mithilfe dieser Verfahren konnte mittlerweile sowohl nachgewiesen werden, dass bei den Betroffenen mit Legasthenie Hirnareale anders funktionieren als auch welche Hirnareale dabei besonders betroffen sind. ${ }^{77}$ Bestimmte Hirnregionen arbeiten bei Legasthenie wenig oder gar nicht mit, während andere, die von „Normallesern“ nicht aktiviert werden, zusätzlich mitarbeiten. ${ }^{78}$ Die neurobiologische Forschung zeigt weiter auf, dass Regionen des Großhirns (linker temporo-parietaler Bereich), die bei der Wahrnehmung und Unterscheidung von Sprachreizen und Lauten aktiviert werden, bei Legasthenikern deutlich geringer als bei einem Normalprobanden aktiviert werden. Es liegt also nachweislich eine Störung der zentralen auditiven Wahrnehmung vor. ${ }^{79}$ Ferner haben neurobiologische Untersuchungen aufgezeigt, dass der occipitale und temporale Cortex bei Legasthenikern im Verhältnis zu Kontrollpersonen verzögert und geringer aktiviert wird. Es finden sich zudem Hinweise, dass spezifische Funktionen von Neuronen des großzelligen Systems, welches durch sich bewegende Reize aktiviert wird, bei Legasthenikern gestört sind. Es liegt also auch eine Störung der visuellen Wahrnehmung vor. Aus medizinischer Sicht ist daher anzunehmen, dass der Störung Legasthenie ein körperlicher Befund zugrunde liegt, der von dem Kind nicht durch vermehrtes Üben oder Lernen ausgeglichen werden kann.

Weniger erforscht ist die Dyskalkulie. Erste medizinische und psychologische Forschungen gibt es seit Anfang der 1990er Jahre. ${ }^{80} 2002$ beschäftigte sich erst eine kleine Gruppe von Spezialisten mit diesem Thema. ${ }^{81}$ Es gibt sehr

\%20und\%20Rechtschreibung-D.htm und http://www.zv.uniwuerzburg.de/forschungsbericht/FOBE-akt/LS-36208000/MG-02\%20Legasthenie-D.htm; Poelmans/Buitelaar/Pauls/Franke, Molecular Psychiatry 2011, S. $365 \mathrm{ff}$

Umfassend zum Stand der Forschung Schulte-Körne, in: Legasthenie und Dyskalkulie 2011, S. 50 ff; Schulte-Körne/Amorosa, in: Legasthenie 2002, S. 14 f sowie http://www.kjp.med.unimuenchen.de/forschung/legasthenie/legasthenie.php

77 Zusammenfassung und Überblick über die Forschung: Maurer, Zeitschrift des BVL, 2009, S. $15 \mathrm{ff}$ und Schulte-Körne, in: Legasthenie und Dyskalkulie 2011, S. $50 \mathrm{ff}$ Maurer, Zeitschrift des BVL, 2009, 15 ff, S. 15 
deutliche Hinweise, dass die Dyskalkulie ebenso wie die Legasthenie eine neurobiologische Störung ist. ${ }^{82}$ Der Einfluss genetischer Faktoren ist sehr wahrscheinlich ${ }^{83}$ Hirnorganisch wird vermutet, dass der Zahlensinn in einem speziellen Bereich des Gehirns sitzt und dort auch Rechenstörungen erzeugbar sind. ${ }^{84}$ Es ist wahrscheinlich, dass basis-numerische Vorläuferfertigkeiten für die Entwicklung der Rechenleistung wie z.B. visuell-räumliche Wahrnehmung oder mengen- und zahlenbezogenes Vorwissen Einfluss auf die Entstehung einer Dyskalkulie haben. ${ }^{85}$ Ergebnisse aus fMRI-Studien haben gezeigt, dass das Gehirn von Kindern mit Dyskalkulie bei basisnumerischen Aufgaben anders funktioniert als bei Kindern ohne Dyskalkulie, weil der parietale Kortex atypisch funktioniert. ${ }^{86}$ Daher ist anzunehmen, dass auch der Dyskalkulie ein körperlicher Befund zugrunde liegt, der vom Kind nicht durch vermehrtes Üben oder Lernen ausgeglichen werden kann. ${ }^{87}$

\subsection{Medizinische Diagnostik nach ICD 10 und Multiaxialem Klassifikationsschema (MAS)}

Um zu wissen, welche Schüler von Legasthenie und Dyskalkulie betroffen sind, ist es notwendig, diese Schüler überhaupt zu identifizieren und ihre Schwierigkeiten festzustellen. Die Diagnose besteht in der Zuordnung von Beschwerdebildern - diagnostischen Zeichen oder Symptomen - zu einem Krankheitsbegriff oder einer Symptomatik. Die medizinische Diagnostik von Legasthenie und Dyskalkulie basiert auf der ICD-10 und wird von Fachärzten für Kinder- und Jugendpsychiatrie und -psychotherapie im Rahmen einer umfangreichen, interdisziplinären Untersuchung festgestellt. ${ }^{88}$ Die konkreten Anforderungen an die Diagnostik ergeben sich aus den Leitlinien der Deutschen Gesellschaft für Kinder- und Jugendpsychiatrie und Psychotherapie von 2007. ${ }^{89}$ Sie beruhen auf

82 Landerl/Kaufmann, Dyskalkulie, S. 12, zu den Erklärungsmodellen s. Hain, Familiendynamik bei Belastungen durch umschriebene Lern- und Leistungsstörungen, S. $27 \mathrm{ff}$

83 Jacobs/Petermann, in: Lehrbuch der klinischen Kinderpsychologie, S. 211; Steinhausen, Psychische Störungen bei Kindern und Jugendlichen, S. 166; Warnke/Plume/Oehler, in: Therapie psychischer Störungen, S. 163

84 Jacobs/Petermann, in: Lehrbuch der klinischen Kinderpsychologie, S. 211

85 Jacobs/Petermann, in: Lehrbuch der klinischen Kinderpsychologie, S. 211; Steinhausen, Psychische Störungen bei Kindern und Jugendlichen, S. 166; Pixner/Kaufmann, in: Legasthenie und Dyskalkulie 2011, S. 200; Ansari, in: Legasthenie und Dyskalkulie 2011; S. 238 m.w.N.

86 Ansari, in: Legasthenie und Dyskalkulie 2011, S. $240 \mathrm{f}$

87 Vgl. Wehrmann, in: Legasthenie und Dyskalkulie 2011, S. 252

88 Umfassend dazu: Eggers/Fegert/Resch, Psychiatrie, S. 110 ff; für die Rechenstörung Jacobs/ Petermann, in: Lehrbuch der klinischen Kinderpsychologie, S. 212; Steinhausen, Psychische Störungen bei Kindern und Jugendlichen, S. 161 f; für die Legasthenie Warnke/Schulte-Körne, in: Therapie psychischer Störungen, S. $155 \mathrm{f}$ 
dem etablierten multiaxialen Klassifikationsschema (MAS) für psychische Störungen des Kindes- und Jugendalters und umfassen die Untersuchung von sechs Achsen: Achse 1: klinisch-psychiatrisches Syndrom, Achse 2: umschriebene Entwicklungsstörungen, Achse 3: Intelligenzniveau, Achse 4: körperliche Symptomatik, Achse 5: aktuelle abnorme psychosoziale Umstände, Achse 6: Globalbeurteilung der psychosozialen Anpassung. ${ }^{90}$ Maßgeblich für die Diagnose "Legasthenie" und/oder "Dyskalkulie“ ist zunächst, dass die Schwierigkeiten nicht dadurch erklärt werden können, dass auf den Achsen 1, 4, 5 und 6, solche Bedingungen vorliegen, die die Schwierigkeiten verursacht oder bedingt haben. ${ }^{91}$ Erst wenn derartige Bedingungen ausgeschlossen sind, erfolgt die Diagnose auf der Grundlage des sog. doppelten Diskrepanzkriteriums, d.h. es müssen zwei Diskrepanzen vorliegen:

1. Diskrepanz: das Niveau im Lesen und in der Rechtschreibung oder im Rechnen ist mangelhaft oder ungenügend im Vergleich zu altersgleicher Schulpopulation;

2. Diskrepanz: das Niveau im Lesen und in der Rechtschreibung oder im Rechnen ist wesentlich niedriger als das gemessene Intelligenzniveau. ${ }^{92}$

Diese Beurteilung erfolgt mithilfe der Achsen 2 und 3:

Achse 2 erfordert die Abklärung umschriebener Entwicklungsstörungen wie Legasthenie und Dyskalkulie vor allem durch standardisierte Lesetests, Rechtschreibtests und Rechentests. ${ }^{93}$ Die Tests vergleichen auf der Basis einer Skala von Prozenträngen von 0 bis 100, wie sich die Leistung der getesteten Person im Vergleich zu 100 getesteten Personen darstellt.

Achse 3 dient der Ermittlung des Intelligenzniveaus des Kindes. ${ }^{94}$ Dieses wird festgestellt durch psychologische Intelligenz- und Leistungsdiagnostik, wobei eine erhebliche Intelligenzminderung sicher ausgeschlossen werden muss. Intelligenztests messen auf einer Skala mit dem Messwert IQ = Intelli-

90 Eggers/Fegert/Resch, Psychiatrie, S. 126, s. dazu auch Wiesner, in: SGB VIII, § 35 a, RN 92 ff; Münder/Baltz/Kreft, in: Frankfurter Kommentar SGB VIII, § 35 a RN 18 ff Warnke/Hemminger/Roth/Schneck, Legasthenie - Leitfaden für die Praxis, S. $39 \mathrm{ff}$; umfassende Darstellung der zu erfassenden Aspekte und Daten: Eggers/Fegert/Resch, Psychiatrie, S. 114 ff; Warnke/ Hemminger/Roth/Schneck, Legasthenie - Leitfaden für die Praxis, S. 57

91 Auf die Achsen werde ich bei der Frage des Datenschutzes noch einmal eingehen. S. dazu unten 10. Teil: 1. Kapitel: 1.1.: Bekanntgabe der Ergebnisse der einzelnen Achsen, insbesondere der Achsen 2 und 3 des multiaxialen Klassifikationsschema (MAS), S. 312

92 Warnke/Hemminger/Plume, Lese-Rechtschreibstörungen, S. 1-2; Steinhausen, Psychische Störungen bei Kindern und Jugendlichen, S. 162, 165; Warnke/Schulte-Körne, in: Therapie psychischer Störungen, S. 155

93 Zu den Gütekriterien psychologischer Tests umfassend Bundschuh/Heimlich/Krawitz, in: Wörterbuch Heilpädagogik, S. $71 \mathrm{ff}$, zur rechtlichen Zulässigkeit psychologischer Tests s. die Zusammenfassung bei Zier, Recht für Diplom-Psychologen, S. $136 \mathrm{ff}$ 
genzquotient. Der Durchschnittswert liegt hier bei 100, bei einer Standardabweichung $+/-10$. Eine geistige Behinderung (= Intelligenzminderung) wird international angenommen, wenn der IQ bei unter 70 liegt. In Deutschland wird bei einem IQ von unter 85 eine Lernbehinderung angenommen. ${ }^{95}$ Eine hohe Intelligenz wird ab etwa IQ 115 angenommen, eine Hochbegabung etwa ab IQ $130 .^{96}$

Erstens muss also das Niveau in den Leistungstests Lesen, Rechtschreiben, Rechnen deutlich unterdurchschnittlich sein. Für die Leistungstests gilt, dass eine Person mit einem Prozentrang (PR) von 50 genau im Durchschnitt liegt. Eine Legasthenie und Dyskalkulie wird diagnostisch jedenfalls dann bejaht, wenn die Leistung des Kindes bei Prozentrang 10 und weniger liegt. Das bedeutet, dass $90 \%$ der Kinder gleichen Alters in dem getesteten Bereich bessere Leistungen aufweisen. Teilweise haben die betroffenen Kinder einen Prozentrang von 1 , d.h. sie sind fast nicht fähig zu lesen, zu schreiben oder zu rechnen.

Zweitens muss die Lese-/Rechtschreibleistung oder Rechenleistung eine deutliche Diskrepanz zur Intelligenz aufweisen. Die erreichten Prozentränge aus den Leistungstests müssen signifikant von dem IQ abweichen. Nach Nr. 2.5 der oben genannten Leitlinien ${ }^{97}$ soll „die Rechtschreib- bzw. Leseleistung mindestens 1,2 Standardabweichungen (zwischen 1-1,5 Standardabweichungen) unterhalb dessen liegen, was aufgrund der Intelligenz zu erwarten ist. Dies kann auch in Zeugnisnoten Ausdruck finden. Schließlich sollte eine Diagnose nicht bei Vorliegen einer Intelligenzminderung $(I Q<70)$ gestellt werden. Das Diskrepanzkriterium ist in der Regel das geeignete Maß zur Stützung der klinischen Diagnose einer Lese-Rechtschreibstörung." Um insbesondere die Benachteiligung von Kindern mit höherer Intelligenz durch ein einfaches Diskrepanzkriterium, das methodisch nicht angemessen ist, zu verhindern, wird international ein Regressionskriterium eingesetzt. ${ }^{98}$ Das Regressionsmodell relativiert die Prozentränge, die für die Diagnostik maßgeblich sind. Danach sind die für die Diagnose zulässigen Prozentränge umso höher, je intelligenter ein Kind ist. So ist etwa bei einem Kind mit einem IQ von 120 die Legasthenie zu bejahen, wenn der Prozentrang 17 beträgt (also deutlich über 10 liegt). ${ }^{99}$ Das führt dazu, dass bei einem Kind mit einem Prozentrang von 25 medizinisch eine

95 Biermann/Goetze, Sonderpädagogik, S. 202; Steinhausen, in: Schule und psychische Störungen, S. 158

96 Einen Überblick über das Verfahren bei Intelligenztestungen gibt die Broschüre des $B M B F$, Begabte Kinder finden und fördern, S. $11 \mathrm{ff}$

97 Leitlinien, Leitlinien Diagnostik und Therapie

98 Schulte-Körne/Deimel/Remschmidt, Kinder- und Jugendpsychiatrie und Psychotherapie 29 (2001), S. 113 ff; Leitlinien, Leitlinien Diagnostik und Therapie, Nr. 2.5; Warnke/SchulteKörne, in: Therapie psychischer Störungen, S. 155

99 http://www.kjp.med.uni-muenchen.de/forschung/legasthenie/diagnose.php 
Legasthenie verneint wird, wenn der IQ zwischen 104 und 110 liegt, aber bejaht wird, wenn das Kind einen IQ von etwa 131 aufweist.

Das Modell vermittelt den Eindruck, als sei eine Legasthenie oder Dyskalkulie mathematisch errechenbar. Es wird jedoch von den Fachärzten darauf hingewiesen, dass die Diagnostik Fachwissen erfordert und sich nicht auf eine bloße Berechnung der Störung beschränkt. ${ }^{100}$ Die Leitlinien stellen daher eine bedeutsame Vorgabe für den Facharzt dar, sind aber nicht die allein entscheidenden Anhaltspunkte in der Diagnostik. ${ }^{101}$ Deshalb muss es als zumindest bedenklich angesehen werden, wenn Gerichte die Legasthenie unter Anwendung der Leitlinien selbst berechnen ${ }^{102}$ oder selbst feststellen. ${ }^{103}$

Legasthenie und Dyskakulie sind demnach Störungen, die die Anforderungen der medizinischen Diagnostik nach der ICD 10 erfüllen.

\section{Pädagogische Diagnostik}

Die Pädagogik beschäftigt sich mit Förderansätzen, unterscheidet dabei aber nicht zwischen Schülern und Schülerinnen mit Legasthenie/Dyskalkulie und Schülern und Schülerinnen, die aus sonstigen Gründen nicht lesen, schreiben

100 http://www.kjp.med.uni-muenchen.de/forschung/legasthenie/diagnose.php

101 Zur rechtlichen Bedeutung der Leitlinien im Arzthaftungsrecht s. Deutsch/Spickhoff, Medizinrecht, Rn. $215 \mathrm{ff}$

102 So z.B. VG Göttingen, Urteil v. 26.21.2006, Az.: 2 A 161/05, www.rechtsprechung.niedersachsen.de (= JAmt 2006, S. $150 \mathrm{ff}$ ), in dem die Diagnostik anhand der ICD 10 und der Leitlinien ausführlich dargelegt wird. Das VG Göttingen versagt den Anspruch aus $\S 35$ a SGB VIII, weil der PR nicht eindeutig unter 10 liegt (S. 5). 1 1/2 Jahre später „aktualisiert" das VG Göttingen seine Rechtsprechung (Urteil v. 10.7.2007, Az.: 2 A 483/05, www.rechtsprechung.niedersachsen.de), wendet nun die aktualisierten Leitlinien an und bejaht aufgrund eigener Berechnungen eine Legasthenie bei einem PR von 14 bei einem Kind mit einem IQ von 120 (S. 5). Auch das VG Darmstadt hat im Urteil v. 28.4.2009, Az.: $7 \mathrm{~K}$ 687/08.DA 3 (nicht veröffentlicht), die Legasthenie selbst festgestellt (S. 12 ff), ohne Hinzuziehung eines Sachverständigen oder Anhörung der diagnostizierenden Psychologin. Das OLG Celle hat in einem Amtshaftungsverfahren nach Aktenlage festgestellt, dass das betroffene Kind definitionsgemäß kein Legastheniker ist, weil es angeblich einen erheblichen Schielfehler hatte. Dieser, so hat das OLG Celle ohne sachverständige Unterstützung entschieden, sei die Ursache für die Lese-Rechtschreibschwierigkeiten, OLG Celle, Beschluss v. 17.2.2010 Az.: 16 U 62/09 (nicht veröffentlicht). Woher das Gericht diese Sach- und Fachkenntnis hat, hat es nicht ausgeführt. Zur Frage, ob Sehfehler eine Legasthenie bewirken, ob sog. Prismenbrillen o.ä. eine Legasthenie beseitigen können, zur Abgrenzung von Sehstörungen und visuellen Störungen sowie zur wissenschaftlichen Fundierung verschiedener Therapieangebote wie Prismengläser, Irlengläser usw. s. Bundesverband Deutscher Augenärzte, Legasthenie und Sehvermögen und Suchodoletz, in: Therapie Lese-Rechtschreib-Störung, S. $179 \mathrm{ff}$, insbesondere S. $184 \mathrm{ff}:$ „Die berichteten Therapieerfolge halten einer kritischen Überprüfung nicht stand. Negative Folgen des Tragens von Prismenbrillen überwiegen gegenüber einem eventuellen Nutzen." (S. 188) 
und rechnen können. Die Schüler und Schülerinnen werden zusammengefasst unter dem Oberbegriff der "Schüler und Schülerinnen mit Schwierigkeiten". Dies wird besonders deutlich in den verschiedenen Ländervorschriften, die die Problematik als „,besondere Schwierigkeiten beim Erlernen des Lesens, Schreibens und Rechnens" bezeichnen. ${ }^{104}$ Die Bezeichnung "Lese-Rechtschreibschwierigkeiten" wird in der Pädagogik benutzt "als Sammelbegriff für eine Vielzahl von Problemen, die SchülerInnen beim Erlernen des Lesens, Schreibens und Rechtschreibens und späterhin beim Gebrauch der Schriftsprache aufweisen, um zu signalisieren, dass es sich dabei nicht um eine im Kind liegende Schwäche handelt, sondern um auftauchende Schwierigkeiten, die unterschiedlich bedingt sein können, sei es durch organisch-endogene Faktoren, sei es durch häusliche oder schulische Bedingungen. ${ }^{\text {105 }}$ "LRS wird ... nicht als Lernbehinderung, sondern als Behinderung des Lernens, nicht als Beeinträchtigung, sondern als Beeinträchtigung der Lernmöglichkeiten angesehen. ${ }^{106}$ Dieser Sammelbegriff umfasst mithin alle Schwierigkeiten, aus denen sich beim Erlernen des Lesens, Schreibens und Rechtschreibens Probleme ergeben können: soziale Probleme, erzieherische Probleme, Migrationshintergrund und daraus resultierende schlechte Deutschkenntnisse, unangemessene Beschulung, geringe Intelligenz und die individuell-körperlichen Anlagen. Mit der Beschulung und Förderung dieser Kinder beschäftigt sich vor allem die Sonderpädagogik.

Die Diagnose von Legasthenie oder Dyskalkulie ist auf dieser pädagogischen Grundlage indes ausgeschlossen, auch deshalb, weil es die Begriffe Legasthenie und Dyskalkulie in der Pädagogik nicht gibt. Allenfalls möglich ist die Diagnose von Schwierigkeiten, ohne dass hinsichtlich der Ursachen der Schwierigkeiten differenziert werden kann. Eine Diagnose von körperlichen Ursachen durch Pädagogen ist per se nicht möglich.

7. Legasthenie versus Lese-Rechtschreib-Schwäche; Dyskalkulie versus Rechenschwäche

Legasthenie und Dyskakulie sind Störungen, die die Anforderungen der medizinischen Diagnostik nach der ICD 10 erfüllen. Die medizinische Diagnostik setzt per Definition eine Abweichung von der Norm voraus. Die Bestimmung der Norm und der Grenzwerte im Bereich der Medizin ist indes relativ willkürlich ${ }^{107}$

\footnotetext{
104 KMK-Lesen/Rechtschreiben/Rechnen, BB VV-LRSR; HB LRS-Erlass; HH Förder-Richtlinie; HE VO-GestSchulVerh; MV VV-LRR; NI LRR-Erlass; NRW LRS-Erlass; RP VV-LRS; SL VV-LRS; SH Legasthenie-Erlass

105 Amelow, Lese- und Rechtschreibschwierigkeiten, S. 10

106 Valtin, in: LRS und Legasthenie, S. 57 f, kritisch dazu Biermann/Goetze, Sonderpädagogik, S. 8

107 Umfassend dazu Eggers/Fegert/Resch, Psychiatrie, S. 110 ff; s. auch Schulte-Körne auf der Internetseite der KJP München unter Diagnose, http://www.kjp.med.uni-muenchen.de/
} 
und orientiert sich vor allem an Kriterien, die für Forschungszwecke entwickelt wurden. ${ }^{108}$

Die Kehrseite dieser Diagnostik ist, dass leichtere Formen der Störung, die auf den gleichen Ursachen beruhen, aber die Diagnosekriterien nicht erfüllen, keine Legasthenie oder Dyskalkulie sind. Ebenfalls aus der Diagnose ausgeschlossen sind diejenigen Kinder, deren Schwierigkeiten beim Erlernen des Lesens, Rechtschreibens oder Rechnens nicht auf eine Teilleistungsstörung im obigen Sinne zurückgeführt werden können, sondern wo die Ursachen in einer verzögerten Entwicklung des Kindes oder in seinem familiären, schulischen oder auch sozialen Umfeld liegen. Dabei handelt es sich um nicht altersgemäße Leistungen ohne neuropsychologische Funktionsstörungen - häufig vor dem Hintergrund sozioökologischer Benachteiligungen bzw. Belastungen, für die in der angloamerikanischen Literatur der Begriff des allgemeinen Rückstands von Lese- und Rechtschreibfertigkeiten („general reading backwardness“) eingeführt worden ist. ${ }^{109}$ Schließlich fallen aus der Diagnose diejenigen Kinder heraus, deren Schwierigkeiten vorübergehend sind, etwa aufgrund besonderer familiärer Belastungen. Diese Gruppe der betroffenen Schüler und Schülerinnen wird in Bayern als Schüler und Schülerinnen mit Lese-Rechtschreib-Schwäche bezeichnet ${ }^{110}$, so dass sich dort eine Differenzierung zwischen Störung und Schwäche herausgebildet hat. Analog lässt sich dies auf die Dyskalkulie übertragen, wo die Dyskalkulie i.S.d. ICD 10 als Störung und die schwächere Form als Rechenschwäche bezeichnet wird.

Der Nachteil dieser Unterscheidung in Störung und Schwäche ist, dass die Gruppe, der eine "Schwäche" attestiert wird, sehr heterogen ist. Sie enthält sowohl diejenigen Fälle, in denen körperliche Ursachen nachweisbar sind, als auch alle anderen Fälle. Der Begriff "Schwäche“ funktioniert als negative Definition in dem Sinne, dass "Schwäche" alles das ist, was nicht Legasthenie oder Dyskalkulie ist. Problematisch ist dabei, dass vor allem diejenigen Betroffenen, die körperliche Ursachen haben, aber nicht die Diagnosekriterien erfüllen, kategorisch aus der Diagnose ausgenommen werden, obwohl die Übergänge in der Betroffenheit immer fließend sind und es, so die Untersuchung von Engelmann/Plaum ${ }^{111}$, keine sicheren Unterscheidungsmerkmale gibt. Ebenso werden aus der Diagnose diejenigen Betroffenen ausgenommen, die aufgrund anderer

forschung/legasthenie/diagnose.php; Landerl/Kaufmann, Dyskalkulie, S. 95 zeigt auf, dass die ICD 10 Forschungskriterien festlegt, wonach maximal 3 \% der Gesamtpopulation die Diagnose erhalten sollen.

$108 \mathrm{Vgl}$. Landerl/Kaufmann, Dyskalkulie, S. 95

109 Steinhausen, in: Schule und psychische Störungen, S. 161

110 BY KMBek Nr. I

111 Engelmann/Plaum, report psychologie 2008, S. 16 ff; s. auch Ehlert/Schroeders/FritzStratmann, Lernen und Lernstörungen 1 (2012), S. $169 \mathrm{ff}$ 
Faktoren, wie z.B. soziale Bedingungen, Schwierigkeiten mit dem Lesen, Rechtschreiben und Rechnen haben. Wegen dieser Abgrenzungsschwierigkeiten zwischen Kindern mit Legasthenie im Sinne der Diskrepanzdefinition von Kindern mit einem Entwicklungsrückstand der Lese-Rechtschreib-Fertigkeiten, ist zumindest im angloamerikanischen Raum für die Ausweitung der Diagnose Dyslexie plädiert worden. ${ }^{112}$

In Deutschland haben die Abgrenzungsschwierigkeiten und die verschiedenen Schwerpunkte der forschenden Disziplinen zu sehr unterschiedlichen Sichtweisen auf die Problematik Legasthenie und Dyskalkulie und zu einer erregten Debatte über das „Konstrukt Legasthenie ${ }^{\text {“113 }}$ geführt, die sogar als "Anti-Legasthenie-Bewegung "114 in die Literatur eingegangen ist. ${ }^{115}$ Die Vehemenz dieser Diskussion erschwert den sachlichen Zugang auf die Problematik. Die KMK hat versucht sich aus der Diskussion herauszuhalten und in den Empfehlungen von 1978 und von 2003/2007 darauf verwiesen, dass die pädagogische, psychologische und medizinische Forschung auf dem Gebiet kontrovers und nicht abgeschlossen ist. ${ }^{116}$ Das führt aber auch dazu, dass sich die KMK bisher zu einer grundsätzlichen Anerkennung der Legasthenie/Dyskalkulie in allen Schulstufen und für alle Schulabschlüsse nicht durchringen konnte.

Versucht man aus der Diskussion die wesentlichen Standpunkte jenseits gesellschaftspolitischer Kritik herauszufiltern, so stellt man fest, dass sie in ihrem Kernanliegen gar nicht so weit voneinander abweichen, wie die vehemente Diskussion glauben lässt. Alle setzen sich nämlich dafür ein, das Kind nicht nur mit seiner Legasthenie/Dyskalkulie und damit seinem Defizit wahrzunehmen, sondern es mit allen seinen Anlagen und Befähigungen zu sehen und ihm die Möglichkeit zu geben, das Lesen, Rechtschreiben und Rechnen zu erlernen. ${ }^{117}$ Das Dilemma ergibt sich für alle Ansichten daraus, dass in der Schule diese Fähigkeiten bewertet werden und dies naturgemäß dazu führt, dass das be-

112 Steinhausen, in: Schule und psychische Störungen, S. 162

113 Valtin, in: LRS und Legasthenie

114 Hartmann, VHN 2008, S. 123 ff, S. 124

115 So spricht z.B. Schubenz davon, dass „.... es eine Interessenvertretung für das legasthene Kind, die sich aus mittleren und höheren gesellschaftlichen Gruppen rekrutierte, schaffte, ihren Schützlingen die Benotung der schriftsprachlichen Leistungen ... zu ersparen" (Ruß, Legasthenie und Hochbegabung, Vorwort von Schubenz, S. XIV), und Valtin behauptet, dass die Berücksichtigung von Legasthenie/Dyskalkulie „zu einem Zwei-Klassen-System der Förderung [führt], weil sich nun vor allem Oberschicht-Eltern die Förder-Privilegien erkämpfen werden." (Valtin, Brauchen wir die Legasthenie?, S. 5) KMK-Lesen/Rechtschreiben/Rechnen

117 S. z.B. Bundesverband Legasthenie und Dyskalkulie e.V.: http://www.bvllegasthenie.de/index.php5?p=/bundesverband/entwicklung; Valtin, Brauchen wir die Legasthenie?; Büchner/Kortländer/Werner/Robering/Schönweiss, ,'Legasthenie - eine Krankheit, eine Behinderung, eine Störung?" 
troffene Kind auf diesen Gebieten schlechte Noten erzielt. Erst oder besonders dadurch bekommen die Schwierigkeiten oder Defizite ihre enorme Bedeutung und werden die vorhandenen Fähigkeiten und Stärken des Kindes immer weniger wahrgenommen. ${ }^{118}$ Schlee, einer der heftigsten Kritiker des LegasthenieBegriffs, setzte insbesondere an dieser Bewertung an und meinte, dass die Diskussion um den Begriff Legasthenie nichts dazu beitragen würde, gerade die seiner Ansicht nach grundlegende Frage zu beantworten, welche Bedeutung vor allem die Rechtschreibung für den Schulerfolg haben darf. Er sah in der Bewertung der Rechtschreibung und ihrer Auswirkungen auf den Schulerfolg das entscheidende Manko der deutschen Schule. ${ }^{119}$ Hier trifft er sich mit Vanselow/Dummer-Smoch, die im Hinblick auf die Legasthenie zwar eine grundsätzlich andere Sichtweise als Schlee vertreten, aber auch detailliert und auf der Basis statistischer Auswertungen herausstellen, welchen enormen Stellenwert vor allem die richtige Rechtschreibung in Deutschland für den Schulerfolg und damit für die beruflichen Ausbildungsmöglichkeiten und Lebenschancen eines Kindes hat, während andere Fähigkeiten, wie z.B. mathematische und naturwissenschaftliche viel weniger entscheidend sind. ${ }^{120}$ Letztendlich liegt der Diskussion die meist unausgesprochene Frage zugrunde, ob vor allem die Rechtschreibzensur ein gewichtiges Beurteilungs- und Auslesekriterium sein sollte ${ }^{121}$ Die Beantwortung dieser Frage ist jedoch eine schulpolitische Frage, die in dieser Arbeit nicht zu behandeln ist.

Von dieser schulpolitischen Frage ist jedoch die für diese Arbeit maßgebliche rechtliche Frage zu trennen, ob jedenfalls Schüler und Schülerinnen mit Legasthenie und/oder Dyskalkulie, deren Beeinträchtigung nach medizinischen Kriterien messbar und auf der Grundlage der ICD 10 diagnostizierbar ist, aus den Grundrechten und hier vor allem aus Art. 3 III 2 GG, Art. 3 I GG, Art. 12 GG, Art. 2 । GG und Art, 2 || GG sowie für ihre Eltern aus Art. 6 । GG den verfassungsrechtlichen Anspruch haben, dass auf ihre Beeinträchtigung Rücksicht genommen wird. Die weitere Frage ist, ob wenn dies der Fall ist, nur Legasthenie/ Dyskalkulie zu berücksichtigen sind oder ob auch Formen der Beeinträchtigung, die unter dem Oberbegriff "Schwäche" zusammengefasst werden, einzeln oder insgesamt zu berücksichtigen sind. ${ }^{122}$

118 Vgl. dazu: Schlichte-Hiersemenzel, Hochbegabte Kinder, S. $44 \mathrm{f}$

119 Schlee, Legasthenieforschung am Ende?, S. 160, s. auch Ruß, Legasthenie und Hochbegabung, S. $101 \mathrm{ff}$

120 Vanselow/Dummer-Smoch, Vernachlässigung individueller Begabungsstrukturen, insbesondere S. $68 \mathrm{ff}, 254 \mathrm{ff}$

121 Schlee, Legasthenieforschung am Ende?, S. 159

122 S. dazu unten 3. Teil: 2. Kapitel: 3.1.4.: Defizit der medizinischen Diagnostik - das Diskrepanzkriterium, S. 100 
8. Welche Folgen haben Legasthenie und/oder Dyskalkulie in Schule und Beruf?

Schüler und Schülerinnen mit Legasthenie/Dyskalkulie erkennen zunächst nur, dass sie größere Schwierigkeiten damit haben, das Lesen, Schreiben und Rechnen zu erlernen als ihre Klassenkameraden. Warum dies so ist, bleibt innen verborgen, meistens halten sie sich für dumm. ${ }^{123}$ Sie entwickeln Methoden, um ihre Schwierigkeiten zu kompensieren. Allerdings ist ihnen nicht bewusst, dass es sich dabei eben um eine Kompensation handelt und andere "Normalbegabte" auf einem viel einfacheren Weg zum Ziel kommen. Sie halten ihren Weg für den „normalen“ Weg. Darin werden sie durch Bemerkungen wie „Warum nicht gleich so?" bestärkt. In neuen Lernsituationen müssen die Betroffenen ihre Kompensationsmethode erst der neuen Aufgabe anpassen, um einen Lösungsweg zu finden; vor allem unter Zeitdruck und in Stresssituationen wird es aber immer schwieriger, ständig zu kompensieren. ${ }^{124}$ Der erheblich größere Aufwand durch das ständige Kompensieren wird meist weder von den Betroffenen noch von Eltern oder Lehrern wahrgenommen und daher auch nicht entsprechend gewürdigt. ${ }^{125}$

Probleme im Lesen und Rechtschreiben führen zunächst zu Misserfolgen im Fach Deutsch. Kinder mit Legasthenie stehen spätestens in der 3. und 4. Klasse in der Gefahr, nicht nur im Fach Deutsch, sondern auch in anderen Schulfächern nicht die ihrer Begabung entsprechende Leistung zu erbringen, da Leistungserhebungen oft in schriftlicher Form erfolgen. Obwohl ein Kind mit einer Legasthenie beispielsweise über gute Rechenleistungen verfügt, kann es in Textaufgaben versagen, da es Schwierigkeiten hat, die Aufgabe zu lesen und den Inhalt des Gelesenen zu verstehen. Im Sachkundeunterricht bringt ein legasthener Schüler sein vorhandenes Wissen nur fehlerhaft zu Papier, so dass die Leistungsbewertung letztendlich negativ ausfallen kann. Aufgrund der wesentlichen Bedeutung der schriftsprachlichen Leistungen kommt es nicht selten am Ende der Grundschulzeit oder auf weiterführenden Schulen zu einem „generalisierten Lernversagen" im schulischen Leistungsbereich. ${ }^{126}$ Dies hat wiederum zur Folge, dass ein nicht geringer Teil der Kinder mit einer Legasthenie

123 So z.B. das Kind in VG Hamburg, Urteil v. 24.11.2009, Az.: 13 K 4032/07, BeckRS 2009 42354, S. 8 Punkt 2.3.3

124 Roos/Schöler, Schriftspracherwerb; S. 159; Geist, in: Legasthenie und Dyskalkulie 2011, S. 167. Dies übersieht das VG Darmstadt, Urteil v. 28. 4. 2009, Az.: 7 K 687/08.DA (3), S. 17 (nicht veröffentlicht), wenn es meint, der Kläger könne in Klausuren keinen "Notenschutz" erhalten, weil er [auch in den Klausuren] davor bewahrt werden müsse, sich auf diesem Schutz auszuruhen und die Übung für die Automatisierung seiner Kenntnisse zu verlieren. Vanselow/Dummer-Smoch, Vernachlässigung individueller Begabungsstrukturen, S. $176 \mathrm{f}$

Plume/Warnke, in: Schule und psychische Störungen, S. 201 
wenigstens eine Klassenstufe wiederholen muss ${ }^{127}$. Nach der Studie von Jansen/Skowronek mussten $43 \%$ der Risiko-Kinder eine Klasse und 18 \% sogar zwei Klassen wiederholen. ${ }^{128}$

In der Sekundarstufe stellt der Erwerb einer Fremdsprache legasthene Kinder vor eine besondere Aufgabe. ${ }^{129}$ Eine wenig lautgetreue Sprache wie z.B. Englisch ist für Kinder mit einer Lese-Rechtschreibstörung meist besonders schwierig zu erlernen. ${ }^{130}$

Die schulische Entwicklung von Schülern mit Legasthenie ist in den letzten 25 Jahren vor allem von der Kinder- und Jugendpsychiatrie und der Psychologie umfassend untersucht und erforscht worden. ${ }^{131}$ Sie haben gezeigt, dass ein gutes Lese-Rechtschreibvermögen ein besserer Prädiktor für schulischen Erfolg ist als das intellektuelle Potential. Die Heidelberger Längsschnittstudie ${ }^{132}$ zeigte, dass Schüler mit Legasthenie eine niedrigere schulische Qualifikation erlangen als Schüler ohne Lernstörung trotz gleichen Begabungsniveaus. Trotz durchschnittlicher allgemeiner Begabung erreichten die Personen mit Rechtschreibstörung nahezu keine weiterführenden Schulabschlüsse. Nur 7 bis $9 \%$ hatten einen Realschulabschluss und $0-2 \%$ das Abitur erreicht. Dieses Ergebnis entsprach nicht der Verteilung des Intelligenzniveaus. In der Stichprobe von Esser und Schmidt hatte $20 \%$ der Schüler mit Lese-Rechtschreibstörung im Alter von 8 Jahren mangelhafte Leistungen in mindestens einem der Kernfächer und etwa die Hälfte dieser Kinder hatte bereits eine Schulklasse wiederholt - trotz durchschnittlicher Intelligenztestwerte. Die Rate der Schulabbrüche korrelierte mit dem Schwergrad der Lese- und Rechtschreibstörung. Auch im Hinblick auf das berufliche Fortkommen kann die Legasthenie den Betroffenen behindern. In der Mannheimer Studie waren $26 \%$ der Personen, die im achten Lebensjahr die Diagnose einer Lese-Rechtschreibstörung erhalten hatten, im 25. Lebensjahr arbeitslos im Vergleich zu einer gleichaltrigen Kontrollgruppe, in der die Arbeitslosenrate bei $4 \%$ lag. ${ }^{133}$

127 S. Pressemitteilung Elternumfrage 2009, die zwar nicht repräsentativ ist, aber dennoch beeindruckt: $25 \%$ der Kinder wiederholen eine Klasse, $20 \%$ müssen die Schule wechseln.

Zitiert nach Vanselow/Dummer-Smoch, Vernachlässigung individueller Begabungsstrukturen, S. 71

Sellin, Wenn Kinder mit Legasthenie Fremdsprachen lernen; Sellin, in: Legasthenie; Gantner, in: Legasthenie; Will, in: Legasthenie; Wölms, in: Legasthenie und Dyskalkulie 2007 m.w.N. Schulte-Körne/Stieglitz, in: Adoleszenzpsychiatrie, S. 477; a. A. VGH Kassel, Beschluss v. 8.12.2011, Az.: 7 A 2621/10.Z, juris, Rn. 17

131 S. dazu die nachfolgenden Studien und die Nachweise bei Schulte-Körne/Stieglitz, in: Adoleszenzpsychiatrie, S. $477 \mathrm{f}$ und $485 \mathrm{ff}$

132 Haffner/Zerahn-Hartung/Pfuller/Parzer/Streh/ow/Resch, Zeitschrift für Kinder- und Jugendpsychiatrie und Psychotherapie 26 (1989), S. $124 \mathrm{ff}$

133

Esser/Wyschkon/Schmidt, Zschr. klin. Psychol. Psychiatr. Psychother 31 (2002), S. 235 ff, S. 238 
Tatsächlicher Problemaufriss

Die Legasthenie gefährdet daher trotz vorhandener durchschnittlicher Begabung die ganze Leistungsfähigkeit und den weiteren Lebensweg der Betroffenen. ${ }^{134}$

Über die langfristigen Auswirkungen einer Dyskalkulie auf Schullaufbahn und berufliche Entwicklung ist dagegen bisher sehr wenig bekannt, sie könnten aber möglicherweise noch massiver sein als die dokumentierten negativen Langzeiteffekte von Legasthenie. Eine englische Studie hat gezeigt, dass in einer Stichprobe von Männern im Alter von 37 Jahren 48 \% der Männer mit auffällig schwachen Rechenleistungen arbeitslos waren. Diese Quote lag noch über der Quote von $41 \%$ bei arbeitslosen Männern mit schwachen Leseleistungen. ${ }^{135}$

\section{Welche psychischen Auswirkungen haben Legasthenie und/oder Dyskalkulie?}

Die täglichen Belastungen eines Kindes mit Legasthenie/Dyskalkulie sind erheblich. In der Rechtsprechung zu § 35 a SGB VIII lassen sich die psychischen Beeinträchtigungen dieser Kinder eindrucksvoll nachlesen, auch wenn sie von den Gerichten in der Regel nicht als so gravierend angesehen werden, dass sie als "drohende Beeinträchtigung der Teilhabe" anerkannt werden. ${ }^{136}$ Die Auswirkungen vor allem der Legasthenie auf die psychische Entwicklung der Betroffenen sind seit Jahren Gegenstand vieler internationaler und nationaler Studien, die sich z. T. über Jahrzehnte erstreckt haben und daher wissenschaftlich fundierte Ergebnisse der Langzeitauswirkungen von Legasthenie liefern. ${ }^{137}$ Kinder

134 Diese Einschätzung teilen auch die Berufsunfähigkeitsversicherungen, die in den Gesundheitsfragen ausdrücklich nach Legasthenie fragen und Menschen mit Legasthenie nicht versichern: Financial Times Deutschland vom 16.4.2010: „Diskussion um Berufsunfähigkeitspolicen - Annahmepflicht für Versicherer gefordert";

http://www.ftd.de/unternehmen/versicherungen/:diskussion-um-

berufsunfaehigkeitspolicen-annahmepflicht-fuer-versicherer-gefordert/50101621.html

Landerl/Kaufmann, Dyskalkulie, S. 100

136 Z.B. VG Hamburg, Urteil v. 24.11.2009, Az.: 13 K 4032/07, BeckRS 2009 42354, S. 8 Punkt 2.3.3. Das VG Hamburg verneint im Ergebnis den Anspruch aus $\S 35$ a SGB VIII mangels Teilhabebeeinträchtigung, da die Probleme im entschiedenen Fall nur im häuslichen Bereich auftreten würden, das Kind viel Unterstützung durch die Familie erfahre, sowie eine MutterKind-Kur geplant sei. Dies zeige, dass das Familiensystem noch funktioniere. Vgl. auch VGH Kassel, Urteil v. 20.8.2009, Az.: 10 A 1874/08 juris, in dem die alltägliche, sehr belastende Situation eines 9-jährigen Kindes dargestellt wird. Das vorgängige VG Darmstadt hatte die Klage auf Eingliederungshilfe nach $\S 35$ a SGB VIII trotz der massiven Schwierigkeiten des Kindes abgewiesen, da eine Beeinträchtigung der seelischen Gesundheit nicht gegeben sei. Der VGH Kassel gab der Klage statt.

137 Zusammenfassung bei Gasteiger-Klicpera/Julius/Klicpera, in: Hdb. Sonderpädagogik, m.w.N.; Esser/Wyschkon/Schmidt, Zschr. klin. Psychol. Psychiatr. Psychother 31 (2002), S. 235 ff; 
mit einer Legasthenie werden häufig in der Schule von den Lehrkräften nicht emotional unterstützt und von Mitschülern gehänselt. ${ }^{138}$ Für den deutschen Sprachraum ist nachgewiesen, dass Kinder mit Lese- und Rechtschreibproblemen, die im Kindergarten noch normal in die Gruppe integriert waren, bereits in der 1. Klasse, wenn eine Diagnostik noch gar nicht möglich ist, in eine Sonderrolle geraten und sich die Tendenz erhöht, dass sie sich zurückzuziehen. In den höheren Klassen geraten sie häufig in eine Außenseiterstellung und fühlen sich selbst niedergeschlagener und einsamer. Zudem ist das globale Selbstwertgefühl in der 4. Klasse deutlich geringer als jenes von Kindern ohne Schulschwierigkeiten. ${ }^{139}$ Das gleiche gilt für Kinder mit Rechenschwierigkeiten, die in hohem Maße mit emotionalen Problemen belastet sind. ${ }^{140}$

Die Verknüpfung von Legasthenie und Dyskalkulie mit Begleitstörungen wird in den Leitlinien der Deutschen Gesellschaft für Kinder- und Jugendpsychiatrie und Psychotherapie von 2007 zur Diagnostik besonders aufgeführt (Nr. 2.3). Charakteristisch ist, dass die Komorbidität bzw. die Begleitstörungen im Laufe der ersten Schulklasse bzw. in späteren Grundschuljahren im Zusammenhang mit schulischen Anforderungen verstärkt auftreten und z.B. an Wochenenden oder in Ferienzeiten geringer ausgeprägt erscheinen. ${ }^{141}$ Bei vielen betroffenen Kindern und Jugendlichen treten emotionale Probleme, depressive Störungen, Störungen des Sozialverhaltens und Hyperaktivitätssyndrome auf, verbunden mit niedrigem Selbstwertgefühl, Anpassungsproblemen in der Schule und Hausaufgabenkonflikten, Demotivierung und Schulunlust sowie somatischen Beschwerden wie Kopf- und Bauchschmerzen. ${ }^{142}$

Bei Rechenstörungen treten als Begleitstörungen sprachliche Entwicklungsdefizite und/oder visuell-räumliche und optische Verarbeitungsstörungen und

Klicpera/Heyse/Warnke/Kutschera/Schwaiger, Fortschritte der Neurologie Psychiatrie 1981, S. 193 ff; Schulte-Körne/Stieglitz, in: Adoleszenzpsychiatrie, S. 478 f; Daniel/Walsh/Goldston/ Arnold/Reboussin/Wood, Journal of Learning Disabilities 2006, S. 507 ff; s. z.B. die umfassende langjährige Studie in Neuseeland Klicpera/Gasteiger-Klicpera, in: Hdb. Sonderpädagogik, S. 360

Warnke/Schulte-Körne, in: Therapie psychischer Störungen, S. 160; Eggers/Fegert/Resch, Psychiatrie, S. 764

139 Klicpera/Gasteiger-Klicpera, in: Hdb. Sonderpädagogik, S. $360 \mathrm{f}$

140 Klicpera/Gasteiger-Klicpera, in: Hdb. Sonderpädagogik, S. 354

141 Steinhausen, Psychische Störungen bei Kindern und Jugendlichen, S. 159 f; Schulte-Körne, in: Legasthenie und Dyskalkulie 2011, S. 48; zu chronisch zwischenmenschlichen Belastungen im Zusammenhang mit Schule Beyer, Kognitive und psychosoziale Faktoren, S. 107

Gasteiger-Klicpera/Julius/Klicpera, in: Hdb. Sonderpädagogik, S. 354 f; Beyer, Kognitive und psychosoziale Faktoren, S. 29 f; Suchodoletz/Amorosa, in: Therapie Lese-RechtschreibStörung, S. 269; Steinhausen, Psychische Störungen bei Kindern und Jugendlichen, S. 159; Warnke/Schulte-Körne, in: Therapie psychischer Störungen, S. 155; Plume/Warnke, in: Schule und psychische Störungen, S. 199 
emotionale Störungen ${ }^{143}$ auf. Häufig entwickelt sich eine negative Spirale aus schwacher Leistungsfähigkeit, schulischen Misserfolgen, negativen Konsequenzen und zunehmender Resignation und Leistungsverweigerung. ${ }^{144}$

Als Folge der Lese-Rechtschreibstörung/Rechenstörung ergibt sich daher nicht nur eine generalisierte Lern-Leistungsbeeinträchtigung, sondern auch eine emotionale Entwicklungsgefährdung. Diese generalisierend pathogenetische Auswirkung ist ebenfalls international durch vielfältige Studien erwiesen. Nach der Mannheimer prospektiven epidemiologischen Längsschnitt-Studie ${ }^{145}$ wiesen die Schüler mit Legasthenie eines Geburtsjahrgangs im Vergleich zu den Schülern des Geburtsjahrgangs ohne Legasthenie im Alter von 8 Jahren mit 43,2 \%, im Alter von 13 Jahren zu 44,1\%, im Alter von 18 Jahren zu 34,4\% und im Alter von 25 Jahren noch zu 29 \% signifikant häufiger psychische Störungen auf. Charakteristisch waren Symptome der motorischen Unruhe, Konzentrationsstörungen und insbesondere dissoziale Verhaltensprobleme. Im Alter von 18 Jahren erschien die Suizidgefährdung erhöht (13,3 \% gegenüber 3,9 \% in der Kontrollgruppe). Nach einer Studie von Klicpera ${ }^{146}$ hatten die ehemals kinderpsychiatrisch stationär im Max-Planck-Institut für Psychiatrie in München behandelten Patienten mit einer Lese-Rechtschreibstörung 2 bis 10 Jahre nach der stationären Behandlung im Vergleich zu anderen Diagnosegruppen die höchste Rate psychopathologischer Symptome. Die Schwierigkeiten können so massiv werden, dass die betroffenen Kinder nicht mehr beschulbar sind. ${ }^{147}$

143 Steinhausen, Psychische Störungen bei Kindern und Jugendlichen, S. 165

144 Jacobs/Petermann, in: Lehrbuch der klinischen Kinderpsychologie, S. 212

145 Esser/Wyschkon/Schmidt, Zschr. klin. Psychol. Psychiatr. Psychother 31 (2002), S. $235 \mathrm{ff}$

Klicpera/Heyse/Warnke/Kutschera/Schwaiger, Fortschritte der Neurologie Psychiatrie 1981, S. $193 \mathrm{ff}$

147 So der Werdegang eines Kindes, der nachzuvollziehen ist u.a. aus den Urteilen des VG Lüneburg, Beschluss v. 12.8.2004, Az.: 4 B 123/04 (nicht veröffentlicht); OLG Celle, Urteil v. 4.11.2008, Az.: 16 U 70/07, (nicht veröffentlicht), Kammerbeschluss des BVerfG, Az.: 1 BvR 1711/09, v. 21.11.2012, juris, Rückverweisung an das OLG Celle und den Urteilen des LSG Niedersachsen-Bremen, Urteil v. 14.1.2009, Az.: L 9 U 129/06 Revision vom BSG zurückgewiesen, Urteil v. 27.4.2010; Az.: B 2 U 13/09, juris, und dem Nichtannahmebeschluss des BVerfG zur Verfassungsbeschwerde, BVerfG, Beschluss v. 8.6.2012; Az.: 1 BvR 2853/10, http://www.bverfg.de/entscheidungen/rk20120608_1bvr285310.html 


\section{Kapitel: Legasthenie und Dyskalkulie in der Schule}

\section{Beschulung von betroffenen Schülern und Schülerinnen}

Da sich die Störungen Legasthenie/Dyskalkulie erst in der Schule zeigen, werden Schüler und Schülerinnen mit Legasthenie/Dyskalkulie normal eingeschult. Häufig zeigen sich bereits in den ersten Wochen Schwierigkeiten beim Erlernen des Lesens, Schreibens und Rechnens. Einige Kinder sind aber in der Lage, ihre Schwierigkeiten bis in höhere Klassen zu kompensieren und daher nicht aufzufallen. Eine präzise Feststellung von Schwierigkeiten ist vor allem im ersten Schuljahr schwierig, weil alle Kinder ein unterschiedliches Lerntempo und Lernverhalten haben und deshalb die Unterscheidung zwischen Kindern, die etwas langsamer lernen und solchen, die manifeste Schwierigkeiten haben, nur schwer möglich ist. Vielfach erkennen Lehrkräfte die Probleme nicht. So ergab die IGLU-Studie 2006, dass Lehrkräfte die Lesekompetenz bei nur 14 \% der Schüler mit Legasthenie und 27 \% der Schüler mit Leseschwäche auch tatsächlich als mangelhaft oder schlechter einschätzten. ${ }^{148}$ Wenn die Probleme auffallen, die betroffenen Kinder aber oft einen aufgeweckten und intelligenten Eindruck machen, wird häufig angenommen, dass sich die Probleme "auswachsen werden“. ${ }^{49}$

Werden die Schwierigkeiten in der Grundschule größer, wird häufig eine Untersuchung des sonderpädagogischen Förderbedarfs in die Wege geleitet, die feststellen soll, ob das Kind auf eine Förderschule umgeschult werden soll. ${ }^{150} 16 \%$ der Kinder mit Legasthenie besuchen trotz allgemein durchschnittlicher Intelligenz Förderschulen. ${ }^{151}$

Schüler und Schülerinnen mit Legasthenie/Dyskalkulie, die auf der Regelschule verbleiben, werden nach dem Regellehrplan unterrichtet.

148 Valtin/Hornberg/Buddeberg/Voss/Kowoll/Potthoff, in: Bos, IGLU 2006, S. $76 \mathrm{f}$

149

Geist, in: Legasthenie und Dyskalkulie 2011, S. 167 mit der Feststellung: „von wegen, dass sich das auswachse"; Löffler/Meyer-Schepers/Lischeid, in: Legasthenie und Dyskalkulie 2007, S. 275, mit dem Hinweis darauf, dass sich das Problem erheblich verschlimmert statt sich zu verbessern; Klasen, Legasthenie, S. 68; s. dazu auch die Selektion der Lehrkräfte in der Studie von Schulte-Körne/Deimel/Remschmidt, Zeitschrift für Kinder- und Jugendpsychiatrie und Psychotherapie 31 (2003), Punkt 3.1: Kinder mit Schwächen ausschließlich im Rechtschreiben wurden eher als entwicklungsverzögert eingeschätzt (,das wächst sich aus') und nicht für die Förderung vorgeschlagen.

150 So ausdrücklich Kultusministerium SN: http://www.sachsen-macht-schule.de/schule/ 1792.htm (Abruf 3.3.2010); „Hat ein Schüler Probleme beim Lesen und Schreiben, beim Erarbeiten mathematischer Zusammenhänge und beim selbstständigen Anwenden des Erlernten, kommt eine sonderpädagogische Förderung in Betracht"; zu den schulischen Bestrebungen, die Kinder auf Förderschulen zu überweisen, s. Klasen, Legasthenie, S. 97

Schleider, Lese- und Rechtschreibstörungen, S. 81 
Tatsächlicher Problemaufriss

\section{Bewertungs- und Benotungsvorschriften}

Das Bundessozialgericht hat $1979^{152}$ die Schwierigkeiten beim Lesen und Rechtschreiben mit Störungen beim Erwerb musischer Kenntnisse oder dem Einüben sportlicher Fähigkeiten gleichgesetzt und deshalb einen Krankheitswert verneint. Störungen auf diesen sehr verschiedenen Gebieten können jedoch nur gleichgesetzt werden, wenn sie auch in ihrer Auswirkung vergleichbar sind. Der entscheidende Unterschied zwischen den Fähigkeiten im Lesen, Schreiben, Rechnen und in Musik und Sport ergibt sich aus dem Stellenwert, den diese Fähigkeiten in der Schule, insbesondere in Bezug auf die Schullaufbahn und mögliche Schulabschlüsse, haben. Musik und Sport sind durchgängig Nebenfächer, die sich in aller Regel nicht auf die mögliche Schullaufbahn und den möglichen Schulabschluss auswirken. ${ }^{153}$ Demgegenüber sind Lesen, Schreiben und Rechnen Grundvoraussetzungen der Schulbildung und die Fähigkeiten auf diesen Gebieten von erheblicher Relevanz sowohl für die mögliche Schullaufbahn als auch für den Schulabschluss. Mit anderen Worten, es macht für die Schullaufbahn und den Schulabschluss nichts aus, wenn jemand unsportlich oder unmusikalisch ist, es wirkt sich aber erheblich aus, wenn jemand schlecht liest, schreibt (rechtschreibt) oder rechnet. Dass schlechte Noten in diesen Fächern für die Schullaufbahn und den Schulabschluss relevant sind, ist offensichtlich. Wie umfassend ihre Relevanz ist, ist jedoch - auch Gerichten ${ }^{154}$ - nicht immer bekannt, so dass die wesentlichen Auswirkungen hier kurz vorgestellt werden sollen.

\subsection{Grundschule und Bildungsempfehlung}

Die Bildungsempfehlung im Anschluss an die Grundschule stellt die ersten Weichen für spätere Berufs- und Lebensmöglichkeiten und ist damit eine „Richtungsentscheidung ${ }^{\prime 155}$, der eine sehr hohe Bedeutung zukommt. Erhält das Kind nicht die Bildungsempfehlung für eine Realschule oder ein Gymnasium, ist es bereits von einer Vielzahl daran anknüpfender Berufsausbildungen ausgeschlossen. Diese Schullaufbahnempfehlung ist in Deutschland außerordentlich

152 BSGE 48, $258 \mathrm{ff}$

153 Ausnahme sind nur Schulabschlüsse, die insbesondere auf die Fächer Musik und Sport ausgerichtet sind, etwa an musischen oder sportlichen Schulen. Diese Ausnahmen können hier jedoch vernachlässigt werden.

154 Vgl. OVG Lüneburg, Beschluss v. 10.7.2008, Az.: 2 ME 309/08, www.rechtsprechung.niedersachsen.de, Rn. 14; OVG Berlin-Brandenburg, Beschluss v. 16.6.2009, Az.: 3 M 16.09, juris Rn. 4; auch Kischel, in BeckOK GG, Art. 3, Rn. 219.3: Sie gehen davon aus, dass die Rechtschreibung nur in den Fächern gewertet wird, in denen sie auch Teil der Leistung ist, und in den Naturwissenschaften und Mathematik nicht Prüfungsgegenstand ist oder sich nur rudimentär auswirkt. 
unterschiedlich ausgestaltet. ${ }^{156}$ Die Tendenz geht weg von einer Entscheidung der Eltern hin zu einer höheren Gewichtung der Lehrerempfehlung ${ }^{157}$, vielfach ausdrücklich gebunden an bestimmte Durchschnittsnoten, i.d.R. 2,0 in den Fächern Deutsch und Mathematik. ${ }^{158}$ Die Sprachkompetenz ist eines der zentralen Elemente für die Empfehlung. ${ }^{159}$

Automatisiertes Lesen und Schreiben ist für die Bildungsempfehlung und alle nachfolgenden Bildungsangebote zentral. ${ }^{160}$ Lesefähigkeiten werden vor allem in der Grundschule im Fach Deutsch benotet. Schlechte Lesefähigkeiten beeinträchtigen jedoch indirekt auch die Leistungen in anderen Fächern, weil insbesondere in Prüfungen die Aufgaben nicht so schnell erlesen werden wie dies bei normalen Lesern der Fall ist. ${ }^{161}$ Zudem können Lesefehler zu Fehlern in der Aufgabenbearbeitung führen, vor allem in den in der Grundschule wichtigen Fächern Mathematik und Sachkunde.

Die Rechtschreibung wird in der Grundschule ebenfalls benotet und ist ein Teil der Deutschnote. Inwieweit Fehler in der Rechtschreibung zu schlechteren Noten in anderen Fächern wie Sachkunde und Mathematik führen, hängt von den landesrechtlichen Vorschriften bzw., wo solche nicht existieren, von der Bewertungspraxis der jeweiligen Lehrkraft ab. In Sachsen-Anhalt enthält bereits der Erlass zur Leistungsbewertung in Grundschulen die Vorschrift, dass gravierende Verstöße gegen grammatische und orthografische Regeln, schwerwiegende Mängel im Ausdruck und im normgerechten Schreiben zu Punktabzug bzw. zu einer Herabsetzung um bis zu einer Notenstufe führen können. ${ }^{162}$

Die Fähigkeit zur Rechtschreibung hat jedoch noch einen weiteren, viel höheren Stellenwert, vor allem für das Fach Deutsch. Je besser die Rechtschreibung ist, desto besser ist die Deutschnote, dies nicht deshalb, weil das Gewicht der Rechtschreibnote so hoch ist, sondern weil von der Fähigkeit im Rechtschreiben unbewusst auf die Sprachkompetenz des Kindes geschlossen wird. ${ }^{163}$

156 S. dazu Informationsunterlage des Sekretariats der KMK -, Übergang von der Grundschule in Schulen des Sekundärbereichs I" Oktober 2010

Bericht IGLU 2006, s. z.B. NI Broschüre des KM: Schullaufbahnempfehlung - Informationen Anregungen - Hilfen, S. 7: Gymnasium Deutsch, Mathematik, Sachkunde 1 - 2; Realschule gleiche Fächer 2 - 3, http://www.ms.niedersachsen.de/download/4438

BW Aufnahmeverordnung§ 4, BY: ab 2010 2,33 in Deutsch/Mathe/Sachkunde für das Gymnasium und 2,66 in den gleichen Fächern für die Realschule, http://www.km.bayern.de/eltern/schularten/uebertritt-schulartwechsel.html, HB AufnahmeVO § 10 IV 2: Leistungen über dem Regelstandard in Deutsch und Mathematik; ST Sek IÜg-VO§ 1 VI; TH SchuO §§ 124, 125 Muñoz, Report Special Rapporteuer on the right to education, Rn. 54

160 Roos/Schöler, Schriftspracherwerb, S. 158

161 Klicpera/Schabmann/Gasteiger-Klicpera, Legasthenie, S. 126

162 ST Erlass Grundschulbewertung Nr. 3.1.9.

163 Roos/Schöler, Schriftspracherwerb, S. 129, 130 
Die Bedeutsamkeit der Rechtschreibung für die Deutschnote verstärkt sich noch zwischen der 2. und 4. Klasse und wirkt sich deutlich auf die Bildungsempfehlung aus: Je besser ein Kind im Rechtschreiben abschneidet, desto höher ist die Chance auf die Bildungsempfehlung für das Gymnasium oder die Realschule. ${ }^{164}$ Dementsprechend hat bereits die Rechtschreibleistung am Anfang der zweiten Klasse eine bedeutende Wirkung für den späteren Schulerfolg. ${ }^{165} \mathrm{Im}$ Umkehrschluss folgt daraus, dass allein eine schlechte Rechtschreibung sowohl die Chancen verringert gute Noten in Deutsch zu erzielen als auch die Chancen, eine Bildungsempfehlung für Realschule oder Gymnasium zu bekommen.

Mathematik ist das Fach, das neben Deutsch die Möglichkeiten des Übergangs auf eine weiterführende Schule am meisten mitbestimmt. In der Regel erfordert der Übergang auf das Gymnasium ein "gut" in Mathematik, für den Übergang auf die Realschule mindestens ein "befriedigend“. ${ }^{166}$ Schüler mit Dyskalkulie haben Schwierigkeiten insbesondere im Erlernen der Grundrechenarten, also des Stoffes, der in der Grundschule gelehrt wird. Deshalb sind ihre Noten in Mathematik im Grundschulbereich besonders schlecht. Häufig erreichen sie nur mit äußerster Anstrengung ein „ausreichend“. Damit ist ein Übergang auf eine andere Schulart als die Hauptschule praktisch ausgeschlossen. ${ }^{167}$

\subsection{Sekundarstufe I}

Im Sekundarbereich I sind die Anforderungen bezüglich der Rechtschreibung nicht einheitlich. Einige Bundesländer schreiben die Berücksichtigung von Rechtschreibfehlern in Klassenarbeiten zwingend vor $^{168}$, andere Bundesländer haben dazu keine Regelung. Deshalb ist es falsch anzunehmen, dass die Rechtschreibung nur in den Fächern gewertet wird, in denen sie auch Teil der Leistung ist und in den übrigen Fächern nicht gewertet wird. ${ }^{169}$

Schlechte Leistungen im Rechnen wirken sich im Sekundarbereich I naturgemäß auf die Note in Mathematik aus. In den höheren Klassen kommt es zu Schwierigkeiten in Physik und Chemie sowie in Fächern wie Erdkunde, kaufmännisches Rechnen, Hauswirtschaft oder ähnlichen.

164 Roos/Schöler, Schriftspracherwerb, S. 132

165 Vanselow/Dummer-Smoch, Vernachlässigung individueller Begabungsstrukturen, S. 20, 71; s. auch für die 4. Klasse Schleider, Lese- und Rechtschreibstörungen, S. 81

166 S. Nachweise Fn. 157 und 158

167 So die Regelung in Bremen: s. HB LRS-Erlass, Teil II, zweitletzter Absatz unter Verweis auf $\S 6$ a HB SchVwG

168 Z.B. NRW APO-S-I § 6 VI, HH Förder-Richtlinie Nr. 4.2 (Absenkung um eine Note); § 21 II; SN SOGY

169 So aber Kischel, in: BeckOK GG, Art. 3, Rn. 219.4; OVG Lüneburg, Beschluss v. 10.7.2008, Az.: 2 ME 309/08, www.rechtsprechung.niedersachsen.de, Rn. $14 \mathrm{f}$ 
Beim Wechsel von der Hauptschule auf die Realschule oder von der Realschule auf das Gymnasium während der Sekundarstufe I sind in der Regel gute Noten in den Fächern Deutsch, Fremdsprache und Mathematik erforderlich. ${ }^{170}$ Diese guten Leistungen können Betroffene nicht erbringen, weil die Noten in Deutsch und der Fremdsprache wegen der erheblichen Auswirkungen der schlechten Rechtschreibung auf die Note meistens allenfalls ein befriedigendes Niveau erreichen. Die Folge ist, dass den Betroffenen ein Wechsel auf diese Schulen nicht möglich ist.

\subsection{Sekundarbereich II - Oberstufe}

Die Voraussetzungen für den Wechsel vom Sekundarbereich I in die Oberstufe eines Gymnasiums ergeben sich aus der "Vereinbarung über die Schularten und Bildungsgänge im Sekundarbereich I" der KMK. ${ }^{171}$ Der Besuch der Oberstufe und damit die Möglichkeit, die allgemeine Hochschulreife zu erlangen, setzen voraus, dass der Schüler die besonderen Eintrittsbedingungen erfüllt. Geht er bereits ins Gymnasium muss er in allen versetzungsrelevanten Fächern mindestens die Note „ausreichend" erzielen (Nr. 6.3. (a) KMK-Sekl). Hat der Schüler den Mittleren Schulabschluss an einer anderen Schule erworben, so muss er im Durchschnitt mindestens befriedigende Leistungen in Deutsch, einer Fremdsprache und Mathematik und in allen versetzungsrelevanten Fächern im Durchschnitt befriedigende Leistungen aufweisen (Nr. 6.3.(b) KMK-SekI). Diese Voraussetzung können Betroffene wegen der Bewertung der Rechtschreibung, der schlechten Leseleistung und/oder im Rechnen ${ }^{172}$ in der Regel nicht erbringen.

Die gymnasiale Oberstufe ist stärker als die Schulen des Sekundarbereichs I durch Vereinbarungen der KMK geregelt. Die Ausgestaltung erfolgt nach der „Vereinbarung zur Gestaltung der gymnasialen Oberstufe in der Sekundarstufe

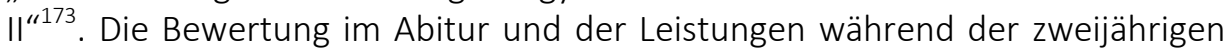
Qualifikationsphase erfolgen nach der Vereinbarung über die Abiturprüfung der gymnasialen Oberstufe in der Sekundarstufe II. Diese sieht in $\S 6$ Abs. 2 KMK-Abitur-Gymnasiale-Oberstufe ${ }^{174}$ vor, dass gehäufte Verstöße gegen die sprachliche Richtigkeit zu einer Abwertung der Note um 1 bis 2 Punkte der

\footnotetext{
170 Z.B. MV VKDVO § 11; NI DVO; § 9; SN SOMIAP § 9 II; ST Sek I-Üg-VO § 2 II; TH SchulO § 125

171 KMK-Sek I, s. Anlage 2: Beschlüsse und Empfehlungen der KMK

172 VG Düsseldorf, Beschluss v. 16.11.2009, Az.: 18 L 1654/09, juris, Rn. 5, 19 - die Schülerin mit Dyskalkulie konnte nicht auf die Oberstufe übertreten, weil sie in Mathematik „ausreichend“ hatte. Eine Berücksichtigung der Dyskakulie lehnte das Gericht mit dem Hinweis auf den Grundsatz der Chancengleichheit ab.

173 KMK-Sek II

174 KMK-Abitur-Gym-Oberstufe
} 
einfachen Wertung führen. ${ }^{175}$ Es kann aber auch zu Abzügen von bis zu 4 Punkten kommen. ${ }^{176}$

Die Fächer werden noch nach den „Einheitlichen Prüfungsanforderungen im Abitur" (EPA) ${ }^{177}$ der KMK unterrichtet, die in den Ländern als Grundlage der fachspezifischen Anforderungen in der Abiturprüfung nach den Gegebenheiten der jeweiligen Abiturbestimmungen übernommen werden (Nr. 1 KMK-VB-EPA) und die es zurzeit für 42 Fächer gibt. ${ }^{178}$ Die EPA enthalten genaue Vorgaben zum Themenumfang, zu den Prüfungen und zur Leistungsbewertung. Die Vorschrift, dass gehäufte Verstöße gegen die sprachliche Richtigkeit zu Abzügen führen, enthalten fast alle EPA, meist unter Punkt 3.5, auch die für bildende Kunst, Musik und Sport. Die Rechtschreibung ist damit als Prüfungsgegenstand aller Fächer festgelegt. Ausnahmen sind nur die Fächer Chinesisch, Japanisch und Darstellendes Spiel. Weil die Fächer in der Qualifikationsphase bereits auf der Grundlage der EPA erteilt werden, gilt die Vorschrift auch schon in dieser Zeit.

Die KMK hat nunmehr durch Beschluss vom 18.10.2012 verbindliche Bildungsstandards für die Allgemeine Hochschulreife in den Fächern Deutsch, Mathematik und Fortgeführte Fremdsprache Englisch/Französisch beschlossen. ${ }^{179}$ Diese werden die EPA ersetzen und sollen spätestens im Schuljahr 2016/17 in allen Bundesländern im Abitur umgesetzt werden. Auch diese Bildungsstandards enthalten jeweils unter Punkt 3.1.2 die Vorgabe, dass schwerwiegende und gehäufte Verstöße gegen die sprachliche Richtigkeit oder gegen die äußere Form zu einem Abzug von bis zu zwei Punkten in einfacher Wertung führen.

Hinzu kommen Vorgaben für die Fremdsprachen. Die EPA sehen spezielle fachspezifische Grundsätze vor und differenzieren dabei zwischen der inhaltlichen und der sprachlichen Leistung. ${ }^{180}$ Bei der Bildung der Gesamtnote kommt der sprachlichen Leistung, die die Rechtschreibung beinhaltet, die größere Bedeutung zu. Sog. Sperrklauseln bewirken, dass eine Gesamtnote von besser

175 Zu den Auswirkungen s. z.B. VG Hannover, Beschluss v. 13.12.2010, Az.: 6 B 5596/10, www.rechtsprechung.niedersachsen.de, S. 2

176 So der Sachverhalt in VG Frankfurt a.M., Beschluss v. 10.09.2009 5 K 1501/09.F(1), S. 6 (nicht veröffentlicht), nachgängig VGH Kassel, Beschluss v. 17.11.2010, Az.: 7 A 2970/09.Z, juris), der Schülerin wurden in Deutsch durchgängig die Maximalpunktzahl von 4 Punkten und in Wirtschaft und Politik die Maximalpunktzahl von 2 Punkten abgezogen.

177 KMK-VB-EPA

178 Zur Rechtmäßigkeit der Verweisung der landesrechtlichen Prüfungsverordnungen auf die EPA: OVG Bautzen Urteil v. 18.8.2010, Az.: 142/09, Sächs.VBI. 2011, S. 35 ff, S. 37

179 Beschluss der KMK vom 18.10.2012, Standards s. Anhang ॥

180 Z.B. Nr. 3.5.2 EPA Englisch; Nr. 3.5.2 EPA Französisch; Nr. 3.5.2 Italienisch; Nr. 3.5.2 Spanisch 
als 3 Punkten nicht mehr möglich ist, wenn eine ungenügende Leistung im sprachlichen oder inhaltlichen Teil vorliegt. ${ }^{181}$

Die Abiturnote setzt sich aus den Leistungen in der Qualifikationsphase und denen der Abiturprüfung zusammen. Die Leistungen der vier Schulhalbjahre der Qualifikationsphase und die Leistungen der Abiturprüfung fließen im Verhältnis 2:1 in die Gesamtnote ein. ${ }^{182}$ Die Noten haben daher Relevanz, soweit sie in die Gesamtqualifikation einfließen. ${ }^{183}$ So ist es durchaus wahrscheinlich, dass sich z.B. Fehler in der Rechtschreibung im Fach Mathematik durchgängig verschlechternd auswirken, weil die Noten in den Klausuren durchgängig um zwei Punkte abgewertet werden. Deshalb fällt dann die Halbjahreszensur um zwei Punkte geringer aus, als es an sich dem Leistungsstand entspricht. Im Leistungskurssystem verstärkt die Mehrfachwertung der Leistungskursfächer diesen Abzug noch einmal. De facto kann dies dazu führen, dass vier bis sechs Punkte weniger erzielt werden, als ohne Bewertung der Rechtschreibung. Über den Zeitraum der Oberstufe summiert sich dieser Punktabzug weiter und führt durch die Verpflichtung zur Einbringung dieser Noten in die Abiturnote zu einer Verschlechterung der Abiturnote.

\subsection{Abitur}

Der Abschluss der Qualifikationsphase berechtigt nicht automatisch zur Abiturprüfung. Es bedarf vielmehr der Zulassung, die an Mindestnoten gekoppelt ist. Schließt ein Schüler in der Qualifikationsphase einen zwingend zu belegenden Kurs, etwa in Mathematik, mit „ungenügend" (= 0 Punkte) ab, so kann er nicht zum Abitur zugelassen werden. ${ }^{184}$

Die meisten Länder haben das „klassische“ Grund- und Leistungskurssystem abgelöst durch ein System von drei bis sechs vierstündigen Fächern auf erhöhten und weiteren Fächern auf grundlegendem Anforderungsniveau. Dabei werden die Fächer Deutsch, Mathematik und eine Fremdsprache in der Regel auf dem erhöhten Anspruchsniveau, d.h. im Leistungskursniveau, unterrichtet und

181 S. dazu auch die Darstellung der Bewertung in VG Schleswig Urteil v. 10.6.2009, Az.: 9 A 208/08, BeckRS 200938123

182 KMK-SekII Nr. 9.3.2.

183 KMK-SekII Nr. 9.3.3.; das OVG Berlin-Brandenburg, Beschluss vom 16.6.2009, Az.: 3 M 16.09, juris, Rn. 5, sieht die Regelung wohl als verhältnismäßig an, weil der Punktabzug begrenzt ist und nicht mehr als 2 Punkte betragen kann; fraglich könnte das aber in Hessen sein, wo ein Abzug bis zu vier Punkten in Deutsch möglich ist, s. VG Frankfurt a. M., Beschluss v. 10.09.2009 5 K 1501/09.F(1), S. 6 (nicht veröffentlicht), nachgängig VGH Kassel, Beschluss V. 17.11.2010, Az.: 7 A 2970/09.Z, juris.

KMK-Sek II Nr. 9.3.6.: „Dabei dürfen unter den eingebrachten Schulhalbjahresergebnissen höchstens $20 \%$ mit weniger als 5 Punkten und darf kein Ergebnis mit 0 Punkten bewertet sein.", s. z.B. § 34 III 4 HE SchulG 
müssen als Kernfächer verpflichtend im Abitur geschrieben werden. ${ }^{185}$ Die Abiturnote setzt sich zusammen aus Noten, die in den zwei letzten Schuljahren erzielt wurden, und den Noten aus der Abiturprüfung.

In der Abiturprüfung führen Fehler in der sprachlichen Richtigkeit wegen der Regelungen in den EPA zu Punktabzügen von mindestens 2 Punkten. ${ }^{186}$ Ausdrücklich ist das z.B. in Brandenburg für das Zentralabitur $2011^{187}$ geregelt: Bei einem Fehlerquotienten von 3,0 (= 3 Fehler auf 100 Wörter) wird 1 Punkt abgezogen. Der Abzug kann bis zu 2 Punkte betragen. Diese Abzugsregelung gilt für die Fächer Geografie, Geschichte, Politische Bildung, Mathematik, Physik, Chemie und Biologie. Für die Fremdsprachen gelten besondere Regelungen. Im Leistungskurs Englisch führt ein Fehlerquotient von 5,6 (= $5-6$ Fehler auf 100 Wörter) dazu, dass die Leistung nur noch mit maximal 3 Punkten bewertet werden kann. ${ }^{188}$ Schlechte Rechtschreibung kann hier schon dazu führen, dass der Schüler nicht zum Abitur zugelassen wird, weil er die Voraussetzungen zur Zulassung zum Abitur nicht erfüllt. ${ }^{189}$ Sie wirkt sich auch umfassend auf die Noten aus der Abiturprüfung und damit auf die Durchschnittsnote aus.

Für Schüler mit Legasthenie war es im alten Leistungskurssystem noch möglich, durch eine geschickte Fächerauswahl zumindest zu vermeiden, dass sie zwei sprachliche Leistungskurse absolvieren mussten. Damit konnte die Gefahr, dass die Legasthenie sich massiv auf die Leistungen in den Abiturfächern auswirkt, etwas verringert werden. In den Ländern, die jetzt zwingend die Abiturprüfung in den Fächern Deutsch und einer Fremdsprache auf Leistungskursniveau vorschreiben, werden sich die schlechten Leistungen im Lesen und Rechtschreiben erheblich auswirken ${ }^{190}$ : Die Schüler müssen in zwei sprachlichen Fächern einen erhebliches Lesepensum vor allem in den Prüfungen bewältigen. Ihre Arbeiten werden wegen der schlechten Rechtschreibung regelmäßig abgewertet und in den Fremdsprachen kommt es wegen der o.g. Sperrklauseln dazu, dass wegen schlechter Leistungen im sprachlichen Teil selbst bei sehr guten Leistungen im inhaltlichen Teil kein "ausreichend" mehr erzielt werden kann. Deshalb wird es für sie schwierig sein, die Vorgabe zu erfüllen, dass in mindestens zwei bzw. drei schriftlichen Abiturfächern (je nachdem ob das Abitur aus vier oder fünf Fächern besteht) mindestens 5 Punkte erzielt werden. ${ }^{191}$

185 KMK-Sek II Nr. 8.3.

186 S. z.B. HH APO-AH § 10 II

187 BB RS 7/09 Leistungsbewertung Abitur 2011

188 BB RS 7/09 Leistungsbewertung Abitur 2011

189 KMK-Sek II Nr. 9.3.6.

190 S. dazu die umfassende Darstellung der Abituranforderungen in SH im neuen System im Urteil VG Schleswig Urteil v. 10.6.2009, Az.: 9 A 208/08, BeckRS 200938123

191

KMK-Sek II Nr. 9.3.7. 
Für Schüler und Schülerinnen mit Dyskalkulie wird es kaum möglich sein, einem Mathematik-Unterricht auf erhöhtem Anforderungs- bzw. Leistungskursniveau zu folgen, so dass die große Gefahr besteht, dass sie an der 0 Punkte-Klausel ${ }^{192}$ scheitern.

3. Die schulrechtliche Regelung zur Berücksichtigung von Legasthenie in Bayern

Bayern hat 1999 eine sehr viel weiter reichende Regelung zur Berücksichtigung von Schwierigkeiten beim Lesen und Rechtschreiben erlassen als die anderen Bundesländer: Die Kultusministerielle Bekanntmachung (KMBek) ${ }^{193}$ unterscheidet zwischen der Legasthenie und einer Lese-Rechtschreibschwäche. Die Legasthenie ist durch ein kinder- und jugendpsychiatrisches Gutachten nachzuweisen und dann durch den zuständigen Schulpsychologen für die Schule festzustellen. Mit der Feststellung des Schulpsychologen muss die Legasthenie in der Schule bis einschließlich zum Abitur berücksichtigt werden. Den Schülern muss in den schriftlichen Arbeiten ein Zeitzuschlag gewährt werden. Hinzu kommen weitere Formen des Nachteilsausgleichs je nach individuellem Bedarf. Darüber hinaus wird die Rechtschreibung bei den betroffenen Schülern in keinem Fach gewertet. Die Gewichtung der schriftlichen und mündlichen Leistungen in den Fremdsprachen erfolgt im Verhältnis 1:1. Eine Lese-Rechtschreibschwäche kann demgegenüber allein durch den Schulpsychologen festgestellt werden. Die Regelungen, die bei Legasthenikern zwingend anzuwenden sind, stehen bei Schülern mit Lese-Rechtschreibschwäche im Ermessen der Lehrkräfte. Allerdings reduziert die Feststellung des Schulpsychologen das Ermessen der Lehrkräfte nahezu auf null.

4. Grundzüge der Beschlüsse der KMK und der Landesvorschriften zur Berücksichtigung von Legasthenie und Dyskalkulie - mit Ausnahme von Bayern

Die Tatsache, dass es Schüler und Schülerinnen gibt, die erhebliche Schwierigkeiten im Erlernen des Lesen, Schreibens, Rechtschreibens und/oder Rechnens haben, hat sowohl die KMK als auch die Bundesländer dazu veranlasst, für diesen Bereich besondere Regelungen zu erlassen. ${ }^{194}$ Bis auf Bayern unterscheiden diese Regelungen nicht zwischen Schülern und Schülerinnen mit Schwierigkeiten im Lesen, Rechtschreiben und Rechnen und Schülern und Schülerinnen mit Legasthenie und Dyskalkulie, sondern gelten für alle Schüler und Schü-

\footnotetext{
S. z.B. § 34 III 4 HE SchulG

193 BY KMBek Legasthenie

194 S. Anhang 3: Schulrechtliche Vorschriften der Bundesländer
} 
lerinnen mit Schwierigkeiten in diesen Bereichen. Der Rechtscharakter der Regelungen ist sehr unterschiedlich: In Berlin finden sich die Vorschriften in den allgemeinen Schulordnungen ${ }^{195}$, Baden-Württemberg hat eine allgemeine Verwaltungsvorschrift für "Kinder und Jugendliche mit Behinderungen und besonderem Förderbedarf" erlassen. ${ }^{196}$ In Thüringen gelten fachliche Empfehlungen für Kinder und Jugendliche mit besonderen Lernschwierigkeiten in den allgemein bildenden Schulen (außer Förderschule), wozu auch Verhaltensstörungen zählen ${ }^{197}$, i.V.m. § $59 \mathrm{~V}$ und VI TH SchulO.

Die Vorschriften regeln die Grundsätze der Förderung, des Nachteilsausgleichs und des Abweichens von bestimmten Leistungsbewertungen für Schüler mit Schwierigkeiten im Lesen, Rechtschreiben und Rechnen. Überwiegend findet sich keine eigenständige Definition der Schwierigkeiten, zum Teil werden die Schwierigkeiten angenommen, wenn die schulischen Leistungen über einen bestimmten Zeitraum schlechter als "ausreichend" sind. ${ }^{198}$ Zusätzlich finden sich in den Regelungen Begriffe wie "besonders schwere Beeinträchtigung" (KMK), „besonders begründete Ausnahmefälle“ (Baden-Württemberg), „gravierende Lese-Rechtschreibstörung" und "gravierende Lese-Rechtschreibstörung gutachterlich festgestellt" (Berlin), "veränderungsresistente Schwierigkeiten" (Bremen), „ausgeprägte Schwierigkeit", „besonders und lang anhaltende Schwierigkeiten" (Rheinland-Pfalz) und "schwere Lese-Rechtschreibschwäche" (Thüringen). Keiner dieser Begriffe wird in den Regelungen definiert.

Sind die Schwierigkeiten festgestellt, liegt es im Ermessen vor allem der Deutschlehrer und der Klassenkonferenzen, ob und in welcher Weise auf die Schwierigkeiten Rücksicht genommen wird.

Je höher die Klassenstufe ist, desto strenger werden die Voraussetzungen für die Berücksichtigung. Für die Legasthenie wird der Anspruch auf Ausgleich häufig zeitlich und auch schulformspezifisch begrenzt. Ab der 5 . Klasse wird die Gewährung von Ausgleich an immer strengere Kriterien geknüpft und bis auf wenige Ausnahmen mit der 10. Klasse beendet. Einige Länder gewähren Ausgleich in der Hauptschule, lassen inn aber in der Realschule und dem Gymnasium oder ab Klasse 7 des Gymnasiums nicht zu. ${ }^{199}$ Einige Länder lassen Ausgleich in der Sekundarstufe I zu, nicht aber in den Abschlussklassen und Ab-

\footnotetext{
195 BE GsVO §§ 14, 16; BE Sek I - VO §§ 14, 16; BE VO-GO § 31 II

196 BW VV Besonderer Förderbedarf

197 TH Fachliche Förderempfehlung

198 BW VV Besonderer Förderbedarf Nr. 2.3.2; HB LRS Erlass Nr. 4.2., SL VV-LRS Nr. 2.1, 4 und 4.5

199 SL VV-LRS Nr. 5.1 - bis Klasse 9; SN VV-LRS Nr. 4.3, 4.4 bis Oberstufe möglich; NRW LRSErlass Nr. 4 (bis Klasse 6, begründete Einzelfälle bis 10, keine Unterscheidung der Schulform); HH Förder-Richtlinie Nr. 4 (bis Klasse 8)
} 
schlussprüfungen dieser Schulstufe. ${ }^{200}$ Überwiegend wird Ausgleich in der Sekundarstufe II nicht mehr gewährt. ${ }^{201}$ Ausnahmen gelten für Brandenburg ${ }^{202}$, Bremen ${ }^{203}$, Hessen ${ }^{204}$, Mecklenburg-Vorpommern ${ }^{205}$, Niedersachsen ${ }^{206}$, Sachsen-Anhalt ${ }^{207}$, Schleswig-Holstein ${ }^{208}$ und Thüringen. ${ }^{209}$

Ein gebundener Anspruch auf Berücksichtigung der Legasthenie/Dyskalkulie besteht, mit Ausnahme von Bayern, nicht. Überwiegend sehen die Regelungen vor, dass die Schwierigkeiten im Lesen und Rechtschreiben bei sonst angemessener Gesamtleistung kein Grund sein sollen, den Schüler von einem Wechsel an eine weiterführende Schule auszuschließen. ${ }^{210}$

\section{Berücksichtigung der Dyskalkulie in der Schule}

Die KMK-Empfehlung vom 4.12.2003 hat erstmals Schwierigkeiten im Rechnen mit aufgenommen und die Berücksichtigung zumindest in der Grundschule ermöglicht. Inzwischen ist die Berücksichtigung von Schwierigkeiten in Mathematik in der Grundschule und teilweise auch im Sekundarbereich I möglich in Baden-Württemberg ${ }^{211}$, Brandenburg $^{212}$, Bremen $^{213}$, Hamburg $^{214}$, Hessen $^{215}$,

200 BW VV Besonderer Förderbedarf Nr. 2.3.2; BE Sek I-VO § 14 III; NI LRR-Erlass Nr. 4.2; SN VVLRS Nr. 4.4; ST VO Sonderpäd. Förderung § 7 III; TH Fachliche Förderempfehlung L.u.Z.

201

202

203

BE VO-GO (nicht vorgesehen); NRW LRS-Erlass Nr. 4; RP VV-LRS Nr. 1; SN VV-LRS Nr. 4.4

BB VV-LRSR Nr. 7।

HB LRS-Erlass Nr. 4.1 und 4.4.1 und 4.4.2. - die Berücksichtigung ist in der Abschlussprüfung Mittlerer Schulabschluss ausgeschlossen, denn dann gelten die verbindlichen Korrekturanweisungen (Nr. 4.4.1)

HE VO-GestSchulVerh § 39 IV - aber nicht mehr im Abitur - so VGH Kassel, Beschluss V. 5.2.2010, Az.: 7 A 2406/09, juris

MV VV-LRR Nr. 7

NI LRR-Erlass Nr. 4.2 - nicht in Abschlussprüfung

ST VO Abschlüsse $\S 20$

SH Legasthenie-Erlass Nr. 1.1.1

THSchulO § 110 S. 2 i.V.m. § 59: nur Nachteilsausgleich aber kein Aussetzen der Bewertung des Rechtschreibens oder Rechnens im Abitur

BE GsVO § 16 IX - allerdings unpräzise; BB VV-LRSR Nr. 2 VII; BY KMBek Legasthenie Nr. 3.5; HB LRS-Erlass Nr. 4.3 und HB AufnahmeVO § 10 IV 3; HH Förder-Richtlinie Nr. 4.4; HE VOGestSchulVerh § 37 V; MV VV-LRR Nr. 7 II; NI LRR-Erlass Nr. 4.2; NRW LRS-Erlass Nr. 4.3; RP VV-LRS Nr. 4.5; SL VV-LRS Nr. 5.4, s. dazu bereits 1972 VG Hannover, Urteil v. 10.2.1972, Az.: VI A 197/69, SPE 800, das die Überweisung einer Schülerin auf die Sonderschule aufhob, weil die Sonderschule zur Förderung der Legastheniker ungeeignet sei.

BW VV Besonderer Förderbedarf Nr. 2.2 - Grundschule

BB VV-LRSR Nr. 6 II - Förderung auch in der Sek II, Nr. 7 I - Nachteilsausgleich nur bis KI. 10

HB LRS-Erlass Teil II - Grundschule, nicht aber beim Übergang auf die weiterführende Schule

- zweitletzter Absatz - Verweis auf $§ 6$ a BremSchVwG

HH Förder-Richtlinie Nr. 4 - Grundschule 
Tatsächlicher Problemaufriss

Mecklenburg-Vorpommern ${ }^{216}$, Niedersachsen ${ }^{217}$, Sachsen-Anhalt ${ }^{218}$, SchleswigHolstein $^{219}$ und Thüringen. ${ }^{220}$ Die sonstigen Voraussetzungen entsprechen den Regelungen für Schwierigkeiten im Lesen und Rechtschreiben. In Bayern ist die Dyskalkulie nicht als Lernschwierigkeit anerkannt, die zu einem Nachteilsausgleich berechtigt. ${ }^{221}$

\section{Förderschule statt Regelschule?}

Angesichts der Schwierigkeiten der Schüler und Schülerinnen mit Legasthenie/ Dyskalkulie und der Notwendigkeit, sie speziell zu fördern, könnte man auf die Idee kommen, die betroffenen Schüler und Schülerinnen auf Förderschulen zu beschulen. Für die meisten Menschen ist Sonderbeschulung behinderter oder beeinträchtigter Menschen keine Diskriminierung, sondern notwendige Folge einer körperlichen, geistigen oder seelischen Beeinträchtigung. ${ }^{222}$ Tatsächlich

215 HE VO-GestSchulVerh § 39 IV 3 - Grundschule

216 MV VV-LRR Nr. 7 III - Grundschule und Sekundarstufe

217 NI LRR-Erlass Nr. 3.2 - einschließlich Sekundarbereich I

218 ST VO Abschlüsse § 20 - Sekundarstufe I

219 SH Rechenschwäche-Erlass

220 TH Fachliche Förderempfehlung F.b.b.L. - einschließlich Sekundarstufe I. Dazu steht in Widerspruch, dass $\S 59 \vee \mathrm{TH}$ SchulO einen Nachteilsausgleich bei Rechenschwäche nicht erwähnt. § 59 TH SchulO wird allerdings vom KM Thüringen sehr weit ausgelegt und auch als Grundlage für Nachteilsausgleich bei nicht ausreichenden Deutschkenntnissen angesehen, s. dazu Fachliche Empfehlungen zum Schulbesuch und zur Förderung von Schülerinnen und Schülern nichtdeutscher Herkunftssprache in Thüringen (Stand Juli 2012), S. 9 f, http://www.thueringen.de/imperia/md/content/tmbwk/bildung/schulwesen/empfehlungen /fachliche_empfehlung_schueler_auslaendischer_herkunft.pdf, so dass dennoch auch die Rechenschwäche von § 59 TH SchulO umfasst ist. http://www.schulberatung.bayern.de/schulberatung/bayern/fragen_paed_psy/lern_leis tungsschwierigkeiten

222 S. die Nachweise bei Degener, ZaöRV 2005, S. 887 ff, S. 897; Rothfritz, UN-BRK, S. 384. Die Entscheidung des VGH Kassel, Beschluss v. 12.11.2009, Az.: 7 B 2763/09, juris, Rn. 41, zeigt, dass die Rechtsprechung einen solchen defizitorientierten Ansatz vertritt: Der VGH Kassel ordnet nämlich die Überweisung auf die Förderschule als eine erlaubte, geradezu geforderte besondere Maßnahme i.S. d. Art. 5 Abs. 4 BRK ein, die zur Beschleunigung oder Herbeiführung der tatsächlichen Gleichberechtigung von Menschen mit Behinderung erforderlich sei (Rn. 45). Nach Art. 5 Abs. 4 BRK gelten „,[b]esondere Maßnahmen, die zur Beschleunigung oder Herbeiführung der tatsächlichen Gleichberechtigung von Menschen mit Behinderungen erforderlich sind, ... nicht als Diskriminierung." Damit sind nach dem Handbuch zur BRK jedoch „positive actions“ wie spezielle Subventionen oder Quoten gemeint (United Nations, From Exclusion to Equality, S. 66 ff; Degener, ZaöRV 2005, S. 887 ff, S. 915), die den Menschen mit Behinderung ausdrücklich gegenüber Menschen ohne Behinderung bevorzugen, um insgesamt die Gleichberechtigung der Menschen mit Behinderung voranzutreiben. In der Überweisung eines Kindes auf eine Förderschule eine derartige „Bevorzugung“ zu sehen, 
besuchen $16 \%$ der Kinder mit Legasthenie trotz allgemein durchschnittlicher Intelligenz Förderschulen. ${ }^{223}$ Da es nur mit ganz wenigen Ausnahmen Schulen mit spezieller Förderung bei Legasthenie oder Dyskalkulie gibt ${ }^{224}$, kommt als Förderschwerpunkt nur der Schwerpunkt "L“, also Lernbehinderung in Betracht. ${ }^{225}$ Die Hoffnung ist, dass die Schüler und Schülerinnen dort „dringend erforderliche Entwicklungsschritte" machen und ihnen "Frustrationserlebnisse“ erspart bleiben. ${ }^{226}$ Diese Hoffnung erfüllt sich aber, ausweislich einer Studie von Wocken, durchgängig nicht: „Je länger ein Schüler in der Förderschule zugebracht hat, desto schlechter sind sowohl seine Rechtschreibleistungen als auch seine Intelligenztestwerte. ${ }^{\text {“227 }}$ Dagegen spricht auch, dass grundsätzlich anerkannt ist, dass die betroffenen Schüler und Schülerinnen zu angemessenen Gesamtleistungen fähig und damit prinzipiell zum Besuch weiterführender Schulen geeignet sind. ${ }^{228}$ Unter diesen Umständen wäre eine Überweisung auf eine Förderschule "L" unverhältnismäßig.

Da die Förderschulen „L“ den Besonderheiten der Schüler und Schülerinnen mit Legasthenie/Dyskalkulie nicht gerecht werden können, kämen nur neue Förderschulen in Betracht. Es ist inzwischen durch viele Untersuchungen nachgewiesen, dass der Besuch von Sonderschulen stigmatisiert und insbesondere die Schüler der Sonderschulen für Lernbehinderte keine bleibenden positiven Effekte beim Selbstbewusstsein erreichen, die ihre Stigmatisierung kompensieren. ${ }^{229}$ Vor allem die Sonderschule für Lernbehinderte ist eine Sackgasse für die

verschließt die Augen vor sämtlichen Erkenntnissen über die negativen Wirkungen von Förderschulen (vgl. dazu Wocken, Andere Länder, andere Schüler? und Schumann, „Ich schäme mich ja so!").

223 Schleider, Lese- und Rechtschreibstörungen, S. 81

224 Spezielle LRS-Klassen für die zweite bzw. dritte Klasse gibt es in Sachsen (SN VV-LRS, Nr. 4.2 Aufspaltung der 3. Klasse in die Klassen $3 \mathrm{I}$ und II) und in Mecklenburg-Vorpommern (MV VVLRR Nr. 6 II - selbständige Klassen in den Klassenstufen 2 und 3)

Obwohl als primäres Merkmal einer Lernbehinderung eine unterdurchschnittliche Intelligenz (d.h. IQ < 85) gilt Biermann/Goetze, Sonderpädagogik, S. 202, und diese gerade ein Ausschlusskriterium für die Diagnose einer Legasthenie ist (s. o. S. 5 und S. 16) So die Begründung des VG Braunschweig, Beschluss v. 20.8.2008, Az.: 6 B 196/08, BeckRS 200841195 für eine Sonderschulüberweisung, kritisch dazu: Marwege, RdJB 2009, S. 229 ff, S. 240; zu den negativen Auswirkungen der Beschulung auf Sonder- oder Förderschulen s. Schumann, "Ich schäme mich ja so!“; Pfahl/Powell, Gemeinsam leben - Zeitschrift für integrative Erziehung 2005, 68 ff; Mokatef, Menschenrecht auf Bildung und Schutz vor Diskriminierung, S. 39 ff; grundsätzlich zur Sonderschule als segregierende Institution: Powell, in: Erziehungswissenschaft

227 Wocken, Andere Länder, andere Schüler?, S. 58

228 S. die Nachweise in Fn. 210

229 Umfassend dazu Schumann, „Ich schäme mich ja so!", insbesondere S. 84 ff; s. auch Mokatef, Menschenrecht auf Bildung und Schutz vor Diskriminierung, S. 39; Ennuschat, Die Rechte stotternder Menschen in Schule, Ausbildung und Studium, S. 47, Fn. 67 
betroffenen Kinder, an der sie in zehn Bundesländern keinen Hauptschulabschluss machen können ${ }^{230}$ und aus der ihnen eine Rückkehr in die Regelschule nur außerordentlich selten gelingt. ${ }^{231}$ Die Separierung von Schülern und Schülerinnen in Sonderschulen ist daher generell ein Maßnahme, die nicht neutral wirkt, sondern eine Benachteiligung ist ${ }^{232}$, weil die Einrichtung gleichwertiger, aber getrennter öffentlicher Einrichtungen diskriminierend bleibt. ${ }^{233}$

\section{Fördermöglichkeiten}

\subsection{Schulische Fördermöglichkeiten}

Zentrales Anliegen von Lehrkräften und Schule ist es, Schüler und Schülerinnen zu befähigen, zu lesen, richtig zu schreiben und zu rechnen. Lernen die Kinder diese Grundfertigkeiten nicht im normalen Schulunterricht, sollen sie individuell gefördert werden. ${ }^{234}$ Fördern ist ein grundlegender Auftrag von Schule, dabei wird Fördern verstanden als ein "Weiter-nach-vorn-Bringen" in der individuellen Persönlichkeits-, Lern- und Leistungsentwicklung. ${ }^{235}$

Ist ein Kind beim Erlernen des Lesens, Schreibens und/oder Rechnens auffällig, so wird zunächst versucht den Schwierigkeiten mit binnendifferenzierten Methoden oder durch vermehrtes Üben zu begegnen. ${ }^{236}$ Halten die Schwierigkeiten an, erhält das Kind in der Regel zunächst Förderung in Form der allgemeinen Sprach- oder Deutschförderung. ${ }^{237}$ Vielfach versuchen vor allem die Mütter ihre Kinder zu Hause bei den Hausaufgaben und mit häuslichen Übungsaufgaben zu unterstützen ${ }^{238}$ und werden auch von der Schule gezielt

230 Konsortium Bildungsberichterstattung, Bildung in Deutschland 2008, S. 70, $89 \mathrm{ff}$

231 S. dazu Hohmann-Dennhardt, in: FS Renate Jaeger S. 257 Buch, Grundrecht der Behinderten, S. 109; Berlit, RdJB 1996, S. 145 ff, S. 150 Sachs, RdJB 1996, S. 154 ff, S. 168; Castendiek, in: GS Jean d'Heur, S. 350; König/Peters, in: Grote/Marauhn EMRK/GG, Kapitel 21: Das Diskriminierungsverbot, Rn. 50; umfassend Höller, Beschulung behinderter Kinder und Jugendlicher an öffentlichen Schulen, $194 \mathrm{ff}$ KMK-Lesen/Rechtschreiben/Rechnen, Abschnitt I

235 Hanke/Hein, in: Arnold/Graumann/Rakhhochkine, Hdb. Förderung, S. 390

236 Schleider, Lese- und Rechtschreibstörungen, S. 31 f; vgl. Rahn, Legasthenie bei Erwachsenen, S. 24 f; Typische Beispiele für die schulischen Ansätze, der Problematik zu begegnen, finden sich bei Lenhard/Küspert/Becker, in: Legasthenie

237 Vgl. VG Hamburg, Urteil v. 24.11.2009, Az.: 13 K 4032/07, BeckRS 2009 42354, S. 3 - Tatbestand

238 Z.B. VG Hamburg, Urteil v. 24.11.2009, Az.: 13 K 4032/07, BeckRS 2009 42354, S. 8 Punkt 2.3.3; VG Düsseldorf, Urteil v. 5.3.2008, Az.: 19 K 1659/07, www.justiz.nrw.de; s. dazu die Berichte in Anderson/Meier-Hedde, International Case Studies 
zur täglichen Unterstützung des schulischen Förderprogramms herangezogen. $^{239}$

Eine separate Förderung erfolgt meist erst dann, wenn sich die Leistungen verschlechtern und das Kind schlecht genug für diese Förderung ist. ${ }^{240}$ Diese ist in der Regel keine spezielle Förderung, die am individuellen Problem des Kindes ansetzt ${ }^{241}$, sondern Förderung in einer kleineren Lerngruppe mit Schülern, die ebenfalls Schwierigkeiten im Fach Deutsch haben. Die Zusammensetzung der Gruppe ist meist im Hinblick auf Ursache und Umfang der Schwierigkeiten sehr heterogen. ${ }^{242}$

Die Problematik der allgemeinen Förderung besteht darin, dass Schüler und Schülerinnen mit Legasthenie/Dyskalkulie eine spezifische Förderung brauchen, die präzise an ihren Defiziten ansetzt. Ein Kind, das nicht schätzen und Mengen nicht erfassen kann, ist mit jedem Rechenvorgang überfordert und lernt nichts, wenn der Schulstoff nur wiederholt wird. ${ }^{243}$ Ein Kind, das Laute nicht erkennen kann, lernt nichts, wenn es Diktate übt. ${ }^{244}$ Schwer betroffene Kinder mit Legasthenie brauchen z.B. zunächst Handzeichen oder Lautgebärden, um überhaupt Laute in Schrift umsetzen zu können. ${ }^{245}$ Sie sind darauf angewiesen, dass ihnen beispielweise die Rechtschreibung nicht durch Übung, sondern durch einen Unterricht vermittelt wird, der innen die Rechtschreibstrukturen erläutert und

239 OVG Münster, Beschluss v. 8.9.2008, Az.: 12 A 1752/08, www.justiz.nrw.de, Rn. 16; zum Nutzen eines solchen Übungsprogramms: Schulte-Körne, Elternratgeber Legasthenie, S. 119, der ausführt, dass die Übungseinheiten nur zweimal die Woche erfolgen sollen, weil sie sonst das Kind überfordern.

So ausdrücklich die Aussage der Deutschlehrerin in VG Hamburg, Urteil v. 24.11.2009, Az.: 13 K 4032/07, juris, S. 5 - Tatbestand; zum schulischen Förderangebot s. OVG Münster, Beschluss v. 8.9.2008, Az.: 12 A 1752/08, www.justiz.nrw.de: das schulische Förderangebot bestand aus einer Wochenstunde in einer Gruppe von 9 Schülern. Dieses schulische Förderkonzept beinhaltete auch, dass die Schüler 3 - 4 mal pro Woche unter Kontrolle ihrer Eltern 10 bis 15 min etwas schreiben, dabei auch vor sich hinlesen und versuchen sollten, den Text selbst zu korrigieren (Rn. 16), für die notwendige psychische Betreuung verwies das OVG den Schüler auf eine psychotherapeutische Behandlung (Rn. 18)

Zur Notwendigkeit einer individuellen Förderung Reichelt, Analphabeten, S. 6; Steinhausen, Psychische Störungen bei Kindern und Jugendlichen, S. 164; s. auch Klicpera/Schabmann/ Gasteiger-Klicpera, Legasthenie, S. 267 Tacke, in: Legasthenie und Dyskalkulie 2007, S. 136; s. die Schilderung schulischer Fördermaßnahmen von Klasen, Legasthenie, S. $90 \mathrm{ff}$ Eggers/Fegert/Resch, Psychiatrie, S. 768; s. die Modelle der Entwicklung der Rechenleistung bei Landerl/Kaufmann, Dyskalkulie, S. 86 ff und S. 200, und der Überblick über die Module einer Dyskalkulie-Therapie bei Jacobs/Petermann, in: Lehrbuch der klinischen Kinderpsychologie, S. $215 \mathrm{ff}$

244 Ligges, in: Legasthenie und Dyskalkulie 2007, S. 237 ff; zu den vielfältigen Möglichkeiten, die Kinder zu verwirren, s. Götzinger-Hiebner, Rechtschreibverwirrbuch 
in dem sie spezifische Aufgabenstellungen bekommen. ${ }^{246}$ Üben hilft ihnen nicht, erweist sich z. T. sogar als Nachteil. ${ }^{247}$ Voraussetzung für eine wirksame Förderung ist, dass die Schwierigkeiten des Kindes umfassend untersucht werden und nach den spezifischen Ursachen geforscht wird. Eine solche umfassende Untersuchung der Schwierigkeiten durch die Schule unterbleibt jedoch vor allem in den ersten beiden Grundschulklassen vielfach ${ }^{248}$, auch deshalb, weil die Lehrkräfte das Kind nicht mit der Feststellung von Schwierigkeiten vorverurteilen wollen. ${ }^{249}$ Generell werden eindeutige Untersuchungen und Feststellungen sehr kritisch gesehen: „Die Vorteile der Arbeit mit lernzielnahen, didaktisch orientierten Modellen bestehen ... darin, dass auf weitergehende riskante diagnostische Zuschreibungen (z.B. Legasthenie ...) verzichtet und damit der Gefahr des Etikettierens vorgebeugt wird. “250 Dies hat zur Folge, dass Fördermethoden eingesetzt werden, die, wenngleich durchaus wohlgemeint, für diese besonderen Schwierigkeiten unpassend oder jedenfalls nicht effektiv sind. ${ }^{251}$ Insgesamt ist die Gefahr groß, dass Lehrer, Eltern und Nachhilfelehrer „nur ein oberflächliches Herumdoktern an Symptomen leisten“. ${ }^{252}$

Die bestehende Förderung von Schülern und Schülerinnen mit Legasthenie/ Dyskalkulie in Schulen geht mithin vielfach an ihren besonderen Bedürfnissen vorbei. ${ }^{253}$ Sie sind vielmehr auf eine Förderung - sei sie schulisch oder außerschulisch - angewiesen, die bei ihnen die Voraussetzungen für das Erlernen von Lesen, Schreiben und Rechnen erst schafft.

Zudem ist die Wirksamkeit von Fördermethoden kaum evaluiert: es gibt einen erheblichen Mangel an wissenschaftlichen deutschsprachigen Studien über die Wirksamkeit von schulischen Fördermaßnahmen. ${ }^{254}$ Bei Kindern mit Legas-

246 Roos/Schöler, Schriftspracherwerb, S. 194 m.w.N.

247 Roos/Schöler, Schriftspracherwerb, S. $193 \mathrm{f}$

248 Kany/Schöler, Diagnostik, S. 25 f

249 Aussage der unterrichtenden Lehrkraft im Verfahren LG Lüneburg, Urteil v. 20.4.2009, Az.: 2 O 220/08 (nicht veröffentlicht) - bestätigt durch OLG Celle Beschluss v. 17.2.2010, Az.: $16 \mathrm{U}$ 62/09 (nicht veröffentlicht); zur Notwendigkeit des Screenings ganzer Klassen s. Klicpera/ Schabmann/Gasteiger-Klicpera, Legasthenie, S. 266

250

251 Klasen, Legasthenie, S. $91 \mathrm{f}$, die darauf verweist, dass der Förderunterricht auch bei dem besten Willen der Lehrkräfte unfachmännisch und fachlich unzureichend erteilt wird.

252 Rahn, Legasthenie bei Erwachsenen, S. 25; umfassend Schulte-Körne/Deimel/Remschmidt, Zeitschrift für Kinder- und Jugendpsychiatrie und Psychotherapie 31 (2003), S. $267 \mathrm{ff}$

S. z.B. die angebotenen schulischen Fördermaßnahmen im Fall VG Düsseldorf, Urteil v. 22.1.2001, Az.: 19 K 11140/98, www.justiz.nrw.de, Rn. 82

254 chotherapie 31 (2003), Punkt 1: Einleitung; s. jetzt die Metaanalyse Ise/Engel/Schulte-Körne, Kindheit und Entwicklung 21 (2012), S. $122 \mathrm{ff}$ 
thenie sind die Fördererfolge "deprimierend“. ${ }^{255}$ Langfristige Erfolge, z.B. in Form einer eindeutigen größeren Steigerung der Lesefähigkeit, wären nur mit therapeutischen Maßnahmen, die eine sorgfältige Diagnostik voraussetzen, zu erzielen. ${ }^{256}$ Sie zeigen sich nur bei einer Förderung, die nicht nur einfach intensiver, sondern anders als normale schulische Förderung ist. ${ }^{257}$ Ein Fördererfolg kann sich nur dann einstellen, wenn die Gruppen möglichst leistungshomogen und störungsspezifisch sind und die Gruppengröße maximal 5 Kinder beträgt. ${ }^{258}$

Schließlich sehen sich viele Lehrkräfte, obwohl ihnen die Schwierigkeiten des Kindes bewusst sind und sie die Notwendigkeit der Förderung einräumen, nicht in der Lage, diese Förderung auch tatsächlich durchzuführen. ${ }^{259}$ Von den betroffenen Schülern und Schülerinnen erhalten nur 30 \% eine Förderung in der Schule ${ }^{260}, 40 \%$ eine außerschulische Förderung oder Nachhilfe; etwa $37 \%$ der legasthenen Kinder erhalten weder in der Schule noch außerschulisch Förderung oder Nachhilfe. ${ }^{261}$

\subsection{Außerschulischen Therapie und ihre Finanzierung}

Überwiegend herrscht das Verständnis vor, dass Kinder mit Legasthenie oder Dyskalkulie in der Schule gefördert werden sollen, um ihr Handicap zu überwinden. Wird die Förderung in der Schule nicht angeboten, ist sie nicht effektiv genug oder sieht sich auch die Schule nicht in der Lage, den besonderen Bedürfnissen des Kindes gerecht zu werden, so werden außerschulische Therapien oder Hilfsangebote in Anspruch genommen. Der Markt an außerschulischen Förder- und Therapiemöglichkeiten für Kinder mit Legasthenie/Dyskalkulie ist enorm und unüberschaubar. ${ }^{262}$ Er reicht von Nachhilfestunden und -instituten sowie lerntherapeutischen Ansätzen über Bachblütentherapien, Spezialbrillen, Kinesiologie bis hin zu qualifizierten Psychologen. Ein anerkanntes Be-

\footnotetext{
255 Tacke, in: Legasthenie und Dyskalkulie 2007, S. 136

Steinhausen, Psychische Störungen bei Kindern und Jugendlichen, S. 160

257 Klicpera/Gasteiger-Klicpera, in: Hdb. Sonderpädagogik, S. 361; Warnke/Schulte-Körne, in: Therapie psychischer Störungen, S. 157: Kinder mit Legasthenie benötigen z.B. Regeltraining, der im normalen schulischen Förderunterricht weniger als $1 \%$ ausmacht. Warnke/Schulte-Körne, in: Therapie psychischer Störungen, S. 160

259 Roos/Schöler, Schriftspracherwerb S. 198: „Also man ist da auch als Lehrer ein bisschen überfordert, immer, selbst wenn man es will, auf die Probleme jedes einzelnen einzugehen"; „Man hat gar nicht die Zeit."; „Das ist nicht mein Ding." Zum Anspruch auf Förderung und dem Rechtsweg bei Ablehnung der Förderung Verfassung BVerfG, Beschluss v. 8.6.2012; Az.: 1 BvR 2853/10, http://www.bverfg.de/entscheidungen/rk20120608_1bvr285310.html, Rn. 9 Valtin/Hornberg/Buddeberg/Voss/Kowoll/Potthoff, in: Bos, IGLU 2006, S. 76 
rufsbild des Legasthenie- oder Dyskalkulie-Therapeuten existiert nicht. ${ }^{263}$ Die Wirksamkeit insbesondere von alternativen Therapieansätzen ist teilweise sehr umstritten ${ }^{264}$, auch weil sie überwiegend nicht evaluiert sind. ${ }^{265}$ Lehrkräfte, Schulen, Schulverwaltungen, Eltern und auch die Gerichte ${ }^{266}$ gehen mehr oder weniger selbstverständlich davon aus, dass das Kind, wenn die schulische Förderung nicht ausreicht oder nicht angeboten wird, Anspruch auf eine (qualifizierte) außerschulische Therapie hat und die Kosten zweifellos von der Krankenkasse oder von den Jugendämtern übernommen werden. Dies ist jedoch nicht der Fall.

\subsubsection{Finanzierung durch die Krankenkassen nach § 27 SGB V}

Die Krankenkassen übernehmen die Kosten einer Legasthenie/DyskalkulieTherapie grundsätzlich nicht. Zunehmend, so wird berichtet, weigern sich viele Krankenkassen auch, die Kosten für die Diagnostik zu bezahlen. Diese Haltung gründet sich auf ein Urteil des Bundessozialgerichts von 1979 267 , in dem es verneint hat, dass es sich bei Legasthenie um eine Krankheit handelt. Damit besteht nach § 27 SGB V keine Leistungspflicht der gesetzlichen Krankenkassen für die Diagnose und Behandlung. Das BSG hat in seinem Urteil festgestellt: „Unter einer Legasthenie ist eine Schwäche im Erlernen des Lesens und des Rechtschreibens bei hinreichender Intelligenz und sonst regelrechtem neurologischen Befund zu verstehen. ... Das wesentliche Merkmal dieser Erscheinung besteht darin, dass das Kind beim Erwerb gewisser Fähigkeiten Schwächen und Störungen zeigt. Vergleichbare Störungen können sich allerdings auch beim Erwerb anderer Fähigkeiten einstellen, wie z.B. beim Erlernen mathematischer Kenntnisse, bei der Erlernung von Fremdsprachen, beim Erwerb musischer Kenntnisse oder bei der Einübung sportlicher Fähigkeiten; solchen Erscheinungen kommt kein Krankheitswert i.S.d. § 182 RVO (heute § 27 SGB V) zu. ... Eine Störung der natürlichen körperlichen und/oder geistigen Funktionen eines Menschen wird erst dann zur Krankheit i.S. des Gesetzes mit der Folge der Leistungspflicht der GKV, wenn diese Funktionen über eine bestimmte Bandbreite individueller Verschiedenheiten hinaus in einem so beträchtlichen Maße einge-

263 Der Bundesverband Legasthenie und Dyskalkulie e.V. versucht mit der Zertifizierung von Fortbildungsinstituten einen einheitlichen Standard in der Weiterbildung zu setzen. Therapeuten, die an diesen Instituten ihre Ausbildung gemacht haben, dürfen sich Dyslexie- bzw. Dyskalkulie-Therapeut nach BVL nennen. http://www.bvl-legasthenie.de/index.php5?p=/zertifizierung

264 S. dazu umfassend: Suchodoletz, Therapie der Lese-Rechtschreib-Störung S. $15 \mathrm{ff}, 167 \mathrm{ff}$

265 Warnke/Schulte-Körne, in: Therapie psychischer Störungen, S. 158

266 So das VG Hamburg, Urteil v. 24.11.2009, Az.: 13 K 4032/07, BeckRS 2009 42354, S. 10 Punkt 5.

267 BSGE 48, $258 \mathrm{ff}$ 
schränkt sind, dass ihre Wiederherstellung der Mithilfe des Arztes bedarf. "268 Diese Auffassung, die sich damals allein auf die Definition im medizinischen Wörterbuch Pschyrembel stützte, ist heute unter Berücksichtigung der neueren wissenschaftlichen Erkenntnisse zu den Ursachen und zu den genetischen Faktoren der Legasthenie nicht mehr haltbar. Hinzu kommt, dass meines Wissens Legasthenie und Dyskalkulie die einzigen Störungen bzw. Krankheiten sind, deren Behandlung nicht von den gesetzlichen Krankenkassen übernommen wird, obwohl sie im ICD 10 verzeichnet sind, der nach $\S 295$ Abs. 1 SGB V die Grundlage für die Abrechnung der Krankenkassen ist.

Eine Revision des Urteils des BSG würde im Moment die Lage jedoch nicht verändern, denn die Zahlung der Legasthenie/Dyskalkulie-Therapien durch die Krankenkassen ist durch die Heilmittel-Richtlinien ausgeschlossen. Der gemeinsame Bundesausschuss der Ärzte und Krankenkassen hat nach $\S 5$ HeilM-RL in der Anlage zu den Heilmittel-Richtlinien ${ }^{269}$ unter Buchst. b, Ziff. 4 die Indikation Lese-Rechtschreibschwäche selbst bei nachgewiesenem therapeutischem Nutzen vom Anwendungsbereich ausgenommen. Wird die Therapie durch einen Pädagogen durchgeführt, entfällt die Leistungspflicht der gesetzlichen Krankenkasse auch deshalb, weil dieser nicht unter ärztlicher Leitung handelt und vor allem eine pädagogische Leistung erbringt. ${ }^{270}$ Allerdings werden psychotherapeutische Behandlungen, die als Folge der Legasthenie/Dyskalkulie erforderlich werden, vom BSG als sekundäre Folgestörung und Krankheit anerkannt, die von der Krankenkasse zu zahlen ist. ${ }^{271}$

268

269

BSG, BSGE 48, 258, 264

Richtlinie des Gemeinsamen Bundesausschusses über die Verordnung von Heilmitteln in der vertragsärztlichen Versorgung (HeilM-RL) in der Fassung vom 20. Januar 2011/19. Mai 2011, veröffentlicht im Bundesanzeiger 2011; Nr. 96 (S. 2247) in Kraft getreten am 1. Juli 2011; http://www.g-ba.de/downloads/62-492-532/HeilM-RL_2011-05-19_bf.pdf; Anlage zu den Richtlinien des Gemeinsamen Bundesausschusses über die Verordnung von Heilmitteln in der vertragsärztlichen Versorgung (Heilmittel-Richtlinien) vom 21. 12. 2004

BSG SozR 2200 Nr. 48 zu § 182 RVO, LSG Bayern, Urteil v. 23. 3. 2006; Az.: L 4 KR 279/04, JAmt 2006, S. 314 - 316; LSG Baden-Württemberg, Urteil v. 28.1.2000 - L 4 KR 4592/98 ELSG KR-169 S. 3; OLG Saarbrücken, Urteil v. 16.1.2008, 5 U 287/07, VersR 2008, S. 1382 ff, BGH Beschluss v. 11.2.2009, IV ZR 28/08, VersR 2009, S. 533

BSG, Urteil vom 25. 7. 1979 - 3 RK 45/78 SozR 2200 Nr. 48 zu § 182 RVO; mit anderen Worten: Die Behandlung der Grundstörungen Legasthenie/Dyskalkulie durch eine geeignete Therapie ist keine Kassenleistung, wohl aber die Behandlung der teilweise massiven psychischen Folgestörungen, die sich aus der nicht therapierten Legasthenie/Dyskalkulie ergeben. Vergleichbar wäre es, wenn die Krankenkassen die Behandlung von motorischen Beeinträchtigungen mit Ergotherapie oder Stottern mit Logopädie ablehnen würden, wohl aber die Kosten für die Behandlung der psychischen Schäden übernehmen würden, die die unbehandelte motorische Beeinträchtigung oder das unbehandelte Stottern hervorruft; sehr kritisch dazu Warnke/Wewetzer, Berufsverband der Ärzte für Kinder- und Jugendpsychiatrie und Psychotherapie Deutschland e.V. 1997, 61 ff, S. 71. 


\subsubsection{Finanzierung durch die Jugendämter nach $\S 35$ a SGB VIII}

Die Finanzierung der außerschulischen Hilfen und der außerschulischen Legasthenie/Dyskalkulie-Therapien kann noch über $\S 35$ a SGB VIII durch die Jugendämter erfolgen. ${ }^{272}$ Nach $\S 10$ Abs. 1 SGB VIII sind die Jugendämter zwar nur nachrangig zuständig, wenn aber weder Krankenkassen noch Schulen die Förderung leisten, bleibt es bei der Zuständigkeit der Jugendämter. ${ }^{273}$

Das Verhältnis zwischen Jugendhilfe und Schule ist schwierig und konfliktbeladen. Die Jugendhilfe sieht die Schulen vorrangig in der Pflicht, notwendige Förderangebote für Legasthenie/Dyskalkulie anzubieten, hält die Nichterfüllung dieser Pflicht für rechtswidrig und beruft sich deshalb häufig auf die Nachrangklausel des $\S 10$ SGB VIII. ${ }^{274}$ Sie empfindet sich als „Ausfallbürge“ für die Schule. ${ }^{275}$

Die Eingliederungshilfe für seelisch behinderte Kinder und Jugendliche gem. $\S 35$ a SGB VIII hat das Ziel, die Integration derjenigen jungen Menschen, die aufgrund einer Beeinträchtigung ihrer seelischen Gesundheit an der altersgemäßen umfassenden Teilhabe am Leben in der Gesellschaft beeinträchtigt sind, zu ermöglichen. ${ }^{276}$ Nach ganz h.M. reicht allein die Diagnose Legasthenie/Dyskalkulie für einen Anspruch nach $\S 35$ a SGB VIII nicht aus. ${ }^{277}$ Deshalb finanzieren die Jugendämter grundsätzlich keine Therapien allein wegen der Diagnose Legasthenie/Dyskalkulie.

Praxis und Rechtsprechung legen die Anspruchsvoraussetzungen des $\S 35 \mathrm{a}$ SGB VIII außerordentlich eng aus. Die Gerichte vertreten zunehmend die Ansicht, dass die Diagnose Legasthenie/Dyskalkulie noch nicht ausreicht, sondern eine seelische Störung i.S.d. § 35 a Abs. 1 S. 1 Nr. 1 SGB VIII erst dann vorliegt, wenn sekundäre Folgen, die zu der Legasthenie/Dyskalkulie hinzutreten müssen, die seelische Gesundheit beeinträchtigen. ${ }^{278}$ Zusätzlich muss die seelische

272 Zur Notwendigkeit wegen der Vorgaben der BRK die für Kinder je nach Behinderungsart bisher im SGB IX und im SGB VIII getrennt geregelte Eingliederungshilfe zusammenzuführen s. Banafsche, SGb 2012, S. 373 ff, S. $440 \mathrm{ff}$, S. $443 \mathrm{f}$

273 VGH Kassel, Urteil v. 20.8.2009, Az.: 10 A 1874/08, juris, Leitsatz und Rn. 47

274 Meysen, JAmt 2003, S. 53 ff, S. 54 ff; sehr kritisch dazu Mrozynski, in: SGB VIII, § 35 a, Rn. 8

275 Meysen, JAmt 2003, S. $53 \mathrm{ff}$ Harnach-Beck, in: Jans/ Happe/ Saurbier/ Maas KJHG, § 35 a, Rn. 1

Riehle, in: Krug/ Riehle SGB VIII, § 35 a, 8 (5); Meysen, in: Frankfurter Kommentar SGB VIII, § 10, Rn. 24; Vondung, in: Kunkel SGB VIII, § 35 a, Rn. 19; Harnach-Beck, in: Jans/Happe/Saurbier/Maas KJHG, § 35 a, Rn. 39; Wiesner, in: SGB VIII § 35 a, Rn. 71

Abweisend z.B.: OVG Koblenz, Beschluss in PKH-Verfahren v. 26.3.2007, 7 E 10212/07, NJW 2007, S. 1993 ff; OVG Münster, Beschluss v. 23. 1. 2009, Az.: 12 A 2897/09, BeckRS 2009 34706; OVG Lüneburg, Beschluss v. 4.2.2009, Az.: 4 LC 514/07, www.rechtsprechung.niedersachsen.de; OVG Weimar, Beschluss v. 17.5.2010, Az.: 1 EO 854/10, juris; VGH München, Urteil v. 24.6.2009, Az.: 12 B 09.602, juris; VGH Kassel, Urteil v. 20.8.2009, Az.: 10 A 1799/08, juris; OVG Bautzen, Beschluss v. 20.8.2009, Az.: 1 B 432/09, 
Störung die Teilhabe am Leben beeinträchtigen. Nach der Rechtsprechung reichen bloße Schulängste, wie sie jedes normale Kind gelegentlich hat, nicht aus, um eine seelische Behinderung zu bejahen. Eine drohende seelische Behinderung wird erst dann angenommen, wenn eine auf Versagensängsten beruhende Schulphobie, totale Schul- und Lernverweigerung, Rückzug aus jedem sozialen Kontakt oder Vereinzelung in der Schule vorliegen. ${ }^{279}$ In der Realität bedeutet dies, dass die Kinder in ihrem sozialen Verhalten bereits massiv beeinträchtigt sein müssen, bevor eine Therapie bewilligt wird. ${ }^{280}$

\subsubsection{Finanzierung durch die Eltern}

Infolgedessen wird der ganz überwiegende Anteil an Legasthenie- oder Dyskalkulie-Therapien von den Eltern privat bezahlt. ${ }^{281}$ Eltern nehmen diese Kosten

BeckRS 2009 39566; VGH München; Beschluss v. 14.9.2010; Az.: 12 CE 10.1981, juris; VGH München, Beschluss v. 9.11.2010; Az.: 12 ZB 09.1251

stattgebend: VG Augsburg, Urteil v. 24.3.2009, Az.: Au 3 K 08.1139; VG Frankfurt (Oder), Beschluss v. 1.7.2009, Az.: 6 K 50/05, juris; VGH Kassel, Urteil v. 20.8.2009, Az.: 10 A 1874/08, juris, OVG Lüneburg v. 25.3.2010, Az.: 4 LA 43/09, juris, Rn. 2, vorgehend VG Braunschweig, 18. 12. 2008, Az: 3 A 55/08, juris; OVG Bautzen, Beschluss v. 9.6.2009, Az.: 1 B 288/09; www.justiz.sachsen.de; VG Köln, Urteil v. 21.1.2010, Az.: 26 K 4295/09, juris; VG Aachen, Urteil v. 30.9.2010; Az.: 1 K 1858/08, juris; VG Aachen, Urteil v. 8.6.2010; Az.: 2 K 1357/07, juris; s. auch Schwengers, Eingliederungshilfe $\S 35$ a SGB VIII und sehr kritisch Mrozynski, in: SGB VIII, § 35 a , Rn. $7 \mathrm{ff}$

BVerwG, Urteil v. 26. 11.1998, Az.: 5 C 38.97, FEVS 33, 457 zu § 39 BSHG; VG Oldenburg, Beschluss vom 10. 6. 2004, AZ: 13 B 1332/04; Beck-RS; VG Göttingen, Urteil vom 29.8.2006, Az.; 2 A 184/05, www.rechtsprechung.niedersachsen.de. Hier ergibt sich eine erhebliche Diskrepanz zwischen der juristischen und der medizinischen Einschätzung für die Notwendigkeit einer Therapie überhaupt. Die von der Rechtsprechung für erforderlich gehaltenen Beeinträchtigungen werden nämlich in der Kinder- und Jugendpsychiatrie für so schwerwiegend gehalten, dass sie nicht nur Indikation für Therapie überhaupt, sondern bereits Indikation für ansonsten restriktiv gehandhabte teilstationäre oder sogar stationäre Langzeittherapien sind Eggers/Fegert/Resch, Psychiatrie, S. 149, 766; Mierau, in: Legasthenie und Dyskalkulie 2011, S. 37

S. dazu die massiven Beeinträchtigungen, die dem Fall VGH Kassel, Urteil v. 20.8.2009, Az.: 10 A 1874/08, juris, zugrunde lagen, die aber dem VG Darmstadt dennoch nicht ausreichten, um eine Abweichung von der seelischen Gesundheit zu bejahen.

281 Zur Frage, ob diese Kosten im Rahmen der Grundsicherung übernommen werden müssen, s. SG Halle, Beschluss v. 19.3.2010, Az.: S 7 AS 1072/10 ER, juris. Das SG Halle hat die Übernahme der Kosten durch die Grundsicherung prinzipiell bejaht (Rn. 20), im Einzelfall aber abgelehnt, weil die durchgeführte Form der Förderung in Form einer "klassischen Nachhilfe" in einer Gruppe mit 5 Mitschülern bei Legasthenie nicht hilfreich und deshalb auch nicht im Rahmen der Grundsicherung zu übernehmen sei. Bei Legasthenie sei vielmehr eine gezielte Legasthenietherapie in Form einer Einzeltherapie erforderlich (Rn. 21), sowie SG Hildesheim, Beschluss v. 15.7.2010, Az.: S 55 AS 1010/10, BeckRS 2010, 72100, bejahend, und LSG Niedersachsen-Bremen, Beschluss v. 5.8.2010, Az.: L 8 SO 143/10 B ER, juris, ablehnend 
Tatsächlicher Problemaufriss

auch deshalb über Jahre hinweg auf sich, weil das Schulrecht die Berücksichtigung einer Legasthenie davon abhängig macht ${ }^{282}$, dass das betroffene Kind gefördert wird und diese Förderung regelmäßig nachgewiesen wird. ${ }^{283}$ Da Schulen in der Praxis jedoch spätestens ab etwa der 7. Klasse keine Förderung für die betroffenen Kinder mehr anbieten, kann der schulrechtlich geforderte Nachweis über eine Förderung nur über die privat bezahlten außerschulischen Therapien erbracht werden. ${ }^{284}$

Der finanzielle Aufwand, den Eltern für die Therapie betreiben müssen, ist erheblich. Er liegt bei einem Förderzeitraum von etwa 3 Jahren bei einer monatlichen Belastung von ca. $180 € €^{285}$ Ob die Eltern diesen Betrag steuerlich als außergewöhnliche Belastung geltend machen können, hängt davon ab, ob sie

282 S. KMK-Lesen/Rechtschreiben/Rechnen, Punkt: Abschlüsse und Prüfungen (S. 5)

283 Z.B. HE VO-GestSchulVerh $\S 42$ I: die Berücksichtigung der Legasthenie bei der Leistungsfeststellung und Leistungsbewertung ist nur möglich, wenn eine andauernde Förderung des Schülers erfolgt ist, wobei nach HE VO-GestSchulVerh § 40 II 2 auch eine außerschulische Förderung zu berücksichtigen ist.

So die Sachlage im Fall VG Frankfurt, Urteil v. 10.9.2009, Az.: 5 K 1501/09.F, S. 10 (nicht veröffentlicht)

285 Berechnung bei 40 Therapiestunden pro Jahr, 120 Stunden Förderung und einem Stundensatz von $45,00 €$ plus 8,55 € MWSt (= 53,33 €/Stunde). Zum Umfang der Förderung s. Reuter-Liehr, in: Legasthenie und Dyskalkulie 2007, S. 129; vgl. VG Hamburg, Urteil v.

24.11.2009, Az.: 13 K 4032/07, BeckRS 2009 42354, S. 1 Tatbestand; zum Stundensatz s. die Erhebung im Raum Leipzig im Urteil des VG Leipzig v. 22.11.2007; Az.: 5 K 1733/05, juris, Rn. 43. Die ermittelten Sätze liegen zwischen 25,00 € und 55,45€. Der VGH Kassel hat den beklagten Landkreis im Urteil v. 20.8.2009, Az.: 10 A 1874/09, juris, ausdrücklich zur Übernahme von $42,00 € /$ Therapiestunde verurteilt. Es scheint durchaus üblich zu sein, dass die Therapieeinrichtungen mit den Jugendämtern Stundensätzen abrechnen, die ihnen von den Jugendämtern vorgegeben werden, und dann von den Eltern die Differenz zum tatsächlichen Stundensatz verlangen, so OVG Lüneburg, Urteil v. 12.10.2006, Az.: 2 A 173/05, www.rechtsprechung.niedersachsen.de: der bekl. Landkreis erstattete aufgrund eigener Richtlinie 23,00 € bzw. 31,00 € pro Behandlungseinheit, die Therapeuten verlangten jedoch erheblich mehr. Das OVG Lüneburg stellte fest, dass diese Praxis der Rechtslage bei $\S 35 \mathrm{a}$ SGB VIII widerspricht; zur Umsatzsteuerpflicht s. BFH Urteil v. 18.8.2005 V R 71/03, Rn. 20 www.bundesfinanzhof.de unter Bezugnahme auf das oben dargestellte Urteil des BSG von 1979, BSGE 48, 258, 264: Legasthenie-Behandlungen grundsätzlich nicht nach § 4 Nr. 14 UStG steuerfrei, da Legasthenie keine Krankheit im versicherungsrechtlichen Sinn ist. Mit der Legasthenie-Behandlung wird daher auch keine Heilbehandlung erbracht, die eine Befreiung von der Umsatzsteuer begründen könnte. Die Jugendämter sind bei diesen Leistungen allerdings - im Gegensatz zu den Eltern - nach Artikel 13 Teil A Abs. 1 Buchstabe g der europäischen Richtlinie 77/388/EWG von der Umsatzsteuer befreit. 
den Nachweis erbringen können, dass die Therapie medizinisch indiziert ist ${ }^{286}$, weil die Legasthenie Krankheitswert hat. ${ }^{287}$

\section{Formen der Berücksichtigung in der Schule}

Die Berücksichtigung von Legasthenie/Dyskalkulie in der Schule lässt sich auf drei Bereiche aufteilen: Förderung, Nachteilsausgleich und Abweichung von allgemeinen Grundsätzen der Leistungsfeststellung und -bewertung. ${ }^{288}$ Für die möglichen Formen der Berücksichtigung muss zwischen dem Unterrichtsalltag, Prüfungen und spezieller Förderung unterschieden werden.

Im Unterrichtsalltag geht es um eine Gestaltung des Unterrichts, die auf die besonderen Belange der betroffenen Kinder Rücksicht nimmt. Diese Möglichkeiten der Berücksichtigung sind, unabhängig von der Frage der Einzelförderung, vielfältig und angesichts der stetig fortschreitenden technischen Entwicklung sehr wandelbar ${ }^{289}$ : Beispiele täglicher Hilfestellungen sind: Der schriftliche Arbeitsanteil wird reduziert wird. Die Lehrkraft schreibt Aufgabenblätter nicht mit der Hand sondern benutzt Schriftarten, die keine Serifen enthalten und daher besser lesbar sind ${ }^{290}$, der Zeilenabstand wird vergrößert, die Arbeitsvorlagen werden vergrößert (z.B. Kopie von Din A4 auf Din A3). ${ }^{291}$ Texte werden vorgelesen, auf umfangreiche „Abschreibeaufgaben“ wird verzichtet. Beim

286 BFH, Urteil v. 11.11.2010, Az.: VI R 17/09, juris, Rn. 26: „medizinisch indiziert ist jedes diagnostische oder therapeutische Verfahren, dessen Anwendung in einem Erkrankungsfall hinreichend gerechtfertigt (angezeigt) ist"

Zum Nachweis der medizinischen Indikation ist nach der Rspr. des BFH, Urteil v. 11.11.2010, Az.: VI R 17/09, juris, Rn. 18, 24, 26, jetzt nicht mehr erforderlich, dass diese durch ein vorheriges amtsärztliches Gutachten nachgewiesen wird. Aufgabe der bisherigen ständigen Rspr. des BFH, s. z.B. Beschluss v. 19.5.2009, Az.: VI B 159/08, juris, Rn. 6; BFH, Urteil v. 3.3.2005, II R 64/ 03, BFH/NV 2005, 1286-1287

S. dazu Deutschen Gesellschaft für Lesen und Schreiben, Zehn Rechte der Kinder: http://www.dgls.de/die-dgls/zehn-rechte-der-kinder.html, und der Forderungskatalog des Bundesverbandes Legasthenie und Dyskalkulie e.V.: http://www.bvllegasthenie.de/index.php5? $\mathrm{p}=/$ schule/schulfoerderunglegas\#02

S. z.B. die Vorschläge für die Unterrichtsgestaltung bei autistischen Kindern: http://www.sghl.de/forum/index.php?page=Attachment\&attachmentID=99\&h=ffb06baa42a ba9b9c7a59a4ed76988e10a54f03a und Richter, in: Legasthenie; s. auch die umfassenden Hilfeleistungen in den Niederlanden, die die Bezahlung eines Laptops mit entsprechender Software, die Ausleihe von Büchern und gesprochenen Schulbüchern beinhalten, http://www.steunpuntdyslexie.nl/sitemanager.asp?pid=1801. In Norwegen erhalten legasthene Schüler einen Laptop mit einem speziellen norwegischen Rechtschreibprogramm für Legastheniker http://lingit.de/aktuelles/neues/eine_eins_in_der_muttersprache_-_mit_ legasthenie

290 Schriftart mit Serifen (z.B. Palatino); Schriftart ohne Serifen (z.B. Arial, Calibri, Tahoma)

291 S. dazu umfassend: Verband Dyslexie Schweiz, Richtlinien Produktion Lernmaterialien, der die niederländischen Richtlinien übernommen hat. 
Abschreiben der Hausaufgabenliste wird geholfen, Schüler müssen Tafelanschriften nicht abschreiben, sondern dürfen sie fotografieren oder - etwas altmodischer - ein Mitschüler schreibt mit Hilfe von Kohlepapier die Tafelanschrift für den beeinträchtigten Schüler mit usw. ${ }^{292}$ Die Kinder dürfen Hefte mit anderer Lineatur (z.B. Lineatur der 3. Klasse), Lesehilfen (spezielle Lineale u.ä.) oder Zählmaterialien benutzen. Sie sitzen so im Klassenzimmer, dass das Abschreiben von der Tafel leichter ist. Technische Geräte wie z.B. Laptops, MP3Player oder auch Diktiergeräte werden eingesetzt.

Bei Prüfungen wird zwischen Nachteilsausgleich und sog. Schutzmaßnahmen differenziert. ${ }^{293}$ Als Nachteilsausgleich kommen z.B. in Betracht: besondere Gestaltung von Aufgabenblättern (nicht handgeschrieben, besondere Schrift und Schriftgröße, besonderer Zeilenabstand), Vorlesen von Aufgaben, Umwandlung von schriftlichen Aufgabenstellungen in MP3-Formate, die von MP3Playern abgehört werden können, Einsatz von Diktiergeräten und Laptops (mit oder ohne Rechtschreibprogramm), Zeitzuschläge in schriftlichen Prüfungen, Benutzung von Taschenrechnern und Formelsammlungen, Benutzung von konkretem, didaktischen Material, Anpassung der Aufgabe an den gesicherten Leistungsstand des Schülers (z.B. bei Diktaten oder in Mathematik, d.h. Aufgaben der Klassenstufe, aber nur in dem Zahlenraum, den das Kind sicher beherrscht (z.B. Hunderterraum)), Lückendiktate, Ersatz von schriftlichen Prüfungen durch mündliche Prüfungen (z.B. Vokabeltests) usw. Welcher Nachteilsausgleich erforderlich ist, hängt von den konkreten Schwierigkeiten und der konkreten Prüfung ab: So ist z.B. das Erlesen eines Aufgabentextes, den die Lehrerin in Französisch handschriftlich und mit der in Frankreich üblichen Handschrift schreibt, für einen Schüler mit Legasthenie viel schwieriger bis unmöglich als das Erlesen des gleichen Aufgabentextes, wenn dieser getippt und auf die Größe A 3 vergrößert wurde. Das Erlesen eines Textes für eine Inhaltsangabe ist für die betroffenen Schüler sehr zeitaufwändig, weil sie langsam lesen. Wird ihnen der Text auf MP3-Player gespielt, können sie den Text abhören und dann ebenso eine Inhaltsangabe schreiben wie die anderen Schüler.

Als sog. Schutzmaßnahmen werden angesehen: Befreiung von üblichen Prüfungsteilen, wie Diktat oder Mathematik-Diktat, Befreiung vom Vorlesen oder Vorrechnen an der Tafel, Nicht-Bewertung des Lesens, Schreibens, Rechnens, abweichende Gewichtung von mündlichen und schriftlichen Noten bei der Bildung von Gesamtnoten und Versetzung oder Zulassung zu Prüfungen trotz ungenügender Leistung. ${ }^{294}$

\footnotetext{
292 S. dazu die Auflistung der möglichen Hilfestellungen des Landesverbandes Legasthenie und Dyskalkulie Hessen e. V LVL Hessen, Empfehlung Nachteilsausgleich

293 Die Differenzierung beruht auf dem Gutachten von Langenfeld, RdJB 2007, S. $211 \mathrm{ff}$

294 S. z.B. auch die Auflistung möglicher Maßnahmen in HE GestSchulVerh § 7 ॥
} 
Im Bereich der Förderung wird eine Förderung gefordert, die an den speziellen Bedürfnissen der betroffenen Schüler und Schülerinnen ansetzt und von qualifizierten Lehrern und Lehrerinnen durchgeführt wird.

\section{Kapitel: Zusammenfassung}

Legasthenie und Dyskalkulie sind Störungen, die nach medizinischen Kriterien diagnostiziert werden können und die Vorgaben der ICD 10 erfüllen. Eine präzise Abgrenzung von schwächeren Formen der Legasthenie und Dyskalkulie ist kaum möglich, vor allem weil der Begriff der "Schwäche" eine Vielzahl von Problemen unabhängig von deren Ursachen und Gründen umfasst.

Die schlechten Leistungen der Betroffenen im Lesen, Rechtschreiben und Rechnen wirken sich erheblich auf die Noten und damit auch auf die Schullaufbahnmöglichkeiten bzw. Schulabschlüsse aus. Die Betroffenen können deshalb häufig keinen begabungsgerechten Schulabschluss machen, sie bleiben deutlich hinter ihren intellektuellen Fähigkeiten zurück. Die Schwierigkeiten in der Schule führen zu psychischen Beeinträchtigungen, die teilweise so massiv sind, dass die Betroffenen nicht mehr beschulbar sind. Die Störung beeinträchtigt und behindert die Teilhabe der Betroffenen an der schulischen Ausbildung und anschließend auch an den beruflichen und gesellschaftlichen Möglichkeiten. 



\section{Teil: Rechtlicher Problemaufriss}

Die Problematik von Schülern und Schülerinnen mit Legasthenie/Dyskalkulie liegt darin, dass sie mit nicht-betroffenen Schülern formell gleichbehandelt werden und die allgemeinen schulischen Anforderungen und Vorschriften uneingeschränkt auch für sie gelten. Nach den vorangegangenen tatsächlichen Erörterungen wäre indes eine Veränderung der Prüfungsbedingungen für die betroffenen Kinder und damit eine Ungleichbehandlung mit Schülern und Schülerinnen ohne diese Beeinträchtigung angezeigt. Es ginge also dann um ein Recht auf Berücksichtigung der Differenz. ${ }^{295}$ Fraglich ist daher, ob sich aus den Grundrechten ein solcher Anspruch auf Ungleichbehandlung ergibt. Für die Beantwortung dieser Frage reicht es nicht aus, so wie bei anderen Grundrechten, darauf abzustellen, ob hier Eingriffe der öffentlichen Gewalt in die Freiheitssphäre des Bürgers abgewehrt werden sollen, denn das Verhältnis zwischen Schülern und Schule ist mehrdimensional und vielschichtig. Der Schüler braucht die Schule, damit sie ihm grundlegende Kenntnisse vermittelt, ihn ausbildet und inm Lebensmöglichkeiten eröffnet. Schule soll Schüler individuell fördern, kann sich aber wiederum nicht nur auf einzelne Schüler konzentrieren. Sie muss die Ausbildung vieler Schüler und Schülerinnen bewirken und vielfältige Interessen der Schüler wie auch der Gesellschaft berücksichtigen. Deshalb sind zunächst die Grundlagen dieses Sonderstatusverhältnisses Schule darzustellen, in dem dann die Ausgangsfrage zu beantworten ist.

\section{Kapitel: Das Sonderstatusverhältnis Schule}

Das Schulverhältnis ist ein gesetzlich geordnetes öffentlich-rechtliches Rechtsverhältnis, in dem Eingriffe in die Grundrechte gesetzlich legitimiert sein müssen. ${ }^{296}$ Dass die Grundrechte nicht nur auch in der Schule gelten ${ }^{297}$, sondern vielmehr angesichts der erheblichen Einschränkung des Persönlichkeitsrechts

\footnotetext{
295 S. dazu ausführlich Fuerst, Behinderung zwischen Diskriminierungsschutz und Rehabilitationsrecht, S. $85 \mathrm{ff}$

296 Avenarius/Heckel, Schulrecht, Ziff. 24.111

297 Vgl. Langenfeld, RdJB 2007, S. 211 ff, S. 212
} 
des einzelnen Kindes durch die allgemeine Schulpflicht gerade in der Schule ${ }^{298}$, ist inzwischen unstreitig. Ein Grundrecht auf Bildung ist zwar nach ganz herrschender Meinung im Grundgesetz nicht ausdrücklich enthalten ${ }^{299}$, es ist aber in fast allen Länderverfassungen geregelt. ${ }^{300}$ Aus Art. 2 । GG, 3 । GG, 12 I GG und dem Sozialstaatsgebot hat jedoch jedes Kind Anspruch auf Erziehung und Bildung durch Einrichtungen der staatlichen Gemeinschaft. ${ }^{301}$ Darüber hinaus hat Deutschland mit der Ratifikation des 1. Zusatzprotokolls zur EMRK das Recht auf Bildung aus Art. 2 1. Zusatzprotokoll begründet ${ }^{302}$ und dies auch mit der Ratifikation der Behindertenrechtskonvention (BRK) völkerrechtlich noch einmal bekräftigt. ${ }^{303}$ Der Anspruch wird umgesetzt durch den nach allgemeiner Ansicht in Art. 7 I GG begründeten Erziehungsauftrag des Staates. ${ }^{304}$ Ziel dieses Erziehungsauftrages ist es zu gewährleisten, dass allen jungen Bürgern gemäß ihren Fähigkeiten die dem heutigen gesellschaftlichen Leben entsprechenden Bildungsmöglichkeiten eröffnet werden. ${ }^{305}$ Außerdem ermöglicht es der Erziehungsauftrag des Staates, grundlegende Elemente der Gesellschaft an die nachfolgenden Generationen zu vermitteln und die Integration des Gemeinwesens zu pflegen und zu fördern. ${ }^{306}$ Ein entwickeltes öffentliches und kostenloses Schulwesen ist Basis für jede funktionierende moderne Demokratie. ${ }^{307}$

298 Reichenbach, Anspruch behinderter Schülerinnen und Schüler auf Unterricht in der Regelschule, S. 211

299 Langenfeld, Integration und kulturelle Identität zugewanderter Minderheiten, S. 222 m.w.N. in Fn. 52; umfassend sowohl zur völkerrechtlichen Lage als auch zur deutschen Lage sowohl in Bezug auf den Bund als auch auf die Länder: Poscher/Rux/Langer, Recht auf Bildung

300 Art. 11 I Verfassung Baden-Württemberg, Art. 128 I Verfassung Bayern; Art. 20 I 1 Verfassung Berlin; Art. 29 I Verfassung Brandenburg; Art. 27 I Verfassung Bremen; Art. 59 II Verfassung Hessen; Art. 4 I Verfassung Niedersachsen; Art. 8 Verfassung Mecklenburg-Vorpommern; Art. 8 I 1 Verfassung Nordrhein-Westfalen; Art. 31 I Verfassung Rheinland-Pfalz; Art. 27 VI Verfassung Saarland; Art. 29 Verfassung Sachsen; Art. 25 I Verfassung Sachsen-Anhalt; Art. 20 Verfassung Thüringen; Art. 8 II Verfassung Schleswig-Holstein; nur im Schulgesetz § 1 HH SchulG

301 Niehues/Rux, Schulrecht, Rn. 166; zur historischen Entwicklung; Marwege, RdJB 2009, S. $229 \mathrm{ff}$, S. 229

302 Umfassend dazu Langenfeld, RdJB 2007, S. $412 \mathrm{ff}$

303 S. dazu Riedel, Gutachten UN-Behindertenrechtskonvention, S. 7 ff

304 Langenfeld, Integration und kulturelle Identität zugewanderter Minderheiten, S. 213 m.w.N.; Ennuschat, RdJB 2007, S. 271 ff, S. 274, 275, 277

305 BVerfGE, 47, $48 \mathrm{ff}$ - st. Rspr.; vgl. umfassend Langenfeld, Integration und kulturelle Identität zugewanderter Minderheiten, S. $212 \mathrm{ff}$

306 Langenfeld, Integration und kulturelle Identität zugewanderter Minderheiten, S. 217

307 Langenfeld, Integration und kulturelle Identität zugewanderter Minderheiten, S. 218; Ennuschat, RdJB 2007, S. 271 ff, S. 275, S. 277 
Wesentlicher Inhalt des Erziehungsauftrages des Staates ist die unstreitig zulässige Festlegung der allgemeinen Schulpflicht. ${ }^{308}$ Der Staat greift damit zwar erheblich sowohl in das Grundrecht der Kinder aus Art. 2 I GG auf freie Entfaltung der Persönlichkeit als auch in das Grundrecht der Eltern auf Erziehung ihrer Kinder aus Art. 6 II GG ein ${ }^{309}$, dieser Eingriff ist aber regelmäßig gerechtfertigt, weil die Schulpflicht dazu dient, den Bildungsanspruch des Kindes umzusetzen.

Der staatliche Erziehungsauftrag wird allerdings wiederum durch das Recht des Kindes auf eine möglichst ungehinderte Wahrung und Entfaltung seiner Persönlichkeit und damit seiner Anlagen und Befähigungen begrenzt. ${ }^{310}$ Das kindliche Entfaltungsrecht ist insofern ein "konkretisiertes Spezialgrundrecht", das durch die Verbindung mit der Menschenwürde aus Art. 1 GG eine Schutzverstärkung erfährt. ${ }^{311}$ Das Recht auf freie Entfaltung der Persönlichkeit aus Art. 2 । GG ${ }^{312}$ umfasst daher auch die Gewährung einer freien Persönlichkeitsentwicklung ${ }^{313}$, weshalb die Schule so ausgestaltet sein muss, dass sie dies ermöglicht.

Das Schulverhältnis wird weiter durch die Bindung aller Staatsgewalt ${ }^{314}$ an den allgemeinen Gleichheitssatz des Art. 3 I GG geprägt, wonach gleiche Sachverhalte gleich und ungleiche Sachverhalte ungleich behandelt werden müssen. ${ }^{315}$ Das BVerfG hat den Gleichheitssatz des Art. 3 । GG in ständiger Rechtsprechung auf die Formel gebracht, dass er verbietet „wesentlich Gleiches willkürlich ungleich", aber auch „wesentlich Ungleiches willkürlich gleich zu behan-

308 BVerfG, Beschluss v. 29.4.2003; Az.: 1 BvR 436/03, Rn. 7, http://www.bverfg.de/entscheidungen/rk20030429_1bvr043603.html; BVerwG Beschluss v. 8.5.2008, Az.: 6 B 65/07, juris, Rn. 4; Beschluss v. 15.10.2009, Az.: 6 B 27/09, juris, Rn. 4; Urteil v. 25.8. 1993; Az.: 6 C 9/81, juris, Rn. 15

Gröschner, in: Dreier GG, Art. 7, Rn. 53; Langenfeld, RdJB 2007, S. 211 ff, S. 222; Rux, Pädagogische Freiheit des Lehrers, S. 26; Niehues/Rux, Schulrecht, Rn. 125 und Rn. 145 ff zum Elternrecht; Robbers, in: v. Mangoldt/Klein/Starck GG, Art. 7, Rn. 31; Gröschner, in: Dreier GG, Art. 7, Rn. 53; Badura, in: Maunz/Dürig/Herzog GG, Art. 7, Rn. 25; Ennuschat, RdJB 2007, S. $271 \mathrm{ff}$, S. 288; Avenarius/Heckel, Schulrecht, Ziff. 25.12

BVerfGE 45, 400, 417; 58, 257, 272; Langenfeld, in: Grote/Marauhn EMRK/GG, Kap. 23: Das Recht auf Bildung, Rn. 41; Oppermann, in: HStR VI, § 135, Rn. 33; Stefek, Recht auf Bildung in der EG, S. 191 Langenfeld, in: Grote/Marauhn EMRK/GG, Kap. 23: Das Recht auf Bildung, Rn. 20 und 41 Gröschner, in: Dreier GG, Art. 7, Rn. 66; Thiel, Erziehungsauftrag des Staates in der Schule, S. 189; Langenfeld, Integration und kulturelle Identität zugewanderter Minderheiten, S. 223; Oppermann, in: HStR VI, § 135, Rn. 33 Thiel, Erziehungsauftrag des Staates in der Schule, S. 190

314 Kischel, in: BeckOK GG, Art. 3, Rn. 9

315 Kannengießer, in: Schmidt-Bleibtreu/Klein GG, Art. 3, Rn. 14; BVerfGE 3, 58, 135; 18, 38, 46; $72,141,150 ; 84,133,158 ; 98,365,385$, st. Rspr. 
Rechtlicher Problemaufriss

deln. ${ }^{\text {“316 }}$ Der Gleichheitssatz findet im Schulbereich seine besondere Ausprägung im Grundsatz der Chancengleichheit. Dieser wird vom BVerfG ${ }^{317}$ und dem BVerw ${ }^{318}$ sowie von den Obergerichten vielfach nicht mehr eigens begründet, sondern als selbstverständlich vorausgesetzt. ${ }^{319}$

Entsprechend hat die deutsche Schule stets zwei Aufgaben: erstens die pädagogische Aufgabe, das einzelne Kind individuell zu unterrichten und zu fördern, und zweitens die urteilende Aufgabe, die Leistungen des Schülers entsprechend den vorgegebenen Bildungsstandards und Prüfungsvorgaben, die an abstrakter Vergleichbarkeit orientiert sind, zu bewerten. ${ }^{320}$ Diese Doppelfunktion spiegelt sich in den Leistungsanforderungen an die Schüler und Schülerinnen: Sie haben eine Bildungsfunktion, indem sie dem Wissens- und Fähigkeitserwerb dienen, und sie haben eine Berechtigungsfunktion, da sie Grundlage der zu vergebenden Berufszugangsberechtigungen sind. ${ }^{321}$ Schließlich haben auch Lehrkräfte eine doppelte Funktion: sie erziehen und lehren, aber gleichzeitig bewerten sie und entscheiden mit über Berechtigungen wie Versetzungen und Schulabschlüsse. Chancengleichheit muss in beiden Bereichen hergestellt und gewahrt werden.

\section{Unterricht}

Hauptaufgabe der Schule ist der tägliche Unterricht. In diesem Bereich müssen gleiche Chancen für alle Schüler und Schülerinnen im Unterrichtsalltag gewährleistet werden. Im Schulbereich wird zwar grundsätzlich davon ausgegangen, dass Kinder bei Schuleintritt im Wesentlichen die gleichen Voraussetzungen mitbringen und deshalb gleich zu behandeln sind. ${ }^{322}$ Es ist jedoch auch selbstverständlich, dass eine schematische, formale Gleichbehandlung im Bereich des Unterrichts, in dem es um die vielfältigen Formen der Vermittlung von Wissen, des Einübens von Fähigkeiten oder auch des Erlebens und Vorlebens von sozialen Anforderungen geht, den Bedürfnissen der Kinder und auch der Aufgabe von Schule nicht gerecht wird. Vielmehr beinhaltet der Erziehungsauftrag des Staates im Bereich des allgemeinen Unterrichts und der Förderung gerade, dass vorhandene Unterschiede ausgeglichen werden und so sichergestellt

316 Kannengießer, in: Schmidt-Bleibtreu/Klein GG, Art. 3, Rn. 14; st. Rspr. BVerfGE 3, 58, 135; 18, 38,$46 ; 49,148,165 ; 72,141,150 ; 84,133,158 ; 98,365,385$

317 BVerfGE 84, 34, 50

318 BVerwGE 80, 282, 284

319 Lindner, BayVBI 1999, S. 100 ff, S. 101; s. auch die Nachweise in der folgenden Fußnote

320 Eichholz, R\&B 2007, S. 3 ff, S. 8, zu den Standards s. Anhang 2: Beschlüsse und Empfehlungen der KMK-Bildungsstandards und $B M B F$, Bildungsstandards

321 Ossenbühl, Schulzeugnisse, S. 20; Richter, Recht im Bildungssystem; S. 99; Oppermann, in: HStR VI, § 135, Rn. 43

322 Kritisch dazu: Dür/Fürth/Griebler, in: Gesundheitliche Ungleichheit, S. 426 
wird, dass jedermann gleichen Zugang zur Bildung sowie die gleiche Chance bei der Ausbildungs- und Berufswahl erhält. ${ }^{323}$ Chancengleichheit ist hier "etwas anderes als ,Gleichmacherei', nämlich ein vielgestaltiges Grundrecht, nach dem ,Jedem das Seine' nach Maßgabe seiner persönlichen Befähigung und Leistung zu geben ist. ${ }^{\prime 324}$ Hier zeigt sich, dass rein formale Gleichbehandlung der Kinder in hohem Maße ungerecht sein kann, weil die Kinder sehr verschiedene Voraussetzungen mitbringen. Eine, gerechte' Beschulung ist mithin nur gegeben, wenn die grundsätzliche Ungleichbehandlung ungleicher Tatbestände zulässig ist. ${ }^{325}$ Das rechtfertigt nicht nur, sondern erfordert sogar, dass individuell auf jedes Kind eingegangen wird, um den unterschiedlichen Fähigkeiten, Begabungen, Ausgangsbedingungen und Vorkenntnissen durch individuelle Ansprache, Förderung und Unterricht Rechnung zu tragen. Die pädagogische Leistung der Lehrkraft liegt gerade darin, nicht von allen Kindern dieselbe Leistung zu verlangen, sondern auf die Begabungsschwerpunkte des einzelnen Kindes einzugehen, und, wenn sich Schwächen zeigen, helfend einzugreifen. ${ }^{326}$ Eine solche Ungleichbehandlung der Kinder dient ihrer Förderung und beeinträchtigt sie nicht. Deshalb ist im Unterrichtsalltag jeder individuelle Ansatz, jede "Ungleichbehandlung" durch individuelle Förderung erlaubt, wenn sie zu dem Ziel führt, dass das Kind die geforderten Fähigkeiten erlernt. ${ }^{327}$

Die Förderung eines Kindes im Unterricht ist deshalb auch nicht davon abhängig, dass bei einem Kind eine besonderer Förderbedarf, eine Beeinträchtigung oder auch eine Behinderung festgestellt worden ist. Vielmehr ist schon die subjektive Einschätzung der Lehrkraft ausreichend, dass dem Kind eine Förderung gut tun würde. Diesen, auch rechtlichen Hintergrund übersehen jene, die meinen, dass eine Förderung bei Legasthenie/Dyskalkulie nur zulässig ist, wenn ein Förderbedarf festgestellt wurde. ${ }^{328}$ Vielmehr entspricht es dem

323 Zur inklusiven und barrierefreien Ausgestaltung des Unterrichts s. KMK-Inklusive Bildung Punkt II. und II.1

324 Oppermann, in: HStR VI, § 135, Rn. 40, s. auch Glotz/Faber, in: Hdb. des Verfassungsrechts, $\S 28, \mathrm{Rn} .12$

Stern, in: FS Günter Dürig, S. 210 Pietzcker, Verfassungsrechtliche Anforderungen an staatliche Prüfungen, S. 36

Starck, in: v. Mangoldt/Klein/Starck GG, Art. 3, Rn. 37. Besonders deutlich wird das an den vielschichtigen Besonderheiten von Kindern mit Migrationshintergrund, die häufig die deutsche Sprache nicht in dem Maße beherrschen, dass sie problemlos und gleichberechtigt dem deutschsprachigen Unterricht folgen können und die deswegen zunächst einmal ein gleichberechtigtes Sprachniveau erreichen müssen, um dann alle Bildungsmöglichkeiten wahrnehmen zu können; grundlegend Langenfeld, Integration und kulturelle Identität zugewanderter Minderheiten Bezüglich Legasthenie/Dyskalkulie wird dieser Zusammenhang von Feststellung einer Behinderung und Förderung insbesondere von Valtin behauptet. Sie meint, dass die Berücksichtigung von Legasthenie/Dyskalkulie „zu einem Zwei-Klassen-System der Förderung [führt], 
Grundsatz der Chancengleichheit, wenn Schüler und Schülerinnen mit Legasthenie/Dyskalkulie im täglichen Unterricht, in dem die Lehrkräfte einen weiten Gestaltungsfreiraum haben, ihren Bedürfnissen und Fähigkeiten entsprechend ungleich behandelt werden. Meistens ist eine solche differenzierte Unterrichtung und Leistungsanforderung in diesem Bereich auch relativ unproblematisch.

\section{Prüfungen}

Das zweite große Aufgabenfeld der Schule ist der Bereich der Prüfungen und die damit verbundene Vergabe von Berechtigungen in Form von Schulzuweisungen oder -empfehlungen, Versetzungen und Schulabschlüssen.

Die Gestaltungsfreiheit des Staates im Schulwesen auf der Grundlage von Art. 7 I GG umfasst neben der Organisation der Schule auch die inhaltliche Festlegung der Ausbildungsgänge, Unterrichtsziele, Prüfungen und des Prüfungsstoffes. ${ }^{329}$ Zum formalen Schulauftrag gehört die Vermittlung von Bildungsabschlüssen, die berechtigenden Charakter haben oder doch zumindest die Berufschancen erhöhen. Berechtigungen enthalten schematisierte und formalisierte Eignungsvermutungen und sind erforderlich, um Zugang zur nächsten Jahrgangsstufe, zu weiterführenden Schulen oder höheren Stufen des Bildungswesens zu erhalten oder zu bestimmten beruflichen Laufbahnen. Nicht nur der Staat (vgl. § 17 BBG, § 14 Nds.BG; Art. 31 ff BayBG), sondern auch die Privatwirtschaft knüpft bei Einstellungen an qualifizierte staatliche Schulabschlüsse an. ${ }^{330}$ Schulen sind daher in Deutschland auch "Verteilerstellen für Berufs- und Lebenschancen“. ${ }^{331}$ Daher hat der Staat ein legitimes Interesse an Prüfungen, denn ein leistungsorientiertes Gemeinwesen kommt ohne Leistungskontrollen, Prüfungen und Berechtigungen, um Eignungen und Befähigungen festzustellen, nicht aus. Ohne Prüfungen würden sich andere informelle und dem Verdacht der Willkür ausgesetzte Mechanismen herausbilden, die

weil sich nun vor allem Oberschicht-Eltern die Förder-Privilegien erkämpfen werden. "Valtin, Brauchen wir die Legasthenie?, S. 5. Diese Ansicht von Valtin beruht auf Erfahrungen aus den 1970er Jahren. Damals hing die Förderung von Kindern mit Schwierigkeiten im Lesen und Schreiben davon ab, dass sie als Legastheniker anerkannt waren. Kinder, die diese Anerkennung nicht hatten, wurden nicht gefördert. Dieses - zutreffend - als ungerecht empfundene Vorgehen, verstieß gegen den allgemeinen Auftrag der Schule, alle Kinder zu fördern, und gegen Art. 3 । GG, denn es gibt keinen sachlichen Grund, nur Kinder mit Legasthenie/ Dyskalkulie zu fördern und andere Kinder mit Schwierigkeiten oder auch ohne Schwierigkeiten nicht zu fördern. Zimmerling/Brehm, Prüfungsrecht, Rn. 348

330 Oppermann, in: HStR VI, § 135, Rn. 5

331 Vgl. BVerfGE 33, 303, 338, Rn. 75, 346, Rn. 92; Niehues, Schul- und Prüfungsrecht, Rn. 1; Ossenbühl, Schulzeugnisse, S. 21; Avenarius/Heckel, Schulrecht, Ziff. 4.21; zur Geschichte des sog. Berechtigungswesens: Richter, Recht im Bildungssystem, S. $95 \mathrm{f}$ 
gegenüber Prüfungen, insbesondere formalisierten Prüfungen, keine Vorteile haben. ${ }^{332}$

\section{Kapitel: Gleichheit und Ungleichheit von Schülern und Schülerinnen in Prüfungen}

Weil die Berechtigungen den Zugang zu weiteren schulischen oder beruflichen Möglichkeiten eröffnen, haben die Schulen und Lehrer in diesem Bereich eine große Verantwortung. Dies ist den Verantwortlichen sehr bewusst. Deshalb ist im Prüfungsbereich ein prinzipieller Grundsatz der Kultusverwaltungen, die strikte formale Gleichbehandlung der Prüflinge zu gewährleisten, um die Konkurrenz so fair wie irgend möglich zu gestalten. ${ }^{333}$ Allerdings ist allgemein anerkannt, dass diese strikt formale Gleichbehandlung aller Prüflinge zu Ungerechtigkeiten führen und es Prüflinge geben kann, die ungleich zu behandeln sind. Geprüft wird, ob ein Prüfling mit dem gedachten "Normalkandidaten" vergleichbar ist. Ist der beeinträchtige Prüfling mit diesem nicht vergleichbar, in der Terminologie des BVerfG also "wesentlich ungleich", dann wird er ungleich behandelt und seine Prüfungsbedingungen müssen entsprechend angepasst werden. ${ }^{334}$

Die schwierige Frage ist, nach welchen Kriterien festgestellt wird, ob ein Schüler/eine Schülerin mit Legasthenie/Dyskalkulie gegenüber dem "Normalschüler" wesentlich ungleich ${ }^{335}$ und ihre Chancengleichheit also beeinträchtigt ist.

Für die Feststellung, ob Sachverhalte wesentlich gleich und deshalb gleich zu behandeln sind, kommt es darauf an, welchem gemeinsamen Oberbegriff sie zuzuordnen sind. ${ }^{336}$ Schüler und Schülerinnen sind dem Oberbegriff "alle Schüler, die eine bestimmte Prüfung ablegen" zuzuordnen. Sie werden in ihrer jeweiligen Schulart und Jahrgangsstufe nach gleichen Lehrplänen und mit gleichen Lernzielen unterrichtet und sind deshalb in diesem Bereich gleich. Ihre

\footnotetext{
332 Langenfeld, RdJB 2007, S. 211 ff, S. 219

333 Vgl. Osterloh, in: Sachs GG, Art. 3, Rn. 58

334 BVerwG, Beschluss v. 13.12.1985, Az.: 7 B 210.85, juris, Rn. 6; BVerwG, Urt. v. 30.08.1977 VII C 50.76 -, Buchholz 421.0, Prüfungswesen, Nr. 85; vgl. auch OVG Koblenz, Urt. v. 16.01.1980 - 2 A 49/79 -, DVBI. 1981, S. 591; OVG Weimar, Beschluss v. 17.5.2010, Az.: 1 EO 854/10, juris, Rn. 28 f; Niehues, Prüfungsrecht, Rn. 249

$335 \mathrm{Vgl}$. Reichenbach, Anspruch behinderter Schülerinnen und Schüler auf Unterricht in der Regelschule, S. 210

336 Pieroth/Schlink, Grundrechte, Rn. 463 ff; vgl. dazu grundlegend Hufen, Gleichheitssatz und Bildungsplanung, S. 126 ff; kritisch Kischel, in: BeckOK GG, Art. 3, Rn. 15.2
} 
formale Gleichbehandlung bei Prüfungen sichert die Gleichwertigkeit und Vergleichbarkeit der Berechtigungen, die sie erwerben.

Für die Frage, wann eine Abweichung von der Gleichbehandlung und damit eine Ungleichbehandlung innerhalb dieser Gruppe gerechtfertigt ist, hat das BVerfG verschiedene Formeln entwickelt ${ }^{337}$, die sich nicht ausschließen, sondern nebeneinander fortbestehen und aufeinander aufbauen. ${ }^{338}$ Nach der „Willkürformel" sind Ungleichbehandlungen unzulässig, wenn sich „ein vernünftiger, sich aus der Natur der Sache ergebender oder sonst wie sachlich einleuchtender Grund nicht finden lässt. ${ }^{\text {339 }}$ Diese Rechtsprechung hat das Gericht auf die „neue Formel“340 erweitert, nach der Art. 3 I GG dann als verletzt ist, „wenn eine Gruppe von Normadressaten im Vergleich zu anderen Normadressaten anders behandelt wird, obwohl zwischen beiden Gruppen keine Unterschiede von solcher Art und solchem Gewicht bestehen, dass sie die ungleiche Behandlung rechtfertigen könnten. ${ }^{1341}$ Nach der ",neuesten Formel“ dann, die das Gericht 1993 entwickelt hat, ist die Bindung des Gesetzgebers „umso enger, je mehr sich die personenbezogenen Merkmale den in Art. 3 Abs. 3 GG genannten annähern und je größer deshalb die Gefahr ist, dass eine an sie anknüpfende Ungleichbehandlung zur Diskriminierung einer Minderheit führt. Die engere Bindung ist jedoch nicht auf personenbezogene Differenzierungen beschränkt. Sie gilt vielmehr auch, wenn eine Ungleichbehandlung von Sachverhalten mittelbar eine Ungleichbehandlung von Personengruppen bewirkt. Bei lediglich verhaltensbezogenen Unterscheidungen hängt das Maß der Bindung davon ab, inwieweit die Betroffenen in der Lage sind, durch ihr Verhalten die Verwirklichung der Merkmale zu beeinflussen, nach denen unterschieden wird. Überdies sind dem Gestaltungsspielraum des Gesetzgebers umso engere Grenzen gesetzt, je stärker sich die Ungleichbehandlung von Personen oder Sachverhalten auf die Ausübung grundrechtlich geschützter Freiheiten nachteilig auswirken kann. ${ }^{3342}$

Für die hier vorliegende Problematik ergibt sich mithin folgendes Bild: Knüpft die Gleich- oder Ungleichbehandlung nicht an rein sachliche, sondern an personenbezogene Kriterien an, ist zunächst die "neue Formel" zu prüfen. Sind diese personenbezogenen Kriterien jedoch durch Art. 3 Abs. 3 GG besonders geschützt, weisen sie eine enge Nähe zu dessen Schutzbereich auf oder geht es um Sachverhalte, deren Regelung mittelbar eine geschützte Gruppe

337 S. dazu ausführlich Straßmair, Der besondere Gleichheitssatz aus Art. 3 Abs. 3 Satz 2 GG, S. $192 \mathrm{ff}$

338 Kischel, in: BeckOK GG, Art. 3, Rn. 29.1

339 St. Rspr. BVerfGE 1, 14, 16, 52

340 Kannengießer, in: Schmidt-Bleibtreu/Klein GG, Art. 3, Rn. 17

341 BVerfGE 55, 72, 88

342 BVerfGE 88, 87, 96, Rn. 35 
betrifft, so ist die strengere "neueste Formel" zu prüfen. Ungleichbehandlungen in Prüfungen sind nach der "neuesten Formel“ also nur zulässig ${ }^{343}$, wenn sie auf personenbezogenen Kriterien beruhen und für die Ungleichbehandlung Gründe von solcher Art und solchem Gewicht bestehen, dass sie die ungleichen Rechtsfolgen rechtfertigen können. ${ }^{344}$ Verfolgt der Staat den Zweck, vorhandenen Unterschieden Rechnung tragen, ist darauf abzustellen, ob die Unterschiede tatsächlich bestehen und hinreichend gewichtig sind, um die Ungleichbehandlung zu rechtfertigen. ${ }^{345}$

Ziel der Ungleichbehandlung von Schülern und Schülerinnen in Prüfungssituationen und bei Benotungsregeln ist es, vorhandenen Unterschieden Rechnung zu tragen, also interne Zwecke zu verfolgen. Anknüpfungspunkt müssen stets persönliche Eigenschaften des Schülers, also personenbezogene Kriterien sein.

\section{Personenbezogenes Kriterium: Behinderung}

Das personenbezogene Kriterium, das Anknüpfungspunkt für eine Ungleichbehandlung in Prüfungen ist und eine Ungleichbehandlung rechtfertigt, kann hier sein, dass Schüler und Schülerinnen mit Legasthenie/Dyskalkulie behindert i.S.d. Art. 3 III 2 GG sind. Sind Schüler behindert i.S.d. Art. 3 III 2 GG, so sind sie im Vergleich zu den "Normalschülern" ungleich, so dass eine Ungleichbehandlung gerechtfertigt, wenn nicht sogar geboten ist. In diesem Fall würde nach den Kriterien der "neuesten Formel“ des BVerfG eine weitere Gleichbehandlung an ein personenbezogenes Merkmal aus Art. 3 III GG anknüpfen, nämlich an eine Behinderung, und zu einer Diskriminierung dieser Gruppe führen, was sich erheblich nachteilig auf die Ausübung grundrechtlich geschützter Freiheiten auswirken kann. Sie sind dann mit Schülern und Schülerinnen ohne diese Beeinträchtigung nicht vergleichbar, so dass ein sachlicher Grund für eine Ungleichbehandlung vorliegt, die dann aus Art. 3 I GG i. V. m. Art. 3 III 2 GG gerechtfertigt sein könnte.

\section{Personenbezogenes Kriterium: Schüler und Schülerinnen mit Schwierigkeiten}

Fraglich ist, ob es zwingend erforderlich ist, festzustellen, dass Schüler und Schülerinnen mit Legasthenie/Dyskalkulie behindert sind, um eine Ungleichbehandlung in Prüfungen zu begründen, oder ob es hierfür nicht ausreicht, sie als Normalschüler mit Schwierigkeiten in einem bestimmten Bereich zu sehen, so wie dies bisher überwiegend tatsächlich geschieht.

\footnotetext{
343

Vgl. Epping, Grundrechte, Rn. 796 f; Michael/Morlok, Grundrechte, Rn. 795

344

Epping, Grundrechte, Rn. 804

345

Epping, Grundrechte, Rn. 806
} 
Rechtlicher Problemaufriss

Im Bereich der Pädagogik wird die Einordnung der Legasthenie und Dyskalkulie als Behinderung u.a. aus „ethischen“ Gründen abgelehnt, weil sie zur Etikettierung und Stigmatisierung führe und überflüssig sei. ${ }^{346}$

Wie gezeigt, setzt die Rechtfertigung einer Ungleichbehandlung voraus, dass sie aufgrund von personenbezogenen Kriterien erfolgt. Nur dann, wenn es einen solchen personenbezogenen Unterschied zwischen den Schülerinnen und Schülern mit Legasthenie/Dyskalkulie und den nicht-betroffenen Schülern und Schülerinnen gibt, kann, darf und muss diese Gruppe als ungleich angesehen und ungleich behandelt werden. ${ }^{347}$

Um Schwierigkeiten im Lesen, Rechtschreiben und/oder Rechnen bei Schülern und Schülerinnen als personenbezogenes Kriterium zu definieren, ist es erforderlich, dass diese Schwierigkeiten bestimmbar sind. Denn um die Verschiedenheit von Gruppen zu bestimmen und darauf eine unterschiedliche Behandlung aufzubauen, muss der Staat seine Handlungsmaßstäbe offenlegen und begründen können. Die Wertungen müssen allgemein und konsistent erfolgen und sie müssen verallgemeinerbar sein. ${ }^{348}$ An diese Maßstäbe sind besonders hohe Anforderungen zu stellen, weil sie nicht nur die Rechte der "ungleich" behandelten Schüler und Schülerinnen betreffen, sondern weil vor allem auch die Grundrechte der "Normalschüler" betroffen sein können. Jede Ungleichbehandlung von anderen Schülern und Schülerinnen kann ihre Freiheitsrechte z.B. aus Art. 2 I und 12 I GG beeinflussen. Die Grenzen des Gestaltungsspielraums des Gesetzgebers für die Bestimmung der ungleich zu behandelnden Personengruppe sind daher umso enger, je stärker sich die Ungleichbehandlung von Personen oder Sachverhalten auf die Ausübung grundrechtlich geschützter Freiheiten anderer nachteilig auswirkt. ${ }^{349}$ Dies gilt insbesondere im Schulbereich, weil der Staat im Bereich der Schulen das zumindest faktische Monopol hat und deshalb hier eine besondere Verantwortung trägt. ${ }^{350}$

Die landesrechtlichen Bestimmungen zur Berücksichtigung von Schwierigkeiten im Lesen, Rechtschreiben und Rechnen ${ }^{351}$ dienen dazu, gerade diese Bestimmung vorzunehmen, ob und inwieweit Schüler und Schülerinnen mit solchen Schwierigkeiten mit anderen Schülern und Schülerinnen ungleich und deshalb Veränderungen in den Prüfungen zulässig sind. Fraglich ist jedoch, ob

346 Büchner/Kortländer/Werner/Robering/Schönweiss, „Legasthenie - eine Krankheit, eine Behinderung, eine Störung?", S. 2, 4; Hanert-Möller, Konstrukt Legasthenie, S. 83; Valtin, in: LRS und Legasthenie, S. $58 \mathrm{ff}$; Valtin, Brauchen wir die Legasthenie?, S. $6 \mathrm{f}$

$347 \mathrm{Vgl}$. Michael/Morlok, Grundrechte, Rn. 803

348 Michael/Morlok, Grundrechte, Rn. 781, 805

349 BVerfGE 88, 87 (96)

350 BVerfGE 98, 288, 304, Rn 70

351 S. Sammlung der Vorschriften in Anhang 3 
diese Bestimmungen den oben dargestellten Anforderungen des Maßstabes genügen.

Die bayerische Regelung bestimmt die Ungleichheit auf der Grundlage von kinderpsychiatrischen Gutachten oder Untersuchungen durch die Schulpsychologen, an die die Schulen dann gebunden sind. Da die Diagnostik der Kinderund Jugendpsychiater auf der ICD 10 und den Leitlinien beruht, liegt insofern ein Maßstab vor, der allgemein, konsistent, verallgemeinerbar, nachvollziehbar und wiederholbar ist.

In den anderen Bundesländern ist entscheidendes Kriterium überwiegend, dass der Schüler in dem geforderten Fach Schwierigkeiten hat, die sich insbesondere an schlechten Noten zeigen. ${ }^{352}$ Die Problematik dieser Bestimmung der Verschiedenheit ist jedoch, dass Prüfungen und Noten gerade aufzeigen sollen, welche Leistungen ein Schüler erbringt. Schlechte Noten sind ein Teil dieses Systems und gerade kein Grund, um deswegen die Prüfungsbedingungen zu verändern oder die Benotung auszusetzen. ${ }^{353}$ Allein Schwierigkeiten in einem bestimmten Fach oder Teilfach reichen daher nicht aus, um die Verschiedenheit dieser Gruppe zu bestimmen. Bedenken gegen eine solche Bestimmung ergeben sich auch daraus, dass eine Abgrenzung dieser gedachten Gruppe zu den „Normalschülern", die auch schlechte Noten haben, nicht möglich ist. Wonach ist zu bestimmen, ob der Schüler Schwierigkeiten in dem Fach hat und deshalb „ungleich" ist oder ob der Schüler in dem Fach einfach schlecht ist und zu den „Normalschülern“ gehört? Ein Nachteilsausgleich etwa für allgemein leseschwache Schüler ist unter diesen Umständen rechtlich nicht begründbar. ${ }^{354}$

Darüber hinaus erfolgt die allgemeine Feststellung von Schwierigkeiten von Schülern und Schülerinnen überwiegend im Vergleich mit dem Klassenverbund und als subjektive Einschätzung der Lehrkraft oder der Klassenkonferenz. ${ }^{355}$

352 S. oben 1. Teil: 2. Kapitel: 4.: Grundzüge der Beschlüsse der KMK und der Landesvorschriften zur Berücksichtigung von Legasthenie und Dyskalkulie - mit Ausnahme von Bayern, S. 37

Anders § $6 \mathrm{IV}$ SH ZeugnisVO und SH Legasthenie Erlass Nr. 2.1, die eine Berücksichtigung von besonderen und andauernder Schwierigkeiten im Lesen oder Rechtschreiben auch unabhängig von der förmlichen Feststellung einer Lese-Rechtschreib-Schwäche festlegen

So aber die Forderung von Valtin/Hornberg/Buddeberg/Voss/Kowoll/Potthoff, in: Bos, IGLU 2006, S. 84

BW VV Besonderer Förderbedarf Nr. 2.3.2; BY automatisch, wenn gutachterlich festgestellt, Zeitzuschlag bestimmt Schulleiter; BE GsVO § 16 VII; BB VV-LRSR Nr. 3 I für Lesen und Rechtschreiben, allerdings jetzt mit dem Hinweis, dass Verfahren anzuwenden sind, die der Objektivierung der Feststellung dienen Nr. 3 I S. 3 und Nr. 6 I für Rechnen ohne diesen Hinweis; HB LRS-Erlass Nr. 4; HH Förder-Richtlinie Nr. 3.1 - Testergebnis entscheidet; HE VO-

GestSchulVerh $\S 38$ - der in $\S 4$ II VOLRR noch vorhandene Hinweis auf die Einbeziehung von Fachgutachten wurde gestrichen; MV VV-LRR Nr. 5 III - das staatliche Schulamt; NI LRRErlass Nr. 3; NRW LRS-Erlass Nr. 3.2 - Schulleitung entscheidet; RP VV-LRS Nr. 4.3; SL VV-LRS Nr. 2.1 a); SN VV-LRS Nr. 4.1.4; ST Grundschulbewertungs-Erlass Nr. 4.1.1, ST Sekundarstu- 
Diese Einschätzung ist jedoch nicht objektiv und verallgemeinerbar, ${ }^{356}$ da die Vergleichsgröße für jedes Kind eine andere ist. Kinder, die in einer Klasse als schlecht gelten, können unter Umständen in einer anderen Klassenumgebung als durchschnittlich leistungsfähig angesehen werden. Ihnen bleibt dann die Berücksichtigung von Schwierigkeiten versagt. Ebenso bleibt Kindern die Berücksichtigung von Schwierigkeiten versagt, wenn die Einschätzung der Lehrkräfte dahin geht, dass das Kind weniger begabt ist und die schlechte Leistung deshalb im Erwartungshorizont der Lehrkraft liegt. Andererseits kann diese Auswahl aber auch dazu führen, dass bei solchen Kindern Schwierigkeiten angenommen werden, denen keine belegbaren Probleme zugrunde liegen. Dann werden diejenigen Schüler benachteiligt, die den normalen Leistungsanforderungen unterworfen bleiben.

Die bloße Existenz von Schwierigkeiten ist daher kein personenbezogenes Kriterium, das sachlich eine Ungleichbehandlung dieser Schüler und Schülerinnen mit den „Normalschülern" rechtfertigt. Diese betroffenen Schüler und Schülerinnen unterliegen damit den gleichen Leistungs-, Prüfungs- und Beurteilungsanforderungen wie alle Schüler.

\section{Ergebnis}

Nur die Einstufung von Legasthenie/Dyskalkulie als Behinderung i.S.d. Art. 3 III 2 GG kann ein personenbezogenes Merkmal darstellen, das eine Ungleichbehandlung in Prüfungen rechtfertigt. Liegt eine Behinderung nicht vor, unterfallen die betroffenen Schüler und Schülerinnen den allgemeinen Anforderungen.

Da es eine Analogie bei Grundrechten nicht gibt, entfällt dann auch die Möglichkeit, sie ungleich zu behandeln und ihnen „Hilfen im Sinne eines Nachteilsausgleichs" zu gewähren. ${ }^{357}$

fenbewertungs-Erlass Nr. 5.1.2; SH Legasthenie-Erlass Nr. 2.1; TH Fachliche Förderempfehlung - Förderplan erstellt der Klassenlehrer; anders in MV VV-LRR Nr. 5.1 I, wo die Diagnostik durch den Diagnostischen Dienst nach der MV Diagnostischer Dienst Nr. 2.2 erfolgt.

Ausführlich zu sozialen Bezugsnormen als diagnostische Grundlage: Kany/Schöler, Diagnostik, S. 52 ff; Roos/Schöler, Schriftspracherwerb, S. 120, 164 m.w.N.; ausführlich unten: 9. Teil: 1. Kapitel: 3.3.1.: Relativierung der Diagnostik durch pädagogische Einschätzungen?, S. 263

357 So aber für Niedersachsen Behrens, Nds. SVBI 2006, S. 189 ff, S. 190; s. auch KMKLesen/Rechtschreiben/Rechnen, Punkt Leistungsbewertung, Grundsätze; HB LRS-Erlass Nr. 4.1; MV VV-LRR Nr. 7 III (allerdings unpräzise: mal ist von Nachteilsausgleich (Nr. 7 II), mal von Hilfen iS eines Nachteilsausgleich (Nr. 7 III) die Rede; NI LRR-Erlass Nr. 4.1; RP VV-LRS Nr. 4.2; SL VV-LRS Nr. 5.1; ST Erlass Grundschulbewertung Nr. 5.1.1; SH Legasthenie-Erlass Nr. 1.1.1 


\section{Teil: Anspruch aus Art. 3 III 2 GG}

Ein Anspruch auf Berücksichtigung von Legasthenie/Dyskalkulie in der Schule ergibt sich aus Art. 3 III 2 GG dann, wenn die Nicht-Berücksichtigung oder nicht ausreichende Berücksichtigung von Legasthenie/Dyskalkulie in der Schule eine ungerechtfertigte Benachteiligung wegen einer Behinderung ist.

\section{Kapitel: Verhältnis von Art. 3 III 2 GG und Art. 3 I GG}

Ein Anspruch aus Art. 3 III 2 GG könnte allerdings ausgeschlossen sein, wenn der allgemeine Gleichheitssatz des Art. 3 I GG vorrangig vor Art. 3 III 2 GG zur Anwendung käme und somit der Gleichbehandlung aller Schüler und Schülerinnen Vorrang vor der Berücksichtigung der Behinderung zukäme. Von einem solchen Vorrang geht die Rechtsprechung aus, da sie zwar bei Schülern und Schülerinnen mit Legasthenie/Dyskalkulie das Vorliegen einer Behinderung überwiegend bejaht, aber eine Ungleichbehandlung unter Verweis auf den Grundsatz der Chancengleichheit und damit auf Art. 3 I GG ablehnt. ${ }^{358}$

Die Diskriminierungsverbote des Art. 3 III 2 GG und Art. 3 II GG sind negative, verschärfende Konkretisierungen des allgemeinen Gleichheitssatzes. ${ }^{359}$ In der Literatur wird Art. 3 III 2 GG daher gegenüber Art. 3 I GG als das speziellere Grundrecht angesehen ${ }^{360}$ und es wird nach der allgemeinen Anwendungsregel „lex specialis derogat legi generali“ Art. 3 III 2 GG der Vorrang vor Art 3 I GG

358 OVG Münster, Beschluss v. 4.9.2008, Az.: 19 B 1293/08, www.justiz.nrw.de; VG Köln, Beschluss v. 26.9.2008, Az.: 10 L 1240/08, www.justiz.nrw.de; VG Schleswig Urteil v. 10.6.2009, Az.: 9 A 208/08, BeckRS 2009 38123, S. 7; VG Gießen, Urteil v. 30.6.2009. Az.: 4 K 1052/09.GI, S. 15 (nicht veröffentlicht); VG Aachen, Urteil v. 13.11.2009, Az.: 9 K 25/09, BeckRS 2009 42206, S. 6; OVG Berlin, Beschluss v. 16.11.2004, 8 S 130.04, juris, Rn. 10; OVG Berlin-Brandenburg, Beschluss v. 16.6.2009, Az.: 3 M 16.09, juris, Rn. 4; VG Düsseldorf, Beschluss v. 16.11.2009, Az.: 18 L 1654/09, juris, Rn. 21; VGH Kassel, Beschluss v. 5.3.2010, Az.: 7 A 2406/09, juris, Rn. 43 ff; ebenso Kischel, in: BeckOK GG, Art. 3, Rn. 219.4

359 König/Peters, in: Grote/Marauhn EMRK/GG, Kapitel 21: Das Diskriminierungsverbot, Rn. 46 
eingeräumt, soweit dieser spezielle Gleichheitssatz die fragliche Behandlung abschließend regelt. ${ }^{361}$ Diese Auslegung wird unterstützt durch die Rechtsprechung des BVerfG und des BVerwG zur Berücksichtigung von Art. 3 III 2 GG bei der Anwendung von Art. 33 ॥ GG. ${ }^{362}$ Art. 33 ॥ GG gewährt jedem Deutschen ein grundrechtsgleiches Recht auf den Zugang zu einem öffentlichen Amt, wenn er oder sie geeignet ist. Weil Teil der Eignung vor allem auch die geistige, seelische und körperliche Eignung ist, war streitig, ob der Dienstherr stets die uneingeschränkte Dienstfähigkeit fordern kann, oder ob und in welchem Umfang er bei der Beurteilung dieser Frage dem besonderen Gleichheitssatz aus Art. 3 III 2 GG Rechnung zu tragen hat. Beide Gerichte haben geurteilt, dass Art. 33 II GG nicht vorrangig vor Art. 3 III 2 GG ist, sondern dass bei der Anwendung des Art. 33 || GG das unmittelbar geltende Benachteiligungsverbot des Art. 3 III 2 GG zu beachten und konkret und individuell zu prüfen ist.

Wenn Schüler und Schülerinnen mit Legasthenie/Dyskalkulie unter den Anwendungsbereich des Art. 3 III 2 GG fallen und dadurch diskriminiert werden, dass die allgemeinen Prüfungsbedingungen für sie eine unzulässige Benachteiligung sind, dann regelt Art. 3 III 2 GG diese Frage abschließend und ist daher vorrangig. ${ }^{363}$

\section{Kapitel: Schutzbereich}

Art. 3 III 2 GG schützt davor, wegen einer Behinderung benachteiligt zu werden. Nach ganz herrschender Meinung enthält Art. 3 III 2 GG ein individuelles Abwehrrecht gegen den Staat. ${ }^{364}$

\section{Der verfassungsrechtliche Begriff Behinderung}

Eine eigene Definition des Begriffs der Behinderung enthält Art. 3 III 2 GG nicht. Die Frage ist daher, was unter Behinderung i.S.d. Grundgesetzes zu verstehen

361 Osterloh, in: Sachs GG, Art. 3, Rn. 77 f; Heun, in: Dreier GG, Art. 3, Rn. 141; Kischel, in: BeckOK GG, Art. 3, Rn. 2; Sachs, Besondere Gleichheitsgarantien, in: HStR VIII, § 182, Rn. 19

362 BVerfG, 2 BvR 2571/07 vom 10.12.2008, http://www.bverfg.de/entscheidungen/rk20081210_2bvr257107.html Rn. 11; BVerwG Urteil v. 21.6.2007, Az.: 2 A 6/ 06, http://www.bundesverwaltungsgericht.de/media/archive/5320.pdf Rn. 20 Vgl. Reichenbach, Anspruch behinderter Schülerinnen und Schüler auf Unterricht in der Regelschule, S. 211

364 Castendiek, in: GS Jean d'Heur, S. 340 f; Sacksofsky, in: Umbach GG, Art. 3, Rn. 392; Heun, in: Dreier GG, Art. 3, Rn. 17; Osterloh, in: Sachs GG, Art. 3, Rn. 38 ff; 305; Eckertz-Höfer, in: AKGG, Art. 3, Rn. 128; Kannengießer, in: Schmidt-Bleibtreu/Klein GG, Art. 3, Rn. 59; Beyerlin, RdJB 1999, S. 157 ff, S. 162; Beaucamp, DVBI 2002, S. 997 ff, S. 998; Luthe, Optimierende Sozialgestaltung, S. 118 f; Gubelt, in: v. Münch GG, Art. 3, Rn. 104 b 
ist. Nach ganz herrschender Auffassung muss sich die Auslegung eines verfassungsrechtlichen Begriffs am Schutzbereich desjenigen Grundrechts orientieren, zu dem er gehört. Bei Art. 3 III 2 GG ist die Auslegung des Begriffs Behinderung mithin Mittel zum spezifischen Gesetzeszweck, ggf. bestehende Benachteiligungen aufgrund von Behinderung zu kennzeichnen und zu verhindern. Der Gehalt des Begriffs ist nach den allgemeinen Methoden der Auslegung zu ermitteln ${ }^{365}$, wobei derjenigen Methode der Vorzug zu geben ist, die die juristische Wirkungskraft des Grundrechts am stärksten entfaltet. ${ }^{366}$

\subsection{Vorrang der Verfassung}

Das Bundesverfassungsgericht hat sich in seiner Leitentscheidung zum Verbot der Benachteiligung wegen einer Behinderung im Bereich des Schulwesens, umfassend mit Art. 3 III 2 GG beschäftigt. ${ }^{367}$ In dem Verfahren ging es um die Zulässigkeit der Überweisung eines behinderten Kindes auf eine Förderschule. In dieser Entscheidung hat das BVerfG eine abschließende Bestimmung des Begriffs „Behinderung“ ausdrücklich offen gelassen. ${ }^{368}$ Es hat sich aber auf den damals geltenden $\S 3$ । S. 1 SchwbhG gestützt ${ }^{369}$, der Behinderung als „die Auswirkung einer nicht nur vorübergehenden Funktionsbeeinträchtigung [bestimmt], die auf einem regelwidrigen körperlichen, geistigen oder seelischen Zustand beruht. Regelwidrig ist der Zustand, der von dem für das Lebensalter typischen abweicht." Das BVerfG führte aus: „Behinderung ist ... eine Eigenschaft, die die Lebensführung für den Betroffenen im Verhältnis zum Nichtbehinderten ... grundsätzlich schwieriger macht" ${ }^{370}$

Die staatsrechtliche Literatur zu Art. 3 III 2 GG knüpft überwiegend an § 2 Abs. 1 S. 1 SGB IX an, der inzwischen $\S 3$ SchwbhG abgelöst hat. ${ }^{371}$ Danach ist Behinderung die Beeinträchtigung der Teilhabe am Leben in der Gesellschaft infolge einer Abweichung der körperlichen Funktionen, geistigen Fähigkeiten

\footnotetext{
365 Umfassend dazu Ossenbühl, in: Hdb. Grundrechte I, § 15, S. 595 ff; Larenz, Methodenlehre

371 Kischel, in: BeckOK GG, Art. 3, Rn. 211; Rüfner, in: Bonner Kommentar GG, Art. 3, Rn. 870 f, Heun, in: Dreier GG, Art. 3, Rn. 135; Jarass, in: Jarass/Pieroth GG, Art. 3, Rn. 144; Starck, in: v. Mangoldt/Klein/Starck GG, Art. 3, Rn. 418; Osterloh, in: Sachs GG, Art. 3, Rn. 310; Kannengießer, in: Schmidt-Bleibtreu/Klein GG, Art. 3, Rn. 213; Sacksofsky, in: Umbach GG, Art. 3, Rn. 396
} 
oder der seelischen Gesundheit. ${ }^{372}$ Eine striktere Ansicht vertritt Kischel, der sich auf die Definition des BVerfG auf der Grundlage von $\S 3$ SchwbhG bezieht und den "herkömmlichen“ Begriff für abschließend hält, da der Verfassungsgeber an diesen Begriff angeknüpft habe. ${ }^{373}$

Auch die Instanzgerichte ${ }^{374}$ stellen mehr oder weniger direkt für den Anwendungsbereich des Art. 3 III 2 GG auf $\S \S 2$ SGB IX und 35 a SGB VIIII ${ }^{375}$ ab. Das VG Schleswig hat für die Bestimmung des Schutzbereichs des Art. 3 III 2 GG explizit auf den sozialrechtlichen Begriff aus $\S 2$ SGB IX und dann weiter auf den besonderen Tatbestand des $\S 35$ a SGB VIII zurückgegriffen. ${ }^{376}$ Es meint, dass der Begriff der Behinderung sozialrechtlich und im Fall der Legasthenie durch die Spezialvorschrift des $\S 35$ a SGB VIII definiert wird. ${ }^{377}$ Eine verfassungsrechtlich relevante Behinderung liege nur dann vor, wenn der Tatbestand des $\S 35$ a SGB VIII, der speziell die Eingliederungshilfe für seelisch behinderte Kinder regelt, erfüllt sei. Zudem müsse der Betroffene nachweisen, dass er konkret in der Teilhabe beeinträchtigt ist. ${ }^{378}$ Damit wird nach dem Urteil des VG Schleswig der Begriff der Behinderung nach Art. 3 III 2 GG durch die einfachgesetzliche Spezialvorschrift aus dem SGB VIII determiniert, soweit es um Legasthenie geht.

Ein solcher Rückgriff auf eine einfachgesetzliche Norm zur Auslegung eines Grundrechts ist jedoch nicht nur methodisch bedenklich ${ }^{379}$, sondern unzulässig. Die Verfassung steht nach Art. 20 III GG über dem einfachen Recht, so dass eine Auslegung verfassungsrechtlicher Begriffe nach Maßgabe des einfachen Rechts ausgeschlossen ist. ${ }^{380}$ Vielmehr hat das Verfassungsrecht Distanz zu

372 Sacksofsky, in: Umbach GG, Art. 3, Rn. 397

373 Kischel, in: BeckOK GG, Art. 3, Rn. 211: „In Abkehrung einer bekannten Parole wird man also nicht behindert, sondern ist es", die Kommentierung datiert von 2011

374

OVG Lüneburg, Beschluss v. 10.7.2008, Az.: 2 ME 309/08,

www.rechtsprechung.niedersachsen.de; OVG Weimar, Beschluss v. 17.5.2010, Az.: 1 EO

854/10, juris, Rn. 31 ff; VGH Kassel, Beschluss v. 5.2.2010; Az.: 7 A 2406/09.Z, juris, Rn. 9; VG Hannover, Beschluss v. 13.12.2010, Az.: 6 B 5596/10,

www.rechtsprechung.niedersachsen.de, S. 5

375

So das VG Hannover, Beschluss v. 13.12.2010, Az.: 6 B 5596/10, www.rechtsprechung.niedersachsen.de, S. 5

376 VG Schleswig, Urteil v. 10.6.2009, Az.: 9 A 208/08, BeckRS 2009 38123, S. 7, s. dazu auch oben 1. Teil: 2. Kapitel: 7.2.2.: Finanzierung durch die Jugendämter nach § 35 a SGB VIII, S. 48

377 VG Schleswig; Urteil v. 10.6.2009, Az.: 9 A 208/08, BeckRS 2009 38123, S. 7; diese Ansicht ausdrücklich ablehnend: OVG Weimar, Beschluss v. 17.5.2010, Az.: 1 EO 854/10, juris, Rn. 31

378 VG Schleswig, Urteil v. 10.6.2009, Az.: 9 A 208/08, BeckRS 2009 38123, S. 7

379 Zinsmeister, Mehrdimensionale Diskriminierung, S. 78

380 BVerfGE 12, 45 ff, Rn. 24; Jarass/Pieroth, in: Jarass/Pieroth GG, Einleitung I, Rn. 12; Beaucamp, JA 2001, S. 36 ff, S. 37; Beaucamp, DVBI 2002, S. 997 ff, S. 998; Spranger, br 
einfach-gesetzlichen Definitionen zu halten. ${ }^{381}$ Deshalb sind Gründe, die für die Begrenzung einfachgesetzlicher Leistungstatbestände überzeugend sein mögen, nicht ohne weiteres tauglich für die Begrenzung eines verfassungsrechtlichen Benachteiligungsverbots. ${ }^{382}$ Ebenso wenig wie sich der Anwendungsbereich des Art. 5 III GG über die Landespressegesetze bestimmen lässt, lässt sich die Behinderung über sozialrechtliche Normen bestimmen. ${ }^{383}$ Zudem würde die Grundrechtsbindung des Gesetzgebers aufgehoben, wenn man eine Verfassungsinterpretation am einfachen Gesetz zuließe. ${ }^{384}$ Schließlich ließe eine solche Interpretation des Grundrechts auf der Basis des einfachen Rechts außer Betracht, dass die Grundrechte neben der Abwehrfunktion eine objektivrechtliche Dimension im Sinne einer Wertentscheidung haben. ${ }^{385}$ Diese Wertentscheidung strahlt in das einfache Gesetz aus. ${ }^{386}$ Es ist gerade die Wechselwirkungslehre des BVerfG im Hinblick auf das Verhältnis von einfachem Recht und Verfassungsrecht, die es nicht ausreichen lässt, einfaches Recht zur Interpretation der Grundrechte heranzuziehen, sondern die verlangt, dass das einfache Gesetz der Wertentscheidung des Grundgesetzes gerecht wird. ${ }^{387}$ Das aber ist nur möglich, wenn die Auslegung des Grundrechts sich vom einfachen Gesetz löst.

\subsection{Wandelbarkeit des verfassungsrechtlichen Begriffs "Behinderung"}

Voraussetzung für eine verfassungsrechtliche Bestimmung des Begriffs der Behinderung ist ferner, dass sie der Wandlung der gesellschaftlichen und rechtlichen Auffassungen gerecht wird und nicht auf den "herkömmlichen" Begriff der Behinderung, der bei der Aufnahme des Art. 3 III 2 in das Grundgesetz vorherrschte und in $\S 3$ SchwbhG normiert war, reduziert bleibt. ${ }^{388}$

Maßggeblich für die Auslegung einer Verfassungsnorm ist der in der Norm zum Ausdruck kommende objektivierte Wille des Normgebers ${ }^{389}$, denn die Legitimationsgrundlage der Verfassung liegt in der Gegenwart und nicht in der

1998, S. 92 f; Reichenbach, Anspruch behinderter Schülerinnen und Schüler auf Unterricht in der Regelschule, S. 111; Heun, in: Dreier GG, Art. 3, Rn. 135; so auch ausdrücklich OVG Weimar, Beschluss v. 17.5.2010, Az.: 1 EO 854/10, juris, Rn. 31

Luthe, in: Rehabilitationsrecht, Rn. 3

Osterloh, in: Sachs GG, Art. 3, Rn. 310; Boysen, in: v. Münch GG 2012, Art. 3 GG, Rn. 194

Spranger, br 1998, S. 92 f, S. 92

Reichenbach, Anspruch behinderter Schülerinnen und Schüler auf Unterricht in der Regelschule, S. 111

Umfassend: Bryde, in: Hdb. Grundrechte I, § 17, Rn. $28 \mathrm{ff}$

BVerfGE 7, 198, 205 - Lüth

Bryde, in: Hdb. Grundrechte I, § 17, Rn. 37

S. die Bezugnahme des BVerfGE 96, $288 \mathrm{ff}, \mathrm{Rn} .48$ auf $\S 3$ SchwbhG

Münch, von, in: v. Münch GG, Vorb. Art. 1 - 19, Rn. 50 
Vergangenheit. ${ }^{390}$ Deshalb ist es allgemeine Ansicht, dass die Verfassung offen ist gegenüber gewandelten Erkenntnissen, Anschauungen und Bewertungen. ${ }^{391}$ Sie markiert keinen status quo, sondern entwickelt sich mit der Gesellschaft. ${ }^{392}$ So hat z.B. der Begriff der Familie über die Jahrzehnte einen grundlegenden Auffassungswandel erlebt, dem das BVerfG in seinen Entscheidungen Rechnung getragen hat. ${ }^{393}$

Ein solcher gesellschaftlicher Wandel hat sich auch beim Begriff „Behinderung" vollzogen und vollzieht sich weiterhin. ${ }^{394}$ Die Bezeichnung der betroffenen Menschen hat sich geändert, weg von den frühen im Wesentlichen pejorativen Bestimmungen als Krüppel, Schwachsinnige, Idioten usw., hin zu dem heute gebräuchlichen Begriff "Menschen mit Behinderungen“. ${ }^{395}$ Diese Entwicklung ist aber ihrerseits noch nicht abgeschlossen, sondern weiterhin im Fluss (siehe Präambel Buchst. e UN-Behindertenrechtskonvention (BRK) ${ }^{396}$ ).

Schon aufgrund der vom BVerfG offen gelassenen Begriffsbestimmung der „Behinderung" wurde gefordert, dass der verfassungsrechtliche Begriff nicht an der defizitorientierten Sichtweise des SchwbhG festhalten dürfe, sondern dass, vor allem auch für den Bereich des Schulrechts, eine "Fähigkeitsorientierung" notwendig sei. ${ }^{397}$

Der Wandel der gesellschaftlichen Auffassungen zeigt sich nicht zuletzt daran, dass das gesamte Fürsorge- und Sozialrecht in die verschiedenen Bücher des SGB überführt wurde und sich dabei der Begriff Behinderung vom personalen zum sozialen Begriff wandelte. ${ }^{398} \S 3$ Schwbh wurde aufgehoben und durch die veränderten Begriffsbestimmungen in §§ 2 I SGB IX, 19 SGB III, 35 a SGB VIII, 53 SGB XII und 3 BGG ersetzt. Allgemein wird darin ein Paradigmenwandel von einer defizitorientierten Sicht zu einer menschenrechtlichen Sicht

\footnotetext{
390 Windthorst, Verfassungsrecht, Rn. 35

391 Kunig, in: v. Münch GG, Art. 1, Rn. 19

392 Michael/Morlok, Grundrechte, S. $46 \mathrm{f}$

393 Welti, Behinderung und Rehabilitation im sozialen Rechtsstaat, S. 438 f, Coester-Waltjen, in: v. Münch GG, Art. 6, Rn. 4

394 Vgl. Präambel Buchst. e BRK

395 Historischer Überblick bei Zinsmeister, Mehrdimensionale Diskriminierung, S. 71 ff, s. auch Höller, Beschulung behinderter Kinder und Jugendlicher an öffentlichen Schulen, S. $120 \mathrm{ff}$

396 Internationales Übereinkommen über die Rechte von Menschen mit Behinderungen (BRK), BGBI. II 2008, S. 1419; Convention on the Rights of People with Disabilities, http://www.un.org/disabilities; weiterführende Informationen unter Deutsches Institut für Menschenrechte: http://www.institut-fuer-menschenrechte.de und Netzwerk Artikel 3, http://www.netzwerk-artikel-3.de

397 Füssel, RdJB 1998, S. 250 ff, S. 252 f

398 Welti, Behinderung und Rehabilitation im sozialen Rechtsstaat, S. $77 \mathrm{ff}$ und zur historischen Entwicklung insgesamt S. $229 \mathrm{ff}$
} 
von Behinderung gesehen. ${ }^{399}$ Die Politik für behinderte Menschen entwickelt sich damit immer mehr in Richtung Integration der Menschen mit Behinderung und dazu, innen ein selbstbestimmtes Leben zu ermöglichen. Die Bundesregierung hat in der Denkschrift zur UN-BRK die Ausrichtung des Begriffs Behinderung auf das soziale Modell bestätigt und bekräftigt. ${ }^{400}$

Mit einer solchen Veränderung der gesellschaftlichen Wirklichkeit wäre es unvereinbar, wenn die Begriffsbestimmung auf der Grundlage des überholten Behinderungsbegriffs, wie er in $\S 3$ SchwbhG normiert war, erfolgen würde.

\subsection{Völkerrechtsfreundliche Auslegung des Begriffs Behinderung}

Weiter ist die Frage, inwieweit die Auslegung von Art. 3 III 2 GG durch völkerrechtliche Verpflichtungen der Bundesrepublik, insbesondere der UN-Behindertenrechtskonvention (BRK) beeinflusst wird. Der Bund hat der BRK mit Gesetz vom 21. 12. 2008 zugestimmt $^{401}$ und sie ratifiziert. Sie ist am 26. März 2009 für die Bundesrepublik Deutschland in Kraft getreten. ${ }^{402}$ Die Länder haben im Ratifizierungsprozess ihre Zustimmung gemäß dem sog. Lindauer Abkommen erklärt ${ }^{403}$, so dass der Vertragsabschluss rechtmäßig und wirksam erfolgte. ${ }^{404}$

Eine besondere Bedeutung der BRK ergibt sich daraus, dass sie als erste völkerrechtliche Konvention von der EU als Gemeinschaft ratifiziert wurde ${ }^{405}$, so

399 Welti, Behinderung und Rehabilitation im sozialen Rechtsstaat, S. 58 f; Luthe, in: Rehabilitationsrecht, Rn. 7; s. auch: Denkschrift der Bundesregierung zu dem Übereinkommen vom 13. Dezember 2006 über die Rechte von Menschen mit Behinderung, BT Drs. 16/10808, S. 46 und Mathe, br 2011, S. 34 ff, S. 34

Denkschrift der Bundesregierung zu dem Übereinkommen vom 13. Dezember 2006 über die Rechte von Menschen mit Behinderung, BT Drs. 16/10808, S. 46

401

Gesetz zu dem Übereinkommen der Vereinten Nationen vom 13. Dezember 2006 über die Rechte von Menschen mit Behinderungen sowie zu dem Fakultativprotokoll vom 13. Dezember 2006 zum Übereinkommen der Vereinten Nationen über die Rechte von Menschen mit Behinderungen, vom 21. Dezember 2008, BGBI 2008, Teil II Nr. 35, S. 1419

402 S. dazu: Aichele, UN-BRK und Fakultativprotokoll; Degener, RdJB 2009, S. 200 ff; Latham/ Watkins, Gutachten zur UN-BRK; Riedel, Gutachten UN-Behindertenrechtskonvention; Rux, RdJB 2009, S. 220 ff; Masuch, in: FS Renate Jaeger, S. 245 ff und VGH Kassel, Beschluss V. 12.11.2009, Az.: 7 B 2763/09, juris; zur Entstehung der BRK s. United Nations, From Exclusion to Equality

Detailliert zum Zustimmungsverfahren: Latham/Watkins, Gutachten zur UN-BRK, S. 23 f; die Zweifel des VGH Kassel, Beschluss v. 12.11.2009, Az.: 7 B 2763/09, juris, Rn. 20 f an der ausreichenden Zustimmung des Landes Hessen sind danach verfehlt. Riedel, Gutachten UN-Behindertenrechtskonvention, S. 34 Beschluss des Rates vom 26.11.2009 über den Abschluss des Übereinkommens der Vereinten Nationen über die Rechte von Menschen mit Behinderungen im Namen der Gemeinschaft (Art. 1 des Beschlusses 2010/48/EG), http://eur-lex.europa.eu/LexUriServ/LexUriServ.do?uri=OJ:L:2010:023:0035:0061:DE:PDF; s. 
dass sie gem. Art. 25 GG i.V.m. Art. 4 EUV im Kompetenzbereich der EU den gliedstaatlichen Gesetzen vorgeht ${ }^{406}$ und für Deutschland auch als Mitgliedstaat der EU Geltung hat.

Es ist allgemein anerkannt, dass dem GG die Verfassungsentscheidung für eine "offene Staatlichkeit" zugrunde liegt, die es gebietet, bei der Anwendung des deutschen Rechts die völkerrechtlichen Verpflichtungen der Bundesrepublik zu beachten, es völkerrechtsfreundlich auszulegen und bei etwaigen Konflikten die Gesetze im Einklang mit den völkerrechtlichen Verpflichtungen auszulegen. ${ }^{407}$ Voraussetzung dafür ist allein, dass die Bundesrepublik einen völkerrechtlichen Vertrag geschlossen hat. Dieser bindet die Bundesrepublik als Völkerrechtssubjekt nach Art. 32 I, 59 I GG i.V.m. Art. 26, 27, 46 der Wiener Vertragsrechtskonvention (WVK) auf völkerrechtlicher Ebene.

Fraglich aber kann sein, ob die BRK auch bei der Auslegung der Grundrechte zu beachten ist. Das BVerfG hat die Frage, ob völkerrechtliche Rechtsquellen bei der Interpretation zu berücksichtigen sind, etwa für die Europäische Menschenrechtskonvention (EMRK) bejaht: „Der Konventionstext und die Rechtsprechung des Europäischen Gerichtshofs für Menschenrechte dienen auf der Ebene des Verfassungsrechts als Auslegungshilfen für die Bestimmung von Inhalt und Reichweite von Grundrechten ...., sofern dies nicht zu einer ... Einschränkung oder Minderung des Grundrechtsschutzes nach dem Grundgesetz führt. ${ }^{\prime 408}$ In der jüngsten Entscheidung zur Sicherheitsverwahrung ${ }^{409}$ hat das BVerfG diese Position bekräftigt und ausgeführt, dass den Garantien der Menschenrechtskonvention in der Bundesrepublik Deutschland möglichst umfassend Geltung zu verschaffen ist und damit verdeutlicht, dass die Grundrechte auch als Ausprägung der Menschenrechte zu verstehen sind. ${ }^{410}$ Die BRK ist ein menschenrechtlicher Vertrag, mit dem nicht neue Rechte für Menschen mit

auch Verhaltenskodex zwischen dem Rat, den Mitgliedstaaten und der Kommission zur Festlegung interner Regelungen für die Durchführung des Übereinkommens der Vereinten Nationen über die Rechte von Menschen mit Behinderungen durch die Europäische Union und für die Vertretung der Union in Bezug auf das Übereinkommen v. 15.12.2010; 2010/C 340/08, http://eur-lex.europa.eu/LexUriServ/LexUriServ.do?uri=OJ:C:2010:340:0011: 0015:DE:PDF Vgl. Geiger/Khan/Kotzur EUV/AEUV Art. 4 EUV, Rn. 11, $27 \mathrm{ff}$

407 St. Rspr. BVerfGE 75, 17; 111, 307, 328; Geiger, Völkerrecht, S. 170; Voßkuhle, NVwZ 2010, S. 1 ff, S. 3 f; ausführlich Proelss, in: Rechtsprechung BVerfG, S. 553 ff; zur BRK insbesondere Riedel, Gutachten UN-Behindertenrechtskonvention, S. 38

BVerfGE 111, 307, 317, Rn. 32, http://www.bverfg.de/entscheidungen/rs20041014_2bvr148104.html BVerfG, Beschluss 4.5.2011; Az.: 2 BvR 2365/09, http://www.bverfg.de/entscheidungen/rs20110504_2bvr236509.html, Rn. 88 410 BVerfG, Beschluss 4.5.2011; Az.: 2 BvR 2365/09, http://www.bverfg.de/entscheidungen/rs20110504_2bvr236509.html, Rn. 90 
Behinderung geschaffen, sondern bestehende Menschenrechte so konkretisiert werden, dass Menschen mit Behinderung sie ebenso ausüben und genießen können wie alle anderen. ${ }^{411}$ Das BVerfG hat dies für die BRK bejaht ${ }^{412}$, so dass die BRK als Auslegungshilfe für die Grundrechte heranzuziehen ist. ${ }^{413}$ Dies gilt insbesondere für das spezielle Grundrecht für Menschen mit Behinderung aus Art. 3 III 2 GG, das in der BRK sein völkerrechtliches Pendant findet. Art. 3 III 2 GG ist daher unter Berücksichtigung der BRK völkerrechtsfreundlich auszulegen. ${ }^{414}$

In welchem Umfang der Grundsatz der völkerrechtsfreundlichen Auslegung zur Anwendung kommt, ist im Einzelnen streitig. ${ }^{415}$ Auf diesen Streit kommt es jedoch nicht an, wenn schon die engste Auslegung dieses Grundsatzes seine Anwendung gebietet. Die engste Auslegung geht davon aus, dass es sich bei dem Grundsatz der völkerrechtsfreundlichen Auslegung um eine Konfliktvermeidungsregel handelt, die zur Anwendung gelangt, wenn eine innerstaatliche Regelung und eine völkerrechtliche Norm mehrere Deutungen zulassen. ${ }^{416}$ In einem solchen Fall ist derjenigen Interpretation der Vorrang zu geben, die gleichzeitig den Anforderungen des Völkerrechts gerecht wird. ${ }^{417} \mathrm{Zu}$ untersuchen ist daher jeweils, ob es einen Konflikt zwischen der Auslegung des Behinderungsbegriffs des Art. 3 III 2 GG und demjenigen der BRK gibt, der dann im Zweifel zugunsten der völkerrechtlichen Norm zu entscheiden ist.

Die BRK definiert den Begriff Behinderung nicht ${ }^{418}$, sondern beschreibt die Behinderung explizit als Wechselwirkung zwischen dem Individuum und der Umwelt. Art. 1 II BRK führt aus: „Zu den Menschen mit Behinderungen zählen Menschen, die langfristige körperliche, seelische, geistige oder Sinnesbeeinträchtigungen haben, welche sie in Wechselwirkung mit verschiedenen Barrieren an der vollen, wirksamen und gleichberechtigten Teilhabe an der Gesell-

411 Aichele, UN-BRK und Fakultativprotokoll, S. 4; Mathe, br 2011, S. 34 ff, S. 38; Masuch, in: FS Renate Jaeger, S. 246; Denkschrift der Bundesregierung zu dem Übereinkommen vom 13. Dezember 2006 über die Rechte von Menschen mit Behinderung, BT Drs. 16/10808, S. 46 BVerfG Beschluss v. 23.3.2011; Az.: 2 BvR 882/09, http://www.bundesverfassungsgericht.de/entscheidungen/rs20110323_2bvr088209.html, Rn. 52; ebenfalls bejahend: LSG Sachsen, Beschluss v. 3.6.2010, Az.: L 7 SO 19/09 B ER, www.sozialgerichtsbarkeit.de, S. 6; s. auch Proelss, in: Rechtsprechung BVerfG, S. 564; Michael/Morlok, Grundrechte, Rn. 115; Geiger, Völkerrecht, S. 170

BVerfG 2 BvR 882/09 vom 23.3. 2011, Rn. 52; so auch VG Freiburg, Urteil v. 25.3.2009, Az.: 2 K 1638/08, juris, Rn. 58; Krajewski/Bernhard, BayVBI 2012, S. 134 ff, S. 139; umfassend Aichele, Anwaltsblatt 2011, 727 ff, S. 730 S. dazu ausführlich Proelss, in: Rechtsprechung BVerfG, S. $557 \mathrm{ff}$

Proelss, in: Rechtsprechung BVerfG, S. 557 f, S. 560 
schaft hindern können. ${ }^{419}$ Die BRK hat damit ein sehr weites Verständnis von Behinderung. Riedel weist darauf hin, dass eine Ausklammerung von Behinderungsformen und -erfahrungen aus der BRK völkerrechtlich ausgeschlossen ist, da dies Inhalt und Geist der Konvention fundamental widersprechen würde. ${ }^{420}$ Ergänzend ist darauf hinzuweisen, dass sich die Ansicht durchsetzt, dass $\S 2$ SGB IX überarbeitet werden muss, um den Anforderungen der BRK zu genügen und bereits jetzt die völkerrechtsfreundliche Auslegung bei sozialrechtlichen Ansprüchen zu einer weiteren, über den Wortlaut des $\S 2$ SGB IX hinausgehenden Auslegung zwingt. ${ }^{421}$

\subsection{Grammatische Auslegung}

Im Rahmen der Auslegung eines verfassungsrechtlichen Begriffs ist zunächst nach dem Wortsinn zu fragen. Die Alltagssprache ist mit Begriffen des Behindertseins besetzt, die zwar metaphorisch gemeint sind, aber eine allgemein negative Einstellung gegenüber Behinderungen enthalten. In der Jugendsprache ist "Behinderung" ein Schimpfwort, das benutzt wird, um andere herabzu-

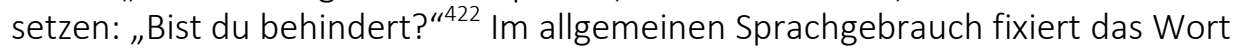
Abweichungen und (re)produziert Ausgrenzung, schafft potentiell und faktisch Trennlinien. ${ }^{423}$ Behinderung ist meist ein Skandalon, mit dem regelmäßig Eigenschaften der Unfähigkeit und des Nichtkönnens verbunden werden. ${ }^{424}$

Der Begriff der Behinderung ist alltagssprachlich daher kein Begriff, auf den das Recht zurückgreifen könnte. ${ }^{425}$ Als Rechtsbegriff ist Behinderung einer der ungeklärtesten unbestimmten Rechtsbegriffe, nicht nur in der deutschen, sondern auch in anderen Rechtsordnungen. ${ }^{426}$ Seine Auslegung muss den Wandel in der gesellschaftlichen und auch rechtlichen Auffassung berücksichtigen.

419 Wortlaut der amtlichen deutschen Übersetzung. Der Wortlaut der deutschen Schattenübersetzung, die vom Netzwerk Artikel 3 mit dem Anspruch veröffentlicht wurde, (angebliche) Übersetzungsfehler der deutschen Übersetzung zu korrigieren, ist: „Zu den Menschen mit Behinderungen zählen Menschen, die langfristige körperliche, seelische, geistige oder Sinnesbeeinträchtigungen haben, die in Wechselwirkung mit verschiedenen Barrieren ihre volle und wirksame Teilhabe gleichberechtigt mit anderen an der Gesellschaft behindern können." http://www.netzwerk-artikel-3.de/index.php?view=article\&catid=42:internationalevereinbarungen-un-gleichstellung\&id=93:international-schattenuebersetzung\&format=pdf Riedel, Gutachten UN-Behindertenrechtskonvention, S. 6

Banafsche, SGb 2012, S. 373 ff, S. 440 ff, S. 374 f; Hirschberg, DIMR -Behinderung, S. 3; Aichele/ Althoff, Kommentar zur UN-BRK, Nicht-Diskriminierung, Rn. 38

422 Dederich, Zwischen Ausschluss und Anerkennung, S. 103

423

425 Welti, Behinderung und Rehabilitation im sozialen Rechtsstaat, S. 113

426 S. den Überblick bei Degener, ZaöRV 2005, S. 887 ff, S. 905, $907 \mathrm{ff}$ 
Ursprünglich basierte der Rechtsbegriff „Behinderung“ auf einem medizinischdefektorientierten Ansatz, der von einer Krankheit oder sonstigen Abweichung vom Normalen ausging. ${ }^{427} \S 3$ SchwbhG definierte als Behinderung den regelwidrigen körperlichen, geistigen oder seelischen Zustand. Die Definition setzte bei dem betroffenen Menschen und seiner Abweichung von der Norm der Nichtbehinderten an. ${ }^{428}$ Die Zuschreibung der "Behinderung" diente insbesondere dazu, die Gruppe derjenigen zu definieren, die Anspruch auf bestimmte staatliche Leistungen haben. Aufgabe des Menschen mit Behinderung ist es nach diesem Verständnis, dieses Problem selbst zu lösen. ${ }^{429}$ Dementsprechend war es das Ziel sozialrechtlicher Normen, die Behinderung zu überwinden und den behinderten Menschen dazu zu befähigen, den allgemeinen Anforderungen zu genügen. Die Behinderung wurde als „problem to be fixed“430 angesehen. Dieser Defizitansatz hat auch das Schulrecht geprägt, wie z.B. die Ausführungen von Lambert zeigen: „Hintergrund ist folgende einfache Überlegung: Die Behinderung ist sicherlich ein Nachteil, aber ein Nachteil, der von der Natur vorgegeben ist, den die Rechts- und Gesellschaftsordnung also vorfindet und nicht schafft. Es müssen Hilfsmittel und Fördermaßnahmen bereitgestellt werden, um diesen Nachteil zu beheben oder zu mildern. Es handelt sich insoweit aber um Vorteile, die dem behinderten Schüler gewährt werden. ${ }^{\text {“431 }}$

Das BVerfG hat sich in seiner Sonderschulentscheidung ebenfalls auf diesen defektorientierten Ansatz gestützt, die abschließende Bestimmung des Begriffs „Behinderung" aber ausdrücklich offen gelassen. ${ }^{432}$ In den folgenden Entscheidungen zu Art. 3 III 2 GG hat es an diesem defektorientierten Ansatz festgehalten, denn es hat jeweils geprüft, ob die jeweilige Benachteiligung durch behinderungsbezogene Besonderheiten gerechtfertigt seien.

So nahm das BVerfG die Verfassungsbeschwerden zweier Mädchen, die an der Glasknochenkrankheit leiden, gegen die Nichtaufnahme in einen integrativen Regelkindergarten nicht zur Entscheidung an und führte aus, dass es aus verfassungsrechtlichen Gründen nicht zu beanstanden sei, dass der Staat die zielgleiche wie die zieldifferente integrative Erziehung unter den Vorbehalt des organisatorisch, personell und von den sächlichen Voraussetzungen her Möglichen stelle. ${ }^{433}$ Die angegriffene Regelung des niedersächsischen Kindertages- 
stättengesetzes sei verfassungsgemäß, da der Anspruch auf Aufnahme in einen Regelkindergarten bestehe, wenn das behinderte Kind nach der Art seiner Behinderung in der Lage sei, ohne besondere Hilfe an den vorhandenen Betreuungsmöglichkeiten des Regelkindergarten teilzuhaben. Sei es dazu wegen der Art seiner Behinderung nicht fähig, sei es nicht zu beanstanden, dass es in eine integrierte Gruppe oder eine teilstationäre Einrichtung verwiesen würde. ${ }^{434}$ Das BVerfG orientiert sich hier an dem körperlichen Defizit der betroffenen Mädchen und prüft, ob dieses Defizit die Betroffenen daran hindert, sich in eine Regeleinrichtung zu integrieren. ${ }^{435}$ Die Anpassungsleistung liegt damit nur bei den Betroffenen, während die Regeleinrichtung keine Anpassung an die Bedürfnisse der Betroffenen leisten muss.

In der gesellschaftlichen und auch internationalen Diskussion ${ }^{436}$ wurde jedoch immer mehr wahrgenommen, dass Behinderungen keineswegs von der Natur vorgegeben und persönliche Defizite sind, sondern durch Rollenzuweisungen und Benachteiligung erst entstehen ${ }^{437}$, mithin auch von der Rechtsordnung geschaffen werden können. ${ }^{438}$ Statt Behinderung als individuelles Problem zu definieren, wurde das soziale Modell von Behinderung entwickelt, das den Blick auf die Umwelt und die Gesellschaft und deren Umgang mit Differenz richtet. ${ }^{439}$ Die Behinderung wird damit beschrieben als soziales Verhältnis zwischen behindertem Menschen und behindernder Umwelt. Infolgedessen wird mit Behinderung nicht mehr eine abgrenzbare Menge von behinderten Menschen bezeichnet. Vielmehr wird auf die gesellschaftlichen Zustände gesehen, in denen der betroffene Mensch agiert. ${ }^{440}$ Die Vorstellung, was Menschen normalerweise können und leisten sollen, welches Verhalten "normal" und welches "abweichend" ist, wird wesentlich von ökologischen, ökonomischen und kulturellen Faktoren bestimmt. ${ }^{441}$ Wandeln sich gesellschaftliche Erwartungen, so wandelt sich auch das Verständnis davon, wer "normal“ und wer „behindert" ist. Die gesellschaftlichen Verhältnisse bestimmen daher entscheidend, was eine Behinderung ist und was nicht. Dieses soziale Modell der Be-

434 BVerfG Beschluss v. 10.2.2006 Az.: 1 BvR 91/06, Rn. 13, http://www.bverfg.de/entscheidungen/rk20060210_1bvr009106.html

435

436

Chegener, br 2009, S. $34 \mathrm{ff}, \mathrm{s} .41$

S. dazu auch Denkschrift der Bundesregierung zu dem Übereinkommen vom 13. Dezember 2006 über die Rechte von Menschen mit Behinderung, BT Drs. 16/10808, S. 45 f

437 UN-Behindertenrechtskonvention, S. 3

438 A.A. Lambert, RdJB 1998, S. 384 ff, S. 388

439

440

441

Degener, ZaöRV 2005, S. 887 ff, S. 889; Mathe, br 2011, S. 34 ff, S. 37

Welti, Behinderung und Rehabilitation im sozialen Rechtsstaat, S. 81

Zinsmeister, Mehrdimensionale Diskriminierung, S. 83; s. dazu das Beispiel bei Fuerst, Behinderung zwischen Diskriminierungsschutz und Rehabilitationsrecht, S. 52, Fn. 135 
hinderung hat die WHO in der, International Classification of Functioning, Disability and Health' (ICF) ${ }^{442}$ niedergelegt. Danach wird als Behinderung der Zustand bezeichnet, der im Zusammenwirken von einem Gesundheitsproblem mit personenbezogenen Faktoren oder Umweltfaktoren entsteht. Die Behinderung selber setzt sich aus der Schädigung der Körperfunktionen, der Aktivitätsbeeinträchtigung und der Partizipationsbeeinträchtigung zusammen. Diese drei Komponenten sind nicht kausal miteinander verbunden, sondern stehen in prozeduraler Wechselwirkung zueinander. ${ }^{443}$ Anhand des Kodierungssystems des ICF können die Bereiche, in denen ein Mensch eingeschränkt ist oder in der Partizipation beeinträchtigt ist, beschrieben, festgelegt und in ihrer Ausprägung gewichtet werden. ${ }^{444}$ Dieses Koordinierungssystem macht überdies deutlich, wie sich Veränderungen im gesellschaftlichen Sprachverhalten normativ niederschlagen und wie derartige Normierungen wiederum auf das gesellschaftliche Sprachverhalten zurückwirken. ${ }^{445}$

Mit dem weiten Behinderungsbegriff entsteht ein Modell, das zum Ausdruck bringt, dass ein Mensch mit Behinderung nicht behindert ist, sondern durch die an Nichtbehinderten ausgerichtete Lebens- und Sprachumwelt behindert wird. ${ }^{446}$ Je stärker die Umgebung auf eine körperliche Einschränkung reagiert, desto schwerer wird der Grad der Behinderung für diese körperliche Einschränkung. Andererseits können schwerste körperliche Einschränkungen, auf die die Umgebung nicht reagiert, keine oder fast keine Behinderung bedeuten. Verdeutlichen lässt sich dieses Modell an der Linkshändigkeit: Lange Zeit wurde die Linkshändigkeit als anormal angesehen und mit entsprechend negativer Konnotation auch so benannt. Die Schule tat daher alles, um die Kinder auf rechtshändiges Schreiben umzuerziehen. Die Umerziehung blieb oft nicht ohne erhebliche auch psychische Folgen. Heute ist Linkshändigkeit als Normvariante anerkannt und akzeptiert. ${ }^{447}$ Das körperliche Anderssein ruft keine negative Reaktion der Umwelt mehr hervor. Linkshändigkeit ist sprachlich nicht mehr negativ konnotiert, und eine Behinderung liegt nicht (mehr) vor. Das so-

442 Klassifikation der Funktionsfähigkeit, Behinderung und Gesundheit in der maßgeblichen deutschen Übersetzung durch das DIMDI, http://www.dimdi.de/dynamic/de/klassi/ downloadcenter/icf/endfassung/icf_endfassung-2005-10-01.pdf

Fuerst, Behinderung zwischen Diskriminierungsschutz und Rehabilitationsrecht, S. 50 f; s. dazu auch Meysen, in: Frankfurter Kommentar SGB VIII, § 35 a, Rn. 33 ff

ICF, deutsche Fassung S. 163; So kann z.B. bei einem Kind mit Dyskalkulie dargestellt werden, wie stark es in einem Ausmaß zwischen 1 bis 4 in bestimmten Bereichen, wie z.B. d150 (Elementares Lernen -Rechnen Lernen), d 172 (Wissensanwendung - Rechnen lernen), d 820 (Schulbildung), d 845 (Arbeit erhalten ...) beeinträchtigt ist; zur Maßgeblichkeit dieser Klassifikation s. auch Bundesregierung, Nationaler Aktionsplan zur UN-BRK, S. 27, S. 84 Vgl. hierzu Kupka, Archiv für Rechts- und Sozialphilosophie 2011, S. $44 \mathrm{ff}$

446 Straßmair, Der besondere Gleichheitssatz aus Art. 3 Abs. 3 Satz 2 GG, S. 170

447 Vanselow/Dummer-Smoch, Vernachlässigung individueller Begabungsstrukturen, S. 259 
ziale Modell ermöglicht es auch, nicht nur zu sehen, was ein Mensch mit Behinderung nicht kann, sondern ein Augenmerk darauf zu legen, was ein Mensch mit Behinderung - gerade weil er fehlende oder eingeschränkte Fähigkeiten kompensieren muss - gut oder sogar besser kann als Menschen ohne Behinderung. ${ }^{448}$

Das soziale Modell, das die sozialen Auswirkungen der Behinderung berücksichtigt ${ }^{449}$, wird so auch von der Literatur zu Art. 3 III 2 GG vertreten. ${ }^{450}$ Sie versteht Behinderung als sich wechselseitig bedingende Abhängigkeit zwischen im weitesten Sinne körperlich angelegten Beeinträchtigungen und den sozialen Lebensbedingungen. ${ }^{451}$ Caspar verweist beispielhaft auf die norwegische Definition, die Behinderung versteht als "Diskrepanz" zwischen den Fähigkeiten eines Individuums und den Funktionen, die ihm in der Gesellschaft abverlangt werden. Daher definiere sich die Behinderung weniger über das Nicht-Können, als über die konkreten Anforderungen des täglichen Lebens, aus denen die Behinderung eine soziale Dimension erhalte. ${ }^{452}$

Die Literatur, die sich mit der Problematik der Beschulung von behinderten Schülern beschäftigt, versteht den Behinderungsbegriff noch weiter. Durchgängig wird hier die Ansicht vertreten, dass schon die Feststellung des sonderpädagogischen Förderbedarfs den Begriff der Behinderung erfüllt. ${ }^{453}$ Reichenbach sieht darin eine Behinderung, weil insofern eine seelische Regelwidrigkeit

448 Blinde Menschen können viel besser tasten als Menschen ohne Behinderung. Diese besondere Fähigkeit wird dazu genutzt, blinde Frauen darin auszubilden, Brustkrebs zu ertasten. http://www.discovering-hands.de. Menschen mit Autismus haben besondere Fähigkeiten aufgrund der ihnen angeborenen Detailgenauigkeit Ehrich, Thorkil Sonne, Specialisterne, S. $34 \mathrm{ff}$ und http://www.focus.de/gesundheit/ratgeber/gehirn/krankheiten/tid18198/modernes-leben-teil-2-die-stille-reserve_aid_506761.html; sowie die weiteren Beispiele bei Zinsmeister, in: Antidiskriminierungsrecht, S. $173 \mathrm{f}$

449 Grundlegend dazu Welti, Behinderung und Rehabilitation im sozialen Rechtsstaat, S. 5-115, Überblick bei Zinsmeister, Mehrdimensionale Diskriminierung, S. 70 - 88, Degener, Disability Studies Quarterly 2006; Heiden, in: Benachteiligungsverbot, S. 17 ff; Höller, Beschulung behinderter Kinder und Jugendlicher an öffentlichen Schulen, S. 119 ff; s. auch Fuerst, Behinderung zwischen Diskriminierungsschutz und Rehabilitationsrecht, S. $48 \mathrm{ff}$ So jetzt auch Boysen, in: v. Münch GG 2012, Art. 3 GG, Rn. 192

451 Luthe, in: Rehabilitationsrecht - Verfassungsrecht, Rn. 1 f; Caspar, EuGRZ 2000, S. 135 ff, S. 136; Zinsmeister, Mehrdimensionale Diskriminierung, S. 87 f; Höller, Beschulung behinderter Kinder und Jugendlicher an öffentlichen Schulen, S. 152; Straßmair, Der besondere Gleichheitssatz aus Art. 3 Abs. 3 Satz 2 GG, S. 174 f; Riedel, Gutachten UN-Behindertenrechtskonvention, S. 4; Rux, RdJB 2009, S. 220 ff; Spranger, br 1998, S. 92 f, S. 93 Caspar, EuGRZ 2000, S. 135 ff, S. 136

Höller, Beschulung behinderter Kinder und Jugendlicher an öffentlichen Schulen, S. 144 ff; Reichenbach, Anspruch behinderter Schülerinnen und Schüler auf Unterricht in der Regelschule, S. 166; Reimann, Sicherstellung des Schulbesuchs behinderter Kinder, S. 34 f; Jürgens, DVBI 1997, S. 410 ff, S. 411; Rux, RdJB 2009, S. 220 ff, S. 223 
vorliegen würde. ${ }^{454}$ Reimann hält es aus rechtssystematischen Gründen für verfehlt, bei der Bestimmung des Anwendungsbereichs des Art. 3 III 2 GG zwischen Behinderung und sonderpädagogischem Förderbedarf zu unterscheiden. ${ }^{455}$ Eine Unterscheidung würde dazu führen, dass diejenigen Schüler, deren sonderpädagogischer Förderbedarf nicht auf einer körperlich angelegten Störung, sondern auf anderen Faktoren beruht, im Schutzniveau hinter anderen Schülern zurückstehen würden. Sie würden gegenüber behinderten Schülern schlechter gestellt, obwohl sie die gleichen Benachteiligungen erfahren würden. ${ }^{456}$ Höller legt dar, dass der sonderpädagogische Förderbedarf dem heilpädagogischen Begriff der Behinderung entspricht ${ }^{457}$ und sonderpädagogischer Förderbedarf eine Behinderung i.S.d. Art. 3 III 2 GG ist. ${ }^{458}$

Der Begriff "Behinderung" ist daher sprachlich weit im Sinne des sozialen Modells auszulegen.

\subsection{Historische Auslegung}

Der Aufnahme des Art. 3 III 2 in das Grundgesetz ist eine längere Diskussion darüber vorausgegangen, ob die Aufnahme des Diskriminierungsverbots in das Grundgesetz überhaupt erforderlich sei oder ob die Menschen mit Behinderung nicht bereits umfassend über Art. 1 I GG und das Sozialstaatsprinzip geschützt seien. ${ }^{459}$ Zu einem Stimmungswechsel führte unter anderem ein Urteil des Amtsgerichts Flensburg ${ }^{460}$, das in der Anwesenheit einer Gruppe geistig behinderter Menschen in einem Hotel in der Türkei einen Reisemangel sah. Schließlich fand sich doch die notwendige 2/3-Mehrheit für die GrundgesetzÄnderung. ${ }^{461}$ Aus den Beratungen über das Diskriminierungsverbot und seine sprachliche Fassung ergibt sich, dass der Begriff der Behinderung seinerzeit als hinreichend konkret und der Kreis der Betroffenen als klar abgrenzbar angesehen wurde, so dass der Begriff nicht weiter diskutiert wurde. ${ }^{462}$ Welches Begriffsverständnis der Verfassungsgesetzgeber aber genau hatte, ist kaum feststellbar.

\footnotetext{
454 Reichenbach, Anspruch behinderter Schülerinnen und Schüler auf Unterricht in der Regelschule. S. 166

455 Reimann, Sicherstellung des Schulbesuchs behinderter Kinder, S. 35

456 Reimann, Sicherstellung des Schulbesuchs behinderter Kinder, S. 34

457 Höller, Beschulung behinderter Kinder und Jugendlicher an öffentlichen Schulen, S. 161

458 Höller, Beschulung behinderter Kinder und Jugendlicher an öffentlichen Schulen, S. 168

459 Jürgens, DVBI 1997, S. 410 ff, S. 410

460 AG Flensburg, NJW 1993, S. 272; Az: 63 C 265/92

461 Umfassend zur Entstehungsgeschichte Jürgens, DVBI 1997, S. $410 \mathrm{ff}$, S. $410 \mathrm{f}$

462 Reichenbach, Anspruch behinderter Schülerinnen und Schüler auf Unterricht in der Regelschule, S. 145 ff m.w.N.; Osterloh, in: Sachs GG, Art. 3, Rn. 309 m.w.N.
} 


\subsection{Systematische Auslegung}

Im Grundgesetz findet sich keine weitere Bestimmung bezüglich Menschen mit Behinderungen. Das heißt jedoch nicht, dass Art. 3 III 2 GG allein und ohne Bindung an die grundsätzlichen Wertungen des GG steht.

Maßgebliches Fundament des Grundgesetzes ist die Menschenwürde, deren Inhalt nicht statisch, sondern ebenfalls für gewandelte Erkenntnisse, Anschauungen und Bewertungen offen ist. ${ }^{463}$ Die Menschenwürde ist höchster Rechtswert innerhalb der Verfassungsordnung, ${ }^{464}$ die jeder Mensch besitzt. ${ }^{465}$ Die verfassungsrechtliche Grundentscheidung zur Achtung der Menschenwürde verpflichtet den Staat dazu, Beeinträchtigungen der Würde abzuwehren und solchen Beeinträchtigungen vorzubeugen ${ }^{466}$, und den Menschen nicht zum bloßen Objekt des Staates zu machen. ${ }^{467}$ Die Gefahr, ein bloßes Objekt des Staates zu sein, ist bei Menschen mit Behinderungen größer als bei anderen Menschen. Deshalb ist bei innen der Schutz des Staates besonders erforderlich, so dass die staatliche Verpflichtung zum Schutz der Menschenwürde hier eine besondere Bewandtnis hat. ${ }^{468}$

Das Benachteiligungsverbot für Behinderte steht weiter in engem Zusammenhang mit dem Sozialstaatsprinzip. Die Sozialstaatlichkeit gehört zum Kern des Grundgesetzes. Dieses Prinzip, dass sich aus Art. 20 I GG und Art. 28 I 1 GG ergibt, verpflichtet den Staat, jedem Staatsbürger eine Teilhabe an den für das Staatsvolk typischen, durch staatliche Gesetzgebung herstellbaren Lebensbedingungen anzubieten. ${ }^{469} \mathrm{Zu}$ den wesentlichen Komponenten des Sozialstaatsprinzips gehört u.a. die Verwirklichung der Chancengleichheit. Diese zielt auf die Angleichung der tatsächlichen Voraussetzungen für den Erwerb materieller und immaterieller Güter und soll die Nutzung der Freiheitsrechte ermöglichen. ${ }^{470}$ Das Sozialstaatsprinzip hat durch Art. 3 III 2 GG eine besondere Ausprägung erhalten, weil mit diesem Grundrecht nicht nur ein Konsens festgeschrieben wurde, sondern das Ziel verfolgt wurde, die Integration von Menschen mit Behinderungen zu fördern und voranzubringen. ${ }^{471}$ Teilweise wird die praktische Bedeutung des Art. 3 III 2 GG in der Konkretisierung des Sozial-

\footnotetext{
463 Kunig, in: v. Münch GG, Art. 1, Rn. 19

464 BVerfGE 5, 85, 204; 6, 32, 41; 30, 173, 193; 32, 98, 106

465 BVerfGE 87, 209, 228

466 Kunig, in: v. Münch GG, Art. 1, Rn. 30

467 BVerfGE 9, 89, 95

468 Frowein, Rechtsgutachten, S. 4

469 Herdegen, VSSR 1992, S. 245 ff, S. 249

470 Herdegen, VSSR 1992, S. 245 ff, S. 249; s. dazu BVerfG, 1 BvL 1/09 vom 9.2.2010, http://www.bverfg.de/entscheidungen/Is20100209_1bvl000109.html, Hartz IV-Gesetz

471 Vgl. Herdegen, VSSR 1992, S. 245 ff, S. 247
} 
staatsprinzips gesehen, insbesondere wenn es um besondere staatliche Leistungen geht. ${ }^{472}$

Diese Wertvorstellungen des GG führen konkret dazu, dass der Begriff der „Behinderung“ vor allem die Menschenwürde der Menschen mit Behinderung im Blick haben muss und in ihm die Verwirklichung der Chancengleichheit als Ausprägung des Sozialstaatsgebots angelegt ist. Dies gewährleistet nur das oben erörterte soziale Modell von Behinderung, das die gesellschaftlichen Bedingungen einbezieht, um die Chancengleichheit von Menschen mit Behinderung zu verwirklichen.

\subsection{Teleologische Auslegung}

Die Auslegung einer Verfassungsnorm muss sich auf der Grundlage der Werte des Grundgesetzes, am Ziel der Norm und an der ihr zugrunde liegenden Gerechtigkeitsvorstellung orientieren. ${ }^{473}$ Ziel des Art. 3 III 2 GG ist nach der ausdrücklichen Intention des Verfassungsgesetzgebers nicht nur die Integration all derjenigen Menschen mit Behinderungen, die unter Diskriminierung leiden. ${ }^{474}$ Vielmehr soll durch die Vorschrift auch ein Bewusstseinswandel herbeigeführt werden, der bewirkt, dass sich die soziale Umwelt so verändert, dass sie sich gegenüber Menschen mit Behinderung nicht mehr benachteiligend verhält. ${ }^{475}$ Die Ausgrenzung von Menschen mit Behinderung soll verhindert und überwunden werden. ${ }^{476}$ Mit der Aufnahme des Art. 3 III 2 GG wollte der Verfassungsgeber einerseits den Schutz für Menschen mit Behinderungen verstärken und der staatlichen Gewalt enge Grenzen vorgeben, andererseits verfassungspolitisch ein deutliches Signal setzen und einen Anstoß für einen Bewusstseinswandel in der Bevölkerung geben ${ }^{477}$, weg von einer Diskriminierung im Alltagsleben in Form von Vorurteilen, Stigmatisierung und Kontaktvermeidung, hin zu einer sozial wirksamen Integration. ${ }^{478}$ Auch dieses Ziel des Verfassungsgebers spricht für einen weiten Begriff der Behinderung.

Der deutsche Gesetzgeber hat diese Ziele noch einmal dadurch verdeutlicht, dass er die BRK ratifiziert und für die Bundesrepublik übernommen hat.

\footnotetext{
472 Osterloh, in: Sachs GG, Art. 3, Rn. 305

473 Welti, Behinderung und Rehabilitation im sozialen Rechtsstaat, S. 439

474 Spranger, br 1998, S. 92 f, S. 93

475 BT-Drs. 12/6000, 53 Gubelt, in: v. Münch GG, Art. 3, Rn. 104 a; Fuerst, Behinderung zwischen Diskriminierungsschutz und Rehabilitationsrecht, S. 66

476 BT-Drs. 12/8165, S. 28

477 BT-Drs. 12/6000, S. 53 Gubelt, in: v. Münch GG, Art. 3, Rn. 104 a

478 BT-Drs. 12/6323, S. 11 f; BR-Drs. 12/8165, S. 28 f
} 
Die BRK regelt und konkretisiert so umfassend die Rechte von Menschen mit Behinderung, dass sie eine Konkretisierung von Art. 3 III 2 GG darstellt. ${ }^{479}$

\subsection{Rechtsvergleichende Auslegung}

Die Debatte über den Behinderungsbegriff ist, insbesondere auch wegen der Klassifikationen durch die WHO und die UN-BRK, eine internationale Debat$\mathrm{te}^{480}$, so dass eine Begriffsbestimmung ohne Bezug auf die internationalen Entwicklungen und Verständnisse von Behinderung nicht mehr möglich ist. Das international vorherrschende Modell der Behinderung ${ }^{481}$ ist das soziale Modell. Es setzt die Erkenntnis um, dass jeder Mensch ungeachtet dessen, ob er „normal“ ist oder ob er körperlich, geistig, seelisch oder in den Sinnen beeinträchtigt ist, das gleiche Recht auf volle und wirksame Teilhabe gleichberechtigt mit anderen an der Gesellschaft hat. Deshalb muss sich der beeinträchtigte Mensch nicht mehr den Anforderungen der "normalen“ nicht-behinderten Gesellschaft anpassen. Vielmehr muss sich die Gesellschaft so verändern, dass die beeinträchtigten Menschen an ihr ungehindert teilhaben können.

Entsprechend vertritt auch der EuGH einen weiten Behinderungsbegriff. In der Entscheidung Chacón Navas hat er sich im Rahmen eines Vorabentscheidungsverfahrens, dessen Grundlage eine arbeitsrechtliche Kündigung war, mit dem Behinderungsbegriff beschäftigt und betont, dass das gemeinschaftsrechtliche Behinderungsverständnis unabhängig von den nationalen Rechtsordnungen und einheitlich ist. ${ }^{482}$ Er hat Behinderung dann - entsprechend der arbeitsrechtlichen Ausrichtung des Vorlagebeschlusses - definiert als eine Einschränkung, die insbesondere auf physische, geistige oder psychische Beeinträchtigungen zurückzuführen ist und die ein Hindernis für die Teilhabe des Betreffenden am Berufsleben bildet. ${ }^{483}$

Auch dies spricht für den weiten Behinderungsbegriff in Form des sozialen Modells.

\subsection{Diskussion}

Die hier vorgenommene Auslegung des Art. 3 III 2 GG ergibt, dass Menschen mit Behinderung umfassend geschützt und sozial wirksam integriert werden sollen. Art. 3 III 2 GG muss daher der soziale Behinderungsbegriff zugrunde

\footnotetext{
479 S. dazu Kotzur/Richter, Kommentar zur UN-BRK, Verbindlichkeit, Rn. 2, 10; Aichele/ Althoff, Kommentar zur UN-BRK, Nicht-Diskriminierung, Rn. 38

480 Fuerst, Behinderung zwischen Diskriminierungsschutz und Rehabilitationsrecht, S. 48

481 Degener, RdJB 2009, S. 200 ff, S. 201

482 EuGH, Urteil v. 11.7.2006, Az.: C 13/05, ABI. C 224 v. 16.9.2006, S. 9, http://curia.europa.eu/jcms/jcms/j_6, Rn. 43

483 EuGH, Urteil v. 11.7.2006, Az.: C 13/05, ABI. C 224 v. 16.9.2006, S. 9, http://curia.europa.eu/jcms/jcms/j_6, Rn. 42
} 
gelegt werden, der sich vor allem aus den internationalen Vorgaben des ICF und der BRK ergibt, und der auf eine umfassende Integration und Inklusion der Menschen mit Behinderung ausgerichtet ist. Zu den Menschen mit Behinderungen zählen daher Menschen, die langfristige körperliche, seelische, geistige oder Sinnesbeeinträchtigungen haben, welche sie in Wechselwirkung mit verschiedenen Barrieren an der vollen, wirksamen und gleichberechtigten Teilhabe an der Gesellschaft hindern können.

Das Merkmal "Behinderung" ist damit kein fixes Merkmal wie z.B. Geschlecht (vgl. Art. 3 III $1 \mathrm{GG}$ ), sondern ein relatives Merkmal. ${ }^{484}$ Das führt zu der Schwierigkeit, dass die Auslegung und Subsumtion durch das Vorverständnis des Anwenders, das Larenz als „Vorurteil" bezeichnet ${ }^{485}$, nämlich die Determination der Auslegung durch die unbewusste Befangenheit des Interpreten in seiner sozialen Umwelt, seiner Herkunft, seiner Erziehung und seinen Erfahrungen, beeinflusst wird. Seine subjektiven Wertmaßstäbe, seine subjektiven Erfahrungen und sein Wissen um soziale Zusammenhänge beeinflussen das Ergebnis der Auslegung. Sie sind daraus nicht wegzudenken. ${ }^{486}$ Eine objektive Auslegung ist daher nicht denkbar, deshalb muss das Bemühen umso größer sein, die Auslegung „um der Sache selbst" zu betreiben. ${ }^{487}$ Dabei stehen sich zwei Interessen gegenüber: Während einerseits die Betroffenen ihre Benachteiligung und Diskriminierung als wenig wahrgenommen empfinden und deshalb dazu neigen, alles unter Art. 3 III 2 GG zu subsumieren, neigen andererseits die Nicht-Betroffenen dazu, an hergebrachten Erfahrungen festzuhalten und Benachteiligungen nicht wahrzunehmen, weil sie "notwendigerweise" und "schicksalhaft" mit der Behinderung verbunden und deshalb hinzunehmen sind. ${ }^{488}$

Die Wechselwirkung zwischen den vielfältigen Faktoren und den Barrieren, die Menschen mit Behinderung antreffen, führt dazu, dass das Merkmal „Behinderung" entscheidend durch äußere Faktoren bestimmt wird, nämlich durch die tatsächliche oder rechtliche Gestaltung der Umwelt. Damit ist „Behinderung" jede Maßnahme, Struktur oder Verhaltensweise, die Menschen an der Teilhabe hindern oder einschränken kann ${ }^{489}$, also alles was auf den Menschen

${ }^{484}$ Cloerkes/Felkendorff/Markowetz, Soziologie der Behinderten, S. 9

Larenz, Methodenlehre, S. 211

486 Larenz, Methodenlehre, S. $206 \mathrm{ff}$

487 Larenz, Methodenlehre, S. 211

Jürgens, in: Benachteiligungsverbot, S. 183, so die Entscheidung des BSG, BSGE 48, 258 ff, die Legasthenie mit mangelndem musischem oder sportlichem Talent gleichsetzt und die Beschlüsse des OLG Celle, Urteil v. 4.11.2008, Az.: 16 U 70/07, S. 13 (keine Amtspflichtverletzung des Jugendamtes - nicht veröffentlicht) und Beschluss v. 17.2.2010, Az.: 16 U 62/09 (keine Amtspflichtverletzung der Lehrer - nicht veröffentlicht), die ausdrücklich auf die „bedauerliche, schicksalhafte" Entwicklung des Kindes verweisen. 
wirkt und wodurch er in der Teilhabe beeinträchtigt „wird“. Es kommt dann nicht mehr darauf an, ob der betroffene Mensch durch eine körperliche Disposition selbst beeinträchtigt ist. Das führt jedoch zu dogmatischen Problemen, weil die notwendige Trennung zwischen der zu schützenden Person und ihrer Behandlung aufgehoben wird. ${ }^{490}$ Stellt man allein auf ein solches „Behindertwerden" ab, dann umfasst der Schutzbereich des Art. 3 III 2 GG auch Personen, die zwar benachteiligt werden, aber keine körperlich angelegten Beeinträchtigungen aufweisen, etwa weil man bei ihnen eine Beeinträchtigung vermutet, obwohl dies nicht der Fall ist. ${ }^{491}$ Die verlässliche Bestimmung des schutzbedürftigen Personenkreises würde also nahezu unmöglich ${ }^{492}$ und ist daher zu weit gefasst für einen Rechtsbegriff. ${ }^{493}$ Der Behinderungsbegriff muss es ermöglichen, den Personenkreis abzugrenzen, dem im Gegensatz zur übrigen Gesellschaft ein Nachteilsausgleich und eine Verbesserung ihrer Stellung in der Gesellschaft zuerkannt werden soll. ${ }^{494}$ Erst eine solche Normativität führt zu einer ausreichenden Gleichheit in der Rechtsanwendung und zu einer Konsistenz der Lösungen. ${ }^{495}$

Je enger jedoch der Schutzbereich gefasst wird und je strenger die Voraussetzungen für die Annahme einer Behinderung sind, desto weniger Menschen mit Behinderungen werden vom Schutzbereich erfasst. So führt z.B. die Rechtsprechung des VG Schleswig ${ }^{496}$, das die verfassungsrechtliche Subsumtion des Begriffs Behinderung auf der Grundlage von $\S 35$ a SGB VIII vornimmt, dazu, das z.B. Legasthenie allein keine Behinderung ist, sondern eine Behinderung nur dann vorliegt, wenn zur Legasthenie eine sekundäre Neurotisierung und Schulphobie und totaler Rückzug aus der Gemeinschaft hinzukommen. Eine Behinderung liegt danach nur dann vor, wenn die Beeinträchtigung sowohl einer bestimmten Kategorie zugeordnet werden kann als auch erhebliche nachgewiesene Auswirkungen hat. Damit würde entscheidend auf das Maß der Behinderung abgestellt. Eine solche Begrenzung des Schutzbereichs des Art. 3

490 Degener, ZaöRV 2005, S. 887 ff, S. 908 f; Zinsmeister, Mehrdimensionale Diskriminierung, S. 85

491 Zinsmeister, Mehrdimensionale Diskriminierung, S. 86 f; so z.B. in BAG, Urteil v. 17.12.2009, Az.: 8 AZR 670/08, juris, Rn. 14

492 Luthe, in: Rehabilitationsrecht - Verfassungsrecht, Rn. 5

493 Luthe, in: Rehabilitationsrecht - Verfassungsrecht, Rn. 5; Fuerst, Behinderung zwischen Diskriminierungsschutz und Rehabilitationsrecht, S. 55 und $66 \mathrm{f}$

494 Höller, Beschulung behinderter Kinder und Jugendlicher an öffentlichen Schulen, S. 143; s. dazu das Beispiel bei Fuerst, Behinderung zwischen Diskriminierungsschutz und Rehabilitationsrecht, S. 57 ff, Sutton v. United Airlines - Diskriminierung wegen einer Sehbeeinträchtigung, die durch optische Korrekturen behebbar war.

495 Luthe, in: Rehabilitationsrecht - Verfassungsrecht, Rn. 5

496 VG Schleswig, Urteil v. 10.6.2009, Az.: 9 A 208/08, BeckRS 2009 38123; OVG Weimar, Beschluss v. 17.5.2010, Az.: 1 EO 854/10, juris, Rn. 31 
III 2 GG auf Schwerbehinderungen ist nach der Einfügung des Benachteiligungsverbots in das Grundgesetz diskutiert worden. Die Befürworter dieser Beschränkung meinten, dass Art. 3 III 2 GG nur diejenigen schützt, bei denen eine Schwerbehinderung vorliegt. ${ }^{497}$ Diese Ansicht hat sich jedoch nicht durchgesetzt. Es besteht vielmehr heute Einigkeit in der Literatur, dass es für die Definition der Behinderung nicht auf das $\mathrm{Maß}^{498}$ und eine bestimmte Schwere der Beeinträchtigung ankommt. ${ }^{499}$ Deshalb kommt es für den verfassungsrechtlichen Begriff der Behinderung auch nicht darauf an, dass die Behinderung, so wie es etwa das BVerwG 1998 für $\S 39$ BSHG entschieden hat, ihrer Tiefe, Breite und Ausprägung nach eine bestimmte Wesentlichkeitsschwelle überschritten hat ${ }^{500}$ oder dass eine vorhandene attestierte Störung zusätzlich noch eine sekundäre Neurotisierung bewirkt. ${ }^{501}$ Die Bemerkung des VGH Kassel, dass eine nicht unerhebliche Behinderung bei einem Grad der Behinderung von 30 wegen Legasthenie und depressiver Störungen vorliege, ist deshalb zumindest missverständlich, da sie impliziert, dass Behinderungen einen bestimmten Grad der Erheblichkeit erreichen müssen. ${ }^{502}$ Erforderlich ist nur eine jedenfalls nicht völlig belanglose Beeinträchtigung, nicht jedoch eine bestimmte Schwere der Beeinträchtigung. ${ }^{503}$

Das weite Verständnis von Behinderung im Sinne des sozialen Modells erweitert die hergebrachte Auslegung des Behinderungsbegriffs des Art. 3 III 2 GG, die im deutschen Recht immer noch eher auf dem medizinischen, defektorientierten Ansatz basiert. ${ }^{504}$ Damit wird der Begriff der BRK, die im Rahmen der völkerrechtsfreundlichen Auslegung als Auslegungshilfe für die Bestim-

497

498

499

Jürgens, NVwZ 1995, S. 452; Caspar, EuGRZ 2000, S. 135 ff, S. 136; Osterloh, in: Sachs GG, Art. 3, Rn. 310; Sacksofsky in: Umbach GG, Art. 3, Rn. 398; Rüfner, in: Bonner Kommentar GG, Art. 3, Rn. 871; Neumann, NVwZ 2003, 897 ff, S. 899; Boysen, in: v. Münch GG 2012, Art. 3, Rn. 194; weitere Nachweise bei Höller, Beschulung behinderter Kinder und Jugendlicher an öffentlichen Schulen, S. 150, Fn. 482

500 $\S 39 \mathrm{BSHG}$ sich insoweit auf die Rspr. des BVerwG FEVS 49, 488, 489 bezieht.

502

Art. 3, Rn. 310; Sacksofsky, in: Umbach GG, Art. 3, Rn. 398; Rüfner, in: Bonner Kommentar GG, Art. 3, Rn. 871; Starck, in: v. Mangoldt/Klein/Starck GG, Art. 3, Rn. 419; Beaucamp, JA 2001, 36 ff, S. 38; das Urteil des VG Schleswig 10.6.2009, Az.: 9 A 208/08, BeckRS 2009 38123, S. 7 ist daher evident falsch.

504 
mung des Inhalts und der Reichweite von Art. 3 III 2 GG herangezogen werden muss ${ }^{505}$ übernommen, weil nur eine weite Auslegung des Grundrechts aus Art. 3 III 2 GG gleichzeitig den Anforderungen der BRK gerecht wird. Eine solche weite völkerrechtsfreundliche Auslegung wäre nur dann nicht zulässig, wenn sie dazu führen würde, dass Grundrechte eingeschränkt oder gemindert werden. Eine weite Auslegung des Begriffs Behinderung erweitert das Grundrecht und schränkt es nicht ein oder mindert es.

Inhaltlich bedeutet das soziale Modell von Behinderung vor allem, die Abwendung von dem Maßstab des „Normalen“ als Richtschnur und die Anerkennung der Behinderung als soziales Phänomen. Betrachtet man Behinderung als Abweichung von einem gesellschaftlichen Normalzustand, dann wird der Mensch mit Behinderung stets mit dieser Norm verglichen und die Frage gestellt, ob er in der Lage ist, die Abweichungen und Schäden zu kompensieren und sich an die bestehenden Normen anzupassen. Integration ist dann seine Aufgabe. Betrachtet man Behinderung dagegen als soziales Phänomen, dann wird die Frage nach den außerindividuellen Bedingungen, die Behinderung verursachen, gestellt. Langfristiges Ziel ist es, Behinderung als soziales Phänomen verschwinden zu lassen, indem behindernde Strukturen von vornherein vermieden oder offengelegt und beseitigt werden. Die Integration wird ersetzt durch die Inklusion, in der die Gesellschaft und das System so gestaltet werden, dass alle Bürger, auch die Bürger mit Behinderung, gleiche Entfaltungsmöglichkeiten haben. Inklusion ist somit eine Aufgabe der Gesellschaft und nicht mehr nur eine Aufgabe des Menschen mit Behinderung. ${ }^{506}$

Insbesondere im Hinblick auf das Bildungsrecht aus Art. 24 BRK ist diese Veränderung der Perspektive, die das soziale Modell erfordert, in der deutschen Literatur bereits diskutiert worden. Sie setzt sich kritisch damit auseinander, dass in der deutschen Übersetzung der BRK der zentrale Begriff der "inclusion" mit "Integration" übersetzt worden ist und zeigt auf, dass die Begriffe "Inklusion" und "Integration" nicht identisch sind. ${ }^{507}$ Beim Integrationskonzept besuchen Schüler mit Behinderungen gemeinsam mit Schülern ohne Behinderung allgemeinbildende Schulen und werden lernzielgleich unterrichtet. Das Konzept verlangt als "Mainstreaming" die Anpassungsleistung der Schüler

505 So auch Aichele, Stellungnahme DIMR 2010, S. 10; Riedel, Gutachten UN-Behindertenrechtskonvention, S. 1349; Kotzur/Richter, Kommentar zur UN-BRK, Verbindlichkeit, Rn. 10 Insgesamt Mathe, br 2011, S. 34 ff, S. 35 ff; Boysen, in: v. Münch GG 2012, Art. 3, Rn. 194; Ennuschat, in: FS Klaus Stern, S. 719, S. 724

507 Degener, RdJB 2009, S. 200 ff, S. 211; Latham/Watkins, Gutachten zur UN-BRK, S. 8; maßgeblich für die juristische Interpretation ist nach Art. 50 BRK die authentische Sprachfassung, d.h. der Begriff "Inklusion“. Da die deutsche Fassung nicht dazu gehört, ist sie nicht verbindlich. Umfassend zum Inklusionsbegriff der BRK Wansing, Kommentar zur UN-BRK, Inklusionsbegriff. 
mit Behinderungen an die bestehenden Schulstrukturen. ${ }^{508}$ Entscheidend ist also, ob ein Kind trotz Behinderung die Anforderungen erfüllt, die an ein nichtbehindertes Kind gestellt werden. Das Maß ist das „normale“ Kind.

Das Inklusions-Konzept beruht demgegenüber auf dem Prinzip, alle Schüler ungeachtet ihrer individuellen Unterschiede gemeinsam zu unterrichten. ${ }^{509}$ Strukturen und Didaktik des Unterrichts werden von vornherein auf die Unterschiedlichkeit der Schüler und Schülerinnen und auf ihre individuellen Lernrahmen hin ausgerichtet. Es findet sowohl eine lernzielgleiche als auch eine lernzieldifferente Unterrichtung statt. Ziel ist die Anerkennung und Wahrung der Vielfalt sowie die Bekämpfung diskriminierender Einstellungen und Werte $e^{510}$, also eine Umsetzung des sozialen Modells von Behinderung. Die deutsche Übersetzung wird deshalb dem Anspruch der BRK nicht gerecht. ${ }^{511}$

\subsection{Ergebnis}

Behinderung ist deshalb weit als Wechselwirkung zwischen dem Individuum und der Umwelt zu verstehen. Sie ist zu beschreiben wie in Art. 1 II BRK: „Zu den Menschen mit Behinderungen zählen Menschen, die langfristige körperliche, seelische, geistige oder Sinnesbeeinträchtigungen haben, welche sie in Wechselwirkung mit verschiedenen Barrieren an der vollen, wirksamen und gleichberechtigten Teilhabe an der Gesellschaft hindern können."

Aus der Beschreibung der Behinderung nach dem sozialen Modell folgt, dass sich nicht mehr die Menschen mit Behinderung an die Normalität anpassen müssen, sondern dass sich die sozialen und gesellschaftlichen Bedingungen und Faktoren verändern müssen, um dem Menschen mit Behinderung eine gleichberechtigte Teilhabe am Leben in der Gesellschaft zu ermöglichen.

508 Steinbrück, Die Bedeutung des Übereinkommens der Vereinten Nationen über die Rechte von Menschen mit Behinderungen für die Bildungspolitik in Deutschland, S. 3; Rothfritz, UNBRK, S. 383 f; Latham/Watkins, Gutachten zur UN-BRK, S. 8 ff; Poscher/Langer/Rux, Gutachten zu Art. 24 BRK, S. 19 ff, S. 24

Steinbrück, Die Bedeutung des Übereinkommens der Vereinten Nationen über die Rechte von Menschen mit Behinderungen für die Bildungspolitik in Deutschland, S. 4; Degener, br 2009, S. $34 \mathrm{ff}$, S. $41 \mathrm{f}$

510 Steinbrück, Die Bedeutung des Übereinkommens der Vereinten Nationen über die Rechte von Menschen mit Behinderungen für die Bildungspolitik in Deutschland, S. 4

511 Degener, br 2009, S. 34 ff, S. 41 f; Latham/Watkins, Gutachten zur UN-BRK, S. 8 ff; Poscher/Langer/Rux, Gutachten zu Art. 24 BRK, S. 19 ff, S. 24 
2. Qualifikation von Legasthenie/Dyskalkulie als Behinderung durch:

\subsection{Rechtsprechung}

Das BVerfG hat sich mit der Problematik Legasthenie/Dyskalkulie bisher nicht grundlegend beschäftigt. Verschiedene Verfassungsbeschwerden wurden nicht zur Entscheidung angenommen. In einem Nichtannahmebeschluss aus dem Jahr 2012 hat das BVerfG jedoch zugunsten des Beschwerdeführers unterstellt, dass er aufgrund seiner Legasthenie- und Dyskalkulie-Erkrankung im Sinne des Art. 3 III 2 GG behindert ist. ${ }^{512}$

In seinen sonstigen Entscheidungen zu Art. 3 III 2 GG hat das BVerfG sich nicht eingehend mit dem Behinderungsbegriff auseinandergesetzt, weil die Behinderung jeweils klar auf der Hand lag. ${ }^{513}$ Der Schwerpunkt der Prüfung lag daher auf der Rechtfertigung der Benachteiligungen. So wurde die Verfassungsbeschwerde eines blinden Schöffen, der ausdrücklich wegen seiner Blindheit von der Schöffenliste gestrichen worden war, nicht zur Entscheidung angenommen, weil das BVerfG darin keine Verletzung von Art. 3 III 2 GG sah. Das Diskriminierungsverbot des Art. 3 III 2 GG gelte nicht ohne jede Einschränkung. Fehlten einer Person gerade wegen ihrer Behinderung bestimmte körperliche Fähigkeiten, die unerlässliche Voraussetzung für die Wahrnehmung eines Rechts seien, läge in der Verweigerung dieses Rechts kein Verstoß gegen das Benachteiligungsverbot. Eine rechtliche Schlechterstellung Behinderter sei zulässig, wenn behinderungsbezogene Besonderheiten es zwingend erforderten. ${ }^{514}$ Ebenso wurde die Zuweisung von zwei behinderten Mädchen in einen Förder-Kindergarten für zulässig gehalten. ${ }^{515}$ Nur im Hinblick auf die Testierfähigkeit hat das BVerfG einen Verstoß gegen Art. 3 III 2 GG und damit eine verbotene Benachteiligung wegen einer Behinderung bejaht. ${ }^{516}$ Menschen, die in geistiger Hinsicht zu einer eigenverantwortlichen letztwilligen Verfügung in der Lage seien, dürften nicht allein deswegen an der Testierung von Rechts wegen gehindert werden, weil sie aus körperlichen Gründen nur über eingeschränkte

512 BVerfG, Beschluss v. 8.6.2012; Az.:1 BvR 2853/10 http://www.bverfg.de/entscheidungen/rk20120608_1bvr285310.html, Rn. 6

BVerfG, 1 BvR 9/97 vom 8.10.1997, BVerfGE 96, $288 \mathrm{ff}=$

http://www.bverfg.de/entscheidungen/rs19971008_1bvr000997.html; umfassende Darstel-

lung des Verfahrensgangs, in dem die Sache zwischen dem OVG Lüneburg und dem BVerfG

hin- und herpendelte bei Reichenbach, Anspruch behinderter Schülerinnen und Schüler auf

Unterricht in der Regelschule, S. 72 ff BVerfG Beschluss v. 10.2.2006 Az.: 1 BvR 91/06,

http://www.bverfg.de/entscheidungen/rk20060210_1bvr009106.html

BVerfG Beschluss v. 10.3. 2004 Az.: 2 BvR 577/01,

http://www.bundesverfassungsgericht.de/entscheidungen/rk20040310_2bvr057701.html

515 Ausführlich oben 3. Teil: 2. Kapitel: 1.4: Grammatische Auslegung, S. 76

516 BVerfG, Urteil v. 19.1. 1999 Az.: BvR 2161/94,

http://www.bverfg.de/entscheidungen/rs19990119_1bvr216194.html 
Verständigungsmöglichkeiten verfügten. ${ }^{517}$ Zwingende Gründe für die Benachteiligung der behinderten Menschen, die durch diese Vorschriften von der Testiermöglichkeit ausgeschlossen würden, lägen nicht vor. ${ }^{518}$

Damit hat das BVerfG überwiegend auf die Auswirkungen von Defiziten abgestellt, sich aber nicht mit der Wechselwirkung von Funktionsstörungen und sozialen Folgen beschäftigt. Einen Übergang zum sozialen Modell der Behinderung hat es bisher nicht vorgenommen.

In den schulrechtlichen Verfahren haben die Gerichte das Vorliegen einer Behinderung nach Art. 3 III 2 GG überwiegend nicht umfassend geprüft und erörtert, aber eine Behinderung bei Legasthenie, Dyskalkulie und auch bei ADHS und der Notwendigkeit sonderpädagogischer Förderung schlicht bejaht. ${ }^{519}$ Soweit die Gerichte das Vorliegen einer Behinderung eingehender geprüft haben, ${ }^{520}$ haben sie, mehr oder weniger direkt, für den Anwendungsbereich des Art. 3 III 2 GG auf $\S 2$ SGB IX und 35 a SGB VIII abgestellt. ${ }^{521}$ Verneint worden ist die Behinderung vom VG Schleswig. ${ }^{522}$ Das OVG Berlin-Brandenburg und das

517 BVerfG, Urteil v. 19.1. 1999 Az.: BvR 2161/94, Rn. 46, http://www.bverfg.de/entscheidungen/rs19990119_1bvr216194.html

518

BVerfG, Urteil v. 19.1. 1999 Az.: BvR 2161/94, Rn. 56, http://www.bverfg.de/entscheidungen/rs19990119_1bvr216194.html

S. dazu die Rechtsprechungsübersicht Marwege, RdJB 2009, S. 229 ff; OVG SchleswigHolstein, Beschluss v. 19.8.2002, Az.: 3 M 41.02, juris - Leitsätze; VGH Kassel, Beschluss v. 3. 1. 2006, 8 TG 3292/05, NJW 2006, S. 1608 f; VG Kassel, Beschluss v. 23.3.2006, 3 G 419/06, Sonderheft Recht des BVL: Chancengleichheit herstellen - Diskriminierung vermeiden, 2006, http://www.bvl-legasthenie.de/index.php5?p=/downloads, BFH, Beschluss v. 8.7.2008; Az.: VII B 241/07, juris; OVG Lüneburg, Beschluss v. 10.7.2008, Az.: 2 ME 309/08, www.rechtsprechung.niedersachsen.de; OVG Lüneburg, Beschluss v. 20.9.2012, Az.: 2 LA 234/11, S. 5 (nicht veröffentlicht); OVG Münster, Beschluss v. 4.9.2008, Az.: 19 B1293/08, www.justiz.nrw.de; VG Köln, Beschluss v. 26.9.2008, Az.: 10 L 1240/08, www.justiz.nrw.de; VGH Kassel, Beschluss v. 25.3.2009, Az.: 7 B 772/09, S. 5 (nicht veröffentlicht); VG Frankfurt a. M., Urteil v. 10.9.2009, Az.: 5 K 1501/09.F, S. 5 (nicht veröffentlicht); VGH Kassel, Beschluss v. 5.2.2010; Az.: 7 A 2406/09.Z, juris, Rn. 40 f; OVG Weimar, Beschluss v. 17.5.2010, Az.: 1 EO 854/10, juris, Rn. 31; VGH Kassel, Beschluss v. 17.11.2010, Az.: 7 A 2970/09.Z, juris, Rn. 9; VG Gießen, Urteil v. 2.11.2010, Az.: 7 K 1841/10.Gi, S. 9, 12 (nicht veröffentlicht); VG Hannover, Beschluss v. 13.12.2010, Az.: 6 B 5596/10, www.rechtsprechung.niedersachsen. de, S. 6; OVG Lüneburg, Beschluss v. 25.3.2011, Az.: 2 ME 52/11, S. 3 (nicht veröffentlicht); VG Düsseldorf, Beschluss v. 20.5.2011, Az.: 18 L 707/11, juris, Rn. 11

OVG Lüneburg, Beschluss v. 10.7.2008, Az.: 2 ME 309/08, www.rechtsprechung.niedersachsen.de; OVG Weimar, Beschluss v. 17.5.2010, Az.: 1 EO 854/10, juris, Rn. 31 ff; VGH Kassel, Beschluss v. 5.2.2010; Az.: 7 A 2406/09.2, juris, Rn. 9; VG Hannover, Beschluss v. 13.12.2010, Az.: 6 B 5596/10, www.rechtsprechung.niedersachsen.de, S. 5 So das VG Hannover, Beschluss v. 13.12.2010, Az.: 6 B 5596/10, www.rechtsprechung.niedersachsen.de, S. 5 
VG Aachen haben in Verfahren, in denen eine Legasthenie fachärztlich bescheinigt oder jedenfalls festgestellt worden war, die Frage, ob LRS (so die Bezeichnung in den Urteilen) eine Behinderung ist, ausdrücklich offen gelassen. ${ }^{523}$

Für die Dyskalkulie hat das VG Düsseldorf 2001 den Anspruch auf Eingliederungshilfe nach § 35 a SGB VIII vor allem aus Art. 3 III 2 GG hergeleitet. ${ }^{524}$ Das VG Regensburg hat in einer Entscheidung ${ }^{525}$, die später vor dem VGH München durch Vergleich beendet wurde ${ }^{526}$, bejaht, dass es sich bei der Dyskalkulie wie bei der Legasthenie um eine Behinderung i.S.d. Art. 3 Abs. III S. 2 GG handelt. Die Schule müsse sich damit auseinandersetzen, unabhängig davon, ob es dazu entsprechende Vorschriften des Kultusministeriums gebe oder nicht. Das OVG Weimar hat festgestellt, dass bei summarischer Prüfung bereits gemessen am nationalen Recht keine Bedenken daran bestehen, Dyskalkulie als Behinderung zu bewerten. Die Dyskalkulie sei eine Funktionsstörung i.S.d. ICD 10 und führe dazu, dass die Betroffene von der Teilhabe am Leben in der - vom „rechnenden Geist" geprägten Gesellschaft - beeinträchtigt sei. ${ }^{527}$

Soweit die Rechtsprechung also den Begriff der Behinderung prüft, legt sie zumeist die einfachrechtlichen Normen des $\S 2$ SGB IX oder auch $\S 35$ a SGB VIII zugrunde. Eine spezielle verfassungsrechtliche Auslegung erfolgt nicht, vielmehr wird das einfache Recht zum Auslegungsmaßstab des Grundrechts. Kein Gericht hat sich bisher mit der Veränderung des Behinderungsbegriffs hin zum sozialen Modell beschäftigt, so dass auch keine Auseinandersetzung mit den Wechselwirkungen zwischen der Funktionsstörung und den sozialen Anforderungen erfolgt. Zwar wird die Einschränkung der Teilhabe durch die Legasthenie/Dyskalkulie gesehen, es bleibt aber beim alten Behinderungsbegriff, der auf das Defizit abstellt und von den Schülern mit Behinderung eine Anpassung an die vorgegebenen Normen verlangt. So spricht das OVG Münster ausdrücklich davon, dass Schüler mit Legasthenie wegen dieses Eignungsmangels nicht nachweisen können, dass sie für das Gymnasium befähigt sind ${ }^{528}$, und das VG Düsseldorf urteilt, dass es zu Lasten des Schülers geht, wenn er das Förderziel im Bereich Lesen und Schreiben nicht erreicht. ${ }^{529}$ Ein Paradigmenwechsel hin zum sozialen Modell von Behinderung ist hier bisher nicht erfolgt.

523 VG Aachen, Urteil v. 13.11.2009, Az.: 9 K 25/09, BeckRS 2009 42206, S. 6; OVG BerlinBrandenburg, Beschluss v. 16.6.2009, Az.: 3 M 16.09, juris, Rn. 4

524 VG Düsseldorf, Urteil v. 22.1.2001, Az.: 19 K 11140/98, juris, Rn. $114 \mathrm{ff}$

525 VG Regensburg, Beschluss v. 8. 9. 2006, Az.: Rn. 1 E 06.1610 (nicht veröffentlicht)

526 VGH Bayern, Beschluss v. 20.12.2006, Az.: 7 CE 06.2754, juris

527 OVG Weimar, Beschluss v. 17.5.2010, Az.: 1 EO 854/10, juris, Rn. 35

528 OVG Münster, Beschluss v. 4.9.2008, Az.: 19 B1293/08, www.justiz.nrw.de, Rn. 24

529

VG Düsseldorf, Beschluss v. 20.5.2011, Az.: 18 L 707/11, juris, Rn. 14 


\subsection{Schuladministrative Regelungen}

Im Schulrecht ist der sonderpädagogische Förderbedarf der Leitbegriff von Regeln und Ansprüchen für behinderte Kinder ${ }^{530}$ Als entscheidend für die pädagogische Praxis wird nicht die Behinderung, sondern der sich daraus ergebende Förderbedarf angesehen. ${ }^{531}$ Deshalb wird in schulrechtlichen Regelungen immer der sonderpädagogische Förderbedarf geregelt und nicht ein Anspruch behinderter Schüler und Schülerinnen. Der Begriff der Behinderung wurde im Schulrecht lange nicht definiert. ${ }^{532}$ Inzwischen findet sich jedoch gelegentlich die Bezugnahme auf $\S 2$ SGB IX. So bestimmt z.B. $\S 6$ Abs. 1 SH Zeugnisordnung ${ }^{533}$ bezüglich des Nachteilsausgleichs: „Werden Schülerinnen und Schüler ... mit einer Behinderung nach $\S 2$ Abs. 1 Satz 1 des Neunten Buches Sozialgesetzbuch nach den lehrplanmäßigen Anforderungen einer allgemein bildenden oder berufsbildenden Schule unterrichtet ... hat die Schule der Beeinträchtigung angemessen Rechnung zu tragen (Nachteilsausgleich)."

Die KMK-Empfehlung und viele Länderregelungen sprechen bei Maßnahmen bei Lese-Rechtschreib- und Rechenschwierigkeiten ausdrücklich von „Hilfen im Sinne von Nachteilsausgleich “534 und grenzen sie damit von dem Nachteilsausgleich $a b$, der bei Behinderungen zu gewähren ist. In Niedersachsen wird explizit vertreten, dass es sich bei Lese-Rechtschreib- und Rechenschwierigkeiten nicht um Behinderungen handelt, die Schüler und Schülerinnen aber "Hilfen im Sinne eines Nachteilsausgleichs" erhalten können. ${ }^{535}$ Die Regelung in Schleswig-Holstein sieht Schüler und Schülerinnen mit Legasthenie offenbar als Sonderfall an, denn § 6 Abs. 4 SH Zeugnisordnung bestimmt für sie: „Im Falle besonderer und andauernder Schwierigkeiten im Lesen oder Rechtschreiben beschließt die Klassenkonferenz - auch unabhängig von der förmlichen Feststellung einer Lese-Rechtschreib-Schwäche - über angemessene Maßnahmen im Sinne eines Nachteilsausgleichs." In einem Rechtsstreit vor dem VG Schleswig hat das Kultusministerium SH die Auffassung vertreten, dass Legasthenie nur dann eine Behinderung ist, wenn die Voraussetzungen des $\S 35$ a SGB VIII

530 Welti, Behinderung und Rehabilitation im sozialen Rechtsstaat, S. 48

Welti, Behinderung und Rehabilitation im sozialen Rechtsstaat, S. 49; Behrens/Wachtel, Nds. SVBI 2008, S. 144 ff, S. 144

So Landtag von Baden-Württemberg Drucksache 14 / 630 14. Wahlperiode, 27. 11. 2006, S. 3

SH ZeugnisVO, s. Anhang 3: Schulrechtliche Vorschriften der Bundesländer

KMK-Lesen/Rechtschreiben/Rechnen, Punkt Leistungsbewertung, Grundsätze; HB LRS-Erlass Nr. 4.1; MV VV-LRR Nr. 7 III (allerdings unpräzise: einmal ist von Nachteilsausgleich (Nr. 7 II), einmal von Hilfen i.S. eines Nachteilsausgleich (Nr. 7 III) die Rede; NI LRR-Erlass Nr. 4.1; RP VV-LRS Nr. 4.2; SL VV-LRS Nr. 5.1; ST Erlass Grundschulbewertung Nr. 5.1.1; SH LegasthenieErlass Nr. 1.1.1 
erfüllt sind. ${ }^{536}$ In Hamburg werden Behinderungen und besondere Schwierigkeiten im Lesen und Rechtschreiben bezüglich des Nachteilsausgleichs im Sekundarbereich I in derselben Vorschrift geregelt, die Unterscheidung in Behinderung einerseits und besondere Schwierigkeiten im Lesen und Rechtschreiben andererseits zeigt jedoch, dass Legasthenie offenbar nicht als Behinderung im schulrechtlichen Sinne gesehen wird. ${ }^{537}$ Hessen hat in einem Verfahren vor dem VGH Kassel vorgetragen, die hessische VOLRR ${ }^{538}$ blende bewusst das "Konstrukt Legasthenie" aus und stelle ursachenunabhängig auf Schwierigkeiten beim Erlernen und Gebrauch der Schriftsprache ab, weshalb die medizinische Feststellung der Legasthenie durch einen Kinder- und Jugendpsychiater unerheblich sei. ${ }^{539}$

Im Bereich des Schulrechts ist mithin festzustellen, dass eine konsistente Definition von Behinderung meist nicht vorhanden ist. Soweit doch Definitionen vorhanden sind, werden sie nicht auf Legasthenie/Dyskalkulie angewandt, weil diese Störungen nach dem pädagogischen Verständnis etwas anderes sind als Behinderungen.

\subsection{Literatur}

Für den Bereich der Legasthenie/Dyskalkulie wird das Vorliegen einer Behinderung i.S.d. Art. 3 III 2 GG von der Literatur überwiegend bejaht. Zinsmeister führt aus, dass sich mit der Änderung gesellschaftlicher Erwartungen und Anforderungen auch das Verständnis davon wandelt, wer "normal" und wer "behindert" ist. Mit den steigenden Erwartungen an die Lese- und Schreibfähigkeit der Bevölkerung sei auch der Anteil der als lese-rechtschreibschwach qualifizierten Schüler signifikant gestiegen. ${ }^{540}$ Reichenbach konstatiert, es liege auf der Hand, dass die aus der Legasthenie/Dyskalkulie resultierende Unfähigkeit zu lesen, zu schreiben oder zu rechnen das soziale und auch berufliche Leben erheblich einschränkt, wenn nicht gar unmöglich macht. ${ }^{541}$ Caspar stellt fest, dass es für den Behinderungsbegriff entscheidend darauf ankommt, welche sozialen Folgen mit dem Nicht-Vorliegen der entsprechenden Fähigkeit verknüpft sind. „Mit einer erheblichen Lern- oder Schreibschwäche ist jemand in

536 Bejahend: VG Schleswig, Urteil v. 10.6.2009, Az.: 9 A 208/08, BeckRS 2009 38123; ausdrücklich ablehnend: OVG Weimar, Beschluss v. 17.5.2010, Az.: 1 EO 854/10, juris, Rn. 31 HH APO-AS $\S 3$ IV und HH APO-GrundStGy $\S 6$

538 Inzwischen ersetzt durch HE VO-GestSchulVerh §§ 37-44

539 VGH Kassel, Beschluss v. 17.11.2010, Az.: 7 A 2970/09.Z, juris, Rn. 9, der VGH Kassel hat diese Sichtweise abgelehnt.

540 Zinsmeister, Mehrdimensionale Diskriminierung, S. 83, ebenso Welti, Behinderung und Rehabilitation im sozialen Rechtsstaat, S. 89

541 Reichenbach, Anspruch behinderter Schülerinnen und Schüler auf Unterricht in der Regelschule, S. 165 
der modernen Informationsgesellschaft regelmäßig behindert; während etwa in einer agrarisch strukturierten Gesellschaft seiner Beeinträchtigung keine annähernd gravierende Bedeutung zukäme. ${ }^{1542}$ Langenfeld führt aus, dass sich die Legasthenie in ganz erheblicher Weise auf die Chancen der Betroffenen auswirkt, an den allgemeinen Bildungs- und Lebenschancen der Gesellschaft teilzuhaben und deshalb die Voraussetzungen des $\S 2$ SGB IX, an denen sie Art. 3 III 2 GG orientiert sieht, erfüllt sind. ${ }^{543}$ Ennuschat ordnet Legasthenie und Dyskalkulie „ohne Weiteres" als Behinderungen ein. ${ }^{544}$ Höller stellt fest, dass Art. 3 III 2 GG auch auf die Teilleistungsschwächen wie Legasthenie Anwendung findet, weil vom Vorliegen einer auf gesellschaftlichen Einflüssen beruhenden Regelwidrigkeit und damit von einer Behinderung im verfassungsrechtlichen Sinn auszugehen sei. ${ }^{545}$ Schließlich stellen auch Poscher/Rux/Langer auf der Grundlage der BRK fest, dass Legasthenie und Dyskalkulie unter den Behinderungsbegriff der BRK zu subsumieren sind. ${ }^{546}$ Kischel bezweifelt demgegenüber als einziger die Einordnung als Behinderung vor allem mit dem Hinweis, dass sie, wenn sie denn eine geistige Behinderung sein sollte, kaum von der Minderbegabung abzugrenzen sei, die auch sonst keine Behinderung darstelle. ${ }^{547}$

Mit Ausnahme von Kischel stellen mithin alle Autoren auf die Wechselwirkung von Funktionsstörung und sozialem Anspruch ab. Die Einschränkungen, die sich in schulischer und beruflicher Hinsicht durch die eingeschränkten Fähigkeiten im Lesen, Rechtschreiben und Rechnen ergeben, führen dazu, dass Legasthenie und Dyskalkulie als Behinderung zu qualifizieren sind.

\section{Der Behinderungsbegriff im Einzelnen und seine Anwendung auf Legasthenie/Dyskalkulie}

\subsection{Funktionsstörung}

Für die verlässliche Bestimmung des Schutzbereiches ist, wie dies z.B. in Art. 1 BRK gefordert wird, eine langfristige Beeinträchtigung im körperlichen, seelischen, geistigen Bereich oder im Bereich der Sinne (im Folgenden zusammenfassend: Funktionsstörung) erforderlich. ${ }^{548}$ Fraglich ist dabei, inwieweit hierfür auf medizinische Feststellungen zurückgegriffen werden kann ${ }^{549}$ oder muss ${ }^{550}$,

\footnotetext{
542 Caspar, EuGRZ 2000, S. 135 ff, S. 136

543 Langenfeld, RdJB 2007, S. 211 ff, S. 214

544 Ennuschat, br 2008, S. 93 ff, S. 12

545 Höller, Beschulung behinderter Kinder und Jugendlicher an öffentlichen Schulen, S. 168

546 Poscher/Langer/Rux, Gutachten zu Art. 24 BRK, S. 17

547 Kischel, in: BeckOK GG, Art. 3, Rn. 219.1

548 Zinsmeister, Mehrdimensionale Diskriminierung, S. 86

549 Degener, ZaöRV 2005, S. 887 ff, S. 907 ff
} 
denn mit der medizinischen Diagnose halten das medizinische Modell und der damit verbundene defektorientierte Ansatz wieder Einzug in die Begriffsbestimmung, obwohl beim Behinderungsbegriff dieser Ansatz gerade aufgegeben wurde.

\subsubsection{Medizinische Diagnostik als Grundlage}

Nach Auffassung von Castendiek setzt der Begriff der Behinderung eine wissenschaftliche Belegbarkeit von Unterschieden, an die sachlich gebotene Differenzierungen geknüpft werden können, geradezu voraus. ${ }^{551}$ Zinsmeister ist der Ansicht, dass es allein auf die Behandlung des Menschen und nicht auf seine persönlichen Eigenschaften ankommt. Sie hält jedoch bei der Vergabe von staatlichen Leistungen individuumsbezogene Gründe für notwendig. ${ }^{552}$ Höller stellt auf eine weit verstandene Funktionsbeeinträchtigung ab, die sich auch aus dem gesellschaftlichen Kontext ergeben kann ${ }^{553}$ Luthe wendet sich strikt dagegen, dass es allein auf das Erleben behindernder Situationen ankommt, da damit ein Einfallstor für Subjektivismen jeglicher Art eröffnet würde. ${ }^{554}$

Luthe ist darin zuzustimmen, dass es allein auf die subjektive Sicht des Behindertwerdens nicht ankommen kann. Es würde tatsächlich jeglichem Subjektivismus Tür und Tor öffnen, sollte für die Definition der Behinderung allein das subjektive Empfinden einer Person ausreichend sein. Damit würde das Grundrecht aus Art. 3 III 2 GG verwässert und könnte seine Funktion für den zu schützenden Personenkreis nicht mehr umfassend wahrnehmen. Das Grundrecht will nicht per se jeden vor dem „Behindertwerden“ schützen, sondern individuelle Personen. Sie sind von Diskriminierungshandlungen oder -maßnahmen betroffen, vor denen sie Schutz benötigen. ${ }^{555}$ Es besteht ansonsten die große Gefahr, dass aus Angst vor ausufernder Auslegung der Anwendungsbereich des Art. 3 III 2 GG bereits bei diesem Merkmal außerordentlich eng ausgelegt wird. Dass eine solche Gefahr besteht, zeigt die Entwicklung in den USA, wo die Rechtsprechung den Behinderungsbegriff so eng gefasst hat, dass viele Klagen daran scheiterten. ${ }^{556}$ Dieser engen Auslegung ist inzwischen durch eine Gesetzesänderung der Boden entzogen worden. ${ }^{557}$ Auch in Deutschland besteht die

\footnotetext{
550 Dazu Castendiek, in: GS Jean d'Heur, S. 343

551 Castendiek, in: GS Jean d'Heur, S. 343

552 Zinsmeister, Mehrdimensionale Diskriminierung, S. $86 \mathrm{f}$

553 Höller, Beschulung behinderter Kinder und Jugendlicher an öffentlichen Schulen, S. $143 \mathrm{f}$

554 Luthe, in: Rehabilitationsrecht - Verfassungsrecht, Rn. 5

555 Degener, ZaöRV 2005, S. 887 ff, S. 908

556 Degener, ZaöRV 2005, S. 887 ff, S. 906 m.w.N.

557 ADA Amendments Act of 2008 (ADAAA v. 25. 9. 2008, in Kraft getreten am 1. Januar 2009), http://www.wrightslaw.com/law/504/adaaa.pdf, s. auch http://peoplewise.wordpress.com/2008/11/11/the-adaa-2008
} 
Gefahr, über eine enge Auslegung des Behinderungsbegriffs den Anwendungsbereich des Art. 3 III 2 GG zu beschränken, wie die restriktive Auslegung des Art. 3 III 2 GG durch das VG Schleswig zeigt, dass insoweit den sehr engen Tatbestand des $§ 35$ a SGB VIII für maßgeblich hält ${ }^{558}$

Auf einen individuellen Bezug kann folglich nicht verzichtet werden. Diesen Bezug stellt explizit auch Art. 1 BRK her, indem er darauf abstellt, dass der zu schützende Mensch in seiner körperlichen, geistigen, seelischen Funktion oder in den Sinnesfunktionen betroffen sein muss. Die Funktionsbeeinträchtigung muss daher medizinisch fassbar sein. ${ }^{559}$ Legasthenie/Dyskalkulie sind Funktionsstörungen, die auf der Grundlage der ICD 10 und den diagnostischen Leitlinien der Kinder- und Jugendpsychiater diagnostiziert festgestellt werden können. $^{560}$

\subsubsection{Jede Art der Funktionsstörung?}

Rechtsprechung und Literatur versuchen immer wieder zu bestimmen, ob die Funktionsstörungen Legasthenie und Dyskalkulie die körperliche, geistige oder seelische Gesundheit betrifft. Meistens wird die Legasthenie/Dyskalkulie dann als "geistige" Behinderung bezeichnet. ${ }^{561}$ Fraglich ist, ob es für den Behinderungsbegriff des Art. 3 III 2 GG überhaupt erforderlich ist eine solche Bestimmung vorzunehmen.

Die Einteilung entspricht der überkommenen deutschen Differenzierung in körperliche, geistige und seelische Behinderungen. ${ }^{562} \mathrm{Im}$ Sozialrecht werden zunächst alle Formen der Funktionsstörung unter den Begriff Behinderung gefasst, auch, um einer Beschränkung und Fixierung des Begriffs Behinderung

558 VG Schleswig, Urteil v. 10.6.2009, Az.: 9 A 208/08, BeckRS 200938123

559 Neumann, in: Handbuch SGB IX, § 2 Verfassungsrecht, Rn. 9; Castendiek, in: GS Jean d'Heur, S. 343

560 Bei § 35 a SGB VIII in dessen Abs. 1 a SGB VIII die Diagnostik nach diesen Leitlinien ausdrücklich festgeschrieben wurde, erfüllt die Diagnose einer solchen Funktionsstörung nach der Rechtsprechung nicht das Tatbestandsmerkmal „wenn die seelische Gesundheit ... abweicht". Vielmehr fordert die Rechtsprechung, dass zusätzlich zu der Legasthenie/Dyskalkulie noch weitere psychische Probleme vorhanden sind, die sich auf die seelische Gesundheit auswirken. Dem liegt wieder der Gedanke zugrunde, dass die Legasthenie/Dyskalkulie keine Krankheit i.S.d. deutschen (Krankenversicherungs-)Rechts ist und daher allein den Tatbestand der Beeinträchtigung der Gesundheit nicht erfüllt. OVG Koblenz, Beschluss in einem PKH-Verfahren v. 26.3.2007, 7 E 10212/07 juris; s. oben 1. Teil: 2. Kapitel: 7.2.2. Finanzierung durch die Jugendämter nach $\S 35$ a SGB VIII, S. 48

561 BVerwG FEVS 46, 360, 362; differenzierend OVG Bautzen, Beschluss v. 9.6.2009, Az.: 1 B 288/09; http://www.justiz.sachsen.de/ovgentsch/documents/1B288_09.pdf, S. 4 („geistige Lernstörung“, „abgegrenzter Ausfall von Hirnleistungen“); Ennuschat, in: FS Klaus Stern, S. 713 m.w.N. 
auf die körperliche Funktionsstörung entgegen zu wirken. ${ }^{563}$ Eine Differenzierung erfolgt dann aber bezüglich der Zuständigkeiten und Kostenträger zwischen Hilfen für körperlich und geistig behinderte Menschen im SGB IX und für seelisch behinderte Kinder und Jugendliche im SGB VIII. Diese aufgespaltene Zuständigkeit macht es im Sozialrecht erforderlich, präzise zwischen körperlich/ geistigen oder seelischer Behinderung zu unterscheiden, ${ }^{564}$ obwohl eine solche Abgrenzung für die diagnostizierenden Ärzte fast unmöglich ist. ${ }^{565}$ Die Bestrebungen gehen derzeit dahin diese Differenzierung aufzuheben und eine Lösung für alle behinderten Kinder zu finden. Dies auch vor dem Hintergrund, dass die derzeitige Rechtslage, die die Kinder je nach Funktionsstörung unterschiedlichen sozialen Hilfesystemen zuordnet, nicht mit dem umfassenden Ansatz von Art 7 II BRK und Art. 3 I KRK vereinbar sein dürfte. ${ }^{566}$

Art. 3 III 2 GG schützt alle Arten von Behinderungen in gleicher Weise und nicht nur die körperlichen Behinderungen. Deshalb kommt es für das Verfassungsrecht auf diese Unterscheidung nicht an. Allenfalls könnte man sich erhoffen, dass eine Subsumtion unter eine dieser Kategorien den Umgang mit der Behinderung erleichtert. Dann aber müsste es möglich sein, die Kategorien klar voneinander abzugrenzen. Das ist jedoch aus medizinischer Sicht nicht möglich und gilt im Bereich der Kinder- und Jugendpsychiatrie sogar als überholt. ${ }^{567}$ Am Beispiel der Legasthenie lässt sich dies exemplarisch verdeutlichen. Daraus, dass bei den betroffenen Menschen Hirnregionen anders funktionieren als bei Menschen ohne diese Beeinträchtigung, kann gefolgert werden, dass es sich, da das Hirn ein Teil des Körpers ist, um körperliche Beeinträchtigungen handelt. Meistens wird jedoch von der Tatsache, dass es sich um eine Störung handelt, die im Kopf zu lokalisieren ist, darauf geschlossen, dass es sich um eine geistige Störung handelt. ${ }^{588}$ Die Bezeichnung oder Einordnung der Legasthenie/

563 Welti, in: Welti/Lachwitz/Schellhorn, HK-SGB IX, § 2 Rn. 23; Welti, Behinderung und Rehabilitation im sozialen Rechtsstaat, S. 95

564 S. dazu LSG Niedersachsen-Bremen, Beschluss v. 5.8.2010, Az.: L 8 SO 143/10 B ER, juris, Rn. 13, 14, 16 - ohne Bezug auf die BRK

S. dazu ausführlich Fegert, JAmt 2010, S. 267 ff, S. 269

Banafsche, SGb 2012, S. 373 ff, S. 440 ff, S. 444

Welti, Behinderung und Rehabilitation im sozialen Rechtsstaat, S. 94; vgl. Welti, in:

Welti/Lachwitz/Schellhorn, HK-SGB IX, § 12, Rn. 23, 26; Mrozynski, in: SGB VIII § 35 a, Rn. 1 weist darauf hin, dass die Unterteilung in Körper, Geist und Seele im Bereich der Kinder- und Jugendpsychiatrie naiv und überholt ist.

568 BVerwG FEVS 46, 360, 362; differenzierend OVG Bautzen, Beschluss v. 9.6.2009, Az.: 1 B 288/09; http://www.justiz.sachsen.de/ovgentsch/documents/1B288_09.pdf, S. 4 („geistige Lernstörung", „abgegrenzter Ausfall von Hirnleistungen“); LSG Niedersachsen-Bremen, Beschluss v. 5.8.2010, Az.: L 8 SO 143/10 B ER, juris, Rn. 16; Kischel, in: BeckOK GG, Art. 3, Rn. 219, 219.1, dem insoweit zuzustimmen ist, dass die rechtliche Einordnung mit den bisherigen Begründungen nicht einleuchtet. 
Dyskalkulie als „geistige Störung" führt jedoch zu einer Fehleinschätzung, denn mit "geistiger Behinderung" wird im medizinischen Bereich eine Minderbegabung im intellektuellen Bereich, d.h. ein Intelligenzquotient jedenfalls unter 70, bezeichnet (ICD $10 \mathrm{~F}$ 7). Auch allgemein-sprachlich wird unter "geistiger Störung" eine Intelligenzminderung oder Geistesschwäche verstanden. Eine solche Minderbegabung ist bei Legasthenie/Dyskalkulie jedoch, wie oben ausgeführt, ${ }^{569}$ schon definitorisch ausgeschlossen.

Es reicht daher aus, dass eine Funktionsstörung als solche festgestellt werden kann.

\subsubsection{Kenntnis der Ursachen}

Fraglich ist weiter, ob es für den Begriff der Behinderung darauf ankommt, dass die Ursachen der Funktionsstörung bekannt sind. Die KMK-Empfehlung von 2007 legt dies nahe, da dort gleich zu Beginn vermerkt wird, dass „die pädagogische, psychologische und medizinische Forschung auf diesem Gebiet kontrovers ist und viele Fragen nicht abschließend geklärt sind. “570 Der VGH München hat in seinem Vergleichsvorschlag für ein Verfahren zur Dyskalkulie darauf hingewiesen, dass die Dyskalkulie nach Ursache, Entstehung und Ausprägung nicht annähernd so intensiv erforscht und abgesichert sei, wie die Legasthenie. Ähnlich argumentiert das bayerische Kultusministerium, wenn es darauf verweist, dass bei Dyskalkulie die Ursachen noch nicht so erforscht sind, wie bei der Legasthenie. ${ }^{571}$ Dies erweckt den Eindruck, als hinge die Anerkennung bzw. Einordnung einer Störung als Behinderung davon ab, dass die Ursachen der Funktionsstörung geklärt sind. ${ }^{572}$ Diese Frage taucht, soweit ersichtlich, nur bzgl. der Legasthenie/Dyskalkulie im Schulrecht auf.

Voraussetzung für eine Behinderung ist nur das Vorliegen einer Funktionsstörung. Dafür ist die Kenntnis der Ursachen ohne Belang. ${ }^{573}$ Wollte man die Kenntnis der Ursachen zum Merkmal der Behinderung machen, würden viele Störungen aus dem Anwendungsbereich des Art. 3 III 2 GG herausfallen, etwa Stottern $^{574}$, Mutismus, Autismus, Schizophrenie, aber auch z.B. Diabetes, deren

569 S. oben 1. Teil: 1. Kapitel: 5.2.: Medizinische Diagnostik nach ICD 10 und Multiaxialem Klassifikationsschema (MAS), S. 16

KMK-Lesen/Rechtschreiben/Rechnen, Punkt Nr. I

571 Bay. Landtag, Schriftliche Anfrage Drucksache Nr. 16/5468 vom 06.07.2010, Zu Nr. 6 und Schriftliche Anfrage Drucksache Nr. 16/5698 vom 18.8.2010, Zu. Nr. 5, abrufbar unter: www.bayern.landtag.de Den Eindruck erweckt auch Ennuschat, in: FS Klaus Stern, S. 712 f, der sich ausführlich mit der Frage der Ursachen von Legasthenie, Dyskalkulie und Stottern beschäftigt.

S. dazu auch BSG Urteil v. 30.9.2009, Az.: B 9 SB 4/08, www.sozialgerichtsbarkeit.de, Rn. 30; BSG, Urteil v. 24.4.2008, Az.: B 9/9a SB 7/08, www.sozialgerichtsbarkeit.de, Rn. 14; LSG Berlin-Brandenburg, Urteil v. 3.12.2009, Az.: L 13 SB 235/07, www.sozialgerichtsbarkeit.de, S. 4 
Ursachen ebenfalls nicht vollständig geklärt sind. Der Schutzbereich würde damit massiv eingeschränkt. Es gibt keinerlei Anhaltspunkte dafür, dass das Grundrecht in dieser Weise beschränkt werden sollte.

\subsubsection{Defizit der medizinischen Diagnostik - das Diskrepanzkriterium}

Mit der medizinischen Diagnostik und ihren Grenzwerten werden jedenfalls die Funktionsstörungen erfasst, die von der Medizin als erheblich angesehen werden, also im Bereich Legasthenie/Dyskalkulie die Störungen, die die obigen Diagnosekriterien ${ }^{575}$ erfüllen.

Hier entsteht nun jedoch das Dilemma, dass der Rechtsbegriff der Behinderung es einerseits erfordert, den Personenkreis abzugrenzen, der wegen einer Behinderung benachteiligt sein kann und der deshalb nicht diskriminiert werden $\operatorname{darf}^{576}$, und es andererseits bedenklich ist, die Feststellung der Behinderung an fixe medizinische Kriterien zu binden und diese damit über den Zugang zu grundrechtlichem Schutz entscheiden zu lassen. ${ }^{577}$ Die medizinische Diagnose setzt per Definition eine Abweichung von der Norm voraus. Die Bestimmung der Norm und der Grenzwerte im Bereich der Medizin ist jedoch für rechtliche Differenzierungen nicht so ohne weiteres zu gebrauchen. ${ }^{578}$ Sie orientiert sich vor allem an Kriterien, die für Forschungszwecke Sinn machen. ${ }^{579}$ Diese Kriterien eigenen sich jedoch nicht dafür, davon die Anwendbarkeit eines Benachteiligungsverbots abhängig zu machen. ${ }^{580}$ Hier offenbart sich das Grundproblem des Behinderungsbegriffs: Behinderung ist kein fixes Merkmal wie Geschlecht, Herkunft, Religion, das vorhanden ist oder nicht, sondern ein relatives Merkmal, das fließende Übergänge hat. ${ }^{581}$ Das Problem des Ein- oder Ausschlusses von Beeinträchtigungen aus dem Behinderungsbegriff stellt sich vielfältig: Wann ist eine Sehschwäche eine Behinderung, wann eine Hörschwäche? Ist der Verlust eines Fingers eine Behinderung oder erst der Verlust von mehre-

575 S. oben 1. Teil: 1. Kapitel: 5.2.: Medizinische Diagnostik nach ICD 10 und Multiaxialem Klassifikationsschema (MAS), S. 16; die amerikanische DSM V (s. oben Fn. 20) löst die Diagnose von dem Diskrepanzkriterium (Stichwort: Rationale)

576 Vgl. Höller, Beschulung behinderter Kinder und Jugendlicher an öffentlichen Schulen, S. 143; Leder, Diskriminierungsverbot wegen einer Behinderung, S. 113 ff; Degener, Disability Studies Quarterly 2006, Punkt 2 a; Degener, ZaöRV 2005, S. 887 ff, S. 908 Spranger, br 1998, S. 92 f, S. 93

578 Umfassend dazu Eggers/Fegert/Resch, Psychiatrie, S. 110 ff; s. auch Schulte-Körne auf der Internetseite der KJP München unter Diagnose: http://www.kjp.med.unimuenchen.de/forschung/legasthenie/diagnose.php; Landerl/Kaufmann, Dyskalkulie, S. 95 zeigt auf, dass die ICD 10 Forschungskriterien festlegt, wonach maximal $3 \%$ der Gesamtpopulation die Diagnose erhalten sollen.

579 Vgl. Landerl/Kaufmann, Dyskalkulie, S. 95

580 Leder, Diskriminierungsverbot wegen einer Behinderung, S. 113

581 Leder, Diskriminierungsverbot wegen einer Behinderung, S. $113 \mathrm{f}$ 
ren Fingern $?^{582}$ Wann ist Fettleibigkeit eine Behinderung und wann nicht? ${ }^{583}$ Die Kritik an der bayerischen KMBek zur Legasthenie richtet sich gerade gegen die Ausschlussfunktion der medizinischen Diagnostik. ${ }^{584}$ Würde man nun die Bestimmung der Behinderung allein an den (veränderbaren) medizinischen Kriterien festmachen, wäre man wieder beim überholten medizinischen Modell angekommen, dass die Anerkennung der Behinderung von einem medizinisch gesehen erheblichen Defizit beim Betroffenen abhängig macht. Alle leichteren Fälle einer Störung, die medizinisch die Diagnosekriterien nicht erfüllen, würden aus dem Anwendungsbereich herausfallen. ${ }^{585}$ Das aber widerspricht dem allgemeinen Konsens, dass es auf die Schwere der Beeinträchtigung nicht ankommt. Die medizinischen Kriterien können daher nicht das allein entscheidende Abgrenzungskriterium sein. Sie können nur die Störungen benennen, die jedenfalls geschützt sind. Sie können aber nicht zum Maßstab dafür gemacht werden, dass nur diese Störungen geschützt sind. Vielmehr sind als Funktionsstörung i.S.d. Art. 3 III 2 GG alle medizinisch fassbaren Störungen zu verstehen.

Auch leichtere Formen der Legasthenie/Dyskalkulie, die die medizinischen Kriterien nicht erfüllen und üblicherweise als Lese-Rechtschreibschwäche und Rechenschwäche bezeichnet werden, sind in diesem Sinne medizinisch fassbar. Sie sind daher ebenfalls Funktionsstörungen. ${ }^{586}$ Etwas anderes könnte nur dann gelten, wenn ausgeschlossen wäre, dass aufgrund dieser Funktionsstörungen benachteiligt wird. ${ }^{587}$ Das aber kann nicht allein aufgrund der Funktionsstörung

582 Vgl. Degener, ZaöRV 2005, S. 887 ff, S. 907

583

Leder, Diskriminierungsverbot wegen einer Behinderung, S. $114 \mathrm{ff}$; s. dazu BSG, Urteil v. 24.4.2008, Az.: B 9/9a SB 7/08, www.sozialgerichtsbarkeit.de, Rn. 14; LSG Berlin-Brandenburg, Urteil v. 3.12.2009, Az.: L 13 SB 235/07, www.sozialgerichtsbarkeit.de, S. 4: Fettleibigkeit kann eine eigene Behinderung sein, nicht nur eine zusätzliche Beschwer bei anderen Krankheiten

Die Kritik an der bayerischen KMBek zur Legasthenie richtet sich vor allem gegen die Ausschlussfunktion der medizinischen Diagnostik: Büchner/Kortländer/Werner/Robering/Schönweiss, „'Legasthenie - eine Krankheit, eine Behinderung, eine Störung?"; Valtin, Brauchen wir die Legasthenie?; Engelmann/Plaum, report psychologie 2008, S. $16 \mathrm{ff}$

Ausführlich dazu auch Leder, Diskriminierungsverbot wegen einer Behinderung, S. $111 \mathrm{ff}$

S. zur Aufgabe des Diskrepanzkriteriums für die Berücksichtigung der Behinderung in der Schule in den USA: Individuals with Disabilities Education Act (IDEA) Sec. 300.307 (a) (1): , ,... the criteria adopted by the State must not require the use of a severe discrepancy between intellectual ability and achievement for determining whether a child has a specific learning disability, as defined in Sec. 300.8(c)(10) Child with a disability Dyslexia, mathematical calculations" http://idea.ed.gov/explore/view - Stichwort Regulations; sowie die Überarbeitung des DSM 5, die wegen dieser schulrechtlichen Regelung ebenfalls auf das Diskrepanzkriterium verzichten will, http://www.dsm5.org/ProposedRevisions/Pages/proposedrevision.aspx?rid=84\# 
beantwortet werden, sondern ergibt sich erst daraus, welche Auswirkung die Funktionsstörung hat. Ob die Funktionsstörung eine Behinderung ist, ergibt sich deshalb aus der Wechselwirkung mit dem zweiten Merkmal, nämlich der Hinderung der Teilhabe.

Damit wird der Streit, ob und inwieweit es einen qualitativen Unterschied zwischen Legasthenie/Dyskalkulie und Lese-Rechtschreibschwäche/Rechenschwäche gibt, obsolet. In dieser Arbeit wird daher der Ansatz vertreten, dass sowohl die medizinisch definierte Legasthenie/Dyskalkulie als auch die medizinisch fassbaren leichteren Formen der Legasthenie/Dyskalkulie (meist als LeseRechtschreibschwäche/Rechenschwäche bezeichnet), die die Diskrepanzkriterien der ICD 10 nicht erfüllen, Funktionsstörungen i.S.d. Behinderungsbegriffs sind und daher auch bezüglich der sich aus Art. 3 III 2 GG ergebenden Rechte gleichzubehandeln sind. Es wird deshalb im Weiteren auch nicht zwischen Legasthenie/Dyskalkulie und leichteren Formen davon unterschieden.

Nicht umfasst werden von der hier vorgenommenen Definition der Funktionsstörung indes vorübergehende Einschränkungen und sonderpädagogischer Förderbedarf, soweit er nicht auf körperlichen Funktionsstörungen beruht. Ob eine Behinderung generell zu bejahen ist, wenn sonderpädagogischer Förderbedarf festgestellt wurde, so wohl die h. M. ${ }^{588}$, kann dahingestellt bleiben, weil es darauf für die Frage, ob Legasthenie/Dyskalkulie und leichtere Formen der Legasthenie/Dyskalkulie, die medizinisch fassbar sind, Behinderungen sind, nicht ankommt.

\subsection{Hinderung der Teilhabe}

Die Funktionsstörung muss Auswirkungen auf die Position des Einzelnen in der Gesellschaft haben. ${ }^{589}$ Nach der weiten Beschreibung des Art. 1 BRK kommt es darauf an, ob der betroffene Mensch an der vollen, wirksamen und gleichberechtigten Teilhabe in der Gesellschaft gehindert ist. Bleibt die Funktionsstörung oder Betroffenheit ohne Auswirkungen in der Gesellschaft, liegt keine Behinderung vor. Anders als das Sozialrecht, das Leistungen zuteilt, geht es bei Art 3 III 2 GG darum, Diskriminierungen abzuwehren. Die Gefährdungsschwelle für den Begriff "Behinderung" muss daher eine andere sein als im Sozialrecht. ${ }^{590}$ Deshalb reicht es hier aus, dass die Einschränkung der Teilhabe des

588 Höller, Beschulung behinderter Kinder und Jugendlicher an öffentlichen Schulen, S. 144 f; Reichenbach, Anspruch behinderter Schülerinnen und Schüler auf Unterricht in der Regelschule, S. 166; Reimann, Sicherstellung des Schulbesuchs behinderter Kinder, S. 34 f; Jürgens, DVBI 1997, S. 410 ff, S. 411; Rux, RdJB 2009, S. 220 ff, S. 223

589 Luthe, in: Rehabilitationsrecht - Verfassungsrecht, Rn. 4

590 Luthe, in: Rehabilitationsrecht - Verfassungsrecht, Rn. 4, a. A. das VG Schleswig; Urteil v. 10.6.2009, Az.: 9 A 208/08, BeckRS 2009 38123, S. 7 
betroffenen Menschen bei allgemeiner Betrachtung möglich ist. ${ }^{591}$ Eine „Beeinträchtigung", d.h. eine nachgewiesene, individuelle Einschränkung der Teilhabe am Leben in der Gesellschaft, wie dies z.B. bei § 2 SGB IX notwendig ist, ist nicht erforderlich.

Würde bereits hier auf eine konkrete, individuelle Beeinträchtigung abgestellt, würde das Merkmal der Benachteiligung seinen Inhalt verlieren. Denn die (verbotene) Teilhabebeeinträchtigung ist zwangsläufig das Ergebnis einer Benachteiligung i.S. einer Diskriminierung und daher bei der Benachteiligung zu prüfen. ${ }^{592}$

Legasthenie/Dyskalkulie hindern die betroffenen Schüler und Schülerinnen an der vollen, wirksamen und gleichberechtigten Teilhabe in der Gesellschaft, die bei Kindern maßgeblich über die Schule vermittelt wird. ${ }^{593}$ Weil den Kindern bereits die Wahrnehmungsvoraussetzungen für das Erlernen des Lesens, Schreibens/Rechtschreibens und Rechnens fehlen, können sie schon nach kurzer Zeit dem Unterricht nicht mehr folgen. Der weitere Unterricht geht an ihnen vorbei, weil ihnen die Grundlagen fehlen. ${ }^{594}$ Die Beschulung, die nicht an ihren Erfordernissen ausgerichtet ist, führt dazu, dass sie den Anschluss verlieren. Die nicht vorhandenen oder eingeschränkten Leistungen im Lesen, Rechtschreiben und Rechnen werden ständig benotet. Die schlechten Noten in diesen Leistungen führen zu vielfachen Beschränkungen und Nachteilen, da sie sowohl zur Folge haben, dass Klassen wegen dieser schlechten Leistung wiederholt werden müssen als auch dazu, dass der Zugang zu weiterführenden Bildungsgängen versagt wird. Im Extremfall werden die Kinder, die besonders intensive Förderung brauchen, auf Förderschulen überwiesen und/oder scheitern schulisch vollständig. ${ }^{595}$

Auch die leichteren Störungen Lese- und Rechtschreibschwäche und Rechenschwäche hindern die betroffenen Schüler und Schülerinnen an der gleichbe-

591 Jürgens, NVwZ 1995, S. 452 f, S. 452; Luthe, in: Rehabilitationsrecht - Verfassungsrecht, Rn. 4

Jürgens, ZfSH/SGB 1995, S. 353 ff, S. 359; Luthe, in: Rehabilitationsrecht - Verfassungsrecht, Rn. 4

Vgl. Mrozynski, in: SGB VIII, § 35 a, Rn. 6

Reichelt, Analphabeten, S. 6

595 Exemplarisch das Schicksal eines Jungen, der seit seinem 14. Lebensjahr krank und unbeschulbar ist. Seine Geschichte ergibt sich aus den Urteilen des VG Lüneburg, Beschluss v. 12.8.2004, Az.: 4 B 123/04 (nicht veröffentlicht); OLG Celle, Urteil v. 4.11.2008, Az.: 16 U 70/07, (nicht veröffentlicht), Kammerbeschluss des BVerfG, Az.: 1 BvR 1711/09, v.

21.11.2012, juris, Rückverweisung an das OLG Celle und den Urteilen des LSG Niedersachsen-Bremen, Urteil v. 14.1.2009, Az.: L 9 U 129/06 Revision vom BSG zurückgewiesen, Urteil v. 27.4.2010; Az.: B 2 U 13/09, juris, und dem Nichtannahmebeschluss des BVerfG zur Verfassungsbeschwerde, BVerfG, Beschluss v. 8.6.2012; Az.: 1 BvR 2853/10, http://www.bverfg.de/entscheidungen/rk20120608_1bvr285310.html 
rechtigten Teilhabe. Sie haben die gleichen Nachteile wie die Schüler und Schülerinnen mit Legasthenie/Dyskalkulie, wenn auch nicht immer in der gleichen Intensität und Ausprägung. Tatsächlich kann bei ihnen ihre leichtere Betroffenheit zu gravierenderen Auswirkungen führen. Anders als bei Schülern und Schülerinnen mit Legasthenie/Dyskalkulie wird die Beeinträchtigung in der Schule häufig nicht anerkannt oder die Lehrkräfte üben ihr Ermessen dahin aus, die Schwäche nicht zu berücksichtigen. ${ }^{596}$ In Bayern ergibt sich eine Hinderung der Teilhabe dadurch, dass bei ihnen die Berücksichtigung der Schwäche in der Oberstufe im Regelfall ausgeschlossen ist.

\section{Ergebnis}

Legasthenie/Dyskalkulie sind Behinderungen, weil ihnen Funktionsstörungen zugrunde liegen und sie an der Teilhabe am Leben in der Gesellschaft durch die Bewertung des Lesen, Rechtschreibens und/oder Rechnens hindern. Behinderungen i.S.d. Art. 3 III 2 GG sind auch die Lese-Rechtschreibschwäche und die Rechenschwäche.

\section{5. „Inflation“ der Behinderungen}

Problematisch an dem weiten Begriff der Behinderung kann sein, dass der Begriff der Behinderung so umfassend und unscharf wird, dass nahezu jede Abweichung von einer Norm zur Behinderung wird. ${ }^{597}$

Art. 3 III 2 GG verlangt das Vorliegen einer Behinderung, das Grundrecht steht dann nur dem Menschen mit Behinderung zu, die Behinderung muss daher personalisierbar sein. Was eine Behinderung ist, ist ebenso wie viele andere unbestimmte Rechtsbegriffe in anderen Rechts- und Verfassungsnormen auszulegen und immer wieder neu zu hinterfragen. Viele Rechtsbegriffe beziehen sich nicht auf konstante Personengruppen, sondern auf Situationen. ${ }^{598}$ Deutlich wird dies etwa am Begriff der Familie, der über die Jahre einen grundlegenden Wandel erfahren hat. Ebenso wandelbar ist z.B. der Begriff der Versammlung, der über die Jahre zwar relativ konstant war, inzwischen aber neu ausgelegt werden muss, weil es fraglich ist, ob sog. "flash mobs" unter den Versammlungsbegriff fallen oder nicht. Das Problem, dass das soziale Modell weit gefasst ist und die Differenzierung zwischen Menschen mit Behinderung und solchen ohne Behinderung schwer macht, ist also ein übliches rechtliches Anwendungsproblem.

\footnotetext{
596 So die Regelung in Bayern BY KMBek Legasthenie IV, 1

597 Neumann, NVwZ 2003, 897 ff, S. 899, dagegen Welti, Behinderung und Rehabilitation im sozialen Rechtsstaat, S. 440

598

Welti, Behinderung und Rehabilitation im sozialen Rechtsstaat, S. 440
} 
In der Feststellung einer Behinderung sehen viele allerdings eine Stigmatisierung der betroffenen Kinder und lehnen sie deshalb ab. ${ }^{599}$ Damit wird das Problem der Kinder mit Behinderung aber nicht gelöst. Die Definition „Behinderung" macht die gestörte Beziehung zwischen einem Menschen mit Beeinträchtigungen und seiner Umwelt sichtbar und zeigt damit auf, dass hier eine Veränderung stattfinden muss. ${ }^{600}$ Die Probleme im Umgang zwischen Menschen mit und ohne Behinderung bestehen nicht in der Unterschiedlichkeit und der Benennung dieser Unterschiede, sondern darin, dass bestimmte Umstände Anlass dazu sind, Menschen zu diskriminieren, ihnen fundamentale Menschenrechte vorzuenthalten oder konkrete Lebensvollzüge zu erschweren. ${ }^{601}$ Die Definition und Benennung ist Mittel zum Zweck, um die Diskriminierung zu überwinden. Je mehr Menschen mit Behinderung es gibt, desto klarer treten die Versäumnisse von Staat und Gesellschaft zutage, die Barrieren abzubauen. ${ }^{602}$ Verschließt man jedoch die Augen vor dieser Relation und definiert solche gestörten Beziehungen aus Sorge vor Stigmatisierung nicht als Behinderung, ist schon das wieder diskriminierend, denn die beeinträchtigte Situation des betroffenen Menschen wird bagatellisiert und verschleiert. Zudem fehlt dann, wie sich gerade bei Legasthenie/Dyskalkulie zeigt, der rechtliche Anspruch darauf, dass sich die Lage für die Betroffenen verändert. Liegt keine Kategorisierung als Behinderung vor, können die Betroffenen - wie bisher - nur auf Einsicht hoffen, nicht aber rechtliche Veränderung einfordern.

Das Ziel der Kritiker, die Legasthenie/Dyskalkulie für ein Konstrukt und die Feststellung einer Legasthenie/Dyskalkulie für stigmatisierend halten, ist, dass in der Schule der Blick auf die Fähigkeiten des Kindes zu richten sei, statt auf seine Defizite gerichtet wird und dass ihm eine umfassende schulische Ausbildung trotz oder besser mit der Einschränkung ermöglicht wird. Dieses Ziel wird auch erreicht, wenn die Schule die Behinderung anerkennt, ernst nimmt und berücksichtigt. Ist das der Fall, wird die Beeinträchtigung der Teilhabe der Schüler und Schülerinnen mit Legasthenie/Dyskalkulie abnehmen und schließlich verschwinden. Weil Behinderung kein statischer Begriff ist, sondern sich aus der Relation zwischen dem Menschen und seiner Umwelt ergibt, kann dann

599 Vgl. die Thesen von Büchner/Kort/änder/Werner/Robering/Schönweiss, „Legasthenie - eine Krankheit, eine Behinderung, eine Störung?", S. 2 und 13 und Valtin, Brauchen wir die Legasthenie?, sowie die Haltung von Thiel, Rechenschwäche und Prengel, Sozialextra 2006, 15 ff, S. 16 Vgl. Banafsche, SGb 2012, S. 373 ff, S. 440 ff, S. 375

601 Biermann/Goetze, Sonderpädagogik, S. 8; vgl. auch die Hinweise bei Schulte-Körne/Stieglitz, in: Adoleszenzpsychiatrie, S. 476 über den anderen Umgang mit der Legasthenie in England, wo es durch eine Anerkennung der Legasthenie und gezielte Aufklärungskampagnen und Hilfs- und Fördermaßnahmen gelingt, die Stigmatisierung gerade zu vermeiden. 
auch die Behinderung der Schüler und Schülerinnen mit Legasthenie/Dyskalkulie verschwinden.

\section{Kapitel: Benachteiligung}

In den Schutzbereich des Art. 3 III 2 GG wird eingegriffen, wenn der Mensch mit Behinderung im Vergleich zu nicht behinderten Menschen benachteiligt wird. Synonym für den Begriff „Benachteiligung" wird auch der Begriff „Diskriminierung" verwendet. Der Begriff der Benachteiligung überschneidet sich zum Teil mit dem Begriff der Behinderung, denn dieser enthält bereits das Merkmal, dass sich die körperlich angelegte Beeinträchtigung einschränkend und nachteilig auswirken kann und Behinderung deshalb als solche grundsätzlich nachteilig ist. ${ }^{603}$ Das allein reicht jedoch für eine Benachteiligung i.S.d. Art. 3 III 2 GG nicht aus.

Nach dem modernen Grundrechtsbegriff ist ein Eingriff jedes staatliche Handeln, das dem Einzelnen ein Verhalten, das in den Schutzbereich des Grundrechts fällt, ganz oder teilweise unmöglich macht, unabhängig davon, ob diese Wirkung final oder unbeabsichtigt, unmittelbar oder mittelbar, rechtlich oder tatsächlich, mit oder ohne Zwang eintritt. ${ }^{604}$ Eingriffe in Form einer Benachteiligung sind daher, über die individuell angelegten Probleme des Menschen mit Behinderung hinaus, solche Regelungen oder Maßnahmen, die die Lebenssituation von Menschen mit Behinderung im Verhältnis zur Lebenssituation der Nichtbetroffenen verschlechtern. ${ }^{605}$ Dazu gehören nicht nur Benachteiligungen in Bezug auf Rechte, sondern auch ideelle, wirtschaftliche und emotionale Nachteile. ${ }^{606}$ Der Begriff des Nachteils ist nach herrschender Meinung weit auszulegen, so dass auch geringe Nachteile erheblich sein können. ${ }^{607}$ Entscheidend ist nach der Rechtsprechung des BVerfG, wie sich die Regelung oder Maßnahme materiell auf die tatsächlichen Lebenssachverhalte auswirkt. ${ }^{608}$ Bei Schülern und Schülerinnen mit Legasthenie/Dyskalkulie kann eine Benachteiligung darin liegen, dass sie den gleichen Prüfungsbedingungen un-

603 Castendiek, in: GS Jean d'Heur, S. 350; Rüfner, in: Bonner Kommentar GG, Art. 3, Rn. 872

604

BVerfGE 105, 279, 301; Pieroth/Schlink, Grundrechte, Rn. 253

605 Neumann, in: Handbuch SGB IX, § 2 Verfassungsrecht, Rn. 21

606 Degener, Theresia, in: Antidiskriminierungsrecht, S. 70 m.w.N.; König/Peters, in: Grote/ Marauhn EMRK/GG, Kapitel 21: Das Diskriminierungsverbot Rn. 50

607 BVerfGE 71, 39, 50; Osterloh, in: Sachs GG, Art. 3, Rn. 84 und 312, Jarass, in: Jarass/Pieroth GG, Art. 3, Rn. 9 ff; 10 ff; Eckertz-Höfer, in: AK-GG, Art. 3, Rn. 137; a.A. Kischel, in: BeckOK GG, Art. 3, Rn. 19

608

BVerfGE 49, 148, 165; 50, 217, 231; 85, 238, 245; Osterloh, in: Sachs GG, Art. 3, Rn. 84 m.W.N. 
terliegen und nach den gleichen rechtlichen Regelungen benotet werden, wie Schüler und Schülerinnen ohne diese Behinderung. Eine Benachteiligung kann auch in einer Zeugnisbemerkung liegen, die ich jedoch später separat behandle. ${ }^{609}$

\section{Völkerrechtsfreundliche Auslegung des Begriffs Benachteiligung}

Die völkerrechtsfreundliche Auslegung des Art. 3 III 2 GG gebietet es weiter, den Begriff der Benachteiligung nach den Vorgaben der BRK auszulegen.

Art. 5 II BRK enthält das allgemeine Diskriminierungsverbot der BRK ${ }^{610}$ und verbietet jegliche Diskriminierung in den in Art. 2 BRK definierten Formen. Danach „,bedeutet ,Diskriminierung aufgrund von Behinderung' jede Unterscheidung, Ausschließung oder Beschränkung aufgrund von Behinderung, die zum Ziel oder zur Folge hat, dass das auf die Gleichberechtigung mit anderen gegründete Anerkennen, Genießen oder Ausüben aller Menschenrechte und Grundfreiheiten im politischen, wirtschaftlichen, sozialen, kulturellen, bürgerlichen oder jedem anderen gesellschaftlichen Bereich beeinträchtigt oder vereitelt wird. Sie umfasst alle Formen der Diskriminierung, einschließlich der Versagung angemessener Vorkehrungen." Der Begriff der Benachteiligung ist damit sehr weit gefasst. Art. 24 BRK stellt bezüglich des Bildungsbereichs eine Konkretisierung des Diskriminierungsverbots dar ${ }^{611}$ und enthält umfassende Bestimmungen dazu, welche Rechte Menschen mit Behinderung in Bezug auf Bildung haben und wie ein diskriminierungsfreies Bildungssystem ausgestaltet sein muss. Seine Vorgaben sind bei der Auslegung des Zwecks und des Ziels des Diskriminierungsverbots der BRK zu beachten. Gefordert ist danach ein inklusives Bildungssystem auf allen Ebenen, in dem Menschen mit Behinderung ihre Begabungen voll zur Entfaltung bringen können (Art. 24 I BRK). Die Vertragsstaaten sind dazu verpflichtet $u$. a. sicherzustellen, dass Menschen mit Behinderung weder vom allgemeinen Bildungssystem noch vom Besuch weiterführender Schulen ausgeschlossen werden (Art. 24 II Buchst. a), dass sie Zugang zu inklusivem Unterricht an Grundschulen und weiterführenden Schulen haben (Art. 24 II Buchst. b), angemessene Vorkehrungen für sie getroffen werden (Art. 24 II Buchst. c) und die notwendige Unterstützung geleistet wird, um ihre erfolgreiche Bildung zu erleichtern (Art. 24 II Buchst. d). Im Umkehrschluss heißt dies, dass ein Bildungssystem, welches diese Voraussetzungen nicht erfüllt,

\footnotetext{
609 10. Teil: 2. Kapitel: Zeugnisbemerkung, S. $318 \mathrm{ff}$

610 Degener, RdJB 2009, S. 200 ff, S. 206; Latham/Watkins, Gutachten zur UN-BRK, S. 11; Althoff, Anwaltsblatt 2011, S. 482 ff, S. 483

611

Degener, RdJB 2009, S. 200 ff, S. 216; Latham/Watkins, Gutachten zur UN-BRK, S. 29, vgl. BSG, Urteil v. 6.3.2012, Az.: B 1 KR 10/11 R, www.bundessozialgericht.de, Rn. 29 ff
} 
diskriminiert bzw. benachteiligt. Diese Konkretisierungen sind insbesondere bei der Beurteilung der Benachteiligung zu beachten. ${ }^{612}$

\section{Objektive oder subjektive Bestimmung des Eingriffs}

Für die Beurteilung, ob eine Regelung oder Maßnahme eine Benachteiligung und damit ein Eingriff ist, kommt es weiter darauf an, aus wessen Sicht dies zu bestimmen ist.

Die Vertreter der objektiven Betrachtungsweise ${ }^{613}$ bejahen eine Benachteiligung nur dann, wenn der Mensch mit Behinderung die tatsächliche Fähigkeit hat, eine bestimmte rechtliche oder tatsächliche Möglichkeit zu nutzen. Sie meinen, dass er schon nicht benachteiligt wird, wenn er diese Fähigkeit (objektiv) nicht hat, weil er die Möglichkeit deswegen von vornherein nicht nutzen kann. ${ }^{614}$ Zum Teil wird geprüft, ob nach der Art und Schwere der vorliegenden Behinderung und den für den jeweiligen Bereich geltenden Anforderungen eine Gleichbehandlung mit Nichtbehinderten möglich ist. ${ }^{615}$ Dirnaicher vertritt eine solche Ansicht in Bezug auf die Entscheidung der Beschulung eines Kindes. Hier liege es allein in der Kompetenz der Schulbehörde, darüber zu entscheiden, wie ein behindertes Kind unterrichtet wird. ${ }^{616}$ Das BVerfG geht in der Sonderschulentscheidung davon aus, dass nur aufgrund des Gesamtergebnisses einer Würdigung darüber befunden werden kann, ob eine Maßnahme im Einzelfall benachteiligend ist. ${ }^{617}$ Einige Stimmen in der Literatur schlagen eine vergleichende Prüfung vor, in der geprüft wird, ob die Nachteile einer Ungleichbehandlung durch Vorteile aufgewogen werden können. ${ }^{618}$ Hier gehen objektive Bestimmung und vergleichende Würdigung ineinander über, wobei angenommen wird, dass die diesbezügliche Entscheidung oder Abwägung objektiv erfolgt.

612 Vgl. Althoff, Anwaltsblatt 2011, S. 482 ff, S. 483, zu den konkreten Anforderungen an eine diskriminierungsfreie Bildung s. unten S. $236 \mathrm{ff}$

613 Heun, in: Dreier GG, Art. 3, Rn. 135; Kischel, in: BeckOK GG, Art. 3, Rn. 212; Engelken, DVBI 1997, S. 763; Dirnaicher, BayVBI 1997, S. 545 ff, S. 549; Osterloh, in: Sachs GG, Art. 3, Rn. 312; VGH Kassel, Beschluss v. 12.11.2009, Az.: 7 B 2763/09, juris, Rn. 45 Kischel, in: BeckOK GG, Art. 3, Rn. 212

615 Engelken, DVBI 1997, S. 763, Dirnaicher, BayVBI 1997, S. 545 ff, S. 545; ausführliche Darstellung des Streitstandes bei Höller, Beschulung behinderter Kinder und Jugendlicher an öffentlichen Schulen, S. $188 \mathrm{ff}$

616 Dirnaicher, BayVBI 1997, S. 545 ff, S. 551

617 BVerfGE 96, S. 288 ff, Rn. 52, so auch VG Aachen, Urteil v. 13.11.2009, Az.: 9 K 25/09, BeckRS 2009 42206, S. 6

618 Osterloh, in: Sachs GG, Art. 3, Rn. 312; so auch Engelken, DVBI 1997, S. 763; Dirnaicher, BayVBI 1997, S. 545 ff, S. 549 und VGH Kassel, Beschluss v. 12.11.2009, Az.: 7 B 2763/09, juris, Rn. 45 
Demgegenüber bestimmen die Vertreter der subjektiven Sichtweise die Benachteiligung danach, wie der Mensch mit Behinderung die einzelne Maßnahme oder Behandlung bewertet. ${ }^{619}$ Sie fürchten, dass sich bei einer objektiven Sichtweise die Erfahrungen aus der Geschichte des Behindertenrechts und der Behindertenpädagogik fortsetzen und sich der fürsorgliche Staat und wohlmeinende Dritte eine Entscheidung darüber anmaßen, was für Behinderte vorteilhaft und was nachteilig ist. ${ }^{620}$ Sie sehen in der objektiven Beurteilung einen Zirkelschluss zulasten der Menschen mit Behinderung, denn damit hätte derjenige, der für die Benachteiligung durch Ausgrenzung verantwortlich ist, zugleich die Definitionsmacht darüber, was ein Nachteil ist ${ }^{621}$ und würde selber den Inhalt und die Reichweite des Art. 3 III 2 GG bestimmen. ${ }^{622}$

Tatsächlich ist die Gefahr gegeben, dass mit einer objektiven Betrachtung schon die Eingriffsqualität einer Regelung oder Maßnahme verneint wird, denn der Eingriff wird nicht nur danach beurteilt, ob er im Ergebnis ein Verhalten unmöglich macht oder erschwert, sondern mit der Wertung verbunden, ob dieses Ergebnis auch nachteilig ist. Wird der Nachteil verneint, fehlt es am Eingriff. Dies zeigt sich zum Beispiel an der besonders streitigen Frage, ob die Überweisung eines Kindes auf die Sonderschule ein Nachteil oder ein Vorteil ist. ${ }^{623}$ Wenn die Schulbehörden bestimmen, dass die Beschulung für das behinderte Kind „objektiv ein großer Vorteil ist“624 oder die Gerichte eine Diskriminierung verneinen, weil „eine solche Zuweisung eine besondere Maßnahme darstellen (kann), die zur Beschleunigung oder Herbeiführung der tatsächlichen Gleichberechtigung von Menschen mit Behinderungen erforderlich ist ${ }^{4625}$, dann

619 Herdegen, Diskriminierungsschutz für Behinderte im Grundgesetz, S. 27; Berlit, RdJB 1996, S. 145 ff, S. 150; Castendiek, in: GS Jean d'Heur, S. 345 f; Sachs, RdJB 1996, S. 154 ff, S. 168; Höller, Beschulung behinderter Kinder und Jugendlicher an öffentlichen Schulen, S. 191; Luthe, in: Rehabilitationsrecht- Verfassungsrecht, Rn. 6; Beaucamp, JA 2001, 36 ff, S. 39; differenzierend Neumann, NVwZ 2003, 897 ff, S. 900; sehr umfassend Reichenbach, Anspruch behinderter Schülerinnen und Schüler auf Unterricht in der Regelschule, S. $131 \mathrm{ff}$

Neumann, in: Handbuch SGB IX, § 2 Verfassungsrecht, Rn. 23; Castendiek, in: GS Jean d'Heur, S. 345

Berlit, RdJB 1996, S. 145 ff; Jürgens, DVBI 1997, S. 764; Höller, Beschulung behinderter Kinder und Jugendlicher an öffentlichen Schulen, S. 189

Reichenbach, Anspruch behinderter Schülerinnen und Schüler auf Unterricht in der Regelschule, S. 169; Jürgens, DVBI 1997, S. 764; Beaucamp, DVBI 2002, S. 997 ff, S. 998; ausdrücklich für diese Definitionsmacht Dirnaicher, BayVBI 1997, S. 545 ff, S. 551, da er der Schulbehörde die Kompetenz zuweist allein darüber zu entscheiden, wie ein behindertes Kind unterrichtet wird. Umfassend dazu Reichenbach, Anspruch behinderter Schülerinnen und Schüler auf Unterricht in der Regelschule und Höller, Beschulung behinderter Kinder und Jugendlicher an öffentlichen Schulen, vgl. VGH Kassel, Beschluss v. 12.11.2009, Az.: 7 B 2763/09, juris, Rn. 45 Engelken, DVBI 1997, S. 762

625 VGH Kassel, Beschluss v. 12.11.2009, Az.: 7 B 2763/09, juris, Rn. 45 
wird der Eingriff verneint, obwohl nicht nur die Betroffenen, sondern auch Sonderpädagogen die Überweisung als erheblichen Nachteil ansehen. ${ }^{626}$

Nach dem modernen weiten Eingriffsbegriff kommt es darauf an, ob der Betroffene das Handeln aus seiner subjektiven Sicht als Eingriff empfindet. ${ }^{627}$ Auch wenn es im Grundgesetz immer eine Fremdbestimmung gibt ${ }^{628}$, so führt doch die objektive Bestimmung, ob ein Eingriff vorliegt, hier dazu, dass der allgemeine Eingriffsbegriff verändert und ihm ein wertendes Element hinzufügt wird. Der Staat hätte damit die Möglichkeit staatliche Handlungen gegen den Willen des Betroffenen in dessen "wohlverstandenen Interesse ${ }^{\text {“629 }}$ für nicht nachteilig zu erklären. ${ }^{630}$ Damit würde der Begriff der Benachteiligung schon auf der Tatbestandsebene aufgeweicht. ${ }^{631}$

Gegen die objektive Ansicht ist einzuwenden, dass sie entscheidend auf einer vergleichenden Betrachtung des behinderten Menschen mit dem nichtbehinderten Menschen beruht. Sie entscheidet danach, ob ein Mensch mit Behinderung die gleiche Fähigkeit hat, wie ein Mensch ohne Behinderung, und danach, ob der Mensch mit Behinderung mit dem Menschen ohne Behinderung gleich behandelt werden kann. Damit wird der „normale“ Mensch zum Maßstab des Vergleichs. Der moderne Behinderungsbegriff bemüht sich jedoch gerade, nicht mehr an diesem Vergleich, also an der Abweichung des Menschen mit Behinderung vom "normalen" Menschen und damit am Defizit anzusetzen, sondern stattdessen Behinderung als möglichen Normalzustand zu sehen und den Blick auf Fähigkeiten zu lenken, die der Mensch mit Behinderung sogar besser hat als der "normale" Mensch. ${ }^{632}$ Weil der soziale Behinderungsbegriff den Bezug zum nichtbehinderten Menschen als Maßstab gerade aufgibt ${ }^{633}$, wäre es widersprüchlich, ihn im Bereich der Beurteilung des Eingriffs und der Benachteiligung wieder einzuführen.

626 Zu den Nachteilen der Förderschule vgl. Schumann, „Ich schäme mich ja so!“; Wocken, Andere Länder, andere Schüler?; Pfahl/Powell, Gemeinsam leben - Zeitschrift für integrative Erziehung 2005, S. $68 \mathrm{ff} ;$ Powell, in: Erziehungswissenschaft, S. $213 \mathrm{ff}$ Neumann, in: Handbuch SGB IX, § 2 Verfassungsrecht, Rn. 23; s. auch BVerfG, Beschluss V. 23.3.2011; Az.: 2 BvR 882/09; http://www.bundesverfassungsgericht.de/entscheidungen/ rs20110323_2bvr088209.html, Rn. 44

628 Neumann, in: Handbuch SGB IX, § 2 Verfassungsrecht, Rn. 23

629 Luthe, in: Rehabilitationsrecht - Verfassungsrecht, Rn. 6

630 Beaucamp, JA 2001, 36 ff, S. 38; Castendiek, in: GS Jean d'Heur, S. 350; Herdegen, Diskriminierungsschutz für Behinderte im Grundgesetz, zitiert nach der Internetversion Punkt 5.4

631 Sachs, Besondere Gleichheitsgarantien, in: HStR VIII, § 182, Rn. 126; Neumann, in: Handbuch SGB IX, § 2 Verfassungsrecht, Rn. 23

632 Fuerst, Behinderung zwischen Diskriminierungsschutz und Rehabilitationsrecht, S. 28

633 S. oben 3. Teil: 2. Kapitel: 1.3.: Völkerrechtsfreundliche Auslegung des Begriffs Behinderung, S. 73 
Gegen eine objektive Betrachtung spricht weiter, dass sie gerade in diesem Bereich sehr problematisch ist. Jede „objektive“ Betrachtung setzt die Wertungen desjenigen, der die „objektive“ Entscheidung trifft, an die Stelle des Betroffenen und wird damit subjektiv. ${ }^{634}$ Grundlage der Wertung ist die Vorstellung davon, welche Fähigkeiten tatsächlich vorhanden sein müssen, um eine Möglichkeit wahrzunehmen. Im Fall eines blinden Piloten ist es noch objektiv feststellbar, dass das Sehen für die Tätigkeit unabdingbar ist. Eine objektive Betrachtung ist jedoch nicht mehr möglich, wenn es um den faktischen Ausschluss von Möglichkeiten geht, weil die rechtlichen und gesellschaftlichen Bedingungen Anforderungen stellen, die ein Mensch mit Behinderung nicht erfüllt bzw. nicht erfüllen kann. Der prominente Sänger Thomas Quasthoff ist nicht in der Lage Klavier zu spielen, weil er keine Arme hat. Die Ausbildung am Klavier ist aber zwingend Teil eines Gesangsstudiums. Die objektive Sichtweise argumentiert hier, dass der (tatsächlich erfolgte) Ausschluss von der Hochschule für Musik für ihn kein Nachteil ist, weil er ohnehin nicht Klavierspielen kann. Für ihn selbst war das jedoch ein Nachteil, weil ihm diese Berufsausbildung unmöglich gemacht wurde. Ebenso ist es für einen körperbehinderten Menschen, der Schauspieler werden möchte, ein Nachteil, wenn er vom Schauspielstudium ausgeschlossen wird, weil er etwa nicht reiten und fechten kann. ${ }^{635}$ Auch für autistische Kinder ist es ein Nachteil, wenn sie vom Besuch des Gymnasiums ausgeschlossen werden, weil sie bestimmte Fähigkeiten nicht haben, obwohl sie für bestimmte anschließende Studiengänge besonders geeignet sind. Und schließlich kann auch der Besuch der Förderschule ein Nachteil sein, denn die Nachteile der Förderschule wurden inzwischen mannigfaltig festgestellt ${ }^{636}$, obwohl die Schulbehörden sie für vorteilhaft halten.

Wenn hier festgestellt wird, dass objektiv kein Nachteil vorliegt, weil die Betroffenen Möglichkeiten nicht nutzen können, dann werden subjektive Wertungen getroffen und keine objektiven Tatsachen festgestellt.

Ebenso wenig ist ein objektiver Vergleich dessen möglich, was nachteilig und vorteilhaft ist und ob die Vorteile die Nachteile aufwiegen. Auch hier fußt der vermeintlich objektive Vergleich auf subjektiven Wertungen und Gewichtungen. Die Vorstellungen davon, was ein Vorteil und ein Nachteil ist, sind höchst unterschiedlich. Ob die Vorteile die Nachteile aufwiegen ist ebenso subjektiv.

634 Castendiek, in: GS Jean d'Heur, S. 345

635 S. die Beispiele bei Theben, in: Gesellschaft der Behinderer, S. $79 \mathrm{ff}$

${ }^{636}$ VGH Kassel, Beschluss v. 12.11.2009, Az.: 7 B 2763/09, juris, zu den Nachteilen der Förderschule vgl. Schumann, „Ich schäme mich ja so!“; Wocken, Andere Länder, andere Schüler?; Pfahl/Powell, Gemeinsam leben - Zeitschrift für integrative Erziehung 2005, S. 68 ff; Powell, in: Erziehungswissenschaft, S. $213 \mathrm{ff}$ 
Auch bezüglich der Berücksichtigung von Legasthenie/Dyskalkulie lässt sich die Unmöglichkeit einer objektiven Betrachtungsweise aufzeigen: Objektiv betrachtet könnte man sagen, dass Schüler und Schülerinnen mit Legasthenie/ Dyskalkulie nicht benachteiligt werden, weil ihnen bestimmte Fähigkeiten fehlen, die insbesondere für den Besuch des Gymnasiums oder der Realschule oder für ein bestimmtes Studium ${ }^{637}$ so wichtig sind, dass nicht darauf verzichtet werden kann. Dann wäre der Ausschluss von bestimmten Schulzweigen und Schulabschlüssen keine Benachteiligung, weil die Fähigkeit zum Lesen, Rechtschreiben und Rechnen tatsächlich vorhanden sein muss. Objektiv könnte man aber auch darauf abstellen, ob die Schüler und Schülerinnen mit Legasthenie/ Dyskalkulie die Intelligenz für bestimmte Schularten und ein Studium mitbringen.

Eine objektive Sichtweise versuchen verschiedene landesrechtliche Regelungen zur Berücksichtigung von Schwierigkeiten beim Lesen, Rechtschreiben und Rechnen. So bestimmen die Regelungen in Bremen und Schleswig-Holstein, dass Schwierigkeiten dann anzunehmen sind, wenn das betroffene Kind länger als ein halbes Jahr schlechter als ausreichend benotet wird. ${ }^{638}$ Damit findet sich ein vorgeblich objektiver Anhaltspunkt. Sicherlich ist hier richtig, dass ein Kind, das langandauernd mangelhafte Ergebnisse vorweist, besonderer Aufmerksamkeit und Förderung bedarf und evtl. einen Nachteilsausgleich benötigt. Dieser "objektive“ Ansatz schließt jedoch die behinderten Kinder aus der Anwendung der Regelung aus, die bessere Noten als „ausreichend" schreiben. Dahinter steckt die Wertung, dass Kinder keinen Nachteil erleiden oder nicht behindert sein können, wenn sie sich im Normbereich befinden. Es gibt jedoch Kinder, die sich zwar (noch) im Normbereich befinden, aber aufgrund ihrer Legasthenie/Dyskalkulie beständig abgewertet werden. Würde z.B. bei ihnen die Rechtschreibung nicht bewertet, wären ihre Aufsätze gut oder sehr gut, so aber sind sie befriedigend und schlechter. Das Gefühl des Versagens und des ewigen Scheiterns wird von den Betroffenen subjektiv entscheidend als Nachteil empfunden, von der schulrechtlichen Regelung aber für unbeachtlich erklärt.

In Hamburg sieht die Richtlinie zur LRS vor, dass Kinder mit einem Prozentrang von 10 und weniger eine Förderung bekommen ${ }^{639}$ und nur bei Kindern mit einem Prozentrang von 5 und weniger die Bewertung der Rechtschreibung

637 Kischel, in: BeckOK GG, Art. 3, Rn. 219.3 versagt den Betroffenen „ohne Weiteres“ Ausgleichsmaßnahmen für alle sozial- und kulturwissenschaftlichen Fächer, Rechtswissenschaften, Theologie und Psychologie.

638 HB LRS-Erlass Nr. 4.2; SH Legasthenie-Erlass Nr. 2.1; s. auch SH Rechenschwäche-Erlass Nr. 4.2 und 5.1 "schwach ausreichend oder schlechter" 
ausgesetzt werden kann. ${ }^{640}$ Diese Bewertung weicht von den medizinischen Diagnosekriterien ab, die jedenfalls alle Werte unter Prozentrang 10 für sehr erheblich halten. ${ }^{641}$ Und sie führt eine sonst nicht vorhandene Trennung zwischen schwer (Prozentrang $<10$ ) und sehr schwer (Prozentrang $<5$ ) betroffenen Kindern ein. Im Ergebnis läuft diese "objektive“ Regelung auch auf eine Differenzierung hinsichtlich der Schwere der Behinderung hinaus, die mit dem Behindertenbegriff nicht zu vereinbaren ist.

Schließlich bestimmt sich die vermeintlich objektive Feststellung der Benachteiligung nach den Vorstellungen desjenigen, der entscheidet, welche Anforderungen an das Abitur, an die Lese- und Rechtschreibfähigkeiten in der Schule und im Studium zu stellen sind. In die Feststellung (tatsächlich ist es eine Wertung) fließt mit ein, dass es nur schwer vorstellbar ist, dass ein intelligentes Kind es nicht schafft, sich die Rechtschreibung von Wörtern zu merken und wohl auch die Vorstellung, dass das Kind faul ist oder mit ein bisschen Anstrengung diese leichte Aufgabe doch bewältigen müsste. ${ }^{642}$ Sind es objektive Feststellungen, wenn Kischel ausführt, dass es Fächer gibt, die aufgrund der ihnen eigenen Sachgesetzlichkeit die Fähigkeit verlangen, gerade einen schriftlichen Text (etwa Lehrbücher, Aufsätze oder Schriftsätze) schnell zu verstehen? ${ }^{643}$ Die angeblich objektive Sichtweise auf die Anforderungen der Fächer stellt sich bei genauer Betrachtung als subjektiv heraus, nämlich als die subjektive Ansicht, schnelles Verstehen gehöre zwingend zu einem bestimmten Fach dazu. Es gibt jedoch keine eigene Sachgesetzlichkeit eines Faches, etwas schnell zu verstehen. Das schnelle Verstehen ist zwar hilfreich, aber es ist dem Fach nicht immanent. Und was schnell ist, ist ebenfalls höchst subjektiv. Käme es darauf an, könnten Blinde nicht Rechtswissenschaften studieren, weil sie einen schriftlichen Text erst dann schnell verstehen, wenn er für sie aufbereitet wurde.

Der Eingriff ist daher nicht objektiv, sondern subjektiv zu bestimmen. ${ }^{644}$ Der Begriff der Benachteiligung ist infolgedessen weit zu fassen und erfasst jede aus Sicht des Menschen mit Behinderung bedeutsame Beeinträchtigung, die die allgemeine Handlungsfreiheit oder Optionen, die der Staat Nichtbehinder-

\footnotetext{
640 HH-Förder-Richtlinie Nr. 4, 4.1, 4.2

641 S. oben 1. Teil: 1. Kapitel: 5. : Medizinische Diagnostik, S. 12

${ }^{642}$ Vgl. VG Düsseldorf, Beschluss v. 20.5.2011, Az.: 18 L 707/11, juris, Rn. 14: Förderung dient dazu, dem Schüler die notwendigen Kompetenzen im Lesen und Schreiben zu vermitteln. „[Das] Nichterreichen des Förderziels geht zu Lasten des Schülers".

643 Kischel, in: BeckOK GG, Art. 3, Rn. 291.3

644 Castendiek, in: GS Jean d'Heur, S. 345 f; Sachs, RdJB 1996, S. 154 ff, S. 168; Höller, Beschulung behinderter Kinder und Jugendlicher an öffentlichen Schulen, S. 191; Luthe, in: Rehabilitationsrecht- Verfassungsrecht, Rn. 6; Beaucamp, JA 2001, 36 ff, S. 39
} 
ten einräumt, beschneidet. Das gilt insbesondere für den Zugang zu allgemeinen staatlichen Leistungen im Vergleich zu anderen Gruppen. ${ }^{645}$

Die Berücksichtigung objektiver Anhaltspunkte oder öffentlicher Interessen wird dadurch nicht grundsätzlich ausgeschlossen. Vielmehr ist es eine Frage der Rechtfertigung und nicht schon des Eingriffstatbestands, ob die öffentlichen Interessen die subjektiven Belange des Menschen mit Behinderung überwiegen oder nicht. ${ }^{646}$

\section{Benachteiligung durch Gleichbehandlung?}

Die Benachteiligung der behinderten Schüler und Schülerinnen mit Legasthenie/Dyskalkulie erfolgt gewöhnlich dadurch, dass sie mit den „normalen“ Schülern und Schülerinnen gleichbehandelt werden. Unstreitig ist, dass Benachteiligung i.S.d. Art. 3 III 2 GG jedenfalls jede nachteilige Ungleichbehandlung von Menschen mit Behinderungen im Vergleich zu Menschen ohne Behinderung ist. ${ }^{647}$ Fraglich ist aber, ob Art. 3 III 2 GG auch vor einer Benachteiligung schützt, die auf einer nachteiligen Gleichbehandlung beruht. Der BFH hat dies verneint. Er hat ohne weitere Begründung festgestellt: „Art. 3 Abs. 3 Satz 2 GG räumt nach Wortlaut, Systematik und Zweck dem Behinderten nur ein subjektives Abwehrrecht gegen Benachteiligungen, aber grundsätzlich keinen Anspruch auf bestimmte Vergünstigungen im Vergleich zu Nichtbehinderten ein. Benachteiligung bedeutet nachteilige Ungleichbehandlung ... Eine nachteilige Gleichbehandlung wird dagegen nicht von Art. 3 Abs. 3 Satz 2 GG erfasst. “648

Nach wohl herrschender Ansicht ist Art. 3 III 2 GG durch seine systematische Einordnung in Art. 3 III GG in erster Linie als Verbot benachteiligender Anknüpfung an eine Behinderung zu verstehen. Allgemeine Regelungen, die auf Behinderte keine besondere Rücksicht nehmen, sind danach ebenso wenig von dem abwehrrechtlichen Benachteiligungsverbot umfasst wie Beeinträchtigungen, die bloß typischerweise Menschen mit Behinderungen treffen. ${ }^{649}$ Das hat zur Folge, dass aus Art. 3 III 2 GG kein Anspruch auf Ungleichbehandlung

645 Herdegen, Diskriminierungsschutz für Behinderte im Grundgesetz zitiert nach der Internetversion Punkt 5.4; Beaucamp, JA 2001, S. 36 ff, S. 39

646 Höller, Beschulung behinderter Kinder und Jugendlicher an öffentlichen Schulen, S. 191; Luthe, in: Rehabilitationsrecht, Rn. 6; Neumann, in: Handbuch SGB IX, § 2 Verfassungsrecht, Rn. 23; Buch, Grundrecht der Behinderten, S. 111 BVerfGE 99, 341, 357; Gubelt, in: v. Münch GG, Art. 3, Rn. 104 d; Kischel, in: BeckOK GG, Art. 3, Rn. 212

648 BFH Urteil v. 21.6. 2007, Az.: III R 48/04, juris, Rn. 36 f, die dagegen erhobene Verfassungsbeschwerde wurde nicht zur Entscheidung angenommen: Beschluss BVerfG v. 6.7.2010, Az.: 2 BvR 2164/07, http://dzg-online.de/files/20100805_infotext_bverfg_urteil_3.pdf 
abgeleitet werden kann, weil die gleichbehandelnden Regelungen immer allgemein sind.

Gegen diese Auslegung spricht, dass Art. 3 III 2 GG eine Konkretisierung des Gleichheitssatzes aus Art. 3 । $\mathrm{GG}^{650}$ und insoweit gegenüber Art. 3 । GG das speziellere Grundrecht ist ${ }^{651}$, das die fragliche Behandlung abschließend regelt. ${ }^{652}$ Nach ganz allgemeiner Ansicht enthält der Gleichheitssatz des Art. 3 । GG den Grundsatz, dass Gleiches gleich und Ungleiches ungleich zu behandeln ist. Er verbietet nicht nur Gleiches ungleich zu behandeln, sondern kann sogar gebieten, Ungleiches ungleich zu behandeln. ${ }^{653}$ Weil die Gleichbehandlung ungleicher Tatbestände in hohem Maße ungerecht sein kann, wird nur mit einer Ungleichbehandlung ungleicher Tatbestände der Gerechtigkeit genüge getan. ${ }^{654}$ Deshalb ist der besondere Gleichheitssatz des Art. 3 III 2 GG ebenso auszulegen, wie der allgemeine Gleichheitssatz des Art. 3 । GG, so dass er auch die nachteilige Gleichbehandlung verbietet.

Der Intention der Diskriminierungsverbote des Art. 3 III 2 GG und Art. 3 II GG, den allgemeinen Gleichheitssatz negativ und verschärfend zu konkretisieren ${ }^{655}$, um die betreffenden Personengruppen besonders zu schützen, widerspricht es, wenn Art. 3 III 2 GG dahingehend ausgelegt würde, dass der Schutzbereich dieses Grundrechts enger ist als der des Art. 3 । GG. In dem Fall wäre die praktische Wirksamkeit des Art. 3 III 2 GG gering ${ }^{656}$. Die Tatsache, dass in Art. 3 III 2 GG, im Gegensatz zum Benachteiligungsverbot des Art. 3 III 1 GG, ausdrücklich nur die Benachteiligung, nicht aber die Bevorzugung verboten wird, zeigt, dass der Verfassungsgeber einen umfassenden Schutz wollte, der über die vorhandenen Konkretisierungen des Gleichheitssatzes hinausgeht. ${ }^{67}$ Die allgemeinen Prinzipien des Art. 3 । GG stellen daher eine Generalklausel ${ }^{658}$ dar, die auch für Abs. 3 Satz 2 GG gilt.

Entgegen der herrschenden Meinung erschöpft sich das Benachteiligungsverbot daher nicht in der Anordnung, behinderte und nichtbehinderte Menschen rechtlich gleich zu behandeln. ${ }^{659}$ Dies ergibt sich auch daraus, dass das BVerfG in der Sonderschulentscheidung festgestellt hat, dass Art. 3 III 2 GG

\footnotetext{
650 Gubelt, in: v. Münch GG, Art. 3, Rn. 1

651 Heun, in: Dreier GG, Art. 3, Rn. 141; Kischel, in: BeckOK GG, Art. 3, Rn. 1

652 Osterloh, in: Sachs GG, Art. 3, Rn. 77 f; Heun, in: Dreier GG, Art. 3, Rn. 141; Kischel, in: BeckOK GG, Art. 3, Rn. 2; Sachs, Besondere Gleichheitsgarantien, in: HStR VIII, § 182, Rn. 19

653 Stern, in: FS Günter Dürig, S. 210, S. 212

654 Stern, in: FS Günter Dürig, S. 210

655 König/Peters, in: Grote/Marauhn EMRK/GG, Kapitel 21: Das Diskriminierungsverbot, Rn. 46

656 S. dazu Boysen, in: v. Münch GG 2012, Art. 3, Rn. 196

657 Vgl. Sachs, Besondere Gleichheitsgarantien, in: HStR VIII, § 182, Rn. 17

658 BVerfGE 3, 225, 240 „Generalklausel“

659 BSG, Urteil v. 6.3.2012, Az.: B 1 KR 10/11 R, www.bundessozialgericht.de, Rn. 31
} 
eine unterschiedslose Behandlung von Behinderten ohne Berücksichtigung ihrer spezifischen Bedürfnisse verbietet, sofern sich hieraus Ausschlusswirkungen ergeben. ${ }^{660}$

Diese Auslegung des Art. 3 III 2 GG entspricht auch der Auslegung der Diskriminierungsverbote der BRK, die generell als Auslegungshilfe für die Bestimmung von Inhalt und Reichweite der Grundrechte und speziell für das Verständnis des Art. 3 III 2 GG heranzuziehen ist. ${ }^{661}$ Der BRK liegt ein weites Verständnis des Diskriminierungsverbots zugrunde, wonach auch die unterschiedslose Gleichbehandlung von Menschen mit und ohne Behinderung verboten ist. Dies hat das Committee on the Rights of Persons with Disabilities (CRPD) in seiner ersten Entscheidung im April 2012 in einem Individualbeschwerdeverfahren wegen Verstoßes gegen die BRK ausdrücklich festgestellt. ${ }^{662}$ Die Beschwerdeführerin aus Schweden wandte sich dagegen, dass ihr die Bauerlaubnis für ein Wassertherapiebecken, das sie wegen ihrer Behinderung benötigte, versagt worden war, weil es auf einer Fläche gebaut werden sollte, für die ein absolutes Bauverbot bestand und eine Ausnahme im Gesetz nicht vorgesehen war. Der Staat Schweden machte vor allem geltend, dass die gesetzlichen Vorschriften für Bürger gleich angewendet worden seien, unabhängig davon, ob sie behindert sind oder nicht. ${ }^{663}$ Das CRPD stellte fest, dass ein Gesetz, das neutral formuliert und angewendet werde, einen diskriminierenden Effekt haben könne, wenn es die besonderen Umstände des Einzelnen nicht berücksichtigt. Das Recht, nicht aufgrund der in der BRK garantierten Rechte diskriminiert zu werden, werde verletzt, wenn ein Staat es versäumt Personen, deren Situationen signifikant [also nach deutscher Terminologie "wesentlich"] unterschiedlich sind, ungleich zu behandeln. Dies stelle eine Verletzung von Art. 5 I und 5 III i.V.m. Art. 25, 26 und i.V.m. Art. 3 Buchst. b, d, e und 4 I Buchst. d BRK dar. ${ }^{664}$ Danach umfasst das allgemeine Diskriminierungsverbot der BRK in Verbindung mit den einzelnen speziellen Rechten auch den Fall der nachteiligen Gleichbehandlung durch an sich neutrale Regelungen.

Die in dieser Untersuchung vertretene völkerrechtsfreundliche Auslegung des Art. 3 III 2 GG gebietet daher die Auslegung dahin, dass Benachteiligungen i.S.d. Art. 3 III 2 GG auch nachteilige Gleichbehandlungen sind.

660 BVerfGE 96, 288 ff, Rn. 52; Langenfeld, RdJB 2007, S. 211 ff, S. 224; Sachs, JuS 1998, S. 553 ff, S. 554; Herdegen, Diskriminierungsschutz für Behinderte im Grundgesetz, S. 29 f; so auch ausdrücklich BSG, Urteil v. 6.3.2012, Az.: B 1 KR 10/11 R, www.bundessozialgericht.de, Rn. 31

661 BSG, Urteil v. 6.3.2012, Az.: B 1 KR 10/11 R, www.bundessozialgericht.de, Rn. 31

662 CRPD http://www.ohchr.org/Documents/HRBodies/CRPD/CRPD.C.7.D.3.2011.doc; zum Verfahren s. Althoff, Anwaltsblatt 2012, S. $52 \mathrm{f}$ 


\subsection{Benachteiligung durch Gleichbehandlung bezüglich äußerer Prüfungsbedingungen}

Die Benachteiligung kann darin liegen, dass Schüler und Schülerinnen mit Legasthenie/Dyskalkulie hinsichtlich der äußeren Prüfungsbedingungen gleichbehandelt werden. Dazu gehören insbesondere die Prüfungszeit, aber auch die Gestaltung von Aufgabenblättern, das Erlesen der Aufgaben, das Ersetzen einer schriftlichen durch eine mündliche Prüfung, die Verkürzung von Aufgaben, die Benutzung eines Laptops mit oder ohne Rechtschreibprogramm, das Diktieren von Antworten in ein Aufnahmegerät statt der Niederschrift usw. Im Einzelnen ist die Abgrenzung, was zu äußeren Prüfungsbedingungen gehört und wann eine Veränderung der inhaltlichen Anforderungen vorliegt, streitig. ${ }^{665}$ Das kann aber hier für die Grundsatzfrage dahinstehen.

Das schulische Prüfungsrecht wird geprägt durch die aus dem Grundsatz der Chancengleichheit abgeleitete Maßgabe, dass in Prüfungen gleiche Ausgangsbedingungen durch formale Gleichbehandlung geschaffen werden müssen. ${ }^{666}$ Grundsätzlich ist im Prüfungsrecht jedoch anerkannt, dass die formale Gleichbehandlung einen Prüfling, der beeinträchtigt ist, benachteiligen kann und deshalb seine Prüfungsbedingungen verändert werden müssen, um so materielle Gleichheit herzustellen. ${ }^{667}$ Ob eine solche Benachteiligung gegeben ist, die einen Anspruch auf Ungleichbehandlung begründet, wird geprüft, indem der Prüfling mit einem gedachten „Normalkandidaten“ verglichen wird. ${ }^{668} \mathrm{Maß}$ geblich ist insoweit, ob die Behinderung solche Fähigkeiten einschränkt, die außerhalb der durch die jeweilige Prüfung zu ermittelnden Fähigkeiten liegen und die deshalb den Nachweis einer ansonsten vorhandenen Befähigung erschweren. ${ }^{669}$ Ist das der Fall, so werden die Prüfungsbedingungen so verändert,

665 Ablehnend zum Mitlesen eines Textes durch einen hörgeschädigten Schüler bei einer Nacherzählung Lambert, SchulVerwaltung BW 2002, S. 148 ff, S. 150; ablehnend zur Verkürzung von Aufgaben, Lambert, SchulVerwaltung BW 2002, S. 148 ff, S. 150; zur Zulässigkeit eines Laptops mit Rechtschreibprogramm in der Oberstufe VGH Kassel, Beschluss v. 5.2.2010, Az.: 7 A 2406/09.Z, juris, Rn 46; ablehnend für juristischen Prüfungen: VGH Bayern, Beschluss v. 3.9.2010; Az.: 7 CE 10.2175, juris; VG Ansbach, Beschluss v. 29.2.2008, Az.:AN 2 E 08.00317, juris; VG Augsburg, Beschluss v. 7.5.2008, Az.: Au 3 E 08.442, juris, den Prüflingen wurde lediglich die Hinzuziehung einer juristisch ungebildeten Schreibkraft gestattet; ablehnend zur Beantwortung einer schriftlichen Aufgabe in mündlicher Form VGH München Beschluss v. 4.1.2010, Az.: 7 CE 09.2900, www.la-by.bayern.de, Rn. 22

OVG Lüneburg, Beschluss v. 10.7.2008, Az.: 2 ME 309/08, www.rechtsprechung.niedersachsen.de; Rn. 19

S. dazu bereits oben 2. Teil: 2. Kapitel: Gleichheit und Ungleichheit von Schülern und Schülerinnen in Prüfungen, S. 61

668 S. dazu bereits oben 2. Teil: 2. Kapitel: Gleichheit und Ungleichheit von Schülern und Schülerinnen in Prüfungen, S. 61 
dass der Prüfling die gleichen Startchancen in der Prüfung hat wie die "Normalkandidaten ${ }^{4670}$ und dadurch in die Lage versetzt wird, dieselbe Prüfungsleistung zu erbringen wie der nicht-behinderte Prüfling, ohne die fachlichen, inhaltlichen Anforderungen zu verändern. ${ }^{671}$ Welche Veränderungen im Einzelnen erforderlich sind, hängt von der Art der Behinderung und der Prüfung ab. Ob der vollständige Ausgleich der Störung durch die Abhilfemaßnahme gelungen ist, ist nach der ständigen Rechtsprechung des BVerfG von den Gerichten uneingeschränkt, ggf. mit Hilfe von Sachverständigen ${ }^{672}$, überprüfbar. ${ }^{673}$

In der Rechtsprechung und Literatur ist inzwischen anerkannt, dass Schüler und Schülerinnen mit Legasthenie durch die Gleichbehandlung bezüglich äußerer Prüfungsbedingungen benachteiligt werden, da sie in der "technischen Fähigkeit" zur Darstellung des vorhandenen Wissens durch eine langsamere Lesegeschwindigkeit und die erschwerte handschriftliche Darlegung eingeschränkt sind. ${ }^{674}$

Auch bei Dyskalkulie können die äußeren Prüfungsbedingungen benachteiligen. Schüler und Schülerinnen mit Dyskalkulie rechnen langsamer und umständlicher und brauchen mehr Zeit zum Verständnis der Aufgaben. Sie sind daher nicht mit dem "Normalkandidaten" vergleichbar. Das OVG Weimar hat daher in einem Eilverfahren einer Schülerin mit Dyskalkulie einen Zeitzuschlag von $25 \%$ zugesprochen. ${ }^{675}$ Zwar hielt es das OVG Weimar nach summarischer Prüfung grundsätzlich für wahrscheinlich, dass die Dyskalkulie die Leistungsfähigkeit als solche betreffe, dennoch sei aber ein Zeitzuschlag erforderlich. Damit würde die sehr langsame Rechengeschwindigkeit kompensiert, die darauf

670 Langenfeld, RdJB 2007, S. 211 ff, S. 218 f, V G Kassel, Beschluss v. 23.3.2006, 3 G 419/06, Sonderheft Recht des BVL: Chancengleichheit herstellen - Diskriminierung vermeiden, 2006, http://www.bvl-legasthenie.de/index.php5?p=/downloads; VGH Mannheim, Beschluss v. 26.8.1993, 9 S 2023/93, NVWZ 1994, S. $598 \mathrm{ff}$

671 Z.B. SH ZeugnisVO § 6 I; BW VV Besonderer Förderbedarf Nr. 2.3.1.; HB AP-V§ 6 a S. 4; HH APO-AH § 13; VGH Kassel, Beschluss v. 5.2.2010, Az.: 7 A 2406/09, juris, Rn. 59 f Zimmerling/Brehm, Prüfungsrecht, Rn. 314 ff; VGH Mannheim, Beschluss v. 26.8.1993, Az.: 9 S 2023/93, NVwZ 1994, S. 599; VG Düsseldorf, Beschluss v. 8.5.2003, Az.: 15 L 1418/03, juris, Rn. 8

673 BVerfG Beschluss v. 12.12.1992, Az.: 1 BvR 1295/90, juris, Rn. 19, vgl. dazu die insgesamt ablehnenden Entscheidungen in Bayern zu der Frage, ob in juristischen Prüfungen ein Laptop gestattet wird, VGH Bayern, Beschluss v. 3.9.2010; Az.: 7 CE 10.2175, juris; VG Ansbach, Beschluss v. 29.2.2008, Az.:AN 2 E 08.00317, juris; VG Augsburg, Beschluss v. 7.5.2008, Az.: Au 3 E 08.442, juris, die Prüflinge wurde lediglich die Hinzuziehung einer juristisch ungebildeten Schreibkraft gestattet.

674 Niehues, Prüfungsrecht, Rn. 259; OVG Lüneburg, Beschluss v. 10.7.2008, Az.: 2 ME 309/08, www.rechtsprechung.niedersachsen.de, Rn. 9 ff; OVG Weimar, Beschluss v. 17.5.2010, Az.: 1 EO 854/10, juris, Rn. 28, 41; VGH Kassel, Beschluss v. 3. 1. 2006, 8 TG 3292/05, NJW 2006, S. 1608 f; OVG Schleswig, Beschluss v. 19.8.2002, Az.: 3 M 41.02, juris - Leitsätze 
beruhe, dass die Schülerin sehr komplizierte Rechenwege verwende. Dies sei auch deshalb berechtigt, weil bei Dyskalkulie vor allem die Grundrechenarten, nicht aber die höheren mathematischen Fertigkeiten betroffen seien. ${ }^{676}$ Auch hier erschöpft sich die Veränderung der äußeren Prüfungsbedingungen nicht in einem Zeitzuschlag, sondern kann es auch erforderlich machen, dem Schüler die Benutzung von didaktischem Material oder von Formelsammlungen zu erlauben.

\subsection{Benachteiligung durch Gleichbehandlung bezüglich inhaltlicher Prüfungsanforderungen}

Die Frage ist weiter, ob eine Benachteiligung darin liegt, dass Schüler und Schülerinnen mit Legasthenie/Dyskalkulie den gleichen Bewertungsregeln unterliegen wie Schüler und Schülerinnen ohne diese Behinderung.

Aus dem Grundsatz der Chancengleichheit wird der Schluss gezogen, dass die Bewertung von Leistungen grundsätzlich nach den gleichen Bewertungsmaßstäben ${ }^{677}$ zu erfolgen hat. Nur so kann sichergestellt werden, dass alle Schüler und Schülerinnen die gleichen Chancen in Prüfungen haben. Eine Ungleichbehandlung bei der Bewertung der Prüfungsleistungen und der inhaltlichen Prüfungsanforderungen wird von Rechtsprechung und Literatur abgelehnt, weil dies zu einer unterschiedlichen Bewertung der Schüler und Schülerinnen mit Behinderung und ohne Behinderung führe ${ }^{678}$, was im Hinblick auf die Bedeutung der Schulabschlüsse, vor allem des Abiturs, nicht zulässig sei. Im Ergebnis wird damit eine Benachteiligung durch die inhaltlichen Prüfungsanforderungen und Standards abgelehnt.

\subsubsection{Benachteiligung durch Noten}

Die Gleichbehandlung führt bei Schülern und Schülerinnen mit Legasthenie/ Dyskalkulie dazu, dass sie schlechtere Noten erzielen, weil bei der Benotung die Behinderung nicht berücksichtigt wird. Fraglich ist, ob diese schlechteren Noten eine rechtsrelevante Benachteiligung sind.

\footnotetext{
676 OVG Weimar, Beschluss v. 17.5.2010, Az.: 1 EO 854/10, juris, Rn. $51 \mathrm{f}$

678 VG Sigmaringen, Beschluss v. 2.4.2008, Az.: 4 K 596/08, S. 4 (nicht veröffentlicht); VG Köln, Beschluss 26.9.2008 Az.: 10 L 1240/08, www.justiz.nrw.de; VG Aachen, Urteil v. 13.11.2009, Az.: 9 K 25/09, BeckRS 2009 42206, S. 5; VG Schleswig, Urteil v. 10.6.2009, Az.: 9 A 208/08, BeckRS 2009 38123, S. 10; VG Gießen, Urteil v. 30.6.2009. Az.: 4 K 1052/09.GI, S. 15 (nicht veröffentlicht); OVG Lüneburg, Beschluss v. 10.7.2008, Az.: 2 ME 309/08, www.rechtsprechung.niedersachsen.de, Rn. 19; VGH Kassel, Beschluss v. 5.2.2010, Az.: 7 A 2406/09, juris, Rn. 43; Kischel, in: BeckOK GG, Art. 3, Rn. 219.4; Ennuschat/Volino, br 2009, S. $166 \mathrm{ff}$, S. 167
} 
Geht man von der Definition der Benachteiligung aus Sicht des Menschen mit Behinderung aus, dann sind schlechtere Noten benachteiligend, da sie den Zugang zu bestimmten Schulformen und weiteren Bildungsgängen beschneiden. Noten sind jedoch die logische Konsequenz aus der Bewertung aller Schüler und Schülerinnen nach allgemeinen und gleichen Maßstäben, und der Ausschluss von bestimmten Schullaufbahnen und Schulabschlüssen ist die sich anschließende systemimmanente Folge.

Eine Auslegung, die jede schlechte Note als Nachteil i.S.d. Art. 3 GG ansehen würde, würde den Besonderheiten des Schulsystems nicht gerecht. Es ist eine der wesentlichen Aufgaben der Schule, Leistungen zu bewerten und Berechtigungen zu erteilen und daher auch zwischen "sehr gut" und „ungenügend" zu bewerten. Die Vermittlung der Fertigkeiten und Fähigkeiten gelingt je nach Veranlagung der Schüler und vieler weiterer Faktoren nicht für alle Schüler und Schülerinnen gleich gut, sondern unterschiedlich gut oder schlecht. Es liegt daher in der Natur des Lernens, der Schule und der menschlichen Vielfalt, dass Schüler und Schülerinnen eine Bandbreite an unterschiedlichen Begabungen, Fähigkeiten, Leistungen und Ergebnissen zeigen und das geforderte Pensum unterschiedlich gut und erfolgreich lernen und darbieten können. Fehlen Fähigkeiten, die im Schulalltag relevant sind, oder sind sie nur begrenzt vorhanden, dann liegt es im System, dass der Schüler schlechte Noten erhält und das schulische Leistungsziel nicht oder nur schlecht erreicht.

Allerdings kann die Frage, ob schulrelevante Fähigkeiten fehlen oder nur eingeschränkt vorhanden sind und deshalb die schlechte Note gerechtfertigt und kein Nachteil ist, auch nicht völlig ohne Bezug zur Behinderung beantworten werden. Der Schüler mit Behinderung erzielt die schlechtere Note gerade weil er die Fähigkeiten, die in dem Bereich geprüft werden, behinderungsbedingt nur eingeschränkt oder nicht hat. Es würde dem Sinn und Zweck des Diskriminierungsschutzes zuwider laufen, wenn in diesem Fall bereits die Benachteiligung verneint würde. ${ }^{679}$ Für die Frage, ob eine Benachteiligung durch Noten vorliegt, muss es ebenso wie bei der Prüfung der Vergleichbarkeit von Personen und Sachverhalten darauf ankommen, inwieweit die Betroffenen in der Lage sind, durch ihr Verhalten die Noten zu beeinflussen ${ }^{680}$ und damit eine Benachteiligung durch ihr Verhalten selbst zu vermeiden. Je weniger die Betroffenen in der Lage sind, die Benachteiligung durch eigenes Verhalten zu vermeiden, desto mehr liegt in den Noten eine Benachteiligung. Die Tatsache, dass der Mensch mit Behinderung für seine Einschränkung "nichts kann", ihr ausgeliefert ist und keine Möglichkeiten hat, auf sie einzuwirken und sie zu verändern, unabhängig davon, ob oder wie sehr er sich bemüht und wie sehr er es sich wünscht, ist die Grundlage für die Berücksichtigung von Behinderungen

\footnotetext{
679 A.A. Kischel, in: BeckOK GG, Art. 3, Rn. 212, 214

680

Vgl. BVerfGE 88, 87, 96, Rn. 35
} 
überhaupt und kann daher nicht der Grund sein, um die Behinderung gerade nicht zu berücksichtigen. ${ }^{681}$ Stellt die Schule gerade auf diese Fähigkeiten ab und bewertet und benotet sie, ist dies für den Menschen, der in genau diesen Fähigkeiten eingeschränkt ist, ein Nachteil.

Die Einordnung als Nachteil ergibt sich auch daraus, dass der Eingriff, wie oben dargelegt ${ }^{682}$, subjektiv zu bestimmen ist. Aus Sicht der Betroffenen liegt eine bedeutsame Beeinträchtigung vor, wenn in schulischen Prüfungen gerade die behinderungsbedingt eingeschränkten oder fehlenden Fähigkeiten bewertet werden.

Auch die Rechtsprechung sieht dies so, denn sie hat in den Verfahren, in denen es um einen Anspruch auf Nichtbewertung der Rechtschreibung ging, einen Nachteil durch die Gleichbehandlung bezüglich der inhaltlichen Prüfungsbedingungen zum Teil explizit bejaht ${ }^{683}$, zum Teil als Folge aus der Qualifikation der Legasthenie als Behinderung inzident unterstellt. ${ }^{684}$ Der VGH Kassel schränkt dies dahin ein, dass ein solcher Nachteil nur gegeben sein kann, wenn die Rechtschreibfehler nachgewiesenermaßen auf der Legasthenie beruhen und nicht auf allgemeinen Rechtschreibfehlern. ${ }^{685}$ Unabhängig von der Frage, ob eine derartige Differenzierung überhaupt möglich ist ${ }^{686}$, ergibt sich daraus jedoch, dass die allgemeinen Bewertungsregeln jedenfalls dann einen Nachteil für Schüler und Schülerinnen mit Legasthenie/Dyskalkulie darstellen, wenn die Fehler auf der Behinderung beruhen.

Im Fall von Behinderungen muss daher differenziert werden: Noten sind dann Nachteile i.S.d. Art. 3 III 2 GG, wenn sie die behinderungsbedingt beeinträchtigten Fähigkeiten betreffen. Betreffen sie dagegen Fähigkeiten, die bei einem Schüler nicht behinderungsbedingt beeinträchtigt sind, dann sind sie keine Nachteile. Bei einem Schüler mit Legasthenie ist daher die Note im Diktat ein Nachteil, weil er im Bereich der Rechtschreibung beeinträchtigt ist, die Be-

681 A. A. Kischel, in: BeckOK GG, Art. 3, Rn. 219.1

682 Vgl. 3. Teil: 3. Kapitel: 2.: Objektive oder subjektive Bestimmung des Eingriffs, S. 108

683 VGH Kassel, Beschluss v. 8.12.2011, Az.: 7 A 2621/10.Z, juris, Rn. 13; VG Hannover, Beschluss v. 13.12.2010, Az.: 6 B 5596/10, www.rechtsprechung.niedersachsen.de, Rn. 34; VG Schleswig, Urteil v. 10.6.2009, Az.: 9 A 208/08, BeckRS 2009 38123, S. 9

684 VG Köln, Beschluss 26.9.2008 Az.: 10 L 1240/08, www.justiz.nrw.de; VG Aachen, Urteil v. 13.11.2009, Az.: 9 K 25/09, BeckRS 2009 42206, S. 5; VG Gießen, Urteil v. 30.6.2009. Az.: 4 K 1052/09.GI, S. 15 (nicht veröffentlicht); OVG Lüneburg, Beschluss v. 10.7.2008, Az.: 2 ME 309/08, www.rechtsprechung.niedersachsen.de, Rn. 19

VGH Kassel, Beschluss v. 8.12.2011, Az.: 7 A 2621/10.2, juris, Rn. 20; die Darlegungs- und Beweislast liegt damit beim Schüler. Zu Beweisschwierigkeiten und den notwendigen Erleichterungen für Menschen mit Behinderungen Aichele/ Althoff, Kommentar zur UN-BRK, Nicht-Diskriminierung, Rn. 27 
notung der mündlichen Abfrage von Vokabeln in einer Fremdsprache ist dagegen kein Nachteil, denn die Legasthenie wirkt sich nicht darauf aus, Vokabeln zu lernen und mündlich wiederzugeben.

Benachteiligend können auch die im Schulrecht vielfältig vorhandenen Vorschriften zur Berechnung der jeweiligen Noten in den Zwischenzeugnissen und in den Jahreszeugnissen sein. Diese Zwischen- und Endnoten ergeben sich aus einer Zusammenschau aller Noten eines Schulhalbjahres oder Schuljahres oder aus Ergebnissen von Schulabschlüssen. Wie das Verhältnis von schriftlichen zu mündlichen Noten ist, was mündliche und was schriftliche Noten sind, variiert von Bundesland zu Bundesland und in einigen Bundesländern auch je nach Schule einer Schulform. Auch diese Noten sind benachteiligend, wenn bei ihrer Vergabe die Behinderung nicht berücksichtigt wurde.

\subsubsection{Benachteiligung durch Leistungsstandards}

Die Benachteiligung kann weiter darin liegen, dass Schüler und Schülerinnen mit Legasthenie die gleichen Standards erfüllen müssen wie ihre nicht behinderten Mitschüler. Gelingt ihnen dies nicht, so führt dies zu schlechteren Noten und hat eventuell Konsequenzen für die weitere Schullaufbahn und den Schulabschluss.

Im Schulrecht werden Standards sowohl bezüglich der inhaltlichen Anforderungen für bestimmte Fächer als auch speziell für die Rechtschreibung gesetzt. ${ }^{687}$ Der Standard ist dort selbstverständlich eine fehlerfreie Rechtschreibung. Die Vorschriften zur Bewertung der Rechtschreibung ordnen an, dass bei gehäuften Fehlern in der Rechtschreibung die Note um 1 oder 2 Punkte oder um bestimmte Notenstufen zu reduzieren ist. Diese Vorschriften knüpfen an ein neutrales Merkmal an, nämlich die Rechtschreibleistung, an das dann die Abwertung der Note gekoppelt wird. Die Abwertung erfolgt ohne Rücksicht darauf, ob der Schüler oder die Schülerin Legasthenie hat oder nicht. Da Schüler und Schülerinnen mit Legasthenie nicht in der Lage sind, ihre Rechtschreibleistung zu beeinflussen und damit den Standard der fehlerfreien Rechtschreibung zu erreichen, wird damit eine behinderungsbedingte Fähigkeit bewertet. Der Standard ist daher für sie benachteiligend.

Das Gleiche gilt für die sog. "Sperrklauseln“, die einen bestimmten Standard in den Fremdsprachen sichern sollen. In den Fremdsprachen setzt sich die Bewertung zusammen aus der Bewertung der inhaltlichen und der sprachlichen Leistung, die u.a. die Rechtschreibung umfasst. Die Endnote ergibt sich aus der Gesamtbewertung beider Bereiche. Die "Sperrklausel“ soll sicherstellen, dass gute Noten in den schriftlichen Arbeiten der Sprachfächer nur erzielt werden können, wenn beide Bereiche einem bestimmten Mindeststandard entspre-

687 Detaillierte Darstellung oben 1. Teil: 2. Kapitel: 2.: Bewertungs- und Benotungsvorschriften, S. $30 \mathrm{ff}$ 
chen. Es soll verhindert werden, dass gute Leistungen nur über die inhaltliche Leistung erzielt werden. Deshalb wird die erreichbare Endnote davon abhängig gemacht, dass die sprachliche Leistung ein Mindestniveau erreicht. ${ }^{688}$ Weil Schüler und Schülerinnen mit Legasthenie dieses Mindestniveau wegen ihrer Rechtschreibfehler überwiegend nicht erreichen, führt die mangelhafte Leistung im sprachlichen Teil dazu, dass ihre Arbeiten nur eine bestimmte Mindestnote (meistens "mangelhaft") erreichen können, selbst wenn der inhaltliche Teil sehr gut ist. ${ }^{689}$ Auch mit dieser Bewertung werden behinderungsbedingt eingeschränkte Fähigkeiten bewertet. Diese Sperrklauseln sind daher benachteiligend.

Auch die Benotungsvorschriften im Rechnen sowie die Vorschriften, die die Zulassung zu bestimmten Schulzweigen oder zum Abitur von bestimmten Noten in Mathematik abhängig machen, führen bei Schülern und Schülerinnen mit Dyskalkulie zu ungerechtfertigt schlechteren Noten mit der Konsequenz des Ausschlusses von bestimmten Schulzweigen und -prüfungen. Sie beschneiden damit die Möglichkeiten dieser Schüler und sind damit ebenfalls benachteiligend.

\subsection{Mittelbare Folgen als grundrechtsrelevanter Nachteil}

Ein grundrechtsrelevanter Nachteil kommt weiter in Betracht, wenn die Gleichbehandlung bei inhaltlichen Prüfungsanforderungen dazu führt, dass Schüler und Schülerinnen mit Legasthenie Ausweichstrategien entwickeln, um möglichst wenig Rechtschreibfehler zu machen und so eine Abwertung wegen der Rechtschreibung zu verhindern. Sie schreiben aus Angst vor Rechtschreibfehlern nur kurze Texte und vermeiden anspruchsvolle Wörter, bei deren Schreibung sie unsicher sind. Dies führt zu schlechteren Noten vor allem in Aufsätzen, nicht wegen der schlechten Rechtschreibung, sondern weil die Aufsätze nicht die Anforderungen bzgl. Länge und Wortschatz erfüllen. ${ }^{690}$ Auch dies ist ein Nachteil.

688 Z.B. Nr. 3.5.2 EPA Englisch; Nr. 3.5.2 EPA; Französisch; Nr. 3.5.2 Italienisch; Nr. 3.5.2 Spanisch; auch enthalten in KMK Bildungsstandard für die Allgemeine Hochschulreife in Fortgeführten Fremdsprache Englisch/Französisch v. 18.10.2012 Punkt 3.2.1.3 Az.: 9 A 208/08 BeckRS 2009 38123, S. 9 


\subsection{Ausschluss von Entfaltungsmöglichkeiten}

Eine Benachteiligung kann weiter darin liegen, dass Schüler und Schülerinnen mit Legasthenie/Dyskalkulie durch die unterschiedslose Gleichbehandlung von Entfaltungsmöglichkeiten ausgeschlossen und dadurch benachteiligt werden. ${ }^{691}$

Das BVerfG hat den Begriff der Benachteiligung in der Sonderschulentscheidung sehr weit gezogen, indem es festgestellt hat: „Vielmehr kann eine Benachteiligung auch bei einem Ausschluss von Entfaltungs- und Betätigungsmöglichkeiten durch die öffentliche Gewalt gegeben sein, wenn dieser nicht durch eine auf die Behinderung bezogene Fördermaßnahme hinlänglich kompensiert wird. ${ }^{4692}$ Dem liegt ein erweitertes Verständnis des Begriffs "Benachteiligung“ zugrunde, das eine unterschiedslose Behandlung von Behinderten ohne Berücksichtigung ihrer spezifischen Bedürfnisse verbietet, sofern sich hieraus Ausschlusswirkungen ergeben. ${ }^{693}$ Derartige Ausschlussmöglichkeiten werden in der Literatur vor allem für den Bereich der Überweisung von Schülern und Schülerinnen auf Förder- bzw. Sonderschulen bejaht. ${ }^{694}$

Auch für Schüler und Schülerinnen mit Legasthenie/Dyskalkulie ist diese Form der Benachteiligung zu bejahen, denn sie schließt die betroffenen Schüler und Schülerinnen von Entfaltungs- und Betätigungsmöglichkeiten aus. Schüler und Schülerinnen mit Legasthenie/Dyskalkulie werden dadurch von bestimmten Schulzweigen und von bestimmten Abschlüssen ausgeschlossen, dass für sie etwa der Übergang auf das Gymnasium erheblich erschwert und auch der Übergang auf die Realschule mindestens eingeschränkt ist. Der Wechsel von der Haupt- oder Realschule auf das Gymnasium ist fast nicht möglich. Die Betroffenen werden damit auch überproportional häufig von der Möglichkeit ausgeschlossen, Abitur und damit akademische Berufsausbildungen zu machen. Bei Schülern und Schülerinnen mit Dyskalkulie ist überproportional häufig der Besuch einer anderen Schule als der Hauptschule ausgeschlossen. Darüber hinaus belegen die Langzeitstudien, dass das Ausbildungsniveau der Betroffenen deutlich niedriger ist, als dies angesichts ihrer intellektuellen Fähigkeiten möglich wäre. ${ }^{695}$

691 Zur Ausschlusswirkung von normierten Leistungsstandards bei Kindern mit Beeinträchtigung: Wansing, Kommentar zur UN-BRK, Inklusionsbegriff, Rn. 11

692

Höller, Beschulung behinderter Kinder und Jugendlicher an öffentlichen Schulen, S. 144 ff; Reichenbach, Anspruch behinderter Schülerinnen und Schüler auf Unterricht in der Regelschule, S. 178; Reimann, Sicherstellung des Schulbesuchs behinderter Kinder, S. 34 f; Jürgens, DVBI 1997, S. 410 ff, S. 411; Rux, RdJB 2009, S. 220 ff, S. 223; Riedel, Gutachten UNBehindertenrechtskonvention, S. 6

695

S. oben 1. Teil: 1. Kapitel: 8.: Welche Folgen haben Legasthenie und/oder Dyskalkulie in

Schule und Beruf?, S. 24 
Das BVerfG hat den weiten Begriff der Benachteiligung jedoch dadurch wieder eingeschränkt, dass es eine Kompensation des Ausschlusses von Entfaltungsoder Betätigungsmöglichkeiten durch Fördermaßnahmen, die auf die Behinderung bezogen sind, für möglich hält. ${ }^{696}$

Es ist indes streitig, ob es überhaupt möglich ist, eine Benachteiligung durch Kompensation aufzuheben. ${ }^{697}$ Eingewandt wird, dass die Prüfung einer Kompensation auf der Tatbestandsebene ein Einfallstor für die Ersetzung des Selbstbestimmungsrechts durch das "wohlverstandene Interesse" der Betroffenen, das der Staat bestimmt, geschaffen wird. ${ }^{698}$ Dass dieser Einwand zutrifft, zeigt sich an den Entscheidungen des VG Schleswig und des VGH Kassel. Das VG Schleswig stellt ausdrücklich fest, dass die Bewertung der Rechtschreibung zu Nachteilen führt, da dadurch die beruflichen Möglichkeiten eingeschränkt werden könnten. Es gesteht den Ländern aber einen weiten Einschätzungsspielraum bei der Beurteilung der Frage zu, ob oder wie eine hinreichende Kompensation erfolgt ${ }^{699}$ Der VGH Kassel sieht in der Überweisung auf eine Sonderschule nicht nur eine Kompensation, sondern sogar eine "Maßnahme zur Beschleunigung oder Herbeiführung der tatsächlichen Gleichberechtigung von Menschen mit Behinderung" gem. Art. 5 IV BRK. ${ }^{700}$ Art. 5 IV BRK soll es den Staaten ermöglichen, positive, bevorzugende Maßnahmen zu ergreifen, um die Gleichberechtigung von Menschen mit Behinderungen zu fördern, etwa durch Quoten bei der Einstellung oder auch spezielle finanzielle Zuwendungen. ${ }^{701}$ Die Überweisung auf eine Förderschule ist jedoch keine solche positive und bevorzugende Maßnahme, sondern, wie bereits oben dargestellt, diskriminierend. ${ }^{702}$

Die Prüfung einer Kompensation beim Merkmal der „Benachteiligung" engt den Tatbestand des Diskriminierungsverbots erheblich ein, denn es reicht für die Verneinung der Benachteiligung dann aus, dass die Maßnahme durch den

696 BVerfGE 96, 288, Rn 52, kritisch dazu Riedel, Gutachten UN-Behindertenrechtskonvention, S. 37, der die Rechtsprechung des BVerfG für nicht mehr vereinbar mit der BRK hält.

697

Bejahend: Luthe, in: Rehabilitationsrecht - Verfassungsrecht, Rn. 6, Beaucamp, JA 2001, S. 36 ff, S. 38; Castendiek, in: GS Jean d'Heur, S. 349; ablehnend VGH München BayVBI 1998, S. 180, 183; BayVBI 1997, S. 561, 563; Höller, Beschulung behinderter Kinder und Jugendlicher an öffentlichen Schulen, S. 194 f, Sachs, RdJB 1996, S. 154 ff, S. 168; Castendiek, in: GS Jean d'Heur, S. 350; Osterloh, in: Sachs GG, Art. 3, Rn. 84 und 312

Castendiek, in: GS Jean d'Heur, S. 350

699 VG Schleswig, Urteil v. 10.6.2009, Az.: 9 A 208/08, BeckRS 2009 38123, S. 9

700 VGH Kassel, Beschluss v. 12.11.2009, Az.: 7 B 2763/09, juris, Rn. 45; kritisch dazu Aichele, Stellungnahme DIMR 2010, S. $10 \mathrm{f}$

701 United Nations, From Exclusion to Equality, S. 66, s. dazu auch Degener, ZaöRV 2005, S. 887 ff, S. 915; Degener, in: Antidiskriminierungsrecht, S. 78

702 Vgl. dazu Wocken, Andere Länder, andere Schüler? und Schumann, „Ich schäme mich ja so!“; vgl. auch Riedel, Gutachten UN-Behindertenrechtskonvention, S. 23; s. dazu oben S. 40 f; a. A. Ennuschat, in: FS Klaus Stern, S. 720 
Staat selber als kompensierend oder sogar als positiv eingeordnet wird. Eine Rechtfertigungsprüfung wäre anschließend nicht mehr erforderlich. Darüber hinaus setzt die Entscheidung der Verwaltung oder auch die gerichtliche Entscheidung darüber, ob eine Benachteiligung durch bestimmte Maßnahmen kompensiert wird oder nicht, die Sichtweise der entscheidenden Behörden oder Gerichte an die Stelle der subjektiven Sichtweise und Erfahrung der Menschen mit Behinderung und beeinträchtigt sie dadurch zusätzlich in ihrem Selbstbestimmungsrecht. ${ }^{703}$

$\mathrm{Zu}$ folgen ist daher der Ansicht, die in der Kompensation eine Frage der Rechtfertigung des benachteiligenden Eingriffs sieht. ${ }^{704}$

Für Schüler und Schülerinnen mit Legasthenie/Dyskalkulie ist diese Frage jedoch eher theoretischer Natur, da sie zwar gelegentlich, nicht aber ständig und während der gesamten Schullaufbahn Förderung erhalten. Wenn eine Förderung erfolgt, dann ist diese darauf gerichtet, durch "Nachhilfe“ die Leistungen im Lesen, Schreiben und Rechnen zu verbessern. Die Förderung ändert jedoch nichts an der Bewertung der Leistung in den Prüfungen und den sich daraus ergebenden Folgen für die Schullaufbahn. Insofern findet jedenfalls in diesem Bereich keine Kompensation statt.

Eine Kompensation könnte eventuell dann vorliegen, wenn man unter „Fördermaßnahme" in diesem Sinne auch den Nachteilsausgleich in Prüfungen subsumieren würde. ${ }^{705}$ In diesem Fall würden die „Fördermaßnahmen“ in Form des Nachteilsausgleichs die Benachteiligung dann entfallen lassen, wenn sie die Beeinträchtigung vollständig ausgleichen. Die Kompensation müsste dann jedoch individuell geprüft werden und tatsächlich sämtliche Nachteile der Behinderung aufheben. Dieser Konstruktion kann jedoch aus folgenden Gründen nicht gefolgt werden: Mit der Gleichsetzung von Fördermaßnahmen und Nachteilsausgleich wird die Aussage des BVerfG, dass die Benachteiligung durch „Fördermaßnahmen, die auf die Behinderung bezogen sind" kompensiert wird, außerordentlich extensiv ausgelegt. Das BVerfG hat offensichtlich pädagogische Förderung vor Augen gehabt und nicht die Veränderung von Prüfungsbedingungen. Außerdem wird dadurch sprachlich vermischt, was rechtlich getrennt werden muss: Pädagogische Förderung ist rechtlich etwas anderes als die Veränderung von Prüfungsbedingungen durch die Festsetzung von Nachteilsausgleich.

Die Gleichbehandlung benachteiligt daher, da sie von Entfaltungsmöglichkeiten ausschließt.

\footnotetext{
703 S. dazu oben 3. Teil: 3. Kapitel: 2.: Objektive oder subjektive Bestimmung des Eingriffs, S. 108

704 Osterloh, in: Sachs GG, Art. 3, Rn. 313; Castendiek, in: GS Jean d'Heur, S. 349; Heun, in:

Dreier GG, Art. 3, Rn. 137

So wie dies HE VO-GestSchulVerh $\S 39$ II definiert.
} 


\section{Abgrenzung der Benachteiligung von der Bevorzugung nach}

Art. 3 III 2 GG

Aus der Qualifikation als Nachteil ergibt sich noch nicht, ob und inwieweit die Benachteiligung gerechtfertigt werden kann. ${ }^{706}$ Sie impliziert aber, dass ein nicht zu rechtfertigender Nachteil durch einen Nachteilsausgleich aufzuheben ist.

Literatur $^{707}$ und Rechtsprechung ${ }^{708}$ sehen jedoch die Grenze des Nachteilsausgleichs erreicht, wenn inhaltliche Prüfungsanforderungen verändert werden.

Besonders diskutiert wird diese Frage bezüglich der Nicht-Bewertung der Rechtschreibung, die vielfach als "Notenschutz" bezeichnet wird. Ich verwende diesen Begriff in der vorliegenden Untersuchung jedoch nicht, weil ich ihn schon in seiner Diktion für benachteiligend halte. Er suggeriert, dass dem betroffenen Schüler eine ihn schützende, gewissermaßen behütende Maßnahme zugestanden wird, während es nach meiner Ansicht um die rationale Frage geht, ob die Rechtschreibung des Schülers zu bewerten ist oder nicht.

Die Frage stellt sich aber auch, wenn es darum geht, dass Aufgaben vorgelesen oder Aufgaben mündlich beantwortet werden können, indem sie diktiert oder auf Band aufgenommen oder Laptops benutzt werden. ${ }^{709}$

Langenfeld hat als Erste in ihrem Gutachten die Ansicht vertreten, dass die Nicht-Bewertung der Rechtschreibung nicht mehr als Ausgleich einer Behinderung angesehen werden kann, sondern eine Bevorzugung darstellt, weil damit auf bestimmte Leistungsanforderungen verzichtet würde. Zu einer solchen Bevorzugung könne der Staat allerdings aus Art. 3 III 2 GG verpflichtet sein, da die Maßnahmen des Nachteilsausgleichs das Defizit in der Rechtschreibung nicht ausgleichen würden. ${ }^{710}$

Ennuschat ist dieser Ansicht grundsätzlich gefolgt. Er grenzt den Nachteilsausgleich von der Bevorzugung danach ab, ob Bedingungen niveaugleich oder niveauverschieden modifiziert werden. Niveaugleiche Modifikationen sind solche, bei denen das Anforderungsniveau gleich bleibt und die Art der Prüfungs-

\footnotetext{
706 S. dazu unten 3. Teil: 5. Kapitel: Rechtfertigung, S. 145

OVG Lüneburg, Beschluss v. 10.7.2008, Az.: 2 ME 309/08, www.rechtsprechung.niedersachsen.de; VG Köln, Beschluss v. 26.9.2008, Az.: 10 L 1240/08, DVBI 2009, S. 538, kritisch dazu Marwege, DVBI 2009, S. 538 ff; VGH Kassel, Beschluss v. 5.2.2010, Az.: 7 A 2406/09.Z, juris, Rn 41 f; ähnlich VG Sigmaringen, Beschluss v. 2.4.2008, Az.: 4 K 596/08, S. 3 (nicht veröffentlicht), das in Art. 3 III 2 GG sogar nur einen Programmsatz sieht; OVG Weimar, Beschluss v. 17.5.2010, Az.: 1 EO 854/10, juris, Rn. 38 
leistung verändert wird, etwa indem schriftliche Prüfungen durch mündliche oder umgekehrt ersetzt werden. Hier liegt ein Nachteilsausgleich vor, weil die inhaltlichen Prüfungsanforderungen dabei nicht verändert werden. ${ }^{711}$ Bei Veränderungen der inhaltlichen Prüfungsanforderungen, die eine andere Bewertung der Leistung nach sich ziehen, liegt jedoch eine niveauverschiedene Modifikation vor, die über den Nachteilsausgleich hinausgeht und in eine Bevorzugung umschlägt. ${ }^{712}$

Die Rechtsprechung hat bisher durchgängig einen Anspruch auf Nicht-Bewertung der Rechtschreibung abgelehnt ${ }^{713}$ und dies wesentlich damit begründet, dass die Nicht-Bewertung der Rechtschreibung eine Bevorzugung sei, auf die kein Leistungsanspruch unmittelbar aus Art. 3 III 2 GG bestehe. Ein solcher Anspruch müsse vielmehr vom Gesetzgeber umgesetzt werden. ${ }^{714}$

Nach diesen Ansichten ergibt sich mithin ein Anspruch auf Ungleichbehandlung direkt aus Art. 3 III 2 GG nur für solche Veränderungen, die durch einen Nachteilsausgleich auszugleichen sind. Ist der Nachteilsausgleich jedoch als Bevorzugung anzusehen, dann besteht kein direkter Anspruch aus Art. 3 III 2 GG.

Fraglich ist daher, wann eine Benachteiligung vorliegt, die durch einen Nachteilsausgleich beseitigt werden muss, und wann eine darüber hinausgehende Bevorzugung. ${ }^{715}$ Sinn und Zweck des Nachteilsausgleichs bei schulischen

711 Ennuschat, br 2008, S. 93 ff, S. 96; Ennuschat, Die Rechte stotternder Menschen in Schule, Ausbildung und Studium, S. 105 ff; a.A. VGH München Beschluss v. 4.1.2010, Az.: 7 CE 09.2900, www.la-by.bayern.de, Rn. 22

Ennuschat, br 2008, S. 93 ff, S. 97; Ennuschat, Die Rechte stotternder Menschen in Schule, Ausbildung und Studium, S. 106 f; Ennuschat/Volino, br 2009, S. 166 ff, S. 167 2970/09.Z juris; Beschluss v. 8.12.2011, Az.: 7 A 2621/10.Z, juris; OVG Lüneburg, Beschluss v. 10.7.2008, Az.: 2 ME 309/08, www.rechtsprechung.niedersachsen.de; Beschluss v. 25.11.2011, Az.: 2 ME 52/11 (nicht veröffentlicht); Beschluss v. 20.9.2012, Az.: 2 LA 234/11; VGH München, Beschluss v. 28.6.2012, Az.: 7 CE 12.1324, juris, (zu Prüfungen im Studienfach Bachelor BWL); VG Aachen, Urteil v. 13.11.2009, Az.: 9 K 25/09, www.justiz.nrw.de; VG Düsseldorf, Beschluss v. 16.11.2009, Az.: 18 L 1654/09, juris; Beschluss v. 20.5.2011, Az.: 18 L 707/11, juris; VG Giessen, Urteil v. 2.11.2010, Az.: 7 K 1841/10; VG Köln, Beschluss V. 26.9.008, Az.: 10 L 1240/08, www.justiz.nrw.de; VG Köln, Beschluss 26.9.2008 Az.: 10 L 1240/08, www.justiz.nrw.de; VG Schleswig, Urteil v. 10.6.2009, Az.: 9 A 208/08, BeckRS 2009 38123, S. 10; VG Sigmaringen, Beschluss v. 2.4.2008, Az.: 4 K 596/08 (nicht veröffentlicht); a.A. nur VG Hannover, Beschluss v. 13.12.2010, Az.: 6 B 5596/10, www.rechtsprechung.niedersachsen.de

OVG Lüneburg, Beschluss v. 10.7.2008, Az.: 2 ME 309/08, www.rechtsprechung.niedersachsen.de, Rn. 19; Beschluss v. 25.11.2011, Az.: 2 ME 52/11, S. 4 f (nicht veröffentlicht); Beschluss v. 20.9.2012, Az.: 2 LA 234/11; VGH Kassel, Beschluss v. 5.2.2010, Az.: 7 A 2406/09.Z, juris, Rn 41 f; VG Gießen, Urteil v. 2.11.2010, Az.: 7 K 1841/10, S. 9 ff (nicht veröffentlicht); VG Köln, Beschluss v. 26.9.2008, Az.: 10 L 1240/08, www.justiz.nrw.de, kritisch dazu Marwege, DVBI 2009, S. 538 ff 
Prüfungen ist die Herstellung von Chancengleichheit. Der Begriff der Chancengleichheit bezieht sich auf Konkurrenzverhältnisse, in denen die "Chance" oder "gute Aussicht" bestehen soll, von den Freiheitsrechten effektiv Gebrauch zu machen ${ }^{716}$ und die gleiche "Erfolgsaussicht" zu haben. Doch welche Chancen sind damit genau gemeint? Ist damit, wie dies bei Art. 3 II GG diskutiert wurde, die Gleichheit vor dem Gesetz gemeint oder ist die Chance auf das Ergebnis zu beziehen und erst dann gegeben, wenn sie die reale Möglichkeit gibt, das gleiche Ergebnis zu erzielen ? ${ }^{717}$ Übereinstimmend wird heute bei Art. 3 II GG vertreten, dass die Chancengleichheit nicht auf formale Gleichheit reduziert ist, sondern dem Ausgleich faktischer Nachteile dienen soll. ${ }^{718}$ Schweizer definiert daher für Art. 3 || GG: „Chancengleichheit ernst genommen heißt also: Gleichheit der Chancen im Sinne einer realen Möglichkeit zur Ergebnisgleichheit. ${ }^{1719}$

Chancengleichheit im Schulbereich kann nichts anderes bedeuten. Der Erziehungsauftrag des Staates soll sicherstellen, dass jedermann gleichen Zugang zur Bildung und damit die Möglichkeit zur Entfaltung seiner Persönlichkeit, wie sie in Art. 2 I GG geschützt ist, sowie die gleiche Chance bei der Ausbildungsund Berufswahl erhält. ${ }^{720}$ Entscheidend ist daher, dass alle Schüler und Schülerinnen, behindert oder nicht-behindert, die Chance oder reale Möglichkeit haben, das gleiche Ergebnis zu erreichen, nämlich einen begabungsgerechten Abschluss. Es muss daher gewährleistet werden, dass alle Schüler die gleichen Startbedingungen im Hinblick auf dieses Ziel erhalten. ${ }^{721}$ Gleiche Startbedingungen herrschen nicht schon, wenn alle den gleichen Ausgangspunkt haben (das wäre nur formale Gleichheit), sondern sie müssen vielmehr in Beziehung zum Ziel gesetzt werden. Die Startbedingungen sind erst dann gleich, wenn es für alle Teilnehmer des Wettbewerbs ab dem Start nur noch auf die gleichen Bedingungen ankommt ${ }^{722}$, wenn alle Personen also die gleiche Chance zur Erfüllung der Kriterien haben, von denen die Vergabe der Leistung oder die Erreichung des Ziels abhängt. ${ }^{723}$

Nachteilsausgleich dient dazu, diese gleiche Startbedingungen und damit die Vergleichbarkeit zwischen behinderten und nicht-behinderten Menschen herzustellen, die nicht mehr durch behinderungsbedingte Einschränkungen

\footnotetext{
716 Weber-Dürler, in: FS Ulrich Häfelin, S. 221

717 Umfassend Schweizer, Gleichberechtigungssatz, S. 141 ff, S. 147

718 Osterloh, in: Sachs GG, Art. 3, Rn. 283; Kischel, in: BeckOK GG, Art. 3, Rn. 180 ff; Rüfner, in: Bonner Kommentar GG, Art. 3, Rn. $710 \mathrm{ff}$

719 Schweizer, Gleichberechtigungssatz, S. 148

720 Langenfeld, Integration und kulturelle Identität zugewanderter Minderheiten, S. 217

721 Vgl. Starck, in: v. Mangoldt/Klein/Starck GG, Art. 3, Rn. 33

722 Vgl. Hufen, Gleichheitssatz und Bildungsplanung, S. 49, S. 125

723 Leder, Diskriminierungsverbot wegen einer Behinderung, S. 84
} 
verzerrt ist. ${ }^{724}$ Wie bei einer Waage werden auf Seiten des Menschen mit Behinderung seine Nachteile durch Ausgleichsmaßnahmen so lange ausgeglichen bis seine Waagschale mit der des Menschen ohne Behinderung austariert ist.

Demgegenüber dienen Bevorzugungen dazu, über diese Vergleichbarkeit hinaus, dem Menschen mit Behinderung einen zusätzlichen Vorteil zu verschaffen, um die behinderungsbedingten Nachteile auszugleichen. In die Waagschale des Menschen mit Behinderung wird ein weiteres Gewicht gegeben, das die Waage im Vergleich mit einem Menschen ohne Behinderung zu seinen Gunsten ausschlagen lässt. Eine solche Bevorzugung sind z.B. Einstellungsquoten, weil sie bei gleicher Eignung zu einer bevorzugten Einstellung schwerbehinderter Bewerber führen (sollen). Die Bevorzugung wirkt durch diese fördernde Ungleichbehandlung aktiv der Diskriminierung entgegen. ${ }^{725}$

Eine Bevorzugung liegt aber nicht vor, wenn Barrieren abgebaut werden, die für Menschen ohne Behinderung nicht existieren. ${ }^{726}$

Prüfungsanforderungen und Prüfungsziele sind typische Barrieren im Schulalltag auf dem Weg zur Versetzung, zum Schulwechsel und zum Schulabschluss, die für alle Schüler und Schülerinnen existieren. Sie werden auf einen "Normalschüler", der nicht behindert ist, bezogen. An seinen "normalen" Fähigkeiten des Sprechens, Sehens, Hörens, Gehens, Lesens, Schreibens und Rechnens usw. sind die Prüfungsaufgaben ausgerichtet. Grundlage jeder Prüfung ist, dass der Prüfling das Prüfungsergebnis durch Wissen, Begabung, Talent und Fleiß beeinflussen kann und die Möglichkeit hat, sich mit entsprechender Anstrengung eine gute Note zu erarbeiten. Eine Prüfung, die eine unmögliche Leistung zum Inhalt hätte, wäre unverhältnismäßig. In welcher Form sich die Barriere einer bestimmten Prüfung auf den Schüler auswirkt, lässt sich daher durch den Schüler beeinflussen. Die Belange von Schülern und Schülerinnen mit Behinderung werden bei der Festlegung des Prüfungsziels i.d.R. nicht berücksichtigt. Das ist durchaus nachvollziehbar, denn es ist kaum möglich, angesichts der Vielfalt und Komplexität von Behinderungen alle Prüfungsbedingungen und Prüfungsziele von vornherein auch auf ihre Belange auszurichten. Je nach Behinderung und Prüfungsfach sind die Anforderungen und/ oder das Ziel dann jedoch so gestaltet, dass es Schüler mit Behinderung nicht erreichen können. Die Prüfungsanforderung wird zu einer Barriere, die der Schüler mit Behinderung gerade wegen seiner Behinderung nicht beeinflussen kann. Ist z.B. Prüfungsinhalt die Nacherzählung so hat ein hörgeschädigter Schüler ohne Hilfestellung nicht die Möglichkeit, diese Prüfung zu absolvieren, weil er den vorge-

\footnotetext{
724 Kischel, in: BeckOK GG, Art. 3, Rn. 219.3, sieht auch in den Maßnahmen des Nachteilsausgleichs Hilfsmaßnahmen und Bevorzugungen

725 König/Peters, in: Grote/Marauhn EMRK/GG, Kapitel 21: Das Diskriminierungsverbot, Rn. 75

726 Vgl. Leder, Diskriminierungsverbot wegen einer Behinderung, S. 266
} 
lesenen Text nicht hört. ${ }^{727}$ Ist Prüfungsinhalt eine mündliche Prüfung in einer Fremdsprache, so können Schüler und Schülerinnen, die stottern, dieses Ziel von vornherein nicht in gleicher Weise erreichen wie ihre Mitschüler, weil sie beim Sprechen beeinträchtigt sind. Beim Diktat ist es das Prüfungsziel, die korrekte Rechtschreibung zu überprüfen. Schüler und Schülerinnen mit Legasthenie können dieses Ziel nicht erreichen, weil sie gerade nicht nachweisen können, dass sie die Rechtschreibung beherrschen. Wegen dieser Barriere sind die Chancen der behinderten Prüflinge das Prüfungsergebnis zu erreichen eingeschränkt und von vornherein ungleich.

Die inhaltlichen Prüfungsanforderungen garantieren daher nicht per se gleiche Chancen für alle, sondern haben selber Auswirkungen auf die Chancengleichheit oder können, wie aufgezeigt, ungleiche Chancen bewirken. Sie stellen Barrieren auf für Menschen mit Behinderungen, die für Menschen ohne Behinderung entweder nicht existieren oder von ihnen beeinflusst werden können. Damit Schüler und Schülerinnen mit Behinderungen die Chance erhalten, das gleiche Ergebnis zu erreichen, muss für sie diese behinderungsspezifische Barriere abgebaut werden. ${ }^{728}$ Die Prüfung muss also für den Prüfling „barrierefrei" gestaltet werden, indem eine faktische Barriere abgebaut wird. Der Abbau einer solchen Barriere ist Nachteilsausgleich.

Ist die Erreichung des Prüfungsziels dem Schüler oder der Schülerin wegen der Behinderung nicht möglich, so kann dies bedeuten, dass die Leistungen in diesem spezifischen Bereich nicht bewertet werden, sei es im Sport bei einem körperbehinderten Schüler, in der Musik bei einem tauben Schüler, im Zeichnen bei einem blinden Schüler, in der Rechtschreibung bei einem legasthenen Schüler oder im Rechnen bei einem dyskalkulen Schüler, wenn nur so die Barriere beseitigt werden kann.

Der Abbau dieser Barrieren, durch Nicht-Bewertung der Rechtschreibung und des Rechnens führt nicht zu einer Besserstellung des behinderten Schülers. Seine Leistungen werden nicht "großzügiger" bewertet, sondern es werden die Leistungen unter Berücksichtigung der Behinderung bewertet. Die Noten werden nicht aufgebessert, Leistungsmängel werden nicht außer Acht gelassen. Bei Schülern und Schülerinnen mit Legasthenie wird durch die Nicht-Bewertung tatsächlich nur fingiert, dass die Rechtschreibung unauffällig ist und innerhalb der Fehlertoleranzgrenze liegt. Er wird mit einem Schüler gleichgestellt, der in diesem Bereich keine Beeinträchtigung aufweist. Mit anderen Worten: Im Bereich des Rechtschreibens werden die Arbeiten des Schülers bei inhaltlicher

727 Lambert, SchulVerwaltung BW 2002, S. 148 ff, S. 150, hält es für eine unzulässige Privilegierung, wenn dem Schüler der Text zum Mitlesen gegeben wird. Tatsächlich wird der Schüler ohne eine solche Hilfestellung jedoch vor eine unerfüllbare Aufgabe gestellt.

728 S. dazu die Grundsätze in Art. 3 BRK, zu denen nach Buchst. $f$ auch die Zugänglichkeit gehört - in der Schattenübersetzung mit Barrierefreiheit übersetzt. 
Richtigkeit nicht mehr wegen der Rechtschreibung abgewertet. Bei Schülern und Schülerinnen mit Dyskalkulie wird durch eine Nicht-Bewertung oder auch veränderte Bewertung vor allem verhindert, dass die Bewertung in diesem Fach nicht allein über die weitere schulische Laufbahn entscheidet.

Um das Bild wieder aufzunehmen: In die Waagschale des Schülers mit Legasthenie/Dyskalkulie wird kein zusätzliches Gewicht gelegt, das die Waage zu seinen Gunsten ausschlagen lässt, sondern es wird das Gewicht der schlechten Rechtschreibung/des schlechten Rechnens, das die Waage immer zu seinen Ungunsten ausschlagen lässt, entfernt. Eine Bevorzugung liegt darin nicht, weil Schüler und Schülerinnen ohne Behinderung diese Nachteile nicht haben.

Nur die Veränderung sowohl der äußeren Prüfungsbedingungen als auch der inhaltlichen Prüfungsanforderungen, unabhängig davon, ob sie niveaugleich oder niveauverschieden erfolgt, führt dazu, dass das Ergebnis der begabungsgerechten Schulausbildung und des begabungsgerechten Schulabschlusses barrierefrei erreicht werden kann und der Schüler seine wahren Fähigkeiten zeigen kann. Chancengleichheit für Schüler und Schülerinnen mit Behinderung besteht nur dann, wenn sie die Chance (= Aussicht) haben, den Bildungsgang, für den sie individuell geeignet und begabt sind, erfolgreich zu absolvieren, trotz oder mit Behinderung. ${ }^{729}$

Im Unterschied dazu liegt eine Bevorzugung i.S.d. Art. 3 III 2 GG nur dann vor, wenn über die Herstellung der Barrierefreiheit hinaus Vorteile gewährt werden. In einigen europäischen Ländern wird eine solche Bevorzugung praktiziert, indem Schüler und Schülerinnen mit Legasthenie vom Erlernen einer zweiten Fremdsprache befreit werden. ${ }^{730}$ Ebenso wäre es Bevorzugung, wenn Schüler und Schülerinnen mit Dyskalkulie vom Fach Physik oder anderen Fächern mit viel Mathematik befreit würden. Mit einer solchen Befreiung würden diese Schüler und Schülerinnen tatsächlich bevorzugt, weil ihnen ein Fach, dass zur Schulausbildung gehört und dass sie mit Einschränkungen auch leisten könnten, erlassen wird. Eine solche Privilegierung, insoweit ist der Rechtsprechung und der Literatur ${ }^{731}$ zuzustimmen, steht im Ermessen des Gesetzgebers und erfordert sein Tätigwerden. Bei dieser Ermessensentscheidung ist nach Art. 7 BRK, Art. 3 Kinderrechtskonvention (KRK) und nach Art. 24 II Charta der Grundrechte der Europäischen Union (Grundrechtecharta) das Kindeswohl vorrangig zu berücksichtigen. ${ }^{732}$

729 Reichenbach, Anspruch behinderter Schülerinnen und Schüler auf Unterricht in der Regelschule, S. 212

730 Möglich z.B. in Frankreich, Irland, Norwegen, Polen, Schweiz, Großbritannien, USA Bogdanowicz/Sayles, Rights of Dyslexic Children in Europe, S. 39

731 S. die Nachweise oben Fn. 707 und 708

732 Näher zum Vorrang des Kindeswohl unten S. $238 \mathrm{ff}$ 


\section{Ergebnis}

Sowohl Gleichbehandlung bezüglich der äußeren Prüfungsbedingungen als auch die Gleichbehandlung bezüglich der inhaltlichen Prüfungsanforderungen sind bei Schülern und Schülerinnen mit Legasthenie/Dyskalkulie Benachteiligungen i.S.d. Art. 3 III 2 GG.

\section{Kapitel: Diskriminierungsformen}

Ob und in welchem Maße Benachteiligungen gerechtfertigt werden können, richtet sich nach der Art und Weise, in der die Benachteiligung erfolgt, also der Diskriminierungsform. Bei unmittelbaren Diskriminierungen wird regelmäßig ein strengerer Maßstab angelegt als bei mittelbaren Diskriminierungen. Es ist daher zu untersuchen, welche Diskriminierungsformen hier vorliegen.

\section{Unmittelbare Diskriminierung}

Unmittelbare Diskriminierungen liegen vor, wenn die benachteiligende Regelung oder Maßnahme zielgerichtet an die Behinderung anknüpft ${ }^{733}$ und dadurch die Situation des Menschen mit Behinderung verschlechtert. ${ }^{734}$ Unterscheidungen, die gezielt an eine Behinderung anknüpfen, gibt es vielfach im Sozialrecht, wo sie dazu dienen, Leistungen zu gewähren, und durchweg für die Betroffenen vorteilhaft sind. Demgegenüber kommen unmittelbare Diskriminierungen, die Menschen mit Behinderung gezielt ausschließen oder benachteiligen, kaum noch vor ${ }^{735}$, weil sie gesellschaftlich tabu sind. Eine der wenigen nachteiligen Regelungen die ausdrücklich an eine Behinderung anknüpfen, ist die Zeugnisbemerkung bei Schülern und Schülerinnen mit Legasthenie/Dyskalkulie. Dort wird in den rechtlichen Regelungen explizit formuliert, dass eine Zeugnisbemerkung zu erfolgen hat, wenn eine Legasthenie vorliegt und die Rechtschreibung deshalb nicht gewertet wurde. ${ }^{736}$

Wie gezeigt, ergibt sich die Benachteiligung von Schülern und Schülerinnen mit Legasthenie/Dyskalkulie bei Prüfungen indem Prüfungsvorschriften auf sie unterschiedslos angewendet werden. Diese Regelungen sind allgemein und neutral. Sie knüpfen nicht gezielt und direkt an die Behinderung an, haben vielmehr Behinderungen gar nicht im Blick. Die benachteiligende Wirkung der Regelung ergibt sich erst als ungewollte Nebenfolge durch die unterschiedslose

\footnotetext{
733 Rothfritz, UN-BRK, S. 223; Osterloh, in: Sachs GG, Art. 3, Rn. 255

734 Kischel, in: BeckOK GG, Art. 3, Rn. 212

735 Boysen, in: v. Münch GG 2012, Art. 3, Rn. 196

736 S. dazu ausführlich unten 10. Teil: 2. Kapitel: Zeugnisbemerkung, S. $318 \mathrm{ff}$
} 
Anwendung auf Menschen mit Behinderung. Infolgedessen liegt keine unmittelbare Diskriminierung vor.

\section{Mittelbare Diskriminierung}

Die Gleichbehandlung der Schüler und Schülerinnen mit Legasthenie/Dyskalkulie bei Prüfungen kann folglich nur eine mittelbare Diskriminierung sein. Mittelbare Diskriminierungen liegen vor, wenn eine Regelung oder Maßnahme an andere, neutrale Merkmale anknüpft, die aber überwiegend oder typischerweise bei den Trägern des in Art. 3 III GG geschützten Merkmals vorliegen. ${ }^{737}$ Die Figur der mittelbaren Diskriminierung dient dazu, dem besonderen Diskriminierungsverbot dadurch zur vollen Wirksamkeit zu verhelfen, dass auch solche Ungleichbehandlungen erfasst werden, die die Angehörigen der betroffenen Gruppe tatsächlich in gleicher Weise wie eine unmittelbare Diskriminierung benachteiligen, obwohl sie neutral formuliert sind. ${ }^{738}$

Ob Art. 3 III 2 GG überhaupt mittelbare Diskriminierungen erfasst, ist indes streitig. ${ }^{739}$ Überwiegend wird die Ansicht vertreten, dass Art. 3 III 2 GG auch die mittelbare Diskriminierung erfasst. ${ }^{740}$ Die Rechtsprechung des BVerfG in der Sonderschulentscheidung ${ }^{741}$, wonach Art. 3 III 2 GG auch „berührt“ wird, wenn die Sonderbeschulung durch die Folgen der körperlichen Beeinträchtigung ausgelöst wird, wird bisweilen dahin ausgelegt, dass das BVerfG damit auch die mittelbare Diskriminierung in den Anwendungsbereich des Art. 3 III 2 GG hineinnimmt. ${ }^{742}$ Es gibt aber auch Stimmen, die die Entscheidung dahingehend auslegen, dass das eher nicht der Fall ist. ${ }^{743}$

Nach herrschender Meinung schützen die Gleichheitssätze nicht nur vor direkter (gezielter) Benachteiligung, sondern auch vor indirekten, mittelbaren

737 Osterloh, in: Sachs GG, Art. 3, Rn. 255; Neumann, in: Handbuch SGB IX, § 2 Verfassungsrecht, Rn. 25; König/Peters, in: Grote/Marauhn EMRK/GG, Kapitel 21: Das Diskriminierungsverbot; Eckertz-Höfer, in: AK-GG, Art. 3, Rn. 138; Rothfritz, UN-BRK, S. 223

König/Peters, in: Grote/Marauhn EMRK/GG, Kapitel 21: Das Diskriminierungsverbot, Rn. 62 Kischel, in: BeckOK GG, Art. 3, Rn. 213; Jürgens, ZfSH/SGB 1995, S. 353 ff, S. 357

Heun, in: Dreier GG, Art. 3, Rn. 137; Eckertz-Höfer, in: AK-GG, Art. 3, Rn. 128; Jarass/Pieroth, in: Jarass/Pieroth GG, Art. 3, Rn. 145; Beaucamp, DVBI 2002, S. 997 ff, S. 999; Beyerlin, RdJB 1999, S. 157 ff, S. 162; Castendiek, in: GS Jean d'Heur, S. 341; Osterloh, in: Sachs GG, Art. 3, Rn. 312; Sacksofsky, in: Umbach GG, Art. 3, Rn. 408; Welti, Behinderung und Rehabilitation im sozialen Rechtsstaat, S. 473; offen gelassen Kischel, in: BeckOK GG, Art. 3, Rn. 213; nur für unmittelbare Diskriminierungen Buch, Grundrecht der Behinderten, S. 134 BVerfGE 96, S. 288, S. $312 \mathrm{f}$

742 Osterloh, in: Sachs GG, Art. 3, Rn. 311 
Diskriminierungen. ${ }^{744}$ Allerdings ist streitig, ob im Hinblick auf mittelbare Diskriminierungen Art. 3 । GG oder Art. 3 III GG einschlägig ist. ${ }^{745}$ Besonders streitig war die Einbeziehung der mittelbaren Diskriminierung bei Art. 3 III 1 GG in Bezug auf die Diskriminierung des Geschlechts. ${ }^{746}$ Inzwischen ist es jedoch allgemeine Ansicht, dass Art. 3 III 1 GG auch vor mittelbaren Diskriminierungen schützt. ${ }^{747}$ Das BVerfG hat ausdrücklich entschieden, dass eine an Art. 3 III 1 GG zu messende Ungleichbehandlung auch dann vorliegt, wenn die streitige Regelung nicht auf eine nach Art. 3 III GG verbotene Ungleichbehandlung angelegt ist, sondern in erster Linie - oder gänzlich - andere Ziele verfolgt. ${ }^{748}$ Es führt aus, dass die Regelung an Art. 3 III GG zu messen sei, wenn der vom Gesetzgeber gewählte, durch Art. 3 III 1 GG nicht verbotene sachliche Anknüpfungspunkt in der gesellschaftlichen Wirklichkeit weitgehend nur für eine Gruppe zutrifft, oder die differenzierende Regelung sich weitgehend nur auf eine Gruppe im Sinne einer faktischen Benachteiligung auswirkt, deren Ungleichbehandlung nach Art. 3 III GG strikt verboten sei (mittelbare Diskriminierung). Eine Anknüpfung an das Geschlecht könne deshalb auch dann vorliegen, wenn eine geschlechtsneutral formulierte Regelung überwiegend Frauen trifft und dies auf natürliche oder gesellschaftliche Unterschiede zwischen den Geschlechtern zurückzuführen ist. ${ }^{749}$

Würde der Anwendungsbereich des Art. 3 III 2 GG auf unmittelbare Diskriminierungen beschränkt, würde dies Art. 3 III 2 GG weitgehend leerlaufen lassen. Die Situation der behinderten Menschen wird nicht durch unmittelbare und gezielte Diskriminierungen geprägt, sondern vielmehr dadurch, dass auf ihre Belange aus Unwissenheit, Gedankenlosigkeit oder Nachlässigkeit keine Rücksicht genommen wird. Beschränkungen ergeben sich vor allem daraus, dass die Gesellschaft tatsächliche und rechtliche Gegebenheiten schafft, die die besondere Situation der Menschen mit Behinderung nicht im Blick haben. Die Problematik im Prüfungsbereich liegt dann darin, dass, ohne die Belange von Menschen mit Behinderungen zu bedenken, Anforderungen gestellt werden, die der "normale“ Mensch erfüllen kann, die aber der Mensch mit Behinderung

744 Statt vieler: Osterloh, in: Sachs GG, Art. 3, Rn. 255 f; Eckertz-Höfer, in: AK-GG, Art. 3, Rn. 137; Beaucamp, JA 2001, S. 36 ff, S. 37

König/Peters, in: Grote/Marauhn EMRK/GG, Kapitel 21: Das Diskriminierungsverbot Rn. 64 m.w.N.

Umfassende Darstellung bei Buch, Grundrecht der Behinderten, S. 121 ff; Osterloh, in: Sachs GG, Art. 3, Rn. 255

Statt vieler Kannengießer, in: Schmidt-Bleibtreu/Klein GG, Art. 3, Rn. 54 ff; Kischel, in: BeckOK GG, Art. 3, Rn. 165 http://www.bverfg.de/entscheidungen/Is20080618_2bvl000607.htm 
nicht erfüllen kann. ${ }^{750}$ Es würde den Anwendungsbereich des Art. 3 III 2 GG erheblich einschränken und "Gedankenlosigkeit prämieren “751, wenn es auf die „zufälligen" oder nicht bedachten Folgen einer Handlung nicht ankäme. Der Mensch mit Behinderung wird von der Teilnahme am Leben in der Gesellschaft durch rechtliche Regelungen ausgeschlossen, wenn diese für eine Leistung oder Zulassung Kriterien aufstellen, die die Belange von Menschen mit Behinderung außer Betracht lassen und/oder eine Berücksichtigung der Behinderung ausschließen. Ebenso wie bei der Anwendung des allgemeinen Gleichheitssatzes, der nicht bei der Herstellung formaler Gleichheit stehen bleibt, kommt es daher auch bei Art. 3 III 2 GG auf die materiellen Auswirkungen der Regelung an, so dass auch hier mittelbare Diskriminierungen zu berücksichtigen sind. ${ }^{752}$

Für die Einbeziehung der mittelbaren Diskriminierung in den Anwendungsbereich des Art. 3 III 2 GG spricht weiter die systematische Auslegung. Die einfachgesetzlichen Diskriminierungsverbote gehen generell dahin, auch mittelbare Diskriminierungen zu erfassen. So umfasst das AGG nach § 3 ॥ AGG ausdrücklich auch Benachteiligungen wegen Behinderung. § 81 II SGB IX übernimmt dies, indem es auf das AGG verweist. Ebenso verbietet $\S 7$ ॥ BGG mittelbare Beeinträchtigungen. Es würde dem Grundsatz, dass Grundrechte so auszulegen sind, dass sie ihre größtmögliche Wirkung entfalten können, ${ }^{753}$ widersprechen, wenn der Anwendungsbereich des Art. 3 III 2 GG enger wäre als der des entsprechenden einfachen Rechts.

Ferner spricht für die Einbeziehung der mittelbaren Diskriminierung das Diskriminierungsverbot des Art. 5 II BRK, das für das Verständnis des Art. 3 III 2 GG heranzuziehen ist. ${ }^{754}$ Art. 5 II BRK umfasst ausdrücklich alle Formen der Diskriminierung, auch die mittelbare Diskriminierung, das schlichte Nichtstun, wenn es eine Anpassungspflicht gibt ${ }^{755}$, und die Form der strukturellen Diskriminierung, bei der eine formale Gleichbehandlung de facto zum Ausschluss von Möglichkeiten für Menschen mit Behinderung führt. ${ }^{756}$

Die weitere Frage ist, ob eine mittelbare Diskriminierung auch in der formellen Gleichbehandlung liegen kann, die sich nachteilig für Menschen mit Behinderung auswirkt. Dies ist insofern fraglich, weil die Regelung oder Maßnahme an sich zulässigerweise neutral einen Sachverhalt regelt, der keinen Be-

$750 \mathrm{Vgl}$. Fuerst, Behinderung zwischen Diskriminierungsschutz und Rehabilitationsrecht, S. 144 zu Art. 2 (2) b RL 2000/78/EG

751 Welti, Behinderung und Rehabilitation im sozialen Rechtsstaat, S. 473

752 Osterloh, in: Sachs GG, Art. 3, Rn. 256

753 BVerfGE 6, 55, 72; 43, 154, 167; Jarass/Pieroth, in: Jarass/Pieroth GG, Einleitung, Rn. 12

754

755 Degener, RdJB 2009, S. 200 ff, S. 205; s. auch Mokatef, Menschenrecht auf Bildung und Schutz vor Diskriminierung, S. 12

756 Vgl. Mokatef, Menschenrecht auf Bildung und Schutz vor Diskriminierung, S. 12 
zug zu einer Behinderung aufweist und daher nicht an ein Merkmal anknüpft, das überwiegend oder typischerweise bei den Trägern des in Art. 3 III GG geschützten Merkmals vorliegt. ${ }^{757}$ Die Regelung wirkt sich nur deshalb mittelbar diskriminierend aus, weil mögliche Folgen für Menschen mit Behinderung nicht bedacht worden sind. Deshalb werden solche Regelungen, die rein sachliche Voraussetzungen aufstellen und einheitliche Rechtsfolgen für alle anordnen, teilweise nicht als diskriminierend im Sinne einer Schlechterstellung der Menschen mit Behinderung angesehen. ${ }^{758}$ Bejaht man jedoch in diesen Fällen eine mittelbare Diskriminierung, so kommt es allein auf die Folgen einer Regelung und nicht mehr darauf an, ob ein Sachverhalt geregelt wird, von dem überwiegend oder typischerweise Menschen mit Behinderung betroffen sind.

Die Auslegung, dass auch die formelle Gleichbehandlung eine mittelbare Diskriminierung sein kann, ergibt sich schon aus der Zielsetzung des Art. 3 III 2 GG, materielle Gleichheit zu schaffen. ${ }^{759}$ Diese geht über die formale Gleichbehandlung hinaus und erfordert, dass die Auswirkungen einer Regelung berücksichtigt werden ${ }^{760}$ und tatsächliche, materielle Gleichheit ${ }^{761}$ durch rechtliche Ungleichbehandlung hergestellt wird. ${ }^{762}$

Gestützt wird dieses Ergebnis durch die Entscheidung des CRPD zum Diskriminierungsverbot des Art. 5 II BRK, in der es ausdrücklich bejaht, dass die formelle Gleichbehandlung, die sich nachteilig für Menschen mit Behinderung auswirkt, diskriminierend und verboten ist. ${ }^{763}$ Diese Auslegung ist bei der Auslegung von Art. 3 III 2 GG zu berücksichtigen

Art. 3 III 2 GG erfasst daher auch mittelbare Diskriminierung durch formelle Gleichbehandlung, die sich nachteilig für Menschen mit Behinderung auswirkt. Stellen sich allgemeine und neutrale Regelungen als Barriere dar, die die Chan-

757 Osterloh, in: Sachs GG, Art. 3, Rn. 255; Neumann, in: Handbuch SGB IX, § 2 Verfassungsrecht, Rn. 25; König/Peters, in: Grote/Marauhn EMRK/GG, Kapitel 21: Das Diskriminierungsverbot; Eckertz-Höfer, in: AK-GG, Art. 3, Rn. 138; Rothfritz, UN-BRK, S. 223

Sachs, Besondere Gleichheitsgarantien, in: HStR VIII, § 182, Rn. 25; Straßmair, Der besondere Gleichheitssatz aus Art. 3 Abs. 3 Satz 2 GG, S. 180

Reichenbach, Anspruch behinderter Schülerinnen und Schüler auf Unterricht in der Regelschule, S. 192

Osterloh, in: Sachs GG, Art. 3, Rn. 256

Osterloh, in: Sachs GG, Art. 3, Rn. 44

Reichenbach, Anspruch behinderter Schülerinnen und Schüler auf Unterricht in der Regelschule, S. 193 m.w.N. mit dem Beispiel (S. 192), dass eine allgemeine Regelung diskriminierend sein kann, wenn sie etwa besagt, dass jedermann mit einem Bus befördert wird, tatsächlich aber nur diejenigen befördert werden, die auch dazu in der Lage sind, den Bus zu besteigen. 
cen der Schüler und Schülerinnen mit Behinderung auf einen begabungsgerechten Schulabschluss verringern, so sind sie mittelbar diskriminierend. ${ }^{764}$

Tatsächlich führt diese Auslegung des Diskriminierungsbegriffs der BRK und damit auch des Art. 3 III 2 GG dazu, dass der Diskriminierungsbegriff erheblich ausgeweitet wird. Entscheidend ist danach nicht mehr die unmittelbare oder mittelbare Anknüpfung an ein verbotenes Merkmal, sondern die Auswirkung der Regelung auf den Merkmalsträger. Diese Veränderung ist vor allem darin begründet, dass im Europa- und Völkerrecht sowie im internationalen Menschenrechtsschutz ein vielschichtiger Diskriminierungsbegriff herrscht und die Rechtsprobleme eher über das Diskriminierungsverbot als über den allgemeinen Gleichheitssatz gelöst werden. ${ }^{765}$ Durch das Diskriminierungsverbot der BRK wirkt diese Veränderung auch in die Auslegung des Art. 3 III 2 GG hinein.

\section{Diskriminierung nur bei überwiegender Betroffenheit?}

Es bleibt die Frage zu klären, ob eine mittelbare Diskriminierung nur dann zu bejahen ist, wenn die Schüler und Schülerinnen mit Behinderung davon in einem besonderen Ausmaß betroffen sind. Ist es etwa erforderlich, dass durch statistische Angaben nachgewiesen wird, dass die Regelungen deutlich mehr Schüler und Schülerinnen mit Behinderung als Schüler und Schülerinnen ohne Behinderung betreffen? In diesem Punkt unterscheiden sich schon die Definitionen des Begriffs "mittelbare Diskriminierungen“. Sie stellen zum Teil darauf $a b$, ob der geschützte Personenkreis „überwiegend“766, oder ob er „typischerweise ${ }^{\text {"767 }}$ betroffen ist.

Kischel scheint davon auszugehen, dass Art. 3 III 2 GG, wenn überhaupt, nur die Variante der mittelbaren Diskriminierung erfasst, in der eine scheinbar neutrale Regelung deutlich mehr behinderte Schüler erfasst als nicht-behinderte Schüler. ${ }^{768}$ Es müsste also statistisch nachgewiesen werden, dass die Regelung deutlich mehr behinderte Schüler betrifft als nicht-behinderte Schüler.

Die gleiche Diskussion über das Ausmaß und den Nachweis der Betroffenheit der diskriminierten Gruppe bei mittelbaren Diskriminierungen hat es im Bereich des Allgemeinen Gleichbehandlungsgesetzes (AGG) gegeben. Die herrschende Meinung hat sich dort von der Ansicht, dass eine überwiegende Betroffenheit der diskriminierten Gruppe statistisch nachzuweisen ist, dahin gewandelt, dass der Nachweis einer statistischen Ungleichbehandlung nicht mehr

764 Ebenso für Schüler und Schülerinnen mit Migrationshintergrund: Althoff, Anwaltsblatt 2011, S. $482 \mathrm{ff}$, S. 483

765 Boysen, in: v. Münch GG 2012, Art. 3, Rn. 122

766 Eckertz-Höfer, in: AK-GG, Art. 3, Rn. 138; Rothfritz, UN-BRK, S. 223

767 Osterloh, in: Sachs GG, Art. 3, Rn. 255; Neumann, in: Handbuch SGB IX, § 2 Verfassungsrecht, Rn. 25

768 Kischel, in: BeckOK GG, Art. 3, Rn. 219.3 
für zwingend geboten gehalten wird. ${ }^{769}$ Denn vor allem der Nachweis nur über Statistiken würde zu einer erheblichen Schutzlücke führen, da vielfach entsprechende Statistiken nicht vorhanden sind. Insofern würde das Erfordernis gegen Sinn und Zweck der Vorschrift, nämlich vor mittelbareren Diskriminierungen und Umgehungen zu schützen, verstoßen. ${ }^{770}$ Daher wird der statistische Nachweis nur noch als eine von mehreren Varianten einer mittelbaren Diskriminierung angesehen, der in diesem Fall den Nachweis einer Diskriminierung erleichtert. $^{771}$

Verlangt man, dass für den Nachweis der mittelbaren Diskriminierung dargelegt wird, dass eine bestimmte schulische Vorschrift mehr Schüler und Schülerinnen mit Behinderung benachteiligt als Schüler und Schülerinnen ohne Behinderung so verlangt man etwas Unmögliches. Es gibt im Schulbereich kein statistisches Material dazu, wie viele Schüler und Schülerinnen behindert $\operatorname{sind}^{772}$ und daher erst recht kein statistisches Material dazu, wie viele behinderte Schüler und Schülerinnen von einer bestimmten Regelung betroffen sind.

Ein Vergleich von behinderten Schülern und Schülerinnen mit nicht-behinderten Schülern und Schülerinnen setzt weiter voraus, dass die Gruppen in sich homogen sind und deshalb miteinander verglichen werden können. Ein solcher Vergleich ist möglich, wenn es sich um Gruppen handelt, die die gleichen Merkmale i.S.d. Art. 3 III 1 GG aufweisen, also etwa beim Vergleich von Frauen und Männern oder von Muslimen und Katholiken. Die Besonderheit der Gruppe von Menschen mit Behinderung ist jedoch gerade, dass sie keine homogene Gruppe bilden. Die Gruppe ist vielmehr außerordentlich heterogen, weil es eine Vielzahl von Behinderungen mit jeweils spezifischen Ausprägungen und damit Betroffenheiten gibt. ${ }^{773}$ Dementsprechend sind die Auswirkungen von Regelungen je nach Behinderung sehr unterschiedlich. Was für einen Teil der Menschen mit Behinderung eine besondere Benachteiligung ist, etwa Treppen oder die fehlende Rampe vor einer Schule für Rollstuhlfahrer, ist für Stotterer und Legastheniker keine Einschränkung. Es wird angesichts dieser Vielfalt unmöglich sein eine Regelung zu finden, die deutlich mehr Schüler und Schülerinnen mit Behinderung betrifft als solche ohne Behinderung. Damit würden mittelbare Diskriminierungen im Bereich des Art. 3 III 2 GG praktisch immer am Nach-

\footnotetext{
769 Schrader/Schubert, in: Däubler/Bertzbach AGG, § 3, Rn. 47 m.w.N.; Wendeling-Schröder/ Stein, in: AGG § 3, Rn. $23 \mathrm{f}$

770 Schrader/Schubert, in: Däubler/Bertzbach AGG, §3, Rn. 47; Wendeling-Schröder/Stein, in: AGG, § 3, Rn. 24; Rust/Falke/Bertelsmann, in: Rust/Falke, AGG, § 3, Rn. 10, 34; Bauer/Göpfert/Krieger, in: AGG, § 3, Rn. 26a f; Schrader/Schubert in Däubler/Bertzbach, AGG, § 3, Rn. 49 f; vgl. BVerfGE 85, 191, 206, zu Art. 3, Osterloh, in: Sachs GG, Art. 3, Rn. 252 Roetteken, Nichtigkeit Versorgungsabschlag, Anm. D - Schlusssatz

772 Das vorhandene statistische Material bezieht sich immer auf Schüler mit sonderpädagogischem Förderbedarf.

773 Welti, Behinderung und Rehabilitation im sozialen Rechtsstaat, S. 475
} 
weis der Betroffenheit scheitern. Das Merkmal der „überwiegenden Betroffenheit" ist daher im Bereich des Art. 3 III 2 GG nicht für eine Bestimmung der mittelbaren Diskriminierung geeignet.

Im Bereich des AGG wird eine mittelbare Diskriminierung dann bejaht, wenn eine neutrale Vorschrift einen bestimmten Personenkreis besonders benachteiligt. ${ }^{774}$ Dazu wird teilweise vertreten, dass das neutrale Merkmal leichter von Menschen mit Behinderung erfüllt werden kann ${ }^{775}$, oder es wird der Nachweis verlangt, dass Menschen mit Behinderung deutlich stärker betroffen werden als andere. ${ }^{776}$ Dies aber sind Nuancen der besonderen Betroffenheit. Es reicht für das Grundrecht aus Art. 3 III 2 GG aus, wenn der Mensch mit Behinderung besonders betroffen ist. ${ }^{777}$

Für die Legasthenie/Dyskalkulie bedeutet dies: die allgemeinen schulrechtlichen Regelungen zur Notenbildung, zu den Anforderungen an den Wechsel auf eine weiterführende Schule und zur Bewertung der Rechtschreibung beziehen sich zwar auf alle Schüler und Schülerinnen, sie betreffen jedoch die Kinder mit Legasthenie/Dyskalkulie besonders und zwar deutlich stärker. Durch die Benotung gerade der Bereiche, in denen sie beeinträchtigt sind, nämlich im Lesen, Schreiben und Rechnen, wirken sich die Rechtsfolgen der Regelungen bei ihnen in besonderem Maße und typischerweise nachteilig aus. Dies ist durch die Langzeitstudien zur Entwicklung von Kindern mit Legasthenie umfassend belegt. ${ }^{778}$ Die formelle, unterschiedslose Gleichbehandlung ist daher mittelbar diskriminierend.

\section{Versagung angemessener Vorkehrungen}

Die Gleichbehandlung von Schüler und Schülerinnen mit Legasthenie/Dyskalkulie mit ihren nicht behinderten Mitschülern kann auch deshalb diskriminierend sein, weil ihnen damit inzident andere, angemessene Vorkehrungen versagt werden. Diskriminierung kann auch in Form der Versagung angemessener Vorkehrungen vorliegen. Diese Diskriminierungsform ist nach Art. 5 II i.V.m. 2 BRK verboten und daher im Rahmen der völkerrechtsfreundlichen Auslegung des Art. 3 III 2 GG zu beachten. ${ }^{779}$

\footnotetext{
774 S. oben Fn. 770

775 Schrader/Schubert, in: Däubler/Bertzbach AGG, § 3, Rn. 48

776 Wendeling-Schröder/Stein, in: AGG, § 3, Rn. 24

777 Ähnlich Castendiek, in: GS Jean d'Heur, S. 341; Osterloh, in: Sachs GG, Art. 3, Rn. 311; Welti, Behinderung und Rehabilitation im sozialen Rechtsstaat, S. 475 f

778 S. dazu oben 1. Teil: 1. Kapitel: 9.: Welche psychischen Auswirkungen haben Legasthenie und/oder Dyskalkulie?, S. 26

779 Aichele, Angemessene Vorkehrungen, S. 3; Aichele/ Althoff, Kommentar zur UN-BRK, NichtDiskriminierung, Rn. $41 \mathrm{ff}$
} 
Der Begriff der angemessenen Vorkehrungen gehört weltweit zu den Schlüsselbegriffen des modernen Behindertengleichstellungsrechts. ${ }^{780}$ Die Diskriminierung durch „Versagung angemessener Vorkehrungen" nach Art. 2 BRK, die ein Eckpfeiler der BRK und der staatlichen Gesetzgebung zur Umsetzung der BRK $\operatorname{sind}^{781}$, stellt eine Weiterentwicklung des deutschen (Antidiskriminierungs-) Rechts dar. ${ }^{782}$ Zwar sieht Art. 5 RL 2000/78/EG (Gleichbehandlungsrahmenrichtlinie) für den arbeitsrechtlichen Bereich vor: „Um die Anwendung des Gleichbehandlungsgrundsatzes auf Menschen mit Behinderung zu gewährleisten, sind angemessene Vorkehrungen zu treffen". Dieser Art. 5 wurde aber im AGG nicht ausdrücklich umgesetzt ${ }^{783}$, so dass es bisher im deutschen Recht keine vergleichbare Regelung gab. „Angemessene Vorkehrungen“ weichen die Grenzen zwischen der herkömmlichen Differenzierung der Grundrechte in Abwehr- und Leistungsrechte auf, weil sie ein positives Tun des Staates verlangen. Das moderne Antidiskriminierungsrecht zielt darauf ab, den behinderten Menschen aus seiner Sonderrolle zu holen und ihm Normalität zu verschaffen. Dazu genügt ein bloßes Recht auf Abwehr von diskriminierenden Maßnahmen nicht, sondern es muss Maßnahmen geben, die das Ziel aktiv verfolgen. Damit wird den Schwierigkeiten des reinen Abwehrrechts gegen eine Diskriminierung wegen des diskriminierenden Merkmals begegnet, denn mithilfe von Abwehrrechten gegen mittelbare oder strukturelle Diskriminierungen können nur Teile aus dem System abgewehrt ${ }^{784}$, nicht aber die erforderlichen aktiven Maßnahmen erreicht werden. Mit angemessenen Vorkehrungen sollen behinderungsbedingte Nachteile ausgeglichen werden, um Chancengleichheit im Wettbewerb mit nicht-behinderten Menschen herzustellen. ${ }^{785}$ Derjenige, der zur Berücksichtigung der Behinderung verpflichtet ist, darf diese nicht mehr ignorieren, sondern muss sie im Gegenteil gerade besonders berücksichtigen. ${ }^{786}$ Mithilfe angemessener Vorkehrungen wird der Nachteil der Behinderung ausgeglichen und der Mensch mit Behinderung in die Lage versetzt, die gestellten Anforde-

780 Degener, br 2009, S. 34 ff, S. 38; umfassend dazu Waddington in: Non-Discrimination Law, S. $629 \mathrm{ff}$

781 United Nations, From Exclusion to Equality, S. 60; Aichele, UN-BRK und Fakultativprotokoll, S. 6

782

Degener, RdJB 2009, S. 200 ff, S. 218 und Degener, ZaöRV 2005, S. 887 ff, S. 913; Fuerst, Behinderung zwischen Diskriminierungsschutz und Rehabilitationsrecht, S. 87

783 Fuerst, Behinderung zwischen Diskriminierungsschutz und Rehabilitationsrecht, S. $121 \mathrm{f}, \mathrm{vgl}$. Degener, RdJB 2009, S. 200 ff, S. 218, umfassend zur RL 2000/78/EG und zur damals geplanten Umsetzung in deutsches Recht Leder, Diskriminierungsverbot wegen einer Behinderung, speziell zu „angemessenen Vorkehrungen“ S. $234 \mathrm{ff}$ Fuerst, Behinderung zwischen Diskriminierungsschutz und Rehabilitationsrecht, S. 85, Fn. 279

785 Fuerst, Behinderung zwischen Diskriminierungsschutz und Rehabilitationsrecht, S. $121 \mathrm{f}$ 
rungen erfüllen zu können. ${ }^{787}$ Werden diese Vorkehrungen ungerechtfertigt verweigert, ist dies gleichbedeutend mit einer unmittelbaren Diskriminierung wegen der Behinderung. ${ }^{788}$ Angemessene Vorkehrung sind individuell ausgerichtet, da sie bei einem bestimmten Individuum ansetzen und nicht bei einer Gruppe von beliebigen Betroffenen. ${ }^{789}$ Korrigiert werden damit vor allem die individuellen Nachteile, die sich aus der speziellen Form der Behinderung ergeben. ${ }^{790}$ Jede Form der Behinderung erfordert daher andere angemessene Vorkehrungen. Das Handbuch für Parlamentarier zur BRK erläutert „angemessene Vorkehrungen" und führt dabei aus, dass diese beinhalten, das gesamte Lebens- und Arbeitsumfeld und u.a. auch Test- und Prüfungsverfahren entsprechend anzupassen. ${ }^{791}$

Ganz so fremd, wie einige Autoren meinen ${ }^{792}$, ist dem deutschen Diskriminierungsrecht die Figur der "angemessene Vorkehrungen“ nicht. Im Prüfungsrecht ist anerkannt, dass sich aus dem Grundsatz der Chancengleichheit aus Art. 3 । GG ergibt, dass die Nachteile eines behinderten Prüflings oder Schülers in Bezug auf die äußeren Prüfungsbedingungen individuell ausgeglichen werden müssen. ${ }^{793}$ Dieser Nachteilsausgleich, der in den Schulen bereits gewährt und individuell ausgerichtet wird, ist eine Form der angemessenen Vorkehrungen, da er bei dem einzelnen Schüler mit Behinderung und seinen speziellen Bedürfnissen ansetzt. ${ }^{794}$

Das Recht auf angemessene Vorkehrungen steht in Beziehung zu dem gesamten Bereich, an dem der Mensch mit Behinderung gleichberechtigt partizipieren möchte und soll Chancengleichheit im Wettbewerb mit nicht-behinderten Menschen herstellen. Entscheidend ist, ob die behinderte Person einer spezifischen Benachteiligung ausgesetzt ist, verglichen mit Personen ohne Behinderung in dieser Situation. ${ }^{795}$

787 Leder, Diskriminierungsverbot wegen einer Behinderung, S. 260, S. 266

788 Vgl. Schiek, NZA 2004, S. 873 ff, S. 875; Fuerst, Behinderung zwischen Diskriminierungsschutz und Rehabilitationsrecht, S. 122; Leder, Diskriminierungsverbot wegen einer Behinderung, S. 238

789 Grundlegend Fuerst, Behinderung zwischen Diskriminierungsschutz und Rehabilitationsrecht, S. 88

790 Fuerst, Behinderung zwischen Diskriminierungsschutz und Rehabilitationsrecht, S. 88

791 United Nations, From Exclusion to Equality, S. 60

792 Degener, RdJB 2009, S. 200 ff, S. 218 und Degener, ZaöRV 2005, S. 887 ff, S. 913; Fuerst, Behinderung zwischen Diskriminierungsschutz und Rehabilitationsrecht, S. 87

S. dazu oben 3. Teil: 3. Kapitel: 3.1.: Benachteiligung durch Gleichbehandlung bezüglich äußerer Prüfungsbedingungen, S. 117

794 Aichele/ Althoff, Kommentar zur UN-BRK, Nicht-Diskriminierung, Rn. 54; s. dazu auch die von Waddington in: Non-Discrimination Law zitierten Fälle zur Berücksichtigung von Legasthenie bei Prüfungen in den Niederlanden, S. 659 f, S. 661, S. $708 \mathrm{f}$ 
Das Recht auf angemessene Vorkehrungen ist nach der Entscheidung des CRPD bereits dann verletzt, wenn der Staat notwendige und geeignete Änderungen und Anpassungen an rechtliche Regelungen, die nicht unverhältnismäßig sind, nicht vornimmt. ${ }^{796}$ Das CRPD weitet damit den Anwendungsbereich der "angemessenen Vorkehrungen" über den oben dargestellten individuellen Bezug und über spezielle, aktive Maßnahmen des Nachteilsausgleichs hinaus, auf die Notwendigkeit aus, Ausnahmetatbestände zur Berücksichtigung von Behinderungen in allgemeinen Gesetzen oder Vorschriften zu schaffen.

Schüler und Schülerinnen mit Legasthenie/Dyskalkulie brauchen andere Prüfungsbedingungen und -anforderungen, um ihre ansonsten gegebene Eignung nachweisen und Schulen gleichberechtigt besuchen zu können. In der formalen Gleichbehandlung der Schüler und Schülerinnen mit Legasthenie/Dyskalkulie mit nichtbehinderten Schülern liegt die Versagung angemessener Vorkehrungen und damit eine Diskriminierung im Sinne von Art. 3 III 2 GG.

\section{Kausalität und/oder Konnexität}

Art. 3 III 2 GG verbietet eine Benachteiligung „wegen“ der Behinderung. Die Frage ist daher, in welchem Verhältnis Benachteiligung und Behinderung zueinander stehen müssen.

Diese Frage muss von der logisch vorrangigen Frage getrennt werden, wie das Verhältnis von Funktionsstörung und Beeinträchtigung der Teilhabe beim Behinderungsbegriff selber ist. Dem Behinderungsbegriff liegt das Konzept des sozialen Modells zugrunde, dass sich diese beiden Komponenten gegenseitig beeinflussen und in Wechselwirkung zueinander stehen. ${ }^{797}$ Die personenbezogenen Faktoren und die Umweltfaktoren werden als gleichwertig angesehen, so dass hier ein spezifischer Kausalzusammenhang nicht erforderlich ist. ${ }^{798}$

Für das Verhältnis von Behinderung und Benachteiligung ist nach herrschender Meinung objektive Kausalität erforderlich ${ }^{799}$, ein subjektives Moment, etwa eine Absicht, ist nicht erforderlich. ${ }^{800}$ Ausreichend ist es, wenn das Merkmal der Behinderung in einem weiten Sinne für die Belastung ursächlich ist. ${ }^{801}$

Das Kausalitätserfordernis könnte eng dahin ausgelegt werden, dass eine Benachteiligung „wegen“ einer Behinderung erst vorliegt, wenn eine konkrete

\footnotetext{
796 CRPD, http://www.ohchr.org/Documents/HRBodies/CRPD/CRPD.C.7.D.3.2011.doc, Rn. 8.5 
tatsächliche Einschränkung nachgewiesen ist. Dann aber würde das Merkmal der Kausalität selbst diskriminierend werden, denn der Mensch mit Behinderung müsste nachweisen, dass er im Vergleich zum Menschen ohne Behinderung eingeschränkt ist. Es würde nicht ausreichen, dass die Bedingungen eine solche Einschränkung vermuten oder wahrscheinlich sein lassen. Eine Veränderung der Bedingungen wäre erst dann notwendig, wenn ein Mensch mit Behinderung auf diese Problematik aufmerksam macht und damit explizit darauf hinweist, dass er mit dieser gesellschaftlichen Realität nicht zurechtkommt. Damit würde die Veränderungslast beim Menschen mit Behinderung liegen statt bei der Gesellschaft. Der nicht barrierefreie Bahnhof würde damit erst dann zur Behinderung, wenn Menschen mit Behinderung ihn tatsächlich nutzen wollen. Ohne das enge Kausalitätserfordernis wäre das Gebäude grundsätzlich (be-) hindernd, weil es Menschen mit Mobilitätseinschränkungen nicht berücksichtigt.

Zutreffend führt Welti aus, dass hier eine wertende Zuordnung im Sinne eines Zurechnungszusammenhangs erforderlich ist. ${ }^{802}$ Nur eine solche Wertung kann sicherstellen, dass diejenige Ungleich- oder Gleichbehandlung zugerechnet wird, die gerade Folge des Zusammenspiels von gesundheitlicher Beeinträchtigung und Umwelt ist, welches die Behinderung ausmacht. Diese Wertung gewährleistet aber auch, dass solche gesellschaftlichen Benachteiligungen aus dem Schutzbereich ausgeschlossen werden, die zwar statistisch häufiger bei Menschen mit Behinderung auftreten, für die aber kein wesentlicher, konkreter Zusammenhang zwischen Behinderung und Nachteil zu bejahen ist, etwa die statisch belegte, aber nicht zurechenbare Benachteiligung von Menschen mit Behinderung bei der Einkommensverteilung. ${ }^{803}$

Bei Legasthenie/Dyskalkulie sind die schulischen Bedingungen und Benotungsvorschriften auch bei wertender Betrachtungsweise ursächlich für die Benachteiligungen der betroffenen Schüler und Schülerinnen. Dadurch, dass in der Schule das Lesen, Rechtschreiben oder Rechnen bei einem Kind mit Legasthenie/Dyskalkulie bewertet wird, wird seine spezifische Beeinträchtigung bewertet. Dies hat unmittelbare negative Folgen für die Teilhabe am Leben in der Schule, auf die Schulbildung und auf die anschließenden beruflichen Möglichkeiten.

\section{Ergebnis}

Schüler und Schülerinnen mit Legasthenie/Dyskalkulie werden durch die unterschiedslose Gleichbehandlung mit Schülern und Schülerinnen ohne Beeinträchtigung benachteiligt und mittelbar diskriminiert.

802 Welti, Behinderung und Rehabilitation im sozialen Rechtsstaat, S. 467, S. 477; ähnlich Michael/Morlok, Grundrechte, Rn. 815

803

Welti, Behinderung und Rehabilitation im sozialen Rechtsstaat, S. 477 


\section{Kapitel: Rechtfertigung}

\section{Rechtfertigungsmaßstab}

Die gerade festgestellte Benachteiligung in Form mittelbarer Diskriminierung ist nach Art. 3 III 2 GG verboten. Allerdings ist auch das vorbehaltlose Grundrecht des Art. 3 III 2 GG nicht schrankenlos, sondern findet seine Schranken jedenfalls in den Grundrechten Dritter und in anderen mit Verfassungsrang ausgestatteten Rechtswerten. ${ }^{804}$ Die Benachteiligung durch Gleichbehandlung kann daher gerechtfertigt sein, wenn sich aus den Grundrechten Dritter oder aus anderen Verfassungsgütern die konkrete Pflicht oder Erlaubnis zur Gleichbehandlung ergibt. ${ }^{805}$

Für mittelbare Diskriminierungen, wie sie hier vorliegen, wird zum Teil vertreten, dass diese bereits durch hinreichend sachliche Gründe gerechtfertigt werden können. ${ }^{806}$ Nach h.M. reichen jedoch allgemeinen Erwägungen, wie sie zu Art. 3 । GG toleriert werden, für die Rechtfertigung nicht aus, sondern es gelten die gleichen, strengen Anforderungen aus Art. 3 III 2 GG, da der besondere Gleichheitssatz die staatliche Gewalt einer engeren Bindung unterstellt als in Art. 3 । GG. ${ }^{807}$

Dem ist zuzustimmen. Wie bereits ausgeführt ${ }^{808}$, ergeben sich viele Diskriminierungen von Menschen mit Behinderungen gerade daraus, dass die Umwelt die Behinderung nicht wahrnimmt oder keinen Anlass sieht, sie barrierefrei zu gestalten. Würde hier jeder sachliche Grund für die Rechtfertigung ausreichen, stünde es dem Gesetzgeber frei, Regelungen zu treffen, die sich sachlich mit allgemeinen Erwägungen und vernünftigen Gründen begründen lassen und so die Menschenrechte von Menschen mit Behinderung zu beschränken. Würde man allein sachliche Gründe ausreichen lassen, könnte auch heute noch ein Schüler, der im Rollstuhl sitzt, faktisch vom Besuch eines Gymnasiums ausgeschlossen werden, weil der allgemeine Schulbus nicht behindertengerecht ausgerüstet ist und nur für diesen Schüler umgerüstet werden müsste. ${ }^{809}$ Eine

\footnotetext{
804 Michael/Morlok, Grundrechte, Rn. 71; Beaucamp, JA 2001, S. 36 ff; Welti, Behinderung und Rehabilitation im sozialen Rechtsstaat, 450; Castendiek, in: GS Jean d'Heur, S. $337 \mathrm{ff}$

805

806

807

Rüfner, in: Bonner Kommentar GG, Art. 3, Rn. 876; Straßmair, Der besondere Gleichheitssatz aus Art. 3 Abs. 3 Satz 2 GG, S. 209, 214; Jürgens, ZfSH/SGB 1995, S. 353 ff, S. 358; Jürgens, DVBI 1997, S. 413 und 764; Boysen, in: v. Münch GG 2012, Art. 3, Rn. 200

808

809

Vgl. Epping, Grundrechte, Rn. 834

Kischel, in: BeckOK GG, Art. 3, Rn. 169, 213

S. oben 3. Teil: 4. Kapitel: 2.: Mittelbare Diskriminierung, S. 134

OVG Lüneburg, Urteil v. 16.6.1970, Az: A 41/70, SPE 734
} 
solche Begründung würde das Grundrecht aus Art. 3 III 2 GG konterkarieren, es würde seinen Sinn verlieren. ${ }^{810}$

Die Rechtsprechung des BVerfG stellt nach der oben zitierten „neuesten Formel" für die Rechtfertigung von Gleich- oder Ungleichbehandlungen ${ }^{811}$ auch bei der mittelbaren Diskriminierung darauf ab, wie stark diese den Menschen in seiner Individualität und in seinen grundrechtlich geschützten Freiheiten betrifft. Je stärker die Ungleichbehandlung resp. Gleichbehandlung, desto strenger werden die Anforderungen an den sachlichen Grund. ${ }^{812}$ D.h. die Kontrolldichte steigt, je schwerer die Ungleichbehandlung bzw. Gleichbehandlung ist, bis hin zu einer strengen Verhältnismäßigkeitsprüfung. ${ }^{813}$ Für die Gleichbehandlung ungleicher Sachverhalte hat das BVerfG deshalb allein sachliche Gründe zur Rechtfertigung dann nicht ausreichen lassen, wenn es der Gesetzgeber unterlässt, tatsächliche Ungleichheiten des zu ordnenden Lebenssachverhalts zu berücksichtigen, die so bedeutsam sind, dass sie bei einer am Gerechtigkeitsgedanken orientierten Betrachtungsweise beachtet werden müssen. Wesentlich ist dafür, in welchem Maße sich die Ungleich- oder Gleichbehandlung auf die Ausübung grundrechtlich geschützter Freiheiten nachteilig auswirken kann ${ }^{814}$. Nach der Rechtsprechung des BVerfG ist die Einschränkung des Grundrechts aus Art. 3 III 2 GG nur dann zulässig, wenn dies zur Lösung von Problemen, die ihrer Natur nach nur bei Behinderten auftreten können, zwingend erforderlich ist. ${ }^{815}$ Die nachteiligen Auswirkungen müssen unerlässlich sein, um behinderungsbezogenen Besonderheiten Rechnung zu tragen. ${ }^{816}$ Es genügt nicht, dass die Ungleichbehandlung für die Mehrheit der Bevölkerung plausibel ist. ${ }^{817}$

Der Maßstab der Rechtfertigung wird außerdem durch das besondere Beziehungsgefüge zwischen dem Staat, der Schule sowie der Schulpflicht der Schüler und Schülerinnen beeinflusst. Dies unterscheidet den Schulbereich maßgeblich von anderen Rechtsgebieten, in denen der Betroffene mehr oder weniger frei wählen kann, ob er sich bestimmten staatlichen Regelungen aussetzen will oder nicht.

\footnotetext{
810 Castendiek, in: GS Jean d'Heur, S. 344

811 BVerfGE 88, 87, 96

812 Kischel, in: BeckOK GG, Art. 3, Rn. 29, vgl. auch Fehling/Arnold, RdJB 2011, S. 316 ff, S. 326

813 Kischel, in: BeckOK GG, Art. 3, Rn. 26

814 BVerfGE 110, 141, 167, Rn. 92; St. Rspr. BVerfGE 1. 264, 275 f; 67, 70, 85 f; 98, 365, 385

815 BVerfGE 99, 341, 357; Heun, in: Dreier GG, Art. 3, Rn. 137; Jarass, in: Jarass/Pieroth GG, Art. 3, Rn. 149; Herdegen, Diskriminierungsschutz für Behinderte im Grundgesetz, S. 256

816 vgl. BT-Drs 12/8165, S. 29; BVerfGE 99, 341, 357

817 Rüfner, in: Bonner Kommentar GG, Art. 3, Rn. 876
} 
Zudem muss sich der Staat im schulischen Bereich, in dem er das Ausmaß und die Folgen seiner Regelungen vollständig selber in der Hand hat ${ }^{818}$, die eintretenden Folgen seiner Regelungen in höherem Maße zurechnen lassen als im außerschulischen Bereich. ${ }^{819}$ Je gewichtiger die Folgen einer Regelung im Hinblick auf Art und Intensität der eintretenden Nachteile für den Betroffenen sind, umso gewichtiger müssen die Gründe sein, die diese Regelung rechtfertigen. ${ }^{820}$ Damit ist Ungleichbehandlung resp. Gleichbehandlung jedenfalls nicht durch solche Differenzierungsgründe zu rechtfertigen, die auf Umständen beruhen, die der Staat selbst in der Hand hat. ${ }^{821}$ Auch deshalb reichen bei mittelbaren Diskriminierungen wegen einer Behinderung im Schulbereich allein sachliche Gründe für die Rechtfertigung der Benachteiligung nicht aus. ${ }^{82}$

Für den Rechtfertigungsmaßstab ist aus dem Grundsatz der völkerrechtsfreundlichen Auslegung auch zu beachten, welche Schranken das Diskriminierungsverbot der BRK enthält, weil auch die Wertungsgesichtspunkte der Verhältnismäßigkeit völkerrechtsfreundlich zu konkretisieren sind. ${ }^{823}$ Ausdrückliche Schranken und Rechtfertigungsgründe enthält die BRK zwar nicht, aber die Rechte aus der BRK gelten nicht schrankenlos, denn ihr ist ebenfalls das Verhältnismäßigkeitsprinzip immanent. ${ }^{824}$ Dies ergibt sich z.B. aus Art. 2, Art. 4 und Art. 7 BRK, die jeweils eine Abwägung beinhalten. Zudem sind bei der Verhältnismäßigkeitsprüfung auch die besonderen Staatenverpflichtungen aus der BRK zu berücksichtigen. ${ }^{825}$ Die Zusammenschau dieser Vorschriften zeigt, dass die Einschränkung der Rechte aus der BRK und damit die Benachteiligung wegen einer Behinderung nur aufgrund einer strengen Verhältnismäßigkeitsprüfung zulässig sind.

Im Ergebnis sind Benachteiligungen wegen einer Behinderung, auch dann, wenn sie mittelbar erfolgen, nur zu rechtfertigen, wenn sie aus Gründen, die in

818 BVerfGE 97, 271, 290 f; 103, 192, 193; Dreier, Horst, in: Dreier GG, Rn. 31, 37; Kischel, in: BeckOK GG, Art. 3, Rn. 42 ff; Straßmair, Der besondere Gleichheitssatz aus Art. 3 Abs. 3 Satz 2 GG, S. 236

Straßmair, Der besondere Gleichheitssatz aus Art. 3 Abs. 3 Satz 2 GG, S. 236

BVerfG, Beschluss vom 4.5.2011; Az.: 2 BvR 2365/09, http://www.bverfg.de/entscheidungen/rs20110504_2bvr236509.html, Rn. 94; Michael/ Morlok, Grundrechte, Rn. 86

824 Ausführlich dazu unten: 7. Teil: 1. Kapitel: 6.: Rechtfertigung, Schranken, Verhältnismäßigkeit, S. 237 
der Behinderung selbst liegen, zwingend erforderlich sind oder wenn in der Abwägung Grundrechte Dritter oder andere mit Verfassungsrang ausgestatteten Rechtswerte überwiegen.

\section{Gleichbehandlung bezüglich der äußeren Prüfungsbedingungen}

\subsection{Legasthenie}

Das BVerfG hat festgestellt, dass Prüflinge einen verfassungsrechtlich verbürgten Anspruch auf gleiche Prüfungschancen haben, so dass bei Beeinträchtigungen des Prüflings Kompensationsmaßnahmen erforderlich sind. ${ }^{826} \mathrm{Im}$ Prüfungsrecht ist daher allgemein anerkannt, dass es Eigenschaften gibt, die die Prüflinge voneinander unterscheiden und deshalb zu einer Durchbrechung der formellen Gleichbehandlung aller Prüflinge und zur Ungleichbehandlung in der Form der Veränderung der äußeren Prüfungsbedingungen zwingen. ${ }^{827}$ Unterschieden wird zwischen aktuellen Beeinträchtigungen und generellen Einschränkungen der Leistungsfähigkeit des Prüflings. Aktuelle, vorübergehende (krankheitsbedingte) Beeinträchtigungen, die es dem Prüfling erschweren, seine „wahren“ Kenntnisse und Fähigkeiten zu zeigen, sind in der Prüfung auszugleichen. ${ }^{828}$ Maßgeblich für die Vergleichbarkeit ist, ob die Behinderung solche Fähigkeiten einschränkt, die außerhalb der durch die jeweilige Prüfung zu ermittelnden Fähigkeiten liegen und die deshalb den Nachweis einer ansonsten vorhandenen Befähigung erschweren, und ob die Behinderung in dem angestrebten Beruf durch Hilfsmittel ausgeglichen werden kann. ${ }^{829}$

In der Rechtsprechung und Literatur ist inzwischen anerkannt, dass Schüler und Schülerinnen mit Legasthenie in der "technischen Fähigkeit" zur Darstellung des vorhandenen eigenen Wissens eingeschränkt sind, weil sie eine langsamere Lesegeschwindigkeit haben und die handschriftliche Darlegung erschwert ist. ${ }^{830}$ Weil insoweit nur der Nachweis einer uneingeschränkt vorhan-

826 BVerfG Beschluss v. 12.12.1992, Az.: 1 BvR 1295/90, juris, Rn. 19

827 BVerwG, Beschluss v. 13.12.1985, Az.: 7 B 210.85, juris, Rn. 6; BVerwG, Urt. v. 30.08.1977 VII C 50.76 -, Buchholz 421.0, Prüfungswesen, Nr. 85; vgl. auch OVG Koblenz, Urt. v. 16.01.1980 - 2 A 49/79 -, DVBI. 1981, 591; OVG Weimar, Beschluss v. 17.5.2010, Az.: 1 EO 854/10, juris, Rn. 36; Niehues, Prüfungsrecht, Rn. 259, 444

828 Münch, von, Jura 1980, S. 448

829 Niehues, Prüfungsrecht, Rn. 122 und 259; Langenfeld, RdJB 2007, S. 211 ff, S. 219; OVG Lüneburg, Beschluss v. 10.7.2008, Az.: 2 ME 309/08, www.rechtsprechung.niedersachsen.de, Rn. 11; OVG Weimar, Beschluss v. 17.5.2010, Az.: 1 EO 854/10, juris, Rn. 28

830 Niehues, Prüfungsrecht, Rn. 259; OVG Lüneburg, Beschluss v. 10.7.2008, Az.: 2 ME 309/08, www.rechtsprechung.niedersachsen.de, Rn. 11; OVG Lüneburg, Beschluss v. 20.9.2012, Az.: 2 LA 234/11, S. 5 (nicht veröffentlicht); OVG Weimar, Beschluss v. 17.5.2010, Az.: 1 EO 
denen Befähigung erschwert ist und die eingeschränkten Fähigkeiten in dem angestrebten Beruf durch Hilfsmittel ausgeglichen werden können, besteht ein Anspruch auf Veränderung der äußeren Prüfungsbedingungen. Maßgeblich ist dafür nach Ansicht der Rechtsprechung vor allem, dass mit einem Zeitzuschlag die inhaltliche Prüfungsleistung nicht verändert wird. ${ }^{831}$ Soweit es daher um den Bereich der „äußeren Prüfungsbedingungen“ geht, ist eine Gleichbehandlung aller Schüler und Schülerinnen mit Legasthenie gerade nicht erforderlich, sondern eine Ungleichbehandlung zwingend erforderlich.

Mit der Veränderung äußerer Prüfungsbedingungen werden für Schüler und Schülerinnen mit Legasthenie Barrieren abgebaut, die ihnen ansonsten den Weg zum Prüfungsziel erschweren oder verbauen. ${ }^{832}$ Die Prüfung wird barrierefrei gestaltet, so dass sie die gleichen Startchancen haben wie ihre Mitschüler ohne Behinderung, damit sie ihre Befähigung unbeeinträchtigt nachweisen können.

Grundrechte der Schüler und Schülerinnen ohne Behinderung, insbesondere das Grundrecht aus Art. 3 I GG, stehen dem nicht entgegen. Durch den Nachteilsausgleich wird nur eine materielle Gleichheit aller Schüler hinsichtlich der äußeren Prüfungsbedingungen erreicht. Der Aussagewert der Prüfung wird dadurch nicht verändert, so dass ein Eingriff in Art. 3 I GG nicht gegeben ist.

Andere Werte von Verfassungsrang, die einer Veränderung der äußeren Prüfungsbedingungen entgegenstehen könnten, sind nicht ersichtlich.

Die Benachteiligung durch Gleichbehandlung bezüglich der äußeren Prüfungsbedingungen ist somit nicht gerechtfertigt. Es besteht ein Anspruch auf Ungleichbehandlung, der auch weitgehend anerkannt ist. ${ }^{833}$

\subsection{Dyskalkulie}

Grundsätzlich streitig ist die Frage der Berücksichtigung und damit der Ungleichbehandlung bei der Dyskalkulie.

In einigen Bundesländern wird Dyskalkulie grundsätzlich nicht berücksichtigt. In einigen anderen Ländern wird sie zwar berücksichtigt, die Berücksichtigung endet jedoch i.d.R. mit der Grundschule. ${ }^{834}$ Das OVG Weimar hat in einem

854/10, juris, Rn. 28, 41; VGH Kassel, Beschluss v. 3. 1. 2006, 8 TG 3292/05, NJW 2006,

S. 1608 f; OVG Schleswig, Beschluss v. 19.8.2002, Az.: 3 M 41.02, juris - Leitsätze

OVG Lüneburg, Beschluss v. 10.7.2008, Az.: 2 ME 309/08,

www.rechtsprechung.niedersachsen.de, Rn. 10; OVG Weimar, Beschluss v. 17.5.2010, Az.: 1

EO 854/10, juris, Rn. 28, 41; VGH Kassel, Beschluss v. 3. 1. 2006, 8 TG 3292/05, NJW 2006,

S. 1608 f; OVG Schleswig, Beschluss v. 19.8.2002, Az.: 3 M 41.02, juris - Leitsätze

So inzwischen auch KMK-Inklusive Bildung Punkt II.3

833 S. dazu auch KMK-Inklusive Bildung Punkt II.3

834 Zu den Einzelheiten s. oben 1. Teil: 2. Kapitel: 5: Berücksichtigung der Dyskalkulie in der Schule, S. 39 
Eilverfahren einer Schülerin mit Dyskalkulie einen Zeitzuschlag von 25 \% zugesprochen, um damit die sehr langsame Rechengeschwindigkeit aufgrund von komplizierterer Rechenweise zu kompensieren. ${ }^{835}$ Auch Ennuschat hält entsprechende Maßnahmen des Nachteilsausgleichs für zulässig. ${ }^{836}$ Demgegenüber hält Kischel eine Berücksichtigung der Dyskalkulie prinzipiell für verfehlt. ${ }^{837}$

Für die Veränderung der äußeren Prüfungsbedingungen bei Dyskalkulie kommt es darauf an, dass diese Schüler hinsichtlich solcher Eigenschaften eingeschränkt sind, die sie bei der Erbringung der Leistung beeinflussen, die aber nichts mit der Leistungsfähigkeit als solcher zu tun haben. Dem OVG Weimar ist darin zuzustimmen, dass das Prüfungsziel darin besteht, bestimmte Aufgaben zu lösen. Wenn dies wegen der Behinderung nur auf komplizierten Wegen möglich ist, so hindert das nicht am Erreichen des Ziels überhaupt. Ebenso wie bei anderen Schülern und Schülerinnen mit Behinderungen baut der Nachteilsausgleich in Form von Zeitverlängerungen bezüglich der äußeren Prüfungsbedingungen bei einem Schüler mit Dyskalkulie Barrieren ab und verschafft ihm erst gleiche, barrierefreie Startbedingungen. Die fachlich-inhaltlichen Anforderungen werden dadurch nicht verändert.

Grundrechte der Schüler und Schülerinnen ohne Behinderung, insbesondere das Grundrecht aus Art. 3 I GG stehen dem ebenso wenig entgegen wie bei der Legasthenie. Durch den Nachteilsausgleich wird nur eine materielle Gleichheit aller Schüler in der Prüfung erreicht. Der Aussagewert der Prüfung wird dadurch nicht verändert, so dass ein Eingriff in Art. 3 I GG nicht gegeben ist.

Werte von Verfassungsrang, die einer Veränderung der äußeren Prüfungsbedingungen bei Dyskalkulie im Gegensatz zur Veränderung bei vergleichbaren Behinderungen, insbesondere der Legasthenie, entgegenstehen könnten, sind nicht ersichtlich. ${ }^{838}$

Auch bei Dyskalkulie lässt sich daher eine Benachteiligung durch Gleichbehandlung bezüglich der äußeren Prüfungsbedingungen nicht rechtfertigen, so dass auch hier ein Anspruch auf Ungleichbehandlung besteht.

\footnotetext{
835 OVG Weimar, Beschluss v. 17.5.2010, Az.: 1 EO 854/10, juris, Rn. $51 \mathrm{f}$

Ennuschat, br 2008, S. 93 ff, S. 96

837 Kischel, in: BeckOK GG, Art. 3, Rn. $219 \mathrm{ff}$

838 Vgl. VG Hannover, Beschluss v. 13.12.2010, Az.: 6 B 5596/10, www.rechtsprechung.niedersachsen.de, Rn. 23
} 


\section{Gleichbehandlung bezüglich der inhaltlichen Prüfungsanforderungen}

Die Rechtsprechung hält die hier vorliegende Benachteiligung durch Gleichbehandlung für gerechtfertigt ${ }^{839}$, weil es anerkannt sei, dass persönlichkeitsbedingte Eigenschaften, die die Leistungsfähigkeit prägen, nicht auszugleichen sind. Dazu gehöre auch die Fähigkeit zur Rechtschreibung. ${ }^{840}$ Weiter verstoße eine solche Veränderung der inhaltlichen Prüfungsanforderungen gegen den Grundsatz der Chancengleichheit, weil dadurch die Anforderungen abgesenkt würden und dies die Grundrechte der anderen Schüler verletze. Eine solche Bevorzugung verletze das Übermaßverbot. ${ }^{841}$ Sie führe insbesondere beim Abitur zu einem Abschluss, der dann nicht alle Zielbereiche der Ausbildung erfasse. ${ }^{842}$ Zudem seien Rechtschreibung und Rechnen in bestimmten Prüfungen gerade Prüfungsinhalt, etwa im Diktat, und damit zu prüfende Kernkompe-

839 VGH Kassel, Beschluss v. 5.2.2010, Az.: 7 A 2406/09.z, juris; Beschluss v. 17.11.2010, Az.: 7 A 2970/09.Z juris; Beschluss v. 8.12.2011, Az.: 7 A 2621/10.Z, juris; OVG Lüneburg, Beschluss v. 10.7.2008, Az.: 2 ME 309/08, www.rechtsprechung.niedersachsen.de; Beschluss v.

25.11.2011, Az.: 2 ME 52/11 (nicht veröffentlicht); Beschluss v. 20.9.2012, Az.: 2 LA 234/11; VGH München, Beschluss v. 28.6.2012, Az.: 7 CE 12.1324, juris, (zu Prüfungen im Studienfach Bachelor BWL); VG Aachen, Urteil v. 13.11.2009, Az.: 9 K 25/09, www.justiz.nrw.de; VG Düsseldorf, Beschluss v. 16.11.2009, Az.: 18 L 1654/09, juris; Beschluss v. 20.5.2011, Az.: 18 L 707/11, juris; VG Giessen, Urteil v. 2.11.2010, Az.: 7 K 1841/10; VG Köln, Beschluss v.

26.9.008, Az.: 10 L 1240/08, www.justiz.nrw.de; VG Köln, Beschluss 26.9.2008 Az.: 10 L

1240/08, www.justiz.nrw.de; VG Schleswig, Urteil v. 10.6.2009, Az.: 9 A 208/08, BeckRS 2009 38123, S. 10; VG Sigmaringen, Beschluss v. 2.4.2008, Az.: 4 K 596/08 (nicht veröffentlicht); a.A. nur VG Hannover, Beschluss v. 13.12.2010, Az.: 6 B 5596/10, www.rechtsprechung.niedersachsen.de

VGH Kassel Beschluss v. 5.2.2010, Az.: 7 A 2406/09.z, juris, Rn. 59; VG Düsseldorf, Beschluss v. 20.5.2011, Az.: 18 L 707/11, juris, Rn. 13

OVG Lüneburg, Beschluss v. 10.7.2008, Az.: 2 ME 309/08, www.rechtsprechung.niedersachsen.de, Rn. 19; Beschluss v. 25.11.2011, Az.: 2 ME 52/11, S. 4 f (nicht veröffentlicht); Beschluss v. 20.9.2012, Az.: 2 LA 234/11, S. 5 f (nicht veröffentlicht); VGH Kassel Beschluss v. 5.2.2010, Az.: 7 A 2406/09.z, juris, Rn. 43, 59; VG Aachen, Urteil v. 13.11.2009, Az.: 9 K 25/09, www.justiz.nrw.de, Rn. 56, 70; VG Düsseldorf, Beschluss V. 16.11.2009, Az.: 18 L 1654/09, juris, zur Dyskalkulie, Rn. 20 f; Beschluss v. 20.5.2011, Az.: 18 L 707/11, juris VG Gießen, Urteil v. 2.11.2010, Az.: 7 K 1841/10, S. 12 (nicht veröffentlicht); VG Sigmaringen, Beschluss v. 2.4.2008, Az.: 4 K 596/08, S. 4 (nicht veröffentlicht); offen lassend VG Hannover, Beschluss v. 13.12.2010, Az.: 6 B 5596/10, www.rechtsprechung.niedersachsen.de, Rn. 29; s. auch LSG Baden-Württemberg, Beschluss v. 9.12.2010, Az.: L 13 AL 4629/10 ER-B, juris, Rn. 33: Zugang zur Hochschulbildung nur unter den auch für nichtbehinderte Menschen geltenden Zugangsbedingungen; OVG Bautzen, Beschluss v. 3.11.2008, Az.: 2 B 292/08, S. 6 f zu einer nicht genannten Behinderung (nicht veröffentlicht); VG Saarlouis, Beschluss v. 17.3.2008, Az.: 1 L 169/08, juris 
tenzen, so dass von ihrer Bewertung nicht abgesehen werden könne. ${ }^{843}$ Wenn der Schüler diese Leistungen nicht erbringen kann, gehe dies zu seinen Lasten. ${ }^{844}$ Schließlich stehe der Veränderung auch entgegen, dass die NichtBewertung der Rechtschreibung eine Bevorzugung sei, auf die kein Leistungsanspruch unmittelbar aus Art. 3 III 2 GG bestehe. Ein solcher Anspruch müsse vielmehr vom Gesetzgeber umgesetzt werden. ${ }^{845}$

Die Literatur hält die Veränderung von inhaltlichen Prüfungsbedingungen für eine Form der Bevorzugung, die sich nicht direkt aus Art. 3 III 2 GG ergibt, sondern vom Staat umgesetzt werden muss.

\subsection{Zwingende Gründe aus der Behinderung selbst}

Die Gleichbehandlung bei Prüfungsanforderungen kann gerechtfertigt sein, wenn sie aus Gründen, die in der Behinderung selbst liegen, zwingend erforderlich ist.

Die Gleichbehandlung aller Schüler und Schülerinnen ohne Rücksicht auf die Legasthenie/Dyskalkulie erfolgt jedoch nicht, um Gründen, die in der Behinderung selbst liegen, Rechnung zu tragen. Sie erfolgt vielmehr deshalb, um die formale Gleichheit zwischen Schülern und Schülerinnen mit und ohne Behinderung zu bewahren. Die Auswirkungen für Schüler und Schülerinnen mit Legasthenie/Dyskalkulie ergeben sich aber vor allem mittelbar, nicht jedoch, wie dies für eine behinderungsbezogene Maßnahme notwendig wäre, ziel- und zweckgerichtet. Sie ist daher keine Maßnahme, um behinderungsbezogenen Besonderheiten Rechnung zu tragen und Probleme zu lösen, die sich ihrer Natur nach nur bei Behinderungen ergeben.

\subsection{Zwingender Grund: persönlichkeitsbedingte Eigenschaften, die die Leistungsfähigkeit prägen}

Die Rechtsprechung begründet die Gleichbehandlung hinsichtlich der inhaltlichen Prüfungsanforderungen damit, dass es sich bei der eingeschränkten Leistungsfähigkeit um „persönlichkeitsbedingte Eigenschaften“ handelt, die die

843 OVG Lüneburg, Beschluss v. 20.9.2012, Az.: 2 LA 234/11, S. 6 (nicht veröffentlicht); VGH München, Beschluss v. 28.6.2012, Az.: 7 CE 12.1324, juris, (zu Prüfungen im Studienfach Bachelor BWL), Rn. 24; VG Düsseldorf, Beschluss v. 20.5.2011, Az.: 18 L 707/11, juris, Rn. 12, 14

844 VG Düsseldorf, Beschluss v. 20.5.2011, Az.: 18 L 707/11, juris, Rn. 14

845 OVG Lüneburg, Beschluss v. 10.7.2008, Az.: 2 ME 309/08, www.rechtsprechung.niedersachsen.de, Rn. 19; Beschluss v. 25.11.2011, Az.: 2 ME 52/11, S. 4 f (nicht veröffentlicht); Beschluss v. 20.9.2012, Az.: 2 LA 234/11; VGH Kassel, Beschluss v. 5.2.2010, Az.: 7 A 2406/09.Z, juris, Rn 41 f; VG Gießen, Urteil v. 2.11.2010, Az.: 7 K 1841/10, S. 9 ff (nicht veröffentlicht); VG Köln, Beschluss v. 26.9.2008, Az.: 10 L 1240/08, www.justiz.nrw.de, kritisch dazu Marwege, DVBI 2009, S. $538 \mathrm{ff}$ 
Leistungsfähigkeit prägen und deshalb nicht zu beachten sind. ${ }^{846}$ Nach Ansicht des OVG Münster ergibt sich daraus ein Eignungsmangel des Schülers, so dass er für den Besuch des Gymnasiums ungeeignet ist. ${ }^{847}$ Demgegenüber haben das OVG Schleswig und der VGH Kassel in der Legasthenie keine persönlichkeitsbedingte Eigenschaft gesehen, sondern nur eine Einschränkung in der „technischen" Erbringung der Leistung. ${ }^{848}$ In der neueren Rechtsprechung vertreten der VGH Kassel und das VG Düsseldorf nunmehr die Ansicht, dass es sich bei der Fähigkeit zur Rechtschreibung um eine solche persönlichkeitsbedingte Eigenschaft handelt. ${ }^{849}$

Im Prüfungsrecht hat sich der Grundsatz etabliert, dass auf Dauerleiden nur insoweit Rücksicht zu nehmen ist, als diese nicht als persönlichkeitsbedingte Eigenschaften die Leistungsfähigkeit eines Prüflings prägen. ${ }^{850}$ Bei konstitutionellen oder sonst auf unabsehbare Zeit andauernden Leiden, wie chronischen Krankheiten oder sonstigen in der Person des Prüflings liegenden Anlagen wird demgegenüber ein Eignungsmangel angenommen, der sich zulässigerweise im Prüfungsergebnis widerspiegelt. ${ }^{851}$ Die ganz herrschende Meinung ${ }^{852}$ vertritt die Ansicht, dass persönliche konstitutionelle Leistungsschwächen für die Befähigung des Prüflings generell von Bedeutung und daher auch Gegenstand der Bewertung sind. Mit einer Berücksichtigung würde ansonsten das Prüfungsergebnis verfälscht, denn der Prüfling solle mit der Prüfung auch zeigen, dass er z.B. mit Zeitschwierigkeiten fertig wird und die Grundvoraussetzungen für die Eignung des bestimmten Berufes habe. Dementsprechend prägt ein nicht in

846 VGH Kassel Beschluss v. 5.2.2010, Az.: 7 A 2406/09.z, juris, Rn. 59; VG Düsseldorf, Beschluss v. 20.5.2011, Az.: 18 L 707/11, juris, Rn. 13 OVG Münster, Beschluss v. 4.9.2008; Az.: 19 B 1293/08, www.justizr.nrw.de, Rn. 19, 23 VGH Kassel, Beschluss v. 3. 1. 2006, 8 TG 3292/05, NJW 2006, S. 1608 f; OVG Schleswig, Beschluss v. 19.8.2002, Az.: 3 M 41.02, juris - Leitsätze VGH Kassel Beschluss v. 5.2.2010, Az.: 7 A 2406/09.z, juris, Rn. 59; VG Düsseldorf, Beschluss v. 20.5.2011, Az.: 18 L 707/11, juris, Rn. 13 VII C 50.76 -, Buchholz 421.0, Prüfungswesen, Nr. 85; vgl. auch OVG Koblenz, Urt. v. 16.01.1980 - 2 A 49/79 -, DVBI. 1981, 591; OVG Weimar, Beschluss v. 17.5.2010, Az.: 1 EO 854/10, juris, Rn. 28; VG Saarland, Urteil v. 5.3.2009, Az.: 1 K 643/09, juris, Rn. 63; Niehues, Prüfungsrecht, Rn. 258 BVerwG, Beschluss v. 13.12.1985, Az.: 7 B 210.85, juris, Rn. 6; OVG Münster, Beschluss v. 4.9.2008; Az.: 19 B 1293/08, www.justizr.nrw.de, Rn. 17

852 Starck, in: v. Mangoldt/Klein/Starck GG, Art. 3, Rn. 39; Münch, von, Jura 1980, S. 448; Zimmerling/Brehm, Prüfungsrecht, Rn. 323; sogar Poscher/Langer/Rux, Gutachten zu Art. 24 BRK, S. 56; Niehues, Prüfungsrecht, Rn. 258; VG Augsburg, Beschluss v. 7.9.2005, Az.: Au 3 E 05.00854, juris, Rn. 23; VG Freiburg, Beschluss v. 30.8.2007, Az.: 2 K 1667/07, juris; OVG Weimar, Beschluss v. 17.5.2010, Az.: 1 EO 854/10, juris, Rn. 28, differenzierend jetzt Niehues, Prüfungsrecht, Rn. 256, ablehnend aber in der 4. Auflage, Rn. 121 
absehbarer Zeit heilbares Dauerleiden, z.B. psychischer Art, im Gegensatz zu den akuten krankheitsbedingten Leistungsminderungen das "normale" Leistungsbild des Prüflings, so dass es nach herrschender Ansicht mit dem Sinn und Zweck der Leistungskontrolle und der Eignungsprüfung nicht zu vereinbaren ist, die darauf beruhende Minderung der Leistungsfähigkeit in der Prüfung zu berücksichtigen. ${ }^{853}$

Unter Hinweis auf persönlichkeitsbedingte Dauerleiden hat das VG Freiburg einem Studenten mit Aufmerksamkeitsdefizitsyndrom eine Zeitverlängerung im 1. juristischen Staatsexamen mit der Begründung verwehrt, dass die Bearbeitung eines juristischen Falles in einer vorgegebenen Zeit gerade Inhalt der Prüfung sei. Die Leistungsminderung durch ADS bestimme sein "normales" Leistungsbild mit der Konsequenz, dass sich die Leistungsschwächen (zu Recht) in der Prüfungsleistung niederschlagen würden. ${ }^{854}$ Ebenso hat das OVG Münster die Berücksichtigung einer nach der ICD 10 diagnostizierten Angststörung (F40.2) abgelehnt. ${ }^{855}$

Zu welchen Unstimmigkeiten die Abgrenzung zwischen Eigenschaften, die die Leistungsfähigkeit nicht betreffen, und „persönlichkeitsbedingten Eigenschaften" führt, zeigt der bereits zitierte Beschluss des OVG Münster ${ }^{856}$ : Das Gericht hält eine Lese-Rechtschreibschwäche für berücksichtigungsfähig, denn sie habe keine Auswirkungen auf das Leistungsvermögen und sei, wenn sie vorübergehend und durch schulische und außerschulische Förderung behebbar ist, kein Eignungsmangel. Demgegenüber sei eine Legasthenie ein Eignungsmangel und deshalb nicht zu berücksichtigen. ${ }^{857}$ Mit anderen Worten: Eine schwächere, behebbare Beeinträchtigung wird durch Berücksichtigung geschützt und ermöglicht den Wechsel auf das Gymnasium, während eine schwerere, nicht behebbare Beeinträchtigung nicht geschützt wird, sondern zulässigerweise vom Wechsel auf das Gymnasium ausschließt.

Dieser Grundsatz des Prüfungsrechts schränkt tatsächlich Art. 3 III 2 GG ein, wird aber weder von der Rechtsprechung noch von der Literatur daran gemessen. ${ }^{858}$ Der Grundsatz kann eine Benachteiligung nach Art. 3 III 2 GG nur recht-

853 Ausdrücklich für die Beeinträchtigung aufgrund manisch-depressiver Erkrankung BVerwG Beschluss v. 13.12.1985, Az.: 7 B 210/85, juris; s. auch VGH Kassel, Beschluss v. 10.7.1989, Az.: 6 TP 1542/89; VG Berlin, Urteil v. 30.1.2008, Az.: 12 A 634.05, juris, Rn. 15 VG Freiburg, Beschluss v. 30.8.2007, Az.: 2 K 1667/07, juris, Rn. 11; anders der Beitrag von Tiemann, BayVBI 1976, S. 650 ff, der ausdrücklich auf eine Behinderung durch Konzentrationsschwächen hinweist. S. 651; s. auch VG Saarland, Urteil v. 5.3.2009, Az.: 1 K 643/09, juris OVG Münster, Urteil v. 8.6.2010, Az.: 14 A 1735/09, juris OVG Münster, Beschluss v. 4.9.2008; Az.: 19 B 1293/08, www.justizr.nrw.de, Rn. 19, 23 OVG Münster, Beschluss v. 4.9.2008; Az.: 19 B 1293/08, www.justizr.nrw.de, Rn. 7, 19, 23

858 Einzige Ausnahme bildet insoweit das OVG Weimar, Beschluss v. 17.5.2010, Az.: 1 EO 854/10, juris, Rn. 37 
fertigen, wenn er selber mit Art. 3 III 2 GG vereinbar ist. Bedenken dagegen ergeben sich schon bei oberflächlicher Betrachtung daraus, dass mit diesem Grundsatz ganz bewusst die Berücksichtigung von bestimmten Dauerleiden bei Prüfungen ausgeschlossen wird. Tatsächlich verschleiert die Wortwahl „persönlichkeitsbedingte Eigenschaft" die dahinter liegende Diskriminierung. Formuliert man die Passage um und ersetzt "persönlichkeitsbedingte Eigenschaft" korrekt durch „Behinderung“, wird die diskriminierende Ausrichtung dieser Figur offensichtlich. Dann heißt es nämlich: Wenn dauerhafte Einschränkungen oder Beeinträchtigungen als Behinderung die Leistungsfähigkeit des Prüflings prägen, ist dies nicht zu berücksichtigen. Oder beim Schüler mit Legasthenie: Auf Legasthenie in Prüfungen ist keine Rücksicht zu nehmen, da sie als Behinderung die Leistungsfähigkeit des Schülers prägt und somit zu einem Eignungsmangel des Schülers führt. ${ }^{859}$

Die Nicht-Berücksichtigung sog. Dauerleiden wäre verfassungsrechtlich nur haltbar, wenn Dauerleiden entweder keine Behinderung sind oder es keine Benachteiligung ist, wenn sie in der Prüfung nicht berücksichtigt werden, oder wenn eine solche Benachteiligung gerechtfertigt ist.

Dauerleiden sind in aller Regel auch Behinderungen, weil sich Leiden immer negativ auswirken. Ein Dauerleiden wird jedenfalls dann zur Behinderung, wenn sein Ausgleich in einer Prüfung versagt wird, denn dann ist seinetwegen nur eine eingeschränkte Teilhabe an der Prüfung möglich, so dass es eine negative Wirkung entfaltet.

Durch diese Nicht-Berücksichtigung wird der Prüfling auch benachteiligt. Der Figur der „persönlichkeitsbedingten Einschränkung“ liegt die Vorstellung zugrunde, dass der behinderte Mensch anders als ein akut kranker Prüfling in der Prüfung nicht benachteiligt wird, weil er ohnehin immer eingeschränkt ist. Sein eingeschränktes Leistungsvermögen stellt daher, weil es dauerhaft vorhanden ist, sein "normales" Leistungsbild dar, das zu seiner Persönlichkeit dazu gehört. Er ist daher nicht benachteiligt, wenn "sein" normales Leistungsbild bewertet wird. Eine solche Sichtweise widerspricht jedoch dem Begriff der Benachteiligung bei Art. 3 III 2 GG. ${ }^{860}$ Die subjektive Sichtweise des Menschen mit Behinderung ist das Maß dafür, ob er sich in der Prüfung benachteiligt fühlt. Stellt er (nachvollziehbar) dar, dass seine Behinderung negative Auswirkungen bei der Prüfung hat, dann kann nicht „objektiv" festgestellt werden, dass der Mensch mit Behinderung diesen Nachteil nicht hat.

Die Benachteiligung durch die Nicht-Berücksichtigung der Beeinträchtigung wird ausdrücklich damit gerechtfertigt, dass Dauerleiden, und vor allem psychi-

\footnotetext{
859 Vgl. OVG Münster, Beschluss v. 4.9.2008; Az.: 19 B 1293/08, www.justizr.nrw.de, Rn. 19, 23 
schen Dauerleiden grundsätzlich der Ausgleich zu versagen ist. ${ }^{861}$ Diese Argumentation verstößt gegen Art. 3 III 2 GG, denn Art. 3 III 2 GG schützt vor Benachteiligungen wegen jeder Form der Behinderung, sei sie körperlich, seelisch, geistig oder eine Sinnesbeeinträchtigung. Damit ist es nicht zu vereinbaren, Dauerleiden und vor allem psychische Krankheiten generell nicht zu berücksichtigen.

Die Annahme einer „persönlichkeitsbedingten Einschränkung“ ist zudem in sich widersprüchlich, denn es ist im Prüfungsrecht trotzdem anerkannt, dass Dauerleiden wie Blindheit, Spastiken ${ }^{862}$ oder Herzkrankheiten berücksichtigt werden. Diese Dauerleiden sind aber sicherlich ebenso persönlichkeitsbedingte Eigenschaften, die die Leistungsfähigkeit des Prüflings prägen und die im späteren Berufsleben nicht kompensiert werden können. Sie unterscheiden sich nur dadurch, dass die einen sichtbar und/oder nachvollziehbar und die anderen unsichtbar und schwer nachvollziehbar sind. ${ }^{863}$ Das darf aber nicht zu einer Ungleichbehandlung der verschiedenen Behinderungsformen führen.

Die Figur des "generellen Eignungsmangels" ist deutlich von der Frage geprägt, ob der behinderte Mensch in der Lage ist, die in der Prüfung und später im Beruf geforderten Leistungen wie ein "normaler" Mensch zu erbringen. So führt das VG Freiburg zum Nachteilsausgleich bei ADS in der 1. Juristischen Staatsprüfung aus, dass es gerade Inhalt der juristischen Leistungsfähigkeit ist, einen Sachverhalt aufzunehmen und in gegebener Zeit einer plausiblen Lösung zuzuführen. Die Schwierigkeiten des betroffenen Prüflings gerade unter zeitlichem Druck zu arbeiten, ließen sich nicht durch Hilfsmittel kompensieren. ${ }^{864}$ Mit anderen Worten: Nur wenn der Betroffene in der Lage ist, mit Hilfe von Kompensationsmöglichkeiten die "normalen“ Leistungen zu erbringen, ist seine Behinderung zu berücksichtigen. Es ist der Behinderung jedoch gerade immanent, dass der behinderte Mensch dem „normalen“ unbeeinträchtigten Leistungsbild eines gedachten Normalkandidaten in der Prüfung nicht gerecht werden kann.

De facto wird hier die Nicht-Berücksichtigung der Behinderung mit der "Natur der Sache" der vorliegenden Behinderung begründet. Mit der "Natur der Sache" lässt sich jedoch fast jede Ungleichbehandlung rechtfertigen. ${ }^{865}$ Diese

861 So aber ausdrücklich Niehues in der 4. Auflage, Rn. 121; jetzt differenzierend Niehues, Prüfungsrecht, Rn. 256; VG Berlin, Urteil v. 30.1.2008, Az.: 12 A 634.05, juris, Rn. 15; VG Freiburg, Beschluss v. 14.10.2008, Az.: 1 K 1823/08, www.landesrecht-bw.de

S. dazu das Urteil des VG Lüneburg v. 28.5.2002, Az.: 4 A 246/01, www.rechtsprechung.niedersachsen.de, zum Nachteilsausgleich bei spastischer Diplegie S. zu den nicht sichtbaren Behinderungen Kirchner, Nicht sichtbare Behinderung

864 VG Freiburg, Beschluss v. 30.8.2007, Az.: 2 K 1667/07, juris, Rn. 12, eine Prüfung von Art. 3 III 2 GG und seines Einflusses auf das Gebot der Chancengleichheit fehlt.

865 Neumann, in: Handbuch SGB IX, § 2 Verfassungsrecht, Rn. 27; Höller, Beschulung behinderter Kinder und Jugendlicher an öffentlichen Schulen S. 215 ff, S. 221 
Argumentationsfigur darf aber nicht zur Blankovollmacht für beliebige Ungleichbehandlungen werden. ${ }^{866}$ Sie darf allenfalls herangezogen werden, wenn eine Behinderung die Berücksichtigung auch unter optimalen Bedingungen ausschließt. ${ }^{867}$ Und es ist zu beachten, inwieweit in die Bewertung der Alternativlosigkeit, die subjektive Wertung „das kann nicht sein" einfließt. Vermutlich können sich nur wenige Menschen vorstellen, dass es gehörlose Schauspieler und Sänger ${ }^{868}$ und Lehrer mit Down-Syndrom ${ }^{869}$ gibt. Die Tatsache, dass es sie gibt, zeigt, dass bei optimalen Bedingungen behinderten Menschen sehr vieles möglich ist und der Ausschluss nur sehr selten in Betracht kommt. Allein die „Natur der Sache" ist daher kein ausreichender Rechtfertigungsgrund für die Nicht-Berücksichtigung von Behinderung.

Im Ergebnis führt vor allem auch die Abgrenzung von Lese-Rechtschreibschwäche gegen Legasthenie, wie sie das OVG Münster vornimmt ${ }^{870}$, dazu, dass leichte Formen der Behinderung, die eventuell nur vorübergehend oder verbesserbar sind, stärker geschützt werden als schwere Formen der Behinderung, die dauerhaft und nicht zu beeinflussen sind. Eine solche Auslegung widerspricht Art. 3 III 2 GG.

Gegen die Figur des „generellen Eignungsmangels“ spricht ferner die neueste Rechtsprechung des BVerfG zu den Anforderungen an die gesundheitliche Eignung im Rahmen von Art. 33 ॥ GG. Das BVerfG hat einen Verstoß gegen Art. 33 II GG i.V.m. Art. 3 III 2 GG darin gesehen, dass einer behinderten Beamtin die Beförderung bzw. der Aufstieg in höhere Ämter allein wegen ihrer Behinderung und ohne Prüfung im Einzelfall versagt wurde. ${ }^{871}$ Es stellte fest, dass bei der gesundheitlichen Eignungsbeurteilung auch dem Verbot der Benachteiligung behinderter Menschen aus Art. 3 III 2 GG Rechnung zu tragen ist. Ein Bewerber dürfe daher nur dann von einer Beförderung ausgeschlossen werden, wenn dienstliche Bedürfnisse eine dauerhafte Verwendung in dem angestrebten Amt zwingend ausschließen. ${ }^{872}$ Es hat sich dabei ausdrücklich auf ein Urteil

866 Luthe, in: Rehabilitationsrecht - Verfassungsrecht, Rn. 8

Vgl. Neumann, in: Handbuch SGB IX, § 2 Verfassungsrecht, Rn. 27; Luthe, in: Rehabilitationsrecht - Verfassungsrecht, Rn. 8

Christina Schönfeld s. Theben, in: Gesellschaft der Behinderer S. 81; http://www.ensembleaventure.de/fotoserie3/foto_1.html 81; http://www.taubenschlag.de/html/ssh/1105.htm Pablo Piñeda, näheres s. http://www.focus.de/wissen/wissenschaft/bildung-dieunmoegliche-karriere_aid_198391.html und Tobias Wolf, Bayern, http://www.aktionmensch.de/inklusion/in-der-schule/inklusion-im-kollegium.php OVG Münster, Beschluss v. 4.9.2008; Az.: 19 B 1293/08, www.justizr.nrw.de BVerfG 2 BvR 2571/07 vom 10.12.2008, http://www.bverfg.de/entscheidungen/rk20081210 __bvr257107.html - betrifft eine Beamtin mit Herzerkrankung; ebenso OVG Bautzen Beschluss v. 15.6.2009, 2 A 140/08, juris - betrifft einen Beamten mit Diabetes 
des BVerwG bezogen, in dem dieses für die Frage der gesundheitlichen Eignung festgestellt hat: „Kann ein schwerbehinderter Bewerber für ein Amt die Anforderungen des Amtes gerade aufgrund seiner Behinderung nicht erfüllen, so folgt aus dem unmittelbar geltenden Benachteiligungsverbot des Art. 3 III 2 GG, dass die gesundheitliche Eignung nur verneint werden darf, wenn im Einzelfall zwingende Gründe für das Festhalten am allgemeinen Maßstab sprechen. “873 Das bedeutet, dass allein die Berufung auf eine Behinderung nicht ausreicht, sondern bei jeder Einschränkung der Beschäftigung und des Aufstiegs, die an gesundheitliche Defizite anknüpft, der Nachweis zu führen ist, dass die angestrebte Aufgabe unter Ausschöpfung aller Möglichkeiten dem behinderten Arbeitnehmer nicht übertragen werden kann. ${ }^{874}$ Mit dieser Auslegung von Art. 3 III 2 GG durch das BVerfG ist der pauschale Verweis auf die Nichteignung wegen einer Behinderung und die pauschale Nicht-Berücksichtigung einer Behinderung in einer Prüfung unvereinbar.

Der Grundsatz des Prüfungsrechts, dass „persönlichkeitsbedingte Eigenschaft, die die Leistungsfähigkeit prägen " in der Prüfung nicht zu berücksichtigen sind, ist mit Art. 3 III 2 GG nicht vereinbar und kann daher die Benachteiligung durch Gleichbehandlung bezüglich der inhaltlichen Prüfungsanforderungen nicht rechtfertigen.

\subsection{Art. 7 I GG: Sicherung von Bildungsstandards und Vergleichbarkeit der Schulabschlüsse}

\subsubsection{Zulässigkeit von Standards und standardisierten Abschlüssen}

Kollidierendes Verfassungsrecht, das einen Eingriff in Art. 3 III 2 GG rechtfertigen kann, kann die staatliche Schulaufsicht aus Art. 7 I GG sein. Ob das vorliegend der Fall ist, ist streitig. Nach der Rechtsprechung des BVerfG bindet das Recht aus Art. 7 I GG wie jedes andere Grundrecht die gesamte staatliche Gewalt und kann deshalb nicht selbst die Wirkung der Grundrechte, insbesondere von Art. 3 III 2 GG, begrenzen. ${ }^{875}$ In der Rechtsprechung und auch in der Literatur $^{876}$ wird die Ansicht vertreten, dass die staatliche Schulaufsicht das Grundrecht aus Art. 3 III 2 GG begrenzt. ${ }^{877}$ Andere halten dem entgegen, dass es sich

873 BVerwG Urteil v. 21.6.2007, Az.: 2 A 6/ 06, http://www.bundesverwaltungsgericht.de/media/ archive/5320.pdf Rn. 20 - betrifft einen Soldaten mit chronischer Polyathritis

874 Gagel, Benachteiligung wegen Behinderung, Punkt D

875 BVerfGE 96, 288, 313, Rn. 88

876 Luthe, in: Rehabilitationsrecht - Verfassungsrecht, Rn. 8; Osterloh, in: Sachs GG, Art. 3, Rn. 314; unklar Neumann, in: Handbuch SGB IX, § 2 Verfassungsrecht, Rn. 27

877 BayVGH, Urteil v. 11.12.1996, Az.: 7 B 96.2568, BayVBI 1997, S. 561, 563; Urteil v. 9.7.1997, Az.: 7 B 97.1185, BayVBI 1998, S. 180, 183, s. Höller, Beschulung behinderter Kinder und Jugendlicher an öffentlichen Schulen S. 231, Fn. 730 
bei Art. 7 I GG um eine reine Organisations- und Kompetenznorm handele, die nicht zum kollidierenden Verfassungsrecht gehöre und daher Art. 3 III 2 GG nicht einschränken könne. ${ }^{878}$ Die staatliche Schulaufsicht ist zwar im Bereich der Grundrechte geregelt, beinhaltet aber kein Grundrecht, sondern eine Organisationsnorm. ${ }^{879}$ Das schließt aber nicht prinzipiell aus, daraus ein Recht des Staates auf Ausgestaltung der Bildung mit Verfassungsrang abzuleiten.

Art. 7 । GG ist die zentrale Norm für die schulische Erziehung durch den Staat. Hierin liegt nach allgemeiner Ansicht die bundesverfassungsrechtliche Grundlage für den Erziehungsauftrag des Staates. ${ }^{880}$ Der Erziehungsauftrag des Staates dient ganz wesentlich dem Ausgleich vorhandener Unterschiede und soll sicherstellen, dass jedermann gleichen Zugang zur Bildung und damit die Möglichkeit zur Entfaltung seiner Persönlichkeit, wie sie in Art. 2 I GG geschützt ist, sowie die gleiche Chance bei der Ausbildungs- und Berufswahl erhält. ${ }^{881}$ Der Begriff der Schulaufsicht umfasst die Gesamtheit der dem Staat an der Schule und über die Schule zustehende Rechte und beinhaltet somit die staatlichen Befugnisse zur Organisation, Planung, Leitung und Beaufsichtigung des Schulwesens ${ }^{882}$, wozu auch die organisatorische Gliederung der Schule und die Regelung der allgemeinen Schulpflicht gehören. Zu dem Recht gehört auch die Ausgestaltung des Schulwesens in verschiedene Schullaufbahnen und Schulabschlüsse, die dazu dient, ein differenziertes, begabungsgerechtes und leistungsfähiges Schulsystem vorzuhalten, das dem Recht auf Bildung der Kinder und ihren vielfältigen Begabungen und Neigungen Rechnung trägt. Ein Mittel dazu ist die Differenzierung in verschiedene Schulabschlüsse, die bestimmte Aussagewerte und Folgewirkungen haben und die in Deutschland eine lange Tradition haben. Ganz unstreitig hat der Schulgesetzgeber das Recht, Lehrziele zu setzen, Prüfungsinhalte festzulegen und darüber zu entscheiden, ob und inwieweit ein Schüler diese erreicht hat. ${ }^{883}$

Um die Vergleichbarkeit der Abschlüsse und die Berufsfreiheit zu gewährleisten, ist es notwendig, sicherzustellen, dass Prüfungen und Schulabschlüssen ein gleiches Anforderungsniveau zugrunde liegt. Die Vergleichbarkeit der Schul-

878 Beaucamp, JA 2001, S. 36 ff, S. 39; Welti, Behinderung und Rehabilitation im sozialen Rechtsstaat, S. 450; Castendiek, in: GS Jean d'Heur, S. 338, Fn. 11 und 343

Jürgens/Römer, NVwZ 1999, S. 847 ff, S. 848; Jarass/Pieroth, in: Jarass/Pieroth GG Art. 7, Rn. 41; Höller, Beschulung behinderter Kinder und Jugendlicher an öffentlichen Schulen, S. 233; Buch, Grundrecht der Behinderten, S. 286

Langenfeld, Integration und kulturelle Identität zugewanderter Minderheiten, S. 213 m.w.N.

BVerfGE, 47, $48 \mathrm{ff}$ - st. Rspr.; vgl. umfassend Langenfeld, Integration und kulturelle Identität zugewanderter Minderheiten, S. $212 \mathrm{ff}$, grundsätzlich zu Bildungsstandards Oelkers, RdJB 2009, S. $190 \mathrm{ff}$, S. $193 \mathrm{ff}$ 
abschlüsse ist ein zentrales Anliegen der KMK, die inzwischen Bildungsstandards für die Allgemeine Hochschulreife in den Fächern Deutsch, Mathematik und Fortgeführte Fremdsprache Englisch/Französisch verabschiedet hat. ${ }^{884}$ Diese neuen Bildungsstandards, ein neuer bundesweiter Aufgabenpool mit gleich schweren, standardisierten Abiturprüfungsaufgaben und einheitlichen Kriterien zur Korrektur und Bewertung der Abiturarbeiten, sollen die Vergleichbarkeit und die Qualität der Allgemeinen Hochschulreife verbessern und die Mobilität in Deutschland erleichtern. Darüber hinaus hat sich die KMK auch auf Bildungsstandards für die anderen Schulabschlüsse geeinigt. Schulabschlüsse sind die „Eintrittskarte" in die Berufsausbildung. Nicht nur der Staat (vgl. § 17 BBG, § 14 NI BG; Art. $31 \mathrm{ff}$ BayBG), sondern auch die Privatwirtschaft knüpfen bei Einstellungen an qualifizierte staatliche Schulabschlüsse an. ${ }^{885}$ Vor allem die Abiturnote entscheidet in einem sehr großen Maß über den Zugang zur Hochschulausbildung. Standards dienen daher gerade dazu die Grundrechte aller Schüler und Schülerinnen aus Art. 3 I GG auf Chancengleichheit und aus Art. 2 I GG und Art. 12 I GG auf Entfaltung ihrer Persönlichkeit und Berufsfreiheit zu sichern. Insofern ist es ein legitimes Ziel und eine geeignete Maßnahme des Gesetzgebers, die Vergleichbarkeit der Schulabschlüsse durch Standards zu gewährleisten.

\subsubsection{Abwägung zwischen Art. 7 I GG und Art. 3 III 2 GG}

Die maßgebliche Frage ist dann, ob diese Standards absolut zu setzen sind und eine Veränderung der gesetzten inhaltlichen Prüfungsanforderungen verbieten, auch dann, wenn Grundlage der Veränderung spezifische Behinderungen sind. Die Rechtsprechung vertritt diese Ansicht und hält ein Abweichen von diesen Standards nicht für zulässig. ${ }^{886}$ Sie sieht zwar, dass das ausnahmslose Festhalten an Standards bei Schülern und Schülerinnen mit Legasthenie/Dyskalkulie ein Eingriff in das Grundrecht aus Art. 3 III 2 GG ist, meint aber, dass dem staat-

${ }^{884}$ Beschluss der KMK vom 18.10.2012, Standards s. Anhang ॥

885

Oppermann, in: HStR VI, § 135, Rn. 5

OVG Bautzen, Beschluss v. 3.11.2008, Az.: 2 B 292/08, S. 6 f zu einer nicht genannten Behinderung (nicht veröffentlicht); OVG Lüneburg, Beschluss v. 10.7.2008, Az.: 2 ME 309/08, www.rechtsprechung.niedersachsen.de, Rn. 12; Beschluss v. 25.11.2011, Az.: 2 ME 52/11, S. 4 f (nicht veröffentlicht); Beschluss v. 20.9.2012, Az.: 2 LA 234/11, S. 5 f (nicht veröffentlicht); VGH Kassel Beschluss v. 5.2.2010, Az.: 7 A 2406/09.z, juris, Rn. 43, 59; VG Aachen, Urteil v. 13.11.2009, Az.: 9 K 25/09, www.justiz.nrw.de, Rn. 56, 70; VG Düsseldorf, Beschluss v. 16.11.2009, Az.: 18 L 1654/09, juris, zur Dyskalkulie, Rn. 20 f; Beschluss v. 20.5.2011, Az.: 18 L 707/11, juris VG Gießen, Urteil v. 2.11.2010, Az.: 7 K 1841/10, S. 12 (nicht veröffentlicht); VG Saarlouis, Beschluss v. 17.3.2008, Az.: 1 L 169/08, juris, Rn. 52; VG Sigmaringen, Beschluss v. 2.4.2008, Az.: 4 K 596/08, S. 4 (nicht veröffentlicht); offen lassend VG Hannover, Beschluss v. 13.12.2010, Az.: 6 B 5596/10, www.rechtsprechung.niedersachsen.de, Rn. 29; so auch KMK-Inklusive Bildung Punkt II.4 
lichen Erziehungsauftrag aus Art. 7 GG hier ein überwiegendes Interesse zukommt.

Die Rechtsprechung tendiert daher dahin, die staatliche Verpflichtung zur Erhaltung der Verfassungsinstitution „Schule“ als umfassende Garantie zu verstehen, die die Grundrechte begrenzt. Wenn jedoch der Funktionsfähigkeit oder dem Erhalt einer Verfassungsinstitution in der Abwägung mit Grundrechten vorschnell der Vorrang eingeräumt wird, dann wird der Grundrechtsschutz wesentlich verkürzt und die Schrankensystematik umgangen. ${ }^{887}$ Ein solcher Vorrang würde auch das Grundrecht aus Art. 3 III 2 GG leer laufen lassen ${ }^{888}$, weil es damit der Staat in der Hand hätte, anhand von sachlichen Gründen eine Benachteiligung zu rechtfertigen. ${ }^{889}$ Art. 7 । GG hat daher keinen prinzipiellen Vorrang gegenüber Art. 3 III 2 GG. ${ }^{890}$

Die Schulaufsicht ist nicht Selbstzweck, sondern besteht allein im Interesse der Kinder, die dem Staat zur schulischen Betreuung anvertraut werden. ${ }^{891}$ Die Grundrechte der Kinder verpflichten den Staat dazu, für behinderte Kinder und Jugendliche schulische Einrichtungen bereit zu halten, die ihnen eine sachgerechte schulische Erziehung, Bildung und Ausbildung ermöglichen. ${ }^{892}$ Deshalb ist bereits im Rahmen des Erziehungsauftrags nach Art. 7 Abs. 1 GG die Wertung des Art. 3 III 2 GG zu beachten ${ }^{893}$ und dieser Erziehungsauftrag so wahrzunehmen, dass bei seiner Ausübung das Grundrecht geschützt wird. Grundsätzlich beinhaltet dies die Pflicht des Staates, sich schützend und fördernd für die Gleichheit behinderter Menschen einzusetzen und auf die rechtliche und faktische Gleichheit im Sinne von gesellschaftlicher und sozialer Gleichstellung hinzuwirken. ${ }^{894}$ Weil der Bildungssektor entscheidend durch den Staat geprägt ist und von kaum zu überschätzender Wichtigkeit für die Kinder und Jugendlichen ist, ist der Staat dazu verpflichtet, gerade die Schule seiner Grundordnung

887

888

889

890

Reichenbach, Anspruch behinderter Schülerinnen und Schüler auf Unterricht in der Regelschule, S. 261; Höller, Beschulung behinderter Kinder und Jugendlicher an öffentlichen Schulen, S. 233; Jürgens/Römer, NVwZ 1999, S. 847 ff, S. 848; Buch, Grundrecht der Behinderten, S. 286; Sacksofsky, in: Umbach GG, Art. 3, Rn. 417

Höller, Beschulung behinderter Kinder und Jugendlicher an öffentlichen Schulen, S. 233;

Welti, Behinderung und Rehabilitation im sozialen Rechtsstaat, S. 450

Reichenbach, Anspruch behinderter Schülerinnen und Schüler auf Unterricht in der Regelschule, S. 261; Sacksofsky, in: Umbach GG, Art. 3, Rn. 417

Straßmair, Der besondere Gleichheitssatz aus Art. 3 Abs. 3 Satz 2 GG, S. 261; Castendiek, in: GS Jean d'Heur, S. 347; Caspar, EuGRZ 2000, S. 135 ff, S. 137 
entsprechend zu gestalten und zu erfüllen. ${ }^{895}$ Daher überlagert Art. 7 । GG weder Art. 3 III 2 GG noch verdrängt er ihn. ${ }^{896}$

Es muss also zwischen dem Verfassungsauftrag aus Art. 7 I GG und dem Grundrecht aus Art. 3 III 2 GG abgewogen werden. Aus dem aufgezeigten Rechtfertigungsmaßstab des Art. 3 III $2 \mathrm{GG}^{897}$ folgt, dass es nicht ausreicht, wenn sachliche Gründe für ein Überwiegen des Verfassungsauftrages sprechen. Im schulischen Bereich muss sich der Staat die durch die Gleichbehandlung eintretenden Folgen seiner Regelungen in höherem Maße zurechnen lassen als im außerschulischen Bereich. ${ }^{898}$ Je gewichtiger die Folgen einer Regelung im Hinblick auf Art und Intensität der eintretenden Nachteile für den Betroffenen sind, umso gewichtiger müssen die Gründe sein, die diese Regelung rechtfertigen. ${ }^{899}$ Damit ist Ungleichbehandlung bzw. Gleichbehandlung jedenfalls nicht durch solche Differenzierungsgründe zu rechtfertigen, die auf Umständen beruhen, die der Staat selbst in der Hand hat. ${ }^{900}$ Vielmehr kann der Erziehungsauftrag nur dann überwiegen, wenn der dadurch verursachte Eingriff in das Grundrecht aus Art. 3 III 2 zwingend erforderlich ${ }^{901}$, d.h. unerlässlich und alternativlos ist. ${ }^{902}$ Es darf keine Möglichkeit geben, denselben Erfolg mit einem weniger schweren Eingriff zu erzielen, der Art. 3 III 2 GG fühlbar weniger einschränkt. ${ }^{903}$ Dabei ist wiederum zu bedenken, dass den Staat aus der BRK nicht nur die Pflicht trifft, den Bildungsbereich diskriminierungsfrei zu gestalten, sondern er aus Art. 4 BRK auch allgemeine Schutz- und Förderpflichten hat, die zum Ziel haben, die Grundsätze aus Art. 3 BRK umzusetzen.

Damit ist es ausgeschlossen, allein deshalb die Veränderung von Prüfungsbedingungen abzulehnen, weil Bildungsstandards und standardisierte Schulabschlüsse dem deutschen Bildungssystem entsprechen und einen hohen Verfassungswert haben, denn der Staat hat es in der Hand, diese Bedingungen so zu verändern, dass auf die Belange von Menschen mit Behinderungen Rücksicht genommen wird und damit negative Folgen für sie abgewendet werden. Die Alternative, die die Rechte von Menschen mit Behinderungen wahrt oder zumin-

895 Reichenbach, Anspruch behinderter Schülerinnen und Schüler auf Unterricht in der Regelschule S. $212 \mathrm{f}$

896 Sacksofsky, in: Umbach GG, Art. 3, Rn. 417

897 S. dazu oben 3. Teil: 5. Kapitel: 1.: Rechtfertigungsmaßstab, S. 145

898 Straßmair, Der besondere Gleichheitssatz aus Art. 3 Abs. 3 Satz 2 GG, S. 236

Straßmair, Der besondere Gleichheitssatz aus Art. 3 Abs. 3 Satz 2 GG, S. 237

900 Straßmair, Der besondere Gleichheitssatz aus Art. 3 Abs. 3 Satz 2 GG, S. 237

901 Vgl. BVerfGE 99, 341, 357

902 Vgl. Luthe, in: Rehabilitationsrecht - Verfassungsrecht, Rn. 8

903 BVerfGE 90, 145, 172; Jarass/Pieroth, in: Jarass/Pieroth GG, Art. 20, Rn. 85; Sodan/Ziekow, Öffentliches Recht, § 24, Rn. 41; Stern, Staatsrecht, S. 779; Michael/Morlok, Grundrechte, Rn. 620 
dest weniger beeinträchtigt, ist eine Veränderung der inhaltlichen Prüfungsbedingungen. Im Ergebnis kann eine solche Auslegung allerdings dazu führen, dass die Schule auf vielfältige Behinderungen Rücksicht nehmen muss und damit das Institut der herkömmlichen Schulabschlüsse und der allgemeinen Hochschulreife in Frage gestellt werden könnte. ${ }^{904}$ Die Furcht davor und auch davor, mit der Anerkennung von bestimmten Behinderungen eine Schleuse zu öffnen, ist groß. So hat der Landtag Baden-Württemberg 2006 ausdrücklich die Anerkennung der Legasthenie abgelehnt, „da ja dann über den Gleichheitssatz auch alle anderen Leistungsschwächen zu berücksichtigen wären (z.B. Dyskalkulie, Aufmerksamkeitsdefizitsyndrom, minor brain dysfunction, Hyperaktivität, Milieuschäden)“. ${ }^{905}$

\subsubsection{Vorrang von Art. 7 I GG bei Fehlen zwingend erforderlicher Fähigkeiten}

Das Festhalten an den allgemeinen Standards könnte jedoch dadurch gerechtfertigt sein, dass sich aus der Behinderung Legasthenie/Dyskalkulie zwingend ergibt, dass diesen behinderten Schülern unerlässlich abzuprüfende Fertigkeiten oder Fähigkeiten fehlen und sie deshalb für bestimmte Schulabschlüsse und die sich daraus ergebenden Ausbildungs- und Berufsmöglichkeiten ungeeignet sind. Das VG Düsseldorf ${ }^{906}$ und auch Kischel ${ }^{907}$ gehen davon aus, dass dies bei Schülern und Schülerinnen mit Legasthenie/Dyskalkulie der Fall ist und es deshalb berechtigt ist, darauf keine Rücksicht zu nehmen, weil die Schüler die elementarsten Fähigkeiten nicht besitzen, die Schule überhaupt vermitteln soll. ${ }^{908}$

Das klingt zunächst überzeugend. Es erklärt aber nicht, warum in vielen Fällen, wie Sport bei körperbehinderten Schülern, Musik bei tauben Schülern, Kunst bei blinden Schülern oder Schreiben (und damit auch Rechtschreiben) bei Schülern mit motorischen Schwierigkeiten aufgrund von Spastiken ${ }^{909}$ auf die Bewertung eines Faches oder Teilaspektes eines Faches völlig verzichtet wird und bei Schülern und Schülerinnen mit Legasthenie/Dyskalkulie nicht Äquivalentes gelten soll. Während im ersten Fall die Bewertung wegen der feh-

904 Rux weist daraufhin, dass das Konzept der allgemeinen Hochschulreife mit dem Inklusionskonzept der BRK nicht mehr vereinbar ist. Rux, RdJB 2009, S. 220 ff, S. 228; Poscher/Rux/ Langer, Recht auf Bildung, S. 58, Fn. 153 Landtag Baden-Württemberg, Drs. 14/630 v. 27.11.2006, Antwort auf Frage 4

906 VG Düsseldorf, Beschluss v. 20.5.2011, Az.: 18 L 707/11, juris, Rn. $13 \mathrm{f}$

907 Kischel, in: BeckOK GG, Art. 3, Rn. 219.2

908 Kischel, in: BeckOK GG, Art. 3, Rn. 219.2

909 S. z.B. VG Lüneburg, Urteil v. 28.5.2002, Az.: 4 A 246/01, www.rechtsprechung.nieder sachsen.de, oder ein mir bekannter Schüler mit spastischer Behinderung, der in seiner gesamten Schulzeit bis einschließlich Abitur einen Zivildienstleistenden zur Unterstützung im Unterricht und in den Prüfungen hatte, der auch die Klausuren niederschrieb. 
lenden Fähigkeit völlig wegfällt, wird im zweiten Fall gerade die nicht vorhandene oder eingeschränkte Fähigkeit bewertet. Das VG Düsseldorf hat die unterschiedliche Behandlung damit begründet, dass etwa blinde Schüler auch bei bester Förderung nicht sehen lernen können, während Schüler und Schülerinnen mit Legasthenie durch die Förderung in die Lage versetzt werden sollen, ausreichende Kompetenzen im Lesen und Schreiben zu erreichen. Erreichen sie dieses Förderziel nicht, so gehe dies zu ihren Lasten. ${ }^{910}$ Das Gericht unterscheidet damit zwischen einer behebbaren Behinderung und einer nicht behebbaren. Eine derartige Differenzierung bürdet dem Menschen, der eine (angeblich) behebbare Behinderung hat, die Pflicht auf, sich um die Behebung dieser Behinderung zu bemühen. ${ }^{911}$ Gelingt ihm das nicht, verliert er de facto das Recht aus Art. 3 III 2 GG. Eine solche Interpretation des Behinderungsbegriffs und des Schutzbereichs von Art. 3 III 2 GG ist mit dem oben dargelegten sozialen Behinderungsbegriff nicht zu vereinbaren.

Für die Frage, welche Folge das Fehlen von notwendigen Fähigkeiten hat, kommt es im Schulalltag offenbar darauf an, welches oder wie viele Fächer betroffen sind und wie wichtig dieses Fach im schulischen Lehrplan ist. Ohne dass dies ausdrücklich thematisiert wird, findet eine Abwägung zwischen der Notwendigkeit oder Bedeutung des Faches für die Schullaufbahn bzw. den Schulabschluss und der jeweils eingeschränkten Fähigkeit des Prüflings in diesem Fach statt. Die Abwägung ergibt dann vor allem bei körperbehinderten Schülern und Schülerinnen überwiegend, dass der Wert des Faches als nicht zwingend für die Qualifikation für den Bildungsgang und den Abschluss angesehen wird und deshalb wegen der Behinderung zurücktritt. Die Abwägung ergibt somit ein Überwiegen der Behinderung gegenüber dem betroffenen Fach. Je mehr Fächer betroffen sind und je mehr es sich um nicht sichtbare ${ }^{912}$ Einschränkungen handelt, desto weniger wird die Behinderung berücksichtigt. Bei Legasthenie findet eine solche Abwägung statt, indem in der Literatur und in der Rechtsprechung die Fähigkeit zum Lesen und richtigen Schreiben für so

910 VG Düsseldorf, Beschluss v. 20.5.2011, Az.: 18 L 707/11, juris, Rn. 14. Die Entscheidung zeigt, dass das Gericht, obwohl es die Legasthenie als Behinderung qualifiziert hat, nicht daran glaubt, dass die Fähigkeit zur Rechtschreibung wirklich eingeschränkt und nicht erlernbar ist. Sonst würde es von dem Schüler nicht erwarten, dass er diese in der Förderung in ausreichendem Maße lernt. Auch bei Legasthenie ist die Förderung wie bei einem blinden Schüler darauf gerichtet, den Umgang mit der Behinderung zu lernen und sie soweit wie möglich zu kompensieren, nicht aber das unabänderliche Defizit zu beheben.

911 S. dazu ausführlich 5. Teil: 2. Kapitel: 2.1.: Pflicht zur Teilnahme an Förderung, S. 202

912 Etwa weil sie Mukoviszidose oder Multiple Sklerose oder Stoffwechselerkrankungen haben, s. zum besonderen Problem der nicht sichtbaren Behinderungen Kirchner, Nicht sichtbare Behinderung 
zwingend gehalten werden, dass ihr Fehlen ${ }^{913}$ den Besuch eines Gymnasiums ${ }^{914}$ ausschließt und auf Beeinträchtigungen in diesem Bereich keine Rücksicht genommen werden kann. ${ }^{915}$ Andere sehen in der Beeinträchtigung dagegen eine einseitige Beeinträchtigung, die durch Kompensationen ausgeglichen werden könne und an der generellen Eignung nichts ändere. ${ }^{916}$ Eine offene Abwägung findet auch bei der Berücksichtigung der Dyskalkulie statt. Hier wird von der Kultusverwaltung explizit vertreten, dass das Fach Mathematik so wichtig ist, dass darauf nicht verzichtet werden kann. ${ }^{917}$

Diesem Abwägungsvorgang, der im deutschen Recht bisher nicht dogmatisch aufgearbeitet ist, liegt die Fragestellung zugrunde, ob der behinderte Schüler trotz seiner Behinderung und des dadurch bedingten Ausfalls von Fächern, für geeignet gehalten wird, den anvisierten Bildungsgang zu durchlaufen und den Schulabschluss zu machen. Er lässt sich mit dem im US-amerikanischen Recht herrschenden Abwägungsgebot vergleichen. Section 504 des Rehabilitation Act of $1973^{918}$ besagt: „No otherwise qualified individual with a disability in the United States ... shall, solely by reason of her or his disability, be excluded from the participation in, be denied the benefits of, or be subjected to discrimination under any program or activity receiving Federal financial assistance ...". Dieses allgemeine Benachteiligungsverbot stellt entscheidend darauf ab, ob die Person mit Behinderung „otherwise qualified“, also „ansonsten geeignet" ist. ${ }^{919}$

Dogmatische Grundlage der Prüfung ist in unserem Fall zunächst Art. 3 । GG, wonach alle Schüler und Schülerinnen mit Behinderungen untereinander gleich zu behandeln sind. D.h. der Abwägungsvorgang bezüglich der Veränderung von inhaltlichen Prüfungsanforderungen muss für alle Schüler und Schülerinnen mit Behinderungen gleich sein, unabhängig davon, ob die Behinderungen sichtbar oder unsichtbar, körperlich oder psychisch sind. Dann ist weiter bei allen Schü-

913 Wobei nicht zwischen der eingeschränkten Fähigkeit und dem völligen Fehlen unterschieden wird.

914 OVG Münster, Beschluss v. 4.9.2008; Az.: 19 B 1293/08, www.justizr.nrw.de, Rn. 17, 19, 23

915

Kischel, in: BeckOK GG, Art. 3, Rn. 219.3; VG Düsseldorf, Beschluss v. 20.5.2011, Az.: 18 L

$707 / 11$, juris, Rn. $13 \mathrm{f}$

916 Niehues/Rux, Schulrecht, Rn. 446; Langenfeld, RdJB 2007, S. 211 ff, S. 219 ff; Ennuschat, br 2008, S. 93 ff, S. $96 \mathrm{ff}$

917 KMK-Lesen/Rechtschreiben/Rechnen: Punkt: Schülerinnen und Schüler mit besonderen Schwierigkeiten im Rechnen; Bay. Landtag, Schriftliche Anfrage Drucksache Nr. 16/5468 vom 06.07.2010, Zu Nr. 6 und Schriftliche Anfrage Drucksache Nr. 16/5698 vom 18.8.2010, Zu. Nr. 5, abrufbar unter: www.bayern.landtag.de

918 http://www.epa.gov/ocr/sec504.htm

919 S. dazu ausführlich Fuerst, Behinderung zwischen Diskriminierungsschutz und Rehabilitationsrecht, S. $91 \mathrm{ff}$ 
lern und Schülerinnen mit Behinderungen zu prüfen, ob die Schüler und Schülerinnen mit Behinderungen „ansonsten geeignet" sind.

Die Frage ist daher, ob Schüler und Schülerinnen mit Legasthenie/Dyskalkulie "ansonsten geeignet" sind für die angestrebten Bildungsgänge und Schulabschlüsse. Den höchsten Stellenwert hat das Abitur, deshalb wird an seinen strengsten Anforderungen geprüft, ob die Einschränkung der Fähigkeiten dazu führen, dass die Schüler und Schülerinnen für ein Abitur nicht geeignet sind. Das käme in Betracht, wenn die Fähigkeiten in den Kulturtechniken ein geeignetes Kriterium sind, um auf die allgemeine Eignung und Begabung des Schülers zu schließen. Die vielfachen Urteile zur Berücksichtigung der Legasthenie in der Oberstufe zeigen, dass die Schüler und Schülerinnen mit Legasthenie nicht von vornherein ungeeignet für diese Schulart und für das Abitur sind. Die Urteile des OVG Lüneburg ${ }^{920}$ und des VGH Kassel ${ }^{921}$ zur (Nicht-)Berücksichtigung der Legasthenie in der Oberstufe betreffen Schüler, die überdurchschnittlich begabt bis hochbegabt sind. ${ }^{922}$ Für den Fall des VG Schleswig ${ }^{923}$ ist das aus der Sachverhaltsschilderung zu vermuten. Eine Schülerin mit Dyskalkulie, die in Niedersachsen wegen 0 Punkten im letzten Mathematik-Grundkurs nicht zum Abitur zugelassen wurde, hatte zu diesem Zeitpunkt einen Notenschnitt von 1,8. Alle Länderregelungen zu den Lese-Rechtschreibschwierigkeiten sehen vor, dass die Schwierigkeiten im Lesen und Rechtschreiben bei sonst angemessener Gesamtleistung kein Grund sein sollen, den Schüler von einem Wechsel an eine weiterführende Schule auszuschließen. ${ }^{924}$ Grundsätzlich besteht daher die Auffassung, dass die betroffenen Kinder an sich für alle Schullaufbahnen geeignet und begabt sind. Die grundsätzliche Eignung für alle Schulabschlüsse ergibt sich auch aus der Erfahrung in Bayern, wo die Legasthenie seit 1999 umfangreich berücksichtigt wird und deshalb dort inzwischen zahlreiche Schüler mit Legasthenie Abitur gemacht haben. Es gibt aus Bayern keinerlei Hinweise, etwa aus den Universitäten oder aus der Wirtschaft, dass diese Abiturienten für die anschließenden Studien und Ausbildungen ungeeignet sind. Schließlich ist ein Indiz für die grundsätzliche Eignung der Schüler und Schülerinnen mit Legasthenie/Dyskalkulie auch die Diagnostik der Störungen, die zwingend voraussetzt, dass die Intelligenz mindestens im durchschnittlichen Bereich liegt.

920 OVG Lüneburg, Beschluss v. 10.7.2008, Az.: 2 ME 309/08, www.rechtsprechung.niedersachsen.de

921 VGH Kassel, Beschluss v. 5.2.2010, Az.: 7 A 2406/09, juris

922 Das ist mir persönlich bekannt. Der Schüler des Verfahrens OVG Lüneburg hat sein Abitur als Jahrgangsbester absolviert.

923 VG Schleswig; Urteil v. 10.6.2009, Az.: 9 A 208/08, BeckRS 200938123

924 BE GsVO § 16 IX - allerdings unpräzise; BB VV-LRSR Nr. 2 VII; BY KMBek Legasthenie Nr. 3.5; HB LRS-Erlass Nr. 4.3; HH Förder-Richtlinie Nr. 4.4; HE VO-GestSchulVerh § 37 V; MV VV-LRR Nr. 8 II; NI LRR-Erlass Nr. 4.2; NRW LRS-Erlass Nr. 4.3; RP VV-LRS Nr. 4.5; SL VV-LRS Nr. 5.4 
Möglich wäre, dass insbesondere Rechtschreibkenntnisse etwas darüber aussagen, ob jemand für bestimmte Fächer oder Studiengänge, etwa Rechtswissenschaften, Theologie oder Psychologie, geeignet ist. ${ }^{925}$ Dann müssten sich aus der Rechtschreibung Rückschlüsse auf die Fähigkeiten in diesen Fächern ziehen lassen. Angesichts des erheblichen Curriculums dieser Fächer ist nicht zu erwarten, dass die Rechtschreibleistung eine Aussage darüber zulässt, ob jemand juristische Sachverhalte lösen kann oder darüber, wie geeignet jemand für diese Fächer ist. Wenn man das aber überhaupt in Betracht ziehen will, dann müssten die Rechtschreibfähigkeiten explizit ausgewiesen werden, um daran anknüpfen zu können und jemanden wegen der schlechten Rechtschreibung von einem Studiengang auszuschließen. Das ist aber nicht der Fall.

Fraglich ist weiter, welche Rückschlüsse sich aus schlechten Noten in Mathematik ziehen lassen. Sicherlich lässt sich daraus auf die Eignung für mathematische Studiengänge oder Studiengänge mit sehr viel Mathematik, etwa Physik, schließen, weil die Mathematiknote sehr eindeutig an die mathematischen Fähigkeiten anknüpft und nicht wie die Sprachenfächer aus mehreren Fähigkeitsbereichen zusammengesetzt ist. Die allgemeine Studierfähigkeit umfasst jedoch sehr viel mehr Fähigkeiten, so dass sich allein aus den Noten in Mathematik nicht der Schluss der generellen Ungeeignetheit für ein akademisches Studium ziehen lässt.

Zwingende Gründe für das Festhalten am allgemeinen Maßstab der inhaltlichen Prüfungsanforderungen könnten schließlich vorliegen, wenn die Kulturtechniken nicht kompensierbar wären, es also zwingend wäre, dass die Absolventen diese Fähigkeiten in vollem Umfang selbst (handschriftlich) beherrschen. Dann würde auch die Möglichkeit entfallen, den Nachteil der Behinderung durch angemessene Vorkehrungen zu beseitigen. Die Kompensationsmöglichkeiten im Bereich des Lesens, Schreibens und Rechnens sind inzwischen immens und nehmen stetig zu. Durch Computer und vielfältige Softwareprogramme gibt es vielfache Möglichkeiten, Einschränkungen in diesem Bereich zu kompensieren. Vermehrt kommen Hörbücher auf den Markt ${ }^{926}$ und werden Internetseiten so gestaltet, dass sie sich der Nutzer vorlesen lassen kann. In der Schule können Lehrer mit einfachen Programmen die am PC erstellten Aufgabenblätter und Klassenarbeiten in vorgelesene Texte im MP3-Format umwandeln, die die betroffenen Schüler dann über MP3-Player anhören können. Mithilfe des PCs können Texte vorgelesen, diktiert und auf Rechtschreibung überprüft $^{927}$ werden. Taschenrechner helfen schon bei den Grundrechenarten, Rechenprogramme ermöglichen kompliziertere Rechenoperationen. Sobald die

\footnotetext{
925 In diese Richtung geht Kischel, in: BeckOK GG, Art. 3, Rn. 219.3

926 In Holland können Schulen für Legastheniker Schulbücher als Hörbücher ausleihen. http://www.balansdigitaal.nl/sitemanager.asp?nieuws=28399

927 Lingcheck, http://lingit.de/produkte/lingcheck
} 
Schüler daher in der Lage sind, PCs zu benutzen, können sie lernen ihre eingeschränkten Fähigkeiten zu kompensieren. Damit ist es ihnen möglich, einen relativ fehlerfreien Text zu erstellen und abzugeben. Es ist sicherlich in hohem Maße wünschenswert, aber nicht zwingend erforderlich, dass diese Fähigkeiten eigenhändig beherrscht werden.

Die eingeschränkten oder auch fehlenden Fähigkeiten der Schülerinnen und Schüler mit Legasthenie/Dyskalkulie führen daher nicht dazu, dass diese für die Schullaufbahnen und-abschlüsse nicht geeignet sind und deshalb die Barrieren der inhaltlichen Prüfungsanforderungen nicht abzubauen sind.

\subsubsection{Vorrang von Art. 7 I GG wegen Fehlen oder Schwächen in den Kernkompetenzen}

Literatur $^{928}$ und Rechtsprechung ${ }^{929}$ vertreten die Ansicht, dass Rechtschreibung und Rechnen in bestimmten Prüfungen, etwa im Diktat, gerade Prüfungsinhalt sind und damit zu prüfende Kernkompetenzen, so dass von ihrer Bewertung nicht abgesehen werden kann.

Die Vermittlung grundlegender Kulturtechniken und Grundfertigkeiten im Lesen, Schreiben und Rechnen ist eine der Hauptaufgaben von Staat und Schule, vor allem der Grundschule. ${ }^{930}$ Ihre Vermittlung dient zum einen dem generellen Bildungsrecht des Kindes, sie dient aber auch seinem Recht, in die Lage versetzt zu werden, an der Gesellschaft, die in hohem Maße auf Kommunikation ausgelegt ist, teilzuhaben. Gerade um das zu erreichen, ist der Staat berechtigt, die allgemeine Schulpflicht einzuführen. ${ }^{931}$ Selbstverständlich sind daher Lesen, Schreiben und Rechnen Grundfertigkeiten, die Schüler und Schülerinnen erlernen müssen. Auch die Rechtschreibung gehört zur Sprache und zur Grundausbildung jedes Schülers dazu. Diesen Fähigkeiten wird daher auch in der Schule ein erheblich höherer Stellenwert zugemessen als Leistungen in musischen, künstlerischen und sportlichen Fächern. Schülern und Schülerinnen mit Legasthenie/Dyskalkulie fehlt diese Fähigkeit, mit Ausnahme der schwerstbetroffenen Kinder, nicht völlig. Es fehlt innen aber die Fähigkeit, dies in der geforderten Schnelligkeit, Fehlerlosigkeit und in bestimmten Bereichen (Grundrechenarten) zu tun.

928 Sachs, JuS 1998, S. 263; Avenarius/Heckel, Schulrecht, Ziff. 27. 322, Fn. 55; Kischel, in: BeckOK GG, Art. 3, Rn. 219.2

OVG Lüneburg, Beschluss v. 20.9.2012, Az.: 2 LA 234/11, S. 6 (nicht veröffentlicht); VGH München, Beschluss v. 28.6.2012, Az.: 7 CE 12.1324, juris, (zu Prüfungen im Studienfach Bachelor BWL), Rn. 24; VG Düsseldorf, Beschluss v. 20.5.2011, Az.: 18 L 707/11, juris, Rn. 12, 14

931 S. dazu 2. Teil: 1. Kapitel: Das Sonderstatusverhältnis Schule, S. $55 \mathrm{ff}$ 


\subsubsection{Bewertung von Diktaten}

Sicherlich ist richtig, dass vor allem das Diktat, sei es in Deutsch oder in den Fremdsprachen, die Rechtschreibfähigkeiten bewerten soll. Ebenso richtig ist aber auch, dass ein Schüler mit Legasthenie gerade diese Fähigkeiten nicht hat und nicht erbringen kann. Die Benotung insbesondere des Diktats führt dazu, dass die Fähigkeiten bewertet werden, die behinderungsbedingt eingeschränkt sind. ${ }^{932}$ Die Bewertung der Rechtschreibleistungen hat das Ziel, Schülerinnen und Schüler zu richtigem Schreiben anzuhalten. In der Regel bewirken Noten einen Leistungsanreiz, vielleicht auch einen "heilsamen Druck", weil sich die Schüler um gute Leistungen bemühen. Außerdem ermöglichen sie einen Leistungsvergleich, der im Sinne eines positiven Wettbewerbs auch leistungsfördernd sein kann. Voraussetzung dafür ist allerdings, dass der Schüler die Möglichkeit hat, diese Leistung zu beeinflussen und aus eigenem Antrieb und Einsatz zu verändern. Da Schüler und Schülerinnen mit Legasthenie jedoch nicht in der Lage sind, ihre Lese-, Rechtschreib- oder Rechenleistung zu beeinflussen, können sie ihre Leistungen in diesem Bereich auch nicht durch Üben verbessern. ${ }^{933}$ Das gerade macht ihre Behinderung aus. Ebenso wenig wie die Benotung des Laufens im Sport dazu geeignet ist, einem körperbehinderten Schüler, der nicht laufen kann, das Laufen beizubringen, bewirkt die Benotung des Diktats bei einem Schüler mit Legasthenie, dass er rechtschreiben lernt. Wie auch bei Körperbehinderungen rechtfertigt sich die Nicht-Bewertung der beeinträchtigten Fähigkeit aus der Behinderung. Es darf daher gerade nicht zu ihren Lasten gehen, wenn sie diese Leistungen nicht erbringen können. ${ }^{934}$

\subsubsection{Bewertung des Faches Mathematik}

Die KMK, die Bundesländer und auch Kischel ${ }^{935}$ sehen die Gleichbehandlung im Bereich Mathematik als zwingend an, weil Mathematik ein unverzichtbares Fach im Bereich der schulischen Ausbildung und Bewertung sei und deshalb die

\footnotetext{
932 Vgl. Sachs, JuS 1998, S. 263

933 Eggers/Fegert/Resch, Psychiatrie, S. 763; die Auffassung des VG Düsseldorf, Beschluss V. 20.5.2011, Az.: 18 L 707/11, juris, Rn. 14, die Einrichtung von Förderungen an den Schulen zeige, dass den Schülern ausreichend Kenntnisse vermittelt werden könnten und das Nichterreichen derartiger Kenntnisse dem Schüler anzulasten ist, verkennt die medizinischwissenschaftlichen Erkenntnisse zur Legasthenie.

934 So aber ausdrücklich VG Düsseldorf, Beschluss v. 20.5.2011, Az.: 18 L 707/11, juris, Rn. 14

935 Kischel, in: BeckOK GG, Art. 3, Rn. 219 ff
} 
Berücksichtigung in Form der Veränderung von Prüfungsinhalten bei der Dyskalkulie unmöglich sei. ${ }^{936}$

Natürlich ist für eine breite Allgemeinbildung und für eine breite Entwicklungsmöglichkeit im beruflichen Bereich die Ausbildung in Mathematik wichtig. Fraglich ist, ob bei Dyskalkulie unbedingt das ganze Fach aus der Bewertung herausfallen muss oder ob es ein milderes Mittel gibt. Im Moment herrscht tatsächlich die pessimistische Einschätzung vor, dass man in Mathematik "nichts machen kann". ${ }^{937}$ Diese Einschätzung lässt jedoch außer Betracht, dass die Schwierigkeiten vor allem die Grundrechenarten betreffen, während mathematische Fähigkeiten für den Bereich der höheren Mathematik vorhanden sind. „Es ist für eine Dyskalkulie typisch, dass ... deren Defizit vor allem die Beherrschung grundlegender Rechenfertigkeiten wie Addition, Subtraktion, Multiplikation und Division betrifft, weniger die höheren mathematischen Fähigkeiten, die für Algebra, Trigonometrie, Geometrie oder Differenzial- und Integralrechnung benötigt werden. ${ }^{\prime 938}$ Es gibt also Bereiche, in denen Schüler und Schülerinnen mit Dyskalkulie durchaus mathematische Fähigkeiten haben, und Anhaltspunkte dafür, dass es innen mit entsprechenden Hilfestellungen ermöglicht werden kann, entweder dem Fach Mathematik insgesamt zu folgen oder zumindest mathematisches Leistungsvermögen auf Teilgebieten zu zeigen.

Soweit sich das durch einen Juristen recherchieren lässt, fehlt es bisher jedoch an einer intensiven Beschäftigung mit der Frage, welcher Nachteilsausgleich Schüler und Schülerinnen mit Dyskalkulie in die Lage versetzen würde, zumindest gewisse Rechenleistungen zu zeigen. Allemal aber sind die Überlegungen, welche Barrieren für diese Kinder abgebaut werden müssen und welche Möglichkeiten der Hilfestellung es gibt, pädagogisch-didaktisch noch in keiner Form ausgelotet. Denkbar ist etwa schlicht die Erlaubnis, die Finger zum Zählen benutzen zu dürfen und den Einsatz von Hundertertafeln, Formelsammlungen oder Einmaleins-Tabellen zu gestatten. ${ }^{939}$ Je früher den betroffenen Schülern und Schülerinnen erlaubt wird, Taschenrechner zu benutzen, um die Grundrechenarten darauf zu rechnen, desto eher können sie sich mit der theo-

936 KMK-Lesen/Rechtschreiben/Rechnen: Punkt: Schülerinnen und Schüler mit besonderen Schwierigkeiten im Rechnen, zu den Möglichkeiten des Nachteilsausgleichs für hörgeschädigte Kinder im Bereich Mathematik Lambert, SchulVerwaltung BW 2002, S. 148 ff, S. 150 KMK-Lesen/Rechtschreiben/Rechnen: Punkt: Schülerinnen und Schüler mit besonderen Schwierigkeiten im Rechnen DIMDI, Int. Stat. Klassifikation der Krankheiten: Diagnostik der Rechenstörung Kapitel F81.2; VG Regensburg, Beschluss v. 8.9.2006; RN 1 E 06.1610; VG Schleswig, Urteil v. 11.8.2004, Az.: 15 A 171/03, juris, Rn, 23; OVG Weimar, Beschluss v. 17.5.2010, Az.: 1 EO 854/10, juris, Rn. 52

939 Vgl. dazu der Ansatz der VV-LRS Brandenburg Nr. 7 II b und c, wonach neben Zeitverlängerung Platz für Nebenrechnungen und besondere didaktisch-methodische Hilfsmittel zugelassen werden können. 
retischen Mathematik beschäftigen. In Betracht kommt weiter, die Prüfungsaufgaben an den Zahlenraum anzupassen, den das Kind beherrscht (also etwa Aufgaben in der 3. Klasse nur im Zahlenraum bis 20 oder Hundert), ${ }^{940}$ Änderungen in der Rechenstrategie anzuzeigen oder schematische Lösungsstrategien explizit zu nutzen. In den Niederlanden etwa werden diese Möglichkeiten umfangreich genutzt. ${ }^{941}$ Weil Schüler und Schülerinnen mit Dyskalkulie in höheren Klassen in der Lage sind, den theoretischen Ansätzen im Mathematikunterricht zu folgen, können sie mithilfe solcher Unterstützung in die Lage versetzt werden zu zeigen, dass sie geforderte Rechenoperationen beherrschen. ${ }^{942}$

\subsection{Grundrechte anderer Schüler}

\subsubsection{Nicht-Bewertung der Rechtschreibung und/oder des Rechnens}

Die Rechtsprechung ${ }^{943}$ und die $\mathrm{KMK}^{944}$ sehen in der Veränderung inhaltlicher Prüfungsanforderungen eine Verletzung des Rechts auf Chancengleichheit der nicht-betroffenen Schüler. Ennuschat/Volino ${ }^{945}$ und Kischel $^{946}$ sehen in der Nicht-Bewertung der Rechtschreibung bei Schülern und Schülerinnen mit Behinderung eine mittelbare Diskriminierung der nicht-behinderten Schüler und Schülerinnen. Dem liegt die Überlegung zugrunde, dass auch die "normalen“ Schüler und Schülerinnen den allgemeinen Anforderungen des Lesens, der Rechtschreibung und des Rechnens gelegentlich nicht entsprechen oder bis-

940 Vgl. dazu SH Rechenschwäche-Erlass Nr. 3.3: Lernausgangslage orientierte Aufgaben - aber nicht für Klassenarbeiten oder sonstige schriftliche Leistungserhebungen

941 http://www.balansdigitaal.nl/stoornissen/dyscalculie/regelingen-envoorzieningen/aangepaste-toetsen-en-schoolexamens/

942 S. zu diesen Möglichkeiten die zulässigen Formen des Nachteilsausgleichs bei Dyskalkulie, die u.a. die Anpassung der Aufgaben an das Niveau des Kindes und Markierung von Plus und Minus vorsehen, näher dazu unten 14. Teil: 4. Kapitel: Niederlande, S. 372

OVG Lüneburg, Beschluss v. 10.7.2008, Az.: 2 ME 309/08, www.rechtsprechung.nieder sachsen.de, Rn. 19; Beschluss v. 25.11.2011, Az.: 2 ME 52/11, S. 4 f (nicht veröffentlicht); Beschluss v. 20.9.2012, Az.: 2 LA 234/11, S. 5 f (nicht veröffentlicht); VGH Kassel Beschluss v. 5.2.2010, Az.: 7 A 2406/09.z, juris, Rn. 43, 59; VG Aachen, Urteil v. 13.11.2009, Az.: $9 \mathrm{~K}$ 25/09, www.justiz.nrw.de, Rn. 56, 70; VG Düsseldorf, Beschluss v. 16.11.2009, Az.: 18 L 1654/09, juris, zur Dyskalkulie, Rn. 20 f; Beschluss v. 20.5.2011, Az.: 18 L 707/11, juris VG Gießen, Urteil v. 2.11.2010, Az.: 7 K 1841/10, S. 12 (nicht veröffentlicht); VG Schleswig, Urteil v. 10.6.2009, Az.: 9 A 208/08, BeckRS 2009 38123, S. 10; VG Sigmaringen, Beschluss v. 2.4.2008, Az.: 4 K 596/08, S. 4 (nicht veröffentlicht); offen lassend VG Hannover, Beschluss V. 13.12.2010, Az.: 6 B 5596/10, www.rechtsprechung.niedersachsen.de, Rn. 29 KMK-Lesen/Rechtschreiben/Rechnen Punkt: Abschlüsse, Prüfungssituationen und KMKInklusive Bildung Punkt II.4

945 Ennuschat, br 2008, S. 93 ff, S. 97; Ennuschat/Volino, br 2009, S. 166 ff, S. 169 
weilen Schwierigkeiten mit diesen Bereichen haben. ${ }^{947}$ Es wird als ungerecht angesehen, einige Schüler von der Benotung auszunehmen, andere aber dieser Benotung weiter zu unterwerfen. ${ }^{948}$ Das VG Düsseldorf erklärt ausdrücklich, dass eine Dyskalkulie kein Grund sei, die Mathematikklausur der Betroffenen „großzügiger" zu bewerten als die der Mitschüler. ${ }^{949}$

Eine Verletzung des Rechts auf Chancengleichheit der nicht-betroffenen Schüler und Schülerinnen kann nur vorliegen, wenn die Veränderung von Prüfungsbedingungen für Schüler mit Behinderungen ein Eingriff in das Grundrecht der nicht-betroffenen Schüler ist, weil sie den allgemeinen Prüfungsanforderungen unterworfen bleiben.

Schüler und Schülerinnen ohne Behinderung werden nach den allgemeinen Normen bewertet. Grundlage jeder Bewertung ist, dass die zu bewertende Leistung durch den Schüler beeinflussbar ist. Ist eine Leistung nicht beeinflussbar, dann ist es unverhältnismäßig, sie zu bewerten und zu benoten. Dementsprechend wird für die Lese-, Rechtschreib-, Rechenleistung davon ausgegangen, dass sie gelernt, geübt und beeinflusst werden kann. Insbesondere die durchgängige Bewertung der Rechtschreibleistungen hat das Ziel, Schüler und Schülerinnen zu richtigem Schreiben anzuhalten. Sie können verhindern für nachlässiges Schreiben sanktioniert und abgewertet zu werden, indem sie auf ihre Rechtschreibung achten und richtig schreiben bzw. Fehler korrigieren. Ohne diese Prämisse wäre fraglich, ob die Bewertung der Rechtschreibung überhaupt und in diesem Umfang verhältnismäßig ist.

Schüler und Schülerinnen ohne Behinderung werden nach den allgemeinen Normen bewertet, unabhängig davon, ob dieser Maßstab für Schüler und Schülerinnen mit Behinderungen ebenfalls angewendet wird oder ob für sie gegebenenfalls ein anderer gilt. Die Nichtbewertung bestimmter Lerninhalte bei Schülern und Schülerinnen mit Behinderung wirkt sich bei Schülern und Schülerinnen ohne Behinderung nicht aus. Sie werden dadurch nicht schlechter gestellt. ${ }^{950}$ Dass sie auch schlechte Noten in Teilbereichen bekommen können, die bei einem Schüler mit Behinderung nicht benotet werden, liegt im System der Benotung. Die Nicht-Bewertung des behinderten Schülers ist dafür nicht kausal. Die Veränderung der Prüfungsbedingungen bei Schüler und Schülerinnen mit

947 So ausdrücklich VG Schleswig, Urteil v. 10.6.2009, Az.: 9 A 208/08, BeckRS 2009 38123, S. 10 und VG Gießen, Urteil v. 30.6.2009. Az.: 4 K 1052/09.GI, S. 14 (nicht veröffentlicht)

948

Die Frage, ob bestimmte Bewertungsregeln ungerecht, unangemessen oder unangebracht sind, ob es gerechter wäre, die Rechtschreibung gar nicht zu bewerten usw., ist jedoch keine Frage der Grundrechtskollision zwischen Schülern und Schülerinnen mit und ohne Behinderung, sondern eine Frage dahingehend, ob die allgemeinen Bewertungsregeln des Schulgesetzgebers für gerecht und angemessen gehalten werden.

949 VG Düsseldorf, Beschluss v. 16.11.2009, Az.: 18 L 1654/09, juris, Rn. 23

950 VG Frankfurt a. M., Urteil v. 10.9.2009, Az.: 5 K 1501/09.F (1), S. 7 (nicht veröffentlicht) 
Legasthenie/Dyskalkulie ist daher kein Eingriff in das Grundrecht auf Chancengleichheit der nicht-betroffenen Schüler und Schülerinnen.

Für Schüler und Schülerinnen mit Legasthenie/Dyskalkulie ist demgegenüber die Bewertung nach den allgemeinen Prüfungsgrundsätzen eine Barriere, die sie nicht überwinden können, denn sie sind gerade nicht in der Lage, ihre Rechtschreib- und Rechenleistung zu beeinflussen. ${ }^{951}$ Sie können Rechtschreibfehler nicht erkennen ${ }^{952}$, sich die richtige Rechtschreibung von Wörtern nicht merken und infolgedessen die Sanktion der Abwertung nicht durch eigene Leistung umgehen. Das Gleiche gilt für die Bereiche des Lesens und des Rechnens. Der sachliche Grund für die Ausnahmeregelung ist gerade, dass das betroffene Kind für diese Einschränkung „nichts kann ${ }^{\text {“953 }}$, die Barriere also nicht beeinflussbar ist.

Die Nicht-Bewertung der Rechtschreibung und des Rechnens führt auch nicht zu einer Besserstellung des behinderten Schülers. Die Klausuren werden nicht „großzügiger" bewertet, sondern es werden die Leistungen unter Berücksichtigung der Behinderung bewertet. Die Noten werden nicht aufgebessert, Leistungsmängel werden nicht außer Acht gelassen. Auch wird nicht etwa immer eine positive Versetzungsentscheidung gefällt. ${ }^{954}$ Bei der Rechtschreibung werden die Arbeiten des Schülers bei inhaltlicher Richtigkeit nur nicht mehr wegen der Rechtschreibung abgewertet. Im Bereich des Rechnens wird dafür gesorgt, dass diese Note nicht mehr die alles entscheidende Bedeutung für das weitere Fortkommen, etwa die Versetzung, hat.

Eine mittelbare Diskriminierung der Schüler und Schülerinnen ohne Beeinträchtigung durch diese Ausnahmeregelung wäre nur zu bejahen, wenn die Ausnahmeregelung an neutrale Merkmale anknüpfen würde, die aber überwiegend oder typischerweise bei den nicht-behinderten Schülern ebenso vorliegen. ${ }^{955}$ Das ist jedoch nicht der Fall. Eine Ausnahmeregelung, die an eine Legasthenie oder Dyskalkulie anknüpft, ist nicht neutral, sondern knüpft explizit an bestimmte Ausnahmemerkmale an und kann daher nicht typischerweise

951 Insofern geht das VG Düsseldorf, (Beschluss v. 20.5.2011, Az.: 18 L 707/11, juris, Rn. 14) von falschen Voraussetzungen aus, wenn es meint, der betroffene Schüler müsse mit einer Förderung ausreichende Rechtschreibkenntnisse erreichen können. Der VGH Kassel Beschluss v. 8.12.2011, Az.: 7 A 2621/10.Z, juris, Rn. 16 und 33 meint, dass zwischen legastheniebedingten und nicht legastheniebedingten Fehlern unterschieden werden könne. Das entspricht nicht den medizinischen Erkenntnissen; S. oben 1. Teil: 1. Kapitel: 2: Legasthenie, S. 6

952 Eggers/Fegert/Resch, Psychiatrie, S. 763

953 Vgl. Kischel, in: BeckOK GG, Art. 3, Rn. 219.3, der darin gerade keinen Grund sieht.

954 So die Bedenken des OVG Lüneburg, Beschluss v. 10.7.2008, Az.: 2 ME 309/08, www.rechtsprechung.niedersachsen.de, Rn. 12

955 Zur Definition der mittelbaren Diskriminierung s. oben 3. Teil: 4. Kapitel: 2: Mittelbare Diskriminierung, S. 134 
diejenigen treffen, die die Merkmale nicht aufweisen. ${ }^{956}$ Im Fall der inhaltlichen Veränderung der Prüfungsanforderungen knüpft die Ausnahme daran an, dass der Schüler wegen Legasthenie/Dyskalkulie behindert ist. Ein nicht-behinderter Schüler wird davon nicht erfasst. Gerade deshalb sind Schüler mit Behinderung und ohne Behinderung ungleich und mithin auch ungleich zu behandeln.

Die Grundrechte der nicht-behinderten Schüler und Schülerinnen können allerdings durch diese Ausnahmeregelung beeinträchtigt werden, wenn die Regelung auf Ausnahmemerkmale abstellt, die unbestimmt oder ungeeignet sind. Das wäre der Fall, wenn sie objektiv nicht überprüfbar wären, denn die Wertungen des Staates für Ungleichbehandlungen müssen allgemein und konsistent erfolgen und verallgemeinerbar sein. ${ }^{957}$

\subsubsection{Ausschluss unqualifizierter Schüler und Schülerinnen aus einer Schulart}

Denkbar wäre, dass ein Eingriff in die Grundrechte nicht-betroffener Schülerinnen und Schüler darin liegt, dass unqualifizierte Schüler und Schülerinnen ihren Bildungsgang besuchen und sie dadurch im Lernen beeinträchtigt werden. ${ }^{958}$

Es ist bereits die Frage, ob diese Argumentation angesichts des Inklusionsziels der BRK überhaupt noch haltbar ist. Da die BRK von der gemeinsamen Beschulung aller Kinder ausgeht, die auch für alle Kinder förderlich ist, kann es keinen Anspruch mehr auf homogene Bildungsgänge oder Lernorte geben.

Unabhängig davon sind Legasthenie/Dyskalkulie jedoch nicht notwendig qualitätsmindernd, so dass betroffene Schüler nicht per se als unqualifiziert angesehen werden können. Das zeigt sich vor allem daran, dass die Bundesländer grundsätzlich, wenn auch in unterschiedlicher Ausprägung, die Behinderung Legasthenie und auch Dyskalkulie anerkennen und in der Regelschule berücksichtigen und damit Formen und Regelungen geschaffen haben, wie die Schulen und die Lehrkräfte damit im täglichen Unterricht umgehen sollen. ${ }^{959}$

\subsection{Pädagogische Aspekte speziell bei Legasthenie/Dyskalkulie}

Die Gleichbehandlung von Schülern und Schülerinnen mit Legasthenie/Dyskalkulie und die Versagung spezieller Unterstützung werden schließlich häufig mit pädagogischen Argumenten begründet. So wird zu bedenken gegeben, dass der Stolz der Schüler nicht durch "soziale“ Noten verletzt werden solle. ${ }^{960}$ Das VG Darmstadt führt aus: „Mit einem Notenschutz könnte der Kläger sogar dazu

956 VG Frankfurt a. M., Urteil v. 10.9.2009, Az.: 5 K 1501/09.F (1), S. 7 (nicht veröffentlicht)

$957 \mathrm{Vgl}$. Michael/Morlok, Grundrechte, Rn. 781, 805

958 Guhl, Prüfungen im Rechtsstaat, S. 26; s. dazu die alte Rspr. des BVerfGE 34, 165, 190

(=1973) und des BVerwGE 5, 153, 160 und 5, 164, 165 (beide 1958)

959 S. oben 1. Teil: 2. Kapitel: 4.: Grundzüge der Beschlüsse der KMK und der Landesvorschriften zur Berücksichtigung von Legasthenie und Dyskalkulie - mit Ausnahme von Bayern, S. 37

960 Lambert, SchulVerwaltung BW 2002, S. 148 ff, S. 148 
verleitet werden, sich auf diesem Schutz auszuruhen, und damit die ... geforderte Fähigkeit und Übung zur ,Automatisierung seiner Kenntnisse' verlieren. Das aber dürfte pädagogisch kaum zu vertreten sein, weil er zukünftig in Studium und Beruf mit Sicherheit nicht den Schutz in entsprechendem Umfang erhalten wird und sich in der Schule auf diese verschärfte Situation vorbereiten soll. ${ }^{\prime 961}$

Derartige pädagogische Argumente haben keinen Verfassungsrang und lassen sich auch mit dem Schutzzweck des Art. 3 III 2 GG nicht vereinbaren. Sie unterstellen nämlich, dass die Berücksichtigung der Behinderung und die daraus folgende Ungleichbehandlung nachteilig für das Kind $\operatorname{sind}^{962}$, und es dem Schutz der Persönlichkeit des Kindes mehr entspricht, wenn es gleichbehandelt wird. Der Schutz des Art. 3 III 2 GG zielt jedoch gerade darauf ab, Benachteiligung abzubauen und dem Kind mit Behinderung eine gleiche Teilhabe am Leben zu ermöglichen. Anzunehmen, dass die Berücksichtigung von Behinderungen nachteiliger ist als die Gleichbehandlung ohne Rücksicht auf die Behinderung widerspricht den tatsächlichen Gegebenheiten.

Dadurch wird keineswegs ausgeschlossen, dass bei der Berücksichtigung einer Behinderung im Einzelfall die Frage entsteht, wie das Kind mit der Behinderung umgeht und welchen Schutz es braucht, um eine etwaige Sonderstellung in der Klasse zu akzeptieren.

Das VG Darmstadt formuliert mit seinen pädagogischen Erwägungen eine vielfach geäußerte Sorge: nämlich, dass sich der Schüler auf einem Schutz ausruht, wenn die Prüfungsanforderungen für ihn verändert werden. Es ist zwar richtig, dass Kinder mit Behinderung lernen müssen, mit ihrer Behinderung umzugehen und einen Weg finden, trotz der Behinderung zu lesen, zu schreiben und zu rechnen. Die Frage ist aber, ob es ein legitimer Zweck ist, deshalb gleich zu behandeln, einen behinderungsbedingten Nachteil nicht auszugleichen und vor allem Prüfungsleistungen abzuwerten, damit sich der Mensch mit Behinderung nicht auf einem Nachteilsausgleich "ausruht" ${ }^{963}$ Das wäre nur zu bejahen, wenn es eine Pflicht des Menschen mit Behinderung gibt, sich die größtmögli-

961 VG Darmstadt, Urteil v. 28.4.2009, Az.: 7 K 687/98.DA (3), S. 17 (nicht veröffentlicht) - im Übrigen trifft die Ansicht des VG Darmstadt, dass die Situation in Studium und Beruf gegenüber der Schule verschärft ist, nicht zu, denn dort wird die Rechtschreibung nicht in dem Umfang gewertet wie in der Schule und es gibt umfangreiche Kompensationsmöglichkeiten mit Computern.

962 In diese Richtung geht die Argumentation von Büchner/Kortländer/Werner/Robering/Schönweiss, "Legasthenie - eine Krankheit, eine Behinderung, eine Störung?", S. 2, 4; HanertMöller, Konstrukt Legasthenie, S. 83; Valtin, in: LRS und Legasthenie, S. 58 ff; Valtin, Brauchen wir die Legasthenie?, S. $6 \mathrm{f}$ schluss v. 20.5.2011, Az.: 18 L 707/11, juris, Rn. 14: Pflicht zur Erreichung des Förderziels, das Nichterreichen geht zu Lasten des Schülers. 
che Mühe zu geben, die Behinderung zu überwinden, und ein damit korrelierendes Recht des Staates, dies nicht nur von ihm einzufordern, sondern eine insoweit schlechte Leistung auch durch schlechtere Noten zu bewerten. Eine solche Haltung aber widerspricht dem Recht des Menschen mit Behinderung aus Art. 2 I GG auf freie Entfaltung der Persönlichkeit, die auch umfasst, etwas nicht zu tun. ${ }^{964}$ Im Ergebnis würde damit die Behinderung wieder im Sinne des medizinischen Modells als etwas begriffen, dass es abzustellen gilt. Diese Wertung ist jedoch mit dem sozialen Behinderungsbegriff nicht zu vereinbaren. Von der Frage, einen Nachteilsausgleichs in Prüfungen aus pädagogischen Gründen nicht zu gewähren, ist die Frage zu trennen, ob Schüler und Schülerinnen mit Legasthenie/Dyskalkulie dazu verpflichtet werden können, an Fördermaßnahmen teilzunehmen und in dieser Form an ihrer Behinderung zu arbeiten. Auf diese Frage gehe ich später umfassender ein. ${ }^{965}$

\section{Kapitel: Ergebnis}

Schüler und Schülerinnen mit Legasthenie/Dyskalkulie sind behindert. Sie werden durch die unterschiedslose Gleichbehandlung mit Schülern und Schülerinnen ohne Behinderung mittelbar diskriminiert und damit benachteiligt. Die Benachteiligung ist nicht gerechtfertigt.

Diese Benachteiligung ist nach Art. 3 III 2 GG verboten. Die Betroffenen haben daher einen Anspruch auf Berücksichtigung von Legasthenie/Dyskalkulie in der Schule und zwar hinsichtlich aller Prüfungsbedingungen, sowohl der äußeren als auch der inhaltlichen.

964 BVerfG, Beschluss v. 23.3.2011; Az.: 2 BvR 882/09; http://www.bundesverfassungs gericht.de/entscheidungen/rs20110323_2bvr088209.html, Rn. 48

5. Teil: 2. Kapitel: 2.1.: Pflicht zur Teilnahme an Förderung, S. 202 


\section{Teil: Anspruch aus dem Grundsatz der Chancengleichheit aus Art. 3 । GG i.V.m. Art. 2 I und 12 I GG}

\section{Kapitel: Konkretisierung des Grundsatzes der Chancengleichheit}

Ein Anspruch auf Berücksichtigung der Legasthenie/Dyskalkulie kann sich weiter aus dem Grundsatz der Chancengleichheit nach Art. 3 I GG i.V.m. Art. 2 I und 12 I GG ergeben.

Der Grundsatz der Chancengleichheit erfährt durch den Paradigmenwechsel, den die BRK bewirkt, eine neue Dimension. Das durch die BRK noch in seiner Bedeutung gesteigerte Grundrecht des Art. 3 III 2 GG verpflichtet den Staat resp. den Schulgesetzgeber nicht nur dazu, Diskriminierungen in größtmöglicher Weise zu vermeiden ${ }^{966}$, indem er dafür sorgt, dass sein Handeln keine ungleichen Chancen bewirkt oder bestehende Ungleichheiten verschärft. ${ }^{967}$ Den Staat trifft vielmehr die Pflicht eine inklusive Beschulung aller Schüler und Schülerinnen in der Schule herzustellen. Der Grundsatz der Chancengleichheit beinhaltet danach nicht den Auftrag, formale Gleichheit herzustellen, damit Schüler mit Behinderungen die Anforderungen erfüllen können, die an „Normalschüler" gestellt werden, sondern fordert materielle Gleichheit im Sinne von „Jedem das Seine" ${ }^{968}$ Chancengleichheit besteht nur dann, wenn nicht nur die formalen Startbedingungen, sondern auch die gesetzten Ziele die Belange von Menschen mit Behinderung berücksichtigen.

Allerdings stellt sich die Frage, wie eine solche materielle Gleichheit zu bestimmen ist.

Das OVG Weimar hat hierzu ausgeführt, dass sich aus Art. 3 III 2 GG nicht ergibt, dass im Rahmen der Leistungsmessung der objektiv bestehende Unterschied zwischen dem grundsätzlich Leistungsfähigen (aber u. U. nur hinsichtlich des Nachweises Beeinträchtigten) und dem grundsätzlich nicht leistungsfähi-

\footnotetext{
966 Vgl. Luthe, in: Rehabilitationsrecht - Verfassungsrecht, Rn. 8

967 Weber-Dürler, in: FS Ulrich Häfelin, S. 221

968 Vgl. Wansing, Kommentar zur UN-BRK, Inklusionsbegriff, Rn. 13
} 
gen Behinderten dadurch aufzuheben ist, dass die Leistung des nicht leistungsfähigen Behinderten trotz seiner Nichtleistung als erbracht behandelt wird. Dies würde die Leistungsmessung überhaupt in Frage stellen. ${ }^{969}$ Das OVG Lüneburg hat die Befürchtung geäußert, dass bei festgestellter Legasthenie andere schulische Defizite, Eignungs- und Leistungsmängel außer Acht gelassen würden und über den Nachteilsausgleich die Noten soweit aufgebessert würden, dass grundsätzlich eine positive Versetzungsentscheidung zu erteilen sei oder Prüfungen für bestanden zu erklären seien, und dies ausdrücklich für unzulässig erklärt. ${ }^{970}$ Die Gerichte ziehen damit die Grenze der materiellen Gleichheit durch Ungleichbehandlung da, wo sie zu Ergebnisgleichheit führt. Dem ist zuzustimmen. Materielle Gleichheit als Ergebnisgleichheit würde im Schulbereich die bestehenden Unterschiede in der Leistungsfähigkeit und Leistungsbereitschaft negieren und damit wiederum Ungleiches gleich behandeln. Schule und Bildung können aber nicht alle vorgefundenen Bedingungen und Anlagen ausgleichen. ${ }^{971}$ Das bestehende Konkurrenzverhältnis würde sonst aufgehoben, indem im Ergebnis doch wieder gilt: „allen das Gleiche“. Chancengleichheit soll aber den Wettbewerb gerechter gestalten und nicht den Wettbewerb aufheben. $^{972}$

\section{Kapitel: Grenzen des Grundsatzes der Chancengleichheit}

Wie weit die materielle Gleichbehandlung geht, hängt von dem Prüfungsziel $a b$, zu dem die Startbedingungen in Beziehung gesetzt werden müssen. Entscheidend ist zunächst, was in den einzelnen Fächern das fachlich-inhaltliche Ziel von Prüfungen ist, welche Leistungsfähigkeiten für das Ziel maßgeblich sein sollen und deshalb geprüft werden. ${ }^{973}$ Dieses allgemeine Anforderungsprofil der Schulabschlüsse muss aber bei Schülern und Schülerinnen mit Behinderungen darauf geprüft werden, ob eine inhaltliche Veränderung wegen der Behinderung erforderlich ist oder ob es zwingend, d.h. unverzichtbar und alternativlos i.S.d. Art. 3 III $2 \mathrm{GG}$ ist.

Diese Bestimmung was zum zwingenden Anforderungsprofil gehört und was veränderbar ist, ist sicherlich nicht einfach. Die Rechtsprechung hat sich

\footnotetext{
969 OVG Weimar, Beschluss v. 17.5.2010, Az.: 1 EO 854/10, juris, Rn. 38

970 OVG Lüneburg, Beschluss v. 10.7.2008, Az.: 2 ME 309/08,

www.rechtsprechung.niedersachsen.de, Rn. 12

971 Reichenbach, Anspruch behinderter Schülerinnen und Schüler auf Unterricht in der Regelschule, S. 213

972 Reichenbach, Anspruch behinderter Schülerinnen und Schüler auf Unterricht in der Regelschule, S. 186

973 Vgl. Langenfeld, RdJB 2007, S. 211 ff, S. 220
} 
mit dieser Frage schon beschäftigen müssen und insgesamt Veränderungen ganz überwiegend abgelehnt. ${ }^{974}$ Nur das VG Hannover ${ }^{975}$ hat festgestellt, dass die Vorschriften über pauschale Punktabzüge bei schweren Rechtschreibmängeln kein geeigneter Beurteilungsmaßstab sind, der eine differenzierte Benotung der Sprachkompetenz von Schülern mit Legasthenie ersetzen könnte. Die orthographische Richtigkeit der Sprache sei nur ein Teilaspekt der geforderten sprachlichen Richtigkeit in der deutschen Sprache und der kompetenten schriftlichen Verständigung.

Die Inhalte und Anforderungen für schulische Prüfungen werden unter Bezugnahme auf einen gedachten „Normalschüler ${ }^{\prime \prime 76}$ festgelegt. Die landesrechtlich zuständigen Schulrechtsgeber legen die einzelnen Schularten, Jahrgangsstufen und Abschlüsse fest und regeln im Einzelnen, welche Leistungsfähigkeit und Leistungsbereitschaft erforderlich ist und welches Prüfungsziel verfolgt wird. Die inhaltlichen Vorgaben ergeben sich überwiegend aus den von der KMK verabschiedeten nationalen Bildungsstandards für die Abschlussklassen $^{977}$, auch wenn diese rechtlich unverbindlich sind. Detailliert ergeben sich die inhaltlichen Anforderungen für die schulischen Prüfungen aus den jeweiligen Lehrplänen. Aus diesen lässt sich relativ konkret ableiten, was Inhalt des Faches ist und welche fachspezifischen Kenntnisse, Arbeitstechniken und Fähigkeiten in der Prüfung ermittelt werden sollen und was das Lernziel einer Jahrgangsstufe ist. Der "Normalschüler" ist ein nicht-behinderter Schüler, auf den die geforderte Leistungsfähigkeit und das Prüfungsziel bezogen sind. An seinen „normalen" Fähigkeiten des Sprechens, Sehens, Hörens, Gehens, Lesens,

974 OVG Saarland, Beschluss v. 2.10.2006, Az.: 3 W 12/06, SPE 333 Nr. 13 (Änderungen bei der Prüfung zum „Fachlagerist“); BFH, Beschluss v. 8.7.2008; Az.: VII B 241/07, juris, Rn. 5 (Änderung des Bewertungsmaßstabes bei einem Prüfling mit Legasthenie); VGH München Beschluss v. 4.1.2010, Az.: 7 CE 09.2900, www.la-by.bayern.de, Rn. 22 (kein Ersetzen einer schriftlichen Prüfung durch eine mündliche Prüfung); VGH München, Beschluss v. 28.6.2012, Az.: 7 CE 12.1324, juris, (Ablehnung der Nicht-Bewertung der Rechtschreibung in den Fremdsprachen im Studienfach Bachelor BWL); VG Saarlouis, Urteil v. 5.3.2009, Az.: $1 \mathrm{~K}$ 643/08, juris, Rn. 55, 74, 76 (keine Veränderung der Anforderung im Bereich Texterstellung (Anschlä$\mathrm{ge} / \mathrm{min}$ ) bei Sehnenscheidenentzündung)

975 VG Hannover, Beschluss v. 13.12.2010, Az.: 6 B 5596/10, www.rechtsprechung.niedersachsen.de, 2. Leitsatz Vgl. VG Kassel, Beschluss v. 23.3.2006, 3 G 419/06, Sonderheft Recht des BVL: Chancengleichheit herstellen - Diskriminierung vermeiden, 2006, http://www.bvllegasthenie.de/index.php5?p=/downloads; VGH Mannheim, Beschluss v. 26.8.1993, $9 \mathrm{~S}$ 2023/93, NVwZ 1994, S. 598 ff; OVG Bautzen, Beschluss v. 3.11.2008; Az.: 2 B 292/08, S. 6 (nicht veröffentlicht); s. auch LSG Baden-Württemberg, Beschluss v. 9.12.2010, Az.: L 13 AL 4629/10 ER-B, juris, Rn. 33, das ausführt, dass der Zugang zur Hochschulbildung nur unter den auch für nichtbehinderte Menschen geltenden Zugangsbedingungen ermöglicht werden kann. 
Schreibens und Rechnens usw. sind die Prüfungsaufgaben ausgerichtet. Insofern wird von einer Homogenität der zu prüfenden Schülergruppe ausgegangen. Die Belange von Schülern und Schülerinnen mit Behinderung werden bei der Festlegung nicht berücksichtigt.

Kriterien für die Bestimmung des Prüfungsziels und der dafür erforderlichen Leistungsfähigkeit ergeben sich aus der Rechtsprechung des BVerfG zu Art. 12 GG. ${ }^{978}$ Danach sind Prüfungen, soweit sie sich auf Berufs- und Lebenschancen auswirken, Grundrechtseingriffe ${ }^{979}$ und deshalb nur dann rechtmäßig, wenn sie nicht gegen das Verhältnismäßigkeitsprinzip verstoßen. ${ }^{980}$ Dafür muss bei schulischen Prüfungen der Prüfungsinhalt auf das Prüfungsziel ausgerichtet sein. ${ }^{981}$ Die Prüfung muss geeignet und ausreichend sein, um das Ziel der Leistungskontrolle zu erreichen. ${ }^{982}$ Das Prüfungsziel muss einen Rückschluss auf das damit verbundene Ergebnis, wie z.B. die Versetzung, oder die damit verbundene Berechtigung zulassen. Es dürfen also nur solche Prüfungsanforderungen abverlangt werden, die im Hinblick auf den mit der Prüfung verfolgten Zweck geboten sind. ${ }^{983}$ Bei Abiturprüfungen sieht die Rechtsprechung deren Zweck darin, zwischen solchen Abiturienten, die für ein Hochschulstudium geeignet sind, und solchen, bei denen dies nicht der Fall ist, zu unterscheiden. ${ }^{984}$ Die Anforderungen, die gestellt werden, müssen sich auf diesen Zweck beziehen und dürfen zu ihm nicht außer Verhältnis stehen. Bestimmte Leistungen dürfen daher nur dann Grundlage der Bewertung und daraus folgender Regelungen zum Nicht-Bestehen der Prüfung sein, wenn sie eine zuverlässige Beurteilungsgrundlage für die Prognose der Eignung für das Hochschulstudium sind. ${ }^{985}$ Das ist bei Schulabschlüssen generell schwierig, weil Schulabschlüsse darauf angelegt sind, eine breite Allgemeinbildung zu vermitteln, die viele Berufswege eröffnet. Der Prüfungszweck ist daher sehr breit angelegt um vielen allgemeinen

978 BVerfGE 52, 380, 388; 84, 34, 45; 84, 59, 72 m.w.N.

979 Pietzcker, Verfassungsrechtliche Anforderungen an staatliche Prüfungen, S. 70 ff; Guhl, Prüfungen im Rechtsstaat, S. $21 \mathrm{ff}$; Lampe, Gerechtere Prüfungsentscheidungen, S. 23

980 BVerwG, Urteil v. 17.7.1987, Az.: 7 C 118/86, juris, Rn. 17; VGH Mannheim, Urteil v. 16.5.2000, Az.: 9 S 2537/99, juris; OVG Bautzen, Beschluss v. 11.9.2001, Az.: 4 BS 156/01, juris; OVG Greifswald, Urteil v. 27.2.1997, Az.: 2 L 52/96, juris, Rn. 23; VG Dresden, Beschluss V. 8.8.2001, Az.: 5 K 1571/01, juris; VG Dresden, Beschluss v. 22.7.1998, Az.: 5 K 1705/98, juris; VG Potsdam, Beschluss v. 19.7.2000, Az.: 5 L 967/00, LKV 2001, S. $572 \mathrm{ff}$ Guhl, Prüfungen im Rechtsstaat, S. 102

982 Niehues, Prüfungsrecht, Rn. 374

983 Lampe, Gerechtere Prüfungsentscheidungen, S. 37; Niehues/Rux, Schulrecht, Rn. 136

984 OVG Greifswald, Urteil v. 27.2.1997, Az.: 2 L 52/96, juris, Rn. 25; VG Dresden, Beschluss V. 22.7.1998, Az.: 5 K 1705/98, juris, Rn. 51; VG Potsdam, Beschluss v. 19.7.2000, Az.: 5 L 967/00, LKV 2001, S. 572, 573 
Anforderungen Rechnung zu tragen. ${ }^{986}$ Daraus ergibt sich, dass geprüft werden muss, welchem Zweck die Prüfung dienen soll, welche wahren Leistungen und Befähigungen, welche Kernkompetenzen, in einer Prüfung nachgewiesen werden sollen oder müssen. ${ }^{987}$ Nur wenn darüber Klarheit herrscht, kann bestimmt werden, welche Leistungsfähigkeiten und Kompetenzen für das Ziel maßgeblich sein sollen und deshalb zu prüfen sind. ${ }^{988}$ Ein maßgeblicher Abwägungsgesichtspunkt sind dabei die beruflichen Möglichkeiten, die die Prüfungen eröffnen. Je vielfältiger die Zwecke der Prüfung sind und je mehr berufsbezogenen Möglichkeiten sie eröffnet, desto größer ist das Fähigkeits- und Kompetenzspektrum, dass mit der Prüfung abgeprüft wird. Da nicht alle Fähigkeiten und Kompetenzen für alle beruflichen Möglichkeiten erforderlich sind, sind nicht alle Fähigkeiten und Kompetenzen zwingend erforderlich, so dass die Möglichkeit zu gewichten und auch auf Fähigkeiten zu verzichten, groß ist. ${ }^{989}$ Je konkreter eine Prüfung jedoch ist, desto genauer sind die Fähigkeiten und Kompetenzen beschrieben und auch für den Erfolg der Prüfung erforderlich, so dass die Möglichkeit, auf bestimmte Fähigkeiten zu verzichten, kleiner wird.

Im Ergebnis läuft dies auf die Beantwortung der Frage hinaus, ob der Prüfling für das mit der Prüfung verfolgte Ziel, etwa Ausübung eines bestimmten Berufs oder Studiums, „ansonsten geeignet“ ist, auch wenn bestimmte Prüfungsanforderungen verändert werden. Eine Grenze wird sich da ergeben, wo der Beruf eine ganz bestimmte Leistung erfordert, die behinderungsbedingt nicht geleistet und auch nicht kompensiert werden kann. ${ }^{990}$ In diesen Fällen ist die Möglichkeit auf Fähigkeiten und Kompetenzen zu verzichten, eingeschränkt. Die Abgrenzung ist im Einzelnen schwierig, wie das Urteil des VG Saarlouis zeigt. Es hatte es abgelehnt, für die Kandidatin, die die erforderliche Schreibgeschwindigkeit mit 2300 Anschlägen, die sie krankheitsbedingt nicht erreichen konnte, zu verändern. Entscheidend war, dass es bei dem angestrebten Beruf einer Fachkauffrau für Büromanagement gerade auf die Schreibgeschwindigkeit ankommt und es die Alternative gibt, eine qualitativ gleichwertige Prüfung mit ähnlichen Berufs- und Weiterbildungsmöglichkeiten zur Wirtschaftsfachwirtin zu machen, die die Schreibgeschwindigkeit gerade nicht erfordert. ${ }^{991}$ Demgegenüber gewährt das bayerische Kultusministerium bei vergleichbaren behinderungsbedingten Einschränkungen Nachteilsausgleich auch in Bezug auf die Schreibgeschwindigkeit u.a. im Fach Textverarbeitung, um die

\footnotetext{
986 Vgl. Rux, Pädagogische Freiheit des Lehrers, S. 38

Vgl. Niehues, Prüfungsrecht, Rn. 380; Avenarius/Heckel, Schulrecht, Ziff. 27.33 Langenfeld, RdJB 2007, S. $211 \mathrm{ff}$, S. 220

$990 \mathrm{Vgl}$. Niehues, Prüfungsrecht, Rn. 444

991 VG Saarlouis, Urteil v. 5.3.2009, Az.: 1 K 643/08, juris, Rn. 30
} 
berufliche Qualifikation gerade nicht an diesem Kriterium scheitern zu lassen. ${ }^{992}$ Es entscheidet sich damit im Zweifel für die Berücksichtigung der Behinderung und gegen die Notwendigkeit des Faches.

Die Entscheidung des VG Hannover zeigt exemplarisch, dass es auch beim Abitur nicht allein auf eine einzelne Fähigkeit oder Kompetenz ankommt und dass sogar die einzelnen Fähigkeiten wieder in Teilaspekte aufgeteilt werden können. ${ }^{93}$ Die allgemeine Aussagekraft des Abiturs wird nicht dadurch verfälscht, dass von der Vielfalt der geforderten und insgesamt auch vorhandenen Fähigkeiten und Kompetenzen einzelne Aspekte behinderungsbedingt eingeschränkt sind. Deshalb sind diese Kompetenzen nicht zwingend erforderlich mit der Folge, dass ohne sie das Abitur nicht möglich wäre. ${ }^{994}$

Diese Zweck-Mittel-Relation übersieht der VGH München sowohl in der Entscheidung zur Ersetzung einer schriftlichen Prüfung durch eine mündliche ${ }^{995}$ als auch in der Entscheidung zur Bewertung der Rechtschreibung im Fach Englisch im Studium BWL. ${ }^{996}$ Er setzt die Prüfungsanforderungen, die auf Normalprüflinge ausgerichtet sind, absolut und prüft nicht, ob das Festhalten an den Prüfungsinhalten trotz der Behinderung der Prüflinge zwingend erforderlich ist. ${ }^{997}$

Entscheidend ist daher, wie allgemein die Prüfung oder der Schulabschluss sind und wie vielfältig die daran anknüpfenden beruflichen Möglichkeiten. Fähigkeiten und Kompetenzen können im Hinblick darauf nur dann zwingend erforderlich und damit einer Ungleichbehandlung nicht zugänglich sein, wenn ohne sie sämtliche anschließenden Berufsmöglichkeiten nicht möglich sind. ${ }^{998}$ Die Festlegung des Anforderungsprofils in Prüfungsordnungen ist gerichtlich voll überprüfbar, denn es handelt sich dabei um eine Rechtsfrage und nicht um eine Ermessensentscheidung. Eine solche umfassende Überprüfung existiert im deutschen Recht bereits im Bereich des Arbeits- und Beamtenrechts. ${ }^{999}$ Wäre

992 BY KMBek Berufsschule Behinderung Nr. 3.3 und 3.5, die Reduzierung ist dann jedoch im Zeugnis zu vermerken.

993 VG Hannover, Beschluss v. 13.12.2010, Az.: 6 B 5596/10, www.rechtsprechung.niedersachsen.de, S. 6

994 Poscher/Langer/Rux, Gutachten zu Art. 24 BRK, S. 58

995 VGH München Beschluss v. 4.1.2010, Az.: 7 CE 09.2900, www.la-by.bayern.de, Rn. 22

996 VGH München, Beschluss v. 28.6.2012, Az.: 7 CE 12.1324, juris, (zu Prüfungen im Studienfach Bachelor BWL), Rn. 24

997 Vgl. zur sog. niveaugleichen Modifizierung Ennuschat, Die Rechte stotternder Menschen in Schule, Ausbildung und Studium, S. 106

998 Rux, Pädagogische Freiheit des Lehrers, S. 38; Poscher/Langer/Rux, Gutachten zu Art. 24 BRK, S. 58

999 BAG NZA 2005, S. 870 ff; NZA 2007, S. 507 ff; NZA 2007, S. 1098 ff; Fuerst, Behinderung zwischen Diskriminierungsschutz und Rehabilitationsrecht, S. $123 \mathrm{ff}$, mit ausführlicher Kritik und 132. BVerfG 2 BvR 2571/07 vom 10.12.2008, http://www.bverfg.de/ 
eine solche Überprüfung nicht möglich, würde es im Bereich der Schule dem Schulgesetzgeber freistehen, die Anforderungen so zu setzen, dass ein Mensch mit Behinderung sie auch trotz getroffener Vorkehrungen de facto nicht erfüllen kann.

Diese umfangreiche Berücksichtigung der Belange von Schülern und Schülerinnen mit Behinderungen in Prüfungen führt dazu, dass allgemeine Bildungsund Prüfungsstandards keinen uneingeschränkten Vorrang (mehr) haben, sondern im Einzelfall auch im Hinblick auf eine Behinderung zu überprüfen sind. Sämtliche Behinderungen müssen dann im Schulbereich und bei Prüfungen berücksichtigt werden. Dieser Paradigmenwechsel ${ }^{1000}$, der von der Schule eine Anpassung an den Schüler mit Behinderung verlangt, kann dazu führen, dass das traditionelle Prinzip der Schulabschlüsse und der Allgemeinen Hochschulreife überprüft werden muss. ${ }^{1001}$ Rux ist darin zuzustimmen, dass dies zur Folge haben kann, dass bei den Bildungsabschlüssen und auch bei der Hochschulzugangsberechtigung differenziert wird, etwa bezogen auf bestimmte Studienrichtungen ${ }^{1002}$, so wie dies bereits beim Fachabitur praktiziert wird.

\section{Kapitel: Ergebnis}

Aus dem Grundsatz der Chancengleichheit aus Art. 3 I GG ergibt sich der Anspruch auf Berücksichtigung der Legasthenie/Dyskalkulie hinsichtlich sämtlicher Prüfungsbedingungen, sowohl der sog. „äußeren“ Bedingungen als auch der inhaltlichen Anforderungen.

entscheidungen/rk20081210_2bvr257107.html, BVerwG Urteil v. 21.6.2007, Az.: 2 A 6/ 06; http://www.bundesverwaltungsgericht.de/media/archive/5320.pdf Rn. 20; ebenso OVG Bautzen Beschluss v. 15.6.2009, 2 A 140/08, juris

1000 S. dazu ausdrücklich Ennuschat, in: FS Klaus Stern, S. 724

1001 S. dazu Rux, RdJB 2009, S. 220 ff, S. 228; Poscher/Rux/Langer, Recht auf Bildung, S. 58, Fn. 153

1002 Rux, RdJB 2009, S. 220 ff, S. 228 



\section{Teil: Nachteilsausgleich und Förderung}

\section{Kapitel: Umfang des Nachteilsausgleichs}

Aus dem obigen ergibt sich, dass zur Herstellung von Chancengleichheit von behinderten Menschen deren unterschiedliche Ausgangsbedingungen beachtet werden müssen. ${ }^{1003}$ Weil die Behinderungen vielfältig sind, kann der Vergleich der Ausgangsbedingungen nicht allgemein erfolgen, sondern muss sich auf die jeweilige Prüfung, ihr konkretes Ziel und ihre Prüfungsart sowie auf die individuelle Behinderung beziehen. ${ }^{1004}$ Nur daraus lässt sich ableiten, ob die Startbedingungen von Schülern und Schülerinnen mit Behinderungen wegen dieser Behinderung in der speziellen Prüfung unterschiedlich, also ungleich sind und deshalb ausgeglichen werden müssen. Etwa sind Schüler und Schülerinnen, die gehörlos sind, Epilepsie haben oder im Rollstuhl sitzen mit nicht-behinderten Schülern und Schülerinnen hinsichtlich der Anforderungen des Faches Mathematik gleich, hinsichtlich der Anforderungen des Faches Sport aber ungleich. Ein hörgeschädigter Schüler ist im Fach Deutsch gleich mit nicht-behinderten Schülern, wenn die Inhaltsangabe eines Lesetextes geschrieben wird, aber ungleich, wenn ein Diktat oder eine Nacherzählung geschrieben wird. Schüler und Schülerinnen mit Legasthenie sind im Fach Sport, i.d.R. auch in Musik und Kunst mit nicht-behinderten Schülern gleich, aber in allen Fächern, in denen es auf das Lesen und die Rechtschreibung ankommt, ungleich. Sie sind in den Fremdsprachen mit den nicht-behinderten Schülern gleich, wenn Vokabeln mündlich abgefragt werden, aber ungleich, wenn die gleichen Vokabeln schriftlich abgeprüft werden. Schüler und Schülerinnen mit Dyskalkulie sind in allen Fächern gleich, in denen es nicht auf das Rechnen ankommt, aber ungleich, in

\footnotetext{
1003 Degener, RdJB 2009, S. 200 ff, S. 205

${ }^{1004}$ S. dazu auch die Rechtsprechung des BVerfG und des BVerwG zur Berücksichtigung von Art. 3 III 2 GG bei der Anwendung von Art. 33 II GG, BVerfG, 2 BvR 2571/07 vom 10.12.2008, http://www.bverfg.de/entscheidungen/rk20081210_2bvr257107.html Rn. 11; BVerwG Urteil v. 21.6.2007, Az.: 2 A 6/ 06; http://www.bundesverwaltungsgericht.de/media/archive/5320.pdf, Rn. 20
} 
den Fächern, in denen gerechnet werden muss. Sie sind innerhalb des Faches Sachkunde in der Grundschule gleich, wenn Unterrichtsinhalt das Wissen um Bäume und Blätter ist, aber ungleich, wenn der Unterrichtsinhalt mathematische Bezüge und Schätzungen enthält, wie z.B. beim Lesen von Karten, Höhenangaben und Entfernungen.

Welche Veränderungen im Einzelnen erforderlich sind, kann nicht generell festgelegt werden, weil die Grenzen zwischen den äußeren und inhaltlichen Prüfungsanforderungen fließend sind. Ebenso sind die schulischen Anforderungen je nach Jahrgangsstufe unterschiedlich und auch hier sind die Grenzen fließend. Was in der Grundschule Prüfungsinhalt ist, etwa das Lesen, wird in den höheren Klassen vorausgesetzt. Nach der hier vertretenen Auffassung besteht ein Anspruch auf Nachteilsausgleich sowohl in Bezug auf äußere als auch auf inhaltliche Anforderungen. Für die Umsetzung in der Praxis wird es jedoch hilfreich sein, wenn zumindest Anhaltspunkte dafür bestehen, ob Veränderungen eher im Bereich der äußeren Bedingungen oder im Bereich der inhaltlichen Anforderungen erforderlich sind, um die Chancengleichheit herzustellen.

\section{Veränderungen der äußerer Bedingungen}

Schüler und Schülerinnen mit Legasthenie/Dyskalkulie sind wie Schüler und Schülerinnen ohne Behinderung in der Lage, die "normalen" Prüfungsziele zu erreichen, wenn nicht konkret das Lesen, Rechtschreiben und Rechnen geprüft wird. Sie können ebenso wie ihre nicht-behinderten Mitschüler z.B. Inhaltsangaben und Erörterungen in Deutsch, Vokabeltests in Fremdsprachen, Wissensabfragen in anderen Fächern usw. schreiben. Allerdings sind ihre Startbedingungen in diesen Prüfungen schlechter, weil sie mehr Zeit und eventuell auch andere Darstellungsformen brauchen, um die Aufgabenstellung zunächst zu erfassen und dann mit der Bearbeitung der Aufgabe zu beginnen. Dieser Nachteil muss so lange ausgeglichen werden, bis die Startbedingungen vergleichbar sind.

Bei Legasthenie ist die Erfüllung der "normalen“ inhaltlichen Anforderungen in der Regel in allen Fächern außer in Deutsch, in den Fremdsprachen und in der Textverarbeitung/im Maschineschreiben möglich, weil in ihnen Lesen und Schreiben für das Fach zwar notwendig, aber nicht fachlicher Inhalt sind. Um diese "normalen" inhaltlichen Anforderungen zu erfüllen, müssen die betroffenen Schüler und Schülerinnen in die Lage versetzt werden, ihr erlerntes Wissen auch darzubieten, indem sie die Prüfungsaufgabe verstehen und ihr Wissen niederschreiben können. Dafür ist in der Regel ein Zeitzuschlag für das Erlesen der Aufgabe und für das Niederschreiben der Lösungen erforderlich sowie die Anwendung der vielfältigen Ausgleichsformen. ${ }^{1005}$ Die Startbedingungen sind z.B. in einem Sprachfach, in dem eine Inhaltsangabe geschrieben wird, ver-

${ }^{1005}$ S. oben 1. Teil: 2. Kapitel: 8.: Formen der Berücksichtigung in der Schule, S. 51 
gleichbar, wenn der Schüler in die Lage versetzt wurde, den Text vollständig zu erlesen und dann mehr Zeit für die Schreibarbeit, die ihm ebenfalls schwerer fällt als nicht-behinderten Schülern, bekommt.

Bei Dyskalkulie betrifft das alle Fächer außer Mathematik und diejenigen Fächer, in denen vornehmlich zu rechnen ist. Oft brauchen Kinder mit Dyskalkulie darin gar keinen Ausgleich. Die Übergänge sind aber fließend, so ist z.B. ein Ausgleich im Fach Kunst denkbar, wenn es dort um Architektur und die Bewertung und Einordnung von Formen und mehrdimensionalen geometrischen Figuren geht, weil bei Schülern und Schülerinnen mit Dyskalkulie die räumliche Vorstellungskraft meist beeinträchtigt ist.

\section{Veränderungen der inhaltlichen Anforderungen}

Wenn der Prüfungszweck jedoch gerade auf das Lesen, Rechtschreiben und Rechnen ausgerichtet ist, dann müssen die inhaltlichen Anforderungen verändert werden. Bei Schülern und Schülerinnen mit Legasthenie betrifft das vor allem die Diktate in allen Fächern, die Leseproben in der Grundschule und im Übrigen die Bewertung der Rechtschreibung, insbesondere also die Fächer Deutsch, Fremdsprachen, Textverarbeitung. Bei Schülern und Schülerinnen mit Dyskalkulie betrifft es insbesondere das Fach Mathematik, aber auch alle anderen Fächer, soweit in ihnen gerechnet werden muss.

Die Veränderung kann entweder erfolgen, indem der Prüfungsbereich, der behinderungsbedingt beeinträchtigt ist, nicht bewertet wird oder indem die Aufgaben individuell so angepasst werden, dass sie sich in dem Leistungsbereich bewegen, den der Schüler mit Behinderung beherrscht. So müssen etwa Lesetexte bei einem Schüler der 3. Klasse, der aber nur das Lesevermögen der 1. Klasse hat, dem Schwierigkeitsgrad der 1. Klasse entsprechen. Diktate dürfen nur den Wortschatz beinhalten, den der Schüler sicher beherrscht. In Mathematik muss die Aufgabe ebenfalls an den beherrschten Zahlenraum angepasst werden. Werden z.B. bestimmte Rechenoperationen der 8. Klasse Gymnasium geprüft, so sind die Bedingungen für einen Schüler mit Dyskalkulie nur dann ausgeglichen, wenn die Aufgaben auf den Zahlenbereich zugeschnitten sind, den er beherrscht und wenn er für die Grundrechenarten einen Taschenrechner benutzen kann. Dann ist er in der Lage, das gleiche Ziel zu erreichen, nämlich nachzuweisen, dass er die Rechenoperation beherrscht.

\section{Maß und Grenze des Nachteilsausgleichs}

Die schulrechtlichen Regelungen sehen, soweit sie die Behinderungen Legasthenie/Dyskalkulie berücksichtigen, durchgängig ein abgestuftes System der Berücksichtigung vor. Die Berücksichtigung wird auf die Grundschule oder bestimmte Schulstufen beschränkt, von bestimmten Noten abhängig gemacht, bei durchschnittlichen Noten wieder entzogen, auf bestimmte Fächer begrenzt 
oder Ähnliches. Fraglich ist, ob und wenn ja, inwieweit derartige Beschränkungen verfassungsrechtlich zulässig sind.

\subsection{Ausgleich nur bei schwerer Betroffenheit?}

Die landesrechtlichen Regelungen machen die Gewährung von Nachteilsausgleich bei Legasthenie/Dyskalkulie teilweise von einer gewissen Schwere der Beeinträchtigung abhängig. Die KMK-Empfehlung lässt einen Nachteilsausgleich nur zu, wenn eine "besonders schwere Beeinträchtigung" vorliegt ${ }^{1006}$, BadenWürttemberg verlangt einen besonders begründeten Ausnahmefall ${ }^{100}$, Berlin eine festgestellte gravierende Lese-Rechtschreibstörung ${ }^{1008}$, Hamburg eine besonders schwere Beeinträchtigung, die den Nachweis des Leistungsstandes wesentlich erschwert. ${ }^{1009}$

Eine solche Beschränkung des Ausgleichs auf schwere oder wesentliche Beeinträchtigungen verstößt, wie oben bereits erläutert, gegen Art. 3 III 2 GG. Behinderungen i.S.d. Art. 3 III 2 GG sind alle Formen der Behinderungen, nicht nur besonders schwere. Wegen keiner dieser Behinderungen darf benachteiligt werden. Es ist deshalb unzulässig, Ausgleich nur für schwere Behinderungen zu gewähren und damit in Kauf zu nehmen, dass bei leichteren Behinderungen Benachteiligungen auftreten.

\subsection{Ausgleich nur bei wesentlichen Auswirkungen?}

Hamburg hat in $\S 3$ Abs. 4 APO-AS ${ }^{1010}$ und $\S 13$ APO-AH den Nachteilsausgleich darauf beschränkt, dass der Nachweis des Leistungsstandes wesentlich erschwert ist, d.h. die Behinderung sich in besonderem Maße auswirkt. Das OVG Lüneburg hat ähnlich den Nachteilsausgleich in dem Fall abgelehnt, in dem sich Nachteile nur rudimentär und damit in zu vernachlässigender Weise auswirken. ${ }^{1011}$ Der Schutzbereich des Art. 3 III 2 GG ist jedoch gerade nicht auf "wesentliche" Benachteiligungen beschränkt. ${ }^{1012}$ Infolgedessen muss ein Ausgleich bei jeder Form der Benachteiligung erfolgen. Würde man rudimentäre Wirkun-

\footnotetext{
1006 KMK-Lesen/Rechtschreiben/Rechnen: Punkt Abschlüsse und Prüfungssituationen, S. 4

${ }^{1007}$ BW VV Besonderer Förderbedarf Nr. 2.3.1

1008 BE VO-GO § $31 ॥$

1009 HH APO-AS § 3 IV; APO-AH § 13

1010 HH APO-AS § 3 IV

1011 OVG Lüneburg, Beschluss v. 10.7.2008, Az.: 2 ME 309/08, www.rechtsprechung.nieder sachsen.de, Rn. 14; dass sich die Legasthenie in dem vom OVG Lüneburg entschiedenen Fall nicht nur rudimentär auf die mathematisch-naturwissenschaftlichen Fächer auswirkt, ergibt sich daraus, dass auch in diesen Fächern die Rechtschreibung gewertet wird und Fehler zur Abwertung führen sowie aus dem erheblichen Lesepensum auch in diesen Fächern. S. dazu sogleich unten S. $194 \mathrm{ff}$

1012 S. oben 5. Teil: 1. Kapitel: 3.2.: Ausgleich nur bei wesentlichen Auswirkungen?, S. 188
} 
gen nicht ausgleichen, weil sie zu vernachlässigen sind, würde man schon logisch keine gleichen Bedingungen herstellen, sondern würde den beeinträchtigten Prüfling dazu verpflichten, jedenfalls rudimentäre Beeinträchtigungen zu tragen, also ungleiche Prüfungsbedingungen hinzunehmen. Die viel schwierige Frage, die es dann zu beantworten gilt, ist, was rudimentär ist und wer das feststellt.

\subsection{Ausgleich nur schulformbezogen und für begrenzte Dauer?}

Für die Dyskalkulie wird der Anspruch durch die KMK-Empfehlung auf den Primarbereich beschränkt. Die Bundesländer, die eine Regelung zur Dyskalkulie haben, berücksichtigen sie in der Grundschule und teilweise in der Sekundarstufe I, nicht aber in der Sekundarstufe II. ${ }^{1013}$ Für die Legasthenie wird der Anspruch auf Ausgleich häufig zeitlich und auch schulformspezifisch begrenzt. Ab der 5. Klasse wird die Gewährung von Ausgleich an immer strengere Kriterien geknüpft und bis auf wenige Ausnahmen mit der 10. Klasse beendet. Einige Länder gewähren Ausgleich in der Hauptschule, lassen ihn aber in der Realschule und dem Gymnasium oder ab Klasse 7 des Gymnasiums nicht zu. ${ }^{1014}$ Einige Länder lassen Ausgleich in der Sekundarstufe I zu, nicht aber in den Abschlussklassen und Abschlussprüfungen dieser Schulstufe. ${ }^{1015}$ Überwiegend wird Ausgleich in der Sekundarstufe II nicht mehr gewährt. ${ }^{1016}$ Ausnahmen gelten für Bayern ${ }^{1017}$, Brandenburg ${ }^{1018}$, Hessen ${ }^{1019}$, Mecklenburg-Vorpommern ${ }^{1020}$, Bremen $^{1021}$, Niedersachsen ${ }^{1022}$, Sachsen-Anhalt ${ }^{1023}$, Schleswig-Holstein. ${ }^{1024}$ Meistens haben die Länder darüber hinaus spezielle Vorschriften zur Berück-

\footnotetext{
1013 S. oben 1. Teil: 2. Kapitel: 5.: Berücksichtigung der Dyskalkulie in der Schule, S. 39

1014 SL VV-LRS Nr. 5.1 - bis Klasse 9; SN VV-LRS Nr. 4.3, 4.4 bis Oberstufe möglich; NRW LRSErlass Nr. 4 (bis Klasse 6, begründete Einzelfälle bis 10, keine Unterscheidung der Schulform); HH Förder-Richtlinie Nr. 4 (bis Klasse acht)

1015 BW VV Besonderer Förderbedarf Nr. 2.3.2; BE Sek I-VO § 14 III; NI LRR-Erlass Nr. 4.2; SN VVLRS Nr. 4.4; ST VO Sonderpäd. Förderung § 7 III; TH Fachliche Förderempfehlung L.u.Z.

1016 BE VO-GO (nicht vorgesehen); NRW LRS-Erlass Nr. 4; RP VV-LRS Nr. 1; SN VV-LRS Nr. 4.4

1017 BY KMBek Legasthenie IV. 3.6 - einschließlich Abitur

1018 BB VV-LRSR Nr. 7 I

1019 HE VO-GestSchulVerh § 39 IV - aber nicht mehr im Abitur - so VGH Kassel, Beschluss V. 5.2.2010, Az.: 7 A 2406/09, juris

1020 MV VV-LRR Nr. 7III, IV

${ }^{1021}$ HB LRS-Erlass Nr. 4.1 und 4.4.1 und 4.4.2. - die Berücksichtigung ist in der Abschlussprüfung Mittlerer Schulabschluss ausgeschlossen, denn dann gelten die verbindlichen Korrekturanweisungen (Nr. 4.4.1)

1022 NI LRR-Erlass Nr. 4.2 - nicht in Abschlussprüfung

1023 ST VO Abschlüsse $§ 20$

1024 SH Legasthenie-Erlass Nr. 1.1.1
} 
sichtigung von Behinderungen in Prüfungen (z.B. § 31 RP Abitur), wenden sie aber bei Legasthenie und Dyskalkulie nicht an. Oder der Ausgleich wird auf eine bestimmte Form, z.B. Zeitverlängerung, beschränkt. So regelt $\S 31 \mathrm{Abs} .1 \mathrm{BE}$ VO-GO, dass behinderte Schüler einen individuellen Nachteilsausgleich erhalten, demgegenüber können Schüler und Schülerinnen mit festgestellten gravierenden Lese- und Rechtschreibstörungen nach Abs. 2 nur eine Verlängerung der Bearbeitungszeit für die schriftlichen Prüfungen beantragen. Ein individueller Nachteilsausgleich etwa durch weitere Hilfsmittel ist nicht vorgesehen. Die Schüler und Schülerinnen mit Legasthenie/Dyskalkulie müssen daher in bestimmten Schulformen und ab bestimmten Klassenstufen ihre Prüfungsleistungen ohne Berücksichtigung der Behinderung erbringen.

Die Rechtsprechung sieht dies als berechtigt an. Es sei stimmig, das System der Fördermaßnahmen vor der Abschlussprüfung zwingend zu beenden. ${ }^{1025}$

Die Benachteiligung wegen einer Behinderung ist aber nach der hier vertretenen Auffassung immer verboten, so dass das Grundrecht in allen Schularten und allen schulischen Prüfungen zu berücksichtigen ist. Legasthenie/Dyskalkulie bleiben bis ins Erwachsenenalter erhalten ${ }^{1026}$, es gibt keine Erkenntnisse da$z u$, dass die Störungen ab einem bestimmten Alter behoben sind oder nicht mehr beeinträchtigen. Infolgedessen ist die Behinderung auch in den höheren Schulformen und Abschlüssen noch vorhanden und muss berücksichtigt werden.

\subsection{Berücksichtigung bei durchschnittlichen Noten?}

Die landesrechtlichen Bestimmungen sehen vor, dass die Anerkennung der Legasthenie entzogen wird, wenn die Leistungen des betroffenen Kindes sich verbessern und es zumindest ausreichende Leistungen erbringt. ${ }^{1027}$ Das Schulamt hat im Verfahren vor dem VG Darmstadt ausgeführt, dass es Ziel der Fördermaßnahmen sei, den Schüler zu befähigen, ohne Notenschutz und Nachteilsausgleich jedenfalls durchschnittliche Leistungen zu erbringen. ${ }^{1028}$

Die Regelungen differenzieren zwischen Schülern und Schülerinnen mit Behinderung, die ausreichende Noten und solchen, die bessere Noten erbringen. Dahinter steckt der Gedanke, dass behinderte Schüler und Schülerinnen mit besseren Noten keinen Nachteilsausgleich brauchen, weil sie aus eigener Kraft in der Lage sind, die Klassenziele zu erreichen. Behinderte Schüler und Schüle-

\footnotetext{
1025 VGH Kassel, Beschluss v. 5.2.2010, Az.: 7 A 2406/09, juris, Rn. 36

1026 Steinhausen, Psychische Störungen bei Kindern und Jugendlichen, S. 164 f; Schulte-Körne/ Stieglitz, in: Adoleszenzpsychiatrie, 476

${ }^{1027}$ Z.B. BW VV Besonderer Förderbedarf Nr. 2.3.2; HB LRS-Erlass Nr. 4.2; NI LRR-Erlass Nr. 3.2; NRW LRS-Erlass Nr. 3.1; SL VV-LRS Nr. 3 und 4.5; SH Legasthenie FAQ S. 6 und 14 - Aussetzung des Nicht-Bewertung der Rechtschreibung, wenn die Leistung besser als mangelhaft ist. 1028 VG Darmstadt, Urteil v. 28. 4. 2009, Az.: 7 K 687/08.DA (3), S. 4 (nicht veröffentlicht)
} 
rinnen mit schlechteren Noten brauchen jedoch einen Nachteilsausgleich, weil bei ihnen die Gefahr besteht, dass sie aufgrund der Behinderung das Klassenziel nicht erreichen und nicht versetzt werden. Damit wird differenziert nach dem Ausmaß, wie sehr sich die Behinderung auf die Teilhabe, also die Erreichung des Klassen- oder Prüfungsziels auswirkt. Auf die Schwere der Beeinträchtigung kommt es für den Schutz des Art. 3 III 2 GG aber nicht an, weshalb die Schwere kein zulässiger Differenzierungsgrund ist.

Zulässig wäre die Differenzierung dann, wenn behinderte Schüler und Schülerinnen mit besseren Noten nicht mehr in der Teilhabe eingeschränkt wären. Das wäre der Fall, wenn sich die Behinderung Legasthenie/Dyskalkulie bei innen nicht mehr nachteilig auswirken würde. Wird bei diesen Schülern und Schülerinnen die Behinderung nicht berücksichtigt, werden sie wie Schüler ohne Behinderung behandelt und den allgemeinen Bewertungsregelungen unterworfen. Ihre Leistungen werden dementsprechend wegen der schlechten Rechtschreib- oder Rechenleistung abgewertet. Sie bekommen damit schlechtere Noten als wenn die Behinderung berücksichtigt würde. Das hat zunächst vielfältige psychische Folgen und beeinträchtigt die soziale Teilhabe. Konkret im Schulbereich wirken sich die schlechteren Noten dadurch aus, dass die Schüler und Schülerinnen z.B. die Noten, die für den Übergang auf weiterführende Schulen erforderlich sind, nicht erreichen. In der Oberstufe wirkt sich jede Einzelnote auf die Abiturnote aus. Deshalb führen hier schlechtere Noten automatisch zu einem schlechteren Abiturschnitt und daher auch zu schlechteren Zulassungschancen an Universitäten. Auch bei diesen Schülern und Schülerinnen wirkt sich daher die Nicht-Berücksichtigung nachteilig aus, so dass sie verboten ist.

\subsection{Ausgleich bei erfolgreichem Schulbesuch?}

Das VG Sigmaringen hat die Gewährung von Nachteilsausgleich und die NichtBewertung der Rechtschreibung mit der Begründung abgelehnt, dass der betroffene Schüler auch ohne Berücksichtigung der Beeinträchtigung in der Lage gewesen sei, die schriftlichen Prüfungen zu bestehen und das Klassenziel zu erreichen. Es sei daher nicht glaubhaft gemacht, dass ihm die ordnungsgemäße Absolvierung der Abiturprüfung erheblich erschwert sei. ${ }^{1029}$ Auch das VG Schleswig stellt darauf ab, ob dem betroffenen Schüler "die Erlangung des Abiturs jedenfalls bei der jetzigen Form der Leistungsbewertung möglich ist" ${ }^{1030}$

Wie bereits ausgeführt, kommt es für das grundsätzliche Verbot der Benachteiligung nicht auf das Ausmaß der Teilhabebeeinträchtigung an. Art. 3 III 2

\footnotetext{
${ }^{1029}$ VG Sigmaringen, Beschluss v. 2.4.2008, Az.: 4 K 596/08, S. 4 (nicht veröffentlicht)

1030 VG Schleswig, Urteil v. 10.6.2009, Az.: 9 A 208/08, BeckRS 2009 38123, S. 9
} 
GG verbietet jede Form der Benachteiligung, nicht nur die „existenzgefährdende“. ${ }^{1031}$

\subsection{Legasthenie: zurückhaltende Bewertung der Rechtschreibung?}

In der Literatur ${ }^{1032}$ und Rechtsprechung ${ }^{1033}$ wird vertreten, dass die Rechtschreibung bei Schülern und Schülerinnen mit Behinderung zu bewerten ist, Art. 3 III 2 GG aber insoweit als Auslegungs- und Ermessensdirektive dienen könne. Da die sprachliche Richtigkeit nach den Vorschriften "angemessen“ zu berücksichtigen sei, liege es im Ermessen der Lehrkräfte die Rechtschreibfehler bei Legasthenie gar nicht oder nur zurückhaltend in die Note einfließen zu lassen. ${ }^{1034}$ Zunächst setzt eine solche Ermessensentscheidung voraus, dass die Legasthenie in der Schule als Behinderung anerkannt worden ist und anschließend entschieden wird, ob und inwieweit bei dem betroffenen Schüler die Rechtschreibung bewertet wird. Bisher kommt es in der Regel nicht zu einer solchen Ermessensentscheidung, weil schon die Legasthenie als solche überwiegend nicht anerkannt wird. ${ }^{1035}$

Ist die Behinderung Legasthenie anerkannt, dann spricht für eine solche Ermessensentscheidung, dass es schwierig ist, die genaue Betroffenheit eines Kindes allgemein und vorab festzustellen, weil die Grenzen zwischen verschiedenen Graden der Legasthenie fließend sind. Es liegt daher nahe, die Entscheidung über den Umfang der Bewertung der Rechtschreibung den Lehrkräften anzuvertrauen, die das Kind unterrichten. Gegen eine solche Ermessensentscheidung spricht jedoch, dass die Bewertung der Rechtschreibung, außer im Diktat, immer eine negative Entscheidung ist. Nur im Diktat ist es möglich, wegen guter bzw. fehlerfreier Rechtschreibung eine gute Note zu bekommen. Im Übrigen wirkt es sich jedoch nicht positiv aus, dass die Rechtschreibung gut ist, etwa indem die Endnote aufgewertet wird, sondern es wird negativ entschieden, ob die Fehler in der Rechtschreibung zu einer Abwertung der Note führen. Auch mit einer abgeschwächten Bewertung der Rechtschreibung wird damit ein Bereich benotet, der behinderungsbedingt nicht geleistet werden kann. Die

${ }^{1031}$ S. oben 5. Teil: 1. Kapitel: 3.1: Ausgleich nur bei schwerer Betroffenheit?, S. 188

${ }^{1032}$ Ennuschat/Volino, br 2009, S. $166 \mathrm{ff}$, S. 168

1033 OVG Lüneburg, Beschluss v. 25.3.2011, Az.: 2 ME 52/11 (nicht veröffentlicht)

1034 Ennuschat/Volino, br 2009, S. 166 ff, S. 168; OVG Lüneburg, Beschluss v. 25.3.2011, Az.: 2 ME 52/11, S. 6 (nicht veröffentlicht)

1035 Vgl. VG Gießen, Urteil v. 30.6.2009. Az.: 4 K 1052/09.GI (nicht veröffentlicht); VG Gießen, Urteil v. 2.11.2010, Az.: 7 K 1841/10.GI (nicht veröffentlicht); OVG Lüneburg, Beschluss V. 10.7.2008, Az.: 2 ME 309/08, www.rechtsprechung.niedersachsen.de; VGH Kassel, Beschluss v. 5.2.2010, Az.: 7 A 2406/09, juris, Rn. 36; VG Darmstadt, Urteil v. 28. 4. 2009, Az.: 7 K 687/08.DA (3), (nicht veröffentlicht); VG Sigmaringen, Beschluss v. 2.4.2008, Az.: 4 K 596/08 (nicht veröffentlicht); VG Schleswig, Urteil v. 10.6.2009, Az.: 9 A 208/08, BeckRS 2009 38123, S. 9 
Ermessensausübung wird dann davon abhängen, wie stark der Schüler betroffen ist. Je schlechter ein Kind ist, desto mehr Verständnis dürfte sich für eine Nicht-Bewertung der Rechtschreibung ergeben. Das aber bedeutet, dass bei Kindern mit einer geringeren Ausprägung und damit einer leichteren Behinderung weniger häufiger von diesem Ermessen Gebrauch gemacht werden wird und sie normal gewertet und damit benachteiligt werden. Damit wird wieder zwischen leichten und schweren Behinderungen differenziert, obwohl Art. 3 III $2 \mathrm{GG}$ alle Formen der Behinderung schützt.

Zudem wird es damit in das Ermessen der Lehrkräfte gestellt, ob sie eine festgestellte Behinderung berücksichtigen oder nicht, denn ihre Sicht und Kenntnis der Behinderung bestimmt dann, ob ein Schüler benachteiligt ist oder nicht. Gegen eine solche Sichtweise sprechen zunächst die grundsätzlichen Bedenken gegen die Bestimmung der Benachteiligung aufgrund eines „objektiven" Maßstabs ohne Berücksichtigung der subjektiven Sichtweise des Menschen mit Behinderung im Rahmen von Art. 3 III 2 GG. ${ }^{1036}$ Des Weiteren zeigt die Rechtsprechung, dass eine solche Benachteiligung dann in der Regel verneint wird. In zwei Fällen hat das VG Gießen entschieden, dass eine Benachteiligung des Schülers mit Legasthenie nicht gegeben war, weil die Lehrer der Ansicht waren, dass die betroffenen Schüler nicht so gravierend benachteiligt waren, dass die Rechtschreibung nicht zu bewerten war. ${ }^{1037}$ In beiden Fällen lag allerdings nach den medizinischen Werten hochgradige Legasthenie vor, in einem Fall auch noch belegt durch die Feststellung der Behinderung nach § 2 SGB IX. ${ }^{1038}$ Dieses Ergebnis wird bestätigt durch Untersuchungen, nach denen sich die pädagogische Einschätzung der Lehrkräfte nicht mit den Ergebnissen standardisierter Tests deckt und mit zunehmender Berufserfahrung die Übereinstimmung zwischen der Lehrereinschätzung und dem Test sogar abnimmt. ${ }^{1039}$ Das pädagogische Ermessen ist daher kein verlässlicher Anknüpfungspunkt für die Berücksichtigung einer Behinderung, sondern birgt die ganz erhebliche Gefahr, dass eine anerkannte Behinderung nicht berücksichtigt wird. Zudem besteht die Gefahr, dass das Ermessen entscheidend dadurch geleitet wird, wie sehr sich Eltern für ihre Kinder einsetzen und eine Berücksichtigung fordern. Auch wenn dies für das einzelne Kind ein Vorteil ist, so ergeben sich insgesamt unsachliche, nicht nachprüfbare Entscheidungen, die die Chancengleichheit aller Kinder beeinträchtigt. Es kann daher nicht dem päda-

\footnotetext{
1036 S. oben 3. Teil: 3. Kapitel: 2.: Objektive oder subjektive Bestimmung des Eingriffs, S. 108

${ }^{1037}$ VG Gießen, Urteil v. 30.6.2009. Az.: 4 K 1052/09.GI (nicht veröffentlicht); VG Gießen, Urteil v. 2.11.2010, Az.: 7 K 1841/10.GI (nicht veröffentlicht)

1038 VG Gießen, Urteil v. 30.6.2009. Az.: 4 K 1052/09.GI (nicht veröffentlicht), Prozentrang 2 im Lesen und 4,5 in der Rechtschreibung (s. Fn. 1398); VG Gießen, Urteil v. 2.11.2010, Az.: $7 \mathrm{~K}$ 1841/10.GI, S. 2, 8, Prozentrang 1,4 (nicht veröffentlicht)

1039 Roos/Schöler, Schriftspracherwerb, S. 147, $156 \mathrm{f}$
} 
gogischen Ermessen überlassen bleiben, ob eine Behinderung berücksichtigt wird oder nicht. ${ }^{1040}$

Einige landesrechtliche Regelungen versuchen in diesem Bereich eine Ermessensleitung. So lautet beispielsweise Nr. 9.11 NI EB-AVO-GOFAK ${ }^{1041}$ : „Als Richtwerte sollen gelten: Abzug eines Punktes bei durchschnittlich fünf Fehlern auf einer in normaler Schriftgröße beschriebenen Seite; Abzug von zwei Punkten bei durchschnittlich sieben und mehr Fehlern auf einer in normaler Schriftgröße beschriebenen Seite. Bei der Entscheidung über einen Punktabzug ist ein nur quantifizierendes Verfahren nicht sachgerecht. Vielmehr sind Zahl und Art der Verstöße zu gewichten und in Relation zu Wertzahl, Wortschatz und Satzbau zu setzen ... Ein Punktabzug muss ebenso wie in Grenzfällen ein Verzicht auf Punktabzug begründet werden." Es ist aber völlig unklar, welche Relationen hier gelten, welche Art von Fehlern besonders gravierend sein sollen und wann ein Grenzfall vorliegen könnte. Die Regelung ist daher zu unbestimmt. ${ }^{1042}$

Wenn man für den Bereich der Legasthenie überhaupt an eine Bewertung denken würde, dann wäre dafür erforderlich, dass bestimmt werden kann, welche Rechtschreibung Schüler und Schülerinnen mit Legasthenie beherrschen können und welche nicht, um dann die beherrschbare Rechtschreibung zu benoten. Es müsste also Legasthenie-spezifische Fehler und nicht Legastheniespezifische Fehler geben. Das aber ist nicht der Fall. ${ }^{1043}$ Die betroffenen Schüler beherrschen die Rechtschreibung grundsätzlich nicht und zudem weist jeder Schüler sein eigenes Fehlerspektrum auf. Da die Benachteiligung wegen einer Behinderung in jeder Form verboten ist, kann es daher keinen Maßstab dafür geben, nach welchen Kriterien ein Ermessen ausgeübt werden soll, ob und wann und in welchem Grad die Rechtschreibung bewertet oder nicht bewertet wird, wie die Legasthenie also berücksichtigt oder nicht berücksichtigt wird.

\subsection{Legasthenie: Begrenzung des Ausgleichs auf bestimmte Fächer?}

Das OVG Lüneburg hat in einem Eilverfahren ${ }^{1044}$ den Nachteilsausgleich in Form eines Zeitzuschlags in der Oberstufe auf die nicht mathematisch-naturwissenschaftlichen Fächer begrenzt, da ,in denjenigen Fächern, in denen es nicht pri-

\footnotetext{
1040 VGH Kassel, Beschluss v. 17.11.2010; Az.: 7 A 2970/09.Z, juris, Rn. 9; VG Hannover, Beschluss v. 13.12.2010, Az.: 6 B 5596/10, www.rechtsprechung.niedersachsen.de S. 4

1041 Niedersachsen: Ergänzende Bestimmungen zur Verordnung über die Abschlüsse in der gymnasialen Oberstufe, im Fachgymnasium, im Abendgymnasium und im Kolleg

1042 Nach Geist, in: Legasthenie und Dyskalkulie 2011, S. 170, fühlen sich weder Lehrer noch Eltern mit einer solchen Regelung wohl, sondern empfinden sie als „Wischi-Waschi-Formulierung"

1043 S. dazu oben 1. Teil: 1. Kapitel: 2.: Legasthenie, S. 6

1044 OVG Lüneburg, Beschluss v. 10.7.2008, Az.: 2 ME 309/08, www.rechtsprechung.niedersachsen.de
} 
mär auf die Lese- und Rechtschreibfähigkeiten ankommt, insbesondere in Mathematik und in den Naturwissenschaften, eine festgestellte Legasthenie generell nur rudimentäre und daher zu vernachlässigende Auswirkungen auf die Notenbildung [wird] haben können."

Ausgangspunkt der Überlegungen des OVG Lüneburg ist, dass ein Ausgleich dann nicht erforderlich ist, wenn sich Legasthenie/Dyskalkulie auf die einzelnen Fächer, insbesondere in der Bewertung, nicht auswirken und dort auch nicht zu Nachteilen führen können. Dem OVG Lüneburg ist darin zuzustimmen, dass in dem Fall die Gewährung von Ausgleich überflüssig und auch gleichheitswidrig wäre, weil die Startbedingungen des Schülers mit Legasthenie/Dyskalkulie gegenüber dem nicht-behinderten Schüler de facto verbessert würden. Dafür muss jedoch feststehen, dass sich die Behinderung in bestimmten Fächern in keiner Weise auswirkt. Das ist z.B. für das Fach Sport in der Sekundarstufe I anzunehmen, für die Sekundarstufe II aber zu verneinen, weil dort im Fach Sport auch Klausuren geschrieben werden. Derzeit kann nur für die sehr seltenen Abiturfächer Chinesisch, Japanisch und Darstellendes Spiel angenommen werden, dass ein Nachteilsausgleich in Form der Nicht-Bewertung der Rechtschreibung nicht erforderlich ist, denn in diesen Fächern wird die Rechtschreibung ohnehin nicht gewertet. ${ }^{1045}$

Der Ausschluss eines Ausgleichs in bestimmten Fächern und insbesondere bei Legasthenie in den mathematisch-naturwissenschaftlichen Fächern kommt daher nur in Betracht, wenn sich die Legasthenie in diesen Fächern nicht auswirkt und es auch dort nicht auf die Lese- und Rechtschreibfähigkeit ankommt. ${ }^{1046}$

Inzwischen lassen sich im Internet viele Aufgaben aus Abschlussarbeiten finden. Aus den Aufgaben lässt sich deutlich ablesen, wie hoch der Leseaufwand in einer Prüfung ist. Eine Auswahl von Aufgaben in schulischen Abschlussprüfungen ergibt beispielhaft folgendes Bild des Umfangs der Prüfungsaufgaben:

\footnotetext{
1045 Ein Zeitzuschlag für das Lesen bleibt jedoch erforderlich.

1046 Insofern geht die Annahme von Kischel, in: BeckOK GG, Art. 3, Rn. 219.2, an der Prüfungsund Benotungsrealität vorbei.
} 


\begin{tabular}{|c|c|c|c|}
\hline Bundesland & Abschluss & Fach & Seitenzahl \\
\hline Bayern & Abitur 2011 & Deutsch $^{1047}$ & ca. 19 \\
\hline Bayern & Abitur 2011 G8 & Mathematik ${ }^{1048}$ & 14 \\
\hline Bayern & Abitur 2011 G8 & Physik $^{1049}$ & 13 \\
\hline $\begin{array}{l}\text { Mecklenburg- } \\
\text { Vorpommern }\end{array}$ & Abitur 2009 & $\begin{array}{l}\text { Leistungskurs Mathema- } \\
\text { tik }^{1050}\end{array}$ & 10 \\
\hline $\begin{array}{l}\text { Mecklenburg- } \\
\text { Vorpommern }\end{array}$ & Abitur 2009 & Leistungskurs Physik $^{1051}$ & 7 \\
\hline $\begin{array}{l}\text { Mecklenburg- } \\
\text { Vorpommern }\end{array}$ & Abitur 2009 & Biologie $^{1052}$ & 15 \\
\hline Bayern & Realschule 2010 & Mathematik $^{1053}$ & 6 \\
\hline Bayern & Realschule 2010 & Physik $^{1054}$ & 16 \\
\hline Bayern & $\begin{array}{l}\text { Mittlerer Schulab- } \\
\text { schluss } 2010\end{array}$ & Mathematik $^{1055}$ & 8 \\
\hline Bayern & $\begin{array}{l}\text { Qualifizierender } \\
\text { Hauptschulabschluss } \\
2010\end{array}$ & Mathematik $^{1056}$ & 9 \\
\hline
\end{tabular}

Daran zeigt sich, dass der Leseaufwand für das Erlesen der Aufgabenstellung auch in den mathematisch-naturwissenschaftlichen Fächern erheblich ist. Das schließt es bereits aus, davon auszugehen, dass sich eine Legasthenie, die sich

\footnotetext{
1047 http://www.isb.bayern.de/isb/index.aspx? $\mathrm{MNav}=0 \& Q \mathrm{Nav}=11 \& \mathrm{TNav}=0 \& \mathrm{Nav}=0 \& \mathrm{VTyp}=26 \& \mathrm{Fach}=12 \& \mathrm{VJg}=37$

1048 http://www.isb.bayern.de/isb/download.aspx? DownloadFilelD $=d 32681 f 81 e 49137 e 75 b 683833262 e b 3 a$

1049 http://www.isb.bayern.de/isb/download.aspx? DownloadFileID=37aa802ecdc93eebc8f0cbd96d19d4d6

1050 http://www.bildungsserver-mv.de/download/abitur/abi-09-mathe-mit_cas.pdf

1051 http://www.bildungsserver-mv.de/download/abitur/abi-09-physik.pdf

1052 http://www.bildungsserver-mv.de/download/abitur/abi-09-bio.pdf

1053 http://www.isb.bayern.de/isb/download.aspx?DownloadFileID= 701edb6c24530792dc2f6cd0fb5300dc

1054 http://www.isb.bayern.de/isb/download.aspx?DownloadFileID= 128d4b6370cbc4eaf896aaf458636638

1055 http://www.isb.bayern.de/isb/download.aspx?DownloadFilelD= 6c4045c4dabf2bef2fb2c89526ef3c3f

1056 http://www.isb.bayern.de/isb/download.aspx?DownloadFilelD= $70806092 b d 3 c 9 d 9 a c c d f 67 d 1 c 2 b b 9271$
} 
aus einer Lese- und einer Rechtschreibstörung zusammensetzt, in diesen Fächern nicht auswirkt.

Die Durchsicht der Aufgabentexte zeigt weiter, dass nur in der Aufgabe für den qualifizierenden Hauptschulabschluss in Mathematik nur gerechnet wird. Beim Realschulabschluss und beim Abitur werden dagegen von den Schülern und Schülerinnen sowohl in der Mathematik als auch in den naturwissenschaftlichen Fächern nicht nur Rechnungen, sondern auch Erörterungen, Erläuterungen und Begründungen, also schriftliche Ausführungen, gefordert. Die Ansicht des OVG Lüneburg, dass die Lese- und Rechtschreibfähigkeiten in diesen Fächern keine oder nur rudimentäre Auswirkungen haben, beruht daher auf unrichtigen Vorstellungen von den Aufgabeninhalten und dem Aufgabenumfang in den mathematisch-naturwissenschaftlichen Fächern.

Die Rechtsprechung des OVG Lüneburg lässt außerdem außer Betracht, dass die Abwertungsregel für Fehler in der Rechtschreibung auch für alle naturwissenschaftlich-mathematischen Fächer gilt. Die Rechtschreibung wird auch hier bewertet. ${ }^{1057}$ Deshalb fallen die Noten während der gesamten Oberstufe schlechter aus und die Nachteile potenzieren sich, weil diese Fächer in die Gesamtqualifikation eingebracht werden müssen.

\subsection{Dyskalkulie: Verzicht auf die Zulassungsnote Mathematik}

Um Schülern und Schülerinnen, die ansonsten für eine bestimmte Schullaufbahn geeignet sind, diesen Weg nicht wegen der Behinderung zu verschließen, kommt in Betracht, für die Zulassung auf die Mathematik-Note zu verzichten. Konkret bedeutet dies, dass bei ansonsten gegebener Eignung der Übertritt auf die Realschule oder das Gymnasium zu ermöglichen ist, obwohl in Mathematik nicht das erforderliche „befriedigend“, sondern nur ein „ausreichend" erzielt wurde, dass der Hauptschulabschluss und der Mittlere Schulabschluss erteilt werden, obwohl in Mathematik ein "mangelhaft“ und kein „ausreichend“ erzielt wurde, dass der Schulwechsel in die Sekundarstufe II ermöglicht wird, obwohl kein "ausreichend“ oder „befriedigend" (beim Wechsel von der Realschule) erzielt wurde und dass die Zulassung zum Abitur und das Bestehen des Abiturs nicht an den Qualifikationsvorgaben insbesondere für Mathematik scheitern dürfen. ${ }^{1058}$ Das gleiche gilt für die Fächer, die mathematische Fähigkeiten

1057 Die Frage, ob die Bewertung der Rechtschreibung in diesen Fächern überhaupt verhältnismäßig ist, wird hier ausdrücklich offen gelassen. Die OVG Lüneburg und Berlin-Brandenburg und Kischel gehen selbstverständlich, aber an den rechtlichen Gegebenheiten vorbei, davon aus, dass die Rechtschreibung in Mathematik und den Naturwissenschaften nicht Inhalt der Prüfung ist. OVG Lüneburg, Beschluss v. 10.7.2008, Az.: 2 ME 309/08, www.rechtsprechung.niedersachsen.de; OVG Berlin-Brandenburg, Beschluss v. 16.6.2009, Az.: 3 M 16.09, juris, Rn. 4; Kischel, in: BeckOK GG, Art. 3, Rn. 219.3

${ }^{1058}$ Zu den konkreten Anforderungen für die Sekundarstufe II s. oben 1. Teil: 2. Kapitel: 2.3.: Sekundarbereich II-Oberstufe, S. 33 und für das Abitur 1. Teil: 2. Kapitel: 2.4.: Abitur, S. 35 
voraussetzen, jedenfalls dann, wenn sie versetzungserheblich oder sonst für die Schullaufbahn relevant sind.

\subsection{Verhältnismäßigkeit des Nachteilsausgleichs}

Der Nachteilsausgleich muss den Anforderungen des allgemeinen Gleichheitssatzes aus Art. 3 I GG entsprechen und daher verhältnismäßig sein. Welche Maßnahmen im Einzelfall erforderlich sind, hängt von der individuellen Situation des Kindes ab. Je schwerer ein Kind betroffen ist, desto mehr Nachteilsausgleich braucht es. Je weniger ein Kind betroffen ist, desto geringer ist der Nachteil, der ausgeglichen werden muss. Unterschiede ergeben sich vor allem im Hinblick auf den Zeitzuschlag und die technischen Ausgleichsmöglichkeiten, wie größere Aufgabenblätter, Vorlesetexte usw. Eine differenzierte Bewertung des Rechtschreibens und Rechnens, in dem Sinne, dass ein anderer (herabgesetzter) Bewertungsmaßstab angewandt wird, kommt, wie oben dargelegt wur$\mathrm{de}^{1059}$, nicht in Betracht, weil es hier, anders als bei Zeitzuschlägen usw., nur ein „ganz oder gar nicht" gibt.

Die Ungleichbehandlung durch Nachteilsausgleichfindet dort ihre Grenze, wo die Leistungsfähigkeit und -bereitschaft des Schülers endet und wo sie zur Ergebnisgleichheit führt. Der Nachteilsausgleich dient nicht dazu, Noten soweit aufzubessern, dass grundsätzlich die Versetzung erfolgt oder die Prüfung bestanden wird. Schulische Defizite, Eignungs- und Leistungsmängel bleiben bedeutsam, soweit sie außerhalb der Behinderung liegen. Hat der Schüler seine Vokabeln oder den Stoff in Geschichte oder Erdkunde nicht gelernt oder in dem für ihn gesicherten mathematischen Bereich die Anwendung der Formeln nicht geübt, so erhält er genauso wie alle anderen Schüler und Schülerinnen schlechte Noten und kann genauso sitzen bleiben wie alle anderen. ${ }^{1059}$ S. oben 5. Teil: 1. Kapitel: 3.6.: Legasthenie: zurückhaltende Bewertung der Rechtschrei-
bung?, S. 192 


\section{Kapitel: Verhältnis Nachteilsausgleich und Förderung}

\section{Vorrang des Nachteilsausgleichs vor Nicht-Bewertung von Prüfungsinhalten?}

Die KMK-Empfehlung sieht eine Rangfolge von Nachteilsausgleich und „Abweichen von den allgemeinen Grundsätzen der Leistungsbewertung" vor. Vorrangig ist die Gewährung von Nachteilsausgleich. ${ }^{1060}$ Zudem wird die Abweichung in der Leistungsbewertung in Prüfungen als Ausnahme formuliert, während Nachteilsausgleich immer zu gewähren ist. ${ }^{1061}$ Viele landesrechtliche Regelungen folgendem und lassen die Abweichung von Bewertungsmaßstäben nur als "ultima ratio" zu. ${ }^{1062}$ Sie kommt nach den Regelungen nur in Betracht, wenn Förderung und Nachteilsausgleich nicht gewirkt haben und ist zeitlich zu befristen. Auch die Rechtsprechung ${ }^{1063}$ geht von einer solchen Rangfolge aus. Der VGH Kassel führt aus, dass die Nicht-Bewertung von Leistungen im Hinblick auf das Übermaßverbot nur dann in Betracht kommt, wenn alle denkbaren und möglichen Maßnahmen des Nachteilsausgleichs ausgeschöpft sind und gleichwohl im Einzelfall die bestehende Legasthenie des Schülers nicht angemessen kompensiert werden kann. ${ }^{1064}$ Auch Ennuschat vertritt die Ansicht, dass Abänderungen in der Leistungsbewertung nur als „ultima ratio“ zulässig sind, weil ansonsten der Anspruch der nicht behinderten Schüler auf Chancengleichheit zu sehr beschnitten würde. ${ }^{1065}$ Langenfeld hat demgegenüber eine derartige Rangfolge nicht angenommen. ${ }^{1066}$

1060 Zur Rangfolge innerhalb des Nachteilsausgleichs: VG Ansbach, Beschluss v. 29.2.2008, Az.: AN 2 E 08.00317, juris, Rn. 24 ff - der Betroffenen wurde im 1. Jur. Staatsexamen die Benutzung eines Laptops verweigert, da dies nur die letzte mögliche Maßnahme des Nachteilsausgleichs sei. Stattdessen wurde ihr ohne weitere Zeitverlängerung nur die Unterstützung durch eine Schreibkraft zugestanden, die sie selbst zu suchen hatte; s. auch VG Düsseldorf, Beschluss v. 8.5.2003; Az.: 15 L 1418/03, juris, Rechtsanwaltsfachangestellte als Schreibkraft im 2. Jur. Staatsexamen

1061 KMK-Lesen/Rechtschreiben/Rechnen, Punkt Leistungsbewertung, Grundsätze, S. 3 und Abschlüsse, Prüfungssituationen, S. 5

1062 HH Förder-Richtlinie Nr. 4.1; HE VO-GestSchulVerh § 39 II; NI LRR-Erlass Nr. 4.1; RP VV-LRS Nr. 4.2; SL VV-LRS Nr. 5.1; ST Erlass Grundschulbewertung Nr. 5.1.1

1063 VGH Kassel, Beschluss v. 5.2.2010, Az.: 7 A 2406/09, juris, Rn. 44 ff; VG Aachen, Urteil v. 13.11.2009, Az.: 9 K 25/09, BeckRS 2009 42206, S. 5; differenzierend: VG Kassel, Beschluss v. 23.3.2006, 3 G 419/06, Sonderheft Recht des BVL: Chancengleichheit herstellen - Diskriminierung vermeiden, 2006, http://www.bvl-legasthenie.de/index.php5?p=/downloads, S. 41

1064 VGH Kassel, Beschluss v. 5.2.2010, Az.: 7 A 2406/09, juris, Rn. 44 ff

1065 Ennuschat, br 2008, S. 93 ff, S. 97

1066 Langenfeld, RdJB 2007, S. 211 ff, S. 224 
Grundlage der Überlegungen zur Rangfolge ist, dass auf einen Nachteilsausgleich ein Anspruch aus Art. 3 III 2 GG besteht, während die Nicht-Bewertung der Rechtschreibung als Bevorzugung angesehen wird. Nach der hier vertretenen Ansicht ist indes auch die Nicht-Bewertung der Rechtschreibung oder des Rechnens Nachteilsausgleich, so dass sich die Frage der Rangfolge insoweit nicht stellt. ${ }^{1067}$

Folgt man der hier vertretenen Ansicht nicht, sondern sieht die Veränderung der Leistungsbewertung als Bevorzugung an, dann ist zu prüfen, ob es eine solche Rangfolge zwischen Nachteilsausgleich und Veränderungen der Leistungsbewertung gibt.

Voraussetzung ist, dass der gegebene Nachteilsausgleich für den Menschen mit Behinderung eine vergleichbare, von Behinderung unbeeinflusste Lage schafft, die es ihm ermöglicht, die gleiche inhaltliche Prüfungsleistung zu erbringen wie ein Prüfling ohne Behinderung. Der Nachteilsausgleich bei den äußeren Prüfungsbedingungen schafft jedoch keine Lage, die nicht mehr von der Behinderung beeinflusst wird. Langenfeld hat bereits zutreffend ausgeführt, dass bei einer Legasthenie ein Zeitzuschlag nicht hilft, um das Defizit in der Rechtschreibung auszugleichen. ${ }^{1068}$ Auch bei der Dyskalkulie hilft dem betroffenen Schüler kein Zeitzuschlag, wenn er den geforderten Zahlenraum nicht beherrscht. Erst mit Zeitzuschlag und Nicht-Bewertung der Rechtschreibung resp. und der Umgestaltung der Mathematikaufgaben oder Nicht-Bewertung des Rechnens werden alle behinderungsbedingten Nachteile ausgeglichen. Solange nicht alle Nachteile ausgeräumt sind, wird nur ein Teilaspekt der Behinderung Legasthenie/Dyskalkulie ausgeglichen. Die Teilaspekte der Behinderung, die sich in der Rechtschreibstörung oder im Rechnen manifestieren, werden hingegen nicht ausgeglichen. Vielmehr wird von dem betroffenen Schüler erwartet, dass er sich den normalen Anforderungen anpasst und sich mithilfe eines Nachteilsausgleichs bemüht, diese zu erfüllen. Das widerspricht dem Benachteiligungsverbot, das eine Einpassung des Menschen mit Behinderung gerade nicht mehr fordert.

Sieht man dennoch in der Veränderung der Leistungsbewertung einen Vorteil der Menschen mit Behinderung und damit eine Bevorzugung, so ist es konsequent, dies als Ermessenleistung des Staates zu sehen. In diesem Fall ist der Ermessensspielraum des Staates weit und es steht inm frei, Bevorzugungen in bestimmten Situationen mit Bedingungen zu verknüpfen und auch als „ultima ratio" auszugestalten. Dieser weite Ermessensspielraum des Staates wird je-

\footnotetext{
1067 Vgl. 3. Teil: 3. Kapitel: 4: Abgrenzung der Benachteiligung von der Bevorzugung nach Art. 3 III 2 GG, S. 127

1068 Langenfeld, RdJB 2007, S. 211 ff, S. 224; so auch VG Kassel, Beschluss v. 23.3.2006, 3 G 419/06, Sonderheft Recht des BVL: Chancengleichheit herstellen - Diskriminierung vermeiden, 2006, http://www.bvl-legasthenie.de/index.php5?p=/downloads, S. 41
} 
doch reduziert, wenn die Benachteiligung, so wie hier, nach wie vor besteht und nur mit einer „Bevorzugung" beseitigt werden kann. Dann reduziert sich der weite Ermessensspielraum des Staates auf ein Ermessen, mit dem die Benachteiligung beseitigt wird. Der Staat ist zur "Bevorzugung" in Form der Veränderung der Leistungsbewertung verpflichtet, um die Benachteiligung abzuwenden.

Mit dieser Verpflichtung ist indes eine Rangfolge zwischen einem Nachteilsausgleich bei äußeren Prüfungsbedingungen und Nachteilsausgleich bezüglich der Leistungsbewertung nicht vereinbar, denn eine solche Rangfolge macht die Berücksichtigung der Behinderung von einer gewissen Schwere abhängig, weil die Veränderung der Leistungsbewertung nur erfolgt, wenn der Schüler trotz Nachteilsausgleich massiv schlechte Leistungen in der Rechtschreibung oder im Rechnen zeigt. Schüler, die trotz der Behinderung wenigstens passable Leistungen im Rechtschreiben und Rechnen erbringen, würden demgegenüber normal bewertet mit der Folge der Abwertung ihrer Arbeiten. Damit wird zwischen leichteren und schwereren Formen der Behinderung differenziert, was mit dem Benachteiligungsverbot wegen Behinderung nicht zu vereinbaren ist. ${ }^{1069}$

Eine Rangfolge ist nur dann zulässig, wenn nach dem Ausgleich aller Nachteile eine weitere Berücksichtigung der Behinderung erfolgen soll, etwa indem von einer Fremdsprache oder dem Fach Physik befreit wird ${ }^{1070}$ Hier ist die Lage der Schüler und Schülerinnen mit und ohne Behinderung vergleichbar, so dass es im freien Ermessen des Staates liegt, ob er die Situation der behinderten Schüler und Schülerinnen darüber hinaus verbessern will.

\section{Bindung von Nachteilsausgleich an die Teilnahme an Fördermaßnahmen?}

Vielfach binden die landesrechtlichen Regelungen die Gewährung von Nachteilsausgleich und Nicht-Bewertung der Rechtschreibung an eine Förderung. ${ }^{1071}$ Welchen Umfang und welche Qualität diese Förderung haben soll oder muss, ist nicht geregelt. In Hessen gelten umfangreiche Dokumentationspflichten ${ }^{1072}$. Fehlt es an der Förderung, kann der Schüler keinen Nachteilsausgleich mehr gewährt bekommen.

\footnotetext{
1069 S. dazu bereits oben 3. Teil: 2. Kapitel: 1.9: Diskussion, S. 84

1070 S. dazu oben 3. Teil: 3. Kapitel: 4: Abgrenzung der Benachteiligung von der Bevorzugung nach Art. 3 III 2 GG, S. 127

1071 BW VV Besonderer Förderbedarf 2.3.1; HH APO-GrundStGy § 6 S. 3; HE VO-GestSchulVerh $\S 42$ III Nr. 3; MV VV-LRR Nr. 7 IV; RP VV-LRS Nr. 4.1; SN Nr. 4.3.4; TH Fachliche Förderempfehlung; SH Rechenschwäche-Erlass Nr. 4.2

1072 HE VO-GestSchulVerh § 40
} 
Langenfeld hat dazu festgestellt, dass ein verfassungsrechtlicher Anspruch nicht auf diese Weise entwertet werden kann. ${ }^{1073}$ Das VG Frankfurt hat festgestellt, dass das Grundrecht der Chancengleichheit nicht davon abhängig ist, dass der Schüler gefördert worden ist. ${ }^{1074}$ Demgegenüber hat der VGH Kassel ausgeführt, dass es die Chancengleichheit der anderen Prüflinge aus Art. 3 I GG verlange, dass sich der Betroffene kontinuierlich um eine Überwindung der Einschränkungen bemühe, wenn es nicht ausgeschlossen sei, dass diese durch Anstrengungen des Betroffenen überwunden werden könnten. Nur so könne eine Überkompensation vermieden werden. Die Obliegenheit des Schülers, seinen Beitrag dazu zu leisten, seiner Legasthenie entgegenzuwirken, sei verfassungsrechtlich gefordert. ${ }^{1075}$ Das VG Düsseldorf hat es als Pflicht des Schülers angesehen, ein bestimmtes Förderziel zu erreichen. ${ }^{1076}$

Die Bindung des Nachteilsausgleichs an die Teilnahme an Förderung setzt zweierlei voraus: Erstens müssen Schüler und Schülerinnen überhaupt verpflichtet werden können, an Förderung teilzunehmen, zweitens muss es zulässig sein, wegen der Nicht-Teilnahme an der Förderung den Nachteilsausgleich zu entziehen, also ein Junktim zwischen Nachteilsausgleich und Förderung herzustellen.

\subsection{Pflicht zur Teilnahme an Förderung}

Die Pflicht zur Teilnahme an der Förderung kann sich zunächst aus der allgemeinen Schulpflicht ergeben. Jeder Schüler ist dazu verpflichtet, regelmäßig am Unterricht und an allen anderen, nicht ausdrücklich freiwilligen Veranstaltungen teilzunehmen. ${ }^{1077}$ Förderung ist jedoch keine allgemeine verbindliche Veranstaltung der Schule, so dass sich die Verpflichtung nicht aus der allgemeinen Schulpflicht herleiten lässt.

Eine über die allgemeine Schulpflicht hinausgehende Teilnahmeverpflichtung an einer schulischen Veranstaltung, etwa in Form eines Förderunterrichts, ist, ebenso wie die Schulpflicht, ein Eingriff in das Grundrecht aus Art. 2 I GG ${ }^{1078}$ und bedarf daher einer gesetzlichen Grundlage. Dementsprechend bestimmen die landesrechtlichen Bestimmungen entsprechende Verpflichtungen für den Besuch von Förderunterricht, wenn die Schüler und Schülerinnen diesem von den Lehrkräften zugewiesen wurden. ${ }^{1079}$ Dieser Förderunterricht ist in der Re-

\footnotetext{
1073 Langenfeld, RdJB 2007, S. 211 ff, S. 220

1074 VG Frankfurt a. M., Urteil v. 10.9.2009, Az.: 5 K 1501/09.F (1), S. 10 (nicht veröffentlicht)

1075 VGH Kassel v. 17.11.2010. Az: 7 A 2970/09.7, juris, Rn. 6 und 10

1076 VG Düsseldorf, Beschluss v. 20.5.2011, Az.: 18 L 707/11, juris, Rn. 14

${ }^{1077}$ Avenarius/Heckel, Schulrecht, Ziff. 25.13; Niehues/Rux, Schulrecht, Rn. 245

1078 Vgl. nur Avenarius/Heckel, Schulrecht, Ziff. 25.12

1079 S. z.B. NRW APO-S-I § 3 III; NI Erlass „Arbeit Sek I Gymnasium“, Nr. 5.7.1. i.V.m. 5.5.1 S. 2
} 
gel ein zeitlich befristeter Unterricht, der dazu dienen soll, vorübergehende Schwierigkeiten des Schülers zu beheben. ${ }^{1080}$

Um solche Förderung geht es bei Schülern und Schülerinnen mit einer Behinderung jedoch nicht. Die Behinderung ist nicht heilbar. ${ }^{1081}$ Die dauernde Förderung dient nicht dazu, dass die Behinderung behoben wird, sondern dazu, dass der Mensch mit Behinderung lernt mit seiner Behinderung umzugehen und trotz oder mit der Einschränkung am Leben teilhaben kann. ${ }^{1082}$ Dazu zählen etwa das Erlernen der Braille-Schrift oder der Gebärdensprache und auch das Mobilitätstraining bei Rollstuhlfahrern. Diese Förderungen sind sicherlich verhältnismäßig. Etwas anderes kann sich jedoch ergeben, wenn Ziel der Förderung ist, dass der Betroffene an seiner Behinderung arbeitet und sie überwindet. Gegen diesen Ansatz, dass der Betroffene seine Behinderung überwinden muss, ergeben sich grundsätzliche Bedenken aus dem vorliegend vertretenen sozialen Behinderungsbegriff. Dieser akzeptiert den Menschen mit Behinderung so, wie er ist und verlangt gerade keine Anpassungsleistung mehr von inm. Seine Behinderung ist kein „problem to be fixed “ ${ }^{“ 083}$ mehr, so dass er sich nicht bemühen muss "normal“ zu sein. Deshalb ist die Frage, ob es nicht ein „Freiheit zur Krankheit“1084 oder auch ein „Recht auf Behinderung“ gibt, so dass es unzulässig wäre, einen Menschen mit Behinderung dazu zu verpflichten, an seiner Behinderung zu arbeiten und diese zu überwinden, weil eine solche Verpflichtung gegen den Selbstbestimmungswillen der Menschen mit Behinderung verstoßen würde. Die Freiheitsrechte schließen das Recht ein, von der Freiheit einen Gebrauch zu machen, der - jedenfalls in den Augen Dritter - den wohlverstandenen Interessen des Grundrechtsträgers zuwiderläuft. Deshalb ist es grundsätzlich Sache des Einzelnen, darüber zu entscheiden, ob er sich Maßnahmen unterziehen will, die ausschließlich seiner Besserung dienen. Die grundrechtlich geschützte Freiheit schließt auch die „Freiheit zur Krankheit" und damit das Recht ein, auf Heilung zielende Eingriffe abzulehnen, selbst wenn diese nach dem Stand des medizinischen Wissens dringend angezeigt sind. ${ }^{1085}$ Gegen eine solche Ausweitung des Freiheitsrechts spricht jedoch bei Schülern und Schülerinnen, dass die Schulpflicht bereits eine Entscheidung des Staates dahin ist, bei Kindern und Jugendlichen die Teilnahme an der Bildung für so wesent-

\footnotetext{
1080 Z.B. NRW APO-S-I § 3 III

1081 Bundesregierung, Nationaler Aktionsplan zur UN-BRK, S. 21

1082 Insofern unzutreffend VG Düsseldorf, Beschluss v. 20.5.2011, Az.: 18 L 707/11, juris, Rn. 14

1083 so die Hohe Kommissarin für Menschenrechte Navi Pillay im März 2009, zitiert nach Degener, RdJB 2009, S. 200 ff, S. 201

1084 S. dazu BVerfGE 58, 208, 225 Rn 44 und BVerfG, 2 BvR 2270/96 vom 23.3.1998, Absatz-Nr. (1 - 25), http://www.bverfg.de/entscheidungen/rk19980323_2bvr227096.html

1085 Ausführlich dazu BVerfG, Beschluss v. 23.3.2011; Az.: 2 BvR 882/09; http://www.bundesver fassungsgericht.de/entscheidungen/rs20110323_2bvr088209.html, Rn. 48 ff
} 
lich anzusehen, dass die Einschränkung des Grundrechts aus Art. 2 I GG gerechtfertigt ist. Ebenso dient eine qualifizierte Förderung der Schüler und Schülerinnen mit Legasthenie/Dyskalkulie dazu, ihnen Bildung, Bildungschancen und die Teilhabe am Leben gerade zu ermöglichen soll. Die Nicht-Teilnahme an qualifizierten Förderungen versperrt innen die Teilhabe erst recht.

Grundsätzlich steht daher Art. 3 III 2 GG einer Verpflichtung zur Teilnahme an Förderungen nicht entgegen.

Die Grenzen der Verpflichtung ergeben sich aus dem Grundsatz der Verhältnismäßigkeit, d.h. die Förderung muss einem legitimen Zweck dienen, geeignet, erforderlich und angemessen sein. Ähnliche Verpflichtungen zur Teilnahme an Maßnahmen ergeben sich z.B. auch im Sozialrecht, in dem die Mitwirkungspflicht und ihre Grenzen umfassend geregelt sind ( $\S \S 60 \mathrm{ff}$ SGB I). Das Schulrecht lässt sich mit der Einbindung der Schüler in das System und die Leistung, die der Staat gegenüber den Schülern und auch Eltern erbringt, mit diesem Bereich vergleichen. Die Förderung kann also im weitesten Sinne mit einer Heilbehandlung verglichen werden, so dass es naheliegt, diese Vorschriften zur Konkretisierung der Verhältnismäßigkeit heranzuziehen. ${ }^{1086}$

Der legitime Zweck der Mitwirkung oder Verpflichtung zur Teilnahme bezieht sich auf das Verhältnis zwischen dem Leistenden, hier dem Staat bzw. der Schule, und dem Verpflichteten. Hier dient die Förderung dazu, dass der Staat seinen Bildungsauftrag erfüllen kann und das Kind die Kulturtechniken erlernt. Das ist sicher ein legitimer Zweck. Der VGH Kassel hat es als legitimen Zweck (und im Weiteren als verfassungsrechtlich gefordert, also auch verhältnismäBig) angesehen, dass ein Kind zur Teilnahme an der Förderung verpflichtet wird, um so die eigene Beeinträchtigung zu überwinden, damit dadurch wiederum die Chancengleichheit der Mitschüler gewahrt wird. ${ }^{1087}$ Gedacht ist wohl daran, dass die Chancengleichheit der Mitschüler umso weniger beeinträchtigt wird, je mehr sich die Legasthenie durch die Förderung bessert und deshalb umso weniger Nachteilsausgleich erforderlich ist. Das würde voraussetzen, dass zwischen der Legasthenie des Kindes und der beeinträchtigten Chancengleichheit der Mitschüler ein Kausal- oder wenigstens ein Zurechnungszusammenhang besteht. Die Legasthenie des Schülers ist für die Chancengleichheit der Mitschüler jedoch ohne Auswirkung. Der legasthene Schüler wird aufgrund einer Ausnahmeregelung anders benotet als die Mitschüler. Dieser sachliche Grund gebietet die Ungleichbehandlung, die, wie oben bereits dargestellt, nicht in die Rechte der Mitschüler eingreift. Deren Rechtskreis bleibt unangetastet. Vergleichbar ist das mit der Nicht-Benotung von Sport bei einem Rollstuhlfahrer. Wenn andere Schüler weiterhin im Sport benotet werden und dabei

\footnotetext{
1086 Umfassend auch BVerfG, Beschluss v. 23.3.2011; Az.: 2 BvR 882/09; http://www.bundes verfassungsgericht.de/entscheidungen/rs20110323_2bvr088209.html, Rn. $56 \mathrm{ff}$

1087 VGH Kassel v. 17.11.2010. Az: 7 A 2970/09.7, juris, Rn. 6 und 10
} 
schlechte Noten erzielen, dann ist die Nicht-Benotung des Rollstuhlfahrers dafür weder kausal noch irgendwie zurechenbar. Das eine hat mit dem anderen nichts zu tun. Infolgedessen ist die Lage der Mitschüler kein legitimer Zweck, um das Kind mit Behinderung zu einer Förderung zu verpflichten.

Die Förderung muss weiter geeignet sein. Bei Heilbehandlungen besteht eine Mitwirkungspflicht nach $\S \S 63,65$ II SGB I nur dann, wenn zu erwarten ist, dass sie eine Verbesserung des Zustandes erwarten lassen, weil davon ausgegangen wird, dass sich dadurch die Erbringung von Sozialleistungen erübrigt. ${ }^{1088}$ Welche Förderung bei Legasthenie/Dyskalkulie geeignet ist und eine Besserung erwarten lässt, ist zwischen Schulbehörden, Jugendamt, Medizin und Psychologie in hohem Maße umstritten.

Nach Auffassung der medizinischen Wissenschaften setzen langfristige Erfolge, z.B. eine eindeutige größere Steigerung der Lesefähigkeit, therapeutische Maßnahmen und diese wiederum eine sorgfältige Diagnostik voraus. ${ }^{1089}$ Sie zeigen sich nur bei einer Förderung die nicht nur einfach intensiver, sondern anders als normale schulische Förderung ist. ${ }^{1090}$ Ein Fördererfolg kann sich daher nur dann einstellen, wenn die Gruppen möglichst leistungshomogen und störungsspezifisch sind und die Gruppengröße maximal 5 Kinder beträgt. ${ }^{1091}$

Demgegenüber fördert die Schule bisher im Rahmen ihres allgemeinen Förderauftrages, der nur dem Grunde nach, und nicht als Anspruch auf eine bestimmte Fördermaßnahme besteht, ${ }^{1092}$ da das öffentliche Schulwesen nicht sämtlichen unterschiedlichen individuellen Begabungen und Schwächen voll Rechnung tragen kann. ${ }^{1093}$ Die Wahl der Fördermaßnahme steht im Ermessen der zuständigen Schule bzw. Schulbehörde. ${ }^{1094}$ Eine separate Förderung erfolgt

\footnotetext{
${ }^{1088}$ Mrozynski, SGB I, § 63 SGB I, Rn. 6; Seewald in Leitherer, Kasseler Kommentar Sozialversicherungsrecht, § 64 SGB I, Rn. 9

1089 Steinhausen, Psychische Störungen bei Kindern und Jugendlichen. S. 160

1090 Klicpera/Gasteiger-Klicpera, in: Hdb. Sonderpädagogik, S. 361; Warnke/Schulte-Körne, in: Therapie psychischer Störungen, S. 157: Kinder mit Legasthenie benötigen z.B. Regeltraining, das im normalen schulischen Förderunterricht weniger als $1 \%$ ausmacht.

1091 Warnke/Schulte-Körne, in: Therapie psychischer Störungen, S. 160

1092 Ausführlich dazu Schwengers, Eingliederungshilfe § 35 a SGB VIII, S. 169 ff; Sidortschuk, JAmt 2005, S. 552 f, S. 553

1093 Niehues/Rux, Schulrecht, Rn. 176

1094 Sidortschuk, JAmt 2005, S. 552 f, S. 553; Förderung erfolgt z.B.: in Form von allgemeinem Förderunterricht in Deutsch und als Hausaufgabenhilfe (Z.B. VG Hamburg, Urteil v. 24.11.2009, Az.: 13 K 4032/07, BeckRS 2009 42354, S. 8 Punkt 2.3.1) oder in Form einer Wochenstunde in einer Gruppe von 9 Schülern und häuslichen Übungsaufgaben, die 3 - 4 mal pro Woche unter Kontrolle ihrer Eltern stattfinden sollen und in denen die betroffenen Kinder 10 bis 15 min etwas schreiben, dabei auch vor sich hinlesen und versuchen sollen, den Text selbst zu korrigieren (OVG Münster, Beschluss v. 8.9.2008, Az.: 12 A 1752/08, www.justiz.nrw.de, Rn. 16), demgegenüber hat das SG Halle eine Förderung in Form einer
} 
erst dann, wenn sich die Leistungen verschlechtern und das Kind schlecht genug für diese Maßnahme ist. ${ }^{1095}$ Eine separate Förderung ist jedoch in der Regel auch noch keine spezielle Förderung, die am individuellen Problem des Kindes ansetzt ${ }^{1096}$, sondern Förderung in einer kleineren Lerngruppe mit Schülern, die ebenfalls Schwierigkeiten im Fach Deutsch haben. Die Zusammensetzung der Gruppe ist meist heterogen. ${ }^{1097}$ Die Wirksamkeit dieser Fördermethode ist kaum evaluiert; es gibt einen eklatanten Mangel an wissenschaftlichen deutschsprachigen Studien über die Wirksamkeit von schulischen Fördermaßnahmen. Schulte-Körne hat für 2003 festgestellt, dass eine wissenschaftlich fundierte Studie zum Einsatz eines Rechtschreibtrainings im schulischen Förderunterricht bisher nicht veröffentlicht wurde. ${ }^{1098}$ Bei Kindern mit Legasthenie sind die Fördererfolge "deprimierend“ ${ }^{1099}$ Dass die schulischen Förderformen bei Schülern und Schülerinnen mit Legasthenie/Dyskalkulie nicht zum Erfolg führen, räumt auch die schärfste Kritikerin des Legasthenie-Begriffs, Valtin, ein, so dass „in diesen Fällen die außerschulische Hilfe von Experten notwendig ist." "1100

Der Markt an außerschulischen Förder- und Therapiemöglichkeiten für Kinder mit Legasthenie/Dyskalkulie und damit an "außerschulischen Experten“ ist allerdings unüberschaubar. ${ }^{1101}$ Die Wirksamkeit insbesondere von alternativen

„klassischen Nachhilfe“ in einer Gruppe mit 5 Mitschülern bei Legasthenie nicht für hilfreich gehalten, die deshalb auch nicht im Rahmen der Grundsicherung zu übernehmen sei. Bei Legasthenie sei vielmehr eine gezielte Legasthenietherapie in Form einer Einzeltherapie erforderlich (SG Halle, Beschluss v. 19.3.2010, Az.: S 7 AS 1072/10 ER, juris, Rn. 21)

1095 So ausdrücklich die Aussage der Deutschlehrerin in VG Hamburg, Urteil v. 24.11.2009, Az.: 13 K 4032/07, juris, S. 5 - Tatbestand; zum schulischen Förderangebot s. OVG Münster, Beschluss v. 8.9.2008, Az.: 12 A 1752/08, www.justiz.nrw.de: das schulische Förderangebot bestand aus einer Wochenstunde in einer Gruppe von 9 Schülern. Dieses schulische Förderkonzept beinhaltete auch, dass die Schüler 3 - 4 mal pro Woche unter Kontrolle ihrer Eltern 10 bis 15 min. etwas schreiben, dabei auch vor sich hinlesen und versuchen sollten, den Text selbst zu korrigieren (Rn. 16); für die notwendige psychische Betreuung verwies das OVG den Schüler auf eine psychotherapeutische Behandlung (Rn. 18)

${ }^{1096}$ Zur Notwendigkeit einer individuellen Förderung Reichelt, Analphabeten, S. 6; Steinhausen, Psychische Störungen bei Kindern und Jugendlichen, S. 164; s. auch Klicpera/Schabmann/ Gasteiger-Klicpera, Legasthenie, S. 267

1097 Tacke, in: Legasthenie und Dyskalkulie 2007, S. 136; s. die Schilderung schulischer Fördermaßnahmen von Klasen, Legasthenie, S. $90 \mathrm{ff}$

1098 Schulte-Körne/Deimel/Remschmidt, Zeitschrift für Kinder- und Jugendpsychiatrie und Psychotherapie 31 (2003), Punkt 1

1099 Tacke, in: Legasthenie und Dyskalkulie 2007, S. 136; Klasen, Legasthenie, S. 91 f, die darauf verweist, dass der Förderunterricht auch bei dem besten Willen der Lehrkräfte unfachmännisch und fachlich unzureichend erteilt wird.

1100 Valtin, in: LRS und Legasthenie, S. 62

1101 Suchodoletz, Therapie der Lese-Rechtschreib-Störung, S. 16 
Therapieansätzen, die überwiegend nicht evaluiert sind ${ }^{1102}$, ist teilweise sehr umstritten. ${ }^{1103}$

Angesichts dieses Streitstandes kann festgestellt werden, dass nicht jede schulische und auch nicht jede außerschulische Förderung eine Besserung des Zustandes erwarten lässt und daher geeignet ist. Erforderlich ist es daher, dass Kriterien für die Qualität und Eignung der Förderung bzw. Therapie gefunden werden, die es ermöglichen, deren Eignung festzustellen.

In der medizinischen Versorgung muss die Behandlung den Voraussetzungen des SGB V und hier insbesondere denen des $\S 2$ SGB $\vee$ entsprechen. $\S 2$ I 3 SGB V bestimmt, dass Qualität und Wirksamkeit der Leistungen dem allgemein anerkannten Stand der medizinischen Erkenntnis zu entsprechen und den medizinischen Fortschritt zu berücksichtigen haben. Der Rechtsbegriff "Qualität" zielt auf eine permanente Überprüfung hin, die den gesamten Behandlungsablauf betrifft und sowohl die Prozessqualität, also die Anforderungen für Steuerung und Verfahren für Diagnostik und Therapie, beinhaltet als auch die Ergebnisqualität. ${ }^{1104}$ Wirksamkeit zielt auf die generelle Fähigkeit bei bestimmten Indikationen bestimmbare, spezifische diagnostische und/oder therapeutische Wirkungen zu erzielen. ${ }^{1105}$ Der allgemeine Stand der medizinischen Erkenntnisse und die Berücksichtigung des medizinischen Fortschritts verpflichten jeden, der an dem Prozess teilnimmt, dazu, sein Handeln ständig zu überprüfen und zu evaluieren. ${ }^{1106}$

Aus alldem ergibt sich, dass maßgeblich für jede "Behandlung", die eine Verbesserung eines Zustandes bewirken soll, ist, dass das Verfahren überprüft und evaluiert sein muss. ${ }^{1107}$ Es muss nachgewiesen werden, dass die Methode wirksam ist, um bestimmte therapeutische Ergebnisse zu erzielen. Nur dann ist sie auch geeignet. ${ }^{1108}$ Weiter ist die Maßnahme nur geeignet, wenn die Förderung von entsprechend fachlich qualifiziertem Personal durchgeführt wird. Sonst verliert sie ihre Wirksamkeit und ist damit ungeeignet.

\footnotetext{
1102 Warnke/Schulte-Körne, in: Therapie psychischer Störungen, S. 158

${ }^{1103}$ S. dazu umfassend: Suchodoletz, Therapie der Lese-Rechtschreib-Störung S. $15 \mathrm{ff}, 167 \mathrm{ff}$

${ }^{1104}$ Plagemann in Schlegel/Engelmann/Voelzke, JurisPK SGB V, § 2, Rn. 45 ff; Nebendah/ in Spickhoff, Medizinrecht, § 2, Rn. 5

1105 Plagemann in Schlegel/Engelmann/Voelzke, JurisPK SGB V, § 2, Rn. 47

1106 Plagemann in Schlegel/Engelmann/Voelzke, JurisPK SGB V, § 2, Rn. 51

1107 Vgl. Ennuschat, RdJB 2007, S. 271 ff, S. 280, wenn schon die Einführung von Ganztagsschulen wissenschaftlich evaluiert sein muss, gilt dies erst recht für den Bereich der individuellen Förderung

1108 Nur damit kann auch verhindert werden, dass Lehrer, Eltern und Nachhilfelehrer „nur ein oberflächliches Herumdoktern an Symptomen leisten“. Rahn, Legasthenie bei Erwachsenen, S. 25; umfassend Schulte-Körne/Deimel/Remschmidt, Zeitschrift für Kinder- und Jugendpsychiatrie und Psychotherapie 31 (2003), S. 85 ff
} 
Die Förderung muss weiter erforderlich, ein milderes Mittel also nicht vorhanden sein. Das wird in der Regel gegeben sein.

Schließlich muss die Förderung angemessen sein. Dabei ist der Nutzen der Förderung ins Verhältnis zur Belastung des Kindes durch die Förderung zu setzen. Vielfach ist die Verpflichtung der Kinder, Förderkurse zu besuchen, für sie eine zusätzliche Belastung, weil sie meist im Anschluss an den regulären Schulunterricht stattfindet, wo das Kind nicht mehr so aufnahmefähig ist ${ }^{1109}$, und weil sie außerdem durch die dann noch anstehenden Hausaufgaben sehr belastet sind. ${ }^{1110}$ Weiter ist zu berücksichtigen, dass es möglich ist, dass das Kind nach umfangreichen Förderungen oder Therapien "austherapiert" oder auch "therapiemüde" ist. Dann bewirken Förderung und Therapie nichts mehr, erzielen eventuell sogar eine nachteilige Wirkung. Infolgedessen kann eine ununterbrochene Förderung unangemessen sein. Weil die Förderung dazu dient, dass der Staat seinen Bildungsauftrag wahrnimmt, sind die Kosten Teil der allgemeinen schulischen Ausbildung. Schulausbildung muss kostenfrei erfolgen ${ }^{1111}$, deshalb sind die Kosten einer qualifizierten Therapie in der Schule auch von der Schule zu tragen.

Im Ergebnis ist daher festzuhalten, dass Schüler und Schülerinnen mit Legasthenie/Dyskalkulie dazu verpflichtet werden können an einer schulischen Förderung teilzunehmen, wenn diese eine qualifizierte, evaluierte Therapie bei Legasthenie/Dyskalkulie ist und von qualifiziertem Personal durchgeführt wird. Die Verpflichtung zur Teilnahme ist analog der Mitwirkungspflicht im SGB I eine Obliegenheit und daher nicht mit Zwangsmitteln durchsetzbar. ${ }^{1112}$

\subsection{Entzug des Nachteilsausgleichs wegen fehlender Förderung}

Des Weiteren ist zu klären, ob der Nachteilsausgleich entzogen werden kann, wenn die Förderung nicht stattfindet oder nicht wahrgenommen wird.

Im Sozialrecht sind die Folgen einer fehlenden Mitwirkung in $\S 66$ SGB I geregelt. Die Vornahme der Mitwirkungshandlung kann nicht erzwungen werden, aber der Leistungsträger kann dem Berechtigten die Leistung teilweise oder ganz entziehen. Damit wird dem Leistungsträger die Möglichkeit eingeräumt, mittelbar Druck auf den Leistungsempfänger auszuüben, damit dieser seiner Mitwirkungspflicht nachkommt. ${ }^{1113}$ Die Mitwirkungspflichten stehen systema-

\footnotetext{
1109 Schulte-Körne, Elternratgeber Legasthenie, S. 124

1110 Tacke, in: Legasthenie und Dyskalkulie 2007, S. 135

${ }^{1111}$ Avenarius/Heckel, Schulrecht, Ziff. 31.4

1112 S. zum SGB I Mrozynski, SGB I, § 60, Rn. 2; Seewald in Leitherer, Kasseler Kommentar Sozialversicherungsrecht, Vor $\S 60$, Rn. 19; Kampe in Schlegel/Engelmann/Voelzke, JurisPK SGB V, $\S 66$, Rn. 15

${ }^{1113}$ Seewald in Leitherer, Kasseler Kommentar Sozialversicherungsrecht, § 66 SGB I, Rn. 2
} 
tisch den Mitverschuldensregeln und der Schadensminderungspflicht im Zivilrecht nahe. ${ }^{1114}$

Findet in der Schule eine qualifizierte Förderung statt, zu dessen Besuch das Kind verpflichtet worden ist, dann bedeutet die Koppelung des Nachteilsausgleichs an die Förderung, dass das Grundrecht aus Art. 3 III 2 GG mit einer Bedingung verknüpft und somit beschränkt wird. Das Grundrecht aus Art. 3 III 2 GG wird schrankenlos gewährt, so dass sich Schranken nur aus spezifischen behinderungsbedingten Gründen und aus kollidierendem Verfassungsrecht ergeben. ${ }^{1115}$ Behinderungsbedingte Gründe, die es zwingend erforderlich machen, wegen der Behinderung Legasthenie/Dyskalkulie - anders als bei allen anderen Schülern und Schülerinnen mit Behinderung - den Nachteilsausgleich nur bedingt zu gewähren, sind nicht ersichtlich. Als kollidierendes Verfassungsrecht kommt, da Grundrechte der Mitschüler, wie gerade ausgeführt wurde, nicht betroffen sind, nur der Erziehungsauftrag des Staates in Betracht. Zweck der Koppelung ist es, die Schüler und Schülerinnen mit Legasthenie/Dyskalkulie zur Mitarbeit zu verpflichten und die Verweigerung zu sanktionieren. Die Sanktion besteht jedoch darin, dass das Grundrecht aus Art. 3 III 2 GG nicht nur eingeschränkt, sondern für diesen Bereich entzogen wird. Der Entzug ist eine direkte und gezielte Benachteiligung wegen der Behinderung, die nicht legitimiert werden kann. ${ }^{1116}$

Wird eine Förderung durch die Schule nicht angeboten bzw. durchgeführt, dann hat das Kind mit Behinderung dies nicht zu vertreten, so dass ihm deshalb auch nicht der Nachteilsausgleich entzogen werden kann.

Denkbar wäre, das Kind bei fehlender schulischer Förderung dazu zu verpflichten, an außerschulischen Förderungen teilzunehmen. Weil damit die Schulpflicht noch einmal ausgeweitet würde, wäre das allerdings ein erheblicher Eingriff in das Grundrecht des Kindes aus Art. 2 I GG. Kinder mit Behinderungen haben häufig umfangreiche therapeutische Förderung, z.B. Logopädie oder Physiotherapie, die jedoch nie mit der schulischen Berücksichtigung der Behinderung verknüpft wird. Es sind keine verfassungsrechtlichen Gründe ersichtlich, die eine solche Verknüpfung gerade für Legasthenie/Dyskalkulie rechtfertigen würden. Die Verpflichtung zum Besuch außerschulischer Förderung scheitert auch daran, dass diese Förderung in der Regel nicht von den Krankenkassen als Heilbehandlung ( $§ 27$ SGB V) oder den Jugendämtern als Eingliederungshilfe ( $\$ 35$ a SGB VIII bezahlt wird. Die außerschulische Förderung müsste dann von den Eltern gezahlt werden. ${ }^{1117}$ Das Sozialstaatsprinzip

\footnotetext{
${ }^{1114}$ Kampe in Schlegel/Engelmann/Voelzke, JurisPK SGB V, § 66, Rn. 8; Mrozynski, SGB I, § 60, Rn. 1; Maydell, von, Sozialrechtshandbuch, Rn. 152

1115 S. oben 3. Teil: 5. Kapitel: 1.: Rechtfertigungsmaßstab, S. 145

${ }^{1116} \mathrm{Vgl}$. Michael/Morlok, Grundrechte, Rn. 818

1117 Die Belastung liegt bei etwa $180 €$ pro Monat, s. dazu oben S. $49 \mathrm{f}$
} 
gebietet aber, dass die Schulbildung keinerlei Kosten verursachen darf. ${ }^{1118}$ Es darf schon im Bereich der Lehrmittelfreiheit kein Druck aufgebaut werden, freie Lernmittel auf eigene Kosten anzuschaffen. ${ }^{1119}$ Deshalb ist es noch weniger mit dem Sozialstaatsprinzip zu vereinbaren, wenn Eltern zu erheblichen Kosten für außerschulische Förderung verpflichtet werden, um in der Schule den Nachteilsausgleich zu erhalten. ${ }^{1120}$

Darüber hinaus ist die Koppelung aber auch nicht verhältnismäßig. Sie mag zwar geeignet und erforderlich sein, um den Besuch der Förderung durchzusetzen, sie ist aber nicht angemessen. Die Mitwirkungspflicht dient in diesem Fall in allererster Linie dem Schüler mit Behinderung. Er soll von der Förderung profitieren. Der staatliche Erziehungsauftrag soll damit umgesetzt werden. Nimmt der Schüler aber nicht an der Förderung teil, dann wird dadurch der staatliche Erziehungsauftrag nicht beeinträchtigt. Ein "Schaden“, den der Schüler mitverantworten würde, tritt nur beim Schüler und nicht beim Staat ein. Diese geringe Betroffenheit des Staates rechtfertigt es nicht, deshalb das Grundrecht des Schülers einzuschränken.

\footnotetext{
1118 Avenarius/Heckel, Schulrecht, Ziff. 31.4

1119 Avenarius/Heckel, Schulrecht, Ziff. 31.423

1120 A.A. VGH Kassel, Beschluss v. 8.12.2011, Az.: 7 A 2621/10.Z, der es als rechtmäßig ansieht, dass der betroffene Schüler seinen Anspruch auf Berücksichtigung der Legasthenie durch eine außerschulische Therapie erhalten hat. Der VGH wirft weiter die Frage auf, ob die außerschulische Therapie inhaltlich und zeitlich mit einer schulischen Förderung, die offenbar nicht stattgefunden hat, gleichzusetzen ist.
} 


\section{Teil: Weitere betroffene Grundrechte}

\section{Kapitel: Anspruch aus Art. 2 I GG und 12 I GG}

\section{Schutzbereich}

Ein Anspruch auf Berücksichtigung der Legasthenie/Dyskalkulie kann sich weiter aus den Grundrechten nach Art. 12 I GG, Art. 2 I GG ergeben. Diese Grundrechte enthalten ein „Abwehrrecht gegen Freiheitsbeschränkungen im Ausbildungsbereich" und ein Teilhaberecht ${ }^{1121}$, indem sie umfassende Bildungsfreiheit und Teilhabe an staatlichen Einrichtungen im Bereich aller Formen der Bildung und Ausbildung gewähren. ${ }^{1122}$ Art. 12 । 1 GG schützt die Berufsfreiheit und dabei u. a. die freie Wahl der Ausbildungsstätte und über den Wortlaut hinaus die gesamte Freiheit der berufsbezogenen Ausbildung. Zu den berufsbezogenen Ausbildungsstätten gehören weiterführende Schulen, insbesondere die Sekundarstufe II des Gymnasiums. ${ }^{1123}$ Grundrechtlich geschützt ist dabei nicht nur die Teilnahme an den Abschlussprüfungen, sondern auch der Zugang zu diesen Ausbildungsstätten. ${ }^{1124}$ Nicht zu den beruflichen Ausbildungsstätten gehören der Grund- und Hauptschulbereich. ${ }^{1125}$ Im Einzelnen ist die Zuordnung

\footnotetext{
${ }^{1121}$ BVerfGE 33, 303, 329

1122 Langenfeld, in: Grote/Marauhn EMRK/GG, Kap. 23: Das Recht auf Bildung, Rn. 18; Langenfeld, Integration und kulturelle Identität zugewanderter Minderheiten, S. 226 f; Jarass, in: Jarass/Pieroth GG, Art. 12, Rn. 93; Ruffert, in: BeckOK GG, Art. 12, Rn. 46; Umbach, in: Umbach GG, Art. 12, Rn. 61

1123 BVerfGE 58, 257, 273; Hofmann, in: Schmidt-Bleibtreu/Klein GG, Art. 7 und Art. 12, Rn. 38; Umbach, in: Umbach GG, Art. 12, Rn. 53; Wieland, in: Dreier GG, Art. 12, Rn. 61; a.A. Ruffert, in: BeckOK GG, Art. 12, Rn. 45.2

1124 Jarass, in: Jarass/Pieroth GG, Art. 12, Rn. 98; Ruffert, in: BeckOK GG, Art. 12, Rn. 46; Wieland, in: Dreier GG, Art. 12, Rn. 66

1125 Umbach, in: Umbach GG, Art. 12, Rn. 53
} 
einzelner Schularten zum Schutzbereich des Art. 12 GG streitig. ${ }^{1126}$ Soweit es nicht um berufsbezogene Ausbildung geht, kommt jedenfalls Art. 2 I GG zum Tragen. ${ }^{1127}$ Im Ergebnis kommt es hier auf die Abgrenzung nicht an, weil die Ausbildung an sämtlichen Schularten entweder über Art. 12 GG oder über Art. 2 I GG geschützt ist. ${ }^{1128}$

\section{Eingriff}

Nach allgemeiner Ansicht sind schulische Prüfungen, die sich auf Berufs- und Lebenschancen auswirken und die Beschränkung des Zugangs zu bestimmten Schularten und damit zu bestimmten Bildungsgängen und möglichen Schulabschlüssen bewirken, Eingriffe in das Grundrecht aus Art. 12 । GG ${ }^{1129}$ bzw. Art. 2 । GG, die der Rechtfertigung bedürfen. ${ }^{1130}$

Bei an sich verhältnismäßigen Prüfungen kann sich ein Eingriff weiter daraus ergeben, dass die Behinderungen Legasthenie/Dyskalkulie nicht berücksichtigt werden und dadurch die Ausübung der Ausbildungs- und Berufsfreiheit erschwert oder unmöglich gemacht wird. ${ }^{1131}$ Die Nicht-Berücksichtigung von Legasthenie/Dyskalkulie in Prüfungen, bei Zulassungsentscheidungen für weiterführende Schulen oder Schulwechsel und in Schulabschlussprüfungen führt dazu, dass bestimmte weiterführende Schulen nicht besucht und bestimmte Schulabschlüsse nicht erreicht werden können, wodurch die weiteren Bildungsund Lebenschancen der Betroffenen beeinflusst werden. Insbesondere Schülern und Schülerinnen mit Dyskalkulie wird eine akademische Ausbildung erheblich erschwert, weil sie i.d.R. nur die Hauptschule besuchen können. Die Nicht-Berücksichtigung von Legasthenie/Dyskalkulie in Prüfungen und Zulassungsentscheidungen ist daher ein mittelbarer Eingriff in die Ausbildungs- und Berufsfreiheit im weitesten Sinne. ${ }^{1132}$

\footnotetext{
${ }^{1126} \mathrm{Vgl}$. die Zusammenfassung bei Langenfeld, RdJB 2007, S. 211 ff, S. 221; ablehnend für alle allgemeinbildenden Schulen Manssen, in: v. Mangoldt/Klein/Starck GG, Art. 12, Rn. 61; Ruffert, in: BeckOK GG, Art. 12, Rn. 45.2

${ }^{1127}$ Jarass, in: Jarass/Pieroth GG, Art. 12, Rn. 93

1128 Jarass, in: Jarass/Pieroth GG, Art. 12, Rn. 93; Jarass/Pieroth, in: Jarass/Pieroth GG, Art. 2, Rn. 6

1129 Rux, RdJB 2009, S. 220 ff, S. 221; Umbach, in: Umbach GG, Art. 12, Rn. 59

1130 BVerfGE 52, 380, 388; 84, 34, 45; 84, 59, 72; VG Hannover, Beschluss v. 13.12.2010, Az.: 6 B 5596/10, www.rechtsprechung.niedersachsen.de S. 4; Poscher/Rux/Langer, Recht auf Bildung, S. 88; Pietzcker, Verfassungsrechtliche Anforderungen an staatliche Prüfungen, S. 70 ff; Guhl, Prüfungen im Rechtsstaat, S. 21 ff; Lampe, Gerechtere Prüfungsentscheidungen, S. 23; Niehues, Prüfungsrecht, Rn. 19

${ }^{1131}$ Vgl. VG Hannover, Beschluss v. 13.12.2010, Az.: 6 B 5596/10, www.rechtsprechung.niedersachsen.de, S. 4

1132 Vgl. Ruffert, in: BeckOK GG, Art. 12, Rn. 68; Niehues/Rux, Schulrecht, Rn. 135 m.w.N.
} 


\section{Rechtfertigung}

Die Ausbildungsfreiheit kann durch Gesetz oder aufgrund eines Gesetzes eingeschränkt werden. Hier geschieht dies durch die vielfältigen schulrechtlichen Regelungen zu Prüfungen, Schulabschlüssen und Übertrittsverfahren. Diese Regelungen müssen selbst verhältnismäßig sein. Im Bereich der Ausbildungsfreiheit ist nach der Rechtsprechung des BVerfG zwischen objektiven und subjektiven Beschränkungen zu unterscheiden. ${ }^{1133}$ Objektive Beschränkungen sind von außen wirkende, unbeeinflussbare Größen, die dem Einzelnen entzogen sind wie etwa ein Numerus clausus. ${ }^{134}$ Sie sind nur zulässig zum Schutz eines überragend wichtigen Gemeinschaftsgutes. Subjektive Beschränkungen liegen vor, wenn diese durch persönliche Faktoren oder Qualifikationen beeinflussbar sind. ${ }^{1135}$ Das BVerfG geht davon aus, dass Prüfungen subjektive Beschränkungen sind, die durch den Prüfling beeinflussbar sind. ${ }^{1136}$ Sie sind zulässig zum Schutz eines wichtigen Gemeinschaftsgutes. Die aktuelle Rechtsprechung des BVerfG hält nicht mehr starr an dieser Stufentheorie fest, sondern wendet den allgemeinen Verhältnismäßigkeitsgrundsatz mit der Maßgabe an, dass es auf die Schwere des Eingriffs für die Betroffenen ankommt. Je empfindlicher die Betroffenen in ihrer Berufs- oder Ausbildungsfreiheit betroffen sind, desto stärker müssen die Interessen des Gemeinwohls sein, denen die Regelung zu dienen bestimmt ist. ${ }^{1137}$ Diese Grundsätze zum Zugangsrecht gelten auch für andere Ausbildungsstätten, soweit der Staat dort ein faktisches, "nicht beliebig aufgebbares" oder ein rechtliches Ausbildungsmonopol besitzt. ${ }^{1138}$ Das gilt z.B. für die weiterführenden Schulen der Sekundarstufen I und II und für die berufsbildenden Einrichtungen. ${ }^{1139}$ Auch wenn das Zugangsrecht von gewissen, insbesondere subjektiven Gegebenheiten abhängig gemacht wird, wie insbesondere Begabung und Leistung, sind doch diese Einschränkungen wiederum daraufhin zu überprüfen, ob sie dem Entfaltungsrecht des Betroffenen hinreichend Rechnung tragen. ${ }^{1140}$

\footnotetext{
1133 BVerfGE 33, 303, 337 f; 43, 291, $313 \mathrm{f}$

1134 Leibholz,/Rinck/Hesselberger/Burghart, in: Leibholz/Rinck, GG, Art. 12, Rn. 341, 361; Umbach, in: Umbach GG, Art. 12, Rn. 85, 88; Ruffert, in: BeckOK GG, Art. 12, Rn. 97, 99

1135 Jarass, in: Jarass/Pieroth GG, Art. 12, Rn. 35 f; Ruffert, in: BeckOK GG, Art. 12, Rn. $97 \mathrm{ff}$

1136 BVerfGE 13, 97, 106 f; 34, 71, $77 \mathrm{f}$

1137 Ruffert, in: BeckOK GG, Art. 12, Rn. $100 \mathrm{ff}$

1138 S. auch Glotz/Faber, in: Hdb. des Verfassungsrechts, § 28, Rn. 13

1139 Langenfeld, Integration und kulturelle Identität zugewanderter Minderheiten, S. $226 \mathrm{f}$

1140 Langenfeld, Integration und kulturelle Identität zugewanderter Minderheiten, S. 227; Niehues/Rux, Schulrecht, Rn. 135
} 


\subsection{Verhältnismäßigkeit von Prüfungen}

Der Staat hat ein legitimes Interesse an Prüfungen, denn ein leistungsorientiertes Gemeinwesen kommt ohne Leistungskontrollen, Prüfungen und Berechtigungen, um Eignungen und Befähigungen festzustellen, nicht aus. ${ }^{1141}$ Die Prüfungen müssen sowohl hinsichtlich des Verfahrens als auch hinsichtlich des Inhalts bestimmte Anforderungen erfüllen, um verhältnismäßig zu sein. Das Prüfungsverfahren muss so gestaltet sein, dass die wahren Kenntnisse und Fähigkeiten des Prüflings genau ermittelt werden, um so die Grundlage für eine zutreffende Bewertung zu schaffen. ${ }^{1142}$ Der Prüfungsinhalt muss hinreichend bestimmt sein, dass daran der Prüfungserfolg, nämlich die Feststellung, ob der Prüfling für die Zulassung zu einer bestimmten Schulart, für einen bestimmten Schulabschluss oder für eine bestimmte Jahrgangsstufe geeignet ist, gemessen werden kann. ${ }^{1143}$ Die Prüfungsaufgaben müssen sowohl nach Inhalt als auch nach Form geeignet sein, Prüflinge dahingehend zu unterscheiden, ob sie eine bestimmte Eignung nachgewiesen haben oder nicht. ${ }^{1144}$ Der Gegenstand der Leistungsbewertung muss inhaltlich auf dieses Ziel ausgerichtet sein, es aber auch entsprechend begrenzen. ${ }^{1145}$

\subsubsection{Legitimer Zweck}

Zulassungen zu weiterführenden Schulen (wie Grundschulnoten am Ende der 4. Klasse, Grundschulempfehlungen, Jahresnoten, die für den Wechsel auf die Realschule oder die Sekundarstufe II erforderlich sind), zu nächsthöheren Klassen (Versetzungen) oder zum Übergang von einer Schulart in die andere (etwa Wechsel von der Hauptschule auf die Realschule) dienen dem Ausbildungszweck der jeweiligen Schulart bzw. Schulstufe. Mit der Prüfung bzw. in der Grundschule mit dem Empfehlungsverfahren, soll ermittelt werden, wer die nötigen Eigenschaften mitbringt, um ohne Behinderung des Ausbildungsbetriebes die Anforderungen dieses Bildungsganges beginnen, erfüllen und abschlieBen zu können. ${ }^{1146}$ Zweck ist damit der Schutz des jeweiligen Bildungsganges vor unqualifizierten Schülern und Schülerinnen und auch der Schutz der anderen Schüler und Schülerinnen davor, durch unqualifizierte Mitschüler im Lernen beeinträchtigt zu werden. ${ }^{1147}$ Um dies ermitteln zu können, setzt der Gesetzge-

\footnotetext{
1141 Langenfeld, RdJB 2007, S. 211 ff, S. 219

1142 Niehues, Prüfungsrecht, Rn. 133

1143 Niehues, Prüfungsrecht; Rn. 375, 380

1144 Niehues, Prüfungsrecht, Rn. 380

1145 Niehues, Prüfungsrecht, Rn. 380

1146 Pietzcker, Verfassungsrechtliche Anforderungen an staatliche Prüfungen, S. 81; Guhl, Prüfungen im Rechtsstaat, S. 26; Langenfeld, in: Grote/Marauhn EMRK/GG, Kap. 23: Das Recht auf Bildung, Rn. 17

1147 Guhl, Prüfungen im Rechtsstaat, S. 26
} 
ber Maßstäbe für die insoweit geforderten Leistungen und etwaige Ausnahmen von diesen Anforderungen fest.

Es ist bereits zweifelhaft, ob ein solches Ziel noch mit der BRK vereinbar ist, die von einer gemeinsamen Beschulung aller Kinder ausgeht. Danach ist eine Differenzierung in qualifizierte und nicht qualifizierte Schüler und Schülerinnen nicht mehr zulässig.

Zweifel an dem legitimen Zweck ergeben sich weiter daraus, dass dieser auf der Prämisse fußt, dass begabte Schüler durch die gemeinsame Beschulung mit weniger begabten Schülern benachteiligt werden, weil sie nicht ihrem Potential entsprechend lernen und gefördert werden. Diese Prämisse ist jedoch durch die empirische Bildungsforschung nicht belegbar. ${ }^{1148}$ Es gibt ebenfalls Studien, die ergeben haben, dass sich eine Leistungsheterogenität nicht auf den Lernerfolg der Schüler auswirkt. ${ }^{1149}$ Da die Auswirkungen jedoch nicht sicher prognostizierbar sind, steht dem Gesetzgeber ein weiter Entscheidungsspielraum $z^{1150}$, so dass der legitime Zweck noch zu bejahen ist.

Der Schulgesetzgeber verfolgt weiter das legitime Ziel, mit bestimmten Abschlüssen auch bestimmte Fähigkeiten, Fertigkeiten und Lernziele zu dokumentieren $^{1151}$, auf eine spätere berufliche Tätigkeit vorzubereiten und dafür die notwendigen Grundkenntnisse zu vermitteln. Mit den Schulabschlüssen ist die Erwartung verbunden, dass die folgende berufliche Ausbildung erfolgreich absolviert werden kann.

\subsubsection{Geeignetheit, Erforderlichkeit, Angemessenheit}

Die schulischen Prüfungen sind nur dann verhältnismäßig, wenn der Prüfungsinhalt auf das Prüfungsziel ausgerichtet ist ${ }^{1152}$. Die Prüfung muss geeignet und ausreichend sein, um das Ziel der Leistungskontrolle zu erreichen. ${ }^{1153}$ Das Prüfungsziel selbst muss ebenfalls wieder verhältnismäßig sein. Es dürfen also nur solche Prüfungsanforderungen abverlangt werden, die im Hinblick auf den mit der Prüfung verfolgten Zweck geboten sind. ${ }^{1154}$

Das Prüfungsziel muss einen Rückschluss auf das damit verbundene Ergebnis, wie z.B. die Versetzung, oder die damit verbundene Berechtigung für Berufswege zulassen. Für juristische Prüfungen, wie oben schon erwähnt, hat die Rechtsprechung für die Verhältnismäßigkeit von Anforderungen, darauf abge-

\footnotetext{
1148 Orth, NVwZ 2011, S. 14 ff, S. 17

1149 Orth, NVwZ 2011, S. 14 ff, S. 17

1150 Orth, NVwZ 2011, S. 14 ff, S. 18

1151 Ennuschat, RdJB 2007, S. 271 ff, S. 275

1152 Guhl, Prüfungen im Rechtsstaat, S. 102, s. dazu VG Hannover, Beschluss v. 13.12.2010, Az.: 6

B 5596/10, www.rechtsprechung.niedersachsen.de, S. 6

1153 Niehues, Prüfungsrecht, Rn. 380

${ }^{1154}$ Lampe, Gerechtere Prüfungsentscheidungen, S. 37; Niehues/Rux, Schulrecht, Rn. 136
} 
stellt, dass sich die juristische Prüfung an den Anforderungen der Berufe ausrichten muss, die die Befähigung zum Richteramt voraussetzen. ${ }^{1155}$ Bei Abiturprüfungen sieht sie deren Zweck darin, zwischen solchen Abiturienten, die für ein Hochschulstudium geeignet sind, und solchen, bei denen dies nicht der Fall ist, zu unterscheiden. ${ }^{1156}$ Die Anforderungen, die gestellt werden, müssen sich daher auf diesen Zweck beziehen und dürfen zu ihm nicht außer Verhältnis stehen. Bestimmte Leistungen dürfen deshalb nur dann Grundlage der Bewertung und daraus folgender Regelungen zum Nicht-Bestehen der Prüfung sein, wenn sie eine zuverlässige Beurteilungsgrundlage für die Prognose der Eignung für das Hochschulstudium sind. ${ }^{1157}$ Allgemein wird angenommen, dass die bestehenden Anforderungen an die Schulabschlüsse und vor allem an das Abitur eine solche Prognose zulassen. Die Erfahrungen mit dem Abitur, das seit Jahrzehnten den Zugang zur akademischen Ausbildung regelt, sprechen sicherlich für eine solche prinzipielle Prognosefähigkeit. Allerdings ist zu bedenken, dass die Anforderungen im Abitur nicht statisch sind, sondern gesellschaftlichen und politischen Veränderungen unterliegen. Sowohl die Veränderungen des Abiturs in den 1970er Jahren zu einer Sekundarstufe II und einem Abitur mit Wahlmöglichkeiten zwischen Leistungs- und Grundkursen als auch die in den letzten Jahren erfolgte Verkürzung der Oberstufe, die vielerorts erfolgte Aufhebung des Leistungs- und Grundkurssystem und die Festschreibung von vier oder fünf Pflichtfächern im Abitur zeigen auf, dass die inhaltlichen Anforderungen an das Abitur variabel sind. Damit ist auch die Vorstellung davon, welche Leistungen im Abitur nachgewiesen werden müssen, um die Zulassung für ein akademisches Studium zu erhalten und damit zu prognostizieren, dass ein Studium erfolgreich sein wird, der steten Veränderung unterworfen. Hinzu kommt, dass die schulischen Prüfungen in der Regel zeitlich weit vor einer beruflichen Festlegung liegen und dass jeder Beruf nicht nur auf eine spezielle Tätigkeit vorbereitet, sondern eine Vielzahl von Betätigungen eröffnet. ${ }^{1158}$ Deshalb gibt es keine unumstößlichen, dauerhaften, eindeutigen, stabilen und überprüfbaren Kriterien dafür, wann eine Berufs- oder Hochschuleignung gegeben ist. ${ }^{1159}$ Vielmehr ist eine Gesamtschau einer Vielzahl von Kriterien und damit Prüfungs-

\footnotetext{
1155 BVerwG, Urteil v. 17.7.1987, Az.: 7 C 118/86, juris, Rn. 17 (Fragen zum Staat Mali); VGH Mannheim, Urteil v. 16.5.2000, Az.: 9 S 2537/99, juris, Rn. 22

1156 OVG Greifswald, Urteil v. 27.2.1997, Az.: 2 L 52/96, juris, Rn. 25; VG Dresden, Beschluss v. 22.7.1998, Az.: 5 K 1705/98, juris, Rn. 51; VG Potsdam, Beschluss v. 19.7.2000, Az.: 5 L 967/00, LKV 2001, S. 572, 573

1157 Vgl. VG Potsdam, Beschluss v. 19.7.2000, Az.: 5 L 967/00, LKV 2001, S. 572, 573

1158 Der Jurist kann Richter, Anwalt, Manager, Journalist, Professor usw. werden, die Eignung ist für jedes Tätigkeitsfeld unterschiedlich. Ein Richter ist nicht unbedingt zum Manager geeignet, ein juristischer Journalist nicht unbedingt zum Anwalt.

$1159 \mathrm{Vgl}$. Skowronek zitiert nach Pietzcker, Verfassungsrechtliche Anforderungen an staatliche Prüfungen, S. 52
} 
inhalten und -zielen vorzunehmen, aus deren Bestehen der Rückschluss auf eine allgemeine Eignung gezogen wird.

Der Prüfungsinhalt muss auf das Prüfungsziel ausgerichtet sein. Inhalte und Anforderungen für schulische Prüfungen werden durch die landesrechtlich zuständigen Verordnungsgeber für die einzelnen Schularten, Jahrgangsstufen und Abschlüsse festgelegt. Sie regeln im Einzelnen, welche Leistungsfähigkeit und Leistungsbereitschaft erforderlich ist und welches Prüfungsziel verfolgt wird. Maßgeblich wird dies beeinflusst durch die von der KMK verabschiedeten nationalen Bildungsstandards für die Abschlussklassen ${ }^{1160}$, auch wenn diese rechtlich unverbindlich sind. Sie sind auf bestimmte Prüfungsziele und Prognosen ausgerichtet und enthalten abgestufte Regelungen zum Bestehen und NichtBestehen der Prüfung, sowie bis zu einem gewissen Grad Ausgleichsmöglichkeiten innerhalb der Fächergruppen. Ziel ist es, durch einheitliche Vorgaben und Bewertungsmaßstäbe die Vergleichbarkeit der Leistungen und Abschlüsse herzustellen und zu gewährleisten. Weil sich der Prüfungszweck nicht exakt abgrenzen lässt, ist anerkannt, dass sowohl ein gewisser „Überschuss" an Prüfungsanforderungen als auch die Bewertung von allgemeinen Grundkenntnissen in Fächern, in denen diese nicht zwingend Teil des Faches sind, zulässig ist. ${ }^{1161}$ Mittelbar gehören die allgemeinen Grundkenntnisse wie Lesen, Schreiben und Rechnen zu jedem Fach dazu und werden dort auch vorausgesetzt, etwa indem die Aufgaben zu erlesen sind oder einfache Rechnungen durchgeführt werden müssen.

Nicht nur die Prüfungsinhalte und -anforderungen, sondern auch die Bestimmungen, die das Nicht-Bestehen der Prüfung regeln, müssen verhältnismäßig sein. Die Verhältnismäßigkeit ist nur gegeben, wenn sich aus dem Versagen gerade in diesen Fächern oder Prüfungsteilen ableiten lässt, dass der Prüfling für das mit der Prüfung angestrebte Ziel nicht geeignet ist. ${ }^{1162}$ Dafür ist es erforderlich zu bestimmen, was denn die Anforderungen der Prüfung sind, welche wahren Leistungen und Befähigungen, welche Kernkompetenzen, in einer Prüfung nachgewiesen werden sollen oder müssen. ${ }^{1163}$ Nur wenn darüber Klar-

\footnotetext{
1160 KMK zu Bildungsstandards, s. Anhang 2

1161 Lampe, Gerechtere Prüfungsentscheidungen, S. 37; Niehues, Prüfungsrecht, Rn. 391, 394

1162 BVerwG, Urteil v. 17.7.1987, Az.: 7 C 118/86, juris, Rn. 17; BVerwG Beschluss v. 9.6.1995; Az.: 6 B 100/94, juris, Rn. 4; VGH Mannheim, Urteil v. 16.5.2000, Az.: 9 S 2537/99, juris, Rn. 23; OVG Greifswald, Urteil v. 27.2.1997, Az.: 2 L 52/96, juris, Rn. 25; VG Göttingen, Urteil v. 11.2.2009, Az.: 1 A 306/07, www.rechtsprechung.niedersachsen.de, S. 2; VG Dresden, Beschluss v. 8.8.2001, Az.: 5 K 1571/01, juris; VG Dresden, Beschluss v. 22.7.1998, Az.: 5 K 1705/98, juris, Rn. 54; VG Potsdam, Beschluss v. 19.7.2000, Az.: 5 L 967/00, LKV 2001, S. 572, S. 573; Niehues, Prüfungsrecht

${ }^{1163}$ Vgl. Niehues, Prüfungsrecht, Rn. 380 ff; Avenarius/Heckel, Schulrecht, Ziff. 27.33 Langenfeld, RdJB 2007, S. $211 \mathrm{ff}$, S. 220
} 
heit herrscht, kann bestimmt werden, welche Leistungsfähigkeiten und Kompetenzen für das Ziel maßgeblich sein sollen und deshalb zu prüfen sind. ${ }^{1164}$

Nach diesen Maßstäben sind die geltenden fachlich-inhaltlichen Anforderungen für schulische Prüfungen insgesamt verhältnismäßig. Zweifel könnten sich jedoch hinsichtlich der Verhältnismäßigkeit der Bewertung der Rechtschreibung in allen Fächern ergeben.

Sicherlich ist die Rechtschreibung wichtig und es ist zulässig, sie in allen Arbeiten und Fächern zu korrigieren. Fraglich ist aber, ob es angemessen ist, dass die Rechtschreibung in der Form benotet wird, dass eine erhöhte Fehlerzahl in allen Fächern eine Abwertung der Endnote bewirkt. ${ }^{1165}$ Angemessen wäre die durchgängige Bewertung, wenn die Rechtschreibung Teil der Leistung dieses Faches ist und sich daher aus den Fehlern in der Rechtschreibung ein Versagen in dem Fach herleiten lässt. ${ }^{1166}$ Davon wird allgemein bei Sprachfächern ausgegangen. Die schulrechtlichen Regelungen stellen diese Prämisse jedoch für sämtliche Fächer auf, also auch für die Naturwissenschaften und Mathematik. Diese Prämisse wird allerdings von der allgemeinen Meinung nicht nur nicht geteilt, sondern implizit verneint. Rechtsprechung ${ }^{1167}$ und Literatur ${ }^{1168}$ halten es für offensichtlich, dass die Rechtschreibung in den Naturwissenschaften und in Mathematik nicht Prüfungsgegenstand ist, ${ }^{1169}$ d.i. sich dort gar nicht oder nur rudimentär auswirkt. ${ }^{1170}$ Die anders lautenden schulrechtlichen Vorschriften zur Bewertung der Rechtschreibung in diesen Fächern und deren Auswirkungen sind offenbar nicht bekannt. Angesichts dieser Diskrepanz kann jedenfalls nicht von einem allgemeinen Konsens ausgegangen werden, dass die Rechtschreibung in jedem Fach einen Rückschluss auf die Beherrschung des Faches zulässt. Jedenfalls in diesen Fächern ist es dann unverhältnismäßig, wenn die Rechtschreibung die Endnote entscheidend beeinflusst.

Zweifel bezüglich der Angemessenheit der umfangreichen Bewertung der Rechtschreibung in allen Fächern ergeben sich weiter daraus, dass die Rechtschreibung zum K.O.-Kriterium für das Bestehen oder Nicht-Bestehen eines Abschlusses werden kann. Es ist möglich, dass allein die Fähigkeit zur Rechtschreibung in den Fremdsprachen über die allgemeine Hochschulreife des

\footnotetext{
${ }^{1164}$ Vgl. Langenfeld, RdJB 2007, S. 211 ff, S. 220

1165 S. dazu ausführlich oben S. $30 \mathrm{ff}$

1166 Vgl. Lampe, Gerechtere Prüfungsentscheidungen, S. 37; Niehues/Rux, Schulrecht, Rn. 136

1167 OVG Lüneburg, Beschluss v. 10.7.2008, Az.: 2 ME 309/08, www.rechtsprechung.niedersachsen.de, Rn. 12 ff; OVG Berlin-Brandenburg, Beschluss v. 16.6.2009, Az.: 3 M 16.09, juris, Rn. 4

1168 Kischel, in: BeckOK GG, Art. 3, Rn. 219.3

1169 Kischel, in: BeckOK GG, Art. 3, Rn. 219.3

1170 OVG Lüneburg, Beschluss v. 10.7.2008, Az.: 2 ME 309/08, www.rechtsprechung.niedersachsen.de, Rn. 14
} 
Schülers entscheidet. ${ }^{1171}$ Alle anderen Noten bleiben ohne Einfluss, selbst ansonsten gute und sehr gute Noten in anderen Fächern führen zu keiner anderen Wertung und können das Manko in der Rechtschreibung nicht aufwiegen. Im Prüfungsrecht ist anerkannt, dass es sog. K.O.-Kriterien geben kann, d.h. Voraussetzungen, die zwingend erfüllt sein müssen, um ein Prüfungsziel zu erreichen, und deren Nicht-Erfüllung dazu führt, dass die Prüfung nicht bestanden ist. ${ }^{1172}$ Der Grundsatz der Verhältnismäßigkeit erfordert hinsichtlich dieser Kriterien, dass sich aus dem Versagen gerade in diesen Fächern oder Prüfungsteilen ableiten lässt, dass der Prüfling für das mit der Prüfung angestrebte Ziel offensichtlich nicht geeignet ist. ${ }^{1173}$ Voraussetzung ist also, dass dieses Fach oder dieser Prüfungsgegenstand für die Befähigung eine solch wichtige, nahezu unverzichtbare Bedeutung hat, sich auf zentrale Fragen des Prüfungsgebietes bezieht und daraus der Schluss der offenbaren Ungeeignetheit gezogen werden kann. ${ }^{1174}$ Danach darf sich die Rechtschreibung nur dann als K.O.-Kriterium für das Bestehen eines Schulabschlusses auswirken, wenn sich aus dem Versagen gerade in der Rechtschreibung ableiten lässt, dass der Prüfling für ein Hochschulstudium offensichtlich nicht geeignet ist. Das ist kaum vorstellbar. Es ist vielmehr davon auszugehen, dass ein derartiger Schluss, vor allem bei ansonsten guten Noten, nicht gezogen werden kann und deshalb die Wirkung der Rechtschreibung als K.O.-Kriterium unangemessen ist.

\subsection{Verhältnismäßigkeit der Nicht-Berücksichtigung von Legasthenie/Dyskalkulie}

Die Frage der grundsätzlichen Verhältnismäßigkeit der Bewertung der Rechtschreibung in allen Fächern muss hier jedoch nicht entschieden werden, wenn jedenfalls bei Schülern und Schülerinnen mit Legasthenie/Dyskalkulie ihre Behinderung zu berücksichtigen ist, so dass die Bewertung des Lesens, Rechtschreibens und/oder Rechnens entweder nicht zulässig ist oder jedenfalls die Endnote nicht durchgängig maßgeblich beeinflussen darf.

\footnotetext{
1171 So die Konstellation im Fall VG Schleswig, Urteil v. 10.6.2009, Az.: 9 A 208/08, BeckRS 2009 38123, S. 2 und 3

1172 Niehues, Prüfungsrecht, Rn. 514

1173 BVerwG, Urteil v. 17.7.1987, Az.: 7 C 118/86, juris, Rn. 17; BVerwG Beschluss v. 9.6.1995; Az.: 6 B 100/94, juris, Rn. 4; VGH Mannheim, Urteil v. 16-5-2000, Az.: 9 S 2537/99, juris, Rn. 23; OVG Greifswald, Urteil v. 27.2.1997, Az.: 2 L 52/96, juris, Rn. 25; VG Dresden, Beschluss v. 8.8.2001, Az.: 5 K 1571/01, juris; VG Dresden, Beschluss v. 22.7.1998, Az.: 5 K 1705/98, juris, Rn. 54; VG Potsdam, Beschluss v. 19.7.2000, Az.: 5 L 967/00, LKV 2001, S. 572, 573; Niehues, Prüfungsrecht; Rn. 167, 541

1174 Niehues, Prüfungsrecht, Rn. 541; VG Göttingen, Urteil v. 11.2.2009, Az.: 1 A 306/07, www.rechtsprechung.niedersachsen.de, S. $3 \mathrm{f}$
} 


\subsubsection{Legitimer Zweck}

Die Nicht-Berücksichtigung der Legasthenie/Dyskalkulie dient dem legitimen allgemeinen Zweck von Prüfungen und der Dokumentation bestimmter Fähigkeiten und Abschlüsse. Diese legitimen Zwecke werden jedoch durch das Grundrecht aus Art. 3 III 2 GG begrenzt. ${ }^{1175}$ Die Verhältnismäßigkeit der Prüfung muss daher unter Beachtung von Art. 3 III 2 GG im Hinblick auf den Schüler mit Behinderung bestimmt werden. Die generelle Nicht-Berücksichtigung der Behinderung dient daher keinem legitimen Zweck.

\subsubsection{Geeignetheit}

Folgt man dieser Ansicht nicht und bejaht einen legitimen Zweck, so ist die Nicht-Berücksichtigung der Legasthenie/Dyskalkulie geeignet, einheitliche Prüfungsanforderungen zu gewährleisten.

Sie ist aber nicht geeignet, wie bereits ausgeführt ${ }^{1176}$, eine Aussage dazu zu treffen, ob die Schüler und Schülerinnen mit Legasthenie/Dyskalkulie für bestimmte höhere Klassenstufen, Schulabschlüsse und Berufsmöglichkeiten befähigt und geeignet sind, weil die Rechtschreib- und Rechenleistungen keinen Schluss auf diese Eignung zulassen.

Die Nicht-Berücksichtigung müsste ferner geeignet sein, eine Aussage darüber zu treffen, ob die betroffenen Schüler und Schülerinnen für bestimmte berufliche Ausbildungen geeignet oder nicht geeignet sind. Dann müsste sich aus dem Umfang der Fähigkeiten im Lesen, Rechtschreiben und Rechnen ein Rückschluss auf die Berufseignung ziehen lassen. Das ist jedoch aufgrund der fast unüberschaubaren Berufsvielfalt nicht möglich. Aus den Fähigkeiten im Lesen, Rechtschreiben und Rechnen lässt sich weder ableiten, wer für sämtliche vielfältigen Berufsmöglichkeiten, die sich an einen Schulabschluss anschließen, geeignet noch wer ungeeignet ist. Je nach Beruf ist es außerordentlich unterschiedlich, auf welche Fähigkeiten es besonders ankommt und in welchem Umfang die Fähigkeiten zum Lesen, Schreiben und Rechnen vorhanden sein müssen. Das Fehlen oder nur eingeschränkte Vorhandensein der Fähigkeit könnte nur dann von Bildungsgängen und Abschlüssen ausschließen, wenn sie "conditio sine qua non" und nicht kompensierbar sind. Nur dann könnte an das Fehlen der Fähigkeit die Folge geknüpft werden, dass bestimmte Ausbildungen nicht absolviert und Berufe nicht gelernt werden dürfen. Dass eine solche „behinderungsbedingte Unfähigkeit" bei Legasthenie/Dyskalkulie nicht vorliegt, wurde bereits festgestellt. Deshalb ist die Nicht-Berücksichtigung nicht geeignet.

\footnotetext{
1175 VG Hannover, Beschluss v. 13.12.2010, Az.: 6 B 5596/10, www.rechtsprechung.niedersachsen.de, S. 4

${ }^{1176}$ S. oben 3. Teil: 5. Kapitel: 3.3.3.: Vorrang von Art. 7 I GG bei Fehlen zwingend erforderlicher Fähigkeiten, S. 163
} 


\subsubsection{Erforderlichkeit}

Die Erforderlichkeit wäre gegeben, wenn es kein milderes Mittel als die NichtBerücksichtigung der Legasthenie/Dyskalkulie gäbe, um Schüler und Schülerinnen von dem Besuch eines Bildungsganges auszuschließen, für den sie nicht qualifiziert sind. Das Ziel lässt sich jedoch auch dann erreichen, wenn Legasthenie/Dyskalkulie berücksichtigt werden.

Für Schüler und Schülerinnen ohne Legasthenie/Dyskalkulie ändert sich hinsichtlich der Beurteilung ihrer Qualifikation nichts. Bei Schülern und Schülerinnen mit Legasthenie/Dyskalkulie verschiebt sich der Schwerpunkt der Beurteilung ihrer Qualifikation. Maßstab der Beurteilung ihrer Qualifikation und der Prognose, ob sie für einen bestimmten Bildungsgang geeignet sind, sind dann nicht die Fähigkeiten im Lesen, Schreiben und Rechnen, sondern die inhaltlichen Fähigkeiten in den Fächern, d.h. maßgeblich wird z.B. in Deutsch die sprachliche Ausdrucksfähigkeit, die Fähigkeit grammatische Strukturen zu lernen und nicht die (Un-)Fähigkeit zu lesen bzw. richtig zu schreiben. ${ }^{1177}$ Erreichen die betroffenen Kinder die Anforderungen nicht, die insgesamt gefordert werden, sind sie für den bestimmten Bildungsgang nicht qualifiziert.

Der mit der Nicht-Berücksichtigung einhergehende Ausschluss von Schülern und Schülerinnen mit Legasthenie/Dyskalkulie von weiteren schulischen Möglichkeiten ist auch deshalb nicht erforderlich, weil sich aus den Schwierigkeiten im Lesen, Rechtschreiben und Rechnen nicht ableiten lässt, dass die betroffenen Schüler und Schülerinnen für die Vielzahl an beruflichen Möglichkeiten, die sich aufgrund eines bestimmten Schulabschluss ergeben, ungeeignet sind. ${ }^{1178}$ Beim Übertritt in die weiterführenden Schulen und bei den Schulabschlüssen ist noch völlig unklar, welchen Beruf ein Schüler erlernen möchte und welche Voraussetzungen er dafür mitbringen muss. Der Schüler mag am Ende seiner Schullaufbahn wegen seiner behinderungsbedingten Einschränkungen von bestimmten Berufsbildern ausgeschlossen sein, das aber macht es nicht erforderlich, ihn von dem gesamten Bildungsgang auszuschließen.

\section{Ergebnis}

Die Nicht-Berücksichtigung von Legasthenie/Dyskalkulie in der Schule ist ein Eingriff in die Grundrechte der betroffenen Schüler und Schülerinnen aus Art. 12 I und 2 I GG. Dieser Eingriff dient weder einem legitimen Zweck noch ist er erforderlich. Die Behinderungen Legasthenie/Dyskalkulie sind daher auch zur

\footnotetext{
1177 Vgl. zu einer solchen Differenzierung VG Hannover, Beschluss v. 13.12.2010, Az.: 6 B 5596/10, www.rechtsprechung.niedersachsen.de, S. 6 f

1178 Ausführlich oben 3. Teil: 5. Kapitel: 3.3.3.: Vorrang von Art. 7 I GG bei Fehlen zwingend erforderlicher Fähigkeiten, S. 163, zur begrenzten Aussagefähigkeit von Schullaufbahnempfehlungen s. Tiedemann/Billmann-Mahecha, Z Erziehungswiss 2010, S. 649 ff, S. 655 f, nach Modellrechnungen liegt die Quote der Fehlzuweisungen bei 30 bis $40 \%$ (S. 656)
} 
Wahrung der Grundrechte aus Art. 12 I und 2 । GG bei Prüfungen zu berücksichtigen.

\section{Kapitel: Anspruch aus Art. 2 I und 6 || GG - Schulpflicht}

Der Anspruch auf Berücksichtigung der Legasthenie/Dyskalkulie kann sich weiter aus dem Grundrecht des Kindes aus Art. 2। GG auf freie Entfaltung seiner Persönlichkeit und dem Grundrecht der Eltern, dem sog. „Elternrecht" ${ }^{\text {1179 }}$ aus Art. 6 ॥ GG, ergeben, wenn die Schulpflicht des Kindes nur zulässig ist, wenn die Behinderung beim Schulbesuch berücksichtigt wird.

Unstreitig ist, dass der Staat das Recht hat, die Schulpflicht festzulegen ${ }^{1180}$, denn jeder Bürger kann die ihm in der Gesellschaft zustehenden Rechte erst dann wahrnehmen, wenn er eine Bildung und Ausbildung erhalten hat. ${ }^{1181}$ Ebenso unstreitig ist aber auch, dass der Staat mit der Festlegung der allgemeinen Schulpflicht schwerwiegend sowohl in das Grundrecht der Kinder aus Art. 2 I GG auf freie Entfaltung der Persönlichkeit als auch in das Grundrecht der Eltern auf Erziehung ihrer Kinder aus Art. 6 II GG eingreift ${ }^{1182}$, denn er nimmt direkten Einfluss auf die Entwicklung der Kinder und Jugendlichen und teilt mit dem Prüfungswesen weit in die Zukunft weisende Lebensführungschancen $\mathrm{zu}^{1183}$

Ein Eingriff in die Rechte des Kindes mit Legasthenie/Dyskalkulie und seiner Eltern durch die Schulpflicht könnte darin liegen, dass die Beschulung der betroffenen Schüler und Schülerinnen ihrer Behinderung nicht gerecht wird und damit inadäquat ist. Der Eingriff kann im Unterlassen einer behinderungsgerechten, adäquaten Beschulung bestehen, wenn eine evidente Gefährdungsla-

\footnotetext{
1179 Schmitt-Kammler/Coelln, in: Sachs GG, Art. 6, Rn. 45

1180 BVerfG, Beschluss v. 29.4.2003; Az.: 1 BvR 436/03, Rn. 7, http://www.bverfg.de/entscheidungen/rk20030429_1bvr043603.html; BVerwG Beschluss v. 8.5.2008, Az.: 6 B 65/07, juris, Rn. 4; Beschluss v. 15.10.2009, Az.: 6 B 27/09, juris, Rn. 4; Urteil v. 25.8. 1993; Az.: 6 C 9/81, juris, Rn. 15

1181 Umfassend: BVerfG, Beschluss v. 29.4.2003; Az.: 1 BvR 436/03, Rn. 7, 8http://www.bverfg.de/entscheidungen/rk20030429_1bvr043603.html;Rux, Pädagogische Freiheit des Lehrers, S. 26, s. dazu oben S. $107 \mathrm{f}$

1182 Vgl. Gröschner, in: Dreier GG, Art. 7, Rn. 53; Langenfeld, RdJB 2007, S. 211 ff, S. 222; Rux, Pädagogische Freiheit des Lehrers, S. 26; Niehues/Rux, Schulrecht, Rn. 125 und Rn. $145 \mathrm{ff}$ zum Elternrecht; Robbers, in: v. Mangoldt/Klein/Starck GG, Art. 7, Rn. 31; Gröschner, in: Dreier GG, Art. 7, Rn. 53; Badura, in: Maunz/Dürig/Herzog GG, Art. 7, Rn. 25; Ennuschat, RdJB 2007, S. 271 ff, S. 288; Avenarius/Heckel, Schulrecht, Ziff. 25.12; Niehues, Prüfungsrecht, Rn. 49

1183 Luthe, Bildungsrecht, S. 16
} 
ge besteht. ${ }^{1184}$ Die notwendige staatliche Garantenstellung ergibt sich insbesondere im Bereich der Schulpflicht aus dem besonderen Näheverhältnis. ${ }^{1185}$ Daher kann auch eine voraussehbare und in Kauf genommene Nebenfolge staatlichen Handelns Grundrechte verletzen. ${ }^{1186}$

Schüler und Schülerinnen mit Dyskalkulie, deren Dyskalkulie z.T. über Jahre hinweg unerkannt bleibt und die deshalb, dem Mathematikunterricht nicht folgen können, so dass sie ohne Abschluss oder mit einem erheblich schlechteren Abschluss als möglich wäre, die Schule verlassen ${ }^{1187}$, werden im mathematischen Bereich in einer Weise beschult, die ihrer Behinderung nicht gerecht wird und als Nebenfolge erhebliche Einbußen in den Lebenschancen nach sich ziehen. Ebenso ist es bei Schülern und Schülerinnen mit Legasthenie, die die Schule z.T. ohne ausreichende Lese- und Schreibkenntnisse verlassen, weil der Unterricht nicht auf ihre Behinderung Rücksicht nimmt und sie nicht entsprechend fördert. Mit der inadäquaten Beschulung und damit dem Unterlassen einer adäquaten Beschulung in einem Bereich, der evident von der Qualität der Beschulung abhängt und für den weiteren Lebensweg der Betroffenen von größter Bedeutung ist, wird in die Grundrechte aus Art. 2 I und 6 || GG eingegriffen.

Ein Eingriff liegt weiter darin, dass schulische Regelungen bestehen, die grundsätzlich eine Behinderung des Kindes nicht berücksichtigen, wodurch die die freie Entfaltung des behinderten Kindes und das Erziehungsrecht der Eltern beeinträchtigt wird.

Ein Eingriff liegt auch in den schulischen Regelungen, die behinderte Schüler und Schülerinnen gerade wegen ihrer Behinderung nicht erfüllen kann, weil die Behinderung nicht berücksichtigt wird. Dadurch wird ihre Möglichkeit, weiterführende Schulen zu besuchen, eingeschränkt oder unmöglich gemacht.

Die Frage ist weiter, ob diese Eingriffe gerechtfertigt sind. Nach ganz herrschender Meinung stehen der staatliche Erziehungsauftrag und das elterliche Erziehungsrecht nicht in einem Über-Unterordnungsverhältnis zueinander, sondern sind gleichgeordnet. ${ }^{1188}$ "Der Staat muss deshalb in der Schule die Verantwortung der Eltern für den Gesamtplan der Erziehung ihrer Kinder achten. Er darf nie den ganzen Werdegang des Kindes festlegen. ${ }^{.1189}$ Er darf aber einen

\footnotetext{
${ }^{1184}$ Luthe, Bildungsrecht, S. 26

1185 Michael/Morlok, Grundrechte, Rn. 520

1186 Luthe, Bildungsrecht, S. 27 m.w.N.

${ }^{1187}$ Leserbrief in der Zeitschrift des BVL, 1/2011, S. $50 \mathrm{f}$

1188 BVerwG Beschluss v. 7.10.2003, Az.: 6 B 41/03, juris, Rn. 3; Badura, in: Maunz/Dürig/Herzog GG, Art. 7, Rn. 23; Robbers, in: v. Mangoldt/Klein/Starck GG, Art. 6, Rn. $219 \mathrm{ff}$

1189 BVerfGE 34, 165, 183, s. auch VGH Mannheim, Beschluss v. 23.7.2007, Az.: 9 S 1298/07, www.landesrecht-bw.de, Rn. $11 \mathrm{ff}, 18$, zur Verletzung des Elternrechts durch die Festschreibung unterschiedlicher Fremdsprachen als erste Fremdsprache je nach Region des Bundeslandes
} 
eigenen Erziehungsauftrag ausüben. ${ }^{1190}$ Das Recht auf freie Entfaltung der Persönlichkeit unterliegt einer sehr weiten Grundrechtsschranke.

Da das Recht des Kindes aus Art. 2 I GG und das Elternrecht aus Art. 6 II GG nur durch verhältnismäßige Regelungen eingeschränkt werden dürfen, müssen die Schulpflicht und die mit ihr zusammenhängenden Funktionen verhältnismäBig sein. ${ }^{191}$ Die Ausgestaltung der Schulpflicht und die Begrenzung der Freiheit des Schülers muss im Hinblick auf die Ziele des Art. 7 I GG und vor allem im Hinblick auf das Entfaltungsrecht der Schüler sachgerecht und geeignet sein. ${ }^{1192}$

Die in der Schulpflicht liegende Freiheitsbeschränkung betrifft den Kernbereich der Person des Schülers unmittelbar ${ }^{1193}$ und steht in unmittelbarer Wechselbeziehung zu dem Zugewinn an Entfaltungsmöglichkeiten, den der Einzelne durch Bildung und Erziehung erhält. ${ }^{1194}$ Sinn und Zweck des Erziehungsauftrages ist es, die Voraussetzungen dafür zu schaffen, dass der Einzelne das Recht auf freie Entfaltung der Persönlichkeit und auf freie Wahl des Berufs wahrnehmen kann. Wesentlicher Bestandteil der schulischen Bemühungen muss daher die gezielte Förderung individueller Gaben und Fähigkeiten der Schüler und Schülerinnen im Bildungs- und Erziehungsprozess sein. ${ }^{1195}$ Die Schulpflicht und schulische Ausbildung muss daher so angelegt sein, dass die Schüler und Schülerinnen diese Fähigkeiten und Kenntnisse, die sie für ihren weiteren Lebensund Berufsweg brauchen, erwerben können. ${ }^{1196}$ Die Schulpflicht ist daher prinzipiell mit dem Anspruch verbunden, dass Schule auch gelingt. ${ }^{1197}$ Wird Schule diesem Anspruch nicht gerecht, dann ist die Anordnung der Schulpflicht unverhältnismäßig, weil dem Zwang zum Schulbesuch kein entsprechender Gewinn gegenüber steht.

Nimmt der schulische Unterricht auf Behinderungen keine Rücksicht, so ist der Unterricht nicht so angelegt, dass die Schüler und Schülerinnen die Fähigkeiten, die sie erwerben sollen, überhaupt erwerben können. Der Schulunterricht geht an innen vorbei, weil sie nicht in der Lage sind, bei diesem Unterricht etwas zu lernen, Schule gelingt insoweit nicht. Ein solcher inadäquater Unterricht ist nicht verhältnismäßig. ${ }^{1198}$

\footnotetext{
1190 BVerwG Beschluss v. 7.10.2003, Az.: 6 B 41/03, juris, Rn. 3

1191 BVerfG, Beschluss v. 29.4.2003; Az.: 1 BvR 436/03, Rn. 7, http://www.bverfg.de/entscheidungen/rk20030429_1bvr043603.html; Robbers, in: v. Mangoldt/Klein/Starck GG, Art. 7, Rn. 31

1192 Jarass, DÖV 1995, S. 674 ff, S. 678; Glotz/Faber, in: Hdb. des Verfassungsrechts, § 28, Rn. 11

1193 Luthe, Bildungsrecht, S. 28

1194 Langenfeld, Integration und kulturelle Identität zugewanderter Minderheiten, S. 234

1195 Langenfeld, Integration und kulturelle Identität zugewanderter Minderheiten, S. 215

1196 Niehues/Rux, Schulrecht, Rn. 137

1197 Luthe, Bildungsrecht, S. 20; s. dazu auch Forkel, SächsVBI 2010, S. 282 ff

${ }^{1198}$ Vgl. Luthe, Bildungsrecht, S. 29
} 
Die Wechselbeziehung zwischen dem Entfaltungsrecht der Schülerinnen und Schüler und der Schulpflicht wird verkannt, wenn Behinderungen prinzipiell nicht berücksichtigt werden. Diese Nicht-Berücksichtigung verstößt gegen Art. 3 III 2 GG.

Eine Schulpflicht ist unverhältnismäßig, wenn sie Zulassungsanforderungen für weiterführende Schulen und schulische Ausbildungen stellt, die behinderte Schüler gerade wegen ihrer Behinderung nicht erfüllen können, und damit diese Gruppe von weiterführenden Bildungsmöglichkeiten ausschließt, obwohl sie diese schulische Ausbildung aufgrund ihrer Begabung und Neigung prinzipiell absolvieren können. ${ }^{1199}$ Die unverhältnismäßigen Anforderungen verletzen auch das Elternrecht aus Art. 6 ॥ GG, denn den Eltern wird die Bestimmung über den weiteren Lebensweg ihres (behinderten) Kindes völlig entzogen. ${ }^{1200}$

Die Zulässigkeit des Eingriffs durch die Schulpflicht kann nur dadurch hergestellt werden, dass die Behinderung sowohl im Unterrichtsalltag als auch bei Prüfungen und Zulassungsentscheidungen berücksichtigt wird. ${ }^{1201}$ Infolgedessen besteht auch aus Art. 2 I GG und Art. 6 II GG ein Anspruch auf Berücksichtigung der Legasthenie/Dyskalkulie in der Schule.

\footnotetext{
1199 Langenfeld, Integration und kulturelle Identität zugewanderter Minderheiten, S. 234; Langenfeld, RdJB 2007, S. 211 ff, S. 222

1200 Vgl. Glotz/Faber, in: Hdb. des Verfassungsrechts, § 28, Rn. 22

1201 Langenfeld, RdJB 2007, S. 211 ff, S. 222
} 



\section{Teil: Anspruch aus Völker- und Europarecht}

\section{Kapitel: Anspruch aus Art. 24 BRK - Recht auf Bildung}

Ein Anspruch auf Berücksichtigung der Legasthenie/Dyskalkulie in der Schule und auf den insoweit erforderlichen Nachteilsausgleich kann sich auch unmittelbar aus dem Recht auf Bildung aus Art. 24 BRK i.V.m. dem Diskriminierungsverbot aus Art. 5 BRK ergeben.

\section{Innerstaatliche Geltung der BRK}

Erforderlich ist dafür zunächst, dass die BRK innerstaatlich anwendbar ist. Das gilt insbesondere für Art. 24 BRK, dessen Regelungsbereich die Gesetzgebungskompetenz der Länder betrifft. ${ }^{1202}$

Aus dem Lindauer Abkommen und dem Gebot der Bundestreue nach Art. 20 III GG ${ }^{1203}$ sind die Länder verpflichtet, ihr Landesrecht an die Anforderungen der BRK anzupassen, soweit es um ihre Gesetzgebungszuständigkeit geht. Das gilt insbesondere für Art. 24 BRK. ${ }^{1204}$ Diese Pflicht wird dadurch verstärkt, dass Art. 4 Abs. 5 BRK ausdrücklich bestimmt, dass die Bestimmungen der BRK ohne Einschränkung oder Ausnahme für alle Teile eines Bundesstaats gelten.

Von dieser Anpassungspflicht ist jedoch die Frage zu trennen, ob die BRK schon aufgrund des Vertragsgesetzes des Bundes umfassend im deutschen Recht gilt oder ob sie erst dann auf Länderebene gilt, wenn diese sie im Bereich ihrer Gesetzgebungskompetenz umgesetzt haben. Dies ist streitig. ${ }^{1205}$ Das BVerwG $^{1206}$ und weitere Gerichte ${ }^{1207}$ sowie weite Teile der Literatur ${ }^{1208}$ vertre-

\footnotetext{
1202 Vgl. Lorz, Vorrang Art. 3 UN-KRK, S. 13 m.w.N.

1203 Näher hierzu Latham/Watkins, Gutachten zur UN-BRK, S. 24

1204 Degener, RdJB 2009, S. 200 ff, S. 217; Poscher/Langer/Rux, Gutachten zu Art. 24 BRK, S. 16; Riedel, Gutachten UN-Behindertenrechtskonvention, S. 34 f; Latham/Watkins, Gutachten zur UN-BRK, S. $23 \mathrm{f}$

1205 Umfassend zur Rechtslage Kotzur/Richter, Kommentar zur UN-BRK, Verbindlichkeit, Rn. 4 ff 1206 BVerwG, Beschluss v. 18.1.2010, Az.: 6 B 52/09, juris, Rn. 4
} 
ten die Auffassung, dass dem Bund für die Umsetzung insbesondere des Art. 24 BRK in innerstaatliches Recht die Gesetzgebungskompetenz fehlt. Deshalb sei die BRK erst dann im Landesrecht anwendbar, wenn die Landesgesetzgeber sie in Landesrecht transformiert haben. Solange diese Transformation nicht erfolgt sei, sei die BRK auf Landesebene im Schulrecht nicht anwendbar. ${ }^{1209}$ Überwiegend wird vertreten, dass sich ohne die notwendige landesrechtliche Umsetzung im Schulrecht aus der BRK keine unmittelbaren Ansprüche insbesondere auf eine inklusive Beschulung und somit auf eine Beschulung auf der Regelschule ableiten lassen. ${ }^{1210}$

Demgegenüber vertritt ein anderer Teil der Literatur die Ansicht, dass die BRK durch das Vertragsgesetz unbeschränkt im deutschen Recht anzuwenden ist. $^{1211}$

1207 VGH Kassel, Beschluss v. 12.11.2009, Az.: 7 B 2763/09, juris; OVG Lüneburg, Beschluss v. 16.9.2010, Az.: 2 ME 278/10, www.rechtsprechung.niedersachsen.de; OVG Münster, Beschluss v. 3.11.2010; Az.: 19 E 533/10, juris; VG Düsseldorf, Urteil v. 16.12.2010, Az.: $18 \mathrm{~K}$ 5702/10, BeckRS 2011, 45848; VG Arnsberg, Beschluss v. 17.8.2010, Az.: 10 L 397/10, juris; vgl. auch OVG Münster, Beschluss v. 9.10.2007, Az.: 15 A 1596/07, www.justiz.nrw.de, Rn. $36 \mathrm{ff}$ und BVerwG, Urteil v. 29.4.2009, Az.: 6 C 16/08, Rn. $46 \mathrm{ff}$, www.bundesverwaltungsgericht.de, zur Geltung des UN-Sozialpakts bei der Festlegung von Studiengebühren; LSG Baden-Württemberg, Beschluss v. 9.12.2010, Az.: L 13 AL 4629/10 ERB, juris, Rn. 33

1208 Huster/Rux, in: BeckOK GG, Art. 20, Rn. 32a.1; Krajewski, JZ 2010, S. 120 ff, S. 124; Faber/ Roth, DVBI 2010, S. 1193 ff, S. 1196; Latham/Watkins, Gutachten zur UN-BRK, S. 23; Riedel, Gutachten UN-Behindertenrechtskonvention, S. 35 f; Ennuschat, in: FS Klaus Stern, S. 721

${ }^{1209}$ VGH Kassel, Beschluss v. 12.11.2009, Az.: 7 B 2763/09, juris, Rn. 7 und 22; OVG Lüneburg, Beschluss v. 16.9.2010, Az.: 2 ME 278/10, www.rechtsprechung.niedersachsen.de, S. 4

1210 Poscher/Langer/Rux, Gutachten zu Art. 24 BRK, S. 33; Latham/Watkins, Gutachten zur UNBRK, S. 24 f; Riedel, Gutachten UN-Behindertenrechtskonvention, S. 36; Die von der Bundesregierung im Vorfeld abgegebene Denkschrift, in der subjektive Ansprüche abgelehnt wurden (BR Drs. 760/08 v. 17.10.2008, S. 48), ist insoweit allerdings unerheblich Rux, RdJB 2009, S. 220 ff, S. 220, a.A. VGH Kassel, Beschluss v. 12.11.2009, Az.: 7 B 2763/09, juris, Rn. 41. Die Bundesregierung scheint von dieser Haltung abgerückt zu sein, da der Koalitionsvertrag für die 17. Legislaturperiode festlegt, dass politische Entscheidungen an der BRK zu messen sind. Koalitionsvertrag Punkt 7.4, S. 83 f, http://www.heute.de/ZDFheute/download/0,6741,7012935,00.pdf

1211 Degener, RdJB 2009, S. 200 ff, S. 212; Aichele, Stellungnahme DIMR 2010, S. 11 und Aichele, Anwaltsblatt 2011, 727 ff, S. 727 m.w.N., Bernstorff, RdJB 2011, S. 203 ff, S. 206 f; Ennuschat, Die Rechte stotternder Menschen in Schule, Ausbildung und Studium, S. 83; Welti, NVwZ 2012, S. 725 ff, S. 726; zur gleichen Fragestellung bezüglich der Geltung des Weltkulturerbeabkommens s. u.a. Brüggen, Kurzgutachten Waldschlösschenbrücke; Fastenrath, Ulrich: Rechtsgutachterliche Stellungnahme zur Zulässigkeit des Baus der Waldschlösschenbrücke; Bundesregierung: Gutachten betreffend die innerstaatliche Bindungswirkung des UNESCOÜbereinkommens zum Schutz des Natur- und Kulturerbes der Welt 
Dieser Streit braucht indes hier nicht entschieden werden, denn die Bundesländer sind durchweg dabei, im Bereich des Schulrechts die BRK umzusetzen ${ }^{1212}$, so dass die Anwendbarkeit der BRK in den Ländern nur noch eine Frage der Zeit sein wird. ${ }^{1213}$ Es spricht im Übrigen mehr dafür, dass es der Umsetzung der BRK in die Länder, sei es durch Transformation, sei es durch Anwendungsbefehl bedarf, weil nur damit das Kompetenzgefüge des Grundgesetzes beachtet wird. ${ }^{1214}$

Hier reicht es, auf die Frage einzugehen, inwieweit sich nach einer Umsetzung der BRK in Landesrecht aus Art. 24 BRK unmittelbare Ansprüche herleiten lassen oder jedenfalls Vorgaben für eine völkerrechtsfreundliche Auslegung der Schulgesetze gewonnen werden können.

\section{Unmittelbare Anwendbarkeit der BRK}

Voraussetzung für unmittelbare Ansprüche aus Art. 24 BRK ist zunächst, dass dieser unverändert durch ein Gesetz in Landesrecht transformiert wurde oder durch innerstaatliches Recht der Anwendungsbefehl für diese Norm erteilt wird. Wird Art. 24 BRK nicht in seiner vollständigen Fassung übernommen, sondern wird er zum Anlass genommen, um das Schulgesetz zu ändern und an die BRK anzupassen ${ }^{1215}$, kommt eine unmittelbare Anwendung nicht in Betracht, weil die Norm dann nicht direkt in innerstaatliches Recht übernommen wurde. In Betracht kommt dann nur die völkerrechtsfreundliche Auslegung des Schulgesetzes, um Konflikte zwischen der BRK und dem deutschen Schulrecht zu vermeiden. ${ }^{1216}$

Wird Art. 24 BRK dagegen unverändert in Landesrecht übernommen, dann kann ein direkter Anspruch auf Berücksichtigung der Legasthenie/Dyskalkulie in

1212 Z.B. BE §§ 18, 20 SopädVO; BY Art. 2 II „Inklusiver Unterricht ist Aufgabe aller Schulen“, 30b, 41 BayEUG, BE Gesamtkonzept Inklusion: http://www.berlin.de/imperia/md/content/senbildung/foerderung/sonderpaedagogische_foerderung/gesamtkonzept_inklusion.pdf?start\& ts $=1296483030 \&$ file=gesamtkonzept_inklusion.pdf; BB SchulG 2011; HB SchulG v. 2009, insbesondere $\S \S 34,35 ;$ HH SchulG § 12, Anspruch von Kindern und Jugendlichen mit sonderpädagogischem Förderbedarf auf Regelbeschulung; HE Schulgesetz §§ 51, 54; MV SchulG, $\S 1$ II, 34, 35; NI Schulgesetz $\S \S 4$ „Die öffentlichen Schulen ermöglichen allen Schülerinnen und Schülern einen barrierefreien und gleichberechtigten Zugang und sind damit inklusive Schulen“, § 183 c Übergangsvorschrift; NRW - Umsetzung vorgesehen zum 1.8.2013, Aktionsplan, S. $198 \mathrm{ff}$, http://www.mais.nrw.de/08_PDF/003/121115_endfassung_nrwinklusiv.pdf; SH SchulG § 4 XI-s. dazu auch Beschluss der KMK v. 18.11.2010: Pädagogische und rechtliche Aspekte der Umsetzung der Behindertenrechtskonvention in der schulischen Bildung

1213 So auch Krajewski/Bernhard, Kommentar zur UN-BRK, Art. 24, Rn. 8 f

1214 Ennuschat, in: FS Klaus Stern, S. 721

1215 So HE SchulG

1216 S. dazu oben S. $73 \mathrm{f}$ 
der Schule aus ihm nur hergeleitet werden, wenn er unmittelbar anwendbar ist. Der VGH Kassel hat eine unmittelbare Anwendbarkeit der BRK verneint, weil die BRK grundsätzlich nicht auf unmittelbare Anwendung angelegt sei, sondern nur politische Zielsetzungen enthalte. Das Diskriminierungsverbot des Art. 5 BRK sei zwar unmittelbar anwendbar, werde aber durch die speziellen Regeln des nicht unmittelbar anwendbaren Art. 24 BRK verdrängt. ${ }^{1217}$ Das OVG Lüneburg hat sich dem angeschlossen und vertreten, dass die Bestimmungen des Art. 24 BRK für eine unmittelbare Anwendbarkeit zu unbestimmt seien. ${ }^{1218}$

Voraussetzung für die unmittelbare Anwendbarkeit einer Völkerrechtsnorm ist, dass diese bestimmt genug ist, um in der konkreten Anwendungsfrage Rechtsfolgen bewirken zu können. ${ }^{1219}$ Dies ist nicht für den Vertrag insgesamt oder einen kompletten Normkomplex, sondern im Hinblick auf einzelnen vertragsrechtliche Normen und ihren spezifischen Anwendungskontext zu prüfen. ${ }^{1220}$ Ist die Norm nach Wortlaut, Zweck und Inhalt geeignet und hinreichend bestimmt, um rechtliche Wirkungen im konkreten Fall zu entfalten, so ist sie unmittelbar anwendbar oder auch „self-executing“. ${ }^{1221}$ Die unmittelbare Anwendbarkeit einer Norm wird schließlich nicht grundsätzlich dadurch ausgeschlossen, dass der Vertrag Formulierungen wie "States parties shall take measures to ....", "shall enable“ oder „shall ensure" enthält ${ }^{1222}$, denn derartige Formulierungen können auch konkretisierende Wirkung haben. ${ }^{1223}$ Für die meisten Normen der EMRK wird inzwischen von der unmittelbaren Anwendbarkeit ausgegangen. ${ }^{1224}$ Soweit teilweise vertreten wird, dass wirtschaftliche, soziale und kulturelle Rechte, etwa die des UN-Sozialpaktes, anders als die bürgerlichen und politischen Menschenrechte generell nur nach und nach vollziehbar und deshalb nicht unmittelbar anwendbar sind ${ }^{1225}$, ist diese Ansicht inzwischen

\footnotetext{
1217 VGH Kassel, Beschluss v. 12.11.2009, 7 B 2763/09, juris, Rn. 21; 26; 27, 31, 34, 40, 45, 46

1218 OVG Lüneburg, Beschluss v. 16.9.2010, Az.: 2 ME 278/10, www.rechtsprechung.niedersachsen.de, S. 5 und bestätigend im Beschluss v. 20.9.2012, Az.: 2 LA 234/11, S. 5 (nicht veröffentlicht); im Ergebnis ebenso Poscher/Langer/Rux, Gutachten zu Art. 24 BRK, S. $32 \mathrm{f}$

1219 Lorz, Vorrang Art. 3 UN-KRK, S. 11

1220 Bernstorff, RdJB 2011, S. 203 ff, S. 209 f

1221 BVerfGE 29, 348, 360; BVerfG Beschluss v. 19.9.2006, 2 BvR 2115/01, Rn. 53; Bernstorff, RdJB 2011, S. 203 ff, S. 210, Kirchhof, EuGRZ 1994, S. 16 ff, S. 27; Eichholz, „Gibt es ein Recht auf eine Schule für alle?", S. $7 \mathrm{f}$

1222 So die Argumentation des VGH Kassel, Beschluss v. 12.11.2009, 7 B 2763/09, juris, Rn. 31

1223 Riedel/Arend, NVwZ 2010, S. 1346 ff, S. 1347; Bernstorff, RdJB 2011, S. 203 ff, S. 212

1224 Geiger, Völkerrecht, S. 346; Bernstorff, RdJB 2011, S. 203 ff, S. 211

1225 VGH Kassel, Beschluss v. 12.11.2009, 7 B 2763/09, juris, Rn. 32 ff; Krajewski, JZ 2010, S. $120 \mathrm{ff}$, S. 123
} 
überholt. ${ }^{1226}$ Auch solche Normen können einen unmittelbaren justiziablen Inhalt haben. ${ }^{1227}$

Grundsätzlich können daher die Normen der BRK unmittelbar anwendbar sein. ${ }^{1228}$ Die unmittelbare Anwendbarkeit scheidet entgegen der Rechtsprechung des VGH Kassel und des OVG Lüneburg ${ }^{1229}$ nicht deshalb aus, weil Art. 4 II BRK den Progressivitätsgrundsatz für die Verwirklichung der Rechte aus der BRK anerkennt, denn dieser Grundsatz wird im letzten Halbsatz dahin eingeschränkt, dass er nur „unbeschadet derjenigen Verpflichtungen, die nach Völkerrecht unmittelbar anwendbar sind" gilt. Damit ist ausdrücklich die Möglichkeit der unmittelbaren Anwendung von Normen geschützt. ${ }^{1230}$

\subsection{Art. 24 I BRK}

Art. 24 BRK umfasst alle speziellen Aussagen der BRK zur Bildung. Die unmittelbare Anwendbarkeit kann hinsichtlich der einzelnen Absätze der Norm unterschiedlich sein, da sie konkret für einen jeweiligen Normbestandteil und dessen Anwendungsfall zu bestimmen ist. Sie ist nicht deshalb ausgeschlossen, weil die Norm aus mehreren Absätzen besteht, von denen einer oder mehrere nicht unmittelbar anwendbar sind. ${ }^{1231}$

Nach Art. 24 I BRK anerkennen die Vertragsstaaten "das Recht von Menschen mit Behinderungen auf Bildung. Um dieses Recht ohne Diskriminierung und auf der Grundlage der Chancengleichheit zu verwirklichen, gewährleisten die Vertragsstaaten ein integratives Bildungssystem auf allen Ebenen und lebenslanges Lernen ...". Das Recht auf Bildung wird verknüpft mit dem Verbot der Diskriminierung, steht also nicht allein, sondern ist im Kontext mit dem Diskriminierungsverbot des Art. 5 II BRK zu lesen. Der VGH Kassel hat Art. 5 II BRK allerdings für nicht anwendbar gehalten, da Art. 24 BRK insoweit Sonderregelungen enthalte, die einen Rückgriff auf die allgemeinen Bestimmungen ausschließe. ${ }^{1232}$ Dabei hat der VGH Kassel jedoch übersehen, dass es sich bei dem Diskriminierungsverbot des Art. 5 II BRK nicht um eine eigenständige Rechtsgrundlage, sondern um ein akzessorisches Diskriminierungsverbot handelt, das

\footnotetext{
${ }^{1226}$ Aichele, Stellungnahme DIMR 2010, S. 6; Riedel/Arend, NVwZ 2010, S. 1346 ff, S. 1347; Bernstorff, RdJB 2011, S. $203 \mathrm{ff}$, S. $211 \mathrm{f}$

1227 Riedel/Arend, NVwZ 2010, S. 1346 ff, S. 1347

1228 Allgemein Bernstorff, RdJB 2011, S. 203 ff, S. 217

1229 VGH Kassel, Beschluss v. 12.11.2009, 7 B 2763/09, juris, Rn. 32 ff OVG Lüneburg, Beschluss v. 16.9.2010, Az.: 2 ME 278/10, www.rechtsprechung.niedersachsen.de, S. 5, Rn. 32 ff; Krajewski, JZ 2010, S. 120 ff, S. 123

1230 Riedel/Arend, NVwZ 2010, S. 1346 ff, S. 1347 f; Bernstorff, RdJB 2011, S. 203 ff, S. 215; Aichele, Stellungnahme DIMR 2010, S. 7; Masuch, in: FS Renate Jaeger, S. 250

1231 Riedel/Arend, NVwZ 2010, S. 1346 ff, S. 1347

1232 VGH Kassel, Beschluss v. 12.11.2009, 7 B 2763/09, juris, Rn. 46
} 
unmittelbar anwendbar ist ${ }^{1233}$, auf die spezielle Vertragsnorm ausstrahlt und ihren subjektiven Kern mit begründet. ${ }^{1234}$ Andererseits beeinflusst die Wertentscheidungen des Art. 24 BRK wiederum das Diskriminierungsverbot des Art. 5 BRK. ${ }^{1235}$ Der Kern des Art. 24 I BRK ist das diskriminierungsfreie Recht auf Bildung. Dieses ist eindeutig und klar, bedarf keiner weiteren normativen Ausführung und ist daher unmittelbar anwendbar. ${ }^{1236}$

Für Schüler und Schülerinnen mit Legasthenie/Dyskalkulie ergibt sich damit unmittelbar aus Art. 24 I BRK der Anspruch auf diskriminierungsfreie Teilhabe am Bildungssystem. Dabei muss dieses, entgegen der deutschen Übersetzung und insbesondere wegen der maßgeblichen englischen Fassung ein inklusives und nicht nur ein integratives Schulsystem sein, das sich den Bedürfnissen der Schüler und Schülerinnen mit Behinderung anpasst und nicht umgekehrt eine Anpassung der Schüler und Schülerinnen an das System verlangt. ${ }^{1237}$

\subsection{Art. 24 II BRK}

Art. 24 I BRK wird in Art. 24 II BRK konkretisiert. Danach stellen die Staaten sicher: (a) dass "Kinder mit Behinderungen nicht aufgrund von Behinderung vom unentgeltlichen und obligatorischen Grundschulunterricht oder vom Besuch weiterführender Schulen ausgeschlossen werden“, (b) dass sie „gleichberechtigt ... Zugang zu einem integrativen, hochwertigen und unentgeltlichen Unterricht an Grundschulen und weiterführenden Schulen haben", (c) dass „angemessene Vorkehrungen für die Bedürfnisse des Einzelnen getroffen werden“, (d) dass "die notwendige Unterstützung geleistet wird, um ihre erfolgreiche Bildung zu erleichtern“, und (e), dass „in Übereinstimmung mit dem Ziel der vollständigen Integration wirksame individuell angepasste Unterstützungsmaßnahmen in einem Umfeld, das die bestmögliche schulische und soziale Entwicklung gestattet, angeboten werden."

Fraglich ist, inwieweit diese Bestimmungen unmittelbar anwendbar sind. Die Bestimmungen der Buchst. (a) und (b) lassen keinen Entscheidungsspielraum zu. Kinder mit Behinderungen dürfen nicht wegen der Behinderung vom Grundschulunterricht oder von weiterführenden Schulen ausgeschlossen werden und haben gleichberechtigten Zugang zu inklusivem Unterricht. Diese Best-

\footnotetext{
1233 Masuch, in: FS Renate Jaeger, S. 250

1234 Riedel/Arend, NVwZ 2010, S. 1346 ff, S. 1349; Bernstorff, RdJB 2011, S. 203 ff, S. 215; Degener, RdJB 2009, S. 200 ff, S. 217; Latham/Watkins, Gutachten zur UN-BRK, S. 11

1235 Latham/Watkins, Gutachten zur UN-BRK, S. $18 \mathrm{f}$

${ }^{1236}$ Riedel/Arend, NVwZ 2010, S. 1346 ff, S. 1347; Bernstorff, RdJB 2011, S. 203 ff, S. 215; Latham/Watkins, Gutachten zur UN-BRK, S. 16 f; a.A. Poscher/Langer/Rux, Gutachten zu Art. 24 BRK, S. 32 f; Ennuschat, in: FS Klaus Stern, S. 722

${ }^{1237}$ Zu diesem Streit ausführlich Latham/Watkins, Gutachten zur UN-BRK, S. 8 f; s. auch Degener, RdJB 2009, S. 200 ff, S. 211 f; Riedel/Arend, NVwZ 2010, S. 1346 ff, S. 1348
} 
immungen sind daher unmittelbar anwendbar. Jedes Kind mit Behinderungen hat daher das Recht, trotz seiner Behinderung eine weiterführende Schule zu besuchen.

Ebenso unmissverständlich ist der Anspruch auf angemessene Vorkehrungen aus Abs. 2 Buchst. (c). Die Versagung angemessener Vorkehrungen ist nach Art. 2 BRK eine Form der Diskriminierung. Da das Diskriminierungsverbot unmittelbar gilt, gilt es auch in dieser Ausprägung im Bereich des Art. 24 BRK unmittelbar. ${ }^{1238}$

Die Bestimmungen der Buchst. (d) und (e) sind weit gefasste Staatenverpflichtungen, die nicht unmittelbar anwendbar sind. ${ }^{1239}$

Folglich haben Schüler und Schülerinnen mit Legasthenie/Dyskalkulie unmittelbar aus Art. 5 und Art. 24 I sowie aus II Buchst. a, b und c BRK Anspruch darauf, wegen der Behinderung nicht diskriminiert zu werden und müssen gleichberechtigten Zugang zu den allgemeinen Schulen und zu weiterführenden Schulen haben sowie angemessene Vorkehrungen erhalten.

\section{Völkerrechtliche Auslegung der Schulgesetze}

Übernimmt das Landesrecht Art. 24 BRK nicht wortgleich, sondern passt es sein Schulgesetz nur der BRK an, so ist für die unmittelbare Anwendbarkeit von Art. 24 BRK kein Raum. Dann allerdings ist das Landesgesetz völkerrechtsfreundlich auszulegen, so dass bei seiner Anwendung ein Verstoß gegen die BRK ausgeschlossen wird. Die Tatbestände des Schulgesetzes sind dann so auszulegen, dass sie dem Diskriminierungsverbot im Bereich der Bildung die größtmögliche Wirkung verleihen. ${ }^{1240}$

\section{Vorgaben des Art. 24 i.V.m. 5 BRK}

Der Anspruch der betroffenen Schüler und Schülerinnen geht dahin, hinsichtlich des Rechts auf Bildung nicht diskriminiert zu werden. Zu prüfen ist, welche Vorgaben Art. 24 i.V.m. Art. 5 BRK für eine diskriminierungsfreie Bildung enthält, die entweder im Rahmen der unmittelbaren Anwendbarkeit des Art. 24 BRK oder im Rahmen der völkerrechtsfreundlichen Auslegung zu beachten sind. Der wesentliche Gedanke der BRK ist die Ausrichtung des Bildungswesens auf inklusive Bildung. Gesellschaftliche Strukturen sollen so gestaltet werden, dass sie der realen Vielfalt menschlicher Lebenslagen von Anfang an gerecht werden. Statt der Assimilation von Menschen mit Behinderung steht die An-

\footnotetext{
1238 Aichele, UN-BRK und Fakultativprotokoll, S. 4; Latham/Watkins, Gutachten zur UN-BRK, S. 17; Degener, RdJB 2009, S. 200 ff, S. 218; insgesamt zu Art. 24 Schulte, br 2011, S. 41 ff, S. 44

1239 Latham/Watkins, Gutachten zur UN-BRK, S. 17

1240 Ausführlich dazu oben S. 73ff; Krajewski/Bernhard, Kommentar zur UN-BRK, Art. 24, Rn. 25
} 
passung des Systems im Vordergrund. ${ }^{1241}$ Damit ist eine Haltung, wonach die Zulassung zur Bildung nur unter den auch für nichtbehinderte Menschen geltenden Zulassungsbedingungen ermöglicht werden kann, nicht mehr vereinbar. ${ }^{1242}$

Das Recht auf Bildung ist bereits in Art. 13 UN-Sozialpakt enthalten und dort durch die Allgemeinen Bemerkungen Nr. 13 entscheidend ausgeformt worden. Bei Allgemeinen Bemerkungen (Generell Comments) handelt es sich um Interpretationshilfen des UN-Ausschusses für wirtschaftliche, soziale und kulturelle Rechte, mit denen die Bestimmungen des UN-Sozialpakts konkretisiert und standardisiert werden. ${ }^{1243}$ Sie beschreiben in autorisierter Form die Standards in der Praxis des Sozialausschusses, dienen damit als Interpretationshilfe und prägen so das Verständnis der vertraglichen Rechtsbegriffe durch die Vertragsstaaten mit. ${ }^{1244}$ Die zu Art. 13 UN-Sozialpakt entwickelten vier Strukturelemente werden mit Blick auf die englischen Begriffe auch als "4-A-Scheme" bezeichnet: availability = Verfügbarkeit, access = Zugang, acceptability $=$ Annehmbarkeit und adaptability = Anpassungsfähigkeit. ${ }^{1245}$ Diese Elemente werden durch Art. 24 BRK weiter spezifiziert und konkretisiert und sind daher bei der Auslegung von Art. 24 BRK zu beachten. ${ }^{1246}$ Die genannten vier Aspekte stehen in Wechselbeziehung zueinander. Das Diskriminierungsverbot bezieht sich auf alle Elemente. ${ }^{1247}$

Die allgemeine Verfügbarkeit (availability) von Bildung verlangt, dass Schulen in ausreichendem Maß zur Verfügung stehen und funktionsfähig sind. ${ }^{1248}$ Für Schüler und Schülerinnen mit Behinderung sind Bildungseinrichtungen nur dann verfügbar und funktionsfähig, wenn sie imstande sind, die besonderen Belange dieser Schüler und Schülerinnen in dem Maße zu berücksichtigen, dass innerhalb der Regelschulen die notwendige Unterstützung vorhanden ist, um sie wie ihre Mitschülern ohne Behinderung entsprechend ihren individuellen Möglichkeiten zu fördern. ${ }^{1249}$

1241 Mathe, br 2011, S. 34 ff, S. 38; Schulte, br 2011, S. 41 ff, S. 43

1242 So aber ausdrücklich LSG Baden-Württemberg, Beschluss v. 9.12.2010, Az.: L 13 AL 4629/10

ER-B, juris, Rn. 33, wie hier: Rux, der darauf hinweist, dass das Konzept der allgemeinen Hochschulreife mit dem Inklusionskonzept der BRK nicht mehr vereinbar ist. Rux, RdJB 2009, S. $220 \mathrm{ff}, \mathrm{S} .228$

1243 Riedel/ Söllner, JZ 2006, S. 270 ff, S. 270

1244 BVerwG, Urteil v. 29.4.2009, Az.: 6 C 16/08, Rn. 48 m.w.N

1245 Mokatef, Menschenrecht auf Bildung und Schutz vor Diskriminierung, S. $16 \mathrm{f}$

1246 Riedel, Gutachten UN-Behindertenrechtskonvention, S. 16; s. auch Aichele, Stellungnahme DIMR 2010; Eckpunkte

${ }^{1247}$ Mokatef, Menschenrecht auf Bildung und Schutz vor Diskriminierung, S. 17

1248 Mokatef, Menschenrecht auf Bildung und Schutz vor Diskriminierung, S. 16; Riedel, Gutachten UN-Behindertenrechtskonvention, S. 14 ff; Lohrenscheit, in: Recht auf Bildung, S. $42 \mathrm{ff}$

1249 Riedel, Gutachten UN-Behindertenrechtskonvention, S. 14 
Bildung ist zugänglich (access), wenn sie nach dem Gesetz und de facto für alle zugänglich ist, insbesondere für die schwächsten Gruppen. Dafür muss sie ohne Diskriminierung sein, physisch erreichbar, d.h. in zumutbarer Entfernung, und wirtschaftlich erschwinglich. ${ }^{1250}$ Für Schüler und Schülerinnen mit Behinderung ist Zugänglichkeit nur gewahrt, wenn sie im Regelschulsystem die gleichen Bildungsmöglichkeiten haben ${ }^{1251}$, sie also de facto die gleichen Zugangsrechte zu allen Schulen haben, ohne dass aus unzulässigen Gründen diskriminiert wird. ${ }^{1252}$ Die Zugänglichkeit wird in Deutschland überwiegend unter dem Gesichtspunkt der Zulassung von Förderschülern an die Regelschulen diskutiert. ${ }^{1253}$ Damit sind jedoch bei Weitem nicht alle Fälle erfasst. Die Zugänglichkeit betrifft vielmehr alle behinderten Schüler, die Regelschulen besuchen wollen, die auf Regelschulen verbleiben und nicht auf Förderschulen verwiesen werden wollen und die Schulen besuchen wollen, die ihrer Begabung entsprechen, ohne dass die Behinderung zu einer Zuweisung an eine nichtbegabungsgerechte (Regel-)Schule führt. ${ }^{1254}$ Barrieren, die die Zugänglichkeit einschränken, sind daher nicht nur physische Barrieren, sondern auch tatsächliche Barrieren, wie das Fehlen bestimmten Unterrichtsmaterials für Schüler mit Behinderung, etwa Braille-Schrift, rechtliche Barrieren und auch negative Einstellungen und Vorurteile von Lehrkräften, Mitschülern, aber auch Eltern anderer Schüler, die den Zugang für Schüler und Schülerinnen mit Behinderung faktisch erschweren. ${ }^{1255} \mathrm{Im}$ Ergebnis kann die Zugänglichkeit nur mit einem inklusiven Unterricht gewahrt werden.

Die Angemessenheit (acceptability) betrifft die Form und den Inhalt von Bildung. Sie muss für alle Schüler relevant, kulturell angemessen und hochwertig sein. ${ }^{1256}$ Eine hochwertige Bildung muss sich auf die Bildungsziele ausrichten und diesen Wirksamkeit verleihen. Die Zielsetzung wird für Schüler und Schülerinnen mit Behinderung in Art. 24 I S. 2 BRK konkretisiert, der als wesentliche Elemente die Würde, die volle Entfaltung des Selbstwertgefühls, die Stärkung der Achtung der menschlichen Vielfalt, die Entfaltung der Begabung und Krea-

\footnotetext{
1250 Mokatef, Menschenrecht auf Bildung und Schutz vor Diskriminierung, S. 16; Riedel, Gutachten UN-Behindertenrechtskonvention, S. 14 ff; Lohrenscheit, in: Recht auf Bildung, S. $42 \mathrm{ff}$

1251 Riedel, Gutachten UN-Behindertenrechtskonvention, S. 15

1252 Mokatef, Menschenrecht auf Bildung und Schutz vor Diskriminierung, S. 17

1253 Die bereits zitierte Rechtsprechung (s. o. Fn. 1207) betrifft fast nur solche Fälle

1254 S. z.B. für Schüler mit ADS, Geist, in: Legasthenie und Dyskalkulie 2011, S. 178 ff: „Der Autor gibt zu, lange so gedacht zu haben, wie man das von einem gymnasialen Schulpsychologen erwartet: ,ADS ist doch nichts, was am Gymnasium vorkommt, das ist etwas für Haupt- und Sonderschulen. “" (S. 178)

1255 Riedel, Gutachten UN-Behindertenrechtskonvention, S. 17

1256 Mokatef, Menschenrecht auf Bildung und Schutz vor Diskriminierung, S. 16; Riedel, Gutachten UN-Behindertenrechtskonvention, S. 14 ff; Lohrenscheit, in: Recht auf Bildung, S. $42 \mathrm{ff}$
} 
tivität sowie der geistigen und körperlichen Fähigkeiten nennt. Inhalte und Material des Unterrichts und der Prüfungen müssen deshalb darauf zugeschnitten sein, alle Schüler im Regelschulsystem auf den Bildungsstand zu bringen, der ihren individuellen Möglichkeiten entspricht. ${ }^{1257}$

Die Anpassungsfähigkeit (adaptability) erfordert, dass sich Bildung an die Erfordernisse sich verändernder Gesellschaften anpasst und den von vielfältigen sozialen und kulturellen Gegebenheiten geprägten Bedürfnissen der Schüler Rechnung trägt. ${ }^{1258}$ Die Adaptierbarkeit der Bildung verlangt eine Anpassung des Bildungssystems an geänderte gesellschaftliche Erfordernisse und damit die Anpassung des Systems an die Erkenntnis, dass Menschen mit Behinderung in das reguläre System einbezogen werden müssen. ${ }^{1259}$ Erforderlich sind individuell angepasste Unterstützungsmaßnahmen, die die bestmögliche schulische und soziale Entwicklung ermöglichen. ${ }^{1260}$

\section{Diskriminierung}

Der Anspruch auf Berücksichtigung von Legasthenie/Dyskalkulie ergibt sich dann, wenn die Nicht-Berücksichtigung eine Diskriminierung, ein Eingriff in den dargestellten Schutzbereich ist.

Schüler und Schülerinnen mit Legasthenie/Dyskalkulie werden dadurch, dass ihre Behinderungen nicht berücksichtigt werden und ihre Leistungen im Lesen, Rechtschreiben und/oder Rechnen bewertet werden, schlechter benotet und dadurch von bestimmten Schulformen und Schulabschlüssen ausgeschlossen oder auch auf Förderschulen überwiesen. Sie werden damit aufgrund ihrer Behinderung unterschieden, ausgeschlossen oder beschränkt, was zur Folge hat, dass sie in ihrem Recht auf Bildung beeinträchtigt werden. Die Diskriminierung erstreckt sich auf alle Elemente des Rechts auf Bildung. Für Schüler und Schülerinnen mit Legasthenie/Dyskalkulie sind die Bildungseinrichtungen nicht verfügbar und funktionsfähig, weil durch die Nicht-Berücksichtigung die besonderen Belange dieser Schüler und Schülerinnen gerade nicht in dem Maße berücksichtigt werden, dass sie innerhalb der Regelschulen die notwendige Unterstützung erhalten und wie ihre Mitschüler ohne Behinderung entsprechend ihren individuellen Möglichkeiten gefördert werden. Die Zugänglichkeit ist nicht gewahrt, weil sie im Regelschulsystem nicht die gleichen Bildungsmöglichkeiten und vor allem die gleichen Zugangsrechte zu allen Schulen haben,

\footnotetext{
1257 Riedel, Gutachten UN-Behindertenrechtskonvention, S. 15

1258 Mokatef, Menschenrecht auf Bildung und Schutz vor Diskriminierung, S. 16; Riedel, Gutachten UN-Behindertenrechtskonvention, S. 16 f; Riedel, Gutachten UN-Behindertenrechtskonvention, S. $14 \mathrm{ff} ;$ Lohrenscheit, in: Recht auf Bildung, S. $42 \mathrm{ff}$

1259 Riedel, Gutachten UN-Behindertenrechtskonvention, S. 16; Mokatef, Menschenrecht auf Bildung und Schutz vor Diskriminierung, S. 14

1260 Riedel, Gutachten UN-Behindertenrechtskonvention, S. 17
} 
sondern wegen der Behinderung von Schulformen und -abschlüssen ausgeschlossen werden. Sie erhalten keine hochwertige Bildung, da insbesondere ihre Möglichkeiten, ihre Begabung und geistigen Fähigkeiten zu entfalten eingeschränkt werden. Zudem führt die Nicht-Berücksichtigung der Legasthenie/ Dyskalkulie zu erheblichen weiteren Beeinträchtigungen, etwa des Selbstwertgefühls und der Würde. Inhalte und Material des Unterrichts und der Prüfungen sind nur diskriminierungsfrei, wenn sie darauf zugeschnitten sind, alle Schüler im Regelschulsystem auf den Bildungsstand zu bringen, der ihren individuellen Möglichkeiten entspricht. ${ }^{1261}$ Das ist bei Nicht-Berücksichtigung der Behinderung nicht der Fall. Schließlich werden sie auch im Bereich der Adaptierbarkeit der Bildung diskriminiert, weil das System nicht so angepasst ist, dass sie in das reguläre System einbezogen sind.

Die Diskriminierung ist eine Form des Nichtstuns oder der Versagung angemessener Vorkehrungen, da aus der BRK und aus Art. 3 III 2 GG, eine Pflicht zur Anpassung besteht. Sie ist vor allem aber auch eine Form der mittelbaren Diskriminierung, weil die formale Gleichbehandlung, insbesondere bezüglich der Bewertungsvorschriften, de facto zu einem Ausschluss von Schülern und Schülerinnen mit Legasthenie/Dyskalkulie aus bestimmten Schularten führt.

\section{Rechtfertigung, Schranken, Verhältnismäßigkeit}

Ausdrückliche Schranken und Rechtfertigungsgründe enthält die BRK nicht. Allerdings gelten die Rechte aus der BRK auch nicht schrankenlos, denn auch der BRK ist das Verhältnismäßigkeitsprinzip immanent. ${ }^{1262}$ Dies ergibt sich z.B. aus Art. 2, Art. 4 und Art. 7 BRK, die jeweils eine Abwägung beinhalten. Bei der Prüfung der Geeignetheit, Erforderlichkeit und Angemessenheit sind zudem die besonderen Staatenverpflichtungen aus der BRK zu berücksichtigen. ${ }^{1263}$ Die Zusammenschau dieser Vorschriften zeigt, dass die Einschränkung der Rechte aus der BRK und damit die Benachteiligung wegen einer Behinderung nur aufgrund einer strengen Verhältnismäßigkeitsprüfung zulässig ist.

\subsection{Die Rechte der Mitschüler}

Das Recht auf Bildung aus Art. 24 BRK steht i.V.m. Art. 13 UN-Sozialpakt allen Kindern zu. Immanente Schranke des Rechts aus Art. 24 BRK sind daher die Rechte der Mitschüler. Eine generelle Aussage dahin, dass die gemeinsame Beschulung von Schülern und Schülerinnen mit und ohne Behinderung immer die Rechte der nicht-behinderten Mitschüler übermäßig beeinträchtigt, ist sozial-

\footnotetext{
1261 Riedel, Gutachten UN-Behindertenrechtskonvention, S. 15

1262 Aichele/ Althoff, Kommentar zur UN-BRK, Nicht-Diskriminierung, Rn. 9

1263 Staatenverpflichtungen sind als objektives Recht verbindlich: Eichholz, „Gibt es ein Recht auf eine Schule für alle?", S. 5
} 
wissenschaftlich nicht belegbar und daher auch juristisch nicht anzunehmen. ${ }^{1264}$

Eine Einschränkung des Rechts auf Bildung des Schülers mit Behinderung kann sich ergeben, wenn die gemeinsame Unterrichtung die Rechte der Mitschüler übermäßig beeinträchtigt. Das kann nur angenommen werden, wenn alle begleitenden und unterstützenden Maßnahmen ausgeschöpft wurden, um eine inklusive Beschulung zu ermöglichen und dennoch eine Gefährdung der Mitschüler gegeben ist, etwa bei dauerhaftem, massiv aggressivem Verhalten. ${ }^{1265}$

\subsection{Kindeswohl des Kindes mit Behinderung}

Im Rahmen der Verhältnismäßigkeitsprüfung ist nach Art. 7 II BRK bei allen Maßnahmen, die Kinder mit Behinderungen betreffen, das Wohl des Kindes vorrangig zu berücksichtigen. ${ }^{1266}$ Diese Vorschrift entspricht Art. 3 KRK der Kinderrechtskonvention und Art. 24 II Grundrechtecharta, die ebenfalls bestimmen, dass das Wohl des Kindes vorrangig zu berücksichtigen ist. ${ }^{1267}$ Der Vorrang des Kindeswohls hat damit im internationalen Vergleich einen beispiellosen Schutzgehalt und scheint sich zu einer allgemeinen völkerrechtlichen Zielbestimmung herauszubilden. ${ }^{1268}$ Es ist deshalb deutlich zu kurz gegriffen, das Kindeswohl als Schranke der Rechte aus der BRK auszulegen. ${ }^{1269}$ Das Kindeswohl ist keine Schranke der Rechte aus der BRK, sondern dient dazu, Kindern mit Behinderungen die Durchsetzung ihres Anspruchs auf gesellschaftliche Inklusion zu erleichtern und zu gewährleisten ${ }^{1270}$, denn die BRK sieht die Inklusion als den Regelfall an und die Exklusion, sei es durch die Überweisung auf eine Förderschule oder durch Nicht-Berücksichtigung der Behinderung, als Ausnahmefall. ${ }^{1271}$ In dem üblichen Konflikt zwischen pädagogischen, organisatori-

1264 Riedel, Gutachten UN-Behindertenrechtskonvention, S. 25; vgl. Poscher/Langer/Rux, Gutachten zu Art. 24 BRK, S. $54 \mathrm{f}$

1265 Poscher/Langer/Rux, Gutachten zu Art. 24 BRK, S. 31; Faber/Roth, DVBI 2010, S. 1193 ff, S. 1198; Krajewski, JZ 2010, S. 120 ff, S. 122; Poscher/Langer/Rux, Gutachten zu Art. 24 BRK, S. 54; Krajewski/Bernhard, Kommentar zur UN-BRK, Art. 24, Rn. 22

${ }^{1266}$ So auch Art. 24 EU-Grundrechte Charta und Art. 3 KRK, s. dazu Eichholz, R\&B 2007, S. 3 ff, 6 f

1267 Ausführlich dazu Lorz, Vorrang Art. 3 UN-KRK und Lorz, Rücknahme Vorbehaltserklärung, S. $18 \mathrm{ff}$

1268 Lorz, Rücknahme Vorbehaltserklärung, S. 22

1269 So Krajewski, JZ 2010, S. 120 ff, S. 123; Krajewski/Bernhard, BayVBI 2012, S. 134 ff, S. 137 f; Latham/Watkins, Gutachten zur UN-BRK, S. 17 f; Poscher/Langer/Rux, Gutachten zu Art. 24 BRK, S. 35

1270 Riedel, Gutachten UN-Behindertenrechtskonvention, S. 24; Aichele, Stellungnahme DIMR 2010, S. 33

1271 Riedel/Arend, NVwZ 2010, S. 1346 ff, S. 1349; Riedel, Gutachten UN-Behindertenrechtskonvention, S. 24; Krajewski/Bernhard, BayVBI 2012, S. 134 ff, S. 137 
schen, personellen, ökonomischen, juristischen, gesellschafts- und staatspolitischen Gesichtspunkten ist das Wohl des Kindes kein Aspekt unter vielen, sondern derjenige, der aus der subjektiven Sicht des einzelnen Kindes zu sehen ${ }^{1272}$ und vorrangig zu beachten ist. ${ }^{1273}$ Die Belange des Kindes können sich nur dann nicht durchsetzen, wenn in der Gesamtbetrachtung die anderen Interessen in der Summe ein derartiges Gewicht haben, dass sich die Kindesinteressen trotz eines eingeräumten Vorrangs nicht durchsetzen können. ${ }^{1274}$ Eine solche Entscheidung, die nach Ansicht Riedels nach der Systematik der BRK nahezu ausgeschlossen ist ${ }^{1275}$, ist umfassend $z u$ begründen, die Behörde trifft die volle Darlegungs- und Beweislast. ${ }^{1276}$ Im deutschen Recht entspricht das in etwa dem zwingenden Grund, der sich gegenüber einem Grundrecht durchsetzt. Die Entscheidung gegen eine inklusive Beschulung kann daher nicht (mehr) mit überwiegend schulpolitischen, schulorganisatorischen oder schulaufsichtlichen Erwägungen begründet werden. ${ }^{1277}$

\subsection{Vorbehalt der unverhältnismäßigen oder unbilligen Belastung}

Nur die besondere Form der Diskriminierung durch Versagung angemessener Vorkehrungen steht nach Art. 2 BRK unter dem Vorbehalt, dass dies keine unverhältnismäßige oder unbillige Belastung darstellt. Für die anderen Diskriminierungsarten gilt dieser Vorbehalt nicht. Diese Schranke könnte dem Vorbehalt des BVerfG entsprechen, wonach Leistungsansprüche im Schulbereich nur im Rahmen des tatsächlich Möglichen und finanziell Vertretbaren bestehen. ${ }^{1278}$ Bei der Prüfung der unverhältnismäßigen oder unbilligen Belastung ist jedoch Art. 4 BRK zu beachten. Dieser verpflichtet die Staaten dazu, die Rechte unter „Ausschöpfung aller verfügbaren Mittel“ zu verwirklichen. Die Staaten sind verpflichtet, ihr gesamtes Ausgabeverhalten und die dabei vorgenommene Prioritätensetzung zu überprüfen. ${ }^{1279}$ Deshalb ist bei der Prüfung der finanziellen Aufwendungen eine Gesamtbetrachtung aller volkswirtschaftlichen Leistungen und Kosten des betreffenden Staates erforderlich und nicht nur eine auf das Schulrecht begrenzte. Rux weist zu Recht darauf hin, dass im Schulbereich auch die Kosten beachtet werden müssen, die im Bereich der Eingliederungshilfen nach $\S \S 53$ SGB XII und 35 a SGB VIII für die Sicherstellung des

\footnotetext{
1272 Riedel, Gutachten UN-Behindertenrechtskonvention, S. 24

1273 Eichholz, R\&B 2007, S. 3 ff, 9, zum gleichlautenden Art. 3 KRK

1274 Eichholz, „Gibt es ein Recht auf eine Schule für alle?", S. 5

1275 Riedel, Gutachten UN-Behindertenrechtskonvention, S. 24

1276 Für den gleichlautenden Art. 3 KRK Lorz, Rücknahme Vorbehaltserklärung, S. 25 f

1277 Eichholz, „Gibt es ein Recht auf eine Schule für alle?", S. 7 f; Krajewski/Bernhard, Kommentar zur UN-BRK, Art. 24, Rn. 22

1278 BVerfGE 96, 288, $306 \mathrm{f}$

1279 Eichholz, „Gibt es ein Recht auf eine Schule für alle?", S. 5
} 
Schulbesuchs anfallen. ${ }^{1280}$ Mit bedacht werden müssen aber auch die gesamtwirtschaftlichen Kosten, die sich später durch eine unzureichende Beschulung ergeben, etwa im Bereich der Arbeitslosenversicherung, Sozialhilfe und auch der Behandlung von Sekundärerkrankungen. Der Ressourcenvorbehalt findet seine Schranke wiederum im Wesensgehalt des Rechts auf diskriminierungsfreie Bildung. ${ }^{1281}$ Deshalb reicht ein Verweis auf den üblichen Haushaltsvorbehalt und den allgemeinen Vorbehalt vorhandener pädagogischer, organisatorischer, personeller und finanzieller Mittel nicht mehr aus. ${ }^{1282}$ Die Darlegungsund Beweislast für das Vorliegen einer unverhältnismäßigen Belastung tragen die Schulbehörden. Im Bereich der Berücksichtigung von Legasthenie/Dyskalkulie wird dieser Vorbehalt jedoch keine Rolle spielen, da durch die Berücksichtigung der Behinderung jedenfalls keine unverhältnismäßigen oder unbilligen Kosten entstehen.

Die Diskriminierung der Schüler und Schülerinnen mit Legasthenie/Dyskalkulie ist demnach nicht gerechtfertigt, so dass sie aus Art. 24 i.V.m. 5 BRK einen unmittelbaren Anspruch auf Berücksichtigung der Behinderung haben.

\section{Der Anspruch im Einzelnen}

Aus den verschiedenen Elementen des Rechts auf Bildung lässt sich für den individuellen Anspruch von Schülern und Schülerinnen mit Legasthenie/Dyskalkulie Folgendes ableiten:

Ihr Recht auf diskriminierungsfreie Bildung wird nur gewahrt, wenn auf ihre individuellen Belange eingegangen wird. Dafür muss der Unterricht in einer Form erfolgen, die es ihnen ermöglicht, ihm zu folgen und die Kulturtechniken zu erlernen, etwa indem ihnen das Alphabet mit Handzeichen oder Lautgebärden vermittelt wird oder indem sie vor dem Erlernen der Rechenvorgänge zunächst schätzen lernen. Bei Prüfungen sind die unterschiedlichen persönlichen Voraussetzungen zu beachten und entsprechende Ausgleichsmaßnahmen festzulegen. Daneben dürfen rechtliche und tatsächliche Barrieren, die de facto den Zugang erschweren, wie etwa die formale Gleichbehandlung mit nichtbehinderten Schülern oder etwa das Vorurteil, sie seien nicht gymnasialtauglich, nicht aufrechterhalten bzw. angewandt werden. Der Inhalt des Unterrichts und von Prüfungen muss auf ihre Bedürfnisse zugeschnitten sein. Sie müssen die Möglichkeit haben, ihre Begabungen und Fähigkeiten zu entfalten und mit-

\footnotetext{
${ }^{1280}$ Rux, RdJB 2009, S. 220 ff, S. 224

${ }^{1281}$ Riedel, Gutachten UN-Behindertenrechtskonvention, S. 28

1282 Degener, RdJB 2009, S. 200 ff, S. 200 ff, S. 218; Riedel, Gutachten UN-Behindertenrechtskonvention, S. 28; § 49 II Nr. 1 HE SchulG verstößt gegen diese Vorschrift, da danach die sonderpädagogische Förderung nur im Rahmen der (vorhandenen) personellen, räumlichen und sächlichen Möglichkeiten besteht, also den Vorgaben der insoweit überholten Rspr. des BVerfG entspricht, s. Fn. 1278
} 
hilfe von Unterstützungsmaßnahmen die beste schulische und soziale Entwicklung erreichen zu können. Dazu sind jeweils angemessene Vorkehrungen im Einzelfall erforderlich. Im Ergebnis müssen sie die Möglichkeit haben, eine begabungsgerechte Schulform zu besuchen und einen begabungsgerechten Schulabschluss zu machen, ohne dass sie davon durch ihre Behinderung abgehalten oder beeinträchtigt werden. Sie dürfen nicht von Schulabschlüssen ausgeschlossen werden, weil ihnen einzelne abzuprüfende Fähigkeiten fehlen, sie aber fähig sind die anschließenden Bildungs- und Ausbildungsgänge auch ohne oder mit Einschränkungen in diesen Fähigkeiten absolvieren zu können.

Insbesondere die Diskriminierungsform der Versagung angemessener Vorkehrungen in der BRK bewirkt eine Verschiebung des Rechtsinhalts des Art. 3 III 2 GG weg von einem Abwehrrecht hin zu einem Leistungsrecht ${ }^{1283}$, denn angemessene Vorkehrungen sind ohne leistungsrechtlichen Inhalt nicht denkbar.

\section{Kapitel: Weitere Völker- und Europarechtliche Regelungen}

Die völker- und europarechtlichen Grundlagen des Rechts auf Bildung ergeben sich aus einer Vielzahl von weiteren völkerrechtlichen und europarechtlichen Bestimmungen ${ }^{1284}$, von denen nur die Wichtigsten erwähnt werden sollen.

\section{Art. 28 Kinderrechtskonvention}

Das Recht auf Bildung wird auch in Art. 28 Kinderrechtskonvention (KRK) ausdrücklich anerkannt. Zur Kinderrechtskonvention hatte die Bundesrepublik Deutschland bei der Ratifikation ausdrücklich erklärt, dass das Übereinkommen innerstaatlich keine unmittelbare Anwendung findet. ${ }^{1285}$ Diesen Vorbehalt hat sie jetzt mit Wirkung zum 15.7.2010 zurückgenommen. Seitdem gelten die in der Konvention enthaltenen Rechte uneingeschränkt in Deutschland. ${ }^{1286}$ Das Recht auf Bildung wird verstärkt durch das Diskriminierungsverbot (Art. 2 I KRK), das ausdrücklich auch die Diskriminierung wegen einer Behinderung verbietet. Auch nach dieser Konvention ist bei Maßnahmen, die das Kind betreffen, das Wohl des Kindes vorrangig zu berücksichtigen (Art. 3 I KRK). ${ }^{1287}$ Allerdings lässt sich im Gegensatz zur BRK aus der KRK kein subjektives Recht auf

\footnotetext{
1283 Vgl. Riedel, Gutachten UN-Behindertenrechtskonvention, S. 37

1284 Umfassend dazu: Poscher/Rux/Langer, Recht auf Bildung, s. auch Langenfeld, RdJB 2007, S. $412 \mathrm{ff}$ und Mahler/Weiß, RdJB 2007, S. $430 \mathrm{ff}$

1285 Vorbehalt zitiert bei Poscher/Rux/Langer, Recht auf Bildung, S. 49

1286 S. hierzu: Lorz, Rücknahme Vorbehaltserklärung, S. 6, 15 m.w.N.

1287 S. zur KRK neben Poscher/Rux/Langer, Recht auf Bildung auch Eichholz, R\&B 2007, 3 ff und Krappmann/Lüscher, RdJB 2009, S. $326 \mathrm{ff}$
} 
inklusive Beschulung ableiten. ${ }^{1288}$ Dennoch verdeutlicht das in der KRK normierte Diskriminierungsverbot bezüglich des Rechts auf Bildung, welch hohen Stellenwert die diskriminierungsfreie Bildung hat und verstärkt dieses Recht.

\section{Recht auf Bildung aus der EMRK}

Maßgeblich ist weiter das Recht auf Bildung aus Art. 2 S. 1 des 1. Zusatzprotokolls zur EMRK. Danach darf niemandem das Recht auf Bildung verwehrt werden. ${ }^{1289}$ Trotz der negativen Formulierung wird darin ein echtes Recht auf Bildung und somit auf Zugang zu bestehenden Bildungseinrichtungen ${ }^{1290}$ in Form eines derivativen Teilhaberechts gesehen. ${ }^{1291}$ Dieses Recht wird verstärkt durch Art. 14 EMRK, das ein akzessorisches Diskriminierungsverbot enthält, welches auch die Diskriminierung wegen einer Behinderung verbietet. ${ }^{1292}$ Das 12 . Zusatzprotokoll zur EMRK, das ein umfassendes Diskriminierungsverbot enthält, hat die Bundesrepublik bis jetzt nur unterschrieben, aber nicht ratifiziert. Es ist jedoch davon auszugehen, dass die Ratifizierung erfolgen wird. Dann ergibt sich aus dem 12. Zusatzprotokoll ein umfassendes Diskriminierungsverbot, das mit Art. 3 GG vergleichbar ist. Die Verpflichtungen der EMRK sind unbedingt einzuhalten und nicht bloße Staatenverpflichtungen. ${ }^{1293}$ Die EMRK wird mittelbar zum verfassungsrechtlichen Prüfungsmaßstab, da alle staatlichen Behörden und Gerichte an sie gebunden sind. Die Auslegung der deutschen Grundrechte im Einklang mit der EMRK und den Entscheidungen des EGMR ist verfassungsrechtliche Pflicht. ${ }^{1294}$ Der EGMR hat bereits aus Art. 14 EMRK und Art. 2 EMRK 1. Zusatzprotokoll ein umfassendes Diskriminierungsverbot für den Schulbereich hergeleitet. ${ }^{1295}$

\footnotetext{
${ }^{1288}$ Rothfritz, UN-BRK, S. 89

1289 Umfassend dazu Langenfeld, in: Grote/Marauhn EMRK/GG, Kap. 23: Das Recht auf Bildung und Langenfeld, RdJB 2007, S. $412 \mathrm{ff}$

1290 Langenfeld, in: Grote/Marauhn EMRK/GG, Kap. 23: Das Recht auf Bildung, Rn. 13; Mahler/ Weiß, RdJB 2007, S. 430 ff, S. 436; Poscher/Rux/Langer, Recht auf Bildung, S. 67

1291 Langenfeld, in: Grote/Marauhn EMRK/GG, Kap. 23: Das Recht auf Bildung, Rn. 13

1292 König/Peters, in: Grote/Marauhn EMRK/GG, Kapitel 21: Das Diskriminierungsverbot, Rn. 164

1293 Poscher/Rux/Langer, Recht auf Bildung, S. 76

1294 umfassend dazu BVerfG, 2 BvR 2365/09 vom 4.5.2011, http://www.bverfg.de/entscheidungen/rs20110504_2bvr236509.html, Rn. 85 ff; BVerfG, Beschluss v. 28.12.2004; Az.: 1 BvR 2790/04, "Görgülü“, Rn. 28 ff http://www.bverfg.de/entscheidungen/rk20041228_1bvr279004.html; Voßkuhle, NVwZ 2010, S. 1 ff, S. 4 f; Poscher/Rux/Langer, Recht auf Bildung, S. 77

1295 EGMR Urteil v. 13.11.2007 - 57325/00, D.H. u.a./ Tschechien, NVwZ 2008, S. 533 ff, S. 536 Ziff. 202; kritisch dazu Heyden/von Ungern-Sternberg, EuGRZ 2009, S. 81 ff
} 


\section{Rechte aus der Europäischen Grundrechtecharta}

Das Recht auf Bildung ist weiter in Art. 14 Grundrechtecharta anerkannt. Die Grundrechtecharta ist am 1.12.2009 als Annex zum Vertrag von Lissabon in Kraft getreten. ${ }^{1296}$ Sie steht den EU-Verträgen gleich (Art. 6 । EUV) und ist streng akzessorisch zu den Kompetenzen der EU. Daher hat sie für das Recht auf Bildung nur eingeschränkte Bedeutung. ${ }^{1297}$ Dennoch zeigt auch diese Vorschrift die Anerkennung des Menschenrechts auf Bildung. Flankiert wird dieses Recht durch den in Art. 26 Grundrechtecharta verankerten Anspruch von Menschen mit Behinderung auf Integration, durch das Diskriminierungsverbot (Art. 21 I Grundrechtecharta) und das Gebot, das Wohl von Kindern bei Entscheidungen, die sie betreffen, vorrangig zu berücksichtigen (Art. 24 ॥ Grundrechtecharta, der Art. 7 BRK entspricht). Die Grundrechte der Grundrechtecharta sind unter Berücksichtigung der BRK auszulegen, da diese von der EU ratifiziert wurde.

\section{Art. 45 AEUV}

Für Kinder mit Legasthenie/Dyskalkulie von EU-Angehörigen, die Rechte aus Art. 45 AEUV wahrnehmen, kommen Rechte aus Art. 45 AEUV i.V.m. Art. 24 II Grundrechtecharta und der BRK in Betracht. Das Diskriminierungsverbot des Art. 45 II AEUV gibt Arbeitnehmern den Anspruch auf Inländergleichbehandlung. Die Kinder des Arbeitnehmers haben das Recht, unter gleichen Bedingungen wie Inländer am allgemeinen Unterricht teilzunehmen. Entspricht das inländische Recht jedoch nicht den Vorgaben der BRK, so gilt für sie darüber hinaus die BRK, denn ihre Rechte leiten sich aus EU-Recht ab, zu dem die BRK gehört, das gegenüber dem deutschen Recht den Anwendungsvorrang genießt.

\footnotetext{
1296 Pressemitteilung der EU-Kommission v. 1.12.2009, http://europa.eu/rapid/pressReleases Action.do? reference=IP/09/1855\&format $=H T M L \&$ aged $=0$ \&language $=\mathrm{DE} \&$ guilanguage $=$ en 1297 Poscher/Rux/Langer, Recht auf Bildung, S. $78 \mathrm{f}$
} 



\section{Teil: Abwehrrecht, Leistungsrecht, Teilhaberecht}

Die bisherige Prüfung hat ergeben, dass aus den Grundrechten aus Art. 3 III 2, 3 I, 2 I, 12 I und 6 || GG i.V.m. der völkerrechtsfreundlichen Auslegung auf der Grundlage von Art. 5, 7, und 24 BRK der Anspruch darauf besteht, dass die Behinderungen Legasthenie und Dyskalkulie in der Schule, im Unterrichtsalltag, bei Prüfungen sowohl hinsichtlich der äußeren Prüfungsbedingungen als auch der inhaltlichen Prüfungsanforderungen und bei Zulassungsentscheidungen berücksichtigt werden. Fraglich ist, wie dieser Anspruch durchzusetzen ist.

Nach ganz herrschender Meinung sind die Grundrechte und insbesondere auch Art. 3 III 2 GG subjektive Abwehrrechte gegen Benachteiligungen durch staatliche Normen ${ }^{1298}$ oder auch gegen ein aktives Handeln. ${ }^{1299}$ Sie statuieren das Recht auf Unterlassung staatlicher Eingriffe $e^{1300}$, begründen aber keine originären grundrechtlichen Leistungsansprüche. ${ }^{1301}$ Ein Leistungsanspruch ist beschränkt auf ein derivatives Teilhaberecht im Rahmen bestehender Leistungssysteme. Damit kann aus den Grundrechten die Nicht-Berücksichtigung der Behinderung abgewehrt werden. Ein solcher Anspruch bleibt jedoch wirkungslos, weil allein die Aufhebung der Nicht-Berücksichtigung noch nicht zu einer grundrechtsadäquaten Berücksichtigung führt. Diese kann nur durch eine Leistung erreicht werden.

Die Rechtsprechung hat hier für die Berücksichtigung von Legasthenie differenziert: Sie erkennt im Bereich der äußeren Prüfungsbedingungen einen Eingriff und einen Anspruch auf Veränderung der äußeren Prüfungsbedingungen

\footnotetext{
1298 Heun, in: Dreier GG, Art. 3, Rn. 17; Osterloh, in: Sachs GG, Art. 3, Rn. 38 ff; 305; EckertzHöfer, in: AK-GG, Art. 3, Rn. 128

1299 Beaucamp, DVBI 2002, S. 997 ff, S. 999

1300 Alexy, Theorie der Grundrechte, S. 395

${ }^{1301}$ Sacksofsky, in: Umbach GG, Art. 3, Rn. 393; Kischel, in: BeckOK GG, Art. 3, Rn. 215; Neumann, in: Handbuch SGB IX, § 2 Verfassungsrecht, Rn. 32; Reichenbach, Anspruch behinderter Schülerinnen und Schüler auf Unterricht in der Regelschule, S. 181; Füssel, RdJB 1998, S. 250 ff, S. 254; Dirnaicher, BayVBI 1997, S. 545 ff, S. 552; Starck, in: v. Mangoldt/Klein/ Starck GG, Art. 3, Rn. 419; Eckertz-Höfer, in: AK-GG, Art. 3, Rn. 128; Alexy, Theorie der Grundrechte, S. 395; Murswiek, in: HStR V, § 112
} 
an. ${ }^{1302}$ In der Veränderung inhaltlicher Prüfungsanforderungen, z.B. durch Nicht-Bewertung der Rechtschreibung, und der darauf beruhenden Zulassungsentscheidung sieht die Rechtsprechung jedoch Ansprüche auf Leistung, die sich aus Art. 3 III 2 GG nicht ergeben, da dieser keinen unmittelbaren Leistungsanspruch enthalte. ${ }^{1303}$ Es bleibe der nicht einklagbaren Entscheidung des jeweils zuständigen Gesetzgebers überlassen, ob und in welchem Umfang er im Rahmen einer leistungsgewährenden Verwaltung Ansprüche begründen wolle. ${ }^{1304}$ Diese Auffassung wird nur für den Bereich der Legasthenie/Dyskalkulie vertreten, denn es gibt keine Rechtsprechung oder Literatur dazu, dass die Veränderung von Prüfungsinhalten durch Befreiung von Prüfungsfächern bei anderen Schülern mit Behinderung eine Leistung i.S.d. Art. 3 III 2 GG ist, die nur durch den Gesetzgeber entschieden werden kann.

Die Grenze zwischen Eingriff und Leistung ist nicht starr: Je mehr ein Grundrechtsträger von staatlichen Leistungen abhängig ist, umso schwieriger wird es, die Grenze zwischen Eingriff und Leistung zu ziehen. Es kommt zu einer Verschiebung zwischen Eingriff und Nichtleistung. ${ }^{1305}$ Insbesondere im Bereich der Schule kann kaum zwischen Eingriff und Leistung unterscheiden werden ${ }^{1306}$, denn der Grundrechtseingriff kann dann nur durch eine Leistung abgewehrt werden, die die Wahrnehmung des Grundrechts auch ermöglicht. ${ }^{1307}$ Tatsächlich wird eine solche Trennung zwischen Eingriff und Leistung im Schulbereich vielfach nicht vorgenommen. Bei Schülern und Schülerinnen wird die undifferenzierte Beschulung von fremdsprachlichen Schülern für unzulässig gehalten. Die notwendigen Sprachfördermaßnahmen werden als Abwehr von Benachteiligungen und nicht als Leistung oder gar Bevorzugung angesehen. ${ }^{1308}$ In der

1302 OVG Lüneburg, Beschluss v. 10.7.2008, Az.: 2 ME 309/08,

www.rechtsprechung.niedersachsen.de; VG Köln, Beschluss v. 26.9.2008, Az.: 10 L 1240/08, DVBI 2009, S. 538, kritisch dazu Marwege, DVBI 2009, S. 538 ff; VGH Kassel, Beschluss v. 5.2.2010, Az.: 7 A 2406/09.Z, juris, Rn 41 f; ähnlich VG Sigmaringen, Beschluss v. 2.4.2008, Az.: 4 K 596/08, S. 3 (nicht veröffentlicht), das in Art. 3 III 2 GG sogar nur einen Programmsatz sieht; OVG Weimar, Beschluss v. 17.5.2010, Az.: 1 EO 854/10, juris, Rn. 52

1303 OVG Lüneburg, Beschluss v. 10.7.2008, Az.: 2 ME 309/08, www.rechtsprechung.niedersachsen.de; VG Köln, Beschluss v. 26.9.2008, Az.: 10 L 1240/08, DVBI 2009, S. 538, kritisch dazu Marwege, DVBI 2009, S. 538 ff; VGH Kassel, Beschluss v. 5.2.2010, Az.: 7 A 2406/09.Z, juris, Rn 41 f; ähnlich VG Sigmaringen, Beschluss v. 2.4.2008, Az.: 4 K 596/08, S. 3 (nicht veröffentlicht)

1304 VGH Kassel, Beschluss v. 5.2.2010, Az.: 7 A 2406/09, juris, Rn. 41; OVG Münster, Beschluss v. 16.11.2007, Az.: 6 A 2171/05, Rn. 13, www.justiz.nrw.de

${ }^{1305}$ Hufen, Staatsrecht $\S 5$ Rn. 10

1306 BVerwGE 55, 155, 158; Luthe, Bildungsrecht, S. 16; Hufen, Staatsrecht § 8, Rn. 14, vgl. auch umfassend Hufen, Gleichheitssatz und Bildungsplanung, S. 147

${ }^{1307}$ Vgl. Schenke, in: Hdb. Grundrechte III, § 78, Rn. 79

1308 Dürig/Scholz in: Maunz/Dürig/Herzog GG, Art. 3, Rn. 73 
Schulpraxis wird in vielen Fällen das Abwehrrecht mit einer leistungsrechtlichen Komponente verknüpft, denn die Prüfungsanforderungen und die Fächerbelegung werden für Schüler und Schülerinnen mit Behinderungen angepasst. Rechtlich sind diese Anpassungen als „angemessene Vorkehrungen“ i.S.d. Art. 24 i.V.m. Art. 5 und 2 BRK zu qualifizieren. ${ }^{1309}$ Dieses Instrument der ",angemessenen Vorkehrungen" bewirkt eine Verschiebung des Rechtsinhalts des Art. 3 III 2 GG weg von einem Abwehrrecht hin zu einem Leistungsrecht, ${ }^{1310}$ denn angemessene Vorkehrungen sind ohne leistungsrechtlichen Inhalt nicht denkbar. Der Anspruch aus Art. 3 III 2 GG lässt sich daher nicht mehr auf ein Abwehrrecht beschränken, sondern beinhaltet auch ein Leistungsrecht. Die Beseitigung ungerechter Verhältnisse im bestehenden System und die gleichberechtigte Teilhabe am staatlichen Bildungsangebot sind Schülern mit Behinderung nur möglich, wenn der Staat die Voraussetzungen für die tatsächliche, wirksame Teilhabe schafft. ${ }^{1311}$

Die Verpflichtung zur Leistung, um diskriminierende Sachverhalte zu beenden und eine diskriminierungsfreie Beschulung zu gewährleisten, ergibt sich auch aus dem objektiv-rechtlichen Gehalt ${ }^{1312}$ des Art. 3 III 2 GG. ${ }^{1313}$ Die Integration von Menschen mit Behinderung kann nur vorangetrieben werden, wenn der Staat auch zur Schaffung der dafür erforderlichen Voraussetzungen verpflichtet ist. ${ }^{1314}$ Ansonsten bliebe ein Unterlassen des Staates folgenlos. Dann hätte der Grundrechtsträger aus dem Abwehrrecht zwar einen Anspruch darauf, dass der Staat die Grundrechtsverletzung durch Unterlassen unterlässt, aber er hätte keinen korrespondierenden Leistungsanspruch darauf, dass der

${ }^{1309}$ S. dazu oben S. $140 \mathrm{ff}$

1310 Vgl. Riedel, Gutachten UN-Behindertenrechtskonvention, S. 37

1311 Vgl. Körting, in: Schule in der Rechtsprechung, S. 11; Kotzur/Richter, Kommentar zur UN-BRK, Verbindlichkeit, Rn. 11; unter diesem Aspekt leuchtet es nicht ein, dass sowohl das VG Schleswig, Urteil v. 10.6.2009, Az.: 9 A 208/08, BeckRS 2009 38123, S. 9, als auch der VGH Kassel, Beschluss v. 5.2.2010, Az.: 7 A 2406/09, juris, Rn. 46, die Benutzung von Laptops bzw. Computern in Prüfungen zulassen, darin aber keine Leistung sehen. Es kann nicht davon ausgegangen werden, dass alle behinderten Schüler und Schülerinnen bzw. deren Eltern in der Lage sind, Laptops zu finanzieren und zur Verfügung zu stellen. Dann muss entweder die Sozialhilfe oder die Schule sie zur Verfügung stellen, was sicher eine Leistung ist. Vgl. BVerfG, Urteil vom 9.2.2010, Az.: 1 BvL 1/09 „Hartz IV“, Absatz-Nr. 197, http://www.bverfg.de/entscheidungen/Is20100209_1bvl000109.html

1312 Zur dogmatischen Herleitung und Problemen des Begriffs s. Ossenbühl, in: Hdb. Grundrechte I, § 15, Rn. 48 ff; Sacksofsky, in: Umbach GG, Art. 3, Rn. 392

1313 Zum objektiv-rechtlichen Gehalt des Art. 3 III 2 GG: Sacksofsky, in: Umbach GG, Art. 3, Rn. 391; Rüfner, in: Bonner Kommentar GG, Art. 3, Rn. 884; Sachs, RdJB 1996, S. 154 ff, S. 170 ff, umfassend: Straßmair, Der besondere Gleichheitssatz aus Art. 3 Abs. 3 Satz 2 GG, S. 149 ff, S. $238 \mathrm{ff}$

1314 Castendiek, in: GS Jean d’Heur, S. 347; Berlit, RdJB 1996, S. 145 ff, S. 147; Beyerlin, RdJB 1999, S. 157 ff, S. 162 
Staat die notwendige Handlung auch vornimmt. ${ }^{1315}$ Besonders deutlich wird dies im Bereich des Diskriminierungsschutzes im Schulrecht: Ein betroffener Schüler kann zwar an sich eine diskriminierende Maßnahme, etwa die Überweisung auf eine Förderschule, abwehren, aber der Erfolg, nämlich die Beschulung auf der Regelschule, nützt ihm nichts, weil er die Förderung, die er braucht, um dort mitkommen zu können, nicht erhält und nicht einklagen kann. ${ }^{1316}$ Ohne flankierende positive Maßnahmen bleibt die Abwehr einer Diskriminierung jedoch nicht nur wirkungslos, sondern wird zum Bumerang. ${ }^{1317}$ Die Betroffenen kämen nicht nur vom Regen in die Traufe, sondern würden ihre Situation massiv verschlechtern. Sie werden sich daher hüten, ihre Rechte aus Art. 3 III 2 GG geltend zu machen. ${ }^{1318}$ Deutlich wird dies auch in den von der Rechtsprechung zur Legasthenie entschiedenen Fällen zum Anspruch auf Nichtbewertung der Rechtschreibung, von der Rechtsprechung als "Notenschutz" bezeichnet. ${ }^{1319}$ Die Verletzung von Art. 3 III 2 GG bleibt folgenlos, weil die Betroffenen nach Ansicht der Gerichte keinen Anspruch auf Leistung in dem Sinne haben, dass der Schulgesetzgeber tätig wird, um die Grundrechtsverletzung zu beseitigen. Die Verfassung manövriert sich damit in eine widersprüchliche Lage, denn ohne leistungsrechtliche Komponente werden die Betroffenen rechtsschutzlos gestellt und das Grundrecht wird „weitestgehend zum Leerlauf verurteilt". ${ }^{1320}$

Der Anspruch auf Berücksichtigung und Schaffung einer diskriminierungsfreien Beschulung kann sich weiter aus dem derivativen Teilhaberecht aus dem allgemeinen Gleichheitssatz in Verbindung mit Art. 12 I GG und dem Sozialstaatsprinzip ergeben. ${ }^{1321}$ Das BVerfG hat in seinem Numerus-Clausus-Urteil das Recht auf Teilhabe auch aus Art. 12 GG hergeleitet und damit deutlich gemacht, dass dieses Freiheitsrecht nicht ausschließlich als Abwehrrecht zu inter-

\footnotetext{
${ }^{1315}$ Vgl. international zu dieser Debatte: Heyning, E.H.R.L.R. 2008, S. 376 ff und Heyden/von Ungern-Sternberg, EuGRZ 2009, S. 81 ff, S. 86 ff

${ }^{1316}$ Zu dieser Problematik Luthe, Optimierende Sozialgestaltung, S. 121; Eichholz, "Gibt es ein Recht auf eine Schule für alle?", S. 10 und Riedel, Gutachten UN-Behindertenrechtskonvention, S. 37 f; das Dilemma, wählen zu müssen zwischen einer Regelschule, die den Belangen der Kinder nicht gerecht wird und einer Sonderschule, die den Bedürfnissen Rechnung trägt, zeigt auch der EGMR auf - Urteil v. 13.11.2007 - 57325/00 (D.H. u.a./Tschechien), NVwZ 2008, S. 533 ff, S. 535, Ziff. 203

1317 Luthe, Optimierende Sozialgestaltung, S. 119

1318 Luthe, Optimierende Sozialgestaltung, S. 119

1319 OVG Lüneburg, Beschluss v. 10.7.2008, Az.: 2 ME 309/08, www.rechtsprechung.niedersachsen.de; VG Köln, Beschluss v. 26.9.2008, Az.: 10 L 1240/08, DVBI 2009, S. 538

${ }^{1320}$ Luthe, Optimierende Sozialgestaltung, S. 121; Beyerlin, RdJB 1999, S. 157 ff, S. 161; Schwengers, Eingliederungshilfe $\S 35$ a SGB VIII, S. 175

${ }^{1321}$ BVerfGE 33, 303, 331; Murswiek, in: HStR V, § 112, Rn. 69, 73
} 
pretieren ist ${ }^{1322}$, denn: „das Freiheitsrecht wäre ohne die tatsächlichen Voraussetzungen, es in Anspruch nehmen zu können, wertlos. “1323 Dieser Grundsatz, dass jeder betroffene Grundrechtsträger einen individuellen Anspruch auf gleichen Zugang zu den tatsächlich der Allgemeinheit zur Verfügung gestellten staatlichen Leistungen hat, gilt selbstverständlich auch im Bereich des Art. 3 III 2 GG. ${ }^{1324}$ Das BVerfG hat in der Sonderschulentscheidung bejaht, dass ein behindertes Kind einen Anspruch auf Beschulung in der Regelschule hat, wenn sie durch einen vertretbaren Aufwand an Förderung möglich ist. ${ }^{1325}$

Das derivative Teilhaberecht beschränkt sich jedoch auf die Teilhabe an vorhandenen Schuleinrichtungen und gibt keinen Anspruch auf Schaffung zusätzlicher Ressourcen. ${ }^{1326}$ Das BVerwG hat die Einrichtung integrativer Klassen als Bereitstellung derartiger zusätzlicher Ressourcen angesehen, auf die sich aus dem Art. 3 III 2 GG kein Anspruch ergibt. ${ }^{1327}$ Auch das OVG Lüneburg meint, unter Bezugnahme auf Entscheidungen, die rein finanzielle Forderungen betrafen ${ }^{1328}$, dass die Nicht-Bewertung der Rechtschreibung eine staatliche Leistung ist, die über die derivative Teilhabe hinausgeht. ${ }^{1329}$ Dabei wird jedoch übersehen, dass es nicht um finanzielle Fragen, sondern um die diskriminierungsfreie Ausgestaltung des bestehenden Schulsystems geht. Wenn die Veränderung von Prüfungsinhalten und damit die Veränderung von Bewertungsvorschriften zur Berücksichtigung von Legasthenie/Dyskalkulie nicht Teil des derivativen Teilhaberechts sind, bedeutet dies, dass sich das Teilhaberecht von vornherein auf die Teilhabe an einem diskriminierenden System beschränken würde ${ }^{1330}$ und es der Entscheidung des Gesetzgeber überlassen bliebe, ob und inwieweit er die verfassungsrechtlich geforderte diskriminierungsfreie Teilhabe am Schulsystem gewährt. ${ }^{1331}$ Eine solche freie Entscheidung kann der Gesetzgeber je-

\footnotetext{
1322 Alexy, Theorie der Grundrechte, S. $399 \mathrm{f}$

${ }^{1323}$ BVerfGE 33, 303, 331

1324 Castendiek, in: GS Jean d'Heur, S. 347, Fn. 41; Straßmair, Der besondere Gleichheitssatz aus Art. 3 Abs. 3 Satz 2 GG, S. 95; Neumann, in: Handbuch SGB IX, § 2 Verfassungsrecht, Rn. 33; BVerwG, Beschluss v. 14.8.1997, Az.: 6 B 34/97, juris, Rn. 7

1325 BVerfGE 96, 288, $306 \mathrm{f}$

${ }^{1326}$ BVerwG, Beschluss v. 14.8.1997, Az.: 6 B 34/97, juris, Rn. 7

1327 BVerwG Beschluss v. 14.8.1997, Az.: 6 B 34/97, juris, Rn. 7

1328 OVG Münster, Beschluss v. 16. 11. 2007, Az.: 6 A 2171/05, www.justiz.nrw.de, OVG Lüneburg, betrifft Kostenübernahme für Gebärdendolmetscher im Rahmen eines ärztlichen Beratungsgesprächs, Beschluss v. 11.9.1998, Az.: 2 L 2640/ 98, juris, Rn. 20

1329 OVG Lüneburg, Beschluss v. 10.7.2008, Az.: 2 ME 309/08, www.rechtsprechung.niedersachsen.de, Rn. 19

1330 So im Ergebnis LSG Baden-Württemberg, Beschluss v. 9.12.2010, Az.: L 13 AL 4629/10 ER-B, juris, Rn. 33

${ }^{1331}$ Vgl. BVerfGE 33, 303, 331
} 
doch im Bereich des Diskriminierungsschutzes nicht treffen. Er hat sicherlich ein Ausgestaltungsrecht im Einzelnen, aber kein Entscheidungsrecht im Hinblick auf die Frage, ob ein System diskriminiert und ob ein diskriminierendes System beibehalten wird oder nicht. Deshalb ist die diskriminierungsfreie Ausgestaltung der Schule ein Anspruch, der gerade aus den Grundrechten und dabei zumindest auch aus dem derivativen Teilhaberecht folgt.

Die Teilhabe steht, entgegen der Rechtsprechung des BVerfG, nicht mehr „unter den Vorbehalt des Möglichen im Sinne dessen, was der Einzelne vernünftigerweise von der Gesellschaft beanspruchen kann. “1332 Denn dieser Ressourcenvorbehalt ist nur noch unter den strengen Voraussetzungen des Art. 4 BRK $^{1333}$ zulässig, so dass der allgemeine Verweis auf fehlende Mittel nicht ausreicht.

Auch aus dem derivativen Teilhaberecht ergibt sich daher der Anspruch der Schüler und Schülerinnen mit Legasthenie/Dyskalkulie auf diskriminierungsfreien Zugang und diskriminierungsfreie Teilhabe an den allgemeinen Schulen. Das bezieht sich auf alle Formen der Diskriminierung, seien es äußere Prüfungsbedingungen, inhaltliche Prüfungsanforderungen oder Zulassungsentscheidungen.

Der Anspruch ist deshalb nicht davon abhängig, dass der Gesetzgeber tätig wird, sondern ergibt sich direkt aus dem Grundrecht und ist nach Art. 1 III GG von der öffentlichen Gewalt unmittelbar zu beachten. Auch ohne gesetzliche Regelungen sind daher bei allen Entscheidungen der Schule die Vorgaben des Art. 3 III 2 GG zu beachten und Nachteile wegen der Behinderung in vollem Umfang auszugleichen. ${ }^{1334}$

\footnotetext{
1332 BVerfGE 33, 303, 333

1333 S. dazu oben S. 239 f, Rux, RdJB 2009, S. 220 ff, S. 224; Eichholz, „Gibt es ein Recht auf eine Schule für alle?", S. $4 \mathrm{f}$

${ }^{1334}$ Vgl. VG Regensburg, Beschluss v. 8. 9. 2006, Az.: Rn. 1 E 06.1610 (nicht veröffentlicht)
} 


\section{Teil: Verfahrensfragen}

Der Anspruch der Schüler und Schülerinnen mit Legasthenie/Dyskalkulie auf Berücksichtigung muss in den Schulen umgesetzt werden. Die Verfahrensanforderungen ergeben sich nach der gefestigten Rechtsprechung des BVerfG vor allem daraus, dass die Grundrechte nicht nur die Ausgestaltung des materiellen Rechts beeinflussen, sondern zugleich Maßstäbe für eine den Grundrechtsschutz effektuierende Organisations- und Verfahrensgestaltung setzen. ${ }^{1335} \mathrm{Da}$ das Verfahren für einen effektiven Grundrechtsschutz ganz entscheidende Bedeutung hat, müssen die Verfahrens- oder Organisationsnormen so beschaffen sein, dass das Ergebnis mit hinreichender Wahrscheinlichkeit und in hinreichendem Maß grundrechtsgemäß ist und auch einer späteren Rechtsschutzüberprüfung genügt. ${ }^{1336}$ Unverzichtbare Mindestanforderungen allgemeiner Art sind insbesondere, dass die Regelungen des Verfahrens angemessen, sachgerecht, geeignet und zumutbar sind und den Betroffenen ein faires Verfahren gewährleisten, also auch dazu geeignet sind, begründete Ansprüche durchzusetzen. ${ }^{1337}$ Für das Benachteiligungsverbot des Art. 3 III 2 GG hat das BVerfG in der Sonderschulentscheidung ausgeführt, dass die Grundrechte durch ein weitgehend objektiviertes und fachliches Entscheidungsverfahren abgesichert sein müssen, in dem die Behörde einer gesteigerten Begründungspflicht unterliegt. ${ }^{1338}$

Das Verfahren zur Berücksichtigung von Legasthenie/Dyskalkulie muss sicherstellen, dass bei allen betroffenen Schülern und Schülerinnen eine Berücksichtigung erfolgt. Es muss ausschließen, dass bei Schülern eine Legasthenie/Dyskalkulie nicht berücksichtigt wird, obwohl sie vorliegt. Denn sonst werden diese Schüler im Vergleich zu anerkannten Schülern mit Legasthenie/Dys-

1335 BVerfGE 69, 315, 355; 53, 30, 65 f, 84, 59, 72; 90, 60, 96; Sodan/Ziekow, Öffentliches Recht, S. 176, Rn. 28; s. auch Lindner, Theorie der Grundrechtsdogmatik, S. 461 ff; Alexy, Theorie der Grundrechte, S. 433

${ }^{1336}$ Alexy, Theorie der Grundrechte, S. 431; Kopp/Ramsauer, VwVfG, Einführung I, Rn. 20

1337 Kopp/Ramsauer, VwVfG, Einführung I, Rn. 21

1338 BVerfGE 96, 288, 310 f; Langenfeld, RdJB 2007, S. 211 ff, S. 226 
kalkulie benachteiligt, weil sie den allgemeinen Beschulungsregeln unterworfen bleiben. Es muss weiter sicherstellen, dass eine mögliche Grundrechtskollision zwischen Schülern und Schülerinnen mit und ohne Behinderung vermieden wird. Die Berücksichtigung von Legasthenie/Dyskalkulie führt zu veränderten Prüfungsbedingungen und -anforderungen, die sachlich gerechtfertigt sein müssen. Fehlt ein solcher sachlicher Grund für die Ungleichbehandlung, dann wird in die Rechte der Schüler und Schülerinnen eingegriffen, die dem normalen Maßstab unterworfen bleiben. Deshalb muss das Verfahren ausschließen, dass bei Schülern eine Legasthenie/Dyskalkulie berücksichtigt wird, obwohl bei innen diese Behinderung nicht vorliegt. Sonst werden sie gegenüber Schülern ohne Behinderung bevorzugt, weil für sie andere Bewertungsregeln gelten.

Für das Verfahren ist zwischen der Grundentscheidung der Anerkennung von Legasthenie/Dyskalkulie und der Berücksichtigung durch Umsetzung von Nachteilsausgleich zu unterscheiden.

\section{Kapitel: Grundentscheidung: Anerkennung von Legasthenie und Dyskalkulie in der Schule}

Die Anwendbarkeit des Grundrechts aus Art. 3 III 2 GG ist, anders als z.B. das Grundrecht auf Asyl aus Art. 16 a GG, nicht davon abhängig, dass der Status erst festgestellt wird, um es sodann in Anspruch nehmen zu können. ${ }^{1339}$ Deshalb ist es für die Anwendung des Art. 3 III 2 im Schulbereich nicht erforderlich, dass die Behinderung rechtsförmlich festgestellt worden ist. Eine Feststellung hat daher keinen konstitutiven, sondern nur einen deklaratorischen Charakter. Die Berücksichtigung einer Behinderung in der Schule kommt jedoch nicht ohne eine geeignete Feststellung und Anerkennung aus. Nur mit einem geeigneten Verfahren kann sichergestellt werden, dass das Benachteiligungsverbot aus Art. 3 III 2 GG für die betroffenen Schüler im schulischen Bereich aktiviert wird, dass etwaige unberechtigte Anträge abgelehnt werden und zum Schutz der Grundrechte der nicht-betroffenen Schüler und Schülerinnen sichergestellt wird, dass ein sachlicher Grund für die Ungleichbehandlung der behinderten Schüler und Schülerinnen vorliegt.

Die Grundzüge des Verfahrens ergeben sich aus dem VwVfG bzw. aus den jeweiligen Verfahrensgesetzen des Bundeslandes, die für die Schulbehörden und die Schulen als Behörden gem. § 1 Abs. 4 VwVfG gelten (§ 1 Abs. 1 $\vee(w \vee f G)^{1340}$, sowie aus den speziellen schulrechtlichen Bestimmungen.

\footnotetext{
1339 Vgl. zu Art. 16 a GG BVerfGE 60, 253, 295 f; s. auch Ennuschat, Die Rechte stotternder Menschen in Schule, Ausbildung und Studium, S. 14

${ }^{1340}$ Avenarius/Heckel, Schulrecht, Ziff. 6.21; vgl. VG München, Beschluss v. 15.10.2002, Az.: M 3 E 02.4698, juris, Rn. 34
} 


\section{Verwaltungsakt: Anerkennung einer Behinderung}

Alle landesrechtlichen Regelungen sehen ein mehr oder weniger stark ausgeformtes Verfahren für die Berücksichtigung von besonderen Schwierigkeiten im Lesen, Schreiben und Rechnen vor. Diese Verfahren haben nach Ansicht der Schulverwaltungen ganz überwiegend nur Innenwirkung. Einen formellen Feststellungsakt kennt bisher ausdrücklich nur der LRS-Erlass Schleswig-Holstein. Dort wird in Nr. 2.2.3.4 i.V.m. Anlage 3 a geregelt, dass die Feststellung der Legasthenie ein Verwaltungsakt ist. In Bayern wird die Feststellung der Legasthenie durch den Schulpsychologen vom Kultusministerium vereinzelt als Verwaltungsakt angesehen. ${ }^{1341}$

Die Entscheidung über die Anerkennung einer Behinderung oder die Versagung der Anerkennung einer Behinderung ist jedoch ein Verwaltungsakt gem. $\S 35 \mathrm{VwVfG}$. Mit der Entscheidung wird durch eine Behörde, meistens die Schulen, auf dem Gebiet des Schulrechts mit unmittelbarer Rechtswirkung nach außen ein Einzelfall geregelt. Angesichts der erheblichen Rechtswirkungen der Anerkennung für den betroffenen Schüler kann diese nicht als eine Maßnahme nur des laufenden Schulbetriebes qualifiziert werden, die unterhalb der Schwelle der Rechtserheblichkeit liegt und daher ohne Rechtswirkungen wäre. ${ }^{1342}$ Die Entscheidung ist vollstreckungsfähig, so dass kein Fall des $\S 44$ a VwGO vorliegt. Dieses Ergebnis stimmt mit der Rechtsprechung zum Prüfungsrecht überein, die in der Gewährung oder Versagung von Nachteilsausgleich in Prüfungen unstreitig einen Verwaltungsakt sieht. ${ }^{1343}$

\section{Rechtsgrundlage}

Der Erlass eines Verwaltungsaktes setzt voraus, dass für die zu treffende rechtliche Regelung in materieller Hinsicht eine gesetzliche Grundlage besteht und die Behörde in der Form des Verwaltungsaktes handeln darf. Die gesetzliche Ermächtigung zum Erlass des Verwaltungsaktes ergibt sich schon aus den allgemeinen schulrechtlichen Bestimmungen zur Berücksichtigung von Behinderungen in der Schule, etwa aus $\S 4$ Abs. 11 SH SchulG oder aus $\S 4$ Abs. 5 HB SchulG, sowie aus den landesrechtlichen Bestimmungen zur Berücksichtigung von Schwierigkeiten beim Lesen, Schreiben und Rechnen, da diese jeweils Bestimmungen über die Anerkennung der Schwierigkeiten enthalten. Bedenken

\footnotetext{
${ }^{1341}$ Ministerialbeauftragter für Oberbayern West, Einzelfallentscheidung, Schreiben vom 8.1.2004 (nicht veröffentlicht)

1342 Vgl. zu dieser Abgrenzung Niehues, Schul- und Prüfungsrecht, Rn. 640

${ }^{1343}$ Vgl. zur Festsetzung des Nachteilsausgleichs bei einer juristischen Prüfung VG Saarlouis, Urteil v. 5.3.2009, Az.: 1 K 643/08, juris, Rn. 44; VG München, Beschluss v. 5.12.2007, Az.: M 4 E 07.5630 (nicht veröffentlicht); VGH München Beschluss v. 7.12.2007. Az.: 7 CE 07.3204 (nicht veröffentlicht) zitiert nach Zimmerling, Prüfungsrecht aus anwaltlicher Sicht, S. 37; Zimmerling/Brehm, Prüfungsrecht, Rn. 326
} 
Verfahrensfragen

bestehen bezüglich der landesrechtlichen Regelung in Thüringen, da dort zwar ein Anspruch aus $\S 59$ TH SchulO auf Nachteilsausgleich besteht, die Feststellung der Schwierigkeit aber nur auf der Grundlage von Fachlichen Empfehlungen zum Umgang mit den Schwierigkeiten erfolgt. Die Pflicht zur Berücksichtigung von Behinderungen ergibt sich hier jedoch direkt aus Art. 20 Thür. Landesverfassung. ${ }^{1344}$

\section{Formelle Rechtmäßigkeit}

Das Verfahren auf Berücksichtigung einer Behinderung ist ein Verwaltungsverfahren nach § 9 VwVfG.

\subsection{Zuständigkeit}

Die Entscheidung muss durch die zuständige Stelle erfolgen. Dies sind die Schulbehörden. Funktionell zuständig können die Schulaufsichtsbehörden, die Schulleitungen, die Konferenzen oder die Fachlehrer sein. Über die Berücksichtigung von körperlichen Behinderungen entscheiden meistens die Schulleiter oder Schulaufsichtsbehörden ${ }^{1345}$, bei Legasthenie/Dyskalkulie nach den bisherigen landesrechtlichen Regelungen überwiegend die Klassenkonferenzen. ${ }^{1346}$

Problematisch ist in diesem Zusammenhang die Übertragung der Zuständigkeit auf eine Konferenz, sei es die Klassenkonferenz oder eine andere Konferenz. Zwar ist die Übertragung der Zuständigkeit auf ein Gremium wie die Klassenkonferenz grundsätzlich zulässig ( $v g l$. § $91 \mathrm{VwVfG}$ ), es ist aber fraglich, in-

\footnotetext{
1344 Art. 20 Landesverfassung Thüringen: „Jeder Mensch hat das Recht auf Bildung. Der freie und gleiche Zugang zu den öffentlichen Bildungseinrichtungen wird nach Maßgabe der Gesetze gewährleistet. Begabte, Behinderte und sozial Benachteiligte sind besonders zu fördern."

1345 Die Zuständigkeit lässt sich bundesweit nicht recherchieren, weil der Begriff der Behinderung dem Schulrecht fremd ist und in der Regel die Behinderung mit der sonderpädagogischen Förderung gleichgesetzt wird. Für Körperbehinderung, Sehstörung und Hörbehinderung gilt z.B. in Bayern: BY KMS VI.8 - 5 S 5300 - 6.108417, v. 08.12.2006, Punkt 5, http://www.schulberatung.bayern.de/imperia/md/content/schulberatung/pdf/nachteilgym. pdf. Für stotternde Schüler hat Ennuschat, Die Rechte stotternder Menschen in Schule, Ausbildung und Studium die Rechtslage für Bayern, Hessen, Nordrhein-Westfalen und Saarland aufgezeigt, S. $122 \mathrm{ff}$

${ }^{1346}$ BW VV Besonderer Förderbedarf Nr. 2.3.2; BY automatisch, wenn gutachterlich festgestellt, Zeitzuschlag bestimmt Schulleiter; BE GsVO § 16 VII; BB VV-LRSR Nr. 3 und 6; HB LRS-Erlass Nr. 4; HH Förder-Richtlinie Nr. 3.1 - Testergebnis entscheidet; HE VO-GestSchulVerh: zuständig für die Feststellung der Schwierigkeiten ist die Klassenkonferenz $\S 39 \mathrm{VI}$, zuständig für die Festlegung des Nachteilsausgleichs der Schulleiter § 7 III; MV VV-LRR Nr. 5.1 III - das staatliche Schulamt und $5.2 \mathrm{II}$ - Leiter des staatlichen Schulamts; NI LRR-Erlass Nr. 3; NRW LRS-Erlass Nr. 3.2 - Schulleitung entscheidet; RP VV-LRS Nr. 4.3; SL VV-LRS Nr. 2.1 a); SN VVLRS Nr. 4.1.4; ST Grundschulbewertungs-Erlass Nr. 4.1.1, ST SekundarstufenbewertungsErlass Nr. 5.1.2; SH Legasthenie-Erlass Nr. 2.1; TH § 59 V und VI SchulO.
} 
wieweit es Sinn macht, ein Gremium mit der Feststellung eines gebundenen Anspruchs $^{1347}$ zu beauftragen. Darüber hinaus sprechen gegen die Zuständigkeit eines Gremiums erhebliche datenschutzrechtliche Gründe. ${ }^{1348}$ Ist die Konferenz jedoch zuständig, dann sind etwaige Eltern- und Schülervertreter ${ }^{1349}$ von der Abstimmung ausgeschlossen. Die an sich zulässige Beteiligung von Erziehungsberechtigten und Schülern an schulischen Entscheidungsprozessen findet ihre Grenze bei Einzelmaßnahmen mit erheblicher Eingriffsrelevanz, die die Betroffenen in besonderem Maße berühren. ${ }^{1350}$ Das ist auf jeden Fall gegeben, wenn über die Anerkennung einer Behinderung und damit über höchstpersönliche Angelegenheiten eines Schülers gesprochen wird.

\subsection{Berücksichtigung auf Antrag oder von Amts wegen?}

Fraglich ist, ob die Schule die Behinderung von Amts wegen berücksichtigen muss oder ein Antrag erforderlich ist. Nach Art. 1 III GG ist die Schule an die Grundrechte als unmittelbar geltendes Recht gebunden und deshalb verpflichtet, das Grundrecht aus Art. 3 III 2 GG zu beachten. Deshalb sind Behinderungen grundsätzlich von Amts wegen zu berücksichtigen und ein Antrag nicht erforderlich. ${ }^{1351}$

Für die Berücksichtigung von Amts wegen spricht auch, dass sich die Behinderungen Legasthenie/Dyskalkulie im Unterschied zu sonstigen Behinderungen erst im Schulbereich zeigen. Sie können von den Eltern und sonstigen Bezugspersonen im außerschulischen Bereich nur schwer erkannt werden. Allerdings kann dies dazu führen, dass dem betroffenen Schüler eine Berücksichtigung aufgedrängt wird, die seine Eltern oder er nicht wünschen. Würde man dies zulassen, würde man dem Schüler mit Behinderung das Selbstbestimmungs-

\footnotetext{
${ }^{1347}$ Dazu sogleich anschließend

1348 S. dazu unten S. $315 \mathrm{ff}$
}

1349 Z.B. gehören nach NI SchulG § 36 II i.V.m. § 35 II der Klassenkonferenz je ein Eltern- und ein Schülervertreter an. Nach HE SchulG § 122 II können Schülervertreter mit beratender Stimme an der Klassenkonferenz ( $\S 135 \mathrm{HE}$ SchulG) mit Ausnahme der Zeugnis- und Versetzungskonferenz teilnehmen. Nach NRW SchulG $\S 71$ III nehmen die Elternsprecher und ab Klasse 7 die Schülervertreter an der Klassenkonferenz teil; sie sind nur ausgeschlossen, wenn es um die Leistungsbewertung eines Schülers geht. Nach SH SchulG § 65 I gehören der Klassenkonferenz ein Elternvertreter und ab Klasse 7 der Klassensprecher an, sie sind nach § $65 \mathrm{IV} 4 \mathrm{SH}$ SchulG ausgeschlossen, wenn die Konferenz als Versetzungs- oder Zeugniskonferenz oder bei Prüfungen tätig wird.

1350 Grundlegend zum Stimmrecht der Eltern- und Schülervertreter in Klassenkonferenzen Nds StGH, Urteil v. 8.5.1996, Az.: StGH 3/94, NVwZ 1997, S. 267 ff, $270 \mathrm{f}$

1351 So z.B. BE SopädVO § 40 I; HB RL Nachteilsausgleich, Punkt Verfahren; SH ZVO LegasthenieErlass Nr. 2.1 I; SH ZeugnisVO § 6 II; Brockmann/Littmann/Schippmann, in: Nds. SchulG, § 68, Ziff. 2.1.1; Ennuschat, Die Rechte stotternder Menschen in Schule, Ausbildung und Studium, S. 171; anders HE 
recht darüber nehmen, wie er mit seiner Behinderung leben will. Notwendig ist die Gratwanderung zwischen Unterstützung und Fremdbestimmung. Um Klarheit und Sicherheit zu schaffen, sollte daher ein Antrag nach $\S 9 \mathrm{VwVfG}$ auf Berücksichtigung der Behinderung erforderlich sein.

Stellen die Eltern keinen Antrag, dann soll die Schule nach § $25 \mathrm{VwVfG}$ die Stellung eines Antrages anregen und die erforderlichen Auskünfte erteilen. Aus dem Schulrecht sind die Lehrkräfte verpflichtet, auf die individuelle Lern- und Leistungsentwicklung des Schülers hinzuweisen. ${ }^{1352}$ Eine weitere Pflicht der Lehrkräfte ergibt sich aus § 61 II SGB IX, wonach sie auf mögliche Behinderungen hinweisen müssen, die sie in ihrer Arbeit wahrnehmen. Diese Obliegenheit verdichtet sich zu einer Informationspflicht der Schule, wenn die Eltern ihr Recht auf Mitwirkung an der schulischen Ausbildung des Kindes, insbesondere durch Nachhilfe oder ärztliche Betreuung, nur nach entsprechender Aufklärung durch die Schule wahrnehmen können. ${ }^{1353}$ Eine Pflicht aber der Eltern, einen Antrag zu stellen, besteht nicht.

\subsection{Diagnostik der Legasthenie/Dyskalkulie}

Die Feststellung und Anerkennung einer Legasthenie/Dyskalkulie in der Schule erfordert eine Diagnostik. Von der Frage der Zuständigkeit und Geeignetheit für die Durchführung der Diagnostik ist die anschließend zu klärende Frage zu trennen, wie und mit welchen Unterlagen die Behinderung dann in der Schule nachzuweisen ist. Wie, in welcher Form und durch wen die Diagnostik der Legasthenie/Dyskalkulie erfolgen soll oder muss, ist zwischen den beteiligten Disziplinen streitig.

Da die Diagnostik der Legasthenie/Dyskalkulie die Grundlage ihrer Berücksichtigung im Unterricht und damit die Grundlage für die Anwendbarkeit des Art. 3 III 2 GG im Schulbereich ist, ist sie von entscheidender Relevanz für die Grundrechtsausübung des betroffenen Schülers. ${ }^{1354}$ Die Feststellung muss daher in einem Verfahren erfolgen, das die Grundrechte der Schüler wahrt. Sowohl die formellen als auch die materiellen Anforderungen, die an ein solches Verfahren zu stellen sind, ergeben sich aus dem VwVfG. Nach § $24 \mathrm{VwVfG}$ ist die Schule formell dazu verpflichtet, den Sachverhalt von Amts wegen zu ermitteln und alle für den Einzelfall bedeutsamen, auch die für die Beteiligten günstigen Umstände zu berücksichtigen. Die Einzelheiten ergeben sich bisher aus den jeweiligen landesrechtlichen Regelungen zur Berücksichtigung. Derzeit lassen sich grob folgende Verfahrensausgestaltungen feststellen:

In Bayern erfolgt die Diagnostik der Legasthenie nur durch Kinder- und Jugendpsychiater und innen gleich gestellte Fachkräfte, auf der Grundlage der

\footnotetext{
1352 Z.B. BayEUG Art. 55 II; NI SchulG § 55; NRW SchulG§ 44 II, V

1353 Niehues, Prüfungsrecht, Rn. 210

${ }^{1354}$ Ennuschat, br 2008, S. 93 ff, S. 97
} 
Vorgaben des $\S 35$ a Abs. 1 a SGB VIII und der ICD 10 in Form der oben dargestellten mulitaxialen Diagnostik. ${ }^{1355}$ Die Schule ist an diese Diagnose gebunden. ${ }^{1356}$

Demgegenüber führt die KMK-Empfehlung von 2007 aus: „Unbestritten ist, dass die Diagnose und die darauf aufbauende Beratung und Förderung der Schülerinnen und Schüler mit besonderen Schwierigkeiten im Lesen und Rechtschreiben zu den Aufgaben der Schule gehört. ${ }^{\text {1357 }}$ Dem liegt ein sonderpädagogisches Verständnis von Diagnose zugrunde. Die schulische „Diagnose“ einer Legasthenie oder Dyskalkulie oder von Schwierigkeiten im Lesen, Schreiben und Rechtschreiben erfordert nach dem Verständnis der KMK und der meisten Kultusministerien weder eine medizinische Diagnostik noch eine spezifische sonderpädagogische Diagnostik. Vielmehr wird überwiegend eine Diagnose durch die Lehrkräfte, die schulpsychologische Dienste oder Beratungsdienste hinzuziehen können ${ }^{1358}$, für ausreichend erachtet. In Hamburg wird die Anerkennung von Schwierigkeiten an die Ergebnisse der Testergebnisse des Rechtschreibtests Hamburger Schreibprobe (HSP), des Hamburger Rechentests (HaReT) und des Intelligenztests CFT gebunden. Andere Testungen sind nicht zugelassen. ${ }^{1359}$ Die Hamburger Richtlinie regelt aber nicht, wer diese Testungen durchführen darf. Mecklenburg-Vorpommern schreibt die Diagnostik der LeseRechtschreibfähigkeiten und eine Intelligenzdiagnostik vor. ${ }^{1360}$ Schleswig-Holstein hat ein förmliches Verfahren zur Feststellung der "Lese-RechtschreibSchwäche" in dem die Diagnostik durch eine qualifizierte Fachkraft erfolgt. ${ }^{1361}$ Für die Diagnostik der Rechenschwäche wird vorgegeben, dass diese durch standardisierte oder informelle Verfahren objektiviert wird und die Handreichungen des IQSH ${ }^{1362}$ beachtet werden. ${ }^{1363}$

1355 BY KMBek Legasthenie Nr. IV, s. dazu auch KMS v. 16.3.2012: Fachliche Voraussetzungen bei approbierten Psychotherapeuten: http://www.schulberatung.bayern.de

1356 VGH München, Urteil v. 17.10.2003, Az.: 7 B 02.2186, juris, Rn. 38

1357 KMK-Lesen/Rechtschreiben/Rechnen, Punkt Nr. 1

1358 BW VV Besonderer Förderbedarf Nr. 1; BE GsVO § 16 III; BB VV-LRSR Nr. 3 I und II, Ausnahme: Nr. 5 III für die Sek II: Feststellung durch Facharzt für Kinder- und Jugendpsychiatrie und Schulpsychologen; HB LRS-Erlass Nr. 1.3; HH Förder-Richtlinie Nr. 2.1 - normierte Tests; HE VO-GestSchulVerh § 38; MV VV-LRR Nr. 5.1 I; NI LRR-Erlass Nr. 1; NRW LRS-Erlass Nr. 2.1; RP die Klassenleitung; SL VV-LRS Nr. 2.1 a); SN VV-LRS Nr. 4.1.2 (Schulpsychologe muss); SH Legasthenie-Erlass 2.2.3.3. nur der Lehrer; TH Fachliche Förderempfehlung

1359 HH Förder-Richtlinie Nr. 2.1 und 2.2

1360 MV VV-LRR Nr. 4 und 5 und MV Diagnostischer Dienst Nr. 2.2

1361 SH Legasthenie-Erlass 2.2.3.3

1362 Institut für Qualitätsentwicklung an Schulen Schleswig-Holsteins, Individuelle Diagnostik bei Kindern, Verfahren und Instrumente, 2011, http://www.schleswigholstein.de/IQSH/DE/IQSH_node.html - Suchwort: Rechenschwäche

1363 SH Rechenschwäche-Erlass Nr. 2.3 
Ob neben der schulischen Diagnose auch außerschulische Befundberichte einbezogen werden dürfen oder müssen, ist sehr unterschiedlich geregelt. In Baden-Württemberg, Hessen, Niedersachsen, Schleswig-Holstein sind bei der schulischen "Diagnose" außerschulische Befundberichte oder Gutachten zu berücksichtigen ${ }^{1364}$, in Niedersachsen darf aber "keinesfalls" die Benotung nur aufgrund eines außerschulischen Gutachtens ausgesetzt werden. ${ }^{1365}$ In den anderen Bundesländern sind vorgelegte kinder- und jugendpsychiatrische Befundberichte mit der Diagnose Legasthenie oder Dyskalkulie nicht zwingend zu berücksichtigen ${ }^{1366}$, häufig werden sie nicht erwähnt. ${ }^{1367}$

Diese Verfahrensausgestaltungen müssen den Anforderungen der $\S \S 24 \mathrm{ff}$ VwVfG sowie an ein fachliches Verfahren genügen, um weiterhin Bestand haben zu können. Das Verfahren muss nach der Rechtsprechung des BVerfG so ausgestaltet sein, dass es auf sorgfältig ermittelten Annahmen und Prognosen über den Sachverhalt beruht. ${ }^{1368}$ Der Gesetzgeber muss bei der Ausgestaltung die vorhandenen Erkenntnisquellen ausschöpfen ${ }^{1369}$ und sachgerecht und vertretbar beurteilen. ${ }^{1370}$ Er muss sich am Stand der wissenschaftlichen Erkenntnisse orientieren, um die voraussichtlichen Auswirkungen seiner Regelung so zuverlässig wie möglich abschätzen zu können und einen Verstoß gegen Verfassungsrecht zu vermeiden. ${ }^{1371} \mathrm{Er}$ muss sogar künftig beobachten, ob sich die Verhältnisse in relevanter Weise ändern. ${ }^{1372}$ Die Sachverhaltsannahmen müssen sich im Rahmen der gerichtlichen Prüfung bestätigen lassen. ${ }^{1373}$ Nach § 26 ।

1364 Die in HE VOLRR § 4 II noch vorgesehene Berücksichtigung wurde in die HE VO-VOGestSchulVerh nicht aufgenommen; BW VV Besonderer Förderbedarf Nr. 1; NI LRR-Erlass Nr. 1; SH Legasthenie-Erlass 2.2.3.3.; SL VV-LRS 2.1 a) nur vom Direktor einer Universitätsklinik

1365 Wolter, Nds. SVBI 2009, S. 271 f, S. 272

${ }^{1366}$ SL VV-LRS 2.1 a) nur vom Direktor einer Universitätsklinik; TH Erlass v. 19.7.2011: außerschulische Gutachten dienen der Information

1367 BW VV Besonderer Förderbedarf Nr. 1; NI LRR-Erlass Nr. 1; SH Legasthenie-Erlass 2.2.3.3, andere Bundesländer nicht erwähnt

1368 BVerfGE 50, 290, 333 f; 88, 40, 58 f; 98, 169, 201, 106, 62, 152; vgl. auch ausführlich zu den Verfahrensschritten zum guten Gesetz Burghart, Pflicht zum guten Gesetz, S. $123 \mathrm{ff}$

1369 BVerfGE 50, 290, 334

1370 Burghart, Pflicht zum guten Gesetz, S. 126

1371 BVerfGE 50, 290, 334, Burghart, Pflicht zum guten Gesetz, S. 125

1372 BVerfG, Urteil v. 24.1.2001, Az.: 1 BvR 2623/95, http://www.bverfg.de/entscheidungen/rs20010124_1bvr262395.html, Rn. 99

1373 BVerfGE 106, 62, 151 - Ein Beispiel für die Gestaltung und Regelung eines fachlichen Verfahrens findet sich in den Regelungen zur Feststellung der Eignung für eine Fahrerlaubnis (§ 11 FeV und Anlage 15), die präzise bestimmen, wann die Eignung mithilfe welcher Gutachten nachgewiesen werden kann. Die zugelassenen Gutachter, ihre Zulassung und der notwendige Inhalt der Gutachten sind eingehend geregelt. Die Gutachten müssen auf anerkannten wissenschaftlichen Grundsätzen basieren (Anlage 15 FeV Nr. 1 c) 
Nr. 2 VwVfG muss die Behörde einen Sachverständigen hinzuziehen, wenn sie die besondere Sachkenntnis, die ein bestimmter Sachverhalt erfordert, nicht hat. Sie hat aber einen gewissen Beurteilungsspielraum hinsichtlich der Frage, ob die eigene Sachkunde ausreicht. ${ }^{1374}$

Die bayerische Regelung setzt eine Diagnostik durch die Kinder- und Jugendpsychiater und ihnen gleichgestellter Fachkräfte in Form der mulitaxialen Diagnostik voraus. Da diese laufend fortgeschrieben wird und wissenschaftlich evaluiert sind, ist bei dieser Diagnostik sichergestellt, dass sie sich an wissenschaftlichen Erkenntnissen orientiert und zu mindestens vertretbaren Ergebnissen kommt. Die Schulen und Schulbehörden bedienen sich damit Sachverständiger gem. § 26 I Nr. 2 VwVfG, so dass ein fachliches Verfahren stattfindet.

Die meisten anderen landesrechtlichen Vorschriften zur Diagnostik genügen diesen Anforderungen indes nicht, weil sie die Diagnose allein den Lehrkräften, die dies allein aus ihrer pädagogischen Befähigung heraus leisten sollen, überlassen. Die Durchführung von standardisierten Tests oder auch nur von bestimmten Überprüfungsverfahren, wie z.B. bestimmten Diktaten oder Ähnlichem, ist überwiegend nicht vorgegeben. Die Feststellung von Schwierigkeiten erfolgt dann nicht anhand von vorgegebenen, nachvollziehbaren und wiederholbaren Kriterien, sondern "schwebt vage im Raum und ist von der Person des Entscheiders abhängig. “1375 "Daraus ergeben sich zwei Probleme: Erstens schöpfen die landesrechtlichen Regelungen die vorhandenen wissenschaftlichen Erkenntnisquellen nicht nur nicht aus, sondern lassen die gesamten wissenschaftlichen Erkenntnisse aus den Bereichen der Medizin, der Psychologie und der Neurowissenschaften außer Betracht. Dies widerspricht den oben dargelegten Grundsätzen der Rechtsprechung des BVerfG zur Berücksichtigung wissenschaftlichen Erkenntnisse und sonstiger vorhandener Erkenntnisquellen. ${ }^{1376}$

Zweitens handelt es sich bei der Lehrereinschätzung um eine fast ausschließlich subjektive Einschätzung. Fraglich ist, ob dies den Anforderungen an eine Diagnostik genügt. Diagnostik muss bestimmten Qualitätsanforderungen genügen, um den Anforderungen an ein objektiviertes und fachliches Verfahren zu genügen. Ein solches liegt nur vor, wenn das gefundene Ergebnis nicht oder so wenig wie möglich von der Untersuchungssituation und nicht von der Person des Feststellenden abhängig ist und wenn es wiederholbar ist. ${ }^{1377}$ Bei der Feststellung von Legasthenie/Dyskalkulie werden im medizinisch-psychologischen Bereich standardisierte psychologische Testverfahren eingesetzt. Psychologische Tests sind hochkomplizierte, in spezielle psychologisch-wissenschaftliche Kontexte eingebundene Verfahren. Sie basieren jeweils auf ganz un-

\footnotetext{
${ }^{1374}$ Kopp/Ramsauer, VwVfG, § 26, Rn. 29

1375 Orthmann Bless, in: Walter/Wember/Borchert/Goetze, Sonderpädagogik des Lernens, S. 98

${ }^{1376}$ Vgl. Burghart, Pflicht zum guten Gesetz, S. 126

1377 Leonhardt, Annette/Wember, Franz B., in: Grundfragen Sonderpädagogik, S. 167
} 
terschiedlichen theoretischen Konzepten, die ein umfassendes fachliches Wissen verlangen. ${ }^{1378}$ Die Objektivität dieser Testverfahren wird dadurch gesichert, dass die Durchführung - bis hin zu präzise vorformulierten Testinstruktionen und Antworten auf mögliche Rückfragen -, Auswertung und Interpretation genau vorgeschrieben wird. Außerdem wird angegeben, wann die Tests durchgeführt werden sollten (Alter und Tageszeit) und wie die Testsituation gestaltet sein sollte. ${ }^{1379}$ Die Vorgaben dienen dazu, die Aussagen vergleichbar zu halten. Außerdem müssen die Testverfahren reliabel (genau und zuverlässig) und valide (aussagekräftig) sein. ${ }^{1380}$ Gegenüber einer solchen testbasierten Diagnostik bestehen im pädagogischen Bereich erhebliche Vorbehalte. Diagnostik wird von Lehrkräften vielfach sehr kritisch gesehen, da sie darin die Gefahr riskanter Zuschreibungen und Etikettierungen sehen. ${ }^{1381}$ Sie wird als nutzlos, unpädagogisch, sogar als unethisch abgelehnt ${ }^{1382}$, weil ihr Normen, die z. T. als inhuman und schädlich bezeichnet werden, zugrunde liegt. Diagnostik ist jedoch immer normorientiert, weil ihr Vergleichsmaßstäbe zugrunde liegen müssen. Professionelle Diagnostik legt die jeweiligen Vergleichsmaßstäbe offen und belässt sie nicht im Ungefähren und intersubjektiv nicht Nachvollziehbaren. ${ }^{1383}$ Nur eine solche nachvollziehbare Diagnostik kann die Voraussetzungen eines objektiven Verfahrens erfüllen. Die subjektive Einschätzung einer Lehrkraft kann derartige vergleichbare Maßstäbe und Ergebnisse offensichtlich nicht vorweisen.

Vielfach besteht die pädagogische "Diagnose" in der Beobachtung des betroffenen Kindes im Klassenverband. Diese Form des Beobachtens wäre nur dann zur Feststellung von Legasthenie/Dyskalkulie geeignet, wenn sie zu objektiven, fachlichen und wiederholbaren Ergebnissen führt. Das Beobachten ist zwar eine diagnostische Methode, aber keine selbstverständliche und vor allem keine einfache diagnostische Methode. Vielmehr ist es die schwierigste diagnostische Methode ${ }^{1384}$, denn im Unterschied zu Alltagsbeobachtungen müssen Beobachtungen, die dazu dienen sollen, Grundlage einer professionellen Diagnose zu sein, systematisch, objektiv und verlässlich und gültig (valide) sein.

\footnotetext{
1378 Zier, Recht für Diplom-Psychologen, S. 137

1379 Leonhardt, Annette/Wember, Franz B., in: Grundfragen Sonderpädagogik, S. 167

${ }^{1380}$ Vgl. zu den Anforderungen im Einzelnen Leonhardt, Annette/Wember, Franz B., in: Grundfragen Sonderpädagogik, S. 167 ff; Kany/Schöler, Diagnostik, S. 65 f

1381 Prengel, Sozialextra 2006, S. 15 ff, S. 16; Thiel, Rechenschwäche; Langfeldt, in: Hdb. Psychologie, S. 631

1382 Langfeldt, in: Hdb. Psychologie, S. 631 m.w.N.; kritisch zu dieser Haltung: Kany/Schöler, Diagnostik, S. 68 und Welti, Behinderung und Rehabilitation im sozialen Rechtsstaat, S. 87, der in Fn. 705 die Ablehnung von Normen durch Bleidieck zitiert: „Der Begriff Norm erfüllt ... nicht das Kriterium der Wissenschaftsgenauigkeit. Sein diffamierender Beiklang macht ihn vollends suspekt. Er ist willkürlich, inhuman und .... schädlich."

1383 Kany/Schöler, Diagnostik, S. 51

${ }^{1384}$ Kany/Schöler, Diagnostik, S. 77
} 
Ihre Ergebnisse müssen eindeutig kommunizierbar und prüfbar sein. Die im Schulalltag vorgenommenen Beobachtungen genügen kaum diesen Standards. ${ }^{1385}$ Nachgewiesen ist, dass die pädagogische Einschätzung der Lehrkräfte ohne Bezugnahme auf standardisierte Tests oder sonstige objektive Verfahren durch vielfältige Annahmen beeinflusst wird, die mit der Fähigkeit zum Lesen, Rechtschreiben und Lesen nichts zu tun haben und die einer wissenschaftlichen Überprüfung nicht zugänglich sind. ${ }^{1386}$ Die Übereinstimmung zwischen dem Lehrerurteil und dem in Leistungstest erzielten Leistungsniveau nimmt mit der Berufserfahrung der Lehrkräfte nicht zu, wie man vermuten würde, sondern ab: je mehr Berufserfahrung die Lehrkraft hat, desto mehr weicht ihr Urteil von dem Ergebnis des Leistungstests ab. ${ }^{1387}$ Die ungeleitete Beobachtung durch die Lehrkraft erfüllt daher nicht die Anforderungen an ein objektives, fachliches Verfahren mit wiederholbaren Ergebnissen.

Denkbar wäre, dass eine sonderpädagogische Diagnostik die Anforderungen an ein objektives, fachliches Verfahren hinsichtlich der Diagnostik einer Legasthenie/Dyskalkulie erfüllen kann. Ob es der Heil- und Sonderpädagogik überhaupt gelungen ist, eine Förderdiagnostik zu etablieren, die auf theoretisch fundierten und empirisch abgesicherten Kriterien beruht und zu reliablen Ergebnissen führt, ist jüngst massiv von Heilpädagogen selbst in Frage gestellt worden. ${ }^{1388}$ Danach ist es trotz 30 Jahren Forschung auf diesem Gebiet bis heute nicht gelungen, eine Förderdiagnostik zu etablieren, die es Praktikern ermöglicht, aufgrund vorhandener Kriterien für einen zu diagnostizierenden

1385 Kany/Schöler, Diagnostik, S. 78

${ }^{1386}$ S. dazu die Selektion der Gruppen in der Studie von Schulte-Körne/Deimel/Remschmidt, Zeitschrift für Kinder- und Jugendpsychiatrie und Psychotherapie 31 (2003), Punkt 3.1. Selektion der Projektgruppen: Die Lehrerinnen selektierten nicht, wie vorgegeben, nach der schlechten Rechtschreibung, sondern nach ganz anderen Kriterien. Gefördert wurden ganz überwiegend Kinder mit durchschnittlichem Rechtschreibvermögen. Kinder mit einer Teilleistungsstörung, d.h. spezifischen Schwierigkeiten im Schreiben und/oder Lesen, kamen eher nicht in die Förderung; auch die IGLU-Studie 2006 kommt zu dem Ergebnis, dass die Förderbedürftigkeit des Kindes sehr häufig nicht erkannt wird. Valtin/Hornberg/Buddeberg/Voss/Kowoll/ Potthoff, in: Bos, IGLU 2006, S. 76; s. auch die umfassende Kritik bei Kany/Schöler, Diagnostik, S. 43 ff, die aufzeigt, dass noch nicht einmal Sonderpädagogen über ausreichende diagnostische Kenntnisse verfügen, Grundschullehrer noch weniger

Roos/Schöler, Schriftspracherwerb, S. 147, 156 f - Als Erklärung wird vermutet, dass jüngere Lehrkräfte eher einen schulübergreifenden Referenzrahmen heranziehen, während Lehrkräfte, die bereits seit vielen Jahren im Schuldienst und im Regelfall auch an derselben Schule unterrichten, sich an das durch das Einzugsgebiet bedingte Leistungsniveau der Schüler/innen gewöhnt haben und eventuell eine gewisse „Betriebsblindheit" entwickeln.

Schlee, Zeitschrift für Heilpädagogik 2008, S. 122 ff, S. 130; Jogschies, Zeitschrift für Heilpädagogik 2008, S. 132 ff, S. 140, s. auch Klemm/Preuss-Lausitz, Gutachten: Perspektiven sonderpädagogische Förderung, S. 21 
Schüler eine Entscheidung über die notwendige Förderung vorzuschlagen. ${ }^{1389}$ Behauptet wird, es stünde im Belieben der jeweiligen Diagnostiker, aus der Datenfülle aussagekräftige und belegbare Ergebnisse zu konstruieren. ${ }^{1390}$ Die pädagogische Literatur weist darauf hin, dass die Gefahr besteht, dass die Diagnose von sachfremden Erwägungen beeinflusst werden kann. Wird die Diagnose durch Sonderpädagogen von Sonderschulen vorgenommen, kann die Existenzsicherung der eigenen Institution in die Empfehlung einfließen. ${ }^{1391}$ Wird sie von der Schulverwaltung vorgenommen, kann sie durch das vorhandene Kontingent an Fördermaßnahmen und/oder -stunden beeinflusst werden. ${ }^{1392}$ Dass die sonderpädagogische Diagnostik wenig nachvollziehbar ist, ergibt sich auch aus den extrem unterschiedlichen Anteilen an Schülern und Schülerinnen an Sonderschulen im Vergleich zu den Regelschulen. Der Anteil schwankt zwischen $4 \%$ in Rheinland-Pfalz und fast $11 \%$ in Mecklenburg-Vorpommern. ${ }^{1393}$ Die Problematik der sonderpädagogischen Diagnostik liegt darin, dass sie sich als „Förderdiagnostik" versteht, die den Schwerpunkt auf mögliche Förderformen, nicht aber auf die gezielte Diagnose der zugrunde liegenden Schwierigkeiten und Ursachen legt. Man ist sich der Problematik wohlbewusst, denn es wird zugestanden, dass Lernstandsanalysen keine Fakten über Kinder hervorbringen können, sondern nur Bilder und Vermutungen, die den zugrunde liegenden Modellen entsprechen. ${ }^{1394}$ Ohne Kenntnis der Fakten ist eine Diagnostik von Legasthenie/Dyskalkulie jedoch nicht möglich. Förderdiagnostische Verfahren erlauben daher keine „punktgenaue Diagnostik“1395 und erfüllen damit ebenfalls nicht die Anforderungen, die an ein objektives und fachliches Verfahren zur Feststellung von Legasthenie/Dyskalkulie zu stellen sind.

Der Verstoß gegen ein objektiviertes und fachliches Verfahren sowie zur Einbeziehung aller Erkenntnisquellen wäre dann hinzunehmen, wenn die pädagogische „Diagnose“ mit sehr hoher Wahrscheinlichkeit zum gleichen Ergebnis kommen würde wie die medizinisch/psychologische Diagnose und die Zahl der differierenden Entscheidungen verschwindend gering wäre. Das ist jedoch nicht der Fall. Es ist wissenschaftlich nachgewiesen, dass die Übereinstimmungsquote zwischen der Diagnostik einer Legasthenie/Dyskalkulie durch einen Facharzt und der Feststellung durch einen Lehrer nur 30 \% beträgt. D.h. in

\footnotetext{
1389 Jogschies, Zeitschrift für Heilpädagogik 2008, S. 132 ff, S. 141

1390 Schlee, Zeitschrift für Heilpädagogik 2008, S. 122 ff, S. 128

1391 Klemm/Preuss-Lausitz, Gutachten: Perspektiven sonderpädagogische Förderung, S. 6; Orthmann Bless, in: Walter/Wember/Borchert/Goetze, Sonderpädagogik des Lernens, S. 98; Langfeldt, in: Hdb. Psychologie, S. 629

1392 Klemm/Preuss-Lausitz, Gutachten: Perspektiven sonderpädagogische Förderung, S. 6

1393 Klemm, Sonderweg Förderschulen, S. 13 und 20

1394 Prengel, Sozialextra 2006, S. 15 ff, S. 16

1395 Vgl. Kany/Schöler, Diagnostik, S. 68 ff
} 
70 \% der Fälle verneint die Lehrkraft eine Legasthenie/Dyskalkulie, obwohl sie medizinisch festgestellt wurde. ${ }^{1396}$ Die Lehrkräfte sind zwar in der Lage festzustellen, wie das Vermögen der Schüler und Schülerinnen ist, zu schreiben, zu lesen und zu rechnen. Sie sind aber wegen der fehlenden Fachkenntnis nicht in der Lage, festzustellen, ob und warum das Vermögen beeinträchtigt ist. ${ }^{1397}$ Es ist innen weder möglich bei schlechter Rechtschreibung eine Legasthenie anzunehmen, denn die schlechte Leistung kann auch auf anderen Faktoren, wie z.B. anderen psychischen Störungen oder neurologischen Erkrankungen beruhen, noch bei durchschnittlicher Leistung eine Legasthenie auszuschließen, denn trotz durchschnittlicher Leistung kann z.B. bei sehr hoher Begabung oder sehr guter Therapie eine Legasthenie vorliegen. ${ }^{1398}$ Gesichert können Legasthenie und Dyskalkulie daher nur über ein objektives, fachliches, reliables und valides Verfahren, das dem Stand der wissenschaftlichen Erkenntnis entspricht, festgestellt werden. Ein solches Verfahren gewährleistet derzeit nur die oben dargestellte multiaxiale Diagnostik nach der ICD 10 und den Leitlinien der Kinder- und Jugendpsychiater.

\subsubsection{Relativierung der Diagnostik durch pädagogische Einschätzungen?}

Die Pädagogik spricht sich dafür aus, dass eine, in einem objektiven und fachlich abgesicherten Verfahren erstellte medizinisch-psychologische Diagnose der Legasthenie/Dyskalkulie aufgrund der subjektiven, pädagogischen Einschätzung

1396 Schulte-Körne, Elternratgeber Legasthenie, S. 44, 124; Schulte-Körne/Deimel/Remschmidt,
Zeitschrift für Kinder- und Jugendpsychiatrie und Psychotherapie 31 (2003), Punkt 3; das
gleiche Ergebnis hat die Pisa Studie Iglu 2006 ergeben Valtin/Hornberg/Buddeberg/Voss/
Kowoll/Potthoff, in: Bos, IGLU 2006, S. 76

1397 Schulte-Körne/Deimel/Remschmidt, Zeitschrift für Kinder- und Jugendpsychiatrie und Psychotherapie 31 (2003), Punkt 3; s. auch Valtin/Hornberg/Buddeberg/Voss/Kowoll/Potthoff, in: Bos, IGLU 2006, S. 76 ff; Geist, in: Legasthenie und Dyskalkulie 2011, S. 167

Derartige Fehleinschätzungen seitens der Lehrkräfte lassen sich in den folgenden, von der Rechtsprechung entschiedenen Fällen nachweisen: VGH Kassel, Beschluss v. 5.2.2010, Az.: 7 A 2406/09, juris. Gesamt-IQ der im überdurchschnittlichen Bereich liegt. Die standardisierten Testverfahren ergeben beim Lesen den Prozentrang (PR) 2 und im Rechtschreiben den Prozentrang (PR) von 4,5. (der Betroffene hat mir dankenswerter Weise erlaubt, diese Werte zu veröffentlichen). VG Gießen, Urteil v. 30.6.2009. Az.: 4 K 1052/09.GI (nicht veröffentlicht), Prozentrang $2 \mathrm{im}$ Lesen und 4,5 in der Rechtschreibung; VG Gießen, Urteil v. 2.11.2010, Az.: 7 K 1841/10.GI, S. 2, 8 (nicht veröffentlicht), Prozentrang 1,4. Das Vorliegen von Legasthenie wird von den Lehrkräften verneint, obwohl die medizinischen Kriterien eindeutig erfüllt und nachgewiesen sind. Die Betroffenheit der hier vorgestellten Schüler mit PR 1,4, PR 2 und PR 4,5 sprechen für schwerste Beeinträchtigungen, s. auch die Ergebnisse der IGLU-Studie, wonach Schüler mit schlechten Leseleistungen aufgrund normierter Tests teilweise Noten von 1 oder 2 in der Leseleistung erhielten, Valtin/Hornberg/Buddeberg/Voss/Kowoll/Potthoff, in: Bos, IGLU 2006, S. 76 
der Lehrkräfte relativiert werden kann. ${ }^{1399}$ Die Rechtsprechung hat derartige subjektive Einschätzungen und Relativierungen ausdrücklich zugelassen. So führt das VG Darmstadt aus: „Es spricht im Übrigen nichts dagegen, die gegenüber den durchgeführten Tests subjektiven Einschätzungen von Lehrkräften in die Entscheidungsfindung einzubeziehen. ... Im Übrigen hält die Kammer die konkreten Beobachtungen der Lehrkräfte immer noch für aussagekräftiger als abstrakte wissenschaftliche Untersuchungen, die in der Regel nicht ohne weiteres auf den Einzelfall übertragbar sind. ${ }^{\prime 1400}$ Das VG Gießen hat geurteilt: „Die Fachlehrer begründen [die Ablehnung der Nicht-Bewertung der Rechtschreibung] ... damit, dass die Rechtschreibleistungen im Vergleich zu den Mitschülern nicht signifikant abweichen. Dieser Einschätzung und Bewertung der Fachlehrer kommt Vorrang zu vor der fachärztlichen Stellungnahme nebst der Bestätigung durch den Schulpsychologen. Der Facharzt kommt zu seiner Bewertung aufgrund einer Untersuchung und eines Testverfahrens, mithin anhand eines nur kurzen Lebensabschnitts. Im Vordergrund stehen medizinische und psychologische Untersuchungen .... Im Vordergrund stehen bei [den Fachlehrern] pädagogische Bewertungen. Es mag sein, dass bei den Fachlehrern die medizinischen Kenntnisse hinsichtlich der Legasthenie nur bedingt vorhanden sind. Andererseits sind sie die Fachleute, die im täglichen Umgang und im Vergleich mit anderen Mitschülern, die nicht im Lesen oder in Rechtschreibung behindert sind, das Vermögen des Klägers zu lesen und fehlerfrei zu schreiben, beobachten und beurteilen können. ${ }^{1401}$ In einem weiteren Verfahren hat das VG Gießen an dieser Linie festgehalten und der Einschätzung der Lehrkräfte den Vorrang gegeben. Es sieht zwar, dass die Klassen zufällig zusammengesetzt sind und deshalb auch die Beurteilungsgrundlage für die Lehrkraft zufällig ist, meint aber, dass diese Zufälligkeit den Lehrern bewusst sein dürfte. ${ }^{1402}$

Beide Gerichte beziehen sich damit auf die soziale Bezugsnorm einer einzigen Klasse, die in aller Regel jedoch eine ungenügende Grundlage bietet und zu gravierenden Fehlern führen kann $^{1403}$, weil die Leistungsunterschiede zwischen Klassen, selbst Parallelklassen, signifikant groß sind. ${ }^{1404}$ Tatsächlich spielt sogar der sozioökonomische Hintergrund der Klasse eine Rolle für die Rechtschreibleistung der gesamten Klasse. ${ }^{1405}$ Nur normierte Verfahren liefern die Grundla-

\footnotetext{
1399 Behrens, Nds. SVBI 2006, S. 189 ff, S. 189

1400 VG Darmstadt, Urteil v. 28. 4. 2009, Az.: 7 K 687/08.DA (3), S. 16 f (nicht veröffentlicht)

${ }^{1401}$ VG Gießen, Urteil v. 30.6.2009. Az.: 4 K 1052/09.GI, S. 13 (nicht veröffentlicht)

1402 VG Gießen Urteil v. 2.11.2010, Az.: 7 K 1841/10.GI, S. 9 (nicht veröffentlicht)

1403 Ausführlich zu sozialen Bezugsnormen als diagnostische Grundlage: Kany/Schöler, Diagnostik, S. $52 \mathrm{ff}$

${ }^{1404}$ Roos/Schöler, Schriftspracherwerb, S. 120, 164 m.w.N.

1405 Roos/Schöler, Schriftspracherwerb, S. 127
} 
ge für den Vergleich des Kindes mit seinen Leistungsmöglichkeiten ${ }^{1406}$, nicht aber die Leistungsmöglichkeiten der Klasse, die als Vergleich genommen wird. ${ }^{1407}$

Diese Rechtsprechung der VG Darmstadt und Gießen steht daher im Widerspruch zur Rechtsprechung des BVerfG, wonach für die Absicherung des Rechts aus Art. 3 III 2 GG ein weitgehend objektives und fachliches Verfahren erforderlich ist. ${ }^{1408}$

Die Lehrereinschätzung ist weiter davon abhängig, ob die Lehrkraft überhaupt Kenntnisse zur Legasthenie/Dyskalkulie besitzt ${ }^{1409}$ Das Ergebnis ist daher weder vergleichbar, noch wiederholbar, reliabel oder valide. Gegen die Einbeziehung der subjektiven Einschätzung spricht schließlich, dass nach Ansicht der Schulverwaltungen und der Rechtsprechung ${ }^{1410}$ von den Lehrern keine Diagnose der Legasthenie (bzw. Dyskalkulie) erwartet werden kann und sie daher in dieser Hinsicht keine Amtspflicht haben. Wenn dem so ist ${ }^{1411}$, dann zeigt dies, dass die Lehrkräfte auf diesem Gebiet nicht gleich kompetent sind wie die Fachärzte, was es ausschließt, ihnen die Befugnis zuzusprechen, ein medizinisches Gutachten zu relativieren.

Die Feststellung der Behinderung ist eine grundlegende Entscheidung für den Schüler in seiner Schullaufbahn und muss justiziabel sein. Wäre eine Diagnose, die in einem objektiven Verfahren ermittelt wurde, durch eine subjektive Einschätzung der Lehrkraft relativierbar, so wäre dies nicht gerichtlich überprüfbar, weil Kriterien für die Einschätzung fehlen. Ein solches Verfahren würde im Ergebnis die Anwendbarkeit des Grundrechts in das Ermessen der Lehrkraft stellen. Das widerspricht jedoch dem Sinn und Zweck von Grundrechten. Nur ein objektives Verfahren auf der Grundlage überprüfbarer und wiederholbarer Kriterien liefert überprüfbare und damit justiziable Ergebnisse.

\subsubsection{Inhaltliche Überprüfung der Diagnose durch die Schule?}

Fraglich ist, ob die Schulbehörden dazu befugt sind, die Diagnose inhaltlich zu überprüfen. Die Schulbehörden verlangen üblicherweise zum Nachweis einer Behinderung einen „geeigneten “1412 oder auch „fachärztlichen“1413 Nachweis.

\footnotetext{
${ }^{1406}$ Kany/Schöler, Diagnostik, S. 53

1407 Kany/Schöler, Diagnostik, S. 57 m.w.N.

1408 Langenfeld, RdJB 2007, S. 211 ff, S. 226

${ }^{1409}$ Das ist nicht zwingend der Fall, weil Kenntnisse über Legasthenie/Dyskalkulie und deren Berücksichtigung im Unterricht nicht zu den Pflichtfächern im Studium gehören, nicht einmal bei Sonderpädagogen.

1410 OLG Celle, Beschluss v. 17.2.2010, Az.: 16 U 62/09 (nicht veröffentlicht)

1411 Näher dazu s. unten 11. Teil: 1. Kapitel: 1.: Amtspflicht zur Feststellung der Legasthenie/Dyskalkulie, S. 334

1412 SH ZeugnisVO $§ 6$
} 
Die genaue Aufschlüsselung der Diagnose, d.h. die Angabe der genauen medizinischen Werte, die die Diagnose stützen, wird nicht gefordert. Eine inhaltliche Überprüfung der Diagnose findet nicht statt.

Die Frage der inhaltlichen Überprüfung von fachärztlichen Stellungnahmen zur Legasthenie/Dyskalkulie ist in Bayern Gegenstand von Urteilen gewesen und nach wie vor, vor allem in der Praxis, umstritten. ${ }^{1414}$ Die Anerkennung der Legasthenie in der Schule erfordert dort ein "Zusammenwirken" von Schulpsychologen und diagnostizierender Fachkraft. ${ }^{1415}$ Fraglich ist immer wieder, wie dieses Zusammenwirken erfolgen soll. Die Schulpsychologen erkennen z. T. die Gutachten der Kinder- und Jugendpsychiater nicht an, einige Schulpsychologen testen die Kinder grundsätzlich neu, um die Werte zu überprüfen, oder halten, dies allerdings sind Einzelfälle, Blutabnahmen oder Hausbesuche für erforderlich. ${ }^{1416}$ Wenn die Schulpsychologen die Diagnose aufgrund der mitgeteilten Werte für falsch halten, wird die Anerkennung der Legasthenie verweigert. Häufig wird dann die in Bayern „mildere Form“ der Lese- Rechtschreibschwäche bestätigt, bei der die Berücksichtigung im Ermessen der Lehrkräfte liegt. ${ }^{1417}$ Der VGH München hat eine inhaltliche Überprüfung der Diagnose des Facharztes nicht für zulässig gehalten und für die bayerische Regelung festgestellt, dass dem Schulpsychologen nicht die Rolle des "Obergutachters" zukommt, der sich ohne Rücksprache mit dem Facharzt über dessen Gutachten hinwegsetzen $\operatorname{kann}^{1418}$, und dass die Feststellung der Legasthenie ausschließlich der zuständige Facharzt trifft. ${ }^{1419}$

Im Prüfungsrecht, wo ärztliche Stellungnahmen insbesondere bei krankheitsbedingtem Rücktritt von der Prüfung vorzulegen sind, findet eine inhaltliche Überprüfung der Diagnose nicht statt. Die Prüfungsbehörde und auch die Schule haben nicht die erforderliche Sachkenntnis, um zu beurteilen, ob ein Prüfling prüfungsunfähig erkrankt ist und daher auch keinen Ermessensspielraum dahin, aufgrund der mitgeteilten Diagnose eine Krankheit zu bejahen

\footnotetext{
${ }^{1413}$ BY KMS VI.8-5 S 5300-6.108417 v. 8.12.2006 S. 2, http://www.best-news.de/?schul_ regelungen

${ }^{1414}$ Marwege, Zeitschrift des BVL, 2007, S. $36 \mathrm{ff}$

1415 BY KMBek Legasthenie Abschnitt IV Abs. 2

${ }^{1416}$ Die Blutabnahme, die ohnehin keinen Aussagewert hätte, da Legasthenie nicht am Blutbild festgestellt werden kann, kann analog § 65 SGB I verweigert werden, weil sie unverhältnismäßig und unzumutbar ist. Für den Hausbesuch fehlt es an der gesetzlichen Ermächtigung für ein derartiges Betretensrecht, s. LSG Hessen, Beschluss v. 30.1. 2006; Az.: L 7 AS 1/06 ER, NJW 2006, S. 1548 f; Bickel/Marwege/Zier, Ratgeber Finanzierung außerschulische Therapien, S. 21

${ }^{1417}$ Marwege, Zeitschrift des BVL, 2007, S. 36 ff, S. 36

${ }^{1418}$ VGH München, Beschluss v. 31. 7. 2001, Az.: 7 ZE 01.103, S. 7 (nicht veröffentlicht)

1419 VGH München, Urteil v. 17.10.2003, Az.: 7 B 02.2186, juris, Rn. 38
} 
oder zu verneinen. ${ }^{1420}$ Die Prüfungsbehörde entscheidet aufgrund der angegebenen Diagnose vielmehr nur über die Rechtsfrage, ob diese Erkrankung zum Rücktritt von der Prüfung berechtigt hat. ${ }^{1421}$ Nur deswegen wird im Prüfungsrecht die Angabe einer Diagnose gefordert und akzeptiert. ${ }^{1422}$

Gegen eine inhaltliche Überprüfungskompetenz der Schule spricht, dass es sich bei dem ärztlichen Attest um ein Sachverständigengutachten handelt. Es ist im Prozessrecht anerkannt, dass sich ein Gericht nur dann über ein Sachverständigengutachten hinwegsetzen kann, wenn es nachvollziehbar nachweist, dass es über Sachkunde verfügt, die dem Sachverständigen überlegen ist ( $\$ 108$ VwGO, § 103 SGG, § 286 ZPO). ${ }^{1423}$ Das Gleiche gilt für ein verwaltungsrechtliches Verfahren, in dem es um die Grundlagen für die Anwendung eines Grundrechts geht. Es ist offensichtlich, dass weder die Schulpsychologen noch die Schulärzte, noch Schulleitung oder Lehrkräfte über mehr Sachkunde verfügen als ein Facharzt, wenn es um die Diagnose der Legasthenie/Dyskalkulie geht. Eine inhaltliche Überprüfung der Diagnose des Facharztes steht innen daher nicht zu. Keiner dieser Personen kommt die Rolle des Obergutachters zu, der sich über das Gutachten des Facharztes hinwegsetzen kann. ${ }^{1424}$ Die Feststellung der Legasthenie/Dyskalkulie trifft ausschließlich der zuständige Facharzt. ${ }^{1425}$ Deshalb reicht es aus, dass der Schule die Diagnose mitgeteilt wird.

\subsubsection{Verhinderung von Gefälligkeitsgutachten?}

Kinder- und Jugendpsychiatern wird bisweilen der Vorwurf gemacht, sie stellten Gefälligkeitsgutachten aus. ${ }^{1426}$ Einer solchen Gefahr könnte entgegen gewirkt werden, wenn die Schule und die Schulbehörden die Diagnosen inhaltlich

1420 OVG Hamburg, Beschluss v. 18.11.2011, Az.: 1 Bs 208/11, NVwZ-RR 2012, S. 240 f; VGH Kassel, Beschluss v. 10.7.1989, Az.: 6 TP 1542/89, juris, Rn. 2; BVerwG, Beschluss v. 6.8.1996, Az.: 6 B 17/96, juris, Rn. 6

${ }^{1421}$ BVerwG, Beschluss v. 14.6.1983, Az.: 7 B 107/82, juris, Rn. 11; BVerwG, Beschluss v. 6.8.1996, Az.: 6 B 17/96, juris, Rn. 6

1422 Zimmerling, MedR 2001, S. 634 ff; einschränkend OVG Hamburg, Beschluss v. 18.11.2011, Az.: 1 Bs 208/11, NVwZ-RR 2012, S. 240 f, wonach ein ärztliches Attest grundsätzlich belegt, dass eine Krankheit vorliegt, die zur Schulunfähigkeit führt.

1423 Bader/Funke-Kaiser/Kuntze/von Albedyll, VwGO, § 108, Rn. 13; MeyerLadewig/Keller,/Leitherer, SGG, § 103, Rn. 7 b; Baumbach/Lauterbach/Albers/Hartmann, ZPO, § 286, Rn. 51

1424 VGH München, Beschluss v. 31. 7. 2001, Az.: 7 ZE 01.103, S. 7 (nicht veröffentlicht)

1425 VGH München, Urteil v. 17.10.2003, Az.: 7 B 02.2186, juris, Rn. 38

${ }^{1426}$ Büchner/Kortländer/Werner/Robering/Schönweiss, „Legasthenie - eine Krankheit, eine Behinderung, eine Störung?", S. 6 f; Valtin, Brauchen wir die Legasthenie?, S. 5; Eine Zurückweisung dieses Vorwurfs durch die Kinder- und Jugendpsychiater ließ sich nicht recherchieren. Es erstaunt sehr, dass die Kinder- und Jugendpsychiater sich nicht gegen diesen massiven Vorwurf wehren, der häufig auch in Verfahren zu § 35 a SGB VIII erhoben wird. 
überprüfen und ihnen dafür nicht nur die Diagnose, sondern auch die detaillierten einzelnen Testergebnisse aus der multiaxialen Diagnostik mitgeteilt würden. Die Mitteilung der detaillierten Testergebnisse des Kindes ist ein erheblicher Eingriff in sein Persönlichkeitsrecht, weil damit sehr persönliche Daten an die Schule weiter gegeben werden. ${ }^{1427}$ Wollte man allein einen Verdacht auf Gefälligkeitsgutachen als ausreichend ansehen, um diesen erheblichen Eingriff in das Persönlichkeitsrecht des Kindes und auch in die ärztliche Schweigepflicht zu rechtfertigen, müsste man konsequenterweise auch in anderen Bereichen wie der Arbeitsunfähigkeitsbescheinigung oder bei Attesten zur Prüfungsunfähigkeit alle Atteste, die Ärzte ausstellen, überprüfen, denn die Gefahr von Gefälligkeitsattesten oder -gutachten besteht in diesen Gebieten ebenso. ${ }^{1428}$ Den ärztlichen Attesten wird jedoch grundsätzlich vertraut. Das Recht auf informationelle Selbstbestimmung und die ärztliche Schweigepflicht reichen im Regelfall sogar so weit, dass noch nicht einmal die Diagnose, die Grundlage des Attests ist, genannt werden muss. ${ }^{1429}$ Die Adressaten des Attestes, z.B. Arbeitgeber oder Dienstherren, sind an das Attest gebunden, eine inhaltliche Überprüfung ist innen mangels Angabe der Diagnose gar nicht möglich. ${ }^{1430}$ Auch im Sozialrecht werden Diagnosen umfassend geschützt und dürfen selbst in Bereichen, in denen von den Diagnosen finanzielle Leistungen abhängen, nicht weitergegeben werden (§35 SGB I, § 69, § 100 SGB X). Üblicherweise sehen sich die Schulen und Schulbehörden an die Nachweise bei anderen Behinderungen gebunden, respektieren dort also die vorgelegten Atteste.

Die Bekanntgabe der Testergebnisse an die Schulen, um diese in die Lage zu versetzen, die Atteste inhaltlich zu überprüfen, würde bedeuten, dass man die diagnostizierenden Fachkräfte, anders als alle anderen Fachärzte und diagnostizierenden Fachkräfte, unter den Generalverdacht stellt, Gefälligkeitsgutachten zu erstellen. Ein solcher Generalverdacht gegen alle Kinder- und Jugendpsychiater ist nicht begründbar und lässt den gebotenen Respekt vor dieser Berufsgruppe vermissen. ${ }^{1431}$ Es ist nicht davon auszugehen, dass gerade diese Ärzte (überwiegend) unrichtige Gesundheitszeugnisse ausstellen, womit sie sich im Übrigen gem. § 278 StGB strafbar machen würden. Dies sieht auch der BFH so, der jüngst seine Rechtsprechung zur Abzugsfähigkeit von Behandlungskosten bei Lese-Rechtschreibschwäche dahin geändert hat, dass nunmehr auch privatärztliche und nicht nur amtsärztliche Gutachten zum Nachweis der Notwendigkeit der Behandlung ausreichen können. Ausdrücklich weist der BFH in

\footnotetext{
${ }^{1427}$ S. dazu auch unter Datenschutz, S. $309 \mathrm{ff}$

1428 S. dazu Niehues, Prüfungsrecht, Rn. 254

1429 Kühne, JA 1999, S. 523 ff, S. 523

1430 Ausführlich dazu Zimmerling, MedR 2001, S. $634 \mathrm{ff}$

${ }^{1431}$ Vgl. VG Ansbach, Beschluss v. 6.9.2007, Az.: AN 14 E 07.02227, juris, Rn. 40
} 
dieser Entscheidung die Vermutung zurück, die freie Ärzteschaft neige dazu, Gefälligkeitsgutachten auszustellen. ${ }^{1432}$

Der Konflikt zwischen dem Überprüfungsbedürfnis ärztlicher Angaben, die Rechtsfolgen auslösen können und dem Geheimhaltungsinteresse des Patienten besteht auch in anderen Bereichen. Im Arbeitsrecht ist er dahin gelöst worden, dass noch nicht einmal die Diagnose genannt werden darf (vgl. § 69 Abs. 4 SGB X), im Prüfungsrecht muss zwar die Diagnose, nicht aber die genaue Diagnostik angegeben werden. In beiden Fällen besteht eine tatsächliche Richtigkeitsvermutung bezüglich der ärztlichen Atteste. ${ }^{1433} \mathrm{Es}$ ist daher nichts dafür ersichtlich, dass es bei der Berufsgruppe der Kinder- und Jugendpsychiater und der Diagnostik der Legasthenie/Dyskalkulie anders und deshalb unerlässlich ist, die Atteste zu überprüfen.

\subsection{4. Überprüfung der Diagnosen durch die Schulen?}

Die Mitteilung von Testergebnissen an die Schule könnte erforderlich sein, weil die Diagnose Legasthenie/Dyskalkulie in der Schule zu einem Anspruch auf Veränderung der Prüfungsbedingungen führt und daher ein öffentliches Interesse daran besteht, diesen Anspruch nur den wirklich Betroffenen und nicht eventuellen "Trittbrettfahrern" zukommen zu lassen. Würden hier die schützenswerten Interessen des betroffenen Kindes und seine Interessen an der Geheimhaltung seiner höchstpersönlichen Daten gegenüber dem öffentlichen Interesse, dass nur bei „wirklich Berechtigten“ die Legasthenie/Dyskalkulie anerkannt wird, zurücktreten, würde dies im Ergebnis wieder auf einen generellen, nicht haltbaren Verdacht gegen die diagnostizierenden Fachkräfte hinauslaufen, dass sie Gefälligkeitsgutachten erstellen. Es liegen daher keine überwiegenden öffentlichen Interessen vor, die den Eingriff unerlässlich machen.

Schließlich kann die inhaltliche Überprüfung der Diagnosen auch nicht dadurch gerechtfertigt werden, dass sie mithilfe der Diagnosedaten auch für Nicht-Fachleute nachzuvollziehen sind und daher angeblich leicht auf ihre Plausibilität überprüft werden können. Dieser Irrglaube beruht darauf, dass die psychologischen Tests zur praktikablen Nutzung einfache Schablonen, Auswertungsschemata und Interpretationsskalen haben und deshalb auch für Laien gut handhabbar sind. Dies verleitet dazu, dass sie auch von Laien angewendet werden, obwohl ihnen i.d.R. das fachliche Hintergrundwissen fehlt. ${ }^{1434}$ Auch die Rechtsprechung ist dieser Versuchung schon erlegen. ${ }^{1435}$ So hat das VG Göttin-

\footnotetext{
1432 BFH, Urteil v. 11.11.2010, Az.:VI R 17/09, juris, Rn. 21

1433 Kühne, JA 1999, S. 523 ff, S. 524

1434 Zier, Recht für Diplom-Psychologen, S. 138

1435 Besonders bemerkenswert ist das Vorgehen des OLG Celle, das in einem Amtshaftungsverfahren nach Aktenlage festgestellt hat, dass das betroffene Kind definitionsgemäß kein Legastheniker ist, weil es angeblich einen erheblichen Sehfehler hatte. Diesen Sehfehler in
} 
gen in einer grundlegenden Entscheidung ${ }^{1436}$ zu § 35 a SGB VIII die Diagnosekriterien der ICD 10 und die Leitlinien der Deutschen Gesellschaft für Sozialpädiatrie und Jugendmedizin dargelegt und selbst angewandt. ${ }^{1437}$ Es verneinte die Legasthenie, weil der Prozentrang des Klägers im Rechtschreibtest bei 12 und nicht unter 10 lag. In einem späteren Urteil hat das VG Göttingen dann seine Rechtsprechung an die neu erlassen Leitlinien angepasst und das Regressionsmodell ${ }^{1438}$, das in der Diagnostik bestimmte Ausnahmefälle erfassen soll, selbst angewandt. ${ }^{1439}$ Auch das VG Darmstadt hat in einem schulrechtlichen Fall die Diagnosekriterien der Leitlinien selbst angewandt und anhand der dargelegten Testwerte dann errechnet, dass eine Legasthenie nicht vorliegt. ${ }^{1440}$ Die Komplexität und die Ergebnisse psychologischer Tests werden jedoch von Laien oft falsch eingeschätzt. ${ }^{1441}$ Deshalb wird von den Fachärzten auch darauf hingewiesen, dass die Diagnostik Fachwissen erfordert und sich nicht auf eine bloße Berechnung der Störung beschränkt. ${ }^{1442}$ Ebenso wenig wie sich die Diagnose des Bluthochdrucks allein aus einer Blutdruckmessung oder des Stotterns allein aus dem Anhören des Betroffenen ergibt, lässt sich die Legasthenie/Dyskalkulie ausrechnen. Sie ergibt sich erst aus der Zusammenschau vieler medizinischer und psychologischer Untersuchungen.

Für die Berücksichtigung der Legasthenie/Dyskalkulie reicht die Diagnose aus, auf die Einzelheiten der Diagnose kommt es nicht an. Infolgedessen ist als Nachweis nur ein Attest der diagnostizierenden Fachkraft über das Vorliegen der Legasthenie/Dyskalkulie ohne Angabe der Testwerte erforderlich.

Form der medizinisch hochumstrittenen Winkelfehlsichtigkeit hatte eine Sonderpädagogin festgestellt. OLG Celle, Beschluss v. 17.2.2010, Az.: 16 U 62/09, S. 4 (nicht veröffentlicht); zur fehlenden eigenen Sachkunde des Finanzamtes oder Finanzgerichts zur Feststellung einer Legasthenie und der Notwendigkeit der Zuziehung eines Sachverständigen s. BFH, Urteil v. 11.11.2010, Az.:VI R 17/09, juris, Rn. 27; zur wissenschaftlichen Fundierung verschiedener „alternativer" Therapieformen: Bundesverband Deutscher Augenärzte, Legasthenie und Sehvermögen und Suchodoletz, Therapie der Lese-Rechtschreib-Störung, S. $184 \mathrm{ff}$

${ }^{1436}$ VG Göttingen, Urteil v. 26.1.2006, Az.: 2 A 161/05, www.rechtsprechung.niedersachsen.de das Urteil ist zwar zu § 35 a SGB VIII ergangen, zeigt aber den Umgang mit den Tests und Diagnosekriterien auf.

${ }^{1437}$ VG Göttingen, Urteil v. 26.1.2006, Az.: 2 A 161/05, www.rechtsprechung.niedersachsen.de, S. 5

${ }^{1438}$ S. oben 1. Teil: 1. Kapitel: 5.2.: Medizinische Diagnostik nach ICD 10 und Multiaxialem Klassifikationsschema (MAS), S. 16

${ }^{1439}$ VG Göttingen, Urteil v. 10.7.2007, Az.: 2 A 483/05, www.rechtsprechung.niedersachsen.de, S. 5

1440 VG Darmstadt, Urteil v. 28. 4. 2009, Az.: 7 K 687/08.DA (3), S. 13 f (nicht veröffentlicht)

${ }^{1441}$ Zier, Recht für Diplom-Psychologen, S. 138

1442 http://www.kjp.med.uni-muenchen.de/forschung/legasthenie/diagnose.php 


\subsubsection{Gleichbehandlung aller Behinderungen}

Bei allen anderen Behinderungen wird die Funktionsstörung durch medizinische Diagnosen nachgewiesen, der Schule steht es nicht zu, diese Diagnose selbst durchzuführen oder zu verändern. Würden die medizinischen Diagnosen Legasthenie/Dyskalkulie durch die pädagogische Einschätzung relativiert oder nur bei innen die Diagnose durch die Schule inhaltlich überprüft, so würden diese Behinderungen gegenüber allen anderen Behinderungen ungleich behandelt. Es ist jedoch kein sachlicher Grund dafür ersichtlich, dass die Behinderungen Legasthenie/Dyskalkulie insoweit anders zu behandeln sind und abweichend von allen anderen Behinderungen hier die pädagogische Einschätzung eines medizinischen Sachverhaltes maßgeblich ist. Eine solche Ungleichbehandlung würde gegen Art. 3 I GG verstoßen.

\subsubsection{Geeignete Fachkräfte für die Diagnostik?}

Erforderlich ist daher eine medizinisch-psychologische Diagnostik der Legasthenie/Dyskalkulie. Das allgemeine Persönlichkeitsrecht aus Art. 2 Abs. 1 GG i.V.m. Art. 1 Abs. 1 GG schützt grundsätzlich vor der Erhebung von Befunden über den Gesundheitszustand, die seelische Verfassung und über den Charakter einer Person. ${ }^{1443}$ Die Untersuchung und Testung ist daher stets ein Eingriff in das Grundrecht des Kindes. ${ }^{1444}$ Nach der Rechtsprechung des BVerfG liegt bereits in der Ankündigung, dass ohne Testung keine Berücksichtigung der Behinderung erfolgt, ein Eingriff. ${ }^{1445}$ Der Eingriff ist besonders schwer, weil es sich nicht nur um eine oberflächliche, äußere Untersuchung handelt, sondern um eine umfassende psychologische Untersuchung. ${ }^{1446}$ Daher sind strenge Anforderungen an die Verhältnismäßigkeit einer Maßnahme im Einzelfall und somit an die Eignung der diagnostizierenden Fachkräfte zu stellen.

Die Frage, wer zur Feststellung von Legasthenie/Dyskalkulie geeignet ist, hat den Gesetzgeber im Rahmen der Eingliederungshilfe nach $\S 35$ a SGB VIII beschäftigt. Dort ist in $\S 35$ a Abs. 1 a SGB VIII ausdrücklich geregelt worden, wer die Diagnostik durchführen darf und dass sie auf der Grundlage der ICD 10 zu erfolgen hat. Auch wenn $\S 35$ a SGB VIII die Eingliederungshilfe für seelische Behinderungen regelt, die nicht deckungsgleich mit einer Legasthenie/Dyskalkulie sind, sind hier doch fachliche Anforderungen und Standards für den Bereich dieser Behinderungen geregelt worden. Es bietet sich daher an, diese Anforderungen und Standards zu übernehmen und analog für den Bereich der Feststellung der Legasthenie/Dyskalkulie anzuwenden. Dafür spricht auch, dass

\footnotetext{
1443 BVerfGE 89, 69, Rn. 51

1444 Zier, Recht für Diplom-Psychologen, S. 139

1445 BVerfGE 89, 69, Rn. 57

${ }^{1446}$ Vgl. zu medizinisch-psychologischen Untersuchung nach FeV BVerfGE 89, 69, Rn. 54 f
} 
Verfahrensfragen

die ICD 10 und die darauf aufbauenden Leitlinien der Deutschen Gesellschaft für Kinder- und Jugendpsychiatrie und Psychotherapie von $2007^{1447}$ den Standard der Diagnostik für die Fachärzte vorgeben und einen haftungsrechtlich relevanten Sorgfaltsverstoß indizieren, wenn der Arzt gegen sie verstoßen hat. ${ }^{1448}$

Allerdings besteht das praktische Problem, dass die Versorgung in Deutschland mit Kinder- und Jugendpsychiatern zum Teil sehr knapp ist. Statistisch kommen auf einen ambulant tätigen Kinder- und Jugendpsychiater ca. 20.700 Kinder. Die Versorgung ist je nach Bundesland sehr unterschiedlich und zum Teil sehr gering, z.B. gab es 2007 in Mecklenburg-Vorpommern nur 7 niedergelassene Kinder- und Jugendpsychiater. ${ }^{1449}$ Insofern würde es zu sehr langen Verfahrensdauern kommen, wenn allein die Kinder- und Jugendpsychiater die Diagnostik durchführen dürften. Bayern hat in einer Ergänzung der bayerischen Regelung den fachlich qualifizierten Personenkreis für diese Diagnostik auf sozialpädiatrische Zentren, Kinderärzte mit kinder- und jugendpsychiatrischer Weiterbildung, approbierte Psychologische Psychotherapeuten und approbierte Kinder- und Jugendlichenpsychotherapeuten, soweit sie entsprechende Kenntnisse nachweisen, erweitert. ${ }^{1450}$ In Betracht gezogen werden kann auch, die Diagnose der Legasthenie/Dyskalkulie durch Dyslexie- oder Dyskalkulie-Therapeuten nach $B V L^{{ }^{1451}}$ oder andere anerkannte Therapeuten zuzulassen, deren Ausbildung zwingend und nachweisbar auch den Bereich der Diagnostik enthält. Ein Interessenkonflikt wie bei § 35 a SGB VIII, der sich daraus ergibt, dass die Diagnose die (vom Jugendamt zu finanzierende) Therapie bei dem diagnostizierenden Therapeuten nach sich zieht, und der zum Ausschluss dieser Berufsgruppen aus dem Kreis der zugelassenen Diagnostiker geführt hat ${ }^{1452}$, ist hier nicht zu befürchten.

Denkbar wäre, die Diagnostik wegen der schulrechtlichen Folgen der Atteste allein Amtsärzten zu übertragen. Voraussetzung dafür wäre jedoch, dass diese für eine Diagnostik gem. der ICD 10 und der Leitlinien der Kinder- und Jugendpsychiater qualifiziert sind. Das wird i.d.R. nicht der Fall sein, weil die wenigsten Amtsärzte von ihrer fachlichen Qualifikation her Kinder- und Ju-

\footnotetext{
1447 Leitlinien, Leitlinien Diagnostik und Therapie

1448 Umfassender Überblick bei Martis/Winkhart-Martis, Arzthaftungsrecht, Rn. B 41 ff, insbesondere Rn. B 72

1449 Berufsverband für Kinder-und Jugendpsychiatrie, Statistische Daten, Folie 7 und 8

1450 KMS IV/1 - 7306/4 - 4/55 382 v. 09.08.2000, http://www.schulberatung.bayern.de/schulberatung/index_05168.asp

${ }^{1451}$ S. dazu die Anforderungen im theoretischen Teil der Ausbildung zum Dyslexie- oder Dyskalkulie-Therapeuten nach BVL ${ }^{\circledR}$ (Bundesverband Legasthenie und Dyskalkulie e.V.), http://www.bvl-legasthenie.de/index.php5?p=/zertifizierung/anforderungen

1452 Wiesner, in: SGB VIII, § 35 a, Rn. 12
} 
gendpsychiater sein dürften. Zudem ist nicht ersichtlich, aus welchen Gründen nur Amtsärzte die erforderliche Sachkunde für die Diagnostik haben sollten. ${ }^{1453}$ Die Beschränkung der Diagnostik auf Amtsärzte würde außerdem das Problem weiter verschärfen, dass es nicht genügend Fachkräfte für die Diagnostik gibt. Die Wartezeiten für die betroffenen Kinder würden sehr lang werden und Hilfen in der Schule könnten erst sehr spät, vielfach wohl auch zu spät, einsetzen.

Fraglich ist, ob die Diagnostik auch durch schulische Fachkräfte durchgeführt werden kann. Mitarbeiter des sonderpädagogischen Dienstes haben i.d.R. keine medizinisch-psychologische Ausbildung, so dass innen die fachliche Kompetenz zur Diagnostik von Legasthenie/Dyskalkulie fehlen dürfte und sie daher i.d.R. nicht geeignet sein werden. ${ }^{1454}$ Geeignete Fachkräfte können insoweit dann nur noch Schulpsychologen sein, wenn sie die Befähigung zur Diagnostik nach ICD 10 besitzen. Ob dies der Fall ist, kann nicht allgemein festgestellt werden, denn es gibt keine einheitliche Ausbildung für Schulpsychologen. Überwiegend sind sie ausgebildete Psychologen, gelegentlich sind sie aber auch Lehrer mit einem Studienschwerpunkt in Psychologie. ${ }^{1455}$ Maßgeblich für die Einschätzung der Eignung einer Fachkraft ist der Sinn und Zweck der Diagnostik und der mit der Untersuchung verbundene Eingriff in das Persönlichkeitsrecht des Kindes. Die Untersuchung durch eine unqualifizierte Person ist ein Eingriff in das Persönlichkeitsrecht des Kindes, der nicht gerechtfertigt werden kann.

Ungeklärt ist die Frage der Finanzierung der Diagnose. Von den Krankenkassen werden Untersuchungen durch Kinder- und Jugendpsychiater, die allein die Diagnose Legasthenie/Dyskalkulie zum Ziel haben, nicht gezahlt, weil Legasthenie/Dyskalkulie nicht als Krankheiten anerkannt sind. ${ }^{1456}$ Eine Kostenübernahme erfolgt nur, wenn das Kind wegen ungeklärter schulischer Probleme vorstellig wird. Deshalb müssen die Eltern die erheblichen Kosten für die Diagnostik, die dann im Rahmen von Privatabrechnungen erfolgt, i.d.R. selber tragen. Das aber wird zwangsläufig dazu führen, dass Kinder aus Familien, die sich diese Ausgabe nicht leisten können, nicht diagnostiziert werden und folglich ihre Behinderung in der Schule auch nicht berücksichtigt wird. Ein solches Ergebnis wäre weder mit dem Sozialstaatsgebot noch mit dem Sinn des Behindertenrechts vereinbar, wonach die Kosten für Leistungen, die erforderlich sind, um ein selbstbestimmtes Leben zu fördern und Benachteiligung zu vermeiden, nicht von den Behinderten selbst zu tragen sind. ${ }^{1457}$

\footnotetext{
1453 Vgl. BFH, Urteil v. 11.11.2010, Az.:VI R 17/09, juris, Rn. 21

1454 VG Weimar, Beschluss v. 3.5.2010, Az.: 2 E 377/10, S. 2 (nicht veröffentlicht)

1455 http://www.bdp-schulpsychologie.de/aktuell/2008/80410_ausbildung.php

1456 S. oben: 1. Teil: 2. Kapitel: 7.2.1.: Finanzierung durch die Krankenkassen nach § 27 SGB VS. 46

${ }^{1457}$ Vgl. VG Regensburg, Urteil v. 10.11.2004, Az.: S 14 KR 38/04, JAmt 2005, S. 89, 90; Mrozynski, in: SGB VIII, § 35 a, Rn. 16
} 
Es kann im Rahmen dieser Arbeit nicht geklärt werden, wie die Finanzierung der Diagnostik zu erfolgen hat. Es lässt sich aber folgender Grundsatz darstellen: Aus dem Grundrechtsschutz durch Verfahren und Organisation folgt als unverzichtbare Mindestanforderung eines Verfahrens, dass es gewährleisten muss, dass begründete Ansprüche durchgesetzt werden können. ${ }^{1458}$ Dies ist aber nur auf der Grundlage einer Diagnostik möglich, so dass auch die Finanzierung der Diagnostik vom Staat geregelt werden muss. Der Anspruch auf Finanzierung der Diagnostik ergibt sich aus Art. 1 Abs. 1 GG i.V.m. dem Sozialstaatsprinzip des Art. 20 Abs. 1 GG, denn ohne Deckung dieser Kosten droht den Kindern der Ausschluss von Lebenschancen, weil ohne die Feststellung der Legasthenie/Dyskalkulie diese in der Schule nicht berücksichtigt wird. Die Deckung dieses Bedarfs gehört zu ihrem existentiellen Bedarf. Es besteht sonst die Gefahr, dass ohne hinreichende staatliche Leistungen ihre Möglichkeiten eingeschränkt werden, am Leben und vor allem an der Schule teilzunehmen. ${ }^{1459}$

\subsubsection{Feststellung nur durch eine von der Schule vorgegebene Fachkraft?}

In der Praxis stellt sich weiter die Frage, ob die Schule bzw. Schulverwaltung vorgeben darf, dass die Diagnostik der Legasthenie/Dyskalkulie nur durch eine von ihr bestimmte Fachkraft oder Institution erfolgen darf. Diese Problematik gibt es nicht nur im Schulrecht, sondern vor allem auch im Sozialrecht. Dort bestimmen $\S 14$ Abs. 5 S. 1 SGB IX und $\S 200$ Abs. 2 SGB VII, dass dem Betroffenen in der Regel mindestens drei Sachverständige zur Auswahl genannt werden müssen. ${ }^{1460}$ Damit wird der Tatsache Rechnung getragen, dass es sich bei der Untersuchung um einen erheblichen Eingriff handelt und der Betroffene wenigstens ein gewisses Wahlrecht haben muss, von wem er die Untersuchung durchführen lässt. Die Vorgabe, sich in sozialrechtlichen Angelegenheiten nur bei einer bestimmten Stelle untersuchen zu lassen, verstößt gegen dieses Wahlrecht. ${ }^{1461}$ Im Schulrecht muss Entsprechendes gelten. Die Beschränkung der Feststellung auf eine bestimmte Fachkraft oder Fachstelle ist daher nicht zulässig.

Die diagnostizierenden Fachkräfte werden als Sachverständige gemäß § 26 VwVfG im Verwaltungsverfahren tätig. ${ }^{1462}$ Möglich ist es daher, dass die Schule bzw. Schulbehörde eine diagnostizierende Fachkraft wegen Befangenheit nach

\footnotetext{
1458 Kopp/Ramsauer, VwVfG, Einführung I, Rn. 21

1459 Vgl. BVerfG, Urteil v. 9.2.2010, Az.: 1 BvL 1/09 „Hartz IV“, http://www.bverfg.de/entscheidungen/ls20100209_1bvl000109.html, Rn. 192, 198

1460 Bickel/Marwege/Zier, Ratgeber Finanzierung außerschulische Therapien, S. 10; Mrozynski, in: SGB VIII, § 35 a, Rn. 15

1461 VG Göttingen, Urteil v. 26.1.2006, Az.: 2 A 161/05, www.rechtsprechung.niedersachsen.de

1462 So auch Ennuschat, Die Rechte stotternder Menschen in Schule, Ausbildung und Studium, S. 172
} 
$\S 21$ VwVfG ablehnt, wenn Gründe vorliegen, die Zweifel an ihrer Unparteilichkeit begründen. Das Verfahren richtet sich nach $\S \S 21 \mathrm{VwVfG}, 54 \mathrm{VwGO}, 44 \mathrm{ff}$ ZPO. Vor einer Ablehnung ist der Sachverständige anzuhören ( $§ 28 \mathrm{VwVfG}$ ). Ob die Ablehnung gegenüber den Beteiligten des Verwaltungsverfahrens, also dem betroffenen Schüler, ein Verwaltungsakt ist, ist streitig. ${ }^{1463}$

\subsection{Nachweis der Legasthenie/Dyskalkulie}

Der Nachweis der Diagnose Legasthenie/Dyskalkulie ist gegenüber der Schule nach § $26 \mathrm{VwVfG}$ zu erbringen.

Üblicherweise genügt zur Anerkennung einer Behinderung in der Schule ein ärztliches Attest. Legasthenie/Dyskalkulie werden von den Kultusministerien jedoch nicht als Behinderung angesehen, deshalb ist die Anerkennung jeweils speziell geregelt. In Bayern ist als Nachweis einer Legasthenie die Stellungnahme eines diagnostizierenden Kinder- und Jugendpsychiaters erforderlich, die dem Schulpsychologen vorgelegt wird. In den anderen Bundesländern ist der Nachweis durch solche außerschulischen Diagnosen nicht möglich, da die Schulen an diese, soweit sie sie überhaupt einbeziehen müssen, nicht gebunden sind.

Da nach der hier vertretenen Ansicht die Diagnostik durch die Kinder- und Jugendpsychiater oder ihnen gleichgestellte Fachkräfte erfolgen muss, ist der Nachweis einer Legasthenie/Dyskalkulie durch eine entsprechende Stellungnahme dieser Fachkräfte zu führen.

Ein Nachweis ist weiter möglich durch einen Bescheid über die Feststellung der Behinderung gem. § 69 Abs. 1 SGB IX und durch die dieser Feststellung gleichgestellten Feststellungen gem. § 69 Abs. 2 SGB IX. Im Verfahren auf Feststellung der Behinderung nach § 69 Abs. 1 SGB IX wird die Behinderung nach den Anhaltspunkten für die ärztliche Gutachtertätigkeit (AHP) auf der Grundlage einer umfassenden Begutachtung der vorgelegten Befundberichte festgestellt. ${ }^{1464}$ In den AHP sind unter Punkt 26.3 auch die Legasthenie und Dyskalkulie (Letztere als isolierte Rechenstörung) aufgeführt. Mit der Feststellung nach $\S \S 2,69$ SGB IX wird daher das Vorliegen der Funktionsstörung Legasthenie/ Dyskalkulie bescheinigt. ${ }^{1465}$ Dieser Bescheid ist nach § 26 । Nr. 3 VwVfG i.V.m.

\footnotetext{
${ }^{1463}$ Vgl. Kopp /Ramsauer, VwVfG, § 21, Rn. 21 m.w.N.

1464 Die rechtliche Legitimation für die AHP ergibt sich inzwischen aus $\S 2$ VersorgungsmedizinVerordnung vom 10.12.2008; zur umstrittenen rechtlichen Legitimation der AHP s. Welti, in: Welti/Lachwitz/Schellhorn, HK-SGB IX, § 2, Rn. $43 \mathrm{ff}$

1465 S. VG Kassel, Beschluss v. 23.3.2006, 3 G 419/06, Sonderheft Recht des BVL: Chancengleichheit herstellen - Diskriminierung vermeiden, 2006, http://www.bvl-legasthenie.de/ index.php5?p=/downloads, S. 40
} 
$\S 418$ ZPO eine öffentliche Urkunde, die gegenüber jedermann den vollen Beweis der Behinderung bzw. Schwerbehinderung erbringt. ${ }^{1466}$

Schulen und Kultusministerien haben die Feststellung der Behinderung nach $\S \S 2,69$ SGB IX häufig nicht als Feststellung ausreichen lassen, weil die Behinderung im Schulrecht anders als im Sozialrecht zu definieren sei. Einen derartigen Widerspruch enthält z.B. § 6 SH ZeugnisVO, der in Abs. 1 auf $\S 2$ SGB IX Bezug nimmt, in Abs. 4 aber eine Sonderregelung für Schüler mit Legasthenie trifft. Wie oben gezeigt, ist der Behinderungsbegriff verfassungsrechtlich auszulegen und sehr weit gefasst. Er ist im Schulrecht nicht anders zu definieren als im Sozialrecht. Daraus ergibt sich, dass jedenfalls die Behinderungen, die nach $\S \S 2,69$ SGB IX festgestellt worden sind, auch im Schulrecht als Behinderungen anzuerkennen sind und daher ihr Nachweis auch durch die Vorlage der Feststellung nach $\S \S 2,69$ SGB IX geführt ist.

\subsection{Mitteilung der Diagnose}

Ebenso wie andere Behinderungen muss die Legasthenie/Dyskalkulie in geeigneter Form gegenüber der Schule nachgewiesen werden. Mit dem Nachweis muss belegt werden, dass der betroffene Schüler eine Funktionsstörung i.S. des Behinderungsbegriffes hat. Problematisch ist hierbei, dass den Schulbehörden eine medizinische Diagnose mitgeteilt werden muss.

Das Recht auf informationelle Selbstbestimmung und die ärztliche Schweigepflicht reichen im Regelfall so weit, dass gegenüber Dritten noch nicht einmal die Diagnose, die Grundlage eines Attestes ist, genannt werden muss ${ }^{1467}$, denn die medizinische Diagnose stellt nach allgemeiner Ansicht ein persönliches Geheimnis des Einzelnen dar. ${ }^{1468}$ Der besondere Schutz dieses Geheimnisses zeigt sich an $\S 203$ StGB, der die Bekanntgabe einer medizinischen Diagnose durch einen Arzt ohne Einwilligung des Betroffenen als Verletzung der Geheimnis- und Individualsphäre des Einzelnen unter Strafe stellt. ${ }^{1469}$ Nur wenn der Patient den Arzt von der Schweigepflicht entbunden hat, ist die Bekanntgabe erlaubt. Der besondere Schutz der Diagnosen zeigt sich auch im Sozialrecht, wo das Sozialgeheimnis aus § 35 SGB I, § 69 und aus 100 SGB X die Weitergabe von Diagnosen prinzipiell verbietet.

Im Schulbereich kann jedoch nicht von der Mitteilung einer Diagnose abgesehen werden, weil nur mit einer Diagnose eine adäquate und gezielte Berücksichtigung der Behinderung in der Schule möglich ist. In der Regel entbinden

\footnotetext{
${ }^{1466}$ Zur Beweiskraft von Urkunden im Verwaltungsverfahren Kopp/Ramsauer, VwVfG, § 26, Rn. 34; Kallerhoff in Stelkens/Bonk/Sachs, VwVfG, § 26, Rn. 88, zur Beweiskraft der Feststellung der Behinderung, BAG, Urteil v. 25.4.2007, Az.: 6 AZR 436/05, juris, Rn. 28

1467 Kühne, JA 1999, S. 523 ff

${ }^{1468}$ Vgl. nur Schünemann, in: Leipziger Kommentar zum StGB, § 203, Rn. 20

1469 Zimmerling, MedR 2001, S. 634 ff, Punkt II. 1.
} 
die Eltern und Schüler den untersuchenden Facharzt von der Schweigepflicht und erlauben inm die Bekanntgabe der Diagnose Legasthenie/Dyskalkulie an die Schule oder die Schulbehörden.

\subsection{Mitwirkungspflicht § $26 \| \mathrm{VwVfG}$}

Nach § 26 II VwVfG haben die Betroffenen die Pflicht, im Verwaltungsverfahren mitzuwirken. Für den Umfang der Mitwirkungspflichten im Schulrecht liegt es nahe, sich an $\S \S 60$ ff SGB I zu orientieren, weil dort ebenfalls höchstpersönliche, individuelle Mitwirkungspflichten sowie ihre Grenzen und die Folgen fehlender Mitwirkung geregelt sind. Demnach besteht eine Mitwirkungspflicht, es sei denn die Pflicht ist unverhältnismäßig (§ 65 I SGB I). Untersuchungen können darüber hinaus nach $\S 65$ I SGB I abgelehnt werden. Die fehlende Mitwirkung kann zur Versagung der begehrten Leistung führen (§ 66 I SGB I), allerdings nur dann, wenn der Betroffene schriftlich und unter Fristsetzung auf diese Mitwirkungspflicht hingewiesen worden ist (§ 66 III SGB I). Die Leistung kann nicht dauerhaft entzogen werden, sondern muss erbracht werden, wenn der Betroffene seine Mitwirkung nachholt ( $\$ 67$ SGB I).

Für den Schulbereich bedeutet dies, dass die Betroffenen bei dem Verfahren zur Anerkennung mitwirken müssen, aber nur zu solchen Handlungen und Untersuchungen verpflichtet sind, die verhältnismäßig und zumutbar sind. ${ }^{1470}$ Auf diese Mitwirkungspflicht muss schriftlich hingewiesen werden, wenn an die fehlende Mitwirkung rechtliche Folgen geknüpft werden sollen.

\subsection{Akteneinsicht}

Es ist für Eltern nicht immer leicht, Akteneinsicht in die Schul- oder Schülerakten ihres Kindes zu erlangen. Gelegentlich wird sie ihnen auch mit dem Hinweis auf den Datenschutz verweigert. Das Recht auf Akteneinsicht steht den Eltern als Beteiligten gem. § 29 VwVfG sowie nach den Landesdatenschutzgeset$z^{14}{ }^{1471}$, den jeweiligen Schulgesetzen ${ }^{1472}$ und weiteren schulrechtlichen Normen zu. ${ }^{1473}$ Es gehört als Verfahrensgrundsatz zum fairen Verwaltungsverfahren. $^{1474}$ Die Akteneinsicht erstreckt sich auf alle einen Verwaltungsakt betreffenden Informationen, insbesondere auch auf "Behördeninterna“, also Besprechungsprotokolle, Bearbeitungsvermerke usw. ${ }^{1475}$ Eine Beschränkung der Ak-

\footnotetext{
1470 Vgl. etwa die Regelung in HH SchulG $\S 34$ II

1471 Z.B. NI DSG § 16; SN DSG § 18

1472 Z.B. HH SchulG § 32 III umfasst i.V.m. § 33 II 4 auch die Einsicht in Untersuchungsunterlagen; NRW SchulG § 122 IV; TH SchuO § 136

1473 Z.B. BY KMBek Datenschutz Nr. 7; NRW VO-DV I § 3

1474 Schmidt-Aßmann, in: Maunz/Dürig/Herzog GG, Art. 19, Rn. 256

1475 Bohl, NVwZ 2005, S. $133 \mathrm{ff}$
} 
teneinsicht auf Teile der Akten kann nach § 29 ॥ VwVfG nur erfolgen, soweit einer vollständigen Akteneinsicht zwingende Normen, insbesondere zu schützende Rechte Dritter entgegenstehen, wobei Behördenmitarbeiter, speziell Lehrkräfte an der Schule, nicht Dritte in diesem Sinne sind.

Ob die Akteneinsicht das Recht beinhaltet, gegen Erstattung der Kosten Kopien aus der Akte zu machen, ist streitig. ${ }^{1476}$ Die herrschende Meinung nimmt an, dass das Recht auf Akteneinsicht keinen solchen Rechtsanspruch gibt, wenn eine spezialgesetzliche Regelung fehlt. ${ }^{1477}$ Die Entscheidung über die Möglichkeit Kopien anzufertigen, steht danach im Ermessen der Behörde. Demgegenüber vertritt der Datenschutzbeauftragte des Landes Brandenburg die Auffassung, dass das Recht auf Akteneinsicht auch das Recht einschließt, die Information in verwendbarer Weise zu erhalten. Es bestehe kein qualitativer Unterschied zwischen der Einsicht, der Fertigung von Notizen oder dem Fotokopieren der eingesehenen Dokumente. ${ }^{1478}$ Die Entwicklung des einfachen Rechts geht dahin, nicht nur den Betroffenen, sondern mehr und mehr sogar jedermann das Recht auf Akteneinsicht einzuräumen (vgl. § 1 Informationsfreiheitsgesetz (IFG) $)^{1479}$, das auch das Recht beinhaltet, sich Notizen zu machen oder Kopien anzufertigen ( $\$ 7$ Abs. 4 IFG). Dies spricht für die Ansicht, dass das Recht auf Akteneinsicht auch das Recht beinhaltet, Kopien anzufertigen. Jedenfalls haben Rechtsanwälte als Organ der Rechtspflege das Recht, Kopien anzufertigen. ${ }^{1480}$

Geht man jedoch mit der herrschenden Meinung von einer Ermessensentscheidung aus, so ist diese regelmäßig aufgrund des Grundsatzes des fairen bürgerfreundlichen Verfahrens auf null reduziert. ${ }^{1481}$ Dieser Grundsatz verlangt, dass die Waffengleichheit der Beteiligten gesichert wird ${ }^{1482}$ und Verfahrensrechte tatsächlich auch wahrgenommen werden können, vor allem dann, wenn die Behörde die notwendigen Informationen monopolisiert hat ${ }^{1483}$ und diese zur effizienten Rechtsverfolgung unverzichtbar sind. ${ }^{1484}$ Die Informationen über

1476 Bohl, NVwZ 2005, S. 133 ff, S. 136

1477 Bonk/Kallerhoff, in: Stelkens/Bonk/Sachs, VwVfG, § 29, Rn. 84; Ritgen, in: Knack/Hennecke VwVfG, § 29, Rn. 36

${ }^{1478}$ Zum Anspruch nach dem DSG Brandenburg: http://www.Ida.brandenburg.de/ sixcms/detail.php?gsid=5lbm1.c.65425.de\&template=themen_e

${ }^{1479}$ Aßmann, in: BeckOK GG, Art. 19 GG, Rn. 256

1480 Bohl, NVwZ 2005, S. 133 ff, S. 137

${ }^{1481}$ VG Freiburg, Beschluss v. 20.11.2009; Az.: 4 K 2096/09, www.landesrecht-bw.de; Rn. 6; vgl. Niehues, Prüfungsrecht, Rn. 201

${ }^{1482}$ Kopp/Ramsauer, VwVfG, Einführung I, Rn. 21

${ }^{1483}$ Aßmann, in: BeckOK GG, Art. 19 GG, Rn. 256

1484 Bonk/Kallerhoff, in: Stelkens/Bonk/Sachs, VwVfG, § 29, Rn. 85; VG Freiburg, Beschluss V. 20.11.2009; Az.: 4 K 2096/09, www.landesrecht-bw.de; Rn. 11; Zimmerling/Brehm, Prüfungsrecht, Rn. 411, $417 \mathrm{f}, 420$ 
den Umgang der Schule mit der Legasthenie/Dyskalkulie des Schülers, ihre Berücksichtigung oder Nicht-Berücksichtigung befinden sich nur in den Akten der Schule. Eltern und die betroffenen Schüler und Schülerinnen selbst können ihre Rechte nur effizient wahrnehmen, wenn sie diese Akten nicht nur einsehen können, sondern zu Beweiszwecken auch kopieren und damit in gerichtlichen Verfahren vorlegen können. Unter diesen Umständen besteht daher trotz des Ermessensspielraums die Pflicht, Kopien gegen Kostenerstattung zu ermöglichen.

Das Akteneinsichtsrecht ist im Wege des vorläufigen Rechtsschutzes nach $\S 123$ VwGO durchzusetzen. § 44 a VwGO steht dem nicht entgegen, da die Verweigerung der Akteneinsicht keine Verfahrenshandlung auf dem Weg zur Sachentscheidung ist, sondern Voraussetzung, um den Anspruch geltend machen zu können. ${ }^{1485}$

\subsection{Weitere Verfahrensrechte}

Eltern haben aus Art. 6 II S. 1 GG einen allgemeinen Informationsanspruch. ${ }^{1486}$ Im Verfahren auf Berücksichtigung einer Behinderung sind sie darüber hinaus als Vertreter ihres Kindes Beteiligte gem. § $13 \mathrm{VwVfG}$ und haben die allgemeinen Verfahrensrechte aus dem VwVfG. Die Beteiligten sind gem. § $28 \mathrm{VwVfG}$ vor Erlass des Verwaltungsaktes anzuhören. Sie können sich gem. § 14 I VwVfG durch einen Bevollmächtigten vertreten lassen. Außerdem können sie sich gemäß § 14 IV VwVfG von einem Beistand zu Verhandlungen und Besprechungen mit der Schule oder Schulbehörde begleiten lassen. Beistand ist dabei jede Person, die den Beteiligten begleitet. Der Beistand kann von der Schule bzw. Schulbehörde nur dann zurückgewiesen werden, wenn die Voraussetzungen des $\S 14$ VI VwVfG gegeben sind, der Beistand also im mündlichen Gespräch nicht zum sachgemäßen Vortrag befähigt ist.

Nach § 37 II VwVfG kann die Entscheidung über die Anerkennung einer Behinderung zwar mündlich ergehen kann, sie ist aber auf Verlangen der Betroffenen schriftlich zu bestätigen ist. Aus Beweisgründen sollte die Entscheidung immer schriftlich erfolgen.

Nach § 39 I VwVfG sind die wesentlichen tatsächlichen und rechtlichen Entscheidungsgründe, sowie bei Ermessensentscheidungen die Ermessensgesichtspunkte auszuführen. ${ }^{1487}$

Der Verwaltungsakt muss eine Rechtsbehelfsbelehrung nach $\S 58$ VwGO halten, wenn er die Frist nach $\S 74$ VwGO in Gang setzen soll. Ansonsten gilt

\footnotetext{
1485 VG Freiburg, Beschluss v. 22.1.2009, Az.: 4 K 2096/09, www.landesrecht-bw.de; Rn. 3

${ }^{1486}$ Avenarius/Heckel, Schulrecht, Ziff. 24.35 Badura, in: Maunz/Dürig/Herzog GG, Art. 7, Rn. 23;

Niehues, Prüfungsrecht, Rn. 210

${ }^{1487}$ Zu den Grundlagen s. Schmidt-Aßmann, in: Maunz/Dürig/Herzog GG, Art. 19, Rn. $253 \mathrm{ff}$
} 
die Jahresfrist nach $\S 58$ II VwGO. Die Bekanntgabe des Verwaltungsaktes muss nach $₫ 41 \mathrm{VwVfG}$ erfolgen.

\section{Materielle Rechtmäßigkeit}

Rechtsgrundlage für den begünstigenden Verwaltungsakt auf Anerkennung der Legasthenie/Dyskalkulie ist Art. 3 III 2 GG i.V.m. den landesrechtlichen Bestimmungen zur Konkretisierung dieses Grundrechts.

\subsection{Grundentscheidung: Gebundener VA oder Ermessensentscheidung?}

Fraglich ist, ob sich aus Art. 3 III 2 GG ein unbedingter Anspruch auf Anerkennung der Behinderung ergibt oder ob es zulässig ist, der Schulverwaltung bei der Anerkennung, also bei der Grundentscheidung, ein Ermessen einzuräumen.

Nur in Bayern besteht bisher ein Anspruch auf Anerkennung der Legasthenie, wenn die Stellungnahme der Kinder- und Jugendpsychiater eine solche bescheinigt. Den am Verfahren beteiligten Schulpsychologen kommt, so der VGH München, nicht die Rolle eines Obergutachters zu, der sich ohne Rücksprache mit dem Facharzt über dessen Gutachten hinwegsetzen kann ${ }^{1488}$, so dass die Feststellung der Legasthenie ausschließlich der zuständige Facharzt trifft. ${ }^{1489}$ Demgegenüber stellen alle anderen landesrechtlichen Regelungen die Anerkennung der Behinderung Legasthenie/Dyskalkulie in das Ermessen der Schule.

Nach ganz herrschender Meinung steht der Prüfungsbehörde beim Ausgleich von Störungen des äußeren Prüfungsablaufs und bei der Festlegung von Nachteilsausgleichen bei Behinderungen kein Ermessensspielraum zu, sondern die Entscheidung ist vollständig gerichtlich überprüfbar ${ }^{1490}$ und daher eine rechtlich gebundene Verwaltungsentscheidung. ${ }^{1491}$ Das BVerfG hat dazu ausgeführt, „.... die Prüflinge haben einen verfassungsrechtlich verbürgten Anspruch auf gleiche Prüfungschancen. Deshalb haben die Gerichte gem. Art. 19 Abs. 4 GG zu kontrollieren, ob die Maßnahmen der Prüfungsbehörde ausreichten, um die Chancengleichheit zu erreichen. Gründe, die für eine Einschränkung dieser Kontrollpflicht sprechen könnten, sind nicht ersichtlich. Ob durch die Kompensationsmaßnahme ein „Ausgleich" gelungen ist und die Chancengleichheit der Kandidaten hergestellt wurde, ist eine Frage der rechtlichen Bewertung, die nach der ständigen Rechtsprechung des BVerfG von den Gerichten uneingeschränkt überprüft werden muss. ${ }^{\text {1492 }}$ Das Gericht hat daher ggf. mit Hilfe von

\footnotetext{
${ }^{1488}$ VGH München, Beschluss v. 31. 7. 2001, Az.: 7 ZE 01.103, S. 7, (nicht veröffentlicht)

1489 VGH München, Urteil v. 17.10.2003, Az.: 7 B 02.2186, juris, Rn. 38

1490 Niehues, Prüfungsrecht, Rn. 476; 899; Zimmerling/Brehm, Prüfungsrecht, Rn. 314 ff; Avenarius/Heckel, Schulrecht, Ziff. 27.31

${ }^{1491}$ VGH Mannheim, Beschluss v. 26.8.1993, Az.: 9 S 2023/93, NVwZ 1994, S. 599; VG Düsseldorf, Beschluss v. 8.5.2003, Az.: 15 L 1418/03, juris, Rn. 8

1492 BVerfG Beschluss v. 12.12.1992, Az.: 1 BvR 1295/90, juris, Rn. 19
} 
Sachverständigen zu überprüfen, ob die Störung durch die Abhilfemaßnahme bzw. die Behinderung durch den festgelegten Nachteilsausgleichs vollständig ausgeglichen wurde. ${ }^{1493}$ Nur für den Bereich der Legasthenie vertritt die Rechtsprechung jedoch - entgegen dieser Rechtsprechung des BVerfG - die Auffassung, dass den Lehrkräften sowohl hinsichtlich des "ob" als auch hinsichtlich des „wie“ des Ausgleichs ein Ermessen zusteht. Nur sie könnten aufgrund der konkreten Beobachtungen im Schulalltag beurteilen, ob ein solcher Ausnahmefall vorliegt, dass der Schüler Ausgleichsmaßnahmen braucht. ${ }^{1494}$ Der VGH Kassel hat in einem Verfahren ebenfalls diese Ansicht vertreten ${ }^{1495}$, sie aber inzwischen dahin modifiziert, dass die Einschätzung der Lehrkräfte, es liege keine Legasthenie vor, das Gericht im Prozess nicht daran hindert, dem vorgelegten psychiatrischen Gutachten, das eine Legasthenie bejaht, zu folgen. ${ }^{1496}$ Ennuschat differenziert und sieht im „ob" des Ausgleichs, also in der Grundentscheidung, eine voll überprüfbare Entscheidung der Schule, während das „wie“ des Ausgleichs, also die Entscheidung über die Ausgestaltung im Einzelnen, im Ermessen der Lehrkräfte steht. ${ }^{1497}$

Schon die Rechtsprechung des BVerfG zur vollen Überprüfbarkeit der Entscheidung der Prüfungsbehörde erfordert es, dass die Grundentscheidung ebenfalls voll überprüfbar ist. Denn wenn schon bezüglich des Umfangs des Nachteilsausgleichs keine Gründe für eine Einschränkung der Kontrollpflicht der Gerichte ersichtlich sind, so gilt dies erst Recht für die zugrunde liegende Entscheidung über das Vorliegen einer Behinderung überhaupt. Die Grundentscheidung, "ob" eine Behinderung vorliegt, ist zudem eine Entscheidung auf der Tatbestandsebene und nicht auf der Rechtsfolgenseite. Der Begriff „Behinderung" ist ein unbestimmter Rechtsbegriff, bei dessen Auslegung es um eine Rechtsfrage und nicht um eine pädagogische Einschätzung geht. Bei Rechtsfragen steht der Behörde kein Beurteilungsspielraum zu, so dass die Anwendung der Norm voll gerichtlich überprüfbar ist, insbesondere dann, wenn Grundrechte im Kernbereich betroffen sind. ${ }^{1498}$ Die besonderen Voraussetzungen für die

1493 Zimmerling/Brehm, Prüfungsrecht, Rn. 314 ff; VGH Mannheim, Beschluss v. 26.8.1993, Az.: 9 S 2023/93, NVwZ 1994, S. 599; VG Düsseldorf, Beschluss v. 8.5.2003, Az.: 15 L 1418/03, juris, Rn. 8

1494 VG Darmstadt, Urteil v. 28. 4. 2009, Az.: 7 K 687/08.DA (3), S. 16 (nicht veröffentlicht) und Urteil v. 2.11.2010, Az.: 7 K 1841/10.GI. S. 8 f (nicht veröffentlicht); VG Gießen, Urteil v. 30.6.2009. Az.: 4 K 1052/09.GI, S. 12 f (nicht veröffentlicht); wohl auch VG Schleswig, Urteil v. 10.6.2009, Az.: 9 A 208/08, BeckRS 2009 38123, S. 9

1495 VGH Kassel, Beschluss v. 25.3.2009, Az.: 7 B 772/09, S. 4 (nicht veröffentlicht)

1496 VGH Kassel, Beschluss v. 17.11.2010; Az.: 7 A 2970/09.Z, juris, Rn. 9

1497 Ennuschat, Die Rechte stotternder Menschen in Schule, Ausbildung und Studium, S. $98 \mathrm{f}$

1498 BVerfGE 88, 40 ff, Rn. 52, 57 (private Grundschule) 
ausnahmsweise Eröffnung eines Beurteilungsspielraums liegen nicht vor. ${ }^{1499} \mathrm{Da}$ Ermessen immer nur auf der Rechtsfolgenseite existiert, kann insoweit auch kein Ermessen eingeräumt werden.

Weil die Grundentscheidung damit eine gebundene Entscheidung ohne Beurteilungsspielraum ist, spricht dies dagegen, diese Entscheidung etwa auf die Klassenkonferenz zu übertragen. Sie hätte nur die Möglichkeit, anzuerkennen oder die Anerkennung abzulehnen. Eine pädagogische Einschätzung, die eine besondere Form der Ermessensausübung durch die Klassenkonferenz ist, ist bei der Grundentscheidung mangels Beurteilungsspielraum nicht zulässig. Die Entscheidung ist daher auch keiner demokratischen Mehrheitsentscheidung zugänglich. Würde die Klassenkonferenz die Anerkennung der Behinderung, trotz Erfüllung des Tatbestandes, versagen, wäre der Schulleiter verpflichtet von seinem Beanstandungsrecht Gebrauch zu machen. ${ }^{1500}$

\subsection{Nebenbestimmungen zum VA}

Des Weiteren stellt sich die Frage, ob die Grundentscheidung mit Nebenbestimmungen, etwa einer Befristung oder einer Bedingung, versehen werden kann.

Ein Verwaltungsakt kann mit einer Nebenbestimmung versehen werden, wenn dies durch Rechtsvorschrift zugelassen ist ( $\$ 36 \mid 1$. Alt. $V w V f G), d . h$. durch Landesgesetze oder Landesverordnungen ${ }^{1501}$ Fehlt eine solche spezielle Regelung des Fachrechts, so sind Nebenbestimmungen bei gebundenen Ansprüchen nur dann zulässig, wenn dadurch sichergestellt werden soll, dass die gesetzlichen Voraussetzungen erfüllt werden ( $\$ 36 \mathrm{Abs} .1 \mathrm{VwVfG}$ ). Ein freies Ermessen zum Erlass von Nebenbestimmungen besteht nicht (vgl. § $36 \| \mathrm{VwVfG}$ ).

\subsection{Befristung der Anerkennung}

Die Anerkennung der Behinderung Legasthenie/Dyskalkulie wird üblicherweise zeitlich und schulformbezogen eingeschränkt. ${ }^{1502}$ Die Berücksichtigung der Dyskalkulie erfolgt, soweit sie erfolgt, in der Grundschule ${ }^{1503}$, teilweise auch im

\footnotetext{
1499 Vgl. dazu Kopp/Ramsauer, VwVfG, § 40, Rn. 71 ff; Stelkens, in: Stelkens/Bonk/Sachs, VwVfG, $\S 40, \mathrm{Rn} .147 \mathrm{ff}$

${ }^{1500}$ Avenarius/Heckel, Schulrecht, Ziff. 7.214 und 7.321; umfassend zu den Eingriffsbefugnissen der Schulaufsichtsbehörden und der Schulleiter Rux, Pädagogische Freiheit des Lehrers, S. $141 \mathrm{ff}$

${ }^{1501} \mathrm{Vgl.} \mathrm{Kopp/Ramsauer,} \mathrm{VwVfG,} \mathrm{§} \mathrm{1,} \mathrm{Rn.} 30$

1502 S. dazu umfassend oben S. $189 \mathrm{f}$

${ }^{1503}$ BW VV Besonderer Förderbedarf Nr. 2.2 - Grundschule; HE VO-VO-GestSchulVerh § 39 IV Grundschule HH Förder-Richtlinie Nr. 4 - Grundschule; MV VV-LRR Nr. 6 III -Grundschule
} 
Sekundarbereich I. ${ }^{1504}$ Legasthenie wird zwar auch in höheren Klassenstufen berücksichtigt, allerdings ist die Anerkennung überwiegend von einer ständigen Überprüfung abhängig.

Beispielhaft seien hier die Regelungen aus Hessen, Hamburg und Bayern dargestellt: In Hessen muss gem. § 40 Abs. 3 HE VO-GestSchulVerh der Lernstand mindestens halbjährlich dokumentiert werden. Die Gewährung von Nachteilsausgleich und von Abweichungen in der Leistungsbewertung erfolgt auf der Grundlage dieser Förderpläne ( $\S \S 40$ Abs. 3, 42 Abs. 2 HE VO-GestSchulVerh). Eine Berücksichtigung in der Sekundarstufe II setzt einen besonders begründeten Ausnahmefall voraus (§ 39 Abs. 4 HE VO-GestSchulVerh). In Hamburg ist für den Anspruch auf Förderung und auf Nachteilsausgleich erforderlich, dass der Schüler im Testverfahren einen Prozentrang von unter 5 erreicht. Dieser Test ist halbjährlich zu wiederholen. (Nr. 2.1 HH Förder-Richtlinie). Darüber hinaus sind in regelmäßigen Abständen Lernstandmessungen $u$. a. durch förderdiagnostische Verfahren durchzuführen (Nr. 3.1 HH Förder-Richtlinie). Die Überprüfung der Behinderung findet in Hessen und Hamburg also halbjährlich statt. Von dem Ergebnis hängt ab, ob weiterhin Förderung, Nachteilsausgleich und evtl. Abweichungen in der Leistungsbewertung erfolgen.

In Bayern wird die Legasthenie ebenfalls in der Regel in der Grundschule festgestellt und anerkannt. Beim Übertritt auf die weiterführende Schule muss das Attest über die Legasthenie erneuert oder bestätigt und den Schulpsychologen der aufnehmenden Schule vorgelegt werden. ${ }^{1505}$ Die Anerkennung erfolgt dann allerdings bis zum Ende der Schullaufbahn. Sie gilt bis zum Abitur, auch wenn zwischenzeitlich ein Wechsel von einer Schulform in die andere, etwa von der Realschule auf eine Fachoberschule, erfolgt. ${ }^{1506}$

Die Begrenzung der Anerkennung der Legasthenie/Dyskalkulie in der Dauer, sei es etwa für die Dauer des Grundschulbesuchs oder bis zum Ende eines Schul(halb)jahres, ist eine Befristung, denn damit wird Anerkennung auf einen bestimmten Zeitraum beschränkt oder zu einem bestimmten Zeitraum beendet. $^{1507}$

Fraglich ist, ob derartige Befristungen, sei es in den Landesgesetzen bzw. Landesverordnungen oder als Nebenbestimmung nach § 36 I VwVfG, zulässig sind. Die Befristung regelt den zeitlichen Geltungsbereich des Verwaltungsak-

\footnotetext{
1504 BB VV-LRSR Nr. 6 Förderung einschließlich Sekundarstufe II, Nr. 7 Nachteilsausgleich nur bis Klasse 10; MV VV-LRR Nr. 7 III und 7 IV; NI LRR-Erlass Nr. 3.2 - einschließlich Sekundarbereich I; ST VO Abschlüsse § 20 - Sekundarstufe I, TH Fachliche Förderempfehlung einschließlich Sekundarstufe I

1505 BY KMBek Nr. IV

1506 Kritisch dazu: Geist, in: Legasthenie und Dyskalkulie 2011, S. 169

1507 Vgl. ausführlich zur Ausgestaltung der Befristung Stelkens, in: Stelkens/Bonk/Sachs, VwVfG, $\S 36, \mathrm{Rn} .70 \mathrm{ff}$
} 
tes. ${ }^{1508}$ Sie ist ein Mittel, um den Erfordernissen des Grundsatzes der Verhältnismäßigkeit Rechnung zu tragen, wenn die künftige Entwicklung der Sachlage nicht hinreichend überschaubar ist. ${ }^{1509}$ Mit ihr soll eine Anpassung der Verwaltungsentscheidung an eine veränderte Sachlage ermöglicht werden und sichergestellt werden, dass nicht auf die Fortgeltung der Regelung über die Befristung hinaus vertraut wird. ${ }^{1510}$ Die Befristung ist daher nur zulässig, wenn die Wahrscheinlichkeit groß ist, dass sich die Sachlage ändert und die einmal getroffene Verwaltungsentscheidung dann diesen Veränderungen nicht mehr Rechnung trägt. Nach Ablauf der Befristung ist folglich der Verwaltungsakt beendet und das Verfahren auf Anerkennung der Behinderung muss erneut durchgeführt werden. ${ }^{1511}$ Für die Legasthenie/Dyskalkulie bedeutet dies, dass eine erneute medizinisch-psychologische Diagnostik und daher erneute psychologische Tests erforderlich sind. Psychologische Testungen sind grundsätzlich belastend und müssen auf ein notwendiges Minimum beschränkt werden. Sie müssen erforderlich sein und dürfen deshalb nicht prophylaktisch erfolgen. ${ }^{1512}$ Die Zulässigkeit einer Befristung hängt daher wesentlich davon ab, ob die erneute Testung des Kindes verhältnismäßig ist.

Die kurze Befristung der Anerkennung wie in Hamburg und Hessen auf ein halbes Jahr wäre zulässig, wenn die Wahrscheinlichkeit groß wäre, dass sich die Legasthenie/Dyskalkulie innerhalb eines halben Jahres so verändert, dass die Anerkennung der Behinderung als nicht mehr gerechtfertigt anzusehen ist. Gegen eine solche Einschätzung spricht, dass nach dem Stand der medizinischen Forschung Legasthenie/Dyskalkulie lebenslange Beeinträchtigungen sind, die die betroffenen Schüler und Schülerinnen während ihrer gesamten Schulzeit und auch später als Erwachsene beeinträchtigen. ${ }^{1513}$ Eine kurzfristige Verbesserung innerhalb eines halben Jahres in der Form, dass eine von einem Kinderund Jugendpsychiater diagnostizierte Legasthenie/Dyskalkulie nicht mehr vorliegt, ist kaum denkbar.

Zudem müsste die insoweit notwendige Testung in halbjährlichem Turnus verhältnismäßig sein. Bedenken dagegen bestehen bereits deswegen, weil es für Testungen innerhalb eines derartig kurzen Abstands nicht genügend standardisierte Tests gibt. Es gibt für jedes Schuljahr etwa einen Test, kaum

\footnotetext{
1508 Stelkens, in: Stelkens/Bonk/Sachs, VwVfG, § 36, Rn. 70

1509 Kopp/Ramsauer, VwVfG, § 36 Rn. 17

1510 Stelkens, in: Stelkens/Bonk/Sachs, VwVfG, § 36, Rn. 74

${ }^{1511}$ Zur Klarstellung sei darauf hingewiesen, dass ein solches neues Verfahren denselben Anforderungen unterliegt, wie die erste Anerkennung.

1512 Maydell, von, Sozialrechtshandbuch, Rn. 161

1513 Schulte-Körne/Stieglitz, in: Adoleszenzpsychiatrie, S. 476 f
} 
mehr. ${ }^{1514}$ Jeder Test kann bei einem Kind nur einmal angewandt werden. Eine Mehrfachnutzung ist nicht möglich, weil damit die Aussagekraft verfälscht wird. Die Testung ist auch nicht angemessen, d.h. zumutbar. Die Testung in kurzen Abständen führt hinsichtlich des Bestehens einer dauerhaften Behinderung kaum zu weiterem Erkenntnisgewinn, ist aber eine erhebliche psychische Belastung für das behinderte Kind. Kinder in Testsituationen wissen, dass es auf ihre Leistung in diesem Bereich ankommt und dass davon die weitere Anerkennung der Legasthenie/Dyskalkulie abhängt. Sie werden damit einem erheblichen Stress ausgesetzt. Außerdem wird ihnen damit vermittelt, dass man nicht daran glaubt, dass sie tatsächlich Legasthenie/Dyskalkulie haben. Warum sonst sollte man in kurzen Abständen nachprüfen, ob die Beeinträchtigung immer noch da ist? Eine solche Skepsis verunsichert die Kinder. Sie lernen nicht, ihre Behinderung zu akzeptieren, sondern sie lernen, daran zu zweifeln. Tatsächlich wird auch getestet, ob die Kinder immer noch schlecht genug sind, um einen Ausgleich für die Behinderung zu bekommen. Schon die Tatsache, dass es auf ein möglichst schlechtes Abschneiden in den Tests ankommt, weil sonst die bisherigen Schwierigkeiten unglaubhaft werden, ist destruktiv und demotivierend. Die ständige Testung des Kindes mit den sich daraus ergebenden psychischen Belastungen und den daran anknüpfenden Folgen der Anerkennung oder Nicht-Anerkennung der Behinderung ist mit dem Wohl des Kindes nicht zu vereinbaren. Dieses ist jedoch gem. Art. 7 BRK bei Maßnahmen, die das behinderte Kind betreffen, vorrangig zu berücksichtigen.

Eine Befristung ist daher nur dann verhältnismäßig, wenn sie sich auf das notwendige Minimum und damit auf das unbedingt Erforderliche beschränkt.

Die Diagnostik der Legasthenie/Dyskalkulie ist in den ersten Grundschuljahren relativ schwieriger als in späteren Klassen, weil sich Kinder sehr unterschiedlich entwickeln und die Abgrenzung zwischen einer langsamen Entwicklung und einer gestörten Entwicklung schwer ist. Je älter das Kind ist, wenn die Legasthenie/Dyskalkulie diagnostiziert wird, desto sicherer ist es, dass dann eine Störung vorliegt. Das spricht dafür, dass die Wahrscheinlichkeit, dass sich die Sachlage zwischen dem Beginn und dem Ende der Grundschulzeit, ausgehend von einer vierjährigen Grundschulzeit, verändert hat, relativ gegeben ist und daher die einmal erfolgte Anerkennung der Legasthenie/Dyskalkulie noch einmal überprüft werden muss. Geist plädiert dafür, eine solche Überprüfung noch einmal am Ende der Unterstufe, also am Ende der siebten Klasse vorzunehmen. ${ }^{1515}$ Wie hoch hier die Wahrscheinlichkeit der Veränderung der Sachlage ist, ist eine Frage der medizinischen Forschung und Diagnostik. Ist aus Sicht der medizinisch-psychologischen Diagnostik eine fundierte Aussage zur Aus-

\footnotetext{
${ }^{1514}$ S. die Auflistung unter http://www.kjp.med.uni-muenchen.de/forschung/legasthenie/ diagnose.php

Geist, in: Legasthenie und Dyskalkulie 2011, S. 169
} 
prägung und eventuellen Veränderung der Legasthenie/Dyskalkulie möglich, so würde dies für eine Befristung und erneute Testung am Ende der Unterstufenzeit sprechen. Insgesamt erscheint ein Überprüfungszeitraum von frühestens drei Jahren nach der letzten Diagnostik noch als verhältnismäßig.

\subsection{Auflösende Bedingung}

Die landesrechtlichen Bestimmungen sehen zum Teil vor, dass die Anerkennung der Legasthenie entzogen wird, wenn die Leistungen des betroffenen Kindes sich verbessern und es zumindest ausreichende Leistungen erbringt. ${ }^{1516}$ Eine solche Verknüpfung der Anerkennung mit bestimmten Leistungen ist eine auflösende Bedingung gem. § 36 II Nr. 2 VwVfG, denn mit dem Eintritt eines ungewissen zukünftigen Ereignisses, nämlich dem Erreichen bestimmter Noten, fällt die Anerkennung der Legasthenie/Dyskalkulie weg. Eine solche Bedingung ist nur zulässig, wenn dadurch sichergestellt wird, dass die gesetzlichen Voraussetzungen erfüllt werden. Wie bereits dargestellt, verstößt es gegen Art. 3 III 2 GG, die Anerkennung der Behinderung beim Erreichen bestimmter Noten zu entziehen. ${ }^{1517}$ Dementsprechend dient eine solche auflösende Bedingung nicht dazu, die gesetzlichen Voraussetzungen sicherzustellen und ist daher unzulässig.

\subsection{Auflagen}

Wird die Anerkennung der Behinderung mit der Verpflichtung verknüpft, eine Förderung zu besuchen, so kann dies eine Auflage gem. § 36 II Nr. 4 VwVfG. Ob der Besuch einer Förderung eine Auflage oder eine Bedingung gem. § 36 II Nr. 2 VwVfG ist, hängt davon ab, wie die Anerkennung und der Besuch der Förderung miteinander verbunden werden. Wird die Anerkennung in der Form mit der Förderung verbunden, dass die Anerkennung entfällt, wenn die Förderung nicht besucht wird, so handelt es sich um eine Bedingung, da der Besuch der Förderung die Anerkennung beeinflusst. Eine solche Bedingung ist unzulässig, da, wie bereits ausgeführt ${ }^{1518}$, ein Junktim von Anerkennung und Förderung die Betroffenen in ihrem Grundrecht aus Art. 3 III 2 GG verletzt.

Demgegenüber ist die Auflage eine selbständig erzwingbare Anordnung, die zusätzlich mit dem Verwaltungsakt verbunden wird. ${ }^{1519}$ Da es grundsätzlich zulässig ist, den Schüler zum Besuch einer qualifizierten, evaluierten Therapie der Legasthenie/Dyskalkulie, die von qualifiziertem Personal durchgeführt wird, zu

\footnotetext{
${ }^{1516}$ Z.B. BW VV Besonderer Förderbedarf Nr. 2.3.2; HB LRS-Erlass Nr. 4.2; NI LRR-Erlass Nr. 3.2; NRW LRS-Erlass Nr. 3.1; SL VV-LRS Nr. 3 und 4.5

1517 S. oben 5. Teil: 1. Kapitel: 3.4.: Berücksichtigung bei durchschnittlichen Noten?, S. 190

1518 S. oben 5. Teil: 2. Kapitel: 2.: Bindung von Nachteilsausgleich an die Teilnahme an Fördermaßnahmen?, S. 201

${ }^{1519}$ Kopp/Ramsauer in: VwVfG, § 36, Rn. 29, 34
} 
verpflichten ${ }^{1520}$, kann der Verwaltungsakt der Anerkennung mit der Auflage des Besuchs dieser Förderung verbunden werden. Es muss allerdings deutlich werden, dass zwischen beiden Entscheidungen kein Junktim besteht.

\section{Kapitel: Ausgestaltung des Nachteilsausgleichs}

Ist die Legasthenie/Dyskalkulie in der Schule dem Grunde nach anerkannt, müssen Form und Umfang des Nachteilsausgleichs festgelegt werden.

\section{Verwaltungsakt}

Die Ausgestaltung des Nachteilsausgleichs muss für jedes betroffene Kind individuell und für die jeweilige Prüfungssituation erfolgen, um seinen individuellen Einschränkungen und dem Schweregrad seiner Behinderung Rechnung zu tragen.

Die Gewährung von Nachteilsausgleich ist daher eine dauernde Aufgabe im schulischen Alltag, die als Maßnahme des laufenden Schulbetriebes zu qualifizieren sein könnte. Gegen eine solche Einordnung spricht jedoch, dass mit der Gewährung oder Nicht-Gewährung und mit der Ausgestaltung des Nachteilsausgleichs eine Entscheidung im Einzelfall getroffen wird, die den betroffenen Schüler in seinen Grundrechten betrifft. Ebenso wie im sonstigen Prüfungsrecht die Entscheidung über den Nachteilsausgleich dem Grunde und der Art nach ein Verwaltungsakt ist, ist diese Entscheidung auch im Schulrecht ein Verwaltungsakt. Für diese Sicht spricht auch, dass dann der Nachteilsausgleich einmal grundlegend für alle Fächer festgelegt wird ${ }^{1521}$ und anschließend eine Hilfestellung für die Lehrkräfte dieses Kindes darstellt, weil sie ersehen können, welche Unterrichts- und Prüfungsformen erforderlich sind, auf welche Besonderheiten sie achten müssen und wie die Prüfungen zu gestalten sind. ${ }^{1522}$

\section{Gebundene Entscheidung oder Ermessensentscheidung}

Fraglich ist, ob die Entscheidung über die Ausgestaltung des Nachteilsausgleichs als gebundene oder als Ermessensentscheidung gestaltet werden sollte. Der Nachteilsausgleichs umfasst viele verschiedene Aspekte, die von grundsätzlichen Entscheidungen, wie der Nicht-Bewertung der Rechtschreibung, des Lesens oder Rechnens und mit dem Anspruch auf Zeitzuschläge über Verände-

\footnotetext{
1520 S. oben: 5. Teil: 2. Kapitel: 2.1.: Pflicht zur Teilnahme an Förderung, S. 202

${ }^{1521}$ Vgl. Geist, in: Legasthenie und Dyskalkulie 2011, S. 170

1522 Geist, in: Legasthenie und Dyskalkulie 2011, S. 170; Erler, Legasthenie Albert-Einstein-SchuleLaatzen, das Merkblatt zeigt das immer wiederkehrende Problem auf, dass Lehrer vielfach nichts über die Legasthenie und nichts über die besonderen Bedürfnisse des Kindes wissen
} 
rungen des Umfangs oder der Darbietung (schriftlich oder mündlich) von Prüfungsleistungen, auf die Ausgestaltung von Prüfungsunterlagen (z.B. größere Schrift, größere Vorlagen, vorgelesener Text), bis hin zu weiteren Formen der Unterstützung (Benutzung von Laptops) reichen. Einige Teile betreffen alle betroffenen Schüler und Schülerinnen gleich, andere Teile müssen individuell an den jeweiligen Schüler angepasst werden. In Bayern wird dieser Variationsbreite dadurch Rechnung getragen, dass die landesrechtliche Bestimmung bestimmte Teile des Nachteilsausgleichs zwingend festschreibt, insbesondere die Nicht-Bewertung der Rechtschreibung und die Grundentscheidung darüber, dass ein Zeitzuschlag zu erfolgen hat, während sie die Bereiche, die die Anpassung an weitere individuelle Bedürfnisse beinhalten, als Ermessensvorschrift fasst.

Wie bereits dargestellt geht das BVerfG davon aus, dass die Festlegung von Nachteilsausgleich der vollen gerichtlichen Überprüfung unterliegt, so dass ein Ermessensspielraum in diesem Bereich nicht besteht. ${ }^{1523}$ Die Rechtsprechung der Verwaltungsgerichte und auch Ennuschat nehmen hier jedoch einen Ermessensspielraum der Lehrkräfte an. ${ }^{1524}$ Für die zwingende Fassung des Nachteilsausgleichs spricht, dass soweit grundrechtliche Ansprüche berührt sind, die Grundrechte selbst und die Rechtsschutzgarantie aus Art. 19 IV GG es erfordern, dass diese umfassend erfüllt werden. Deshalb ist hier die Gesetzesbindung die Regel und die Einräumung von Ermessen die Ausnahme. ${ }^{1525}$ Allerdings ist die Gewährung von Nachteilsausgleich im Schulbereich, anders als bei universitären Prüfungen, eine langfristige und dauernde Aufgabe, die zudem die Entwicklung des Kindes mit beachten muss. Das lässt es unmöglich erscheinen, den Nachteilsausgleich nur einmal festzulegen. Weil der Nachteilsausgleich individuell die Behinderung ausgleichen soll und daher auch besondere Unterrichtsformen oder Prüfungsformen erfasst, ist eine allumfassende schematische Entscheidung für alle betroffenen Schüler und Schülerinnen nicht möglich.

Notwendig ist daher eine differenzierte Betrachtungsweise. Soweit es sich um Nachteilsausgleich handelt, der für alle betroffenen Schüler und Schülerinnen gleich gewährt werden muss, ist dieser in der landesrechtlichen Vorschrift festzulegen und als gebundener Anspruch zu gestalten. Soweit es darüber hinaus auch noch um weitere notwendige Formen des Nachteilsausgleichs geht,

\footnotetext{
1523 S. oben 9. Teil: 1. Kapitel: 4.1.: Grundentscheidung: Gebundener VA oder Ermessensentscheidung?, S. 280

${ }^{1524}$ VGH Kassel, Beschluss v. 25.3.2009, Az.: 7 B 772/09, S. 4 (nicht veröffentlicht); VG Darmstadt, Urteil v. 28. 4. 2009, Az.: 7 K 687/08.DA (3), S. 16 (nicht veröffentlicht); VG Gießen, Urteil v. 30.6.2009. Az.: 4 K 1052/09.GI, S. 12 f (nicht veröffentlicht); wohl auch VG Schleswig, Urteil v. 10.6.2009, Az.: 9 A 208/08, BeckRS 2009 38123, S. 9; Ennuschat, Die Rechte stotternder Menschen in Schule, Ausbildung und Studium, S. $98 \mathrm{f}$

${ }^{1525} \mathrm{Vgl}$. Kopp/Ramsauer, VwVfG, § 40, Rn. 6
} 
um individuelle Einschränkungen auszugleichen, so sind diese als Ermessensnorm auszugestalten.

\section{Ermessen, Ermessensgrenzen, Ermessensfehler}

Es wurde bereits ausgeführt, wie der Nachteilsausgleich praktisch umgesetzt werden kann und welche rechtlichen Anforderungen der Nachteilsausgleich zu erfüllen hat. Hier ist nur noch darauf einzugehen, wie das Ermessen ausgestaltet ist und welchen Begrenzungen es unterliegt.

Soweit die Festlegung des Nachteilsausgleichs eine Ermessensentscheidung ist, ist $\S 40 \mathrm{VwVfG}$ zu beachten. Danach ist das Ermessen an den Zweck der Ermächtigungsgrundlage gebunden, also an Art. 3 III 2 GG und an Art. 24 BRK $^{1526}$, und muss daher dazu dienen, die Behinderung so weit wie möglich und wie nötig auszugleichen. Maßstab für den Ausgleich ist nicht der Normalkandidat, d.h. der Schüler ohne Behinderung, sondern die inhaltlich fachliche Leistung, die mit der Prüfung abgefragt werden soll. Aus den Grundrechten und insbesondere aus dem Grundsatz der Chancengleichheit ergibt sich, dass Prüfungsinhalte und Prüfungsziele entsprechend der Behinderung so anzupassen und zu modifizieren sind, bis eine gleiche Ausgangslage aller Prüflinge besteht. Der Ausgleich muss so gestaltet sein, dass die behinderungsbedingten Nachteile individuell ausgeglichen sind. ${ }^{1527}$ Das versteht sich eigentlich von selbst: Ein schwer betroffener Schüler mit Legasthenie, der etwa kaum lesen kann, braucht andere Formen des Ausgleichs als ein Schüler mit Legasthenie, der relativ gut lesen, dafür aber nur schwer schreiben kann. Ob die Ausgangslage von Schülern im Hinblick auf eine Prüfung gleich ist, entscheidet sich folglich danach, ob die (Start-)Bedingungen für die Erreichung des Ziels gleich sind. Das muss für die verschiedenen Prüfungsverfahren gesondert festgestellt werden. Die Grenze des Ausgleichs ist die Überkompensation der Behinderung, die dazu führt, dass Schüler und Schülerinnen mit Behinderung besser gestellt werden als Schüler und Schülerinnen ohne Behinderung. Insofern ist das richtige Maß zwischen Unterkompensation und Überkompensation zu finden.

Die Ermessensentscheidung ist nur eingeschränkt darauf überprüfbar, ob Ermessensfehler vorliegen. Es gelten die allgemeinen Vorschriften des $\S 40$ VwVfG, wobei dies den Lehrkräften häufig nicht bewusst ist, da sie annehmen, dass sie bei Ermessensentscheidungen völlig frei sind. Beispielhaft seien hier nur einige Probleme erwähnt: Ermessensunterschreitung ist gegeben, wenn die Lehrkraft meint, sie dürfe dem Schüler einen Text nicht mehr vorlesen, wenn er schon Zeitzuschlag bekommt. Ermessensfehlgebrauch liegt vor, wenn die Behinderung grundsätzlich nicht anerkannt wird oder der Nachteilsaus-

\footnotetext{
1526 S. dazu Aichele/ Althoff, Kommentar zur UN-BRK, Nicht-Diskriminierung, Rn. 70

${ }^{1527}$ S. dazu z.B. § 39 BE SopädVO, der eine umfangreiche Auflistung möglicher Beeinträchtigungen und Veränderungen der Anforderungen enthält.
} 
gleich verweigert wird, weil andere Schüler auch schlecht Deutsch können. Ein Ermessensfehler liegt auch vor, wenn einem Schüler zum Ausgleich seiner Behinderung ein Wörterbuch zugestanden wird, damit er seine Fehler korrigieren kann. Der Schüler mit Legasthenie kann gerade seine Rechtschreibfehler nicht erkennen, das Wörterbuch nützt ihm daher nichts. Er ist genauso gestellt, als hätte er gar keinen Ausgleich bekommen. ${ }^{1528}$

Insbesondere beim Zeitzuschlag muss das Ermessen ausgeübt werden. Je nachdem wie schwer die Betroffenheit ist, brauchen die Schüler und Schülerinnen mehr oder weniger Zeitzuschlag. ${ }^{1529}$ Diesem Bedürfnis trägt z.B. die Regelung in Bayern Rechnung, die einen Zeitzuschlag bis zu $50 \%$ vorsieht und es damit ermöglicht, in dieser Spanne unterschiedliche Zeitzuschläge je nach Betroffenheit festzulegen. ${ }^{1530}$ Üblich ist es, den Zeitzuschlag prozentual oder auch in Minuten pro Bearbeitungsstunde festzulegen. Das VG Darmstadt hat eine so festgelegte Zeitzugabe für unzulässig gehalten, weil je nach Klausur die Aufgaben und Anforderungen unterschiedlich seien, so dass eine generelle Regelung nicht möglich sei. Vielmehr sei der Zuschlag für jede Klausur einzeln von der Lehrkraft festzulegen. ${ }^{1531}$ Der VGH Kassel hat darauf hingewiesen, dass es für den Zeitzuschlag auf die Leistungsanforderungen der konkreten Klausur ankommt. ${ }^{1532}$ Eine solche individuelle Festlegung ist zwar sehr gerecht, aber auch sehr aufwändig für die Lehrkräfte. Sie setzt voraus, dass die Lehrkräfte über sehr genaue Kenntnisse verfügen, welche Fähigkeiten des Kindes beeinträchtigt sind und in welchen Bereichen es welchen Ausgleich braucht. Die Lehrkräfte müssten daher sehr genau über die individuelle Behinderung des Kindes Bescheid wissen, was in Bezug auf das Persönlichkeitsrecht des Kindes bedenklich ist. Sie müssten weiter über sehr genaue Kenntnisse über Legasthenie/Dyskalkulie verfügen, um beurteilen zu können, welchen individuellen Nachteilsausgleich das Kind gerade in dieser Prüfungssituation braucht. Noch schwerer wird die individuelle Bestimmung in jeder Prüfungssituation, wenn das Kind mehr-

${ }^{1528} \mathrm{Vgl}$. Eggers/Fegert/Resch, Psychiatrie, S. 763

1529 Nach Auskunft des EPOS (European Personnel Selection Office) wird Personen mit Dyslexia, Dyscalculia, Dyspraxia, Dysgraphia u.ä. im Auswahlverfahren für den Personalpool der Europäischen Institutionen regelmäßig ein Zeitzuschlag von 25 \% gewährt. Entscheidend ist jedoch die Empfehlung des behandelnden Arztes bezüglich der notwendigen Ausgleichmaßnahmen (Auskunft des EPOS, Toralf Katzorke, vom 30.11.2009)

1530 Die Regelung wird allerdings unterlaufen, wenn in einem Gymnasium die schuleigene Regelung erlassen wird, dass alle Schüler mit Legasthenie 10 \% Zeitverlängerung erhalten. Eine solche pauschale Gleichbehandlung aller Schüler mit Legasthenie wird der individuellen Behinderung wieder nicht gerecht und benachteiligt die schwerer betroffenen Schüler. (Schuleigene Regelung an einem Gymnasium in Oberbayern für das Schuljahr 2009/2010, die mir vorgelegt wurde.)

${ }^{1531}$ VG Darmstadt, Urteil v. 28. 4. 2009, Az.: 7 K 687/08.DA (3), S. 10 (nicht veröffentlicht)

${ }^{1532}$ VGH Kassel, Beschluss v. 28.7.2009; Az.: 7 A 1810/09.Z, S. 2 (nicht veröffentlicht) 
fach behindert ist, z.B. durch Legasthenie und ADHS, weil dann beide Behinderungen und ihre jeweiligen Einflüsse aufeinander berücksichtigt werden müssen. Bei zentralen Prüfungen, wie den Abiturklausuren, ist eine individuelle Festlegung des Nachteilsausgleichs fast gar nicht mehr machbar, weil die Lehrkräfte die Aufgaben nicht kennen und weil der Prüfling sich in der Bearbeitungszeit für ein Aufgabe entscheiden kann. Das macht es praktisch unmöglich, den Nachteilsausgleich individuell für diese Klausur festzulegen. Zu bedenken ist auch, dass vor allem Fachlehrer an weiterführenden Schulen häufig eine so große Zahl von Schülern und Schülerinnen unterrichten, dass sie nicht in der Lage sind, vielen besonderen Einzelheiten Rechnung zu tragen. Wenn Lehrkräfte sich wegen der großen Schülerzahlen schon die Gesichter der Schüler und Schülerinnen kaum merken können und deshalb als Gedächtnisstütze Fotos von ihnen machen wollen ${ }^{1533}$, ist es eine Überforderung, von ihnen eine jeweils individuelle Beurteilung des notwendigen Ausgleichs einer Behinderung zu verlangen. Sie brauchen daher möglichst klare Vorgaben, wie sie der Behinderung des Kindes gerecht werden können und welche Formen des Nachteilsausgleichs sie zu gewähren haben. ${ }^{1534}$

Der Zeitzuschlag muss so gewährt werden, dass er den Prüfungszeitraum für den Schüler mit Behinderung effektiv verlängert. Deshalb muss organisatorisch sichergestellt sein, dass in diesem Zeitraum die gleichen Bedingungen, insbesondere bezüglich der Ruhe im Raum, herrschen wie während der allgemeinen Prüfungszeit. ${ }^{1535}$ Wird dies nicht gewährleistet, hat der Schüler mit Behinderung nicht die gleichen Prüfungsbedingungen wie die Schüler ohne Behinderung und wird wieder wegen seiner Behinderung benachteiligt.

Es kommt vor, dass Lehrkräfte in einer Prüfung die Prüfungszeit für die gesamte Klasse verlängern. Eine solche Zeitverlängerung ist nicht auf die individuelle Zeitverlängerung der Schüler und Schülerinnen mit Legasthenie/Dyskalkulie anzurechnen und "verbraucht" diese nicht. Die Lehrkraft verlängert nämlich damit die allgemeine Prüfungszeit und muss dementsprechend den Zeitzuschlag für die betroffenen Schüler noch einmal um ihren Anteil an dieser allen gewährten Zeitverlängerung erhöhen. Wenn die Schüler ohne Beeinträchtigung die Aufgabe nur in einer verlängerten Zeit bearbeiten können, bedeutet dies für die Schüler und Schülerinnen mit Legasthenie/Dyskalkulie, dass sie erst Recht mehr Zeit brauchen.

\footnotetext{
${ }^{1533}$ Was datenschutzrechtlich nicht erlaubt ist - so das Beispiel bei Weichert, in: Schulleitung in den Ländern, Anm. 11.1.2

1534 Geist, in: Legasthenie und Dyskalkulie 2011, S. 170

1535 VG Darmstadt, Urteil v. 28. 4. 2009, Az.: 7 K 687/08.DA (3), S. 10 (nicht veröffentlicht); OVG Weimar, Beschluss v. 17.5.2010, Az.: 1 EO 854/10, juris, Rn. 55
} 


\section{Zeitpunkt der Ermessensentscheidung}

Um den Schüler mit Behinderung nicht nur nicht zu benachteiligen, sondern ihm auch faire und gleichmäßige Chancen in der Prüfung zu geben, muss die Prüfung auch für den Schüler selbst transparent und vorhersehbar sein. Deshalb sollte der Nachteilsausgleich einmal grundlegend für alle Fächer festgelegt werden. ${ }^{1536}$ Die notwendige individuelle Festlegung für die jeweiligen Prüfungen muss so erfolgen, dass der Schüler mit Behinderung ebenso wie der Schüler ohne Behinderung vor der Arbeit die Prüfungsbedingungen kennt. ${ }^{1537}$ Deshalb muss er vor jeder Arbeit darüber informiert werden, wie der Nachteilsausgleich gestaltet ist. Nur dann ist es ihm möglich, hierzu einen eigenen Standpunkt einzunehmen und diesen auch wirksam zu vertreten. ${ }^{1538}$ Wird über den Nachteilsausgleich erst während der Prüfung entschieden ${ }^{1539}$, so schreibt der behinderte Schüler die Prüfung zunächst wie ein nicht-behinderter Schüler mit der Ungewissheit, ob seine Behinderung im Laufe der Prüfung anerkannt wird oder nicht. Wird ihm der Nachteilsausgleich während der Prüfung verwehrt, wird er zusätzlich mit der Entscheidung belastet, ob und wie er sich gegen diese Entscheidung zur Wehr setzen kann. Damit wird er gegenüber den nichtbehinderten Schülern benachteiligt, weil er über die endgültige Ausgestaltung seiner Prüfung im Unklaren gelassen wird und sich in der Prüfungszeit auch noch mit einer anderen Problematik beschäftigen muss. ${ }^{1540}$

\section{Zuständigkeit für die Festlegung}

Geregelt werden muss, wer für die Festlegung der Art und des Umfangs des Ausgleichs zuständig ist. Möglich ist die Entscheidung durch die Lehrkraft, die Klassenkonferenz oder auch die Schulverwaltungsbehörde. ${ }^{1541}$ In Bayern schlägt der Schulpsychologe Form und Umfang des Nachteilsausgleichs vor, die

\footnotetext{
${ }^{1536}$ Vgl. Geist, in: Legasthenie und Dyskalkulie 2011, S. 170; Marwege, RdJB 2009, S. 229 ff, S. 235; Ennuschat, Die Rechte stotternder Menschen in Schule, Ausbildung und Studium, S. 304

${ }^{1537}$ VG Lüneburg v. 28.5. 2002, Az.: 4 A 246/01, www.rechtsprechung.niedersachsen.de, S. 5; VG Darmstadt, Urteil v. 28. 4. 2009, Az.: 7 K 687/08.DA (3), S. 10 (nicht veröffentlicht); OVG Weimar, Beschluss v. 17.5.2010, Az.: 1 EO 854/10, juris, Rn. 55

1538 S. dazu BVerfGE 84, 34, $45 \mathrm{f}$

1539 Dies hat das VG Augsburg Beschluss v. 23.8.2007, Az.: AU 3 E 07.00798, juris, Rn. 32, für zulässig gehalten; ähnlich OVG Nordrhein-Westfalen, Beschluss vom 4.9.2008, Az.: 19 B1293/08, www.justiz.nrw.de, Rn. 14

1540 Marwege, RdJB 2009, S. 229 ff, S. 236 f

${ }^{1541}$ Z.B. Nachteilsausgleich für hörgeschädigte Schüler in Bayern; KMS v. 8.12.2006; Az.: VI.8 - 5 S $5300-6.108417$, http://www.best-news.de/?schul_regelungen; HB RL Assistenz § 2; s. auch Ennuschat, Die Rechte stotternder Menschen in Schule, Ausbildung und Studium, S. 125, 132, 138, 145 (für stotternde Schüler)
} 
letzte Entscheidung trifft jedoch der Schulleiter. Im Übrigen entscheiden ganz überwiegend die Klassenkonferenzen ${ }^{1542}$ darüber, ob und welcher Ausgleich gegeben wird.

Für die Bestimmung der Zuständigkeit ist zunächst zu beachten, dass eine Verteilung der Zuständigkeit auf mehrere Personen und/oder Gremien sehr aufwändig und unübersichtlich ist. Je einfacher und klarer die Zuständigkeitsregelungen sind, desto leichter und einfacher lassen sie sich im Schulalltag umsetzen. Des Weiteren ist für die Bestimmung der Zuständigkeit entscheidend, wer die fachliche Kompetenz besitzt, die Gestaltung des Nachteilsausgleichs zu beurteilen.

Die Entscheidung über den Teil des Nachteilsausgleichs, der im Ermessen steht, muss den individuellen Bedürfnissen des betroffenen Schülers gerecht werden, sie muss seine Rechte auf Datenschutz wahren und Gewähr dafür bieten, dass innerhalb der Gruppe der betroffenen Schüler und Schülerinnen ebenfalls ein gerechter Ausgleich stattfindet, so dass schwer betroffene Schüler einen umfassenderen Ausgleich erhalten als weniger betroffene Schüler. Die Festlegung des Ausgleichs zur Kompensation der Behinderung setzt weiter Kenntnisse über die Art der Behinderung und die möglichen Ausgleichsmaßnahmen voraus. Weil jede Legasthenie/Dyskalkulie anders ist und mit verschiedenen Einschränkungen auch im auditiven, visuellen und graphomotorischen Bereich oder mit weiteren Behinderungen, wie z.B. ADHS, verbunden sein kann, muss der Ausgleich diese berücksichtigen. So muss z.B. ein Kind mit visueller Problematik vermehrt Hörtexte erhalten, während dies bei einem Kind mit einer auditiven Problematik gerade kontraproduktiv ist. Auch der Zeitzuschlag hängt von den individuellen Voraussetzungen des Schülers mit Behinderung ab. Ist er zusätzlich zur Legasthenie/Dyskalkulie motorisch beeinträchtigt, braucht er mehr Zeit als ein Schüler ohne motorische Schwierigkeiten. Das können Lehrkräfte sachverständig nicht für Legasthenie/Dyskalkulie wissen und es kann in diesem Umfang auch nicht von ihnen gefordert werden, denn sie müssten die gleichen Kenntnisse über alle Behinderungen und Krankheiten und auch für

1542 BW VV Besonderer Förderbedarf Nr. 2.3.2; BY automatisch, wenn Gutachterlich festgestellt, Zeitzuschlag bestimmt Schulleiter; BE GsVO § 16 VII; BB VV-LRSR Nr. 5 IV a und Nr. 7 III s. auch Nr. 5 IV b und c für die Sekundarstufe II und die Abiturprüfung; HB LRS-Erlass Nr. 4; HH Förder-Richtlinie Nr. 3.1 - Testergebnis entscheidet; HE VO-GestSchulVerh $§ 7$ III - Entscheidung durch den Schulleiter; MV VV-LRR Nr. 5 III - das staatliche Schulamt II Klassenkonferenz unter Einbeziehung der Eltern; NI LRR-Erlass Nr. 3; NRW LRS-Erlass Nr. 3. 2 - Schulleitung entscheidet; RP VV-LRS Nr. 4.3; SL VV-LRS Nr. 2.1 a); SN VV-LRS Nr. 4.1.4; ST Grundschulbewertungs-Erlass Nr. 4.1.1, ST Sekundarstufenbewertungs-Erlass Nr. 5.1.2; SH LegasthenieErlass Nr. 2.1 und SH Rechenschwäche-Erlass Nr. 4.3 und 5.2; TH § 59 V und VI SchulO 
komplexe Mehrfachbeeinträchtigungen ${ }^{1543}$ haben, die Kinder haben können und die im Unterricht berücksichtigt werden müssen. ${ }^{1544}$

Um diese vielfältigen Interessen zu wahren, ist in Bayern die Entscheidung über den Nachteilsausgleich fast vollständig auf den Schulpsychologen übertragen worden. Die Schulpsychologen erhalten die Stellungnahme der diagnostizierenden Fachkraft und sind in der Grundentscheidung an diese gebunden. Sie erhalten damit einen Einblick in die Schwere der Betroffenheit des Kindes und können diese in Beziehung setzen zu anderen betroffenen Schülern und Schülerinnen. Da sie der Schweigepflicht unterliegen, ist gewährleistet, dass die Diagnose nur nach einer Entbindung von der Schweigepflicht an die Lehrkräfte weitergeleitet wird, die das Kind unterrichten. Der Schulpsychologe gibt dann Hinweise für weiteren Nachteilsausgleich, den das Kind benötigt. ${ }^{1545}$

Ein solches Zusammenwirken im Bereich des individuellen Nachteilsausgleichs wird den Verfahrensanforderungen am besten gerecht und würde dazu führen, dass die jeweiligen Spezialkenntnisse in das Verfahren eingebracht werden.

Die diagnostizierenden Fachkräfte haben spezielle Kenntnisse zur Betroffenheit des Kindes und zu eventuellen weiteren Einschränkungen oder Komorbiditäten des Kindes, so dass sie gezielte Aussagen dazu machen können, wie umfangreich der Zeitzuschlag sein muss und welche sonstigen Einschränkungen auszugleichen sind. Diese Kenntnisse haben weder die Lehrkräfte ohne besondere Ausbildung, noch Sonderpädagogen, die nicht auf den Bereich Legasthenie/Dyskalkulie spezialisiert sind. Bei Schulpsychologen ist davon auszugehen, dass sie Kenntnisse in diesem Bereich haben. Sie können auch die Ausführungen der diagnostizierenden Fachkräfte nachvollziehen und sich einen Überblick über die verschiedenen Schweregrade und Betroffenheiten der Schüler und Schülerinnen machen. Die Lehrkräfte können aus ihrer Erfahrung sicherlich Kenntnisse und Vorgehensweisen einbringen.

Gegen eine Einbindung der Klassenkonferenz in diesen Entscheidungsprozess spricht, dass viele Fachlehrer keine Kenntnisse über Legasthenie/Dyskalkulie haben und deshalb mit der Zuständigkeit für die Festlegung des Nachteilsausgleichs überfordert sind. ${ }^{1546}$ Soweit an den Konferenzen auch Schüler- und

\footnotetext{
1543 Wie muss z.B. der Ausgleich für kleinwüchsiges Kind mit Legasthenie oder für ein Kind mit Herzfehler und Dyskalkulie sein?

1544 S. allein die umfangreiche Darstellung Bundeszentrale für gesundheitliche Aufklärung (BZgA), Chronische Erkrankungen als Problem

1545 Geist, in: Legasthenie und Dyskalkulie 2011, S. 170

${ }^{1546}$ Rux, RdJB 2009, S. 220 ff, S. 227
} 
Elternvertreter teilnehmen, erhalten diese sehr persönlichen Informationen über einen Schüler, die ihnen nicht offenbart werden dürfen. ${ }^{1547}$

\section{Informationspflicht gegenüber den Eltern}

Aus dem Elternrecht und aus Art. 3 III 2 GG ergibt sich eine Informationspflicht der Schule, die Eltern rechtzeitig und umfassend über die Art des geplanten Nachteilsausgleich zu unterrichten. ${ }^{1548}$

\section{Kapitel: Verzicht der Betroffenen auf Berücksichtigung von Legasthenie und Dyskalkulie}

Ist eine Behinderung anerkannt, dann kommt es vor, dass die betroffenen Schüler und Schülerinnen wieder auf die Berücksichtigung verzichten, etwa indem sie Diktate mitschreiben, obwohl sie davon freigestellt sind, indem sie eine innen zustehende Zeitverlängerung nicht ausnutzen oder indem sie in einer Klassenstufe gänzlich auf die Berücksichtigung verzichten. Sie verzichten damit auf ihr Grundrecht.

Beim Grundrechtsverzicht ist zwischen dem Grundrechtsverzicht, der den vollständigen Grundrechtsverlust zur Folge hat, und dem Grundrechtsausübungsverzicht zu differenzieren. Ein Verzicht auf die Grundrechtsfähigkeit oder auf die Fähigkeit Träger eines bestimmten Grundrechts zu sein, der zum vollständigen Grundrechtsverlust führt, ist unstatthaft. ${ }^{1549}$ In Betracht kommt daher nur ein Verzicht, mit dem auf die Ausübung oder Geltendmachung eines Grundrechts für einen bestimmten Zeitraum oder für einen konkreten Fall verzichtet wird. Der Verzichtende disponiert über den Grundrechtsinhalt, indem er auf die Inanspruchnahme des staatlichen Schutzes verzichtet ${ }^{1550}$, bleibt aber Inhaber des Grundrechts. ${ }^{1551}$ Für andere Fälle oder nach Zeitablauf kann das Grundrecht wieder vollumfänglich ausgeübt werden. ${ }^{1552}$ Bei einem freiwilligen Verzicht stellt die staatliche Handlung keinen Grundrechtseingriff dar und die Zurechnung der Grundrechtsbeschränkung zum Staat ist unterbrochen. ${ }^{1553}$

\footnotetext{
1547 S. dazu unten: 10. Teil: 1. Kapitel: 1.: Datenschutz im Verfahren der Grundentscheidung und der Festlegung des Nachteilsausgleichs, S. 310

1548 VG Lüneburg v. 28.5. 2002, Az.: 4 A 246/01, www.rechtsprechung.niedersachsen.de, S. 5; VG Darmstadt, Urteil v. 28. 4. 2009, Az.: 7 K 687/08.DA (3), S. 10 (nicht veröffentlicht); OVG Weimar, Beschluss v. 17.5.2010, Az.: 1 EO 854/10, juris, Rn. 55

1549 Merten, in: Hdb. Grundrechte III, § 73, Rn. 51

1550 Umfassend dazu Merten, in: Hdb. Grundrechte III, § 73 und Stern, Staatsrecht, S. $887 \mathrm{ff}$

1551 Michael/Morlok, Grundrechte, Rn. $534 \mathrm{ff}$

1552 Merten, in: Hdb. Grundrechte III, § 73, Rn. 24, ähnlich Stern, Staatsrecht, S. 915

1553 Merten, in: Hdb. Grundrechte III, § 73, Rn. 13 ff; Michael/Morlok, Grundrechte, Rn. 538 f
} 
Beim Verzicht auf ein Diskriminierungsverbot wird auf das Recht verzichtet, nicht diskriminiert zu werden. ${ }^{1554}$ Das bedeutet umgekehrt: der Verzicht enthält die Erlaubnis diskriminiert zu werden

Ein solcher Ausübungsverzicht ist grundsätzlich zulässig, da das Selbstbestimmungsrecht der Persönlichkeit aus Art. 2 Abs. 1 GG dazu berechtigt, auch über die eigenen Grundrechte zu verfügen. ${ }^{1555}$ Allerdings ist diese Verfügungsfreiheit nicht unbeschränkt, denn der Verzicht ist bei einigen Grundrechten möglich (z.B. Art. 16 I GG, Art. 6 III GG), bei anderen Grundrechten jedoch ausdrücklich ausgeschlossen (Art. 9 III 2 GG). Der Verzicht setzt daher die Disponibilität der betroffenen Rechtsgüter voraus. Grundsätzlich sind zwar alle Grundrechte disponibe ${ }^{1556}$, Unterschiede hinsichtlich des Umfangs der Disponibilität ergeben sich aber aus dem Inhalt des Grundrechts, auf das verzichtet werden soll. ${ }^{1557}$ Das Grundrecht ist umso mehr disponibel, je mehr es der persönlichen Entfaltungsfreiheit dient, und umso weniger disponibel, je mehr es vor allem eine objektiv-rechtliche Funktion hat und Allgemeininteressen dient. ${ }^{1558}$ Das Grundrecht aus Art. 3 III 2 GG ist ein Grundrecht, das sowohl dazu dienen soll, die persönliche Entfaltungsfreiheit der Menschen mit Behinderung zu sichern, als auch dem allgemeinen Grundsatz der Nichtdiskriminierung von Menschen mit Behinderung Rechnung zu tragen. Wegen der objektiv-rechtlichen Funktion des Diskriminierungsverbot als Schutz von Allgemeininteressen hat der EGMR das Diskriminierungsverbot aus Art. 14 EMRK i.V.m. Art. 2 EMRK, 1. Zusatzprotokoll, für unverzichtbar gehalten. ${ }^{1559}$ Die Bedeutung des Grundrechts aus Art. 3 III 2 GG für die Gesellschaft und ihre allgemeinen Interessen und seine unbedingte Schutzrichtung zeigen sich im deutschen Recht u.a. daran, dass nach $\S 31$ AGG und $\S \S 90 \mathrm{ff}$ SGB IX die Regelungen zum Schutz von Menschen mit Behinderungen und zur Abwehr von Diskriminierungen nicht abdingbar sind. Daraus könnte man schließen, dass das Grundrecht nicht in vollem Umfang zur Disposition der Betroffenen steht und daher ein völliger Verzicht auf das Grundrecht ausgeschlossen ist. ${ }^{1560}$ So sieht es z.B. das Land Schleswig-Holstein, wo ein Verzicht auf die Berücksichtigung einer förmlich anerkannten Legasthenie grundsätzlich für unzulässig gehalten wird, da Eltern oder Schüler die Bestimmungen des Legasthenie-Erlasses nicht von sich aus einseitig außer Kraft

\footnotetext{
${ }^{1554}$ EGMR Urteil v. 13.11.2007 - 57325/00, D.H. u.a./ Tschechien, NVwZ 2008, S. 533 ff, S. 536 Ziff. 202

1555 Stern, Staatsrecht, S. 907; Merten, in: Hdb. Grundrechte III, § 73, Rn. 35

1556 Michael/Morlok, Grundrechte, Rn. 535

1557 Stern, Staatsrecht, S. $908 \mathrm{ff}$

1558 Merten, in: Hdb. Grundrechte III, § 73, Rn. 37

1559 EGMR Urteil v. 13.11.2007 - 57325/00, D.H. u.a./ Tschechien, NVwZ 2008, S. 533 ff, S. 536 Ziff. 204

1560 Stern, Staatsrecht, S. 915
} 
setzten können. ${ }^{1561}$ Allerdings würde dies bedeuten, dass ein Mensch mit Behinderung sich immer schützen lassen muss, auch wenn er dies nicht möchte. Das wiederum widerspricht dem Gedanken der Freiheitsrechte, die auch die Freiheit beinhalten, sich nicht schützen zu lassen. ${ }^{1562}$ Daher ist grundsätzlich auch hier ein Verzicht möglich.

Der Ausübungsverzicht erfolgt durch Willenserklärung durch den Grundrechtsträger. ${ }^{1563}$ Dieser muss einsichtsfähig und in der Lage sein, die Folgen seiner Entscheidung zu übersehen. ${ }^{1564}$ Träger des Grundrechts aus Art. 3 III 2 GG ist das betroffene Kind. Dieses ist mangels Geschäftsfähigkeit grundsätzlich nicht in der Lage, selbst auf die Ausübung des Grundrechts zu verzichten. Die Frage, ab wann ein Kind in der Lage ist, allein über die Ausübung seines Grundrechts zu entscheiden, ist eine Frage der Grundrechtsmündigkeit. ${ }^{1565}$ Wann diese eintritt, hängt vom Inhalt des jeweiligen Grundrechts ab, dessen Ausübung troffen ist. Sie hängt weiter ab vom Maß an geistiger Reife, Verantwortungsbewusstsein und Reflexionsfähigkeit, das Minderjährige in einem bestimmten Alter in der Regel aufweisen. ${ }^{1566}$ Angesichts der erheblichen Tragweite für die Schullaufbahn und den weiteren Lebensweg erfordert die selbständige Ausübung dieses Grundrechts ein hohes Maß an geistiger Reife und an Reflexionsfähigkeit, so dass eine Grundrechtsmündigkeit Minderjähriger für dieses Grundrecht allenfalls differenziert in Betracht kommt. Dabei ist zu beachten, dass die Entscheidungen der betroffenen Kinder zur Berücksichtigung von Legasthenie/Dyskalkulie in der Regel erheblich davon beeinflusst werden, dass sie in der Klassengemeinschaft nicht auffallen und keine Sonderrolle einnehmen möchten. Deshalb können sie je nach Alter allenfalls Entscheidungen selbständig treffen, die sich kurzfristig auswirken und jederzeit widerrufbar sind, etwa die Teilnahme an einem bestimmten Diktat und das Nichtausnutzen einer bestimmten Zeitverlängerung in einer Klassenarbeit/einem Test. Entscheidungen, die sich wesentlich auswirken, wie ein längerer Verzicht auf die Berücksichtigung, können sie nicht überblicken und daher auch mit zunehmendem Alter nicht entscheiden.

Infolgedessen haben nur die Eltern als Erziehungsberechtigte die Befugnis, für die Kinder auf die Ausübung des Grundrechts zu verzichten. Das Elternrecht aus Art. 6 II GG ist nicht nur ein Grundrecht, sondern auch eine Pflicht der Eltern. Es ist ein treuhänderisches Recht, das wesentlich im Kindesinteresse

\footnotetext{
1561 SH Legasthenie FAQ, S. 20

1562 S. zum „Recht auf Krankheit“ bereits oben S. $202 \mathrm{ff}$

1563 Merten, in: Hdb. Grundrechte III, § 73, Rn. 38

1564 Michael/Morlok, Grundrechte, Rn. 538

1565 Huber, in: Hdb. Grundrechte II, § 49, Rn. 16

1566 Huber, in: Hdb. Grundrechte II, § 49, Rn. 18
} 
wahrzunehmen ist. ${ }^{1567}$ Grund und Grenze des Elternrechts ist das Kindeswohl. ${ }^{1568}$ Mit dem Wohl des Kindes ist es prinzipiell unvereinbar, wenn Eltern auf das Grundrecht aus Art. 3 III 2 GG, das gerade dem besonderen Schutz des Kindes dient, verzichten. Dies gilt erst Recht bei Legasthenie/Dyskalkulie, weil der Verzicht die Nicht-Berücksichtigung der Behinderung im Unterricht zur Folge hätte, dies aber zu erheblichen psychischen Belastungen und Sekundärerkrankungen des Kindes führen kann. ${ }^{1569}$ Ein Verzicht kommt daher nur in engen Ausnahmesituationen in Betracht, in denen es für die individuelle Situation des Kindes und sein konkretes Wohl besser ist, wenn vorübergehend auf die Berücksichtigung der Behinderung verzichtet wird. Das ist etwa denkbar, wenn ein Kind es nicht ertragen kann, wegen der Legasthenie/Dyskalkulie einen Zeitzuschlag zu erhalten und damit bei Prüfungen in der Klasse als „Sonderfall" aufzufallen. Dann kann die Belastung für das Kind durch die Sonderrolle größer sein, als wenn auf den Zeitzuschlag verzichtet wird, bis sich das Kind an seine besondere Situation gewöhnt hat. ${ }^{1570}$

Der Ausübungsverzicht muss freiwillig erfolgen, muss frei von Täuschung und Willensmängeln, konkret und für den Betroffenen überschaubar ${ }^{1571}$, d.h. in voller Kenntnis der Tatsachen, sein. ${ }^{1572}$ Den Betroffenen müssen eventuelle Auswirkungen ihrer Entscheidung aufgezeigt werden. Fehlt es an der Freiwilligkeit oder beruhte die Erklärung auf einem Irrtum, so ist der Widerruf jederzeit ex tunc möglich. ${ }^{1573}$

Eine freiwillige Verzichtserklärung bewirkt, dass der Grundrechtseingriff, der aufgrund des Verzichts vorgenommen wurde, rechtmäßig ist und durch einen nachträglichen Widerruf nicht rechtswidrig wird. ${ }^{1574}$ Der Widerruf einer wirksam erklärten, freiwilligen Verzichtserklärung ist jedoch jederzeit ex nunc möglich. ${ }^{1575}$

1567 Ennuschat, RdJB 2007, S. 271 ff, S. 276; Jarass/Pieroth, in: Jarass/Pieroth GG, Art. 6, Rn. 36

1568 Lorz, Vorrang Art. 3 UN-KRK, S. 41 f; Dolzer/Waldhoff,/Graßhof, in: Bonner Kommentar GG, Art. 6, Rn. $34 \mathrm{ff}$

1569 S. oben 1. Teil: 1. Kapitel: 9.: Welche psychischen Auswirkungen haben Legasthenie und/oder Dyskalkulie, S. 26

1570 Lenhard/Küspert/Becker, in: Legasthenie, S. 143

1571 Stern, Staatsrecht, S. 914 f; Merten, in: Hdb. Grundrechte III, § 73, Rn. 38 ff

1572 EGMR Urteil v. 13.11.2007 - 57325/00 (D.H. u.a./ Tschechien), NVwZ 2008, S. 533 ff, 535 Ziff. 203

1573 Stern, Staatsrecht, S. 916

1574 Stern, Staatsrecht, S. 915 f

1575 Stern, Staatsrecht, S. 916 unter Verweis auf Robbers, JuS 1985, S. 925 ff 


\section{Verzicht auf Berücksichtigung der Behinderung um die Zeugnisbemerkung zu vermeiden}

Alle landesrechtlichen Regelungen schreiben zwingend eine Bemerkung im Zeugnis vor, wenn die Rechtschreibung nicht bewertet wird. ${ }^{1576}$ Die Bemerkung wird meistens relativ neutral formuliert. Sie lautet z.B.: „Aufgrund von Schwierigkeiten im Lesen und Rechtschreiben wurde die Rechtschreibung nicht bewertet. ${ }^{\prime 1577}$ Diese Zeugnisbemerkung kann nur vermieden werden, wenn auf die Berücksichtigung der Legasthenie verzichtet wird. Bei einem Verzicht erfolgen Bewertung und Prüfung der Leistung dann nach den allgemeinen Regelungen. So ist z.B. in Bayern der Verzicht auf die Berücksichtigung am Ende der Klasse 8 in der Hauptschule möglich. Nachteilsausgleich und Nicht-Bewertung der Rechtschreibung werden dann nicht mehr gewährt. ${ }^{1578}$ Es besteht somit ein Junktim zwischen der Berücksichtigung der Behinderung und der Zeugnisbemerkung. Häufig wird auf die Berücksichtigung der Behinderung verzichtet, um diese Zeugnisbemerkung zu vermeiden.

Unabhängig von der noch zu prüfenden Frage, ob eine solche Bemerkung überhaupt zulässig ist ${ }^{1579}$, stellt sich hier die Frage, ob der Verzicht auf die Berücksichtigung der Behinderung freiwillig ist. Der Verzicht auf die Ausübung eines Grundrechts ist nur dann freiwillig, wenn erstens der Grundrechtsträger die reale Möglichkeit hat, den Grundrechtsschutz in Anspruch zu nehmen, und zweitens der Staat den Grundrechtsverzicht annehmen kann, ohne dabei gegen seine eigene Grundrechtsbindung zu verstoßen. ${ }^{1580}$ Freiwillig ist ein Verzicht daher nur dann, wenn er auf einer eigenen, unabhängigen Entscheidung des Grundrechtsträgers beruht und nicht mit einer Maßnahme gekoppelt ist,

1576 BW VV Besonderer Förderbedarf Nr. 2.3.1; BY KMBek Legasthenie Nr. 3.1; BE GsVO § 16 VIII, BE Sek I-VO § 14 III; BB VV-LRSR Nr. 8 II; HB LRS-Erlass Nr. 4.2; HH Förder-Richtlinie Nr. 4.4; HE VO-GestSchulVerh § 7 IV und 43 II; MV VV-LRR Nr. 8 II, 7 VI; NI LRR-Erlass Nr. 4.2; NRW LRS-Erlass Nr. 4.2; RP VV-LRS Nr. 4.4; SL VV-LRS Nr. 5.1; SN VV-LRS Nr. 4.1.4; ST Grundschulbewertungs-Erlass Nr. 4.1.3, ST Sekundarstufenbewertungs-Erlass Nr. 5.1.3; SH LegasthenieErlass Nr. 3.1, TH Fachliche Förderempfehlung

1577 BW VV Besonderer Förderbedarf Nr. 2.3.1; BY KMBek Legasthenie Nr. 3.1; BE GsVO § 16 VIII, BE Sek I-VO § 14 III; HB LRS-Erlass Nr. 4.2; HH Förder-Richtlinie Nr. 4.4; HE VO-GestSchulVerh $\S 7 \mathrm{IV}$ und $43 \mathrm{II}$; MV VV-LRR Nr. 8 II; NI LRR-Erlass Nr. 4.2; NRW LRS-Erlass Nr. 4.2; RP VV-LRS Nr. 4.4; SL VV-LRS Nr. 5.1; SN VV-LRS Nr. 4.1.4; ST Grundschulbewertungs-Erlass Nr. 4.1.3, ST Sekundarstufenbewertungs-Erlass Nr. 5.1.3; SH Legasthenie-Erlass Nr. 3.1, TH SchulO §§ 59 VI, 60 I S. 2 i.V.m. TH Fachliche Förderempfehlung

1578 BY KMS IV/1 - 7306/4 - 4/55 382 vom 09.08.2000 Punkt 6, http://www.schulberatung.bayern.de/schulberatung/index_05168.asp, demgegenüber wird in Schleswig-Holstein der Verzicht grundsätzlich für unzulässig gehalten, auch dann, wenn er dazu dient, die Zeugnisbemerkung zu vermeiden, s. SH Legasthenie FAQ S. 20)

1579 S. dazu unten S. $318 \mathrm{ff}$

1580 Michael/Morlok, Grundrechte, Rn. 538 
die den Willen des Verzichtenden beeinträchtigt. ${ }^{1581}$ Wird der Verzicht jedoch durch die Gewährung von angeblichen Vorteilen herbeigeführt, die die Entscheidung beeinflussen, liegt keine Freiwilligkeit vor, sondern es bleibt beim Grundrechtseingriff. ${ }^{1582}$ So liegt es hier: Die Schüler verzichten nicht aufgrund einer eigenen, unabhängigen Entscheidung, etwa weil sie meinen, die Berücksichtigung nicht mehr zu benötigen, sondern um einen angeblichen Vorteil zu erlangen, nämlich die Vermeidung der Zeugnisbemerkung. Tatsächlich beinhaltet der Verzicht die Einwilligung des Schülers, den Eingriff in sein Grundrecht in der Form der Nicht-Berücksichtigung zu dulden. Der Schüler entscheidet sich nicht freiwillig, sondern er wägt zwischen zwei Übeln, die inm der Staat anbietet, ab und wählt das scheinbar geringere. Dieser Verzicht auf die Ausübung des Grundrechts erfolgt also, um einen Nachteil zu verhindern und ist deshalb nicht mehr freiwillig und mithin unwirksam. ${ }^{1583}$

Zudem kann der Staat hier den Grundrechtsverzicht auch nicht annehmen, ohne gegen die eigene Grundrechtsbindung zu verstoßen. Nimmt er den Verzicht an, so führt dies dazu, dass die Ausübung des Grundrechts für die Dauer des Verzichts im Schulbereich und damit in dem maßgeblichen Lebensbereich des Schülers mit Behinderung ausgeschlossen ist. Die Schule, die in besonderer Weise dazu verpflichtet ist, niemanden wegen seiner Behinderung zu benachteiligen, erhält mit dem Verzicht die Erlaubnis, wegen der Behinderung zu diskriminieren. Das ist nicht mit dem umfassenden Schutzzweck des Art. 3 III 2 GG zu vereinbaren.

Weil der Verzicht nicht freiwillig ist, bleibt die Nicht-Berücksichtigung der Behinderung, um die Zeugnisbemerkung zu vermeiden, ein Grundrechtseingriff $^{1584}$ in Art. 3 III 2 GG. Die Rechtswidrigkeit kann nur dadurch aufgehoben werden, dass die Kopplung zwischen dem Verzicht auf die Berücksichtigung und der Zeugnisbemerkung aufgehoben wird.

\footnotetext{
1581 Stern, Staatsrecht, S. 914

1582 Jarass/Pieroth, in: Jarass/Pieroth GG, Vorb. Vor Art. 1, Rn. 34 und 35, s. das Beispiel bei Stern, Staatsrecht, S. 914, Fn. 120, wo die Erteilung einer Baugenehmigung davon abhängig gemacht wurde, dass sich der Bauherr "freiwillig" und unter Verzicht auf Rechtsmittel dazu verpflichten sollte, eine bestimmte, an sich zulässige Geschosszahl nicht zu überschreiten.

Damit sind auch die bayerischen Regelungen, die den Verzicht für unwiderruflich halten, nicht vereinbar. s. KMS V/2-S6402/5-5/132 0292542 v. 11.1.2001 (Realschule), KMS III. 6 5S4306.4 - 6.110754 v. 18.10. und 16.11.2007 (Realschule) sowie KMS IV/1 - 7306/4 - 4/55 382 vom 09.08.2000 Punkt 6, III. 6-KMS 5 S 4306.4 - 6.113 140 v. 18 3. 2010 - alle: http://www.schulberatung.bayern.de/schulberatung. Abgegebene Verzichtserklärungen, sind jederzeit widerrufbar; im Übrigen stellt sich die Frage, ob ein derart weitreichender Eingriff in die Grundrechte durch Erlass getroffen werden kann.

${ }^{1584}$ Vgl. Jarass/Pieroth, in: Jarass/Pieroth GG, Vorb. Vor Art. 1, Rn. 34 und 35
} 


\section{Verzicht auf Berücksichtigung der Behinderung durch Nicht- Geltendmachung?}

Das VG Sigmaringen hat 2008 einem Schüler vorgehalten, es sei nicht glaubhaft, dass er für die Abiturprüfung die Berücksichtigung der Legasthenie brauche und durch die Legasthenie beeinträchtigt sei, weil er über sechs Jahre die Berücksichtigung der Legasthenie nicht geltend gemacht habe. ${ }^{1585}$ Die Argumentation des VG Sigmaringen kann so verstanden werden, dass der Betroffene dadurch, dass er sein Grundrecht über einen längeren Zeitraum nicht geltend gemacht hat, darauf verzichtet hat und sich nicht mehr darauf berufen kann.

Fraglich ist, ob die Nicht-Geltendmachung eines Grundrechts über einen längeren Zeitraum dazu führt, dass sich der Grundrechtsträger nicht mehr darauf berufen kann. Zweifelhaft ist an der Argumentation des VG Sigmaringen bereits, ob der Grundrechtsträger überhaupt dazu verpflichtet ist, sein Grundrecht geltend zu machen. Denn der Staat und damit auch die Schule sind immer an die Grundrechte gebunden und nicht nur dann, wenn der Grundrechtsträger sie geltend macht. Unabhängig davon ist die schlichte Nichtausübung eines Rechts dadurch, dass man sich nicht darauf beruft, ein bloßer Nichtgebrauch des Rechts. ${ }^{1586}$ Sie ist ein Fall der Selbstbeschränkung, der Enthaltung oder des Bewendenlassens, nicht aber des „Verzichts“ im Sinne der völligen Aufgabe. ${ }^{1587}$. Der Nichtgebrauch von Grundrechten genießt grundsätzlich den gleichen Schutz wie der Gebrauch. Er ist in gleicher Weise wie dieser Freiheitsausübung $^{1588}$ und beinhaltet keinen rechtsverbindlichen Verzicht. Die Innehabung des Rechts und die Befugnis, es auszuüben bleiben unangetastet, so dass sich der Grundrechtsträger jederzeit wieder auf das Grundrecht berufen kann und an dessen Geltendmachung rechtlich nicht gehindert ist. ${ }^{1589}$

Zudem könnten aus dem Nichtgebrauch eines Grundrechts durch ein Kind nur dann rechtsvernichtende Schlüsse gezogen werden, wenn hierfür ein mindestens konkludenter zulässiger Verzicht der Eltern für das Kind vorliegen würde. An einen konkludenten Ausübungsverzicht auf ein Grundrecht sind erhebliche Anforderungen zu stellen, notwendig ist dafür ein unzweideutiger Wille. ${ }^{1590}$ Ein solcher kann jedoch nicht allein deshalb angenommen werden, weil die Eltern das Grundrecht des Kindes nicht ausgeübt haben. Insbesondere im Fall des VG Sigmaringen kann von einem solchen unzweideutigen Willen nicht aus-

\footnotetext{
1585 VG Sigmaringen, Beschluss v. 2.4.2008, Az.: 4 K 596/08, S. 4 (nicht veröffentlicht)

1586 Merten, in: Hdb. Grundrechte III, § 73, Rn. 3

1587 Merten, in: Hdb. Grundrechte III, § 73, Rn. 9

1588 Merten, in: Hdb. Grundrechte III, § 73, Rn. 7

1589 Merten, in: Hdb. Grundrechte III, § 73, Rn. 3

1590 Stern, Staatsrecht, S. 914
} 
gegangen werden, weil damals in Baden-Württemberg die Berücksichtigung einer Legasthenie nach der Klasse 6 endete und ab der 7. Klasse nur noch in begründeten Einzelfällen zulässig war. ${ }^{1591}$ Da derartige Anträge regelmäßig erfolglos waren, wurden sie von den Eltern nicht gestellt. Im Jahr 2008 hatte sich die Lage durch die neue rechtliche Einschätzung von Langenfeld jedoch entscheidend geändert. ${ }^{1592}$

\section{Kapitel: Anspruch auf Förderung}

Neben dem Ausgleich der Behinderung ist es für die betroffenen Schüler und Schülerinnen besonders wichtig, dass sie in der Schule so gefördert werden, dass sie Lesen, Schreiben und Rechnen so gut, wie es ihnen irgend möglich ist, erlernen. Zu klären ist daher, ob sie neben dem Anspruch auf Berücksichtigung der Behinderung aus den Grundrechten einen Anspruch auf spezifischen Unterricht, besondere Förderung und/oder Einzelförderung haben.

\section{Anspruch auf individuelle, legasthenie-/dyskalkuliespezifische Förderung}

Ein Anspruch auf individuelle, legasthenie-/dyskalkuliespezifische Förderung kann sich aus dem allgemeinen Auftrag der Schule ergeben. Nach ganz allgemeiner Meinung hat die Schule zwar einen Förderauftrag, dieser besteht aber nur dem Grunde nach, und nicht als Anspruch auf eine bestimmte Fördermaßnahme ${ }^{1593}$, weil das öffentliche Schulwesen nicht sämtlichen unterschiedlichen individuellen Begabungen und Schwächen voll Rechnung tragen kann. ${ }^{1594}$ Die Wahl der Fördermaßnahme steht im Ermessen der zuständigen Schule bzw. Schulbehörde. ${ }^{1595}$ Ein individueller Anspruch ergibt sich daraus nicht.

Ein individueller, spezifischer Anspruch lässt sich auch nicht aus den landesrechtlichen Regelungen zur Legasthenie/Dyskalkulie herleiten. Diese legen zwar den Schwerpunkt auf die Förderung dieser Kinder und verpflichten die Lehrkräfte hier zu umfangreichen Anstrengungen. Sie geben aber auch keinen Anspruch auf individuelle, spezifische Förderung.

\footnotetext{
1591 BW VV Legasthenie v. 10.12.1997; Az.: IV/2-6504.2/206, Nr. 3.3 http://www.opti-school.de/ Erlass-BaW_.pdfleB61.htm

1592 Langenfeld, RdJB 2007, S. $211 \mathrm{ff}$

1593 Ausführlich dazu Schwengers, Eingliederungshilfe § 35 a SGB VIII, S. 169 ff; Sidortschuk, JAmt 2005, S. 552 f, S. 553; s. auch Ennuschat, Die Rechte stotternder Menschen in Schule, Ausbildung und Studium, S. 58

1594 Niehues/Rux, Schulrecht, Rn. 176

1595 Sidortschuk, JAmt 2005, S. $552 \mathrm{f}$
} 
Der Anspruch könnte sich aus der Feststellung des sonderpädagogischen Förderbedarfs ergeben. Sonderpädagogischer Förderbedarf ist nach der Empfehlung der $\mathrm{KMK}^{1596}$ anzunehmen bei Kindern und Jugendlichen, die in ihren Bildungs-, Entwicklungs- und Lernmöglichkeiten so beeinträchtigt sind, dass sie im Unterricht der allgemeinen Schule ohne sonderpädagogische Förderung nicht hinreichend gefördert werden können. Er umfasst keine individuelle, legasthenie-/dyskalkuliespezifische Förderung.

Fraglich ist daher, ob sich direkt aus Art. 3 III 2 GG ein Anspruch auf spezifische Förderung ergibt. Nach einer Ansicht lässt sich aus Art. 3 III 2 GG ein solcher Anspruch auf schulische Bildung ohne Benachteiligung mindestens in Form eines unverzichtbaren Mindeststandards herleiten, den die Schule evident verletzt, wenn sie keine angemessene Förderung leistet. ${ }^{1597}$ Nach ganz herrschender Ansicht ergibt sich dagegen aus Art. 3 III 2 GG kein originärer grundrechtlicher Leistungsanspruch ${ }^{1598}$, so dass sich auch kein Anspruch auf eine ganz bestimmte, konkrete Leistung für den Einzelnen herleiten lässt.

Das BVerfG hat diese Frage ausdrücklich offen gelassen. ${ }^{1599}$ Allerdings hat es in einer Entscheidung aus dem Jahr 2012 darauf hingewiesen, dass die Leistungen der Schule bei einem behinderungsbedingten sonderpädagogischen Förderbedarf grundsätzlich durch individuelle Fördermaßnahmen ergänzt werden und dem Schüler der Rechtsweg offensteht, wenn solche Fördermaßnahmen abgelehnt werden. ${ }^{1600} \mathrm{Ob}$ sich das BVerfG damit für einen Anspruch auf individuelle Förderung ausgesprochen hat, bleibt abzuwarten.

\section{Anspruch auf spezifische Förderung aufgrund der Schulpflicht}

Der Anspruch auf individuelle, fachlich geeignete Förderung kann sich aus Art. 2 I i.V.m. Art. 7 । GG ${ }^{1601}$ ergeben. Die Schulpflicht greift in die allgemeine Handlungsfreiheit des Kindes ein. Die Ausgestaltung der Schulpflicht und die Begrenzung der Freiheit des Schülers muss im Hinblick auf die Ziele des Art. 7 I GG und vor allem im Hinblick auf das Entfaltungsrecht der Schüler sachgerecht und

\footnotetext{
1596 KMK-SP Nr. 2 II

1597 VG Düsseldorf, Urteil v. 22.1.2001, Az.: 19 K 11140/98, www.justiz.nrw.de, Rn. 116 ff; Luthe, in: Rehabilitationsrecht - Verfassungsrecht, Rn. 9; zum Anspruch auf lehrplanmäßigen Unterricht vgl. Forkel, SächsVBI 2010, S. 282 ff, S. 283 ff

1598 Sacksofsky, in: Umbach GG, Art. 3, Rn. 393; Kischel, in: BeckOK GG, Art. 3, Rn. 215; Neumann, in: Handbuch SGB IX, § 2 Verfassungsrecht, Rn. 32; Reichenbach, Anspruch behinderter Schülerinnen und Schüler auf Unterricht in der Regelschule, S. 181; Füssel, RdJB 1998, S. 250 ff, S. 254; Dirnaicher, BayVBI 1997, S. 545 ff, S. 552

1599 BVerfGE 96, 288, 304

1600 BVerfG, Beschluss v. 8.6.2012; Az.: 1 BvR 2853/10, http://www.bverfg.de/entscheidungen/rk20120608_1bvr285310.html, Rn. 9

${ }^{1601}$ Vgl. zu dieser Grundlage: Jarass, DÖV 1995, S. 674 ff, S. 677
} 
geeignet sein. ${ }^{1602}$ Sie muss so angelegt sein, dass die Schüler und Schülerinnen diejenigen Fähigkeiten und Kenntnisse erwerben können, die sie für ihren weiteren Lebens- und Berufsweg brauchen. ${ }^{1603}$ Dabei muss die Behinderung beachtet werden ${ }^{1604}$, nur dann ist der Eingriff durch die Schulpflicht verhältnismäßig. ${ }^{1605}$

Werden Schüler und Schülerinnen mit Legasthenie/Dyskalkulie in der Schule nicht mit einer auf ihre Behinderung angelegten speziellen Förderung betreut, erlernen sie schon die Grundlagen nicht, die für das Erlernen des Lesens, Schreibens und Rechnens erforderlich sind. Dann fehlen ihnen die visuellen, auditiven, phonologischen und ähnlichen Fähigkeiten, auf denen der Erwerb der Kulturtechniken aufbaut. Der folgende Unterricht muss an ihnen vorbeigehen. Selbst wenn der Unterricht der Lehrkraft für alle anderen Schüler optimal und sachgerecht ist, bleibt er für diese Schüler nicht sachgerecht und ungeeignet, weil sie mit dieser Unterrichtsgestaltung das Lernziel nicht erreichen können. Vergleichbar wäre es, wenn man blinde oder gehörlose Schüler und Schülerinnen in der Regelschule ohne Rücksicht auf ihre Behinderung beschulen würde. Sie können dem Unterricht dann nicht folgen. Damit verfehlt die Schule bei diesen Schülern ihren Zweck, neben der Erhaltung des Gemeinwesens auch den einzelnen Schülern die Voraussetzung für eine erfolgreiche Lebensgestaltung zu schaffen. Das wäre als Eingriff in das Grundrecht der Schüler unverhältnismäßig und daher rechtswidrig. ${ }^{1606}$

Das wäre gegebenenfalls hinzunehmen, wenn die Schule in der Lage wäre, durch ihr Fördersystem sicherzustellen, dass die Schüler und Schülerinnen mit Legasthenie/Dyskalkulie diejenigen Fähigkeiten und Kenntnisse erwerben können, die sie für ihren weiteren Lebens- und Berufsweg brauchen, auch wenn sie dafür länger brauchen. ${ }^{1607}$ Dem ist aber nicht so. Die Diskussion um den Begriff Legasthenie, die schon mehr als 40 Jahre dauert, zeigt bereits, dass es immer Kinder mit dieser Behinderung gibt und dass es der Schule mit ihren Methoden nicht gelingt, diese Schwierigkeiten aufzufangen. Selbst die schärfste Kritikerin des Legasthenie-Begriffs Valtin räumt ein: „Leider gelingt es aber auch unter günstigen schulischen Voraussetzungen nicht immer, LRS zu verhindern. In diesen Fällen ist die außerschulische Hilfe von Experten notwendig. ${ }^{\text {"1608 }}$

\footnotetext{
1602 Jarass, DÖV 1995, S. 674 ff, S. 678; Glotz/Faber, in: Hdb. des Verfassungsrechts, § 28, Rn. 11

1603 Niehues/Rux, Schulrecht, Rn. 137

1604 S. oben 6. Teil: 2. Kapitel: Anspruch aus Art. 2 I und 6 || GG - Schulpflicht, S. 222; VG Düsseldorf, Urteil v. 22.1.2001, Az.: 19 K 11140/98, juris, Rn. 16

1605 Robbers, in: v. Mangoldt/Klein/Starck GG, Art. 7, Rn. 31

1606 Langenfeld, RdJB 2007, S. 211 ff, S. 222

1607 S. oben 5. Teil: 2. Kapitel: 2.1.: Pflicht zur Teilnahme an Förderung, S. 202

${ }^{1608}$ Valtin, in: LRS und Legasthenie, S. 62
} 
Eine lediglich allgemeine und undifferenzierte Förderung in der Schule ist auch deshalb nicht verhältnismäßig und mit dem Wohl des Kindes unvereinbar, weil sie dem einzelnen Kind nicht gerecht wird, dadurch ggf. Lebenschancen entzieht und es in Kauf nimmt, dass es funktionaler Analphabet bleibt. Jedes Zuwarten mit einer fundierten Förderung verschlechtert die Chancen auf Besserung bei dem Kind erheblich. ${ }^{1609}$ Das Problem „wächst sich nicht aus" sondern wächst, potenziert sich, verschlimmert sich. ${ }^{1610}$ Für bestimmte Entwicklungsund Lernschritte gibt es Zeitfenster, innerhalb derer diese Entwicklungen erfolgt sein müssen. Ist das Zeitfenster geschlossen, sind die Lernschritte nicht mehr aufzuholen. Mit dem Zuwarten, inwieweit die übliche schulische Förderung Erfolg bringt oder nicht, wird das Zeitfenster für das Erlernen der Basisfähigkeiten immer kleiner, bis es sich ganz schließt und die Fähigkeit damit unwiederbringlich verloren ist. Die Förderung muss daher in den ersten beiden Grundschulklassen beginnen. Gerade deshalb ist das Zuwarten und die Sorge vor "riskanten Zuschreibungen und Etikettierungen“1611 für diese Kinder besonders negativ.

Das Ermessen der Schule, das sie grundsätzlich in Bezug auf Fördermaßnahmen hat, reduziert sich bei Schülern und Schülerinnen mit Legasthenie/Dyskalkulie auf eine Förderung die spezifisch auf ihre Behinderung angelegt ist und die individuelle Behinderung des Kindes berücksichtigt.

Ohne spezifische, geeignete Förderung wird dem Kind mit Legasthenie/Dyskalkulie sein "Minimumgrundrecht" auf Teilhabe und Zugang zur Regelschule als "Kehrseite“ der Schulpflicht versagt. ${ }^{1612}$ Der Eingriff in seine Handlungsfreiheit durch die Schulpflicht ist dann nicht gerechtfertigt.

\section{Zuständigkeit der Schule oder anderer Institutionen?}

Es stellt sich allerdings die Frage, ob die Schule zur fachlich erforderlichen Förderung bei Legasthenie/Dyskalkulie überhaupt in der Lage ist. Die bei Legasthenie/Dyskalkulie erforderliche Einzelförderung ist dem Schulsystem eigentlich fremd. Braucht ein Kind mit Behinderung oder auch sonstigen Beeinträchtigungen eine Einzelförderung, z.B. durch Logopädie, Ergotherapie, Krankengymnastik oder sonstige Therapieformen, so findet diese immer außerschulisch statt und wird ganz überwiegend durch die Krankenkassen gezahlt. Bei der Legasthenie/Dyskalkulie ergeben sich jedoch zwei Besonderheiten: Zum einen werden die Therapien bei Legasthenie/Dyskalkulie nicht von den Krankenkas-

\footnotetext{
1609 Meysen, JAmt 2003, S. 53 ff, S. 61; Klasen, Legasthenie, S. 69

1610 S. dazu die Ergebnisse bei Löffler/Meyer-Schepers/Lischeid, in: Legasthenie und Dyskalkulie 2007, S. $268 \mathrm{ff}, 275$

1611 Prengel, Sozialextra 2006, S. $15 \mathrm{ff}$

1612 Vgl. Glotz/Faber, in: Hdb. des Verfassungsrechts, § 28, Rn. 13; VG Düsseldorf, Urteil v. 22.1.2001, Az.: 19 K 11140/98, juris, Rn. 116
} 
sen gezahlt ${ }^{1613}$, zum Anderen sieht Schule die Vermittlung von Lesen, Schreiben und Rechnen als ihre Aufgabe an, die anders als bei Beeinträchtigungen wie z.B. Stottern, Lispeln oder spastischen Beeinträchtigungen, nicht an außerschulische Einrichtungen abgegeben werden soll.

Fest steht, dass Lehrkräfte mit ihrer allgemeinen pädagogischen Ausbildung und ohne Spezialkenntnisse nicht in der Lage sind, Schülern mit Legasthenie/ Dyskalkulie das Lesen, Schreiben und Rechnen zu vermitteln. Dieses räumen auch Kritiker des Legastheniebegriffs ein. ${ }^{1614}$ Es muss daher geklärt werden, ob es dennoch zum (alleinigen) Zuständigkeitsbereich der Schule gehört, die notwendige, individuelle, wissenschaftlich fundierte Förderung und Therapie zu leisten. ${ }^{1615}$ Ist dies der Fall, so ist die Schule vollumfänglich dafür zuständig, muss diese Förderung durch geeignete und qualifizierte Lehrkräfte erbringen ${ }^{1616}$ und auch die haftungsrechtlichen Konsequenzen für den Fall der Nichterbringung tragen. ${ }^{1617}$

Möglich ist aber auch ein Neben- und Miteinander von schulischer Förderung und individueller Therapie, die durch Fachkräfte, die bereits jetzt Therapien nach $\S 35$ a SGB VIII erbringen, erfolgt. In diesem Fall bleibt die Frage zu klären, wer die Kosten trägt. Zuständig dafür könnten, trotz der abweichenden Rechtsprechung des BSG, die Krankenkassen $\operatorname{sein}^{1618}$, denn es handelt sich bei Legasthenie/Dyskalkulie um hirnorganische Störungen. Es ist deshalb nicht einzusehen, warum die Finanzierung der Therapie dieser - inzwischen auch mit bildgebenden Verfahren nachweisbaren - Störung nicht in den Zuständigkeitsbereich der Krankenkassen fallen sollte.

Diese Frage kann im Rahmen dieser Arbeit nicht geklärt werden, denn sie wird durch vielerlei auch finanzielle Interessen, die zudem auf Bund, Länder und Kommunen verteilt sind, bestimmt. Die Verpflichtung dazu, diese Fragen zu klären, ergibt sich für den Staat jedoch auch aus Art. 24 BRK. Dieser verpflichtet ihn, Schülern und Schülerinnen mit Behinderung die notwendige, individuell angepasste Unterstützung zukommen zu lassen, um erfolgreiche Bildung zu erleichtern (Art. 24 Abs. 2 lit c, d und e BRK). ${ }^{1619}$ Die Verpflichtung

\footnotetext{
1613 S. dazu oben S. 46

1614 Valtin, in: LRS und Legasthenie, S. 62

1615 Sidortschuk, JAmt 2005, S. $552 \mathrm{f}$

1616 5. Teil: 2. Kapitel: 2.1.: Pflicht zur Teilnahme an Förderung, S. 202

1617 S. dazu 11. Teil: Amtshaftung aus § 839 BGB i.V.m. Art. 34 GG, S. 333

1618 S. oben 1. Teil: 2. Kapitel: 7.2.1.: Finanzierung durch die Krankenkassen nach § 27 SGB V, S. 46

1619 Die Schattenübersetzung spricht von „ermöglichen“ (Art. 24 Abs. 2 lit d); Arnade, Schattenübersetzung UN-BRK
} 
ergibt sich auch aus der neuesten Rechtsprechung des BVerfG zu „Hartz IV“1620, in der das BVerfG geurteilt hat, dass sich ein Anspruch von hilfebedürftigen Kindern aus Art. 1 Abs. 1 GG i.V.m. dem Sozialstaatsprinzip des Art. 20 Abs. 1 GG darauf ergibt, dass ihr existentieller Bedarf gedeckt wird. Wenn es zum Existenzminimum und existenziellen Bedarf gehört, dass ein hilfebedürftiger Schüler über das Sozialgeld Nachhilfeunterricht erhält, damit er nicht von Lebenschancen ausgeschlossen wird ${ }^{1621}$, muss dies erst recht für Förder- oder Therapieleistungen gelten, die aufgrund einer Behinderung erforderlich sind, um die Fertigkeiten, die für den Schulbesuch erforderlich sind, zu erlernen.

Im Moment ist leider zu konstatieren, dass die Frage auf dem Rücken der Kinder ausgetragen wird, die im Moment gegen keinen der möglichen Kostenträger Krankenkassen Schulen und Jugendämter einen durchsetzbaren Anspruch auf individuelle, spezifische, geeignete Förderung haben. Es wird sowohl hingenommen, dass diese Kinder in der Schule nicht qualifiziert gefördert werden als auch, dass sie durch unqualifizierte Maßnahmen psychisch krank werden. Selbst dann wird ihnen noch Hilfe versagt, weil die psychischen Beeinträchtigungen nicht groß genug sind. Dabei wird im Kompetenzgerangel übersehen: Ein behindertes Kind hat das Recht, dass seine Behinderung in der Schule berücksichtigt wird, es hat das Recht, dass es in der Schule die Kulturtechniken lernt, die es braucht, um an der Gesellschaft teilzuhaben und es widerspricht dem Sozialrecht, dass die Kosten der Therapie von dem Menschen mit Behinderung selbst getragen werden müssen oder den Eltern der Kinder mit Behinderung aufgebürdet werden. ${ }^{1622}$

\footnotetext{
1620 Vgl. BVerfG, Urteil v. 9.2.2010, Az.: 1 BvL 1/09 „Hartz IV“, http://www.bverfg.de/entscheidungen/ls20100209_1bvl000109.html

1621 BVerfG, Urteil v. 9.2.2010, Az.: 1 BvL 1/09 "Hartz IV“, http://www.bverfg.de/entscheidungen/ls20100209_1bvl000109.html, Rn. 192, 197

1622 SG Regensburg, Urteil v. 10.11.2004; Az.: S 14 KR 38/04, JAmt 2005, S. 89 ff, Mrozynski, in: SGB VIII, § 35 a, Rn. 16; umfangreich zu rechtspolitischen Perspektiven Schwengers, Eingliederungshilfe $\S 35$ a SGB VIII, S. $338 \mathrm{ff}$
} 



\section{Teil: Datenschutz und Zeugnisbemerkung}

\section{Kapitel: Datenschutz}

Behinderungen können in der Schule nur berücksichtigt werden, wenn die Schule und die Lehrkräfte überhaupt vom Vorliegen einer Behinderung wissen und wenn sie wissen, was der Schüler/die Schülerin hat. Weil es sich dabei um höchstpersönliche und sehr intime Daten der Schüler und Schülerinnen handelt, ist ein besonders sensibler Umgang mit den Daten notwendig. ${ }^{1623}$

Das Bundesverfassungsgericht hat sich im Volkszählungsurteil umfassend mit der Problematik des Datenschutzes beschäftigt und aus Art. 2 I GG das Recht auf informationelle Selbstbestimmung abgeleitet. Das allgemeine Persönlichkeitsrecht schützt die engere persönliche Lebenssphäre. Es schützt grundsätzlich vor der Erhebung und Weitergabe von Befunden über den Gesundheitszustand, die seelische Verfassung und den Charakter und hat die Aufgabe, diesen Bereich der Privat- und Intimsphäre vor der Öffentlichkeit abzuschirmen. ${ }^{1624}$ Der Einzelne hat das Recht selbst darüber zu bestimmen, wann und innerhalb welcher Grenzen persönliche Lebenssachverhalte offenbart werden. ${ }^{1625}$

Fast alle Schulgesetze der Länder enthalten Datenschutzregelungen. ${ }^{1626}$ Diese sehen vor, dass Schulen personenbezogene Daten von Schülern und Schülerinnen und Erziehungsberechtigten erheben und verarbeiten dürfen, soweit dies zur Erfüllung der schulischen Aufgaben erforderlich ist. Es dürfen

\footnotetext{
${ }^{1623}$ Weichert, in: Schulleitung in den Ländern, Anm. 11.1.7

1624 BVerfGE 65, 1, $41 \mathrm{f}$

1625 Grundlegend das Volkszählungsurteil des BVerfG E 65, 1 ff; 44, 80; 367, 373 ff; Jarass/

Pieroth, in: Jarass/Pieroth GG, Art. 2, Rn. 42

${ }^{1626}$ BW-SchulG § 115; BayEUG Art. 84 f; BE-SchulG 64 f; BB SchulG§§ 65 f; HB-Schuldatenschutzgesetz; HH SchulG §§ 98 f; HE SchulG §§ 83 f; MV-SchulG §§ 70 f; NI SchulG §§ 30 f; NRW-SchulG §§ 120 f; RP-SchulG § 67; SL-SchulG § 20b; ST SchulG § 84a; SH SchulG § 30; TH SchulG $\S \S 57 \mathrm{fUmfassendere} \mathrm{Regelungen} \mathrm{enthalten} \mathrm{z.B.} \mathrm{BW} \mathrm{Erlass} \mathrm{Datenschutz;} \mathrm{SN} \mathrm{VwV}$ Schuldatenschutz; SH SchulG § 30; TH SchuO § 136
} 
nicht mehr Daten als notwendig verarbeitet werden und sie dürfen nur für schulische Zwecke genutzt werden. ${ }^{1627}$ Welche Daten im Einzelnen erhoben, verarbeitet und genutzt werden dürfen, hängt von dem Zweck ab, dem sie dienen. Für den Zweck muss hier zwischen dem Verfahren auf Anerkennung der Behinderung und der Festlegung des Nachteilsausgleichs einerseits und dem Datenschutz im schulischen Alltag andererseits unterschieden werden.

\section{Datenschutz im Verfahren der Grundentscheidung und der Festlegung des Nachteilsausgleichs}

Um die Behinderung berücksichtigen zu können, ist es unverzichtbar, dass der Schule die Diagnose mitgeteilt wird. ${ }^{1628}$ Zu klären bleibt, wie detailliert der Nachweis erfolgen muss.

Die multiaxiale Diagnostik der Legasthenie/Dyskalkulie bringt es mit sich, dass das Kind umfassend untersucht und vielfältige Befunde erhoben werden. ${ }^{1629}$ Nicht nur die Intelligenz und die Lese-/Rechtschreib- und Rechenleistungen werden erfasst, sondern auch der Verlauf der Schwangerschaft, Geburt und frühkindlichen Entwicklung bis hin zur Sauberkeitserziehung, der Beschulung, der Beziehung zu Geschwistern, der familiären Situation, der Beziehung zwischen und zu den Eltern, der sozialen Beziehungen usw. ${ }^{1630}$ Es werden mithin außerordentlich sensible, höchstpersönliche Daten erhoben, um auszuschließen, dass die Schwierigkeiten im Lesen/Schreiben und Rechnen auf nachweisbaren psychosozialen Umständen beruhen.

In Bayern, das insoweit die detaillierteste Regelung hat, ist zunächst von den Schulpsychologen die Forderung erhoben worden, dass die diagnostizierenden Fachkräfte in ihrem Attest für die Schule detaillierte Angaben zu allen oben behandelten Achsen des etablierten multiaxialen Klassifikationsschemas (MAS) für psychische Störungen im Kindes- und Jugendalter machen (Achse 1: klinisch-psychiatrisches Syndrom, Achse 2: umschriebene Entwicklungsstörungen, Achse 3: Intelligenzniveau, Achse 4: körperliche Symptomatik, Achse 5: aktuelle abnorme psychosoziale Umstände, Achse 6: Globalbeurteilung der psychosozialen Anpassung). ${ }^{1631}$ Bedenken gegen diese umfassenden Angaben,

\footnotetext{
1627 Weichert, in: Schulleitung in den Ländern, Anm. 11.1.2; vgl. zu den datenschutzrechtlichen Bedenken gegen umfangreiche Anamnesen bei der Schulfähigkeitsuntersuchung: Giesen, Schutz des Persönlichkeitsrechts, S. 95

${ }^{1628}$ S. oben 9. Teil: 1. Kapitel: 3.5.: Mitteilung der Diagnose, S. 276

${ }^{1629}$ Ausführlich oben 1. Teil: 1. Kapitel: 5.2.: Medizinische Diagnostik nach ICD 10 und Multiaxialem Klassifikationsschema (MAS), S. 16

1630 Sehr knappes Beispiel bei Warnke/Hemminger/Roth/Schneck, Legasthenie - Leitfaden für die Praxis, S. 131

1631 S. dazu Landesverband Bayerischer Schulpsychologen, LBSP-Stellungnahme zur KMBek Legasthenie
} 
vor allem im Hinblick auf die ärztliche Schweigepflicht, führten dann dazu, dass nunmehr in der Regel nur noch Angaben zum Intelligenzquotienten (Achse 3) und zu den Leistungen im Lesen/Rechtschreiben und/oder Rechnen (Achse 2) gemacht werden, die anderen Achsen werden mit „o.B." (= ohne Befund) ausgefüllt. ${ }^{1632}$ In anderen Bundesländern ist davon auszugehen, dass die Eltern den Schulen die umfassenden Stellungnahmen der diagnostizierenden Fachkräfte mit Angaben zu allen einzelnen Achsen und erheblichen persönlichen Angaben, wie sie vor allem für Anträge nach $\S 35$ a SGB VIII erstellt werden, vorlegen. ${ }^{1633}$

Wird der Schule die umfassende Stellungnahme der diagnostizierenden Fachkraft übermittelt, dann wird ihr nicht nur die Diagnose mitgeteilt, sondern sie erhält Auskünfte zu allen sechs Achsen der multiaxialen Diagnostik und damit Informationen über das betroffene Kind und sein persönliches Umfeld, die weit über das übliche Maß hinausgehen. Es ist offensichtlich, dass sich hier ein erhebliches Problem nicht nur in Bezug auf das allgemeine Persönlichkeitsrecht des Kindes und sein Recht auf informationelle Selbstbestimmung (Art. 2 Abs. 1 $\mathrm{GG})$, sondern auch in Bezug auf dieselben Rechte seiner Eltern und Geschwister auftut.

Aus dem allgemeinen Persönlichkeitsrecht ergibt sich, dass der Einzelne das Recht hat, selbst darüber zu bestimmen, wann und innerhalb welcher Grenzen persönliche Lebenssachverhalte offenbart werden. ${ }^{1634}$ Der Schutz ist umso intensiver, je näher die Daten der Intimsphäre des Betroffenen stehen, die als unantastbarer Bereich privater Lebensgestaltung gegenüber aller staatlicher Gewalt Achtung und Schutz beansprucht. ${ }^{1635}$ Je mehr die Befunde nicht rein medizinische Feststellungen, wie etwa den allgemeinen Gesundheitszustand, betreffen, sondern psychologische Befunde sind, die auch Aussagen über die seelische Verfassung, die Selbstachtung und die gesellschaftliche Stellung des Betroffenen enthalten, desto näher stehen sie dem unantastbaren Bereich der Lebensgestaltung und sind noch stärker durch Art. 2 I GG i.V.m. Art. 1 Abs. 1 GG geschützt. ${ }^{1636}$ In das allgemeine Persönlichkeitsrecht kann zwar - aufgrund einer formellen gesetzlichen Grundlage - eingegriffen werden $^{1637}$, an die Ver-

\footnotetext{
${ }^{1632}$ BY KMS IV/1 - 7306/4 - 4/55 382 vom 09.08.2000, Anlage 2, http://www.schulberatung.bayern.de/schulberatung/index_05168.asp

1633 S. z.B. der Ratschlag der Elterngruppe an einer Schule Erler, Legasthenie Albert-EinsteinSchule-Laatzen, S. 2: Gespräch mit dem Lehrer (Gutachten vorlegen); LVL Hessen, Pressemitteilung v. 9.9.2009, „Ein fast normaler Schüler“, http://www.Ivl-hessen.de/pdf/Ein_fast_ normaler_Schueler.pdf, S. 2: „Gutachten wurden kopiert“; Bastian-Becker, in: Recht auf Bildung, S. 227

1634 Grundlegend das Volkszählungsurteil des BVerfG E 65, 1 ff; 44, 80; 367, 373 ff; Jarass/ Pieroth, in: Jarass/Pieroth GG, Art. 2, Rn. $43 \mathrm{ff}$

1635 BVerfGE 89, 69, 82

${ }^{1636}$ Vgl. BVerfGE 89, 69, $82 \mathrm{f}$

1637 Jarass/Pieroth, in: Jarass/Pieroth GG, Art. 2, Rn. 43
} 
hältnismäßigkeitsprüfung ist aber ein besonders strenger Maßstab anzulegen. ${ }^{1638}$ Ein Eingriff in die Privatsphäre ist daher nur verhältnismäßig, wenn er zum Schutz öffentlicher Interessen unerlässlich ist. ${ }^{1639}$

Gesetzliche Grundlage für die Einschränkung der Grundrechte und die Erhebung der Daten sind die Datenschutzbestimmungen in den einzelnen Schulgesetzen sowie die sie konkretisierenden Rechtsverordnungen und/oder Verwaltungsvorschriften. ${ }^{1640}$ Subsidiär gelten die Landesdatenschutzgesetze sowie das Bundesdatenschutzgesetz. Diese Vorschriften sehen regelmäßig vor, dass Schulen und Schulbehörden, personenbezogene Daten der Schülerinnen und Schüler und ihrer Erziehungsberechtigten verarbeiten dürfen, soweit dies zur Erfüllung des Bildungsauftrags der Schule, ihrer Fürsorgeaufgaben oder zur Erziehung oder Förderung der Schülerinnen und Schüler erforderlich ist. (z.B. $\S 31$ Abs. 1 S. 1 NI SchulG). Die Daten über das Vorliegen einer Legasthenie/ Dyskalkulie sind "besondere Arten personenbezogener Daten“ i.S.d. § 3 Abs. 9 BDSG, da sie Angaben über die Gesundheit enthalten. § 3a BDSG bestimmt, dass die Erhebung und Nutzung personenbezogener Daten an dem Ziel auszurichten sind, so wenig personenbezogene Daten wie möglich zu erheben, zu verarbeiten oder zu nutzen.

Für die Zulässigkeit, den Inhalt und den Umfang der Datenerhebung ist zwischen den betroffenen Personen, nämlich Schülern, Eltern und Geschwistern zu unterscheiden.

\subsection{Bekanntgabe der Ergebnisse der einzelnen Achsen, insbesondere der Achsen 2 und 3 des multiaxialen Klassifikationsschema (MAS)}

Erforderlich für den Nachweis der Legasthenie/Dyskalkulie sind Daten des betroffenen Kindes, weshalb der Eingriff in das allgemeine Persönlichkeitsrecht und das informationelle Selbstbestimmungsrecht des Kindes nicht zu vermeiden ist. Die Daten müssen für die Erfüllung der Aufgabe erforderlich sein (vgl. $\S 9$ NI DSG) und es muss das Gebot der Datensparsamkeit des § 3 BDSG beachtet werden. Daraus ergibt sich, dass für den Nachweis nur die Daten gefordert werden dürfen, die erforderlich sind, um die Funktionsstörung zu belegen. Daten, die mit diesem Nachweis nur sekundär zusammenhängen, sind dafür nicht erforderlich. Das betrifft jedenfalls die Daten auf den Achsen 1 (klinisch-psychiatrisches Syndrom), 4 (körperliche Symptomatik), 5 (aktuelle abnorme psychosoziale Umstände) und 6 (Globalbeurteilung der psychosozialen Anpas-

\footnotetext{
${ }^{1638}$ BVerfGE 89, 69, 82 f; Jarass/Pieroth, in: Jarass/Pieroth GG, Art. 2, Rn. 44

1639 BVerfGE 84, 239, 280

1640 § 115 BW-SchG; Art. 84 f BayEUG; § 64 f BE-SchulG; §§ 65 f BB SchulG; HB-Schuldatenschutz-

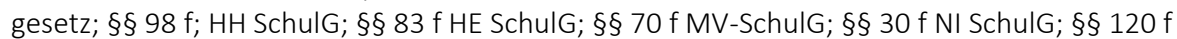
NRW-SchulG; § 67 RP-SchulG; § 20b SL-SchulG; SH SchulG §§ 57 f TH SchulG. Umfassendere Regelungen enthalten z.B. SN VwV Schuldatenschutz; § 30 SH SchulG; § 136 TH SchuO
} 
sung), denn mit den Untersuchungen auf diesen Achsen wird nur ausgeschlossen, dass die Legasthenie/Dyskalkulie auf diese Bereiche zurückzuführen sind. Sind diese Achsen „ohne Befund“, dann sind sie für die Legasthenie/Dyskalkulie nicht kausal und daher für die Diagnose nicht relevant. Die Daten aus diesem Bereich stehen der Schule deshalb nicht zu und sind ihr daher nicht mitzuteilen.

Es stellt sich weiter die Frage, ob nur die Diagnose Legasthenie/Dyskalkulie angegeben werden muss oder ob auch die der Diagnose zugrunde liegenden Testergebnisse (Werte) und damit konkrete Angaben zu den Achsen 2 und 3 angegeben werden müssen. Auf die Angabe der Diagnose kann für die Berücksichtigung der Legasthenie/Dyskalkulie in der Schule nicht verzichtet werden, weil sie Grundlage der Anwendung des Benachteiligungsverbots ist. Die Bekanntgabe von Testergebnissen der Achsen 2: (umschriebene Entwicklungsstörungen) und 3 (Intelligenzniveau) ist jedoch ein Eingriff in das allgemeine Persönlichkeitsrecht des Schülers mit Legasthenie/Dyskalkulie, weil dadurch höchstpersönliche Daten, nämlich die Leistungen in den Bereichen Lesen/ Schreiben/Rechnen und der Intelligenzquotient, gegenüber der Schule preisgegeben werden. Viele Eltern halten die Bekanntgabe der Testergebnisse und vor allem des IQ für unbedenklich und erhoffen sich dadurch, dass die Lehrkräfte daraus ersehen, dass das Kind nicht dumm und faul, sondern begabt und förderungswürdig ist. ${ }^{1641}$ Die Bekanntgabe kann eine solche Reaktion zur Folge haben, sie kann aber auch zur Folge haben, dass das Kind in der Klasse mit seinen Werten konfrontiert wird, etwa durch Bemerkungen wie „Bei deinem IQ hatte ich etwas Besseres erwartet."

Der Intelligenzquotient eines Schülers ist der Schule und den Lehrkräften bei allen anderen Schülern und Schülerinnen nicht bekannt. Er ist nur für die Diagnostik der Legasthenie und Dyskalkulie wichtig, für den schulischen Alltag aber unerheblich. Es ist daher im Interesse des Kindes nicht erforderlich, inn bekannt zu geben.

Die Leistungen in den Bereichen Lesen, Schreiben und Rechnen sind ebenfalls nur für die Diagnostik wichtig, der genaue Wert ist jedoch für die Beschulung unerheblich. Wichtig ist dafür nur, zu wissen, dass die Schwierigkeiten vorliegen und wo besondere Probleme liegen. Gegen die Bekanntgabe der Testergebnisse der betroffenen Schüler und Schülerinnen gegenüber der Schule und den Lehrkräften spricht zudem, dass sich diese Bekanntgabe für sie negativ auswirken kann. Es ist dann nämlich sehr wahrscheinlich, dass nur Schüler und Schülerinnen mit geringen Prozentsätzen und damit schwereren Formen der Legasthenie/Dyskalkulie als beeinträchtigt angesehen werden, während Schüler und Schülerinnen mit höheren Prozentsätzen nicht als beeinträchtigt

${ }^{1641}$ S. dazu das Beispiel bei Bastian-Becker, in: Recht auf Bildung, S. 227 
angesehen werden, obwohl diese eventuell aufgrund ihrer Intelligenz genauso oder sogar noch stärker betroffen sind.

Fraglich ist weiter, ob und inwieweit das Attest eine grundsätzliche Aussage zum Schweregrad und der Ausprägung der Legasthenie/Dyskalkulie enthalten muss. Wie oben dargestellt ${ }^{1642}$, setzt die Festlegung des Nachteilsausgleichs Kenntnisse über die individuelle Situation des Kindes voraus. Um allen betroffenen Kindern an einer Schule gerecht werden zu können, ist es außerdem erforderlich, dass zumindest der Schulpsychologe Kenntnis von dem Schweregrad der Legasthenie/Dyskalkulie hat. Ohne diese Kenntnis kann er weder den richtigen Nachteilsausgleich für ein betroffenes Kind finden, noch zwischen den betroffenen Kindern in sachlicher Weise differenzieren. Deshalb ist es erforderlich, dass das Attest eine Aussage zum Schweregrad der Legasthenie/Dyskalkulie und zu besonderen Ausprägungen, z.B. Schwierigkeiten im auditiven oder im visuellen Bereich oder in der Motorik, enthält. Diese Aussagen dienen gerade auch dem persönlichen Interesse des Kindes und sind daher zulässig.

\subsection{Schutz der Daten der Eltern}

Daten der Eltern bzw. Erziehungsberechtigten dürfen im schulischen Bereich verarbeitet werden, soweit dies zur Erfüllung des Bildungsauftrags der Schule oder der Fürsorgeaufgaben, zur Erziehung oder Förderung der Schülerinnen und Schüler erforderlich ist. Fragen der Schule zu persönlichen Verhältnissen der Eltern sind grundsätzlich unzulässig. ${ }^{1643}$ Die Übermittlung der höchstpersönlichen Daten der Eltern, wie sie in der Diagnostik insbesondere auf den genannten Achsen 5 (aktuelle abnorme psychosoziale Umstände) und 6 (Globalbeurteilung der psychosozialen Anpassung) erhoben werden, wäre daher nur dann gerechtfertigt, wenn sie für den schulischen Auftrag erforderlich ist. ${ }^{1644}$ Der Nachweis der Legasthenie/Dyskalkulie soll (nur) die Funktionsstörung des Kindes nachweisen. Dafür sind nur solche Daten erforderlich, die sich auf das Kind beziehen. Daten der Eltern sind dafür unerheblich. Infolgedessen dürfen Daten aus den Achsen 5 und Achse 6, die u. a. das familiäre Umfeld beschreiben und beurteilen, nicht an die Schulen und Schulbehörden übermittelt werden.

\footnotetext{
1642 S. oben 9. Teil: 2. Kapitel: 5.: Zuständigkeit für die Festlegung, S. 292

1643 Niehues/Rux, Schulrecht, Rn. 469; vgl. auch § 33 II HH SchulG

${ }^{1644} \mathrm{Vgl}$. zu den datenschutzrechtlichen Bedenken gegen umfangreiche Anamnesen bei der Schulfähigkeitsuntersuchung: Giesen, Schutz des Persönlichkeitsrechts, S. 95; s. auch § 99 HH SchulG: Begrenzung des Ergebnisses der Schuleingangsuntersuchung auf das für die Schule maßgebliche Ergebnis
} 


\subsection{Schutz der Daten der Geschwister}

Weder die schulrechtlichen Datenschutzbestimmungen noch die Landesdatenschutzgesetze und das Bundesdatenschutzgesetz lassen die Erhebung von Daten über Geschwister des betroffenen Schülers zu. Eine Ausforschung der familiären Verhältnisse durch Lehrer oder Schule ist nicht zulässig. ${ }^{1645}$ Daher sind Fragen zu den persönlichen Verhältnissen der Geschwister grundsätzlich unzulässig. ${ }^{1646}$ Die Grundrechte der Geschwister werden ohne gesetzliche Grundlage eingeschränkt, wenn Auskünfte über sie an die Schule übermittelt werden. Die Passagen der Diagnostik, die sich mit der familiären Situation des betroffenen Schülers und mit dem Verhältnis zu seinen Geschwistern befassen, also vor allem Informationen zur Achse 5 (aktuelle abnorme psychosoziale Umstände) und Achse 6 (Globalbeurteilung der psychosozialen Anpassung), dürfen daher mangels gesetzlicher Grundlage nicht an die Schule weitergegeben werden.

\section{Datenschutz im schulischen Alltag}

Zu klären ist weiter, wie mit den besonders sensiblen Daten der Schülerinnen und Schüler mit Behinderung im schulischen Alltag umzugehen ist.

\subsection{Geheimhaltung nach $\S 30 \mathrm{VwVfG}$}

Grundsätzlich dürfen die persönlichen Daten der Schüler und Schülerinnen nicht offenbart werden. Nach $\S 30 \mathrm{VwVfG}$ haben die Beteiligten eines Verwaltungsverfahrens Anspruch darauf, dass ihre Geheimnisse von der Behörde nicht unbefugt offenbart werden. § $30 \mathrm{VwVfG}$ ist außerdem Ausdruck eines allgemeinen Rechtsgrundsatzes, der auch außerhalb eines Verfahrens bei sonstigem Verwaltungshandeln zu beachten ist. ${ }^{1647}$ Besondere Geheimhaltungsvorschriften sind vorrangig zu berücksichtigen. Zu den Geheimnissen des $\S 30$ VwVfG gehören alle Privatgeheimnisse, die unter den Schutz des Persönlichkeitsrechts fallen, insbesondere gesundheitliche, familiäre und wirtschaftliche Verhältnisse. ${ }^{1648}$

\subsection{Verschwiegenheitspflicht}

Lehrkräfte unterliegen zudem einer speziellen Verschwiegenheitspflicht aus $\S 37$ BeamtStG sowie aus den Landesbeamtengesetzen und $\S 3$ Abs. 2 TV-L. Die Verletzung der Verschwiegenheitspflicht ist strafbar (§ 203 II StGB). Schulpsy-

\footnotetext{
1645 Avenarius/Heckel, Schulrecht, Ziff. 24.342, s. auch Datenschutz Brandenburg 13. Tätigkeitsbericht: A 7.3 Der Lebenslauf im Deutschunterricht: http://www.Ida.brandenburg.de/ sixcms/detail.php?gsid=Ibm1.c.336155.de\&template=allgemein_Ida

1646 Niehues/Rux, Schulrecht, Rn. 469

1647 Kopp/Ramsauer, VwVfG, § 30, Rn. 3

1648 Kopp/Ramsauer, VwVfG, § 30, Rn. 9
} 
chologen unterliegen darüber hinaus der Schweigepflicht aus § 203 I oder $\S 203$ II StGB. Sie dürfen daher ohne Befreiung von der Schweigepflicht keine Auskunft über den ärztlichen Befund oder auch ihren eigenen Befund geben.

\subsection{Entbindung von der Schweigepflicht}

Werden die Daten über die Behinderung außerhalb des für die Schule absolut erforderlichen Bereichs genutzt, z.B. durch Gespräche mit außerschulischen Einrichtungen oder auch mit der Klasse, ist es erforderlich, dass dazu die Einwilligung der Eltern bzw. des volljährigen Schülers gem. § 4a BDSG oder z.B. § 4 II NI DSG vorliegt. Da es sich bei den Daten zur Legasthenie/Dyskalkulie um besondere personenbezogene Daten handelt, muss sich die Einwilligung ausdrücklich auch auf diese Daten beziehen (§ 4 III BDSG, z.B. § 4 II S. 2 NI DSG). In der Übergabe der Daten (etwa des Befundberichts) an den Lehrer liegt allenfalls eine konkludente, nicht aber eine ausdrückliche Einwilligung zur Weiternutzung und Besprechung dieser Daten. Ohne eine ausdrückliche Einwilligung ist die Weitergabe der Daten rechtswidrig.

\subsection{Umgang mit den Befundberichten}

Bei ärztlichen Befundberichten, die mehr als die Diagnose enthalten, muss sichergestellt werden, dass sie vertraulich behandelt werden. ${ }^{1649}$ Regelungen für den Umgang mit solchen sensiblen Daten enthalten vor allem die Vorschriften zum sonderpädagogischen Förderbedarf, die hier analog angewandt werden können. In der Regel werden die Befundberichte beim schulpsychologischen Dienst geführt. ${ }^{1650}$

Die Aufnahme von Befundberichten in die allgemeine Schülerakte des Schülers mit Behinderung ist nicht zulässig, weil der Befundbericht damit jedem Einsichtnehmenden zugänglich wird ${ }^{1651}$ und die Geheimhaltung nach § 30 VwVfG damit nicht mehr gewährleistet ist.

\subsection{Offenbarung der Behinderung}

Wegen des Geheimnisschutzes aus $\S 30$ VwVfG dürfen die Befundberichte und die Behinderung nicht offen diskutiert werden. ${ }^{1652}$ Es kann sicher notwendig oder förderlich sein, wenn ein bestimmter Personenkreis, etwa die Klassengemeinschaft oder die Klassenkonferenz, über eine Behinderung oder einen Be-

\footnotetext{
1649 S. dazu z.B. die Regelung in SH SchulG § $27 \mathrm{~V}$

1650 Weichert, in: Schulleitung in den Ländern, Anm. 11.1.7

${ }^{1651}$ Weichert, in: Schulleitung in den Ländern, Anm. 11.1.7; SH Legasthenie Datenschutz: Aufnahme in die Schülerakte nur in einem versiegelten Umschlag, der nach Einsicht neu zu versiegeln ist; s. auch BW Erlass Datenschutz Nr. II, 3

1652 Weichert, in: Schulleitung in den Ländern, Anm. 11.1.7
} 
fund unterrichtet wird, weil sich dadurch die Akzeptanz der Behinderung erhöht und der Umgang mit der Behinderung verbessert. Dabei muss jedoch sichergestellt sein, dass die Information über die Behinderung auf das sachlich und für die Schule erforderliche Maß sowie auf den notwendig zu beteiligenden Personenkreis beschränkt wird.

Wegen dieser Pflicht zum sensiblen Umgang mit den höchstpersönlichen Daten des Schülers ist es, wie schon oben erörtert, sehr bedenklich, eine Klassenkonferenz mit der Anerkennung der Behinderung zu betrauen. ${ }^{1653}$ In einigen Ländern gehören den Klassenkonferenzen auch Eltern- und Schülervertreter an, die nach den geltenden gesetzlichen Regelungen bei diesen Entscheidungen nicht von vornherein ausgeschlossen sind, ${ }^{1654}$ deren Beteiligung an dieser Entscheidung aber gegen das Persönlichkeitsrecht und das Recht auf informationelle Selbstbestimmung des Schülers mit Behinderung verstößt. ${ }^{1655}$ Die Notwendigkeit, dass auch die Eltern- und Schülervertreter über die Behinderung und die Art der Behinderung eines Schülers in Kenntnis gesetzt werden, ist nicht ersichtlich. Schüler und Schülerinnen sowie deren Erziehungsberechtigte können gegen die Besprechung der persönlichen Daten in Anwesenheit von Elternvertretern Einwände erheben bzw. widersprechen (z.B. §§ 29 LDSG SH; 17 a Nds. DSG). ${ }^{1656}$

Gelegentlich werden die Daten der betroffenen Schüler auch in der Form offenbart, dass in Lehrerzimmern Listen hängen, in denen die Besonderheiten der Schüler und Schülerinnen aufgeführt sind, z.B. Listen mit den Schülern und Schülerinnen, die eine Legasthenie/Dyskalkulie haben. Solche Listen sind gut gemeint, denn sie sollen die Lehrkräfte, die häufig eine Vielzahl von Schülern unterrichten und sich die Besonderheiten im Einzelfall nicht immer merken können, an die Besonderheiten dieser Schüler erinnern. Die Listen offenbaren jedoch Diagnosen, die allen Personen im Lehrerzimmer bekannt gemacht werden, also auch Lehrkräften, die die Schüler nicht unterrichten, oder schulfremden Personen, die sich im Lehrerzimmer aufhalten. Sie sind daher weder nach $\S 30$ VwVfG noch datenschutzrechtlich zulässig (z.B. §§ 11, 14 LDSG SH). Sie verstoßen ebenso gegen den Datenschutz wie z.B. die Nachricht über die Erkrankung eines Lehrers, in der auch seine Erkrankung angegeben ist. ${ }^{1657}$

\footnotetext{
${ }^{1653} \mathrm{Vgl}$. Weichert, in: Schulleitung in den Ländern, Anm. 11.1.9

1654 S. z.B. HE SchulG § 122 II; NI SchulG § 36 II i.V.m. § 35 II; NRW SchulG § 71 III; SH SchulG § 65 I, IV und oben S. 254

1655 S. dazu bereits oben 9. Teil: 1. Kapitel: 3.1.: Zuständigkeit, S. 254

${ }^{1656}$ S. dazu Unabhängiges Landeszentrum für Datenschutz Schleswig-Holstein, Häufig gestellte Fragen zum Bereich Schule, Punkt V Elternvertretung: https://www.datenschutzzentrum.de/faq/schule2.htm\#v1

${ }^{1657}$ S. dazu Der Hessische Datenschutzbeauftragte: Häufige vorkommende Mängel an Schulen: http://www.datenschutz.hessen.de/ds006.htm
} 
Die Betroffenen selber hingegen sind frei darin, die Daten weiterzugeben. So können z.B. Schüler ihre Fachlehrer an ihre Legasthenie erinnern, indem sie bei schriftlichen Arbeiten neben ihren Namen ein (L) schreiben. Die Lehrkraft wird dann spätestens beim Eintragen der Note daran erinnert, dass dieser Schüler eine Legasthenie hat und dies bei der Korrektur der Arbeit zu berücksichtigen ist.

\section{Kapitel: Zeugnisbemerkung}

Zeugnisse und Zeugnisbemerkungen berühren die Grundrechte der Schülerinnen und Schüler wesentlich. ${ }^{1658}$ Die Zulässigkeit von Zeugnisbemerkungen bewegt sich im Spannungsfeld zwischen der Informations- und Dokumentationsfunktion der Zeugnisse und den negativen und diskriminierenden Auswirkungen von Bemerkungen für den Absolventen.

Überwiegend sehen die landesrechtlichen Regelungen vor, dass ein Nachteilsausgleich nicht im Zeugnis vermerkt wird. Eine Bemerkung bezüglich des Nachteilsausgleichs kann aber in Hamburg ${ }^{1659}$, Niedersachsen ${ }^{1660}$, und Rheinland-Pfalz ${ }^{1661}$ erfolgen, wenn der Nachteilsausgleich als eine Abweichung von der "Leistungserhebung“ bzw. "Leistungsfeststellung" verstanden wird.

Wird allerdings die Rechtschreibung nicht gewertet, so wird in das Zeugnis zwingend eine Bemerkung aufgenommen, weil insoweit von den allgemeinen Leistungsanforderungen abgewichen wird. ${ }^{1662}$ Die Bemerkungen sind meist relativ neutral formuliert und lauten etwa: „Aufgrund von Schwierigkeiten im Lesen und Rechtschreiben wurde die Rechtschreibung nicht bewertet. ${ }^{1663}$

\footnotetext{
1658 Avenarius/Heckel, Schulrecht, Ziff. 26.332; OVG Saarlouis, Urteil v. 19.8.2002, Az.: 3 N 1/01, juris, Rn. 39; OVG Greifswald, Urteil v. 14.10.2008, Az.: 4 K 16/08, NordÖR 2009, 128 ff, 128; VG Braunschweig, Urteil v. 18.2.2004, Az.: 6 A 106/03, juris

1659 HH Förder-Richtlinie Nr. 4.4.: Abweichungen von den allgemeinen Grundsätzen der Leistungserhebung

${ }^{1660}$ NI LRR-Erlass Nr. 4.2: Abweichungen von den allgemeinen Grundsätzen der Leistungsfeststellung

${ }^{1661}$ RP VV-LRS Nr. 4.4: Die Abweichung von den allgemeinen Grundsätzen der Leistungsfeststellung

1662 KMK-Lesen/Rechtschreiben/Rechnen, Punkt Abschlüsse Prüfungsvergünstigungen am Ende und auch KMK-Inklusive Bildung Punkt II 4

${ }^{1663}$ BW VV Besonderer Förderbedarf Nr. 2.3.1; BY KMBek Legasthenie Nr. 3.1; BE GsVO § 16 VIII, BE Sek I-VO § 14 III; BB VV-LRSR Nr. 8 und Anlage 1; HB LRS-Erlass Nr. 4.2; HH FörderRichtlinie Nr. 4.4; HE VO-GestSchulVerh § 7 IV und 43 II; MV VV-LRR Nr. 8 II; NI LRR-Erlass Nr. 4.2; NRW LRS-Erlass Nr. 4.2; RP VV-LRS Nr. 4.4; SL VV-LRS Nr. 5.1; SN VV-LRS Nr. 4.1.4; ST Grundschulbewertungs-Erlass Nr. 4.1.3, ST Sekundarstufenbewertungs-Erlass Nr. 5.1.3; SH Legasthenie-Erlass Nr. 3.1, TH SchulO § 59 VI i.V.m. TH Fachliche Förderempfehlung
} 
Schleswig-Holstein formuliert deutlich negativer ${ }^{1664}$ : „Die Rechtschreibleistungen entsprechen nicht den Anforderungen; sie sind in den Fachnoten nicht enthalten.“ In Bayern lautet sie: „Wegen einer fachärztlich festgestellten Legasthenie wurden Rechtschreibleistungen nicht berücksichtigt." Durch Erlass wurde die Zeugnisbemerkung in Bayern auch auf den Fall ausgeweitet, dass eine Lese-Rechtschreibschwäche ${ }^{1665}$ in der 10. Klasse Gymnasium noch berücksichtigt, in Klasse 11 und 12 aber nicht mehr berücksichtigt wird. ${ }^{1666}$

\section{Unzulässigkeit bei Nachteilsausgleich}

Nach der herrschenden Meinung in der Literatur und den landesrechtlichen Vorschriften ist eine Zeugnisbemerkung unzulässig, wenn mit ihr auf einen Nachteilsausgleich hingewiesen wird. ${ }^{1667}$ Denn mit einem Nachteilsausgleich werden nur die Grundlagen dafür geschaffen, dass der Schüler mit Behinderung die inhaltliche Leistung der Prüfung trotz seiner Behinderung erbringen kann. Nach der hier vertretenen Ansicht ${ }^{1668}$ sind auch die Veränderung inhaltlicher Prüfungsanforderungen und die Nicht-Bewertung der Rechtschreibung Nachteilsausgleich, weshalb schon aus diesem Grund eine Zeugnisbemerkung in diesen Fällen unzulässig ist.

\footnotetext{
1664 SH Legasthenie-Erlass Nr. 3.1

1665 In Bayern per Definition etwas anderes als eine Legasthenie und nach der 10. Klasse nicht mehr berücksichtigungsfähig, s. oben 1. Teil: 2. Kapitel: 3.: Die schulrechtliche Regelung zur Berücksichtigung von Legasthenie in Bayern, S. 37

1666 BY KMS III. 6 - 5 S 4306.4 - 6.113140 v. 18.03.2010, http://www.schulberatung.bayern.de/imperia/md/content/schulberatung/pdf/Irs_gym_jgst _10.pdf - Die Veränderung ergibt sich aus dem Übergang zum G8. Beim G9 erhielten die Schüler am Ende der 10. Klasse ein Zeugnis mit Bemerkung, in der Sek II wurde die LeseRechtschreibschwäche nicht mehr berücksichtigt. Deshalb enthielt das Abiturzeugnis keine Bemerkung mehr. Im G8 ist die 10. Klasse aber eine Übergangsklasse, aus der bereits Noten in das Abiturzeugnis einfließen. Obwohl in den Klassen 11 und 12 die Lese-Rechtschreibschwäche nicht mehr berücksichtigt wird, enthält das Abiturzeugnis nur wegen der Berücksichtigung in Klasse 10 eine Bemerkung. Hier ist neben der grundsätzlichen Zulässigkeit einer solchen Bemerkung vor allem fraglich, ob es angesichts der Grundrechtsrelevanz der Bemerkung zulässig ist, eine solche Regelung durch Erlass vorzunehmen. Wenn es schon gegen den Vorbehalt des Gesetztes verstößt, wenn das Ministerium eine verbindliche mündliche Gruppenprüfung anordnet, obwohl die Prüfungsordnung eine mündliche Einze/prüfung vorsieht, (VG Augsburg, Urteil v. 10.1.2006, Az.: Au 3 K 05.865, juris, Rn. 24), dann muss das erst Recht für die Einführung einer solchen Bemerkung gelten.

1667 Langenfeld, RdJB 2007, S. 211 ff, S. 226; Ennuschat/Volino, br 2009, S. 166 ff, S. 167; KMKLesen/Rechtschreiben/Rechnen, S. 4; KMK-Inklusive Bildung Punkt II 4; z.B. ausdrücklich BE SopädVO § 38 III; BB VV-LRSR Nr. 8 II 2

1668 S. oben 3. Teil: 3. Kapitel: 4.: Abgrenzung der Benachteiligung von der Bevorzugung nach Art. 3 III 2 GGS. 127, S. 127
} 


\section{Zulässigkeit zur Dokumentation der Abweichung von Leistungsstandards}

Die herrschende Meinung in der Literatur und die landesrechtlichen Vorschriften halten die Bemerkung demgegenüber für zulässig und erforderlich, wenn die Rechtschreibung nicht gewertet wird, weil Schüler und Schülerinnen mit Legasthenie insoweit privilegiert würden, wenn die Prüfungsanforderungen verändert wurden. ${ }^{1669}$ Rux hält es trotz des - bisher nur von ihm thematisierten diskriminierenden Charakters der Zeugnisbemerkung für geboten, eine solche Bemerkung aufzunehmen, weil ansonsten Kompetenzen attestiert würden, die nicht bestehen. ${ }^{1670}$ Die Rechtsprechung beschäftigt sich zurzeit in einem Verfahren vor dem VG München mit der Frage. ${ }^{1671}$

\subsection{Eingriff in Art. $3 \| I \mid 2 \mathrm{GG}$}

Zeugnisbemerkungen zu Legasthenie weisen ausdrücklich auf Schwierigkeiten in den Fähigkeiten Lesen, Rechtschreiben und damit auf die Behinderung hin.

Die Bemerkung ist nachteilig. Dies wird auch so in der schulischen Praxis gesehen, denn die Kultusministerien halten es für notwendig, dass die Erziehungsberechtigten ausdrücklich über die Bemerkung informiert werden ${ }^{1672}$ und z.T. auch ausdrücklich ihr Einverständnis damit erklären. ${ }^{1673}$ Von einem Nachteil gehen auch Ennuschat/Volino aus, da sie meinen, dass die Bevorzugung der Schüler und Schülerinnen mit Legasthenie, die in der Nicht-Bewertung der Rechtschreibung läge, durch die Zeugnisbemerkung reduziert wird. ${ }^{1674}$ Das ist nur denkbar, wenn diese ein nicht unerheblicher Nachteil ist. Auch allgemein wird die Bemerkung als Nachteil empfunden. Eltern, Schüler und Schülerinnen, die einen Nachteilsausgleich beantragen, berichten immer wieder davon, dass Schulen von der Berücksichtigung der Legasthenie abraten, weil dann eben eine Zeugnisbemerkung im Zeugnis steht.

Die Bemerkung benachteiligt darüber hinaus, weil sie nicht nur eine Veränderung der Prüfungsanforderungen dokumentiert, sondern auch die Behinderung Legasthenie offenbart. Es wird von der Rechtsprechung schon als nachtei-

\footnotetext{
1669 Langenfeld, RdJB 2007, S. 211 ff, S. 226; Rux, RdJB 2009, S. 220 ff, S. 227; Ennuschat/Volino, br 2009, S. 166 ff, S. 168, 169; Rux in: Ennuschat, Die Rechte stotternder Menschen in Schule, Ausbildung und Studium, S. 59, Fn. 78, der sie hier auch auf stotternde Schüler ausdehnt.

${ }^{1670}$ Rux, RdJB 2009, S. 220 ff, S. 227

${ }^{1671}$ VG München, Urteilstenor v. 26.2.2013, Az.: M 3 K 11.2962 und M 3 K 11.2963, die schriftliche Urteilsbegründung lag bei Drucklegung noch nicht vor

1672 BY KMS IV/1 - 7306/4 - 4/55 382 vom 09.08.2000, Nr. 5, http://www.schulberatung.bayern.de/schulberatung/index_05168.asp

${ }^{1673}$ HB LRS-Erlass Nr. 4.2

1674 Ennuschat/Volino, br 2009, S. 166 ff, S. 169
} 
lig und als hinderlich für ein schulisches und berufliches Weiterkommen angesehen, wenn im Realschulabschluss eine schlechte Note in Deutsch ausgewiesen wird. ${ }^{1675}$ Dann ist ein ausdrücklicher Hinweis auf die Behinderung Legasthenie erst Recht ein Nachteil.

Die Bemerkung knüpft direkt und gezielt an das Unterscheidungsmerkmal der Behinderung an, denn sie erfolgt nur bei Schülern und Schülerinnen mit Legasthenie und wegen der Legasthenie. Es handelt sich damit um eine unmittelbare Diskriminierung wegen der Behinderung. Die Sachlage ist insoweit vergleichbar mit der ausdrücklichen Frage eines Arbeitgebers nach der Behinderung eines Bewerbers. ${ }^{1676}$ Ein solches allgemeines Fragerecht besteht nicht, weil eine solche Frage direkt an die Behinderung anknüpfen würde und damit eine verbotene unmittelbare Diskriminierung wäre. ${ }^{1677}$

Die Benachteiligung erfolgt auch „wegen“ der Behinderung. Die Bemerkungen stellen diesen Zusammenhang ausdrücklich durch die Wörter "wegen“ oder "aufgrund" her. Schon daraus ergibt sich die Unzulässigkeit der Zeugnisbemerkung, denn nach wohl herrschender Ansicht ist Art. 3 III GG als absolutes Anknüpfungsverbot zu verstehen. Danach ist jede Ungleichbehandlung verboten, die auf der Verknüpfung des Diskriminierungsmerkmals mit einer Rechtsfolge basiert. ${ }^{1678}$ Die Rechtsfolge „Zeugnisbemerkung“ knüpft gezielt an das Diskriminierungsmerkmal „Behinderung" an und ist daher verboten. Nach anderer Ansicht ist Art. 3 III GG als Begründungsverbot zu verstehen. Danach ist eine Ungleichbehandlung nur zulässig, wenn sie begründet werden kann, ohne auf das Diskriminierungsmerkmal zurückzugreifen, gelingt dies nicht, ist sie verboten. ${ }^{1679}$ Hier wird die Zeugnisbemerkung ausdrücklich mit der Behinderung begründet. Lässt man die Behinderung als Begründung weg, so kann sie nicht mehr begründet werden, denn einen anderen Grund und damit auch eine andere Begründung für die Bemerkung gibt es nicht.

Diese an sich verbotene unmittelbare Diskriminierung i.S.d. Art. 3 III 2 GG kann, wie oben bereits ausgeführt ${ }^{1680}$, nur durch zwingende Gründe gerechtfertigt, d.h. wenn sie einem legitimen Zweck dient und zwingend erforderlich ist, um behinderungsbezogenen Besonderheiten oder kollidierendem Verfassungsrecht Rechnung zu tragen. ${ }^{1681}$

\footnotetext{
1675 VGH München, Beschluss v. 28.9.2009, Az.: 7 ZB 08.2277, juris, Rn. 8

1676 BAG, Urteil v. 17.12.2009, Az.: 8 AZR 670/08, juris, Rn. 14; LAG Hamm, Urteil v. 19.10.2006, Az.: 15 Sa 740/06, www.justiz.nrw.de, Rn. $54 \mathrm{f}$

1677 LAG Hamm, Urteil v. 19.10.2006, Az.: 15 Sa 740/06, www.justiz.nrw.de, Rn. 55 m.w.N.

1678 Sachs, Besondere Gleichheitsgarantien, in: HStR VIII, § 182, Rn. 29; Osterloh, in: Sachs GG,

Art. 3, Rn. 240; zusammenfassend Fehling/Arnold, RdJB 2011, S. 316 ff, S. 324

1679 Fehling/Arnold, RdJB 2011, S. 316 ff, S. 324

1680 S. oben 3. Teil: 5. Kapitel: 1.: Rechtfertigungsmaßstab, S. 145

1681 S. auch Heun, in: Dreier GG, Art. 3, Rn. 137
} 
Die Bemerkung dient nicht dazu ein behinderungsbedingtes Problem von Schülern und Schülerinnen mit Legasthenie zu lösen, um damit bei ihnen ansetzend behinderungsbezogenen Besonderheiten Rechnung zu tragen. ${ }^{1682}$

Zweck der Zeugnisbemerkung ist es vielmehr, darauf hinzuweisen, dass das Zeugnis des betreffenden Schülers nicht die gleichen Kompetenzen attestiert, wie die Zeugnisse anderer Schüler, weil der Schüler bestimmte Prüfungsanforderungen im Bereich der Rechtschreibung nicht erfüllt. ${ }^{1683}$ Damit wird der Informations- und Dokumentationsfunktion von Zeugnissen gegenüber Nutzern dieser Aussage, wie Universitäten, Hochschulen und Arbeitgebern Rechnung getragen. Sie ersehen aus dem Zeugnis sogleich, dass der Absolvent bestimmte Anforderungen nicht erfüllt und können sich darauf einstellen. Ihr Vertrauen darauf, dass Zeugnisse eine bestimmte Aussagekraft haben und nicht Kompetenzen bescheinigen, die nicht vorhanden oder nur eingeschränkt vorhanden sind, soll geschützt werden. ${ }^{1684}$ Infolgedessen muss das Vertrauen in die Aussagekraft von Zeugnissen ein solch verfassungsrechtliches Gut sein, das es den Eingriff in Art. 3 III 2 GG rechtfertigt und den Menschen mit Behinderung dazu verpflichtet, die Offenbarung seiner Behinderung im Zeugnis hinzunehmen.

Arbeitgeber und Ausbildungsstätten haben ein großes Interesse daran, aufgrund der Zeugnisse den Ausbildungsstand und die Kompetenzen potentieller Bewerber zutreffend einschätzen zu können und möglichst frühzeitig ungeeignete Bewerber aus dem Einstellungs- oder Zulassungsverfahren auszuschließen. Der Gesetzgeber hat die Abwägung zwischen den Interessen des Bewerbers und denen der Ausbildungsstätten und insbesondere der Arbeitgeber bereits in verschiedenen gesetzlichen Bereichen vorgenommen. $\mathrm{Er}$ hat dabei den Belangen der Bewerber und vor allem der Bewerber mit Behinderung grundsätzlich den Vorrang vor den Interessen der Arbeitgeber oder Ausbildungsstätten eingeräumt und sich stets gegen eine Offenbarungspflicht von höchstpersönlichen Angelegenheiten, die sich im Bewerbungsverfahren möglicherweise negativ auswirken können, ausgesprochen.

So ist im Schulbereich ist die Frage problematisiert worden, ob sog. „Kopfnoten", d.h. die Bewertung des Arbeits- und Sozialverhaltens der Schüler, die jedenfalls ein Eingriff in das Persönlichkeitsrecht des Schülers aus Art. 2 I GG und im Bewerbungsverfahren auch ein Eingriff in die Berufsfreiheit aus Art. 12 I GG sein können, zulässig sind. Die allgemeine Meinung ist, dass negative Bemerkungen z.B. über das Sozial- oder Leistungsverhalten des Schülers zulässig sind, solange Zeugnisse (noch) keine Auswirkung auf die beruflichen Möglich-

\footnotetext{
1682 Vgl. BVerfGE 99, 341, 357; Heun, in: Dreier GG, Art. 3, Rn. 137; Jarass, in: Jarass/Pieroth GG, Art. 3, Rn. 149; Herdegen, Diskriminierungsschutz für Behinderte im Grundgesetz, S. 256, vgl. auch BT-Drs 12/8165, S. 29

${ }^{1683}$ Rux, RdJB 2009, S. 220 ff, S. 227

1684 Lambert, SchulVerwaltung BW 2002, S. 148 ff
} 
keiten des Schülers haben. Sobald das Zeugnis jedoch als Grundlage für Bewerbungen dient, werden zwar allgemeine Aussagen über das Verhalten des Schülers für zulässig gehalten (eben „Kopfnoten“) ${ }^{1685}$, nicht aber negative Bemerkungen, die Auswirkungen im Bewerbungsprozess haben können. Deshalb regeln z.B. $\S 9$ VII HH APO-AS und $\S 60$ I S. 1 TH SchulO, dass in den Abschlussund Abgangszeugnissen keine Bemerkungen über das Arbeits- und Sozialverhalten enthalten sein dürfen. In Bayern sind Aussagen zum Sozialverhalten nach Art. 52 III S. 2 BayEUG grundsätzlich zulässig, nicht jedoch in Zeugnissen von Klassenstufen, die zum Eintritt in das Berufsleben dienen können ( $§ 70$ ॥ S. 4 BY GSO; § 64 VIII S. 3 BY RSO; § 50 II S. 2 BY VSO).

Negative Auswirkungen im Bewerbungsverfahren, die sich daraus ergeben können, dass aus dem Zeugnis hervorgeht, dass der Bewerber die Förderschule besucht hat, werden z.B. in Niedersachsen und Mecklenburg-Vorpommern dadurch vermieden, dass problematische Zuordnungen unterbleiben. So regelt z.B. der niedersächsische Runderlass zu Zeugnissen ${ }^{1686}$ in Ziff. 5.7.1.3 und Ziff. 5.7.2.4, dass in den Abschlusszeugnissen der Name der Schule nicht, wie sonst üblich, mit einem Zusatz versehen wird, der auf den Förderschwerpunkt „Emotionale Entwicklung" oder "Lernen“ hinweist. In Mecklenburg-Vorpommern wird das Arbeits- und Sozialverhalten zwar generell bewertet, nicht aber, wenn die Schüler Förderschulen für Erziehungsschwierige und zur individuellen Lebensbewältigung besuchen. ${ }^{1687}$

Obwohl Arbeitgeber sicherlich ein großes Interesse daran haben zu erfahren, ob ein Schüler, der sich bewirbt, behindert ist oder nicht, werden Behinderungen und Einschränkungen in Zeugnissen grundsätzlich nicht wiedergegeben, auch dann nicht, wenn sie sich auf berufliche Kompetenzen auswirken. Dies gilt unabhängig davon, inwieweit der betroffene Schüler die Prüfungsanforderungen in vollem Umfang erfüllt hat. So wird etwa bei Schülern, die eine persönliche Assistenz benötigen, diese nicht aufgeführt und somit auch nicht dokumentiert, welche Leistungsbereiche der Schüler aufgrund der Behinderung nicht erfüllen kann, z.B. selbständiges Schreiben.

Der Schutz des Schülers und Bewerbers geht soweit, dass noch nicht einmal auf den eher minderen Fall von längeren Krankheiten hingewiesen werden darf. Hier ist im Arbeitsrecht ${ }^{1688}$ anerkannt, dass die Ausweisung von entschuldigten Fehlzeiten im Abgangs- bzw. Abschlusszeugnis unzulässig ist. Dies gilt

\footnotetext{
1685 OVG Greifswald, Urteil v. 14.10.2008, Az.: 4 K 16/08, NordÖR 2009, S. 128 ff, VG Braunschweig, Urteil v. 18.2.2004, Az.: 6 A 106/03, juris

1686 NI RdErl. d. MK v. 24.5.2004 -303-83203, zuletzt geändert durch RdErl. v. 8.4.2009 (SVBI. 6/2009, S. 171) - VORIS 22410, www.schure.de

1687 S. OVG Greifswald, Urteil v. 14.10.2008, Az.: 4 K 16/08, NordÖR 2009, S. $128 \mathrm{ff}$

1688 LAG Hamm, Urteil v. 21.12.1993, Az.: 4 Sa 1077/93, juris
} 
auch im Schulrecht ${ }^{1689}$, weil der Schüler keine Möglichkeit hat, den negativen Eindruck, der sich bei künftigen Arbeitgebern aus langen Fehlzeiten ergeben kann, durch eigenes Verhalten zu beeinflussen und er indirekt gezwungen wird, seine Krankheit zu offenbaren. ${ }^{1690}$

Auch im AGG werden die Interessen des Bewerbers mit Behinderung höher gewichtet als das Interesse des Arbeitsgebers an der Kenntnis einer Behinderung. Der Bewerber hat in einem Bewerbungsverfahren grundsätzlich keine Offenbarungspflicht hinsichtlich seiner Behinderung. ${ }^{1691}$ Der Arbeitgeber hat kein allgemeines Fragerecht zu einer Behinderung. ${ }^{1692}$ Ein Fragerecht wird dem Arbeitgeber nur insoweit zugestanden als er ein berechtigtes, billigenswertes und schutzwürdiges Interesse an der Beantwortung der Frage hat. ${ }^{1693}$ Seit der Geltung des AGG ist selbst die Frage nach einer Schwerbehinderung des Bewerbers unzulässig ${ }^{1694}$, obwohl mit der Beschäftigung eines Menschen mit Schwerbehinderung für den Arbeitgeber erhebliche Pflichten einhergehen ( $\S 81$ SGB IX), er also durchaus ein Interesse daran hat, sich auf diese besonderen Pflichten einzustellen. Infolgedessen haben Menschen mit Behinderung im schriftlichen Bewerbungsverfahren prinzipiell keine Pflicht zur Offenbarung ihrer Behinderung, noch nicht einmal bei Schwerbehinderungen.

Insgesamt stellt der Gesetzgeber also den Schutz des behinderten Bewerbers über die Interessen der Arbeitgeber und Ausbildungsstätten. Nur für die Zeugnisbemerkung bei Schülern und Schülerinnen mit Legasthenie wird dies bisher anders gesehen. Hier wird die Abweichung vom Leistungsstandard in der Prüfung für so maßgeblich gehalten, dass fehlende Kompetenzen in diesem Bereich oder die Veränderung von Prüfungsstandards zwingend im Zeugnis ausgewiesen werden müssen. ${ }^{1695}$ Dies führt zu einem Wertungswiderspruch

\footnotetext{
1689 OVG Saarlouis, Urteil v. 19.8.2002, Az.: 3 N 1/01, juris, Rn. 91

1690 OVG Saarlouis, Urteil v. 19.8.2002, Az.: 3 N 1/01, juris, Rn. 85, 90

1691 Joussen, NZA 2007, S. 174 ff, S. 175

1692 BAG, Urteil v. 17.12.2009, Az.: 8 AZR 670/08, juris, Rn. 14; LAG Hamm, Urteil v. 19.10.2006, Az.: 15 Sa 740/06, www.justiz.nrw.de, Rn. $54 \mathrm{f}$

1693 BAG NZA 1996, S. 371; Joussen, NZA 2007, S. 174 ff, S. 175

1694 Joussen, NZA 2007, S. 174 ff, S. 177

1695 Besonders deutlich wird der unterschiedliche Maßstab für die Offenlegung der Behinderung anhand der Berliner Regelungen: Nach § 38 III BE SopädVO besteht bei sonderpädagogischem Förderbedarf ein Anspruch auf Nachteilsausgleich, der nicht im Zeugnis vermerkt werden darf. Demgegenüber wird nach § 14 I BE Sek I-VO die Nicht-Bewertung der Rechtschreibung bei Schülern und Schülerinnen mit Lese-Rechtschreibschwierigkeiten im Zeugnis vermerkt. Ist allerdings bei einem Schüler mit Legasthenie etwa wegen der Schwere der Störung ein sonderpädagogischer Förderbedarf festgestellt, gilt wieder $\S 38$ III BE SopädVO, so dass der Nachteilsausgleich nicht im Zeugnis vermerkt wird. Poscher/Langer/Rux, Gutachten zu Art. 24 BRK, S. 72, Fn. 182; Rux in: Ennuschat, Die Rechte stotternder Menschen in Schule, Ausbildung und Studium, S. 59, Fn. 78 für stotternde Schüler und Schülerinnen sowie Nr. 3.3
} 
zwischen den schulrechtlichen Regelungen und den sonstigen, vor allem arbeitsrechtlichen Gleichbehandlungs- und Nicht-Diskriminierungsgrundsätzen.

Ein solcher Wertungswiderspruch würde nur dann nicht vorliegen, wenn die abweichende Regelung dadurch gerechtfertigt wäre, dass die Behinderung Legasthenie im Vergleich zu allen anderen Behinderungen und Krankheiten derartige Besonderheiten aufweisen würde, dass es deshalb unabdingbar ist, in den Zeugnissen auf diese Behinderung hinzuweisen. Eine derartige Sonderrolle nimmt die Behinderung Legasthenie, insbesondere im Vergleich zu anderen Behinderungen und Schwerstbehinderungen, jedoch offensichtlich nicht ein.

Ebenso wenig wie andere Menschen mit Behinderungen oder Krankheiten diese Behinderung durch ihr Verhalten beeinflussen können, können Schüler und Schülerinnen mit Legasthenie dies tun. Wenn diese anderen Menschen nicht zur Offenbarung ihrer Behinderung gezwungen werden dürfen und die Schule sie durch die Ausweisung entschuldigter Fehlzeiten auch nicht indirekt zur Offenbarung ihrer Behinderung zwingen darf, ${ }^{1696}$ gerade weil sie ihre Behinderung nicht beeinflussen können, ist auch kein Grund ersichtlich, warum bei Schülern und Schülerinnen mit Legasthenie die Behinderung im Zeugnis direkt offenbart werden muss, zumal sich umfassende Bereiche der Behinderung Legasthenie im außerschulischen Bereich durch technische Hilfsmittel wie Computer, Lesesoftware, Rechtschreibprogramme und Diktiergeräte ausgleichen lassen.

Eine Rechtfertigung ergibt sich schließlich nicht daraus, dass der Standard der Schulabschlüsse als kollidierendes Verfassungsrecht überwiegen würde. Eine solche Rechtfertigung ist bereits oben ausgeschlossen worden. ${ }^{1697}$

\subsection{Eingriff in Art. 12 । GG}

Art. 12 I 1 GG schützt die Berufsfreiheit und dabei u. a. die freie Wahl der Ausbildungsstätte und, über den Wortlaut hinaus, die gesamte Freiheit der berufsbezogenen Ausbildung.

Die Zeugnisbemerkung erschwert für die betroffenen Schüler und Schülerinnen vor allem das schriftliche Bewerbungsverfahren. Sie müssen bereits im schriftlichen Bewerbungsverfahren ihre Behinderung offenbaren. Dadurch besteht die erhebliche Gefahr, dass Betroffene schon in diesem Verfahren zurückgewiesen werden, weil die potentiellen Arbeitgeber niemanden mit einer

i.V.m. 3.5 BY KMBek Berufsschule Behinderung, wonach im Bereich Textverarbeitung und Kurzschrift wegen einer Behinderung von den Anforderungen an die Schreibgeschwindigkeit abgesehen werden kann, das dann aber im Zeugnis zu vermerken ist.

1696 OVG Saarlouis, Urteil v. 19.8.2002, Az.: 3 N 1/01, juris, Rn. 85

1697 S. oben 3. Teil: 5. Kapitel: 3.3.: Art. 7 I GG: Sicherung von Bildungsstandards und Vergleichbarkeit der Schulabschlüsse, S. 158 
solchen Problematik einstellen wollen. ${ }^{1698}$ Eine solche Diskriminierungstendenz gegenüber Bewerbern mit Legasthenie ist durch Studien nachgewiesen. ${ }^{1699}$ Sie ist für Bewerber mit Behinderungen generell belegt und nimmt sogar mit der Unternehmensgröße zu. ${ }^{1700}$ Es liegt somit eine subjektive Berufswahlbeschränkung vor.

Ein solcher Eingriff ist nur zum Schutz wichtiger Gemeinschaftsgüter zulässig. In Betracht kommt auch hier der Schutz des Vertrauens der Arbeitgeber auf die Informations- und Dokumentationsfunktion des Zeugnisses. Dieses Vertrauen müsste ein wichtiges Gemeinschaftsgut sein und den Schutz des Menschen mit Behinderung überwiegen. Das ist nicht der Fall. Der einfache Gesetzgeber hat, wie bereits ausgeführt, vor allem im schriftlichen Bewerbungsverfahren die Rechte und Belange der Menschen mit Behinderung über die Interessen potentieller oder auch der tatsächlichen Arbeitgeber gestellt. Eine Offenbarungspflicht von Menschen mit Behinderung besteht erst dann, wenn das schriftliche Verfahren abgeschlossen ist und der Mensch wegen der Behinderung ungeeignet sein wird, die berufliche Tätigkeit auszuüben. ${ }^{1701}$ Deshalb kann sich sogar der blinde Mensch mit seinem Abiturzeugnis für die Ausbildung zum Piloten bewerben und muss erst im persönlichen Vorstellungsverfahren offenlegen, dass er für den Beruf nicht befähigt ist. Es ist kein schützenswerter Belang der Arbeitgeber ersichtlich, der nur bei Legasthenie eine andere Wertung für das schriftliche Bewerbungsverfahren erfordert.

Die Zeugnisbemerkung verletzt daher das Grundrecht der betroffenen Schüler und Schülerinnen aus Art. 12 I GG.

\subsection{Eingriff in Art. 2 I GG durch Benennung der Legasthenie}

Die Zeugnisbemerkungen sind in den Ländern unterschiedlich formuliert. In Bayern wird ausdrücklich auf eine "fachärztlich festgestellte Legasthenie" verwiesen. In Brandenburg werden „besondere Schwierigkeiten im Lesen und Rechtschreiben (LRS)“ ausgewiesen. ${ }^{1702}$ Insbesondere diese Fassungen der Zeugnisbemerkung könnten das Grundrecht aus Art. 2 I GG auf freie Entfaltung der Persönlichkeit und informationelle Selbstbestimmung verletzten. ${ }^{1703}$

\footnotetext{
1698 Vgl. Weuster, Personalauswahl, S. $354 \mathrm{ff}$

1699 S. dazu die Studie speziell zur Auswirkung von Legasthenie im Auswahlprozess von Colella/ DeNisi/Narma, Journal of Applied Psychology 83 (1998), S. $102 \mathrm{ff}$

1700 S. die Nachweise bei Weuster, Personalauswahl, S. $355 \mathrm{ff}$

1701 LAG Hamm, Urteil v. 19.10.2006, Az.: 15 Sa 740/06, www.justiz.nrw.de, Rn. 55 m.w.N.

1702 BB VV-LRSR Nr. 8 i.V.m. Anlage 1

1703 S. dazu bereits ausführlich oben 10. Teil: 1. Kapitel: 1.: Datenschutz im Verfahren der Grundentscheidung und der Festlegung des Nachteilsausgleichs, S. 310
} 
Wie oben bei der Prüfung des Datenschutzes bereits ausgeführt ${ }^{1704}$, gehören Aussagen über den allgemeinen Gesundheitszustand, Krankheiten und psychologische Befunde zum besonders geschützten Bereich des Art. 2 I GG i.V.m. Art. 1 Abs. 1 GG. Sie dürfen nur dann durch staatliche Maßnahmen offenbart werden, wenn dies zum Schutz öffentlicher Interessen unerlässlich ist.

Mit der ausdrücklichen Benennung der Legasthenie in der Zeugnisbemerkung werden gesundheitliche Informationen, die dem besonders geschützten Bereich der Intimsphäre zuzuordnen sind, offenbart. Zudem wird, jedenfalls in der bayerischen Fassung, ausdrücklich darauf hingewiesen, dass es sich um eine medizinische Diagnose und Feststellung („fachärztlich festgestellt") handelt. Damit wird in das Grundrecht auf informationelle Selbstbestimmung eingegriffen.

Ein solcher Eingriff in die Privatsphäre ist nur verhältnismäßig, wenn er zum Schutz öffentlicher Interessen unerlässlich ist. ${ }^{1705}$ Es ist kein öffentliches Interesse ersichtlich, welches es unerlässlich macht, die medizinische Diagnose und die fachärztliche Feststellung konkret zu benennen.

Die genannten Fassungen verstoßen mithin gegen das Grundrecht aus Art. 2 । GG. ${ }^{1706}$

\subsection{Verstoß gegen Art. 4 I BRK}

Die Zeugnisbemerkung könnte außerdem gegen Art. 4 I BRK verstoßen. Danach verpflichten sich die Vertragsstaaten, die volle Verwirklichung aller Menschenrechte und Grundfreiheiten für alle Menschen mit Behinderungen ohne jede Diskriminierung aufgrund von Behinderung zu gewährleisten und zu fördern. Dazu gehört nach Art. 4 I Buchst. d BRK die Verpflichtung, "Handlungen oder Praktiken, die mit diesem Übereinkommen unvereinbar sind, zu unterlassen und dafür zu sorgen, dass die staatlichen Behörden und öffentlichen Einrichtungen im Einklang mit diesem Übereinkommen handeln“. Die ausdrückliche Erwähnung einer Behinderung in einem Zeugnis, ist eine Maßnahme einer staatlichen Behörde, die unmittelbar und gezielt an die Behinderung anknüpft. Sie ist damit eine Diskriminierung, die nach Art. 5 II i.V.m. Art. 2 BRK verboten ist. Sie ist nur gerechtfertigt, wenn der verfolgte Zweck legitim und die Ungleichbehandlung zwingend erforderlich ist. ${ }^{1707}$ Dass dies nicht der Fall ist, wurde bereits festgestellt.

\footnotetext{
1704 Ebenda

1705 BVerfGE 89, 69, 82

1706 So im Ergebnis VG München, Urteilstenor v. 26.2.2013, Az.: M 3 K 11.2962 und M 3 K 11.2963, die schriftliche Urteilsbegründung lag bei Drucklegung noch nicht vor.

1707 Zum Rechtfertigungsmaßstab der BRK in diesem Punkt: Rothfritz, UN-BRK, S. 217
} 


\subsection{Verstoß gegen Art. 7 II BRK, Art. 3 KRK, Art. 24 II Grundrechtecharta}

Die Zeugnisbemerkung kann zudem gegen das Kindeswohl des behinderten Kindes verstoßen. Nach Art. 7 II BRK und den gleichlautenden Vorschriften des Art. 3 KRK und Art. 24 II Grundrechtecharta ist bei allen Maßnahmen, die Kinder mit Behinderungen betreffen, das Wohl des Kindes vorrangig zu berücksichtigen. ${ }^{1708}$ Die Bestimmung dient dazu, Kindern mit Behinderungen die Durchsetzung ihres Anspruchs auf gesellschaftliche Inklusion zu erleichtern und zu gewährleisten. ${ }^{1709}$ Eine Zeugnisbemerkung, die die Behinderung des Kindes offenbart, wird diesem Anspruch nicht gerecht. Sie kann nur dann gerechtfertigt sein, wenn in der Gesamtbetrachtung die anderen Interessen in der Summe ein derartiges Gewicht haben, dass sich die Kindesinteressen trotz eines eingeräumten Vorrangs nicht durchsetzen können. ${ }^{1710}$ Bei einer Gesamtbetrachtung der Interessen können die Interessen des Arbeitgebers, die ansonsten bei Bewerbern mit Behinderung weniger geschützt werden als die Interessen der Menschen mit Behinderung, kein derartiges Gewicht haben, dass sie das Kindeswohl überwiegen. Die Zeugnisbemerkung verstößt daher auch gegen Art. 7 II BRK, Art. 3 KRK, Art. 24 II Grundrechtecharta.

\section{Unzulässigkeit auch bei Dyskalkulie}

Für den Bereich der Mathematik gibt es bisher noch keine Zeugnisbemerkungen in Abschlusszeugnissen, weil Dyskalkulie - wenn überhaupt - nur in der Grundschule anerkannt wird und dort eine Bemerkung keine weitgreifenden Auswirkungen hat. Wenn auch in Abschlussklassen eine Berücksichtigung der Dyskalkulie erfolgt, würde sicherlich gefordert werden, dass - entsprechend den Regelungen bei Legasthenie - eine Bemerkung etwa des Inhalts erfolgt: „Wegen Dyskalkulie wurde Mathematik nicht bewertet".

Bei der Dyskalkulie ergibt sich die Besonderheit, dass bei einer Nicht-Bewertung des Rechnens im Einzelfall das gesamte Fach Mathematik nicht bewertet und ausgewiesen wird. Weil es sich bei Mathematik um ein Kernfach handelt, wird grundsätzlich ein Absehen von der Bewertung hier nicht für möglich gehalten. Dass es jedoch keine zwingenden Gründe gibt, bei einer Behinderung nicht auf die Bewertung von Mathematik zu verzichten, wurde bereits oben ausgeführt. ${ }^{1711}$

\footnotetext{
1708 So auch Art. 24 EU-Grundrechte Charta und Art. 3 KRK, s. dazu Eichholz, R\&B 2007, S. 3 ff, S. $6 \mathrm{f}$

1709 Riedel, Gutachten UN-Behindertenrechtskonvention, S. 24; Aichele, Stellungnahme DIMR 2010, S. 33

${ }^{1710}$ Eichholz, "Gibt es ein Recht auf eine Schule für alle?", S. 5

${ }^{1711}$ S. oben 3. Teil: 5. Kapitel: 3.3.4.2: Bewertung des Faches Mathematik, S. 169
} 
Ebenso wenig wie beim Lesen und bei der Rechtschreibung kann bei Mathematik davon ausgegangen werden, dass es sich bei den Fähigkeiten in Mathematik um derart bedeutsame Fähigkeiten handelt, dass sie es rechtfertigen, gerade und nur diese Behinderung im Gegensatz zu allen anderen Behinderungen in einem Zeugnis zu offenbaren. Die Zeugnisbemerkung ist daher unzulässig.

Das allerdings führt zu der praktischen Schwierigkeit, was im Zeugnis ausgewiesen werden soll. In Fällen von Körperbehinderung wird z.B. darauf verzichtet, das Fach Sport überhaupt in das Zeugnis aufzunehmen. Der Leser muss erst erkennen, dass dieses Fach nicht verzeichnet ist, um dann Rückschlüsse zu ziehen. Das geht im Bereich Mathematik nicht, weil es ein Hauptfach ist und sofort auffällt, wenn es nicht aufgeführt ist. Da eine Bemerkung nicht zulässig ist, käme in Frage, statt der Note einen Strich zu setzen. Es ist aber sicher davon auszugehen, dass eine solche Praxis wiederum zu Nachfragen und Erklärungszwängen führen wird, weil sich jeder potentielle Arbeitgeber erkundigen wird, warum Mathematik nicht bewertet wurde. Auch das wäre wieder eine Benachteiligung, weil sie auf diesem Wege zu einer Offenbarungspflicht bezüglich der Behinderung führt. Eine solche Offenbarungspflicht besteht jedoch nicht.

In Betracht kommt als milderes Mittel die Benotung des Faches „pro forma“ mit "mangelhaft" ${ }^{\text {"1712 }}$, auch wenn diese Lösung immer noch nicht ideal ist. Damit würde die Behinderung nicht offenbart, aber es bliebe deutlich, dass Schwierigkeiten im Bereich Mathematik bestehen. Den Betroffenen steht es dann frei, auf Nachfragen ehrlich zu antworten oder nicht.

\section{Besondere Auswirkungen im Bewerbungsverfahren}

Tatsächlich ist es denkbar, dass sich die Bemerkung im Bewerbungsverfahren für die Betroffenen nicht nur negativ, sondern im Einzelfall auch positiv auswirken kann, weil dem Arbeitgeber die Behinderung explizit bekannt gemacht wird. Die Zeugnisbemerkung führt dann dazu, dass Bewerber mit Legasthenie/ Dyskalkulie anders, eventuell auch positiver behandelt werden müssen als Bewerber mit anderen Behinderungen.

Das Benachteiligungsverbot des AGG gilt nach § 2 । Nr. 1 AGG bereits für das Bewerbungs- und Auswahlverfahren für eine Einstellung. Bei einem Verstoß dagegen ist der Arbeitgeber nach § 15 AGG zum Schadensersatz verpflichtet. Für den Anspruch muss der Bewerber nur Indizien beweisen, die eine Benachteiligung nach dem AGG vermuten lassen. Der Arbeitgeber trägt dann nach $\S 22$ AGG die Beweislast dafür, dass keine Benachteiligung vorgelegen hat. ${ }^{1713}$ Die Indizien für eine Benachteiligung nach dem AGG liegen nach der

\footnotetext{
1712 Eine Bewertung mit „ungenügend“ kommt nicht in Betracht, weil dann der Abschluss gar nicht hätte erteilt werden dürfen.

${ }^{1713}$ BAG, Urteil vom 21.7.2009, Az.: 9 AZR 431/08, juris, Rn. 33
} 
Rechtsprechung des BAG schon darin, dass dem Arbeitgeber die Behinderung bekannt war und er den Bewerber nicht zum Vorstellungsgespräch eingeladen hat. ${ }^{1714}$ Wenn dem Arbeitgeber also mit der Zeugnisbemerkung die Behinderung Legasthenie/Dyskalkulie bekannt gemacht wird, führt dies zur Umkehr der Beweislast im Einstellungsprozess, ohne dass dies vom Bewerber oder Arbeitgeber beeinflusst werden kann. Der Arbeitgeber kann sich dem Verdacht der Benachteiligung nur entziehen, wenn er jeden Bewerber mit Legasthenie/Dyskalkulie zum Vorstellungsgespräch einlädt.

Eine ähnliche Konstellation ergibt sich auch in Bezug auf die besonderen Pflichten für öffentliche Arbeitgeber nach § 82 SGB IX. Danach sind die Dienststellen der öffentlichen Arbeitgeber verpflichtet, schwerbehinderte Menschen, die sich beworben haben, zu einem Vorstellungsgespräch einzuladen. Eine solche Einladung ist nach der Rechtsprechung des BVerwG nur dann entbehrlich, wenn die fachliche Eignung für die Stelle offensichtlich fehlt. Erfolgt eine solche Einladung nicht, benachteiligt der öffentliche Arbeitgeber den Bewerber wegen der Behinderung und macht sich evtl. schadenersatzpflichtig. ${ }^{1715}$ § 82 S. 2 SGB IX wird über den Wortlaut hinaus sowohl auf diejenigen Menschen angewandt, die Menschen mit Schwerbehinderung gleichgestellt sind ${ }^{1716}$ als auch auf Menschen mit Behinderung, die nicht den Grad der Schwerbehinderung erfüllen. ${ }^{1717}$ Begründet wird dies damit, dass kein Grund erkennbar sei, Menschen mit einer "leichten" Behinderung von den Schutzvorkehrungen im Bereich des öffentlichen Dienstes auszuklammern und sie im Verhältnis zu Menschen mit einer "stärkeren" Behinderung wie Menschen ohne Behinderung zu behandeln. Eine solche Verfahrensweise würde gegen Art. 3 III 2 GG und § 7 । AGG verstoBen. ${ }^{1718}$ Infolgedessen müssen die öffentlichen Stellen alle Bewerber, deren Behinderung ihnen bekannt ist oder bekannt sein müsste, zu Vorstellungsgesprächen einladen ${ }^{1719}$, also auch und gerade die Bewerber mit Legasthenie.

\footnotetext{
1714 BAG, Urteil vom 21.7.2009, Az.: 9 AZR 431/08, juris, Rn. 20; VGH Mannheim, Urteil v. 4.8.2009, Az.: 9 S 3330/08, www.landesrecht-bw.de

1715 BVerwG Urteil v. 3. 3. 2011, Az.: 5 C 15.10, Rn. 16, juris, mit Anmerkung Banafsche, br 2011, S. $180 \mathrm{ff}$

${ }^{1716}$ VGH Mannheim, Urteil v. 4.8.2009, Az.: 9 S 3330/08, www.landesrecht-bw.de; BVerwG Urteil v. 3. 3. 2011, Az.: 5 C 15.10 und 5 C 16.10, Rn. 16, juris

1717 LAG Berlin-Brandenburg, Urteil v. 2.6.2009; Az.: 3 Sa 499/09 juris, Rn. 56; Roetteken, Diskriminierende Verfahrensfehler Anm. D

${ }^{1718}$ Roetteken, Diskriminierende Verfahrensfehler, Anm. D; a. A. VG Ansbach, Beschluss v. 14.12.2010; Az.: AN 8 P 10.01772, juris, Rn. 14 für Legasthenie, das den Anspruch auf schwerbehinderte Menschen beschränkt und bei Legasthenie, die sich aus der Zeugnisbemerkung ergibt, keine Nachforschungspflicht bzgl. einer Schwerbehinderung sieht.

1719 Vgl. Roetteken, Diskriminierende Verfahrensfehler, Anm. D, BVerwG Urteile v. 3. 3. 2011, Az.: 5 C 15.10 und 5 C 16.10 , juris
} 
Die Zeugnisbemerkung kann daher zu ganz erheblichen Verpflichtungen der potentiellen Arbeitgeber führen. Dies ist zwar eine für die Betroffenen mit Legasthenie/Dyskalkulie überwiegend positive Folge, die allerdings die negative Folge der zwangsweisen Offenbarung einer Behinderung und das hohe Risiko der Diskriminierung im schriftlichen Bewerbungsverfahren nicht ausgleicht. Für Schüler und Schülerinnen mit anderen Behinderungen ließe sich jedoch fragen, ob diese nicht unter dem Gesichtspunkt der Gleichbehandlung einen Anspruch darauf hätten, dass ihre Behinderung im Zeugnis erwähnt wird, um so auch bei ihnen die Verpflichtung der Arbeitgeber im Bewerbungsverfahren auszulösen.

Tatsächlich dürften diese Verpflichtungen den Landesgesetzgebern nicht bewusst gewesen und nicht das Ziel ihrer Regelungen zur Aufnahme von Zeugnisbemerkungen gewesen sein. Den Arbeitgebern wird mit der Zeugnisbemerkung angesichts der erheblichen Pflichten im Bewerbungsverfahren kein besonderer Gefallen getan. 



\section{Teil: Amtshaftung aus $\S 839$ BGB i.V.m. Art. 34 GG}

Berücksichtigen die Schulen und Lehrkräfte die Legasthenie/Dyskalkulie nicht, können sich für die betroffenen Schüler und Schülerinnen Ansprüche aus Amtshaftung gem. § 839 BGB i.V.m. Art. 34 GG ergeben. ${ }^{1720}$ Danach besteht ein Anspruch auf Schadensersatz, wenn jemand schuldhaft in Ausübung eines öffentlichen Amtes eine Amtspflicht, die im Funktionszusammenhang mit der Amtsausübung steht und einen Drittbezug aufweist, verletzt. Die Anstellungskörperschaft haftet für ihren Amtsträger. Dass ein solcher Anspruch in Betracht kommt, hat der VGH Kassel dem Grunde nach anerkannt ${ }^{1721}$, denn er verweist einen legasthenen Schüler auf einen Schadensersatzprozess, wenn seine Behinderung nicht durch den ihm gewährten Nachteilsausgleich kompensiert worden sein sollte.

Lehrkräfte und Mitarbeiter von Schulbehörden werden im Schulbetrieb hoheitlich tätig und sind insoweit Amtsträger, für die die Anstellungskörperschaft haftet.

\section{Kapitel: Amtspflichten}

Amtspflicht ist jede persönliche Verhaltenspflicht der Amtsträger in Bezug auf ihre Amtsführung. ${ }^{1722}$ Alle Beamten und alle Personen, die hoheitliche Befugnisse ausüben ${ }^{1723}$, haben die Amtspflicht zum rechtmäßigen Handeln. Sie entspricht der sich aus Art. 20 Abs. 3 GG ergebenden Rechts- und Gesetzesbindung der Verwaltung. Beamte und Angestellte haben eine Gehorsamspflicht, die beinhaltet, außer den Gesetzen auch den allgemeinen und im Einzelfall er-

\footnotetext{
${ }^{1720}$ BVerfG, Beschluss v. 8. 6. 2012; Az.: 1 BvR 2853/10, http://www.bverfg.de/entscheidungen/rk20120608_1bvr285310.html, Rn. 9

${ }^{1721}$ Beschluss v. 5.2.2010, Az.: 7 A 2406/09, juris, Rn. 49

1722 Zimmerling, in: jurisPK-BGB, § 839 BGB, Rn. 54

${ }^{1723}$ Zimmerling, in: jurisPK-BGB, $\S 839$ BGB, Rn. 14
} 
lassenen verbindlichen Weisungen der Vorgesetzten zu folgen. ${ }^{1724}$ Lehrkräften obliegt darüber hinaus generell die Amtspflicht, die ihnen anvertrauten Schüler vor Schäden zu bewahren. ${ }^{1725} \mathrm{Im}$ Bereich der Berücksichtigung von Legasthenie/Dyskalkulie kommt die Verletzung verschiedener Pflichten in Betracht.

\section{Amtspflicht zur Feststellung der Legasthenie/Dyskalkulie}

Lehrkräfte und Schulbehörden könnten eine Amtspflicht gegenüber dem Kind auf Feststellung der Legasthenie/Dyskalkulie haben.

Eine Pflicht der Schule zur Feststellung der Legasthenie/Dyskalkulie findet sich in der Empfehlung der KMK von 2003 und 2007, dort allerdings als „Diagnose“ bezeichnet: „Unbestritten ist, dass die Diagnose und die darauf aufbauende Beratung und Förderung von Schülern und Schülerinnen mit besonderen Schwierigkeiten im Lesen und Rechtschreiben zu den Aufgaben der Schule gehört. ${ }^{21726}$ Die landesrechtlichen Regelungen sehen, mit Ausnahme von Bayern, regelmäßig vor, dass die Feststellung der "Schwierigkeiten" durch die Schulen erfolgt. Es liegt nahe, in einer solchen expliziten Aufgabe der Schule auch eine Amtspflicht zu sehen. Das OLG Celle hat in einem Amtshaftungsprozess eine solche Amtspflicht jedoch abgelehnt und entschieden, dass es nicht Aufgabe der Lehrkräfte und der Schule ist, die medizinisch-psychologische Diagnose Legasthenie zu stellen. ${ }^{1727}$

Hier ergibt sich eine Diskrepanz zwischen der Zuständigkeit der Schule für die Feststellung, die Diagnose und ihrer rechtlichen Pflicht dazu. Einerseits sehen Schulen und Schulbehörden die Zuständigkeit der Feststellung einer Legasthenie/Dyskalkulie bei sich und sehen sich nicht an fachärztliche Diagnosen gebunden. Sie erkennen eine Legasthenie trotz vorliegender fachärztlicher Gutachten nicht $a^{1728}$, selbst dann nicht, wenn die Legasthenie nach $\S 2$ SGB IX

${ }^{1724}$ Zimmerling, in: jurisPK-BGB, § 839 BGB, Rn. 78

1725 Papier, in: MüKomm BGB, § 839, Rn. 259

1726 KMK-Lesen/Rechtschreiben/Rechnen, Punkt I

1727 OLG Celle, Beschluss v. 17.2.2010, Az.: 16 U 62/09 (nicht veröffentlicht), vorgängig LG Lüneburg, Urteil v. 20.4.2009, Az.: 2 O 220/08 (nicht veröffentlicht). Derselbe Senat hat auch entschieden, dass keine Amtspflichtverletzung des Jugendamtes vorliegt, wenn die vom Jugendamt bestellte Sachverständige fälschlicherweise eine Legasthenie verneint, OLG Celle, Urteil v. 4.11.2008, Az.: 16 U 70/07 (nicht veröffentlicht). Die letztgenannte Entscheidung wurde vom BVerfG aufgehoben und an das OLG Celle zurückverwiesen, BVerfG Kammerbeschluss, Az.: 1 BvR 1711/09, v. 21.11.2012, juris. In beiden Fällen hat der Senat darauf hingewiesen, dass die „bedauerliche und schicksalhafte Kindheitsentwicklung des Schülers in erster Linie in seiner Person begründet ist und nicht auf ... Versäumnissen von Dritten beruht."

1728 OVG Lüneburg, Beschluss v. 10.7.2008, Az.: 2 ME 309/08, www.rechtsprechung.nieder sachsen.de; VGH Kassel, Beschluss v. 25.3.2009, Az.: 7 B 772/09 (nicht veröffentlicht); VG Gießen, Urteil v. 30.6.2009. Az.: 4 K 1052/09.GI (nicht veröffentlicht); VG Darmstadt, Urteil v. 28. 4. 2009, Az.: 7 K 687/08.DA (3) (nicht veröffentlicht) 
durch das Versorgungsamt festgestellt worden ist. ${ }^{1729}$ Vielmehr halten sie eine pädagogische Würdigung der fachärztlichen Diagnosen für notwendig. ${ }^{1730}$ Dies wird von der Rechtsprechung für zulässig gehalten, denn ihrer Ansicht nach steht Lehrkräften bei der Feststellung einer Legasthenie ein Beurteilungsspielraum $\mathrm{zu}^{1731}$, dem auch dann Vorrang zukommt, wenn bei den Lehrkräften medizinische Kenntnisse zur Legasthenie nur begrenzt vorhanden sind. ${ }^{1732}$ Andererseits lehnen die Schulverwaltungen jedoch die rechtliche Verpflichtung und damit Amtspflicht zur Feststellung ab. Wenn die Schule für sich in dieser Form und in diesem Umfang die Zuständigkeit und Kompetenz für die Feststellung einer Legasthenie/Dyskalkulie in Anspruch nimmt, korrespondiert damit auch die Amtspflicht zur Feststellung. Beruft sich die Schule darauf, dass es sich bei der Feststellung der Legasthenie/Dyskalkulie um eine medizinische Frage handelt, so ist es ein Fall des venire contra factum proprium, wenn sie dennoch, wie zurzeit üblich, medizinische Diagnosen nicht anerkennt. Es ist treuwidriges und widersprüchliches Verhalten, wenn Schulen meinen, dass die genaue Abklärung von Schwierigkeiten nicht von ihnen geleistet werden kann und dafür eine medizinische Untersuchung erforderlich ist, dann aber für den schulischen Kontext eben diese medizinische Diagnose nicht anerkannt wird. Solange die Schulen und Schulbehörden die Aufgabe und Zuständigkeit zur Feststellung und Entscheidung über das Vorliegen einer Legasthenie/Dyskalkulie haben, ist dies auch eine Amtspflicht.

Die Amtspflicht muss auch gegenüber einem Dritten, nämlich dem Schüler bestehen. Die Lehrkraft muss die Pflicht nicht nur im Interesse der Allgemeinheit, sondern auch im Interesse des Schülers beachten. Bei der Pflicht der Lehrkraft aus § 61 II SGB IX auf mögliche Behinderungen hinzuweisen, wird eine Amtspflicht verneint, weil die Lehrkräfte insoweit nur ein Teil des Bezugssystems des Kindes sind und sie nicht alle Behinderungen erkennen können. Liegt jedoch die Zuständigkeit der Diagnose allein bei der Schule, dann muss die Diagnose auch von ihr wahrgenommen werden. Die Diagnose ist für das Kind von eminenter Wichtigkeit für den weiteren schulischen Werdegang, deshalb dient die Diagnose auch dem Schutz des Kindes, so dass die Pflicht drittschützend ist.

Die Amtspflicht zur Diagnose entfällt nur dann, wenn die Schule diese Zuständigkeit generell für sich nicht in Anspruch nimmt, sondern die Zuständig-

${ }^{1729}$ VGH Kassel, Beschluss v. 25.3.2009, Az.: 7 B 772/09 (nicht veröffentlicht), bei dem Kläger war durch Bescheid ein GdB von 30 festgestellt worden.

1730 Behrens, Nds. SVBI 2006, S. 189 ff, S. 189

${ }^{1731}$ VGH Kassel, Beschluss v. 25.3.2009, Az.: 7 B 772/09, S. 4 (nicht veröffentlicht); VG Gießen, Urteil v. 30.6.2009. Az.: 4 K 1052/09.GI, S. 15 (nicht veröffentlicht); VG Darmstadt, Urteil v. 28. 4. 2009, Az.: 7 K 687/08.DA (3), S. 17 (nicht veröffentlicht)

1732 VG Gießen, Urteil v. 30.6.2009. Az.: 4 K 1052/09.GI, S. 13 (nicht veröffentlicht); a.A. VG Weimar, Beschluss v. 3.5.2010; Az.: 2 E 377/10, S. 2 (nicht veröffentlicht); anders jetzt VGH Kassel, Beschluss v. 17.11.2010; Az.: 7 A 2970/09.Z, juris, Rn. 9 
keit der Fachkräfte für die Diagnose anerkennt und diese in der Schule übernimmt. In Bayern lässt sich beobachten, dass Lehrkräfte sich entlastet fühlen, weil sie nicht für die Diagnose zuständig sind, sondern die Eltern zu Fachkräften schicken können.

\section{Amtspflicht zur legasthenie-/dyskalkuliespezifischen Förderung}

Fraglich ist weiter, ob es eine Amtspflicht zur spezifischen Förderung gibt. Dies hängt davon ab, ob diese Pflicht eindeutig dem Zuständigkeitsbereich der Schule zugeordnet oder ausgelagert wird. ${ }^{1733}$ Wenn die notwendige, individuelle und geeignete Förderung für Schüler und Schülerinnen mit Legasthenie/Dyskalkulie so geregelt wird, dass die Schule dafür zuständig ist, dann geht damit die Amtspflicht einher, diese Förderung auch durchzuführen. Das BVerfG sieht hier die Möglichkeit eines Amtshaftungsanspruchs, wenn die Leistung schuldhaft zu Unrecht versagt wird. ${ }^{1734}$

\section{Amtspflicht zur Berücksichtigung der Legasthenie/Dyskalkulie}

Lehrkräfte sind nach $\S \S 33,35$ BeamtStG und $\S 3$ TV-L ihrem Dienstherrn bzw. Arbeitgeber zu Dienst und Treue verpflichtet. Sie müssen als Teil der Verwaltung die Gesetze (Art. 20 Abs. 3 GG) und die dienstlichen Anordnungen ihrer Vorgesetzten befolgen. Aus der Bindung an die Grundrechte aus Art. 1 III GG ergibt sich direkt die Pflicht, die Grundrechte der ihnen anvertrauten Schüler und Schülerinnen zu achten. Unzweifelhaft liegt daher eine Amtspflicht der Schule und der Lehrkräfte darin, eine Behinderung sowohl im Unterricht als auch in Prüfungen zu berücksichtigen, denn das Grundrecht aus Art. 3 III 2 GG beinhaltet die Pflicht, niemanden wegen seiner Behinderung zu benachteiligen. Ist die Behinderung für die Schule darüber hinaus in einem Verfahren anerkannt und sind Formen des Nachteilsausgleichs festgelegt worden, dann ergibt sich die Pflicht zur Berücksichtigung und zur Umsetzung des Nachteilsausgleichs aus der Gehorsamspflicht. ${ }^{1735}$

Darüber hinaus haben Amtsträger die Pflicht, die zur Führung ihres Amtes notwendigen Rechts- oder Verwaltungskenntnisse zu besitzen oder sich zu verschaffen. ${ }^{1736}$ Hat eine Lehrkraft entweder keine Kenntnis von einer Behinde-

\footnotetext{
1733 S. oben 9. Teil: 4. Kapitel: 3.: Zuständigkeit der Schule oder anderer Institutionen?, S. 305

1734 BVerfG, Beschluss v. 8.6.2012; Az.: 1 BvR 2853/10, http://www.bverfg.de/entscheidungen/rk20120608_1bvr285310.html, Rn. 9

1735 Im Verfahren VG München, Beschluss v. 15.10.2002, Az.: M 3 E 02.4698, juris, Rn. 40, verweigerte der Fachlehrer Deutsch ausdrücklich die Berücksichtigung einer festgestellten Legasthenie, weil die Lehrer in Deutsch, Französisch und Englisch und die Schulpsychologin einhellig der Ansicht waren, es liege keine Legasthenie vor. Das Gericht sah darin sachfremde Erwägungen, die die Bewertung der Deutscharbeit unzulässig beeinflussten.

1736 BGH, Urteil v. 9.7.1998, Az.: III ZR 87/97, juris, Rn. 12
} 
rung oder keine Kenntnis vom Umgang mit der Behinderung, so ist es die Pflicht der Lehrkraft, sich diese Kenntnisse zu verschaffen. Verletzt der Amtsträger diese Pflicht, ist dies wiederum eine eigene Amtspflichtverletzung.

Sehen sich Lehrkräfte nicht in der Lage, einer Rechtsvorschrift Folge zu leisten, so haben sie die Pflicht, diese Bedenken vorzutragen und eine Entscheidung des Vorgesetzten herbeizuführen ( $\$ 36$ II BeamtStG). Eine eigene Verwerfungskompetenz der Anordnung steht der Lehrkraft nicht zu. ${ }^{1737}$

Die Amtspflichten sind drittschützend, weil sie als Regelungen des Prüfungsverfahrens dem individuellen Grundrechtsschutz und daher insbesondere dem Schutz des Schülers dienen. ${ }^{1738}$

\section{Pädagogische Freiheit der Lehrkräfte als Grenze?}

Fraglich ist, ob diese Amtspflichten durch die pädagogische Freiheit des Lehrers $^{1739}$, die in allen Schulgesetzen anerkannt ist ${ }^{1740}$, begrenzt oder eingeschränkt werden. Die pädagogische Freiheit ist eine pflichtgebundene Freiheit, deren Grenzen sich aus der Verfassung, den Gesetzen und aus den sonstigen Rechtsvorschriften ergeben. ${ }^{1741}$ Sie wirkt sich vor allem in der Ausgestaltung der Unterrichts- und Erziehungstätigkeit aus. Sie gibt jedoch nicht das Recht, sich bei Entscheidungen über Noten und Bewertungen über die geltenden rechtlichen Vorgaben hinwegzusetzen oder sie individuell auszulegen. Insofern liegt die Nicht-Berücksichtigung einer Behinderung im Unterricht und/oder in Prüfungen nicht im pädagogischen Ermessen einer Lehrkraft. Dieses schränkt die Amtspflicht daher nicht ein. ${ }^{1742}$

\section{Amtspflicht der Schulbehörden auf ordnungsgemäße Organisation}

Ist an den Schulen die Erfüllung der oben dargestellten Amtspflichten nicht möglich, weil ihnen die notwendige personelle und sachliche Ausstattung fehlt, so kommt eine Haftung des Landes aus Organisationsmangel in Betracht. Das Land hat seine Schulen so auszustatten, dass diese ihre Amtspflichten auf ordnungsgemäße Unterrichtung und Förderung der Kinder erfüllen können. Stellt ein Land seinen Schulbehörden die für die Erfüllung dieser Aufgaben erforderlichen Mittel nicht zur Verfügung, so haftet es unter dem Gesichtspunkt des Or-

\footnotetext{
1737 S. dazu Ennuschat/Volino, br 2009, S. 166 ff, S. 168

1738 Vgl. Niehues, Prüfungsrecht, Rn. 512

1739 Umfassend dazu Rux, Pädagogische Freiheit des Lehrers

1740 Vgl. die Nachweise bei Avenarius/Heckel, Schulrecht, Ziff. 19.411, Fn. 97

1741 Avenarius/Heckel, Schulrecht; Ziff. 19.411

1742 S. dazu das Urteil des VG Lüneburg v. 28.5.2002, Az.: 4 A 246/01, www.rechtsprechung.niedersachsen.de zum Nachteilsausgleich bei spastischer Diplegie
} 
ganisationsmangels. Mangelnde Personal- und Sachmittelausstattungen exkulpieren nicht. ${ }^{1743}$

\section{Kapitel: Weitere Voraussetzungen der Amtshaftung}

Die Amtspflichtverletzung muss vorsätzlich oder fahrlässig begangen worden sein, um die Haftung des Staates zu begründen. Für den Nachweis des Verschuldens gilt ein objektivierter Fahrlässigkeitsmaßstab, so dass es auf die Kenntnisse und Fähigkeiten eines durchschnittlichen Beamten ankommt. ${ }^{1744}$ Die Anforderungen sind je nach Amt unterschiedlich und werden mit jedem höheren Amt höher, so dass bei Entscheidungsträgern, wie z.B. Schuldirektoren und Schulaufsichtsbehörden, höhere Anforderungen zu stellen sind als bei Lehrkräften. ${ }^{1745}$ Üblicherweise kann davon ausgegangen werden, dass Amtspflichten nicht vorsätzlich, sondern "nur" fahrlässig verletzt werden. Wird allerdings gegen die Rechtspflicht zur Berücksichtigung einer Behinderung und gegen die Gehorsamspflicht verstoßen, indem eine Behinderung trotz Anerkennung und trotz Festlegung eines Nachteilsausgleichs nicht berücksichtigt wird, ist davon auszugehen, dass dies bewusst, d.h. vorsätzlich, geschieht. ${ }^{1746}$ Im Fall einer vorsätzlichen Amtspflichtverletzung entfällt die Subsidiaritätsklausel des § 839 Abs. 1 S. 2 BGB, d.h. die Lehrkraft kann auch persönlich in Anspruch genommen werden.

Die Amtspflichtverletzung muss kausal für einen Schaden sein. Die Kausalität hat die Rechtsprechung verneint bei rechtswidrigem Unterrichtsausschluss, rechtswidriger Entlassung von der Schule und bei rechtswidriger Überweisung auf die Sonderschule. ${ }^{1747}$ Sie hat sie indes bejaht bei Kosten für Privatunterricht, der notwendig war, weil die Schule pflichtwidrig bei einem Kind mit Schulphobie keinen Sonderunterricht in Form von Hausunterricht organisiert hatte. ${ }^{1748}$ Die Kausalität kann jeweils nur im Einzelfall festgestellt werden. ${ }^{1749}$

\footnotetext{
1743 Grundlegend dazu: BGH, Urteil v. 11.1.2007, Az.: III ZR 203/05 http://juris.bundesgerichtshof.de/cgi-bin/rechtsprechung/document.py?Gericht=bgh\&Art $=e n \& s i d=542 a 812 f 52$ de6e65571a710271c43fc0\&nr=38892\&pos=17\&anz=38, Rn. $19 \mathrm{ff}$

${ }^{1744}$ Zimmerling, in: jurisPK-BGB, § 839 BGB, Rn. 127; BGH, Urteil v. 9.7.1998, Az.: III ZR 87/97, juris, Rn. 12

1745 Zimmerling, in: jurisPK-BGB, § 839 BGB, Rn. 127; vgl. zu den Anforderungen an Beamte des Landesjustizprüfungsamtes OLG München, Urteil v. 17.8.2006; Az.: $1 \cup$ 2960/05, juris

${ }^{1746}$ So z.B. im Fall des VG München, Beschluss v. 15.10.2002, Az.: M 3 E 02.4698, juris, Rn. 40

1747 OLG Hamm Urteil v. 5.11.1999, Az.: 11 U 97/98 juris, Rn. $45 \mathrm{ff}$

1748 OLG Hamm, Urteil v. 23. 3. 1990, Az.: 11 U 108/89 (nicht veröffentlicht)

1749 Vgl. Niehues, Prüfungsrecht, Rn. 513
} 
Als konkreter Schaden kommen vor allem Verdienstausfall und Sozialversicherungsansprüche in Betracht, weil die Berufstätigkeit erst später als nach normalem Verlauf aufgenommen werden kann. Ebenfalls kann ein Anspruch auf Schmerzensgeld nach $\S 253$ BGB bestehen. ${ }^{1750}$ Denkbar sind auch die Kosten für privat bezahlte Nachhilfe oder Therapie, die anstelle der an sich erforderlichen schulischen Förderung getreten sind, Kosten, die durch eine verspätete Aufnahme eines Studiums entstehen, bis hin zu den Kosten, die sich ergeben, wenn ein Studienplatz nicht zugeteilt wird, weil der NC nicht erreicht wird, der Notendurchschnitt aber ausgereicht hätte, wenn die Bewertung rechtmäßig unter Berücksichtigung der Behinderung erfolgt wäre.

Nach $\S 839$ Abs. 3 BGB hat der Verletzte eine Schadensabwendungspflicht, d.h. er muss zunächst versuchen, den Schaden durch Rechtsmittel abzuwenden. Ob dafür auch die Durchführung eines Verfahrens auf vorläufigen Rechtsschutz erforderlich ist, um damit zu erreichen, vorsorglich vor dem Abschluss des Hauptsacheverfahrens eine Wiederholungsprüfung "auf Verdacht" zu absolvieren, hat das BVerwG offengelassen. ${ }^{1751}$

1750 S. dazu OLG München, Urteil v. 17.8.2006; Az.: 1 U 2960/05, juris; s. dazu Niehues, Prüfungsrecht, Rn. 514

1751 BVerwG, Urteil v. 19.12.2001; Az.: 6 C 14/01, NVwZ 2002, S. 1377; ablehnend Faber/Roth, DVBI 2010, S. 1193 ff, S. 1334 ff; dazu auch Niehues, Prüfungsrecht, Rn. 516 



\section{Teil: Rechtsschutz}

Im Hinblick auf den Rechtsschutz in Fällen nichtberücksichtigter Legasthenie oder Dyskalkulie in der Schule soll auf einige ausgewählte Probleme eingegangen werden.

\section{Kapitel: Rechtsschutz im Verfahren der Anerkennung der Behinderung und bei schulischen Prüfungen}

Das Grundrecht aus Art. 19 IV GG sichert den Rechtsschutz gegenüber der Exekutive und ist primär ein Leistungsgrundrecht. ${ }^{1752}$ Das sich daraus ergebende Gebot des effektiven Rechtsschutzes verlangt nicht nur, dass jeder potentiell rechtsverletzende Akt der Exekutive in tatsächlicher und rechtlicher Hinsicht gerichtlich überprüft werden kann, sondern auch, dass die Gerichte den betroffenen Rechten tatsächlich Wirksamkeit verschaffen. Gesetzlich vorgesehene Rechtsbehelfe müssen so ausgestaltet sein, dass der Rechtsschutz, den der Einzelne mit ihnen erreichen kann, für ihn tatsächlich nützlich ist. ${ }^{1753} \mathrm{Zu}$ den wesentlichen Grundsätzen eines rechtsstaatlichen Verfahrens zählt das Recht auf ein faires Verfahren, das die Verpflichtung enthält, dass staatliche Organe korrekt und fair verfahren und dass verfahrensrechtlich "Waffengleichheit" zwischen den staatlichen Organen und dem betroffenen Bürger besteht. ${ }^{1754}$

Der Rechtsschutz ist im Schulbereich besonders problematisch ${ }^{1755}$, weil alle Beteiligten auch während eines Überprüfungsverfahrens weiter miteinander umgehen müssen und sich nicht aus dem Weg gehen können. ${ }^{1756}$ Das Verhältnis zwischen Schule, Schülern und Eltern wird im Falle eines Rechtsstreits meis-

\footnotetext{
1752 Jarass/Pieroth, in: Jarass/Pieroth GG, Art. 19, Rn. $32 \mathrm{f}$

${ }^{1753}$ Vgl. BVerfG, Beschluss vom 30.05.2007 - 2 BvR 2012/05, http://www.bverfg.de/entscheidungen/rk20070530_2bvr201205.html, Rn. 14

1754 BVerfGE 38, 105, 111; 52, 380, 388 f m.w.N.

1755 S. dazu Ennuschat, Die Rechte stotternder Menschen in Schule, Ausbildung und Studium, S. 169; Rux, RdJB 2009, S. 220 ff, S. 227

1756 eine Anleitung für Konfliktgespräche mit Lehrern enthält Enkhardt, Zoff mit der Schule
} 
tens sehr belastet. ${ }^{1757}$ Der betroffene Schüler muss weiterhin die Schule besuchen. Er wird leider häufig mit negativen und abwertenden Bemerkungen über seine Eltern, aber auch über sich selbst konfrontiert. Eltern warten i.d.R. lange $a b$, bis sie sich zu einem Rechtsstreit gegen die Schule entschließen. Wenn es möglich ist, klagen sie erst, wenn das Kind die Schule verlassen hat. Geschwister des Kindes werden leicht in den Konflikt mit hinein gezogen. Lehrkräfte fühlen sich leider oft durch Rechtsmittel persönlich angegriffen. Das Rechtsschutzverfahren dient jedoch der sachlichen Klärung einer rechtlichen Zweifelsfrage. Es ist nicht nur das gute Recht der Eltern (Art. 19 Abs. 4 GG), sondern auch ihre Pflicht (Art. 6 II GG), Eingriffe in die Rechte ihrer Kinder abzuwehren.

Maßnahmen der Exekutive, also der Schule, die der gerichtlichen Überprüfung unterliegen, können sowohl Maßnahmen im Zuge der Grundentscheidung der Anerkennung der Behinderung als auch Maßnahmen im Verlaufe schulischer Prüfungen sein.

Die Anerkennung der Behinderung in der Schule ist ein Verwaltungsakt gem. § 35 VwVfG. Gegen die Ablehnung der Anerkennung einer Legasthenie/ Dyskalkulie ist die Verpflichtungsklage nach $\S 42$ VwGO statthaft. Ob ein Vorverfahren nach $\S \S 68 \mathrm{ff}$ VwGO durchzuführen ist, richtet sich nach den landesrechtlichen Ausführungsbestimmungen zur VwGO.

Schulische Prüfungen sind vielfältig. Schwierigkeiten ergeben sich meist daraus, dass die Behinderung Legasthenie/Dyskalkulie nicht in der festgesetzten Form oder gar nicht berücksichtigt wird. Für den Rechtsschutz in diesen Fällen ist zu differenzieren.

Einzelnoten aus Klausuren, Tests, Referaten, mündlichen Beiträgen usw., sind i.d.R. unselbständige Bestandteile der Endnote resp. des Zeugnisses, so dass sie keine Außenwirkung haben und deshalb nicht als Verwaltungsakte zu qualifizieren sind. ${ }^{1758}$ Rechtsschutz gegen diese Maßnahmen gibt es nur in der Form des Beschwerderechts. Möglich sind Gegenvorstellung, Dienstaufsichtsbeschwerde und Fachaufsichtsbeschwerde sowie z.B. die Anrufung des Datenschutzbeauftragten. ${ }^{1759}$

Verwaltungsakte sind Prüfungsentscheidungen, Versetzungs- und Abschlusszeugnisse, die Nichtversetzung und auch die Nicht-Zulassung zu einem Bildungsgang, z.B. zur Sekundarstufe II. ${ }^{1760}$ Einzelnoten sind nur dann Verwaltungsakte, wenn sie unmittelbar Rechtsfolgen auslösen, etwa weil von ihnen die Zulassung zu einem Bildungsgang abhängt ${ }^{1761}$ oder weil sie sich auf die

\footnotetext{
${ }^{1757}$ Böhm, Elternrechte in der Schule, S. 152

1758 Avenarius/Heckel, Schulrecht, Ziff. 26.333

1759 Avenarius/Heckel, Schulrecht, Ziff. 34.1

1760 Avenarius/Heckel, Schulrecht, Ziff. 34.21

${ }^{1761}$ Avenarius/Heckel, Schulrecht, Ziff. 34.211, 26.333
} 
schulische und berufliche Laufbahn des Betroffenen auswirken. ${ }^{1762}$ Gegen diese Verwaltungsakte ist die Anfechtungsklage nach $\S 42 \mathrm{VwGO}$ statthaft. Weil die Prüfungs- und Versetzungsentscheidungen und die Abschlusszeugnisse auf einer Gesamtbewertung des Schüles auf der Grundlage aller Fächer beruhen, sind dann i.d.R. die Einzelnoten und die diesen zugrunde liegenden einzelnen Prüfungen inzident zu überprüfen. ${ }^{1763}$ Ebenfalls inzident ist im Rahmen der Anfechtungsklage zu prüfen, ob in Prüfungen behinderungsbedingte Nachteile durch Nachteilsausgleich oder mit angemessenen Vorkehrungen ausgeglichen wurden. Der Umfang des Nachteilsausgleichs ist umfassend überprüfbar. Ebenfalls umfassend überprüfbar ist, ob das Anforderungsprofil der Prüfung die Behinderung berücksichtigt oder den Prüfling benachteiligt. Eine solche umfassende Überprüfung und Beweislast bezüglich des Nachweises von Anforderungsprofilen existiert im deutschen Recht bereits im Bereich des Arbeits- und Beamtenrechts. ${ }^{1764}$

\section{Kapitel: Rügepflichten der Eltern/des Schülers bei Nicht- Beachtung der Behinderung}

Aus dem Grundsatz der Chancengleichheit im Prüfungsrecht wird abgeleitet, dass der Prüfling Mängel im Prüfungsverfahren unverzüglich rügen muss. ${ }^{1765}$ Das VG Gießen hat es als Pflicht eines Schülers mit Legasthenie angesehen, bei jeder Klausur zu rügen, wenn ihm eine zugestandene Schreibzeitverlängerung nicht angeboten wurde. ${ }^{1766}$ Das VG Augsburg hat es für zumutbar gehalten, dass ein Grundschüler, der die gymnasiale Schullaufbahn anstrebt, während der Prüfung um das Vorlesen der Aufgaben und um Zeitverlängerung bittet. Wenn er dies aus übermäßiger Scheu nicht tue, dann falle dies in seine Sphäre

1762 VGH München, Beschluss v. 28.9.2009, Az.: 7 ZB 08.2277, juris, Rn. 8, bejaht für die Deutschnote „ausreichend“ im Abschlusszeugnis der Realschule; BVerwG v. 25.4.1983, juris, VGH München, v. 8.9.1999, Az.: 7 B 98.3285, juris; OVG Münster, Beschluss v. 22.1.2001, Az.: 19 A 1901/00, juris, Rn. 20

1763 Z.B. VG Lüneburg, Urteil v. 28.5.2002, Az.: 4 A 246/01, www.rechtsprechung.niedersachsen.de; VG München. Beschluss v. 15.10.2002; Az.: M 3 E 02.4698, juris

1764 BAG NZA 2005, S. 870 ff; NZA 2007, S. 507 ff; NZA 2007, S. 1098 ff; Fuerst, Behinderung zwischen Diskriminierungsschutz und Rehabilitationsrecht, S. $123 \mathrm{ff}$, mit ausführlicher Kritik und 132. BVerfG 2 BvR 2571/07 vom 10.12.2008, http://www.bverfg.de/entscheidungen/ rk20081210_2bvr257107.html, BVerwG Urteil v. 21.6.2007, Az.: 2 A 6/ 06; http://www.bundesverwaltungsgericht.de/media/archive/5320.pdf Rn. 20; ebenso OVG Bautzen Beschluss v. 15.6.2009, 2 A 140/08, juris

1765 Avenarius/Heckel, Schulrecht, Ziff. 27.31

${ }^{1766}$ VG Gießen, Urteil v. 30.6.2009. Az.: 4 K 1052/09.GI, S. 17 (nicht veröffentlicht) 
und sei nicht der Schule anzulasten. ${ }^{1767}$ Das OVG Berlin hat es als eine Obliegenheit der Erziehungsberechtigten angesehen, einen Bildungsplan oder individuelle Fördermaßnahmen, die nach ihrer Auffassung mängelbehaftet oder unzureichend sind, unverzüglich gegenüber der Klassenkonferenz zu rügen. Rügen sie dies nicht, sind entsprechende Einwendungen gegen eine Nicht-Versetzungsentscheidung ausgeschlossen. ${ }^{1768}$

Deshalb ist zu klären, ob und wenn ja in welchem Umfang Schülerinnen und Schüler mit Behinderungen oder ihre Eltern zu Rügen verpflichtet sind, wenn ihre Behinderung im Schuljahr und/oder in Prüfungen nicht berücksichtigt wird.

Weil den Prüfern im Prüfungsrecht ein erheblicher Beurteilungsspielraum hinsichtlich der inhaltlichen Bewertung einer Prüfungsleistung zukommt, der der gerichtlichen Überprüfung entzogen ist ${ }^{1769}$, muss jedenfalls das Prüfungsverfahren rechtsstaatlich und korrekt verlaufen und damit etwaige Nachteile aus dem Bewertungsbereich kompensieren. ${ }^{1770}$ Damit soll gerade den Grundrechten Rechnung getragen und ihr Schutz gewährleistet werden. ${ }^{1771}$ Das formelle Verfahren wird ergänzt durch die Pflicht des Prüflings, auf Mängel im formellen Verfahren hinzuweisen, die sich auf die Rechtmäßigkeit der Prüfung auswirken können. Kommt der Prüfling der Rügepflicht nicht nach, verliert er die Möglichkeit, sich auf den Verfahrensfehler zu berufen.

Die normativen Grundlagen der Rügepflicht werden zum Teil als eine besondere Mitwirkungspflicht des Prüflings im Prüfungsrechtsverhältnis angesehen ${ }^{1772}$, die ihre Grundlage im Grundsatz von Treu und Glauben aus $\S 242$ BGB hat. ${ }^{1773}$ Bei einem Verstoß gegen die Rügepflicht werden die Verfahrensrechte verwirkt. ${ }^{1774}$ Zum Teil wird die Grundlage für den Verlust des Rügerechts in einem „widersprüchlichen Verhalten“ (venire contra factum proprium) gesehen, wenn der Prüfling nicht rechtzeitig gerügt hat. ${ }^{1775}$

In jedem Fall ist die Rügepflicht normativ begründet. Schulkinder sind jedoch zunächst nicht geschäftsfähig und ab sieben Jahren nur eingeschränkt geschäftsfähig, gerade weil ihnen die nötige Einsichtsfähigkeit in viele Vorgänge noch fehlt. ${ }^{1776}$ Minderjährige Schüler können deshalb gegenüber der Schule

\footnotetext{
1767 VG Augsburg, Beschluss v. 23.8.2007, Az.: AU 3 E 07.00798, juris, Rn. 34

1768 OVG Berlin, Beschluss v. 16.11.2004, 8 S 130.04, juris, Rn. 21

1769 S. dazu VGH München, Beschluss v. 28.9.2009, Az.: 7 ZB 08.2277, juris, Rn. 10

1770 Avenarius/Heckel, Schulrecht, Ziff. 27.31 Birnbaum, NVwZ 2006, S. 286 ff, S. 286

1771 Birnbaum, NVwZ 2006, S. 286 ff, S. 286

1772 Niehues, Prüfungsrecht, Rn. $216 \mathrm{f}$

1773 BVerwGE 31, 190, 191; 85, 323; Niehues, Prüfungsrecht, Rn. 216

1774 BVerwG DVBL 1970, S. 928; OVG Greifswald DVBI 1998, S. 972; VGH München DÖV, 1986,

S. 748; Niehues, Prüfungsrecht, Rn. 214

1775 Birnbaum, NVwZ 2006, S. 286 ff, S. 287 m.w.N.

1776 Gitter, in: MüKomm BGB, Vor $\S 104$, Rn. $1 \mathrm{ff}$
} 
keine rechtswirksamen Handlungen vornehmen. ${ }^{1777}$ Wie auch sonst gilt: „Kinder sind keine kleinen Erwachsenen! “1778 Sie können umfassende und sehr abstrakte Pflichten wie die aus Treu und Glauben nicht überblicken und verstehen. Deshalb können sie auch keine Rechte durch „widersprüchliches Verhalten" verlieren. Es ist eine Überforderung von (behinderten) Kindern, insbesondere von Grundschulkindern, von ihnen die Rüge von Verfahrensfehlern zu verlangen. ${ }^{1779}$ Schulkinder können deshalb keine Rügepflicht haben.

Eine Rügepflicht könnte jedoch den Eltern obliegen. ${ }^{1780}$ Voraussetzung für eine solche Rügepflicht, die dazu führt, dass bei Verletzung der Pflicht ein Verfahrensrecht verwirkt wird, ist, dass dieses Verfahrensrecht überhaupt disponibel ist. Sind Verfahrensrechte nicht disponibel, so können der Prüfling und/ oder seine Eltern sie auch dann nicht verlieren, wenn sie ihre Verletzung nicht rügen. ${ }^{1781} \mathrm{Im}$ Prüfungsrecht verlieren aber der Prüfling bzw. seine Eltern das Recht, sich darauf zu berufen, dass die Prüfung mangelhaft war und deshalb entweder das Ergebnis neu zu bewerten oder die Prüfung zu wiederholen ist, wenn nicht sofort gerügt wird. Bei Schülern mit Behinderungen hat die Präklusion nicht nur zur Folge, dass sich der Betroffene nicht mehr auf die Mangelhaftigkeit der Prüfung berufen kann, sondern zudem, dass er das Recht verwirkt, sich darauf zu berufen, dass er wegen seiner Behinderung benachteiligt wurde. Eine solche Verwirkung ist ähnlich zu beurteilen, wie der Verzicht auf das Grundrecht aus Art. 3 III 2 GG. Der Verzicht auf das Grundrecht aus Art. 3 III 2 GG ist nur sehr eingeschränkt und dann auch nur durch ausdrückliche Erklärung möglich ${ }^{1782}$ Das Gleiche muss auch für die Verwirkung gelten, sonst würden Verfahrenshandlungen, die sich auf die Ausübung von Grundrechten auswirken, weniger strengen Voraussetzungen unterfallen als materielle Rechtspositionen. Deshalb ist eine Verwirkung des Verfahrensrechts nur unter den gleichen Voraussetzungen möglich wie der Verzicht, nämlich durch ausdrückliche Erklärung. Das Verfahrensrecht ist daher nur sehr eingeschränkt disponibel. Allein dadurch, dass die mangelhafte Prüfung nicht gerügt wird, kann das Recht, sich auf die Behinderung zu berufen, nicht verwirkt werden. Das Verfahrensrecht ist insoweit nicht disponibel. Die Verletzung der Rügepflicht durch den Prüfling bzw. seine Eltern führt daher nicht dazu, dass diese mit Einwen-

\footnotetext{
1777 Avenarius/Heckel, Schulrecht, Ziff. 24.22

1778 BVerfG, Urteil v. 9.2.2010, Az.: 1 BvL 1/09 - Hartz IV, http://www.bverfg.de/entscheidungen/Is20100209_1bvl000109.html, Rn. 191

1779 so aber VG Augsburg, Beschluss v. 23.8.2007, Az.: Au 3 E 07.00798, juris, Rn. 34, kritisch dazu Marwege, RdJB 2009, S. 229 ff, S. 236

1780 OVG Berlin, Beschluss v. 16.11.2004, 8 S 130.04, juris, Rn. 21

1781 Birnbaum, NVwZ 2006, S. 286 ff, S. 288

1782 S. oben 9. Teil: 3. Kapitel: Verzicht der Betroffenen auf Berücksichtigung von Legasthenie und Dyskalkulie, S. 295
} 
dungen ausgeschlossen werden, die sich auf die Benachteiligung wegen der Behinderung beziehen.

Etwas anderes könnte sich aus der Zwecksetzung der Rügepflicht ergeben. Die Rügepflicht hat verschiedene Aufgaben: Sie soll erstens verhindern, dass sich der Prüfling mit der Geltendmachung des Mangels bis zur Bekanntgabe des Ergebnisses Zeit lässt, um sich im Falle des Nichtbestehens einen zusätzlichen Prüfungsversuch zu verschaffen, der ihm im Vergleich zu den Mitprüflingen nicht zusteht, zweitens die Prüfungsbehörde in die Lage versetzen, Störungen im Prüfungsverlauf abzustellen und ihr drittens ermöglichen, den Sachverhalt zeitnah aufzuklären. ${ }^{1783}$

Die erste Konstellation, dass der Schüler mit einer Rüge so lange wartet, bis er weiß, ob er die Prüfung bestanden hat, um sich dann einen (unzulässigen) weiteren Prüfungsversuch zu verschaffen, ist im Schulrecht nur bei entscheidenden Prüfungen wie der Abiturprüfung oder zentralen Prüfungen zum Abschluss der Realschule oder der Hauptschule denkbar. Es ist jedoch sehr unwahrscheinlich, dass sich ein Schüler hier durch eine späte Rüge einen weiteren Prüfungsversuch verschafft, weil ein solcher dann erst im nächsten Schuljahr im Rahmen der nächsten regulären Prüfung erfolgen kann und der Schüler dafür ein weiteres Jahr zu Schule gehen müsste. Im Schulalltag legen Schüler und Schülerinnen i.d.R. keinen Wert darauf, zusätzliche weitere Prüfungen zu schreiben und sich Prüfungsversuche zu verschaffen. Denn es werden ständig Prüfungen abgehalten und die einzelnen Prüfungen haben keinen solchen Stellenwert, dass die Wiederholung der Prüfung angestrebt oder eine weitere Prüfungschance "erschlichen "1784 wird.

Zweitens soll die Prüfungsbehörde durch die Rüge in die Lage versetzt werden, Störungen im Prüfungsverlauf abzustellen. ${ }^{1785}$ Klassisch gehören dazu Störungen durch Lärm oder durch unzureichende Materialen wie Bücher, Texte etc., also Fälle, in denen die Prüfungsbehörde aufgrund der Vorbereitungen davon ausgeht, dass die Prüfungsbedingungen korrekt sind und sich in der Prüfung herausstellt, dass sie fehlerhaft sind. ${ }^{1786}$ Sind Mängel des Prüfungsverfahrens dagegen offensichtlich, so sind sie auch ohne ausdrückliche Rüge abzustellen, ${ }^{1787}$ denn es ist nicht zulässig, dem Prüfling die Verantwortung für ein ordnungsgemäßes Prüfungsverfahren aufzuerlegen ${ }^{1788}$. Daher hat der Schüler bei einem Fehler, der in der Sphäre der Schule eintritt, keine Mitwirkungspflicht

\footnotetext{
1783 Birnbaum, NVwZ 2006, S. 286 ff, S. 287 f; Avenarius/Heckel, Schulrecht, Ziff. 27.31

${ }^{1784}$ Vgl. BVerwG, Urteil v. 22.6.1994, Az.: 6 C 37/92, NVwZ 1995, S. 492 ff

1785 Birnbaum, NVwZ 2006, S. 286 ff, S. 287 f; Avenarius/Heckel, Schulrecht, Ziff. 27.31

${ }^{1786}$ Avenarius/Heckel, Schulrecht, Ziff. $27.31 \mathrm{f}$

1787 BVerwG, Urteil v. 6.9.1995, Az.: 6 C 16/93, juris, Rn. 44 m.w.N.; VGH Mannheim, Urteil v. 14.9.2010, Az.: 11 S 1415/10, VBIBW 2011, S. $30 \mathrm{f}$

${ }^{1788}$ Niehues, Prüfungsrecht, Rn. 217, 220
} 
und folglich auch keine Rügepflicht. Eine solche Konstellation liegt bei der Nicht-Berücksichtigung einer Behinderung vor: Zu den Pflichten der Lehrkraft gehört die Berücksichtigung der Behinderung in der Prüfung. ${ }^{1789}$ Die Notwendigkeit, eine Behinderung in der Prüfung zu berücksichtigen, ist der Lehrkraft bekannt und sie muss dies und den Umfang des Nachteilsausgleich bereits in der Vorbereitung der Prüfung bedenken, etwa durch entsprechende Gestaltung der Prüfungsaufgabe oder dadurch, dass sichergestellt wird, dass ein Zeitzuschlag auch in der notwendigen Form gegeben und genommen werden kann. Die Nicht-Berücksichtigung ist ein offensichtlicher Mangel, der von Amts wegen zu berücksichtigen ist. ${ }^{1790}$ Insofern hat die Schule bzw. die Lehrkraft Kenntnis davon, dass ohne Berücksichtigung der Behinderung ein Verfahrensfehler eintritt. ${ }^{1791}$

Drittens soll die Rüge, die Prüfungsbehörde in die Lage versetzen, den Sachverhalt zeitnah aufzuklären. ${ }^{1792}$ In diesem Bereich führt die Rügepflicht zu einer Verschiebung der Beweislast vom Prüfling auf die Prüfungsbehörde. Hat der Prüfling die Prüfungsbedingungen gerügt, dann trifft die Behörde die Beweislast dafür, dass diese korrekt waren. ${ }^{1793}$ Versäumt der Prüfling die Rüge, dann wirkt sich dies nur zu seinen Lasten aus, wenn der Sachverhalt nicht vollständig aufgeklärt werden kann. ${ }^{1794} \mathrm{Es}$ wäre unverhältnismäßig, wenn eine solche reine Beweislastregelung zur Folge haben würde, dass sich der Betroffene nicht mehr auf die Behinderung berufen kann und damit in der Ausübung seines Grundrechts eingeschränkt wird.

Auch der Zweck der Rügepflicht rechtfertigt es daher nicht, bei Schülerinnen und Schülern mit Behinderung eine Rügepflicht für Prüfungen zu bejahen, in denen die Behinderung nicht berücksichtigt wurde, mit der Folge der Präklusion, wenn die Rüge nicht erfolgt.

Bejaht man jedoch, anders als hier vertreten, eine Rügepflicht dahin, dass Schüler und Schülerinnen mit Behinderungen die Nicht-Berücksichtigung der Behinderung in Prüfungen zu beanstanden haben, so wird diese begrenzt durch die Fürsorge- und Hinweispflicht der Prüfungsbehörde, hier der Schule. ${ }^{1795}$ Die Schule hat die Pflicht, für einen ordnungsgemäßen Ablauf der Prüfung zu sorgen und Rechtsmängel gar nicht erst entstehen zu lassen. ${ }^{1796}$ Hat die Schule Kenntnis von Mängeln im Verfahren, z.B. darüber, dass eine Lehrkraft

\footnotetext{
1789 Zimmerling/Brehm, Prüfungsrecht, Rn. 1334

$1790 \mathrm{Vgl}$. Niehues, Prüfungsrecht, Rn. 214

1791 Vgl. Birnbaum, NVwZ 2006, S. 286 ff, S. 288

1792 Birnbaum, NVwZ 2006, S. 286 ff, S. 287 f; Avenarius/Heckel, Schulrecht, Ziff. 27.31

${ }^{1793}$ S. auch VG Augsburg, Beschluss v. 23.8.2007, Az.: Au 3 E 07.00798, juris, Rn. 33

1794 Birnbaum, NVwZ 2006, S. 286 ff, S. 291

1795 Vgl. Birnbaum, NVwZ 2006, S. 286 ff, S. 288

${ }^{1796}$ Vgl. BVerwG Urteil v. 6.9.1995, Az.: 6 C 16/93, juris, Rn. 49
} 
den Nachteilsausgleich nicht oder nicht ordnungsgemäß gewährt, so muss sie von sich aus diesen Mangel beseitigen. ${ }^{1797}$ Die Eltern können dann nur die Pflicht haben, einmal darauf hinzuweisen, dass die Pflicht nicht eingehalten wird, denn es muss davon ausgegangen werden, dass Schulen nach einer solchen einmaligen Rüge oder Erinnerung von sich aus alles tun, um sich rechtmäBig zu verhalten. ${ }^{1798}$ Eltern können daher nicht die präkludierende Rügepflicht haben, die Lehrkraft, im schlechtesten Fall immer wieder, auf die Beachtung dieser Pflicht hinzuweisen. Etwas anderes ist eine vorsichtige Erinnerung an diese Pflicht, wie sie die meisten Mütter von Kindern mit Behinderung ohnehin regelmäßig, insbesondere zu Beginn eines jeden Schuljahres, vornehmen. ${ }^{1799}$

Eine Rügepflicht setzt weiter voraus, dass der zur Rüge Verpflichtete Kenntnis von dem Verfahrensfehler hat. Dafür müssen jedoch die Eltern des Schülers den Verfahrensfehler kennen, die Kenntnis des Schülers reicht nicht aus. Die Eltern können Verfahrensfehler nur dann erkennen, wenn sie detailliert über die anstehende Prüfung und über den vorgesehenen Nachteilsausgleich informiert worden sind. Eine solche Information erhalten Eltern jedoch überwiegend nicht. ${ }^{1800}$ Sie können sie auch gar nicht erlangen, wenn der Nachteilsausgleich, so wie z. T. für zulässig gehalten, erst in der Prüfung ${ }^{1801}$ festgelegt wird. Sie können den Verfahrensfehler weiter nur kennen, wenn ihr Kind ihnen davon berichtet, ob und wie in einer Prüfung auf die Behinderung Rücksicht genommen wurde. Die Verfahrensfehler sind innen daher in der Regel nicht oder erst später bekannt. Eine unverzügliche Rüge in der Prüfung und auch im direkten Anschluss an die Prüfung ist den Eltern daher nicht möglich.

Es muss dem Schüler bzw. seinen Eltern weiter im konkreten Fall zumutbar sein, während oder nach Ablauf der Prüfung den Mangel geltend zu machen. ${ }^{1802}$ Wird vom Schüler durch das Prüfungsrecht ein Verhalten in Prüfungen verlangt, dass ihm billigerweise nicht zugemutet werden kann, so verletzt dies den Grundsatz der Chancengleichheit. ${ }^{1803}$

Aus den dargestellten Grundsätzen ergibt sich zunächst, dass es jedenfalls einem Grundschulkind nicht zugemutet werden kann, insbesondere in einer Prüfung, die von einer fremden Lehrkraft mit fremden Mitschülern abgenom-

\footnotetext{
1797 Birnbaum, NVwZ 2006, S. 286 ff, S. $287 \mathrm{f}$

1798 Vgl. VG Hannover, Beschluss v. 13.12.2010, Az.: 6 B 5596/10, www.rechtsprechung.niedersachsen.de, letzter Satz

1799 Vgl. dazu z.B. Erler, Legasthenie Albert-Einstein-Schule-Laatzen, S. 8

1800 S. hierzu VG Lüneburg, Urteil v. 28.5.2002, Az.: 4 A 246/01, www.rechtsprechung.niedersachsen.de

1801 So VG Augsburg, Beschluss v. 23.8.2007, Az.: Au 3 E 07.00798, juris, Rn. 32, 34

1802 BVerwG, Beschluss v. 15.1.1993, Az.: 6 B 45/92, juris, Rn. 9; vgl. Birnbaum, NVwZ 2006, S. $286 \mathrm{ff}$, S. 288

1803 BVerwG, Beschluss v. 15.1.1993, Az.: 6 B 45/92, juris, Rn. 9
} 
men wird, auf Mängel des Prüfungsverfahrens hinzuweisen. Grundschulkinder erkennen nicht, dass es überhaupt Mängel im Prüfungsverfahren geben kann und sind daher ohnehin nicht in der Lage, klassische Mängel im Prüfungsverfahren, wie etwa Lärm oder Hitze o.ä. zu rügen. Das Einfordern von Vorlesen oder eines Zeitzuschlags erfordert von dem Kind nicht nur, dass es erkennt, dass die Prüfung mangelhaft ist und es sich dagegen wehren kann und muss, sondern auch, dass es darüber hinaus den Mut aufbringt, offen und vor allen Mitschülern auf seine Probleme hinzuweisen. Das kann ihm nicht zugemutet werden. ${ }^{1804}$

Maßgeblich für die Zumutbarkeit ist weiter der Kontext der schulischen Prüfungen und die Besonderheiten des Schulsystems, die deutlich andere sind als in akademischen Prüfungen. Die Rügepflicht ist im Prüfungsrecht vor allem zu juristischen und medizinischen Prüfungen entwickelt worden. ${ }^{1805}$ Bei diesen Prüfungen handelt es sich um Prüfungen, die nur an bestimmten Prüfungsterminen durch neutrale Prüfungskommissionen abgenommen werden und an denen die Prüflinge überwiegend anonym teilnehmen. Die Bewertung der Prüfung erfolgt wiederum in einem gesonderten Verfahren durch Prüfer, denen der Verlauf der schriftlichen Prüfung in der Regel nicht bekannt ist. Die Prüflinge, in der Regel Erwachsene ohne Behinderung, sind daher nur zu einer einmaligen Rüge verpflichtet, die sie an eine ihnen in der Regel unbekannte Aufsichtsperson richten und die keine Auswirkung auf die Bewertung der Arbeit haben wird. Mit diesen Prüflingen sind Schüler und Schülerinnen, noch dazu solche mit Behinderungen, nicht vergleichbar. Deshalb können die für berufsbezogene Prüfungen entwickelten Grundsätze nicht ungeprüft und pauschal auf die Schule übertragen werden.

Schüler und Schülerinnen befinden sich in einer ständigen Prüfungssituation. Ihre Eltern müssen die Rüge gegenüber der Lehrkraft vorbringen, die die Leistungen des Kindes bewertet und die das Kind im Verlauf des weiteren Schuljahres weiter unterrichten und bewerten wird. Sowohl die Kinder als auch die Eltern befinden sich gegenüber der Lehrkraft und der Schule in einem Abhängigkeitsverhältnis und nicht in einem Gleichordnungsverhältnis. Eine Rüge, d.h. der explizite Hinweis auf einen Fehler der Lehrkraft, der aus Beweisgründen möglichst auch noch schriftlich erfolgen muss, enthält eine deutliche Kritik an der Lehrkraft und birgt die Gefahr, dass das Verhältnis zwischen Lehrkraft und Schüler erheblich belastet wird. Noch schwieriger wird es, wenn die Lehrkraft die Berücksichtigung der Behinderung vergisst oder ausdrücklich nicht vornehmen will ${ }^{1806}$ und die Eltern wegen der Rügepflicht dazu gezwungen sind,

\footnotetext{
1804 Marwege, RdJB 2009, S. 229 ff, S. 237

1805 Birnbaum, NVwZ 2006, S. 286 ff, S. 289

${ }^{1806}$ So z.B. der Deutschlehrer im Verfahren vor dem VG München, Beschluss v. 15.10.2002, Az.: M 3 E 02.4698, juris, Rn. 40
} 
jedes Mal die Nicht-Berücksichtigung zu rügen. Es ist offensichtlich, dass dadurch das Verhältnis zwischen Lehrer und Schüler massiv beeinträchtigt wird. Die Auswirkungen einer solchen intensiven Rügepflicht lassen sich am Beispiel der Entscheidung des VG Lüneburg ${ }^{1807}$ aufzeigen: Die Lehrkräfte hatten den von der Klassenkonferenz beschlossenen Nachteilsausgleich zum Teil gar nicht, zum Teil in veränderter Form, zum Teil nur nach ständigen Ermahnungen gewährt. Um die Rechte des Kindes zu wahren, hätten die Eltern jeden Fall rügen müssen, in dem der Nachteilsausgleich nicht oder in veränderter Form gegeben wurde oder in dem die Prüfung wegen der ständigen Ermahnungen unter erschwerten Bedingungen erfolgte. ${ }^{1808}$ In Zeiten, in denen viele Klassenarbeiten geschrieben werden, läuft das darauf hinaus, dass Eltern mehrmals wöchentlich schriftlich den Verstoß gegen die Prüfungsbedingungen rügen müssen. Das Lehrer-Schüler-Verhältnis wird dadurch extrem belastet. Es setzt zudem Kinder, die ohnehin durch ihre Behinderung psychischen Belastungen ausgesetzt sind, weiteren psychischen Belastungen aus. Eine derartige Rügepflicht ist daher weder dem Schüler noch den Eltern zuzumuten.

Die Rügepflicht bringt die betroffenen Schüler außerdem in einen Loyalitätskonflikt zwischen ihren Lehrern und ihren Eltern. Weil Schüler und Schülerinnen die Fehler nicht selber rügen können, müssen dies ihre Eltern für sie tun. Diese können Fehler nur rügen, wenn sie durch die Erzählungen ihrer Kinder davon wissen. Die Eltern müssen also von ihren Kindern verlangen, dass sie ihnen bei jeder Prüfung erzählen, ob die Behinderung berücksichtigt worden ist, ob die Lehrkraft sich richtig verhalten hat. Lehrkräfte aber sind für Kinder Autoritätspersonen und sollen dies auch sein. Vor allem Grundschulkinder haben oft eine enge Bindung zu ihren Lehrer/innen und berichten deshalb nur sehr ungern von deren Fehlern. Sie erzählen immer weniger, wenn ihre Erzählungen dazu führen, dass die Eltern sich (wiederholt) mit Beschwerden an die Lehrkraft wenden. ${ }^{1809}$ Im Familienrecht ist eine solche Konstellation explizit in § 1684 Abs. 2 BGB dahin geregelt, dass Eltern die Pflicht haben, alles zu unterlassen, was das Verhältnis des Kindes zum jeweils anderen Elternteil beeinträchtigt oder die Erziehung erschwert und die Kinder in einen Loyalitätskonflikt bringt. Das Wohl der Kinder erfordert es, dass den Kindern ein solcher Loyalitätskonflikt auch in der Beziehung zwischen Elternhaus und Schule erspart wird. Eine faktisch notwendige Erzählpflicht der Kinder, damit die Eltern rügen können, ist für die Kinder unzumutbar.

\footnotetext{
1807 VG Lüneburg, Urteil v. 28.5.2002, Az.: 4 A 246/01, www.rechtsprechung.niedersachsen.de 1808 So auch VG Gießen, Urteil v. 30.6.2009. Az.: 4 K 1052/09.GI, S. 17 (nicht veröffentlicht)

${ }^{1809}$ Vor allem Grundschulkinder empfinden es als „petzen“, wenn sie berichten sollen, ob sich die Lehrkraft richtig verhalten hat oder nicht. Sie kommen in einen Konflikt, weil ihnen üblicherweise von Eltern und Lehrern das „Petzen“ verboten wird.
} 
Die Rügepflicht trifft Schüler und Schülerinnen mit Behinderung und ihre Eltern in besonderem Maße. Das Verfahrensrecht im Prüfungsbereich dient gerade dazu, den Grundrechten in Prüfungen Geltung zu verschaffen. Es muss bei seiner Ausgestaltung daher auch das spezielle Diskriminierungsverbot des Art. 3 III 2 GG beachtet werden. Die Rügepflicht kann jedoch dazu führen, dass das Grundrecht nicht mehr ausgeübt werden kann. Ein Verfahren, dass das behinderte Kind beständig dazu verpflichtet, die Diskriminierung zu rügen oder zumindest, durch Erzählen, die tatsächlichen Voraussetzungen für eine Rüge zu schaffen, wenn es seinen Anspruch auf Nicht-Diskriminierung nicht verlieren will, belastet es jedes Mal wieder damit, auf seine Behinderung aufmerksam machen und die Berücksichtigung einfordern zu müssen. Das ist selbst wieder diskriminierend. Eine solche Ausgestaltung der Rügepflicht verstößt gegen das Wohl des Kindes mit Behinderung (Art. 7 BRK).

Bedenken gegen eine derart ausgedehnte Rügepflicht bestehen auch deshalb, weil sie für Eltern, die die deutsche Sprache nicht beherrschen und auch das deutsche Schulwesen nicht detailliert kennen, oder auch für Eltern, die sich eher weniger um ihre Kinder kümmern (können), nicht erfüllbar ist. ${ }^{1810}$ Sie sind häufig schon nicht in der Lage, ihre Kinder überhaupt schulisch zu unterstützen. Umso weniger sind sie in der Lage, Mängel überhaupt und noch weniger gegenüber der richtigen Stelle zu rügen. ${ }^{1811}$ Ihren Kindern werden damit erhebliche Rechtsschutzmöglichkeiten genommen.

Schließlich ergeben sich bei der Rüge durch die Eltern auch erhebliche Beweisprobleme, weil den Eltern die Fehler in der Prüfung nur aus Erzählungen des Kindes bekannt sein können. Die auf den Aussagen des Kindes basierenden Aussagen der Eltern sind nach der Rechtsprechung mit Unsicherheiten behaftet, so dass innen eine eingeschränktere Aussagekraft zugesprochen wird als der der Lehrkräfte. ${ }^{1812}$ Der dienstlichen Erklärung der Lehrkraft wird demgegenüber ein erhöhter Beweiswert zugesprochen, weil die Lehrkraft dienstrechtlich zur Wahrheit verpflichtet sei. ${ }^{1813}$ Im Ergebnis überwiegt damit vor allem bei Grundschulkindern grundsätzlich die Aussage der Lehrkraft.

${ }^{1810}$ Vgl. zur Frage, inwieweit Eltern aus benachteiligten und wenig gebildeten Gesellschaftsgruppen in der Lage sind, die rechtlichen Auswirkungen einer Einwilligung zu einer Überweisung auf die Sonderschule zu überblicken und zur rechtlichen Bedeutung ihrer Einwilligung s. EGMR Urteil v. 13.11.2007 - 57325/00 (D.H. u.a./ Tschechien), NVwZ 2008, S. 533 ff, Ziff. 203

1811 S. dazu der Beschluss des OVG Berlin, Beschluss v. 16.11.2004, 8 S 130.04, juris, Rn. 21, in dem das OVG eine unverzügliche Rüge gegenüber der Klassenkonferenz für erforderlich hält.

1812 Vgl. dazu VG Augsburg, Beschluss v. 23.8.2007, Az.: Au 3 E 07.00798, juris, Rn. 32; VGH München, Beschluss v. 30.1.2012, Az.: 7 C 11.2261, juris, Rn. 14

1813 VGH München, Beschluss v. 30.1.2012, Az.: 7 C 11.2261, juris, Rn. 14. Die Eltern der Schüler konnten weder mit dem Hinweis, dass die Lehrkraft die schulrechtliche Vorschrift nicht gekannt hatte und daher auch keinen Nachteilsausgleich geben konnte, durchdringen noch mit 
Fraglich ist auch, ob die Rügepflicht für die Abiturprüfungen aufrechterhalten werden kann. Abiturienten werden in der Abiturprüfung zahlreiche Obliegenheitspflichten auferlegt, die den Rügepflichten in berufseröffnenden Prüfungen entsprechen. ${ }^{1814}$ Die Rügepflicht wird hier damit gerechtfertigt, dass die Abiturprüfung eine einmalige Prüfung ist, die gesondert wiederholt werden kann. Außerdem wird vertreten, dass die Abiturienten in aller Regel volljährig und erfahren sind, so dass sie die Konsequenzen einer Rügepflicht erkennen können. ${ }^{1815}$ Eine solche Rügepflicht mag es für das allgemeine Prüfungsrecht geben, für die Nicht-Berücksichtigung der Behinderung ist sie jedoch ausgeschlossen, weil sie auch hier zu der unzulässigen Konsequenz führt, dass die Ausübung des Grundrechts aus Art. 3 III 2 GG beschränkt wird.

Das Gleiche gilt, soweit eine Rügepflicht für Abiturienten des jetzt beginnenden Abiturs nach 12 Schuljahren oder für die zentralen Abschlussprüfungen zum Realschul- oder Hauptschulabschluss in Betracht gezogen wird. Hier gilt angesichts des Alters der Schüler und Schülerinnen von allenfalls 18 Jahren (im Abitur) oder von erst 15 und 16 Jahren (Haupt- oder Realschule) erstrecht, dass ein Verzicht auf das Grundrecht aus Art. 3 III 2 GG nicht möglich ist und die Ausübung daher auch nicht durch nicht erfüllte Rügepflichten versperrt werden kann. ${ }^{1816}$

den Schilderungen der Mitschüler, die betroffenen Schüler hätten keinen Zeitzuschlag bekommen.

1814 Zimmerling/Brehm, Prüfungsrecht, Rn. 1315; VG Dresden, Beschluss v. 22.7.1998, Az.: 5 K 1705/98, juris, Rn. $15 \mathrm{f}$

${ }^{1815}$ Zimmerling/Brehm, Prüfungsrecht, Rn. 1328

1816 Abgesehen davon ist es fraglich, ob Schüler und Schülerinnen im Alter von 15 oder 16 Jahren überhaupt zur Rüge verpflichtet werden können. Sie sind nur in dem Maße grundrechtsmündig, wie sie im Hinblick auf das spezielle Prüfungsrecht und vor allem auf seine erheblichen Auswirkungen auf ihren späteren Lebens- und Berufsweg einsichtsfähig sind (Huber, in: Hdb. Grundrechte, Rn. 18). Es ist nicht nur zweifelhaft, ob sie derartige Verfahrensfragen überblicken können (vgl. Zimmerling/Brehm, Prüfungsrecht, Rn. 1328) oder sich selbst darüber informieren können (zur Informationspflicht des Prüflings in akademischen Prüfungen BVerwG, Urteil v. 22.6.1994, Az.: 6 C 37/92, NVwZ 1995, S. 492 ff, 493), sondern auch, ob sie in der Lage sind, ihre Interessen selbständig zu vertreten, indem sie rügen und sich damit in einen Konflikt mit ihrer Lehrkraft begeben. Für die Schulbehörde ergeben sich aus dem Prüfungsrecht umfassende Beratungs- und Hinweispflichten (Birnbaum, NVwZ 2006, S. 286 ff, S. 286). Dementsprechend könnten Obliegenheitspflichten für Schüler und Schülerinnen ohnehin nur bestehen, wenn die Schule sie vor den Prüfungen umfassend aufgeklärt und auf die Folgen der Nichtbeachtung hingewiesen hat (VG Dresden, Beschluss v. 22.7.1998, Az.: 5 K 1705/98, juris, Rn. 16) 


\section{Kapitel: Rechtsfolgen rechtswidriger Prüfungen}

Fehler in Prüfungen ergeben sich bei Legasthenie/Dyskalkulie vor allem daraus, dass die äußeren Prüfungsanforderungen nicht an die Behinderung angepasst werden, etwa weil kein Zeitzuschlag gegeben wird, die zu erlesenden Texte so gedruckt sind, dass die Betroffenen sie kaum erlesen können (z.B. handschriftliche Aufgabenblätter, schlechte Kopien von Reclam-Texten oder vier ReclamSeiten auf einer Din A4 Seite), Hilfsmittel nicht zur Verfügung gestellt werden oder dass bei der Bewertung der Leistung die Behinderung nicht berücksichtigt wird, vor allem indem die Rechtschreibung bewertet oder in Mathematik keine angepasste Aufgabe gestellt wird. Grundsätzlich haben solche Verfahrensfehler nur dann Rechtsfolgen, wenn sie sich auf das Ergebnis der Prüfung ausgewirkt haben können. ${ }^{1817}$ Dann können sie die Rechtswidrigkeit der Note oder der darauf beruhenden Entscheidung, wie z.B. der Versetzungsentscheidung, nach sich ziehen. ${ }^{1818}$

Allerdings führen Fehler im Verfahren regelmäßig nur zu einem Anspruch auf Wiederholung der Prüfung, nicht aber auf die Feststellung des Bestehens der Prüfung oder auf die Festsetzung einer bestimmten Note. ${ }^{1819}$ Weil bei der Notengebung ein erheblicher pädagogischer Beurteilungsspielraum besteht, kann die Note in der Regel nicht im Nachhinein durch das Gericht festgesetzt werden. ${ }^{1820}$ Geht es bei der Bewertung oder Beurteilung auch um inhaltliche Fragen, so besteht nur ein Anspruch auf fehlerfreie Neubewertung der Prüfungsleistung. ${ }^{1821}$ Nach allgemeiner Meinung kommt es nicht in Betracht, fiktive Leistungen so zu berücksichtigen, dass die Note um einen bestimmten Wert heraufgesetzt wird. ${ }^{1822}$ Etwas anderes gilt, wenn es nicht um eine inhaltliche Bewertung der Leistung, sondern etwa um die Bewertung einer vertretbaren Lösung als unvertretbar ${ }^{1823}$ oder um die Neuberechnung der Prüfungsnote geht. In diesen Fällen kann die Berechnung der Prüfungsnote durch das Gericht selbst erfolgen und auf diesem Wege eine bestimmte Note festgesetzt

\footnotetext{
1817 Avenarius/Heckel, Schulrecht, Ziff. 27.51

1818 VG Lüneburg v. 28.5.2002, Az.: 4 A 246/01, www.rechtsprechung.niedersachsen.de; VG München, Beschluss v. 15.10.2002, Az.: M 3 E 02.4698, juris

1819 Niehues, Prüfungsrecht, Rn. 498 ff; 758 ff; 888

1820 VGH München, Beschluss v. 28.9.2009, Az.: 7 ZB 08.2277, juris, Rn. 10; VG Dresden, Beschluss v. 22.7.1998, Az.: 5 K 1705/98, juris, Rn. 18

1821 Niehues, Prüfungsrecht, Rn. 808

1822 Niehues, Prüfungsrecht, Rn. 759; VG Gießen, Urteil v. 30.6.2009. Az.: 4 K 1052/09.GI, S. 10, 17 (nicht veröffentlicht); VG München, Beschluss v. 8.9.2009, Az.: M 3 E 09.4056, juris, Rn. 23 ff; VG Düsseldorf, Beschluss v. 16.11.2009, Az.: 18 L 1654/09, juris, Rn. 15

1823 VG Dresden, Beschluss v. 22.7.1998, Az.: 5 K 1705/98, juris, Rn. 25
} 
und/oder eine Nicht-Versetzungsentscheidung oder Nicht-Bestehensentscheidung korrigiert werden. ${ }^{1824}$

Für die Fehlerfolgen, die sich ergeben, wenn die Behinderungen Legasthenie/Dyskalkulie nicht berücksichtigt werden, ist deshalb zwischen den einzelnen Fehlern zu differenzieren:

Wird der Zeitzuschlag nicht gegeben oder werden Hilfsmittel nicht zur Verfügung gestellt, so kann dieser Fehler im gerichtlichen Verfahren nicht korrigiert werden. Die Prüfung muss wiederholt werden. ${ }^{1825}$

Werden trotz einer Legasthenie die Rechtschreibleistungen in Prüfungsaufgaben bewertet, so lässt sich dieser Fehler durch eine Neubewertung der Arbeiten beheben. Wenn in der Bewertung die Abwertung wegen Rechtschreibfehlern ausdrücklich ausgewiesen ist, kann diese Neubewertung auch durch das Gericht vorgenommen werden, weil es die Note dann schlicht selbst berechnen kann. ${ }^{1826}$

Werden die Aufgaben in Mathematik trotz einer Dyskalkulie nicht verändert und angepasst, ist eine Neubewertung nicht möglich, weil die Aufgabe nicht angemessen war. In Betracht kommt dann nur eine Wiederholung der Prüfung mit entsprechend angepassten Aufgaben.

Werden bei Legasthenie oder Dyskalkulie die Prüfungsbedingungen oder Prüfungsinhalte nicht angepasst, so kann dieser Fehler nach der Prüfung nicht mehr korrigiert werden. Die Prüfung ist rechtswidrig und muss wiederholt werden.

Daraus ergibt sich, dass nur in wenigen Fällen die Arbeiten neu bewertet oder die Note durch das Gericht festgesetzt werden kann. In diesen Fällen wird die Entscheidungen im Hauptsacheverfahren, aber auch im Verfahren auf vorbeugenden Rechtsschutz nach $\S 123$ VwGO darauf lauten, dass die Prüfungsleistung erneut zu bewerten ist.

In den Fällen, in denen die Prüfungsanforderungen oder -bedingungen nicht angepasst werden, werden die Entscheidungen in der Hauptsache oder im Verfahren nach § 123 VwGO darauf lauten, dass die Schüler und Schülerinnen alle Prüfungen, in denen die Behinderung nicht berücksichtigt wurde, wiederholen

\footnotetext{
${ }^{1824}$ Niehues, Prüfungsrecht, Rn. 807; VG Dresden, Beschluss v. 22.7.1998, Az.: 5 K 1705/98, juris, Rn. 27 ff; VG München. Beschluss v. 15.10.2002; Az.: M 3 E 02.4698, juris; VGH Mannheim, Urteil v. 14.9.2010, Az.: 11 S 1415/10, VBIBW 2011, $30 \mathrm{f}$

1825 Anderer Ansicht VG Lüneburg, Urteil v. 22.6.2011, Az.: 4 A 123/08, S. 6 (nicht veröffentlicht): „nachträgliche Abänderung der erteilten Zeugnisnote nach erfolgter Nachkorrektur der maßgeblichen schriftlichen Leistungsnachweise unter Berücksichtigung einer etwaigen zu kurzen Bearbeitungszeit."

1826 VG München, Beschluss v. 15.10.2002; Az.: M 3 E 02.4698, juris
} 
müssen. ${ }^{1827}$ Es sind aber, soweit die Prüfungen teilbar sind, nur die rechtswidrigen Teile und nicht die gesamte Prüfung zu wiederholen. ${ }^{1828}$

Fraglich ist, ob die Wiederholung aller rechtswidrigen Prüfungen mit dem Gebot des effektiven Rechtsschutzes aus Art. 19 IV GG vereinbar ist. Art. 19 IV GG verlangt nicht nur, dass jeder potentiell rechtsverletzende Akt der Exekutive in tatsächlicher und rechtlicher Hinsicht gerichtlich überprüft werden kann, sondern auch, dass die Gerichte den betroffenen Rechten tatsächlich Wirksamkeit verschaffen. ${ }^{1829}$ Das BVerwG hat dazu für das Prüfungsrecht den Grundsatz entwickelt, dass das Wiederholungsverfahren so zu gestalten ist, dass der Prüfling den geringstmöglichen Nachteil erleidet. ${ }^{1830}$

Soweit es sich um Einzelfälle handelt, bei denen die Berücksichtigung der Behinderung vergessen wurde, ist die Wiederholung von einzelnen Klausuren möglich und zumutbar.

Problematisch ist es jedoch, wenn die Behinderung in vielen Fällen nicht berücksichtigt wurde ${ }^{1831}$, etwa im Abitur oder in anderen zentralen Prüfungen. Dann müssen die betroffenen Schüler alle Prüfungen wiederholen. ${ }^{1832}$ Die betroffenen Schüler und Schülerinnen werden durch diese Ausgestaltung des Prüfungsrechts erheblich benachteiligt. Je nachhaltiger ihr Recht auf Berücksichtigung beeinträchtigt wurde, desto mehr Klausuren und Prüfungen müssen sie wiederholen. Die Wiederholung von Prüfungen bringt zusätzliche Belastungen mit sich, insbesondere wenn die zu wiederholende Prüfung länger zurück liegt. ${ }^{1833}$

Noch weniger effektiv ist ein Rechtsschutz, der zum Abschluss eines Schuljahres oder eines Ausbildungsabschnitts, etwa der Oberstufe, feststellt, dass die Rechte des Schülers durchgängig nicht beachtet wurden und daher eine Vielzahl von Prüfungen zu wiederholen sind. Handelt es sich um Prüfungen

${ }^{1827}$ Vgl. zu einer solchen Konstellation OVG Greifswald, Urteil v. 27.2.1997, Az.: 2 L 52/96, juris, Rn. 29. Das OVG Greifswald löste im konkreten Fall das Dilemma durch den Rückgriff auf den hypothetischen Willen des Normgebers, um dann die alte Prüfungsordnung zugrunde zu legen.

1828 BVerwG, Urteil v. 19.12.2001; Az.: 6 C 14/01, NVwZ 2002, S. 1375 ff

${ }^{1829}$ Vgl. BVerfG, Beschluss vom 30.05.2007 - 2 BvR 2012/05, http://www.bverfg.de/entscheidungen/rk20070530_2bvr201205.html, Rn. 1; Bader/FunkeKaiser/Kuntze/von Albedyll, VwGO, Vor $\S 80, R n .1$

1830 BVerwG, Urteil v. 19.12.2001; Az.: 6 C 14/01, NVwZ 2002, S. 1375; Niehues, Prüfungsrecht, Rn. 503

1831 S. dazu VG Lüneburg v. 28.5.2002, Az.: 4 A 246/01, www.rechtsprechung.niedersachsen.de, VG München, Beschluss v. 15.10.2002, Az.: M 3 E 02.4698, juris, Rn. 40; VG München, Beschluss v. 8.9.2009, Az.: 3 M E 09.4056, juris, Rn. 24; das Gericht merkt ausdrücklich an, dass es wünschenswert gewesen wäre, dass der Behinderung Rechnung getragen worden wäre.

1832 Vgl. OVG Bautzen Urteil v. 18.8.2010, Az.: 142/09, Sächs.VBI. 2011, S. 35 ff

1833 BVerwG, Urteil v. 19.12.2001; Az.: 6 C 14/01, NVwZ 2002, S. 1376 
eines Schuljahres, so kann die Wiederholung in der Form erfolgen, dass der betroffene Schüler die Klausuren in einem kurzen Zeitraum erneut gestellt bekommt, etwa in einer eigenen Prüfungsphase. ${ }^{1834}$ Hier ergeben sich erhebliche Nachteile daraus, dass die betroffenen Schüler und Schülerinnen im Gegensatz zu ihren Mitschülern prüfungsrelevante Kenntnisse über einen längeren Zeitraum auf dem aktuellen Stand halten müssen. ${ }^{1835}$ Zudem haben die Betroffenen erheblich schlechtere Bedingungen als im laufenden Schuljahr, denn üblicherweise werden Schüler durch den Unterricht auf die Klausur vorbereitet und können danach den Prüfungsstoff "abhaken" und sich auf die nächste Klausur vorbereiten. Ein Schüler, der in einem Block nachschreibt, muss demgegenüber alle Gebiete gleichzeitig präsent haben.

Die andere Möglichkeit der Wiederholung ist, dass der Schüler das betreffende Schuljahr wiederholt, um die Prüfungen unter rechtmäßigen Bedingungen abzulegen. Die Wiederholung eines Schuljahres, nur um rechtmäßige Prüfungsbedingungen zu haben, kommt für die allermeisten Schüler jedoch nicht in Betracht. Nach außen trägt eine solche Wiederholung den Makel der Nichtversetzung, der grundsätzlich von der Rechtsprechung als ein generelles Hemmnis für das berufliche Fortkommen aufgefasst wird ${ }^{1836}$ und den daher niemand freiwillig auf sich nehmen wird. Eine solche Ausbildungsverzögerung ist ein erheblicher, grundrechtsrelevanter Nachteil. ${ }^{1837}$

Besonders problematisch ist es, wenn die Berücksichtigung der Behinderung während der Oberstufe und im Abitur nicht richtig gewährt wurde. Weil alle Noten aus der Oberstufe in die Abiturwertung einfließen, kann das dazu führen, dass der Schüler die gesamte Oberstufe wiederholen muss, weil nur so gewährleistet werden kann, dass er ein Abitur unter korrekten Bedingungen macht. Kein Schüler, der das Abitur einmal bestanden hat, wiederholt die Oberstufe, also zwei Schuljahre, um das Abitur noch einmal unter rechtmäßigen Bedingungen zu absolvieren. Dies führte ebenfalls zu einer ganz erheblichen, grundrechtsrelevanten Ausbildungsverzögerung ${ }^{1838}$, denn Zeit hat eine ganz erhebliche Orientierungsfunktion für zukünftige Arbeitgeber. ${ }^{1839}$ Schüler und Schülerinnen werden sich daher regelmäßig gegen die Wiederholung der Prüfungen entscheiden.

\footnotetext{
1834 Z.B. in einem Block in den Ferien

${ }^{1835}$ BVerfG, Beschluss v. 25.7.1996; Az.: 1 BvR 638/96, NVwZ 1997, S. $479 \mathrm{ff}$

${ }^{1836}$ Vgl. OVG Bautzen Urteil v. 18.8.2010, Az.: 142/09, Sächs.VBI. 2011, S. 35 ff, zum Fortbestehen des Rechtsschutzinteresses um den „Makel des Durchgefallenseins" zu beseitigen, s. Niehues, Prüfungsrecht, Rn. 848

1837 BVerfG, Beschluss v. 25.7.1996; Az.: 1 BvR 638/96, NVwZ 1997, S. 481; s. auch OVG Bautzen, Beschluss v. 10.10.2002; Az.: 4 BS 328/02, www.justiz.sachsen.de/ovg/content/803.htm

1838 BVerfG, Beschluss v. 25.7.1996; Az.: 1 BvR 638/96, NVwZ 1997, S. 481

1839 Zimmerling, NVwZ 2004, S. 651 ff, S. 652
} 
Diese Nachteile wiegen umso schwerer, als Schüler und Schülerinnen mit Behinderung dadurch noch einmal benachteiligt werden. Zunächst haben sie den Nachteil zu tragen, dass ihre Behinderung in der Prüfung nicht berücksichtigt wurde und dann den Nachteil, dass sie, um unter rechtmäßigen Bedingungen geprüft zu werden, deswegen Zeit verlieren und evtl. Schuljahre wiederholen müssen.

Will der Schüler diesen Aufwand nicht in Kauf nehmen, so bleibt ihm nach Ansicht des VGH Kassel ${ }^{1840}$ nur ein Amtshaftungsprozess, denn: „Sollten die Beeinträchtigungen des Klägers aufgrund der ... gewährten Hilfen des Nachteilsausgleichs nicht vollständig ausgeglichen gewesen sein, obwohl eine Kompensation durch weitere Hilfen wie etwa die Verwendung eines PCs mit einem Rechtschreibprogramm möglich gewesen wäre, könnte der Kläger allenfalls Schadensersatzansprüche ... geltend machen."

Dieses Ergebnis ist unbefriedigend und verweist die betroffenen Schüler und Schülerinnen auf einen langwierigen Schadensersatzprozess, obwohl der Nachteil auch nach Ansicht des Gerichts nicht ausgeglichen worden ist und die Betroffenen unverzüglich darauf angewiesen sind, dass ihre Rechte gewahrt werden. Die Abschlusszeugnisse gewähren Lebens- und Berufschancen, die die Schüler und Schülerinnen nur direkt nach dem Abschluss in Anspruch nehmen können, wie z.B. die Aufnahme eines Studiums. Es nützt ihnen nichts, wenn sie Jahre später eventuell Schadensersatz bekommen.

Zur Abwendung dieser Nachteile und zur Wahrung der Rechte der betroffenen Schüler und Schülerinnen ist es daher erforderlich bereits im vorbeugenden Rechtsschutz nach $\S 123$ VwGO der besonderen Bedeutung der betroffenen Grundrechte Rechnung zu tragen. ${ }^{1841}$ Grundsätzlich hängt die Gewährung vorläufigen Rechtsschutzes davon ab, wie nach summarischer Prüfung die Erfolgsaussichten im Hauptsacheverfahren sind und ob durch die Entscheidung die Hauptsache unzulässig vorweggenommen wird. ${ }^{1842}$ Insbesondere die Vorwegnahme der Hauptsache ist im Schulrecht ein Problem, weil die Entscheidung im vorbeugenden Rechtsschutz im Prüfungsverfahren weitgehend ein solcher Vorgriff auf die Hauptsache ist, wenn sie effektiv sein soll. ${ }^{1843}$ Allerdings ist es zulässig, die Hauptsache vorwegzunehmen und den Rechtsschutz sogar ohne Berücksichtigung der Entscheidung in der Hauptsache allein aufgrund einer Folgenabwägung zu gewähren, wenn die Versagung des Rechtsschutzes zu schweren und unzumutbaren Nachteilen führt und daher nur mit der Gewäh-

\footnotetext{
1840 VGH Kassel, Beschluss v. 5.2.2010, Az.: 7 A 2406/09, juris, S. 15

1841 Vgl. BVerfG, Beschluss v. 25.7.1996; Az.: 1 BvR 638/96, NVwZ 1997, S. 480, VG Oldenburg, Beschluss v. 17.2.2003, Az.: 5 B 4847/02, www.rechtsprechung.niedersachsen.de; S. 2 und 4

1842 Niehues, Prüfungsrecht, Rn. 909 m.w.N.

1843 Niehues, Prüfungsrecht, Rn. 909
} 
rung ein effektiver Rechtsschutz gewährleistet ist. ${ }^{1844}$ Je schwerer daher die Belastungen sind, die sich aus der Versagung des vorläufigen Rechtsschutzes ergeben, je geringer die Wahrscheinlichkeit ist, dass diese Belastungen beim Obsiegen in der Hauptsache rückgängig gemacht werden können, desto höher ist das Interesse des Betroffenen an vorläufigem Rechtsschutz. ${ }^{1845}$ Wie bereits dargelegt, sind die Nachteile für die betroffenen Schüler und Schülerinnen massiv, wenn sie mehrere Prüfungen wiederholen müssen. Diese Nachteile sind in einem Hauptsacheverfahren auch nicht rückgängig zu machen.

Zeichnet sich ab, dass die Schule die Behinderung nicht berücksichtigt und der Nachteilsausgleich nicht oder in unzureichender Form gewährt wird, so kann der Anspruch auf Berücksichtigung der Behinderung im Verfahren nach $\S 123$ VwGO durchgesetzt werden. Ein Anordnungsanspruch besteht, weil der Anspruch auf Berücksichtigung der Behinderung vorab im Anerkennungsverfahren festgestellt wurde und daher umzusetzen ist. Auch der Anordnungsgrund ist zu bejahen, da dem betroffenen Schüler die oben dargestellten Nachteile für den Fall, dass die Prüfungen nicht ordnungsgemäß durchgeführt werden, nicht zugemutet werden können. Der Anspruch muss glaubhaft gemacht werden. An die Glaubhaftmachung dürfen keine zu hohen Ansprüche gestellt werden. Die Tatsachen werden i.d.R. mithilfe von eidesstattlichen Versicherungen glaubhaft gemacht. ${ }^{1846}$ Da Kinder bis zu ihrem 16. Lebensjahr keine Versicherung an Eides statt abgeben können (§ 393 ZPO), muss ihren Erklärungen auch ohne Beeidigung der gleiche Erklärungswert beigemessen werden wie eidesstattlichen Erklärungen von Erwachsenen, um die Waffengleichheit im Prozess zu wahren. Ein Vorrang der Aussage der Lehrkraft gegenüber der Darstellung der Eltern, die die Schilderung des Kindes an Eides statt darlegen, ist nicht anzunehmen, weil dies die Waffengleichheit verletzen würde. ${ }^{1847}$

\footnotetext{
${ }^{1844}$ BVerfG, Beschluss v. 25.7.1996; Az.: 1 BvR 638/96, NVwZ 1997, S. 480, VG Oldenburg, Beschluss v. 17.2.2003, Az.: 5 B 4847/02, www.rechtsprechung.niedersachsen.de; S. 2 und 4; OVG Bautzen, Beschluss v. 10.10.2002; Az.: 4 BS 328/02, http://www.justiz.sachsen.de/ovg/ content/803.htm, S. 8; Niehues, Prüfungsrecht, Rn. 877 ff, Rn. 909 ff m.w.N.

1845 BVerfG, Beschluss v. 25.7.1996; Az.: 1 BvR 638/96, NVwZ 1997, S. 480

${ }^{1846}$ Bader/Funke-Kaiser/Kuntze/von Albedyll, VwGO, § 123, Rn. 30

1847 So aber VG Augsburg, Beschluss v. 23.8.2007, Az.: AU 3 E 07.00798, juris, Rn. 32
} 


\section{Teil: Notwendigkeit gesetzlicher Regelungen}

Die landesrechtlichen Regelungen zur Berücksichtigung von Legasthenie/Dyskalkulie weisen zurzeit einen sehr unterschiedlichen Rechtsnormcharakter auf. ${ }^{1848}$ In Berlin finden sich die Regelungen in den einzelnen Verordnungen zu den Schulformen. Hessen hat eine eigene Verordnung. Verwaltungsvorschriften bzw. Richtlinien haben Baden-Württemberg, Brandenburg, Hamburg, Mecklenburg-Vorpommern, Rheinland-Pfalz, Saarland und Sachsen. Erlasse haben Bayern, Bremen, Niedersachsen, Nordrhein-Westfalen und SchleswigHolstein. In Sachsen-Anhalt sind die Regelungen in verschiedenen Verordnungen und Erlassen verstreut, Thüringen hat in $\S 59$ TH SchulO den allgemeinen Anspruch auf Nachteilsausgleich geregelt, der durch eine "Fachliche Empfehlung" ergänzt wird. Langenfeld hat bezweifelt, ob diese Praxis der untergesetzlichen Normen mit dem Vorbehalt des Gesetzes vereinbar ist. ${ }^{1849}$ Rux hält es für geboten, die Regelungen zum Nachteilsausgleich zu vereinheitlichen und insbesondere die Verfahrensregelungen klarzustellen. ${ }^{1850}$

\section{Kapitel: Vorbehalt des Gesetzes}

Der Grundsatz des Vorbehalts des Gesetzes verlangt, dass staatliches Handeln in grundlegenden Bereichen durch förmliches Gesetz legitimiert wird. ${ }^{1851}$ Wesentliche Entscheidungen der Verwaltung bedürfen einer gesetzlichen Grundlage, müssen also vom Gesetzgeber selbst getroffen werden. ${ }^{1852}$ Wesentliche Entscheidungen liegen insbesondere dann vor, wenn unterschiedliche, miteinander konkurrierende grundgesetzliche Gewährleistungen gegeneinander ab-

\footnotetext{
1848 S. Anhang 3 - Schulrechtliche Vorschriften der Bundesländer

1849 Langenfeld, RdJB 2007, S. 211 ff, S. 217; Rux, RdJB 2009, S. 220 ff, S. 227

1850 Rux, RdJB 2009, S. 220 ff, S. 227

1851 S. dazu auch Wimmer, RdJB 1997, S. 15 ff und Reuter, RdJB 2003, S. 23 ff

1852 BVerfGE 98, 218, 245; 101, 1, 34 m.w.N.; ausführlich für das Schulrecht Niehues/Rux, Schulrecht, Rn. $26 \mathrm{ff}$
} 
zugrenzen und untereinander auszugleichen sind. ${ }^{1853}$ Deshalb kommt es in erster Linie darauf an, inwieweit die Einzelnen durch die Regelungen in ihren Grundrechten betroffen sind. ${ }^{1854} \mathrm{Im}$ Bereich des Gleichberechtigungsgebots und der besonderen Gleichheitssätze ist anerkannt, dass Ungleichbehandlungen dem Vorbehalt des Gesetzes unterfallen. ${ }^{1855}$

Der Vorbehalt des Gesetzes erstreckt sich auch auf das Schulrecht. ${ }^{1856}$ Das Demokratieprinzip gebietet, dass die Ordnung eines so wichtigen Lebensbereiches wie des Schulwesens zumindest in Grundzügen vom demokratisch unmittelbar legitimierten Gesetzgeber selbst verantwortet wird. ${ }^{1857}$ Hier können sich die Schüler dem Grundrechtseingriff nur beschränkt entziehen und das Konfliktpotential ist wegen der Vielzahl der Beteiligten besonders groß. ${ }^{1858}$ Deshalb ist der Gesetzgeber verpflichtet, die wesentlichen Entscheidungen im Schulwesen selbst zu treffen, und darf sie nicht der Schulverwaltung überlassen. ${ }^{1859} \mathrm{Zu}$ den wesentlichen Entscheidungen gehören jedenfalls die Regelungen über die Ausgestaltung des Prüfungsverfahrens ${ }^{1860}$, die Festschreibung einer bestimmten Fremdsprache als erste Fremdsprache in einer bestimmten Region ${ }^{1861}$, die Versetzung ${ }^{1862}$, die Voraussetzungen für leistungs- oder verhaltensbedingte Schulentlassungen, die Einführung von Sexualkundeunterricht und die Festlegung der Bildungs- und Erziehungsziele. ${ }^{1863}$ Bei Prüfungen bedürfen der Gegenstand der Prüfung, die Leistungsanforderungen in der Prüfung, die Maßstäbe, nach denen die erbrachten Leistungen zu bewerten sind und die Voraussetzungen für Sperrwirkungen nicht ausreichender Leistungen gesetzliche Grundlagen. ${ }^{1864}$

\footnotetext{
1853 BVerfGE 98, 218, 251 f; Badura, in: Maunz/Dürig/Herzog GG, Art. 7, Rn. 9; Niehues/Rux, Schulrecht, Rn. 31

${ }^{1854}$ Niehues/Rux, Schulrecht, Rn. 38; Avenarius/Heckel, Schulrecht, Ziff. 15.331

1855 Neumann, in: Handbuch SGB IX, § 2 Verfassungsrecht, Rn. 26 Osterloh, in: Sachs GG, Art. 3, Rn. 290

1856 VGH Mannheim, Beschluss v. 23.7.2007, Az.: 9 S 1298/07, www.landesrecht-bw.de, Rn. 9

1857 St. Rspr. des BVerfGE 41, 251, $259 \mathrm{f}$

${ }^{1858}$ Niehues/Rux, Schulrecht, Rn. 31

1859 St. Rspr. des BVerfG BVerfGE 58, 257, 268 und des BVerwG BVerwGE 64, 308, 310, so auch VGH Mannheim, Beschluss v. 23.7.2007, Az.: 9 S 1298/07, www.landesrecht-bw.de und auch VG Augsburg, Urteil v. 10.1.2006, Az.: Au 3 K 05.865, juris

1860 VG Augsburg, Urteil v. 10.1.2006, Az.: Au 3 K 05.865, juris, Rn. 24; OVG Bautzen Urteil v. 18.8.2010, Az.: 142/09, Sächs.VBI. 2011, S. 35 ff; ausführlich dazu Niehues, Prüfungsrecht, Rn. $34 \mathrm{ff}$

${ }^{1861}$ VGH Mannheim, Beschluss v. 23.7.2007, Az.: 9 S 1298/07, www.landesrecht-bw.de

1862 BVerfGE 58, 257

${ }^{1863}$ Badura, in: Maunz/Dürig/Herzog GG, Art. 7, Rn. 10

${ }^{1864}$ Niehues, Prüfungsrecht, Rn. 34, 45
} 
Bisher regelt kein Schulgesetz explizit Einzelheiten zur Eingliederung von Schülern und Schülerinnen mit Behinderungen. Zwar werden die Schulgesetze derzeit an die Anforderungen der BRK angepasst, ${ }^{1865}$ regeln aber nicht dezidiert die Ansprüche von Kindern mit Behinderungen. Sie beschränken sich nach wie vor auf allgemeine Bestimmungen zum sonderpädagogischen Förderbedarf und zum Teil allgemeine Regelungen zum Nachteilsausgleich. ${ }^{1866}$ Kein Schulgesetz enthält bisher die Ermächtigung zum Erlass einer Rechtsverordnung zur besseren Eingliederung von Schülern und Schülerinnen mit Behinderung. ${ }^{1867}$

Angesichts der erheblichen Grundrechtsrelevanz der Berücksichtigung von Behinderungen in der Schule und in Prüfungen sind die Regelungen zur Berücksichtigung der Behinderungen Legasthenie/Dyskalkulie "wesentliche" Entscheidungen, die der Schulgesetzgeber selbst zu treffen hat. ${ }^{1868}$ Für die betroffenen Schüler und Schülerinnen hat die Berücksichtigung erhebliche Auswirkungen auf die Verwirklichung ihrer Grundrechte aus Art. 2 I, 3 I, 3 III S. 2 und 12 GG. Nur mithilfe der Berücksichtigung werden sie in die Lage versetzt, wirksam am gesellschaftlichen Leben teilzuhaben und ihr Grundrecht auf Bildung zu verwirklichen. Nur so kann die Grundlage für eine Berufsausbildung und eine spätere selbstbestimmte Lebensführung geschaffen werden. Zu beachten sind aber auch in besonderem Maße die Grundrechte der nicht-behinderten Schüler und Schülerinnen, die durch eine Berücksichtigung von Behinderungen betroffen sein können. Sie sind zwar nicht direkt betroffen, da die Berücksichtigung einer Behinderung sie nicht benachteiligt. ${ }^{1869}$ Auswirkungen ergeben sich für sie aber durch das Verfahren der Anerkennung der Behinderung, weil damit die Schüler und Schülerinnen, die einen Ausgleich erhalten, von denen, die den allgemeinen Regelungen unterworfen bleiben, abgegrenzt werden. Weil sich der Ausgleich, soweit es um die Bewertung der Rechtschreibung oder der Mathematik geht, bei Schülern und Schülerinnen, die sie erhalten ohne behindert zu sein, als ungerechtfertigter Vorteil erweisen kann, ist es erforderlich, die Schüler und Schülerinnen mit Behinderung eindeutig zu bestimmen. Nur dann ist auch das Recht auf Chancengleichheit der nicht-behinderten Schüler und Schü-

\footnotetext{
1865 S. dazu die Nachweise in Fn. 1212

1866 S. der umfassende Überblick über alle landesrechtlichen Regelungen bei Poscher/Langer/ Rux, Gutachten zu Art. 24 BRK, S. $61 \mathrm{ff}$

${ }^{1867}$ Auch nicht das neue SchulG Hessen. §55 HE SchulG weitet nur die Verordnungsermächtigung bzgl. der sonderpädagogischen Förderung auf den inklusiven Unterricht aus; Art. $30 \mathrm{~b}$ III des Gesetzentwurfs zum BayEUG betrifft die Ordnung des Schulprofils „Inklusion“, das aber kein vorgegebenes Profil für alle Schulen, sondern die Möglichkeit eines besonderen Profils für interessierte Schulen ist.

1868 Vgl. Niehues, Prüfungsrecht, Rn. 54

1869 A.A. Ennuschat/Volino, br 2009, S. 166 ff, S. 168
} 
lerinnen gewahrt. Diese Grundrechtsbelange sind daher untereinander abzugrenzen und auszugleichen. ${ }^{1870}$

Dies ist Aufgabe des Gesetzgebers. Er muss dabei die drei wesentlichen Funktionen des Gesetzes beachten: 1. die Sicherung der Position des Bürgers, indem Grundrechtseingriffe und Grundrechtsausgestaltungen gesetzlich gestaltet sind, 2. die Sicherung gleicher Behandlung durch Exekutive und Justiz im Grundrechtsbereich und 3. die Sicherung der Berechenbarkeit. ${ }^{1871}$ Er kann sich dabei eng begrenzter Generalklauseln bedienen ${ }^{1872}$ und auch zu Rechtsverordnungen ermächtigen. In letzterem Fall muss der Gesetzgeber allerdings die Bestimmtheitsanforderungen des Art. 80 I S. 2 GG beachten und das Programm sowie den möglichen Verordnungsinhalt erkennen lassen. ${ }^{1873}$

Die Regelungsdichte ist umso größer, je intensiver der Grundrechtseingriff ist. ${ }^{1874}$ Bei besonders einschneidenden Maßnahmen bedarf es einer abstrakten Umschreibung der einzelnen Tatbestandsmerkmale. ${ }^{1875}$ Weiterhin hat der Gesetzgeber den Grundsatz der Widerspruchsfreiheit zu beachten und Normwidersprüche zu vermeiden. Das rechtliche und tatsächliche Regelungskonzept muss daher mit dem Regelungszweck übereinstimmen und darf ihm nicht widersprechen. ${ }^{1876}$

\section{Kapitel: Mindestumfang gesetzlicher Regelungen}

Das praktische Gewicht der Wesentlichkeitstheorie geht dahin, dass sich für Gesetze besondere Anforderungen des Inhalts und der Bestimmtheit der Normierung ergeben. ${ }^{1877}$ Das BVerfG hat diese Voraussetzungen in der Entscheidung zur Zwangsbehandlung folgendermaßen konkretisiert: Der Gesetzgeber muss seine Vorschrift so bestimmt fassen, wie dies nach der Eigenart des schulischen Lebenssachverhaltes und mit Rücksicht auf den Normzweck möglich ist. Die gesetzesausführende Verwaltung, hier insbesondere die Schulen und Lehrkräfte, müssen für ihr Verhalten steuernde und begrenzende Handlungsmaßstäbe vorfinden. Die Anforderungen an den Grad der Klarheit und Bestimmtheit sind umso strenger, je intensiver der Grundrechtseingriff ist, den die Norm

\footnotetext{
${ }^{1870}$ Vgl. Peine, in: Hdb. Grundrechte III, § 65, Rn. 70

${ }^{1871}$ Lerche, in: Hdb. Grundrechte III, § 62, Rn. $22 \mathrm{ff}$

1872 BVerfGE 33, 1, 11

1873 Lerche, in: Hdb. Grundrechte III, § 62, Rn. 8

1874 Niehues/Rux, Schulrecht, Rn. 43

1875 Peine, in: Hdb. Grundrechte III, § 65, Rn. 57

1876 Lindner, ZG 2007, S. 188 ff, S. 199 f

1877 Vgl. dazu Niehues/Rux, Schulrecht, Rn. $51 \mathrm{ff}$
} 
vorsieht. ${ }^{1878}$ Im Einzelnen müssen daher folgende Bereiche gesetzlich geregelt werden:

Der Anwendungsbereich des Gesetzes muss festgelegt werden, d.h. er muss definiert werden und operationalisierbar sein. ${ }^{1879}$ Bisher fehlt es an Definitionen, die nachvollziehbar und überprüfbar sind. Die untergesetzlichen landesrechtlichen Regelungen definieren ihren Anwendungsbereich nicht in diesem Sinne, sondern beziehen sich vage auf "Kinder mit besonderen, gravierenden oder auch veränderungsresistenten Schwierigkeiten". Verbunden damit, dass eine Diagnostik in der Regel nicht stattfindet, führt dies dazu, dass keine der oben genannten Funktionen eines Gesetzes erfüllt sind: weder wird der Grundrechtseingriff gestaltet, noch die Gleichbehandlung oder Berechenbarkeit gewährleistet. Ohne eine Definition des Anwendungsbereichs stehen sowohl die Berücksichtigung als auch die Nichtberücksichtigung der Behinderung und damit die Beachtung oder Nichtbeachtung und - noch entscheidender - die Verletzung oder Einhaltung des Grundrechts im ungebundenen Ermessen der Lehrkräfte.

Die gesetzliche Regelung muss ferner die wesentlichen Grundzüge des Verfahrens vorgeben. Der Gesetzgeber muss über die notwendige Diagnose, die grundsätzliche Anerkennung der Behinderung und die Grundsätze des Ausgleichs der Prüfungsbedingungen und -inhalte entscheiden. Das bedeutet vor allem, dass der Gesetzgeber grundsätzlich über die Pflicht zur Gewährung von Ausgleich und die Nicht-Bewertung bestimmter Prüfungskomponenten wie Rechtschreibung, Lesen und Rechnen entscheiden muss. ${ }^{1880}$ Zudem sind Vorgaben für die Rechtsverordnung für den Bereich der Zuordnung zu bestimmten Schulzweigen, zur Versetzung und zur Ersetzung bestimmter Prüfungsleistungen durch andere Leistungen notwendig. ${ }^{1881}$

Darüber hinaus muss der Gesetzgeber darüber entscheiden, wann eine Teilnahmepflicht für bestimmte Förderungen oder Maßnahmen statuiert werden soll. Weiterhin ergibt sich aus dem Volkszählungsurteil des BVerfG ${ }^{1882}$, dass der Gesetzgeber über die Pflicht zur Mitteilung einer Diagnose und die Verhältnismäßigkeit dieser Maßnahme zu entscheiden hat. Für die Verpflichtung zur Mitteilung der Diagnose bietet sich an, auf $\S \S 68$ ff SGB X zurückzugreifen.

\footnotetext{
1878 BVerfG, Beschluss v. 23.3.2011; Az.: 2 BvR 882/09, http://www.bundesverfassungsgericht.de/entscheidungen/rs20110323_2bvr088209.html, Rn. 73

$1879 \mathrm{Vgl}$. zur Notwendigkeit maßgebliche Kriterien zu definierten, wenn sie z.B. den bevorzugten Zugang zu einem Studium ermöglichen sollen: Fehling/Arnold, RdJB 2011, S. 316 ff, S. $331 \mathrm{f}$

1880 Langenfeld, RdJB 2007, S. 211 ff, S. 217

${ }^{1881} \mathrm{Vgl}$. zu den Anforderungen an schulgesetzliche Sonderregelungen für Kinder mit Migrationshintergrund: Reuter, RdJB 2003, S. 23 ff, S. $32 \mathrm{ff}$

1882 BVerfGE 65, 1, 44
} 
Aus Art. 4 I BRK ist der Gesetzgeber zudem dazu verpflichtet, den Rechtsschutz für die Betroffenen so auszugestalten, dass sie ihre Rechte effektiv wahrnehmen können. Dazu gehört auch die rechtsverbindliche Verankerung von Schadensersatzansprüchen und, da Diskriminierungen in der Regel nur schwer nachweisbar sind, Erleichterungen hinsichtlich der Beweislast. ${ }^{1883}$

Eine detaillierte Auflistung weiterer wesentlicher Inhalte eines Schulgesetzes, das den Anforderungen der BRK bezüglich eines inklusiven Schulwesens gerecht wird, enthält das „Eckpunkte-Papier" des Deutschen Instituts für Menschenrechte. $^{1884}$

\section{Kapitel: Rechtsverordnung oder Verwaltungsvorschrift?}

Weiter stellt sich die Frage, ob die Einzelheiten in einer Rechtsverordnung geregelt werden müssen oder auch in einer Verwaltungsvorschrift geregelt werden können.

Grundsätzlich steht es dem Gesetzgeber frei, ob er der Verwaltung die Regelungskompetenz für die Ausfüllung der gesetzgeberischen Leitentscheidungen durch Rechtsverordnung oder durch unbestimmte Rechtsbegriffe, die durch Verwaltungsvorschriften ausgefüllt werden, überträgt. ${ }^{1885}$ Die Formfreiheit findet jedoch dort ihre Grenze, wo es um die Begründung, Aufhebung oder Änderung von Rechten und Pflichten im Staat-Bürger-Verhältnis geht. Hier ist stets eine Rechtsverordnung erforderlich. ${ }^{1886}$ Denn nur sie stellt sicher, dass die Anwendung der zu schaffenden Detailregelung von den Betroffenen in allen Einzelheiten deutlich vorhersehbar ist und sie gerade hinsichtlich dieser Details auf ein besonders hohes Maß an Beständigkeit vertrauen dürfen. ${ }^{1887}$ Zudem hat nur die Rechtsverordnung Außenwirkung und schafft somit durchsetzbare Ansprüche für die Betroffenen.

Die Berücksichtigung der Behinderung Legasthenie/Dyskalkulie erfordert detaillierte Regelungen. Davon gehen die Bundesländer schon jetzt aus, da fast alle detaillierte Regelungen zur Berücksichtigung von Schwierigkeiten beim Lesen, Rechtschreiben und Rechnen erlassen haben. Die Detailregelung ist nicht nur für die Betroffenen erforderlich, damit diese die für sie geltenden Prüfungsbedingungen kennen, sondern auch für die Lehrkräfte und für die nichtbehinderten Mitschüler. Geht man von einer Zahl von ca. 5 \% Schülerinnen und Schüler mit Legasthenie und 5 \% Schülerinnen und Schüler mit Dyskalkulie aus,

\footnotetext{
1883 Aichele/ Althoff, Kommentar zur UN-BRK, Nicht-Diskriminierung, Rn. $26 \mathrm{f}$

${ }^{1884}$ Aichele, Stellungnahme DIMR 2010, Anhang 11

1885 Avenarius/Heckel, Schulrecht, Ziff. 15.332

${ }^{1886}$ Avenarius/Heckel, Schulrecht, Ziff. 15.332

${ }^{1887}$ Niehues/Rux, Schulrecht, Rn. 44
} 
befindet sich statisch gesehen in jeder Klasse ein Kind mit Legasthenie und eines mit Dyskalkulie. Das bedeutet, dass sich jede Lehrkraft mit der Berücksichtigung dieser Behinderung beschäftigen muss und dass alle anderen Schülerinnen und Schüler ebenfalls regelmäßig betroffen sind. Weil es sich nicht um ein Einzelphänomen handelt, das gelegentlich auftritt und dann im Einzelfall geregelt werden kann, sondern um ein generell auftretendes Problem, ist eine allgemeine Regelung in Form einer Rechtsverordnung erforderlich. ${ }^{1888}$ Das Regelungsbedürfnis ist vergleichbar mit den besonderen Bestimmungen für die Eingliederung von Schülern und Schülerinnen mit Migrationshintergrund (vgl. etwa § $49 \mathrm{HB}$ SchulG).

\section{Kapitel: Ansprüche bis zum Erlass gesetzlicher Regelungen}

Grundsätzlich hat die Feststellung, dass die verfassungsrechtlich erforderliche gesetzliche Grundlage für eine in die Grundrechte eingreifende Regelung fehlt, zur Folge, dass diese Regelung verfassungswidrig ist und nicht mehr angewandt werden kann. ${ }^{1889}$ Ausnahmsweise räumt das Bundesverfassungsgericht dem Gesetzgeber jedoch für die Schaffung der erforderlichen gesetzlichen Grundlage eine Übergangsfrist ein, während der die rechtlich unzulänglichen Maßnahmen hinzunehmen sind. Voraussetzung dafür ist, dass nur so und soweit ein Zustand vermieden werden kann, der der verfassungsmäßigen Ordnung noch ferner steht als die vorübergehende Hinnahme materiell rechtfertigungsfähiger, gesetzlich aber nicht ausreichend legitimierter Eingriffe. ${ }^{1890}$ Das BVerfG hat dies bejaht, wenn sich allein auf diesem Wege eine sonst eintretende Funktionsunfähigkeit der Schule einstellen würde. ${ }^{1891}$

Hier geht es um Regelungen, die für Schüler und Schülerinnen sowohl positiv als auch negativ sind: positiv, soweit sie die Berücksichtigung der Behinderung überhaupt regeln, negativ, soweit sie die Berücksichtigung unzulässiger Weise einschränken. Auch hier ist eine Abwägung vorzunehmen, welcher Zustand eintritt, wenn die Regelung völlig wegfällt und ob dieser Zustand mit der verfassungsmäßigen Ordnung noch weniger zu vereinbaren ist als die zeitweise Duldung des verfassungswidrigen Zustandes. Mit der verfassungsmäßigen Ordnung ist es nicht zu vereinbaren, wenn die Nicht-Anwendung der landesrechtlichen Regelungen dazu führt, dass die Behinderungen Legasthenie/Dyskalkulie

\footnotetext{
1888 So auch Ennuschat/Volino, br 2009, S. 166 ff, S. 169

1889 BVerfGE Urteil v. 31.5.2006, Az.: 2 BvR 1673/04, http://www.bverfg.de/entscheidungen/rs20060531_2bvr167304.html, Rn. 67

1890 BVerfGE Urteil v. 31.5.2006, Az.: 2 BvR 1673/04, http://www.bverfg.de/entscheidungen/rs20060531_2bvr167304.html, Rn. 67

${ }^{1891}$ BVerfGE 58, 257, $281 \mathrm{f}$
} 
gar nicht mehr anerkannt und berücksichtigt werden. Dann wäre die Benachteiligung der behinderten Schüler und Schülerinnen noch stärker als mit der nicht ausreichend legitimierten landesrechtlichen Regelung. Es ist daher danach zu differenzieren, welche Teile der landesrechtlichen Regelungen verfassungskonform sind und welche nicht. ${ }^{1892}$ Bis zum Erlass einer gesetzlichen Grundlage gelten daher diejenigen landesrechtlichen Regelungen weiter, die verfassungskonform sind. Soweit die landesrechtlichen Regelungen den verfassungsrechtlichen Anspruch begrenzen, sind sie nicht anzuwenden.

Sieht man in dem Anspruch auf Berücksichtigung der Behinderung in der Form der Nicht-Bewertung bestimmter Prüfungsteile oder -komponenten einen von den Grundrechten nicht erfassten Leistungsanspruch, so ist dieser Anspruch davon abhängig, dass der Gesetzgeber tätig wird, ${ }^{1893}$ Das bedeutet, dass die Grundrechtsausübung erst dann möglich ist, wenn der Staat tätig geworden ist. Die Tätigkeit des Staates wirkt insoweit konstitutiv für den Anspruch. Dies stellt die Betroffenen in der Zwischenzeit rechtsschutzlos, weil in ihr Grundrecht aus Art. 3 III 2 GG beständig eingegriffen werden kann. Da eine Frist zum Tätigwerden nicht existiert und ein Tätigwerden des Staates trotz eindeutiger Handlungspflicht bekanntlich oft lange dauern kann ${ }^{1894}$, ist dieser Zustand nicht auf einen überschaubaren Zeitraum begrenzt, in dem dies hingenommen werden müsste.

Das Ziel des Grundrechts aus Art. 3 III 2 GG, vor Benachteiligungen zu schützen, läuft leer, wenn die Betroffenen erst auf eine gesetzliche Ausgestaltung des Grundrechts warten müssen, um dadurch geschützt zu werden. Ebenso wie beim Vorbehalt des Gesetzes ist daher bei einem Leistungsrecht zu fragen, ob die Verpflichtung zur Hinnahme des verfassungswidrigen Zustandes, weil gesetzliche Ausgestaltungen fehlen, verfassungsrechtlich nicht schwerer wiegt, als das Zuwarten auf eine verfassungsmäßige Ausgestaltung des Grund-

1892 Vgl. BVerfGE 41, 251, 267; 58, 257, 282

1893 So OVG Lüneburg, Beschluss v. 10.7.2008, Az.: 2 ME 309/08, www.rechtsprechung.niedersachsen.de; VG Köln, Beschluss v. 26.9.2008, Az.: 10 L 1240/08, DVBI 2009, S. 538; Ennuschat/Volino, br 2009, S. 166 ff S. 168 f, vermischen in ihrer Stellungnahme die Frage des Leistungsanspruchs und des Vorbehalts des Gesetzes. Ein Leistungsanspruch wird durch Gesetz erst geschaffen, demgegenüber regelt der Vorbehalt des Gesetzes, welche grundrechtsrelevanten Entscheidungen der Gesetzgeber selbst zu treffen hat.

1894 S. z.B. die Notwendigkeit, eine gesetzliche Grundlage für die AHP zu schaffen, die seit 1995 vom BVerfG gefordert wurde (BVerfG Beschluss v. 6.3.1995, Az.: 1 BvR 60/95, NJW 1995, S. 3049) und erst mit der Versorgungsmedizin-Verordnung vom 10.12.2008 umgesetzt wurde oder die seit 1976 bekannte Verpflichtung Gesetze für den Jugendstrafvollzug zu erlassen, die erst nachdem das BVerfG eine Frist bis Ende 2007 gesetzt hatte, von den Ländern umgesetzt wurde, BVerfG Urteil v. 31.5.2006, Az.: 2 BvR 1673/04, http://www.bverfg.de/ent scheidungen/rs20060531_2bvr167304.html; s. auch Avenarius/Heckel, Schulrecht, Ziff. 15.392 
rechts durch gesetzliche Regelungen. Dies ist insbesondere dann anzunehmen, wenn dem Gesetzgeber in der Grundsatzentscheidung kein oder kaum Spielraum verbleibt, wenn die Beseitigung des verfassungswidrigen Zustandes kein oder kaum Geld kostet und wenn den Betroffenen ein Zuwarten auf ein gesetzliches Tätigwerden nicht zugemutet werden kann. Gemessen an Art. 3 III 2 GG wiegt die Versagung der Berücksichtigung der Behinderung im Schulbereich schwerer als die Berücksichtigung der Behinderung ohne gesetzliche Grundlage. ${ }^{1895}$ Dem Gesetzgeber verbleibt kein Spielraum hinsichtlich der grundsätzlichen Anerkennung der Behinderung. Sein Gestaltungsspielraum bezieht sich vor allem auf das Verfahren, den Ausgleich im Einzelnen und auf die Ausgestaltung von effektiven Fördermaßnahmen. Die Beseitigung des verfassungsrechtlichen Zustandes durch die Nicht-Berücksichtigung der Behinderung kostet auch kein Geld. Den Betroffenen kann vor allem ein Zuwarten auf eine gesetzliche Regelung nicht zugemutet werden. Die Versagung der Leistung, d.h. die Nicht-Berücksichtigung der Behinderung, führt dazu, dass die betroffenen Schüler während der Schulzeit ihr Grundrecht aus Art. 3 III 2 GG nicht ausüben können. Diese Konstellation gibt es zwar auch bei anderen Grundrechtsverletzungen, etwa fehlerhaften Steuerbescheiden, bei denen ein Zuwarten auf eine gesetzliche Regelung für zulässig gehalten wird, weil die Verletzung rückwirkend, etwa durch Rückerstattungen, geheilt werden kann, sie ist aber nicht auf den Schulbereich übertragbar. Der betroffene Schüler braucht den Schutz des Grundrechtes während des Schulbesuchs, er kann nicht auf eine gesetzliche Regelung warten. Ist der Schulbesuch abgeschlossen, ist eine Heilung oder Wiedergutmachung ausgeschlossen. Deshalb ergibt sich hier aus Art. 3 III 2 GG ein Leistungsanspruch auf voll umfängliche Berücksichtigung der Behinderung.

1895 Ennuschat/Volino, br 2009, S. 166 ff, S. 169 



\section{Teil: Umgang mit Legasthenie/Dyskalkulie in der EU}

Die Problematik des Umgangs mit Legasthenie/Dyskalkulie stellt sich in allen Ländern, in allen Sprachen ${ }^{1896}$, daher auch in allen Ländern der EU. Eine ausführliche Erörterung zum Umgang mit Legasthenie/Dyskalkulie in den einzelnen EU-Ländern würde jedoch den Rahmen dieser Arbeit sprengen. Auch gibt es zwar einige länderübergreifende Forschungsprojekte im Bereich der Neurowissenschaften ${ }^{1897}$ und ein Projekt zur Weiterbildung von Personalfachleuten im Umgang mit Legasthenie ${ }^{1898}$, aber eine detaillierte wissenschaftliche Untersuchung über die rechtlichen Rahmenbedingungen im Schulbereich fehlt bisher. Die European Dyslexia Association, ein Verband der europäischen Selbsthilfeverbände, hat allerdings 2004 eine Umfrage unter den Mitgliedsverbänden durchgeführt und so einige Daten zur Berücksichtigung vor allem der Legasthenie erhoben. ${ }^{1899}$ Und der Bundesverband Legasthenie und Dyskalkulie e.V. hat 2012 eine Broschüre veröffentlicht, in der ein Überblick über die „Best Practices" in fünf europäischen Ländern sowie Kanada und Neuseeland gegeben wird. ${ }^{1900}$ Es sollen daher zumindest die wichtigsten Aspekte abschließend kurz angerissen werden.

\section{Kapitel: Österreich}

In Österreich regelt das Schreiben GZ 36.200/38-SL V/2001 des Bundesministeriums für Bildung, Wissenschaft und Kultur die Leistungsbeurteilung bei Lese-

\footnotetext{
1896 S. dazu http://www.worlddyslexiaforum.org: World Dyslexia Forum 2010 mit Berichten zu den Sprachen Arabisch, Chinesisch, Englisch, Französisch, Russisch und Spanisch

1897 Z.B. Neurodys, http://www.neurodys.com, s. dazu Landerl, in: Legasthenie und Dyskalkulie 2011, und Proread, http://www.kjp.med.uni-muenchen.de/proread/projekt.php

1898 http://www.adystrain.org

1899 Bogdanowicz/Sayles, Rights of Dyslexic Children in Europe

1900 Gronauer, Int. Ländervergleich
} 
Rechtschreibschwäche (LRS) bzw. Legasthenie. ${ }^{1901}$ Danach besteht kein Einwand dagegen, dass Schüler/innen bei der Leistungserbringung - insbesondere in höheren Schulstufen - bei schriftlichen Arbeiten zeitgemäße Hilfsmittel zur Überprüfung der Schreibrichtigkeit zur Verfügung gestellt werden. Sämtliche gängigen Programme zur Textverarbeitung enthalten Rechtschreibprüfungen, durch die die Leistungserbringung erleichtert wird. Es wird davon ausgegangen, dass davon Schüler/innen mit nachweislich legasthenischer Beeinträchtigung besonders profitieren werden. Die Schreibrichtigkeit kann und darf keinesfalls die einzige Grundlage der Leistungsbeurteilung sein.

Bei nachweislich vorliegenden und schwerwiegenden hirnorganischen Störungen, die sich im Sinne einer Körperbehinderung auswirken und das Erlernen und Anwenden der Rechtschreibung beeinträchtigen, kann $\S 18$ Abs. 6 des Schulunterrichtsgesetzes angewendet werden. ${ }^{1902}$ Dieser lautet: „Schüler, die wegen einer körperlichen Behinderung eine entsprechende Leistung nicht erbringen können oder durch die Leistungsfeststellung gesundheitlich gefährdet wären, sind entsprechend den Forderungen des Lehrplanes unter Bedachtnahme auf den wegen der körperlichen Behinderung bzw. gesundheitlichen Gefährdung erreichbaren Stand des Unterrichtserfolges zu beurteilen, soweit die Bildungs- und Lehraufgabe des betreffenden Unterrichtsgegenstandes grundsätzlich erreicht wird."

Der entscheidende Unterschied zur deutschen Rechtslage liegt darin, dass in Österreich die Abwertung wegen Mängeln in der Rechtschreibung nicht zwingend und für alle Fächer vorgesehen ist. Die Leistungsbeurteilung richtet sich nach der Leistungsbeurteilungsverordnung. ${ }^{1903}$ Diese enthält in $\S 15$ Regeln zur Bewertung der Rechtschreibung, nicht aber die Regelung, dass die Arbeit bei gehäuften Verstößen gegen die sprachliche Richtigkeit abzuwerten ist.

Einzelheiten ergeben sich aus der Handreichung des Bundesministeriums für Bildung, Wissenschaft und Kultur sowie aus einzelnen Erlassen der Bundesländer. ${ }^{1904}$

Auch die Dyskalkulie kann im Rahmen des pädagogischen Ermessens berücksichtigt werden. ${ }^{1905}$

\footnotetext{
${ }^{1901}$ http://www.bmukk.gv.at/medienpool/6272/RS31_2001.doc

1902 http://www.bmukk.gv.at/schulen/recht/gvo/schug_teil1.xml\#18(6)

${ }^{1903}$ Verordnung des Bundesministers für Unterricht und Kunst vom 24. Juni 1974 über die Leistungsbeurteilung in Pflichtschulen sowie mittleren und höheren Schulen (Leistungsbeurteilungsverordnung) BGBI. Nr. 371/1974, zuletzt geändert durch BGBI. II Nr. 35/1997, http://www.bmukk.gv.at/schulen/recht/gvo/lb_vo.xml

1904 Handreichung: Die schulische Behandlung der Lese-Rechtschreib-(Rechen-)Schwäche, http://www.schulpsychologie.at/lernen-leistung/lese-rechtschreibschwaeche/handreichung

1905 S. dazu Handreichung Rechenschwäche, S. 37 f, http://www.schulpsychologie.at/uploads/ media/rechenschwaeche.pdf
} 


\section{Kapitel: Schweiz}

In der Schweiz sind die Kantone für die schulischen Regelungen zuständig. In vielen Kantonen gibt es keine Regelungen. ${ }^{1906}$

In Basel gibt es eine sehr umfassende Regelung, die sowohl für Legasthenie als auch für Dyskakulie gilt. ${ }^{1907}$ Die Diagnose richtet sich nach der ICD 10 und dem ICF. Die betroffenen Schüler und Schülerinnen haben Anspruch auf eine differenzierte Beurteilung und auf Nachteilsausgleich. Sie sind von Leistungserhebungen befreit, die ausschließlich der Feststellung eines eng eingegrenzten, die Teilleistungsstörung oder die Behinderung betreffenden Kompetenzbereichs dienen, z.B. dem Kopfrechnen oder dem Diktat. Zu den Möglichkeiten des Nachteilsausgleichs zählen u.a. mehr Zeit in allen zeitlich limitierten Prüfungssituationen, eine Reduktion der Zahl der Prüfungsaufgaben und die Bereitstellung technischer oder anderer Hilfsmittel wie Computer, Taschenrechner, Einmaleins-Tabelle.

Die Kantonsschule Oerlikon in Zürich hat die bayerische Regelung zur Berücksichtigung der Legasthenie fast vollständig übernommen. ${ }^{1908}$

Weitere Informationen finden sich auf dem Schweizer Bildungsserver ${ }^{1909}$, der auch vielfältige Hinweise zum Einsatz von Informations- und Kommunikationstechnologien speziell bei Legasthenie (= Dyslexie) enthält. ${ }^{1910}$

\section{Kapitel: Großbritannien}

In Großbritannien gehören Schüler und Schülerinnen mit Legasthenie zu den Schülern mit "Special Educational Needs" = SEN. Der Begriff SEN, mit dem etwa 19 \% der Schüler im englischen Schulsystem bezeichnet werden, kann nicht mit dem deutschen Begriff „sonderpädagogischer Förderbedarf“ übersetzt werden, sondern entspricht in etwa einem „,besonderen Lernunterstützungsbedarf“ . ${ }^{191}$ Die Definition des Begriffs findet sich im Special Educational Needs and Disabili-

\footnotetext{
1906 http://www.verband-dyslexie.ch/gut-zu-wissen/gut-zu-wissen-dyslexie-legasthenie-unddyskalkulie-in-schule-und-beruf.php

1907 Richtlinien für die öffentlichen Schulen des Kantons Basel-Stadt zur Leistungserhebung und Leistungsbewertung bei attestierten Lernstörungen, Sprachstörungen und Behinderungen v. 10.8.2009, http://www.verband-dyslexie.ch/images/stories/pdf/legasthenie/richtlinienlernstoerungen_basel_stadt.pdf

1908 http://www.verband-dyslexie.ch/images/stories/pdf/LegasthenieRichtlinienKantonsschule Oerlikon.pdf

1909 http://www.educa.ch/de

1910 http://sonderpaedagogik.educa.ch/de/ict-werkzeuge-dyslexie

1911 Plate, Sonderpädagogische Förderung heute 53 (2008), S. 399 ff, S. 401
} 
ty Act 2001 (SENDA). ${ }^{1912}$ Das Gesetz begründet die Rechte von Schülern und Schülerinnen mit Dyslexie (so die Bezeichnung im Englischen) und statuiert ein Diskriminierungsverbot, indem es umfassend regelt, wann eine Diskriminierung gegeben ist (Sect. 11, 12 SENDA). Bei Prüfungen werden die Ausgleichsmaßnahmen zentral durch den Joint Council for Qualification festgelegt ${ }^{1913}$, der umfangreiche Regelungen für mögliche Ausgleichsmaßnahmen aufgestellt hat. ${ }^{1914}$

Das Ministerium für Kinder, Schulen und Familien hat 2008 eine umfassende Studie zur Thematik Dyslexie und Schulen in Auftrag gegeben, deren Ergebnis der detaillierte Rose-Bericht ist. ${ }^{1915}$ Die Umsetzung der Erkenntnisse erfolgt unter anderem durch den Dyslexia Trust, der die Kooperation verschiedener Organisationen steuert.

In England sind mehreren Schülern mit Legasthenie Schadensersatzansprüche aus Amtshaftung zugesprochen worden, weil die Schulpsychologen die Legasthenie nicht erkannt und die Lehrkräfte die Schüler nicht angemessen unterrichtet und gefördert hatten. ${ }^{1916}$

\section{Kapitel: Niederlande}

Die Niederlande haben seit dem 1.1.2009 ein differenziertes Fördersystem, in dem Schulen und Krankenkassen zusammenwirken. Schulen müssen durch regelmäßige Screenings die Lese-Rechtschreibfähigkeiten der Schüler testen. Auffällige Schüler müssen gezielt gefördert werden. Profitiert das Kind nicht von der Förderung wird es zur Diagnose und eventuellen Behandlung an ein Institut außerhalb der Schule geschickt. Das Institut muss eine staatliche Anerkennung haben. Die Kosten für die Diagnose und die Behandlung übernimmt dann die Krankenkasse. In der Schule wird die Legasthenie anerkannt. ${ }^{1917}$ Die Informationen werden gebündelt auf der Internetseite www.masterplandyslexie.nl, die

\footnotetext{
1912 http://www.opsi.gov.uk/acts/acts2001/ukpga_20010010_en_1

1913 S. www.jcq.org.uk

1914 Joint Council for Qualifications, Access Arrangements

1915 Rose, Rose-Review 2009

1916 Judgments - Phelps (A.P.) v. Mayor Etc. of The London Borough of Hillingdon - Anderton (A.P.) v. Clwyd County Council In Re G (A.P.) - Jarvis (A.P.) v. Hampshire County Council, vom 27. 7.2000, http://www.parliament.the-stationery-office.co.uk/pa/ld199900/ldjudgmt/ jd000727/phelp-1.htm; The Supreme Court of Judicature, Court of Appeal (Civil Division), Case No: A2/2005/0762 \& A2/2005/0761, Neutral Citation Number: [2006] EWCA Civ 239, Skipper vs. Calderdale Metropolitan Borough Council and The Governors of Crossley Heath School, vom 15.3.2006, http://www.hmcourts-service.gov.uk/judgmentsfiles/j3992/skipper _v_calderdale_0306.htmhttp://www.bailii.org/ew/cases/EWCA/Civ/2006/238.html

www.masterplandyslexie.nl, Umfassende Informationen auch unter:

http://www.stichtingdyslexienederland.nl und www.balansdigitaal.nl
} 
alle Informationen für die Diagnose und Berücksichtigung der Dyslexie enthält. ${ }^{1918}$ Ferner existiert eine umfassende Handreichung zur Gestaltung von Arbeitsblättern mit 73 Vorschlägen. ${ }^{1919}$

Die besonderen Probleme, die Schüler und Schülerinnen mit Legasthenie in Deutschland durch die Bewertung der Rechtschreibung haben, ergeben sich in den Niederlanden nicht, weil es dort eine solche Bewertungsregel nicht gibt. In Prüfungen erhalten Schüler mit Legasthenie Ausgleichsmaßnahmen nach den jeweiligen Schulordnungen, die im Masterplan Dyslexie in einer Übersicht zusammengestellt sind. ${ }^{1920}$ Z.B. können in der Unterstufe („onderbouw“) Ausnahmen von Teilen der vorgegebenen Lernziele in Niederländisch und Englisch gemacht werden, in der 2. und 3. Fremdsprache können die Kernziele für Schüler mit Legasthenie spezifisch festgelegt werden. Im Gymnasium („,bovenbouw wvo") kann in den Fachrichtungen "Natur und Technik" und "Gesundheit" auf die 2. Fremdsprache verzichtet werden, wenn ansonsten der Abschluss der Schule gefährdet ist. In der zentralen Abschlussprüfung („eindexamen“) entscheidet der Schulleiter über die Ausgleichsmaßnahmen (Art. 55 Eindexamenbesluit) ${ }^{1921}$, wobei er an die Vorschläge der diagnostizierenden Fachkraft gebunden ist. Zugelassen sind für alle Schüler mit Lernbeeinträchtigungen Laptops mit Textverarbeitungs- und Rechtschreibprogrammen.

Für den Bereich der Dyskalkulie ist anerkannt, dass betroffene Schüler Zeitverlängerungen in den Prüfungen bekommen. Außerdem sind bei Dyskalkulie folgende Formen des Nachteilsausgleichs zulässig, wenn die diagnostizierende Fachkraft diese vorschlägt: angepasste Aufgabenblätter an das Niveau des Kindes, Nutzung schematischer Lösungsstrategien, weniger Aufgaben, Angabe von Änderungen der Rechenstrategien (etwa indem minus in blau und plus in rot markiert wird), mündliche Erläuterung oder zusätzliche mündliche Prüfung, Taschenrechner, Formelsammlung. ${ }^{1922}$ Der Nachteilsausgleich ist auch in Abschlussprüfungen zu gewähren und wird dort durch den Schulleiter festgelegt (Art. 55 Eindexamenbesluit). ${ }^{1923}$

${ }^{1918}$ Für den außerschulischen Bereich s. auch Waddington in: Non-Discrimination Law, S. 659 f S. 661, S. $708 \mathrm{f}$

1919 http://www.goedtelezen.nl/richtlijnen; Übersetzung durch Verband Dyslexie Schweiz, Richtlinien Produktion Lernmaterialien

1920 www.masterplansyslexie.nl: Welke wettelijke regelingen zijn er voor leerlingen met dyslexie?

${ }^{1921}$ Eindexamenbesluit vwo-havo-mavo-vbo - Artikel 55. Afwijking wijze van examineren, http://www.examenblad.nl/9336000/1/j9vvhinitagymgn_m7mvh57gltp77x8/vg41h1jpfhtn

1922 http://www.balansdigitaal.nl/stoornissen/dyscalculie/regelingen-envoorzieningen/aangepaste-toetsen-en-schoolexamens

${ }^{1923}$ Eindexamenbesluit vwo-havo-mavo-vbo - Artikel 55. Afwijking wijze van examineren, http://www.examenblad.nl/9336000/1/j9vvhinitagymgn_m7mvh57gltp77x8/vg41h1jpfhtn 


\section{Kapitel: EU}

Das Europäische Parlament hat am 13.11.2007 eine Erklärung „Zur „Dys“kriminierung und sozialen Ausgrenzung dysfunktionaler Kinder" angenommen. ${ }^{1924}$ Darin wird darauf hingewiesen, dass die Dysfunktionen (Dysphasie, Dyspraxis, Dyslexie, Dyskalkulie und Aufmerksamkeits-Defizit-Syndrom usw.), die sehr früh die Kommunikation beeinträchtigen, in vielen Mitgliedstaaten oft unerkannt bleiben und dass nur durch eine frühzeitige, intensive und multidisziplinäre Betreuung in angemessenen Strukturen (im normalen Schulumfeld mit angemessener Begleitung oder in spezialisierten Strukturen) die "Dys"kriminierung dieser Kinder verhindert werden kann. Das Europäische Parlament hat daher die Kommission und den Rat aufgefordert, eine Charta für dysfunktionale Kinder zu erstellen und die Anerkennung der Dysfunktionen als Behinderung zu unterstützen. Zudem sollen bewährte Verfahren gefördert werden u.a. in Bezug auf die frühzeitige Beachtung, Erkennung, systematische Diagnostik und Betreuung und im Hinblick auf effiziente pädagogische Strukturen im normalen oder spezialisierten Umfeld für Kinder, Jugendliche und junge Erwachsene. Das Europäische Parlament geht daher davon aus, dass es sich bei Dyslexie (= Legasthenie) und Dyskalkulie um Behinderungen handelt.

Das European Personnel Selection Office (EPSO), das die Personalauswahl für die EU-Institutionen durchführt, nimmt in seinen Auswahlverfahren auf Behinderung Rücksicht. Bewerber mit Dyslexia, Dyscalculia, Dyspraxia, Dysgraphia u.ä. erhalten im Auswahlverfahren für den Personalpool der Europäischen Institutionen regelmäßig einen Zeitzuschlag von $25 \%$. Entscheidend ist dafür die Empfehlung des behandelnden Arztes bezüglich der notwendigen Ausgleichmaßnahmen. ${ }^{1925}$

\footnotetext{
1924 http://www.europarl.europa.eu/sides/getDoc.do?type=WDECL\&reference=P6-DCL-20070064\&language $=D E$

1925 Auskunft des EPOS, Email Toralf Katzorke vom 30.11.2009 an die Verf.
} 


\section{Teil: Zusammenfassung}

Schüler und Schülerinnen mit Legasthenie/Dyskalkulie haben aus Art. 3 III 2 GG den Anspruch, dass ihre Behinderung im schulischen Alltag und in Prüfungen sowohl hinsichtlich der äußeren Prüfungsbedingungen als auch hinsichtlich der inhaltlichen Prüfungsanforderungen durch Nachteilsausgleich ausgeglichen wird.

Maßgeblich für die Qualifikation von Legasthenie/Dyskalkulie als Behinderung ist der Behinderungsbegriff des Art. 3 III 2 GG. Dieser ist als sozialer Behinderungsbegriff auszulegen, so wie inn Art. 1 II BRK beschreibt. Inhaltlich bedeutet das soziale Modell von Behinderung vor allem die Abwendung vom Maßstab des "Normalen" als Richtschnur und die Anerkennung der Behinderung als soziales Phänomen. Entscheidend ist damit die Wechselwirkung zwischen einer Funktionsbeeinträchtigung und den verschiedenen Barrieren, die die Teilhabe der Betroffenen am gesellschaftlichen Leben hindern. Behinderungen sind aufgrund dieses Modells sowohl die Legasthenie/Dyskalkulie als auch die Lese-Rechtschreibschwäche und Rechenschwäche, soweit sie medizinisch feststellbar sind.

Die Definition der Legasthenie/Dyskalkulie als Behinderung ist Mittel zum Zweck, die Diskriminierung zu überwinden. Sie macht die gestörte Beziehung zwischen einem Menschen mit Beeinträchtigungen und seiner Umwelt sichtbar und zeigt damit auf, dass hier eine Veränderung stattfinden muss. Je mehr Menschen es mit Behinderung gibt, desto klarer treten die Versäumnisse von Staat und Gesellschaft zutage, die Barrieren abzubauen.

Entgegen der herrschenden Meinung erschöpft sich das Benachteiligungsverbot des Art. 3 III 2 GG nicht in der Anordnung, behinderte und nichtbehinderte Menschen rechtlich gleich zu behandeln, sondern umfasst auch das Verbot der unterschiedslosen Gleichbehandlung von Menschen mit und ohne Behinderung, wenn eine unterschiedliche Behandlung aus Gründen der Behinderung erforderlich ist. Diese Auslegung des Art. 3 III 2 GG entspricht der Auslegung der Diskriminierungsverbote der BRK, der ein weites Verständnis des Diskriminierungsverbots zugrunde liegt, und die generell als Auslegungshilfe für 
die Bestimmung von Inhalt und Reichweite der Grundrechte und speziell für das Verständnis des Art. 3 III 2 GG heranzuziehen ist.

Die Benachteiligung der Schüler und Schülerinnen mit Legasthenie/Dyskalkulie liegt in der unterschiedslosen Gleichbehandlung mit nicht-betroffenen Mitschülern bezüglich der Prüfungsbedingungen, sowohl äußerlich als auch inhaltlich, und der entsprechenden Bildungsstandards, insbesondere durch die Bewertung der Rechtschreibung und des Rechnens und durch die Anwendung sog. „Sperrklauseln“ in den Sprachfächern.

Die inhaltlichen Prüfungsanforderungen stellen für Menschen mit Behinderungen Barrieren auf, die von ihnen nicht beeinflusst werden können und die für Menschen ohne Behinderung nicht existieren. Damit Schüler und Schülerinnen mit Behinderungen die gleichen Chancen erhalten, muss für sie diese behinderungsspezifische Barriere abgebaut werden. Die Prüfung muss für den Prüfling „barrierefrei“ gestaltet werden. Der Abbau einer solchen Barriere geschieht durch Nachteilsausgleich.

Behinderungsspezifische Benachteiligungen sind nicht gerechtfertigt, weder hinsichtlich der Gleichbehandlung bei äußeren Prüfungsbedingungen noch hinsichtlich der inhaltlichen Prüfungsanforderungen.

Die Annahme einer „persönlichkeitsbedingten Eigenschaft, die die Leistungsfähigkeit prägt" ist nicht mit Art. 3 III 2 GG vereinbar und berechtigt daher nicht dazu, Behinderungen im Bereich der inhaltlichen Leistungsanforderungen prinzipiell nicht zu berücksichtigen.

Der staatliche Erziehungsauftrag ist kein Selbstzweck, sondern besteht allein im Interesse der Kinder, die dem Staat zur schulischen Betreuung anvertraut sind. Die Grundrechte der Kinder verpflichten den Staat dazu, für behinderte Kinder und Jugendliche schulische Einrichtungen bereit zu halten, die ihnen eine sachgerechte schulische Erziehung, Bildung und Ausbildung ermöglichen. Deshalb ist bereits im Rahmen des Erziehungsauftrags nach Art. 7 Abs. 1 GG die Wertung des Art. 3 III 2 GG zu beachten. Diese kann nur gewahrt werden, wenn bei der Ausgestaltung der allgemeinen Maßstäbe für Prüfungsanforderungen, Prüfungsinhalte und Prüfungsziele die Belange der Menschen mit Behinderung berücksichtigt werden.

Die Berücksichtigung der Dyskalkulie im Fach Mathematik ist möglich, da auch Schüler und Schülerinnen mit Dyskalkulie mathematische Fähigkeiten haben und mit verschiedenen Hilfestellungen und Maßnahmen in die Lage versetzt werden können, entweder dem Fach Mathematik insgesamt zu folgen oder zumindest mathematisches Leistungsvermögen auf Teilgebieten zu zeigen.

Die Nicht-Bewertung der Rechtschreibung und des Rechnens führt nicht zu einer Besserstellung des behinderten Schülers. Die Klausuren werden nicht „großzügiger" bewertet, sondern es werden die Leistungen unter Berücksichtigung der Behinderung bewertet. Die Nichtbewertung bestimmter Lerninhalte 
bei Schülern und Schülerinnen mit Behinderung wirkt sich bei Schülern und Schülerinnen ohne Behinderung nicht aus. Dass sie schlechte Noten in Teilbereichen bekommen können, die bei einem Schüler mit Behinderung nicht benotet werden, liegt im System der Benotung, führt aber nicht dazu, dass sie gegenüber diesen schlechter gestellt werden.

Alle Formen der Berücksichtigung der Behinderung, die erforderlich sind um die Chancengleichheit herzustellen, sind Nachteilsausgleich, auch die Veränderung von inhaltlichen Prüfungsanforderungen.

Eine Bevorzugung liegt nur dann vor, wenn nach der Herstellung der Chancengleichheit durch Nachteilsausgleich bei den Prüfungsbedingungen und bei der Leistungsbewertung zusätzlich auf allgemeine Anforderungen verzichtet wird, um den betroffenen Schülern einen weiteren Ausgleich für ihren behinderungsbedingten Aufwand zu geben, etwa bei Schülern und Schülerinnen mit Legasthenie durch die Befreiung von der zweiten Fremdsprache im Gymnasium oder bei Schülern und Schülerinnen mit Dyskalkulie durch eine Befreiung vom Fach Physik. Eine solche Bevorzugung ist zulässig und muss vom Gesetzgeber speziell festgelegt werden.

Mit Art. 3 III 2 GG nicht zu vereinbaren sind eine Begrenzung der Berücksichtigung, z.B. auf Fälle von schwerer Betroffenheit, Fälle mit wesentlichen Auswirkungen oder auf bestimmte Fächer. Auch ein Entzug der Berücksichtigung bei durchschnittlichen Noten oder erfolgreichem Schulbesuch oder der Ausschluss der Berücksichtigung der Dyskalkulie wegen der Bedeutung des Faches Mathematik sind unzulässig. Ebenso wenig kann der Nachteilsausgleich in Form eines Junktims mit der Pflicht zum Besuch von Förderunterricht oder außerschulischer Förderung verbunden werden. Möglich ist es allerdings, die betroffenen Schüler und Schülerinnen generell zum Besuch einer schulischen Förderung zu verpflichten, wenn diese eine qualifizierte, evaluierte Therapie der Legasthenie/Dyskalkulie ist und von entsprechend qualifiziertem Personal durchgeführt wird.

Der Grundsatz der Chancengleichheit erfährt durch den Paradigmenwechsel, den die BRK bewirkt, eine neue Dimension. Er beinhaltet nicht nur den Auftrag, Gleichheit herzustellen, damit Schüler mit Behinderungen die Anforderungen erfüllen können, die an „Normalschüler" gestellt werden, sondern fordert materielle Gleichheit im Sinne des Grundsatzes „Jedem das Seine“. Chancengleichheit besteht nur dann, wenn nicht nur die formalen Startbedingungen, sondern auch die gesetzten Ziele die Belange von Menschen mit Behinderung berücksichtigen. Schüler und Schülerinnen mit Behinderung müssen die Chance (= Aussicht) haben, den Bildungsgang, für den sie individuell geeignet und begabt sind, erfolgreich zu absolvieren.

Der Anspruch auf Berücksichtigung ergibt sich auch aus Art. 24 BRK, sei es unmittelbar oder durch die völkerrechtsfreundliche Auslegung der Schulgeset- 
ze. Art. 24 BRK normiert positiv die Voraussetzungen, unter denen die Beschulung von Schüler und Schülerinnen mit Behinderungen diskriminierungsfrei ist.

Im Verfahren zur Anerkennung der Legasthenie/Dyskalkulie ist eine Diagnostik auf der Grundlage der ICD 10 und den Leitlinien der Kinder- und Jugendpsychiatrie erforderlich, die nicht durch pädagogische Erwägungen relativiert werden kann. Nur eine solche Diagnostik entspricht den Vorgaben des BVerfG für ein fachliches, objektives Verfahren mit wiederholbaren Ergebnissen.

Die Anerkennung der Legasthenie/Dyskalkulie in der Schule ist ein gebundener Verwaltungsakt. Nebenbestimmungen sind nur in Form von mittelfristigen Befristungen zulässig. Die auflösende Bedingung des Entzugs der Anerkennung bei durchschnittlichen Noten ist unzulässig, ebenso eine Auflage in Form eines Junktims zwischen Nachteilsausgleich und Besuch einer Fördermaßnahme.

Die Ausgestaltung des Nachteilsausgleichs ist eine gebundene Entscheidung, soweit sie etwa die grundsätzliche Nicht-Bewertung der Rechtschreibung oder des Rechnens betrifft. Sie ist eine Ermessensentscheidung, soweit sie die individuelle Anpassung, etwa durch Zeitzuschläge, Nutzung von Hilfsmitteln usw. betrifft, in die die unterrichtenden Lehrkräfte einbezogen werden können.

Ein Verzicht der betroffenen Schüler und Schülerinnen auf die Berücksichtigung der Behinderung ist grundsätzlich möglich.

Eine Zeugnisbemerkung, mit der auf die Legasthenie/Dyskalkulie hingewiesen wird, ist eine unmittelbare Diskriminierung, die nach Art. 3 III 2 GG verboten und nicht gerechtfertigt ist.

Die Rügepflicht im Hinblick auf Mängel im Prüfungsrecht, die im universitären Prüfungsrecht entwickelt worden ist, ist auf den Schulbereich nicht übertragbar. Sie ist eine unverhältnismäßige Belastung von Schülern und Schülerinnen mit Behinderung und ihrer Eltern.

Erforderlich sind gesetzliche Regelungen zur Berücksichtigung der Behinderungen. Bis zum Erlass solcher gesetzlichen Regelungen gelten diejenigen Regelungen der landesrechtlichen Vorschriften fort, die für die betroffenen Schüler und Schülerinnen vorteilhaft sind. 


\title{
Anhang 1: \\ Glossar medizinischer, psychologischer und pädagogischer Fachausdrücke
}

\author{
Assoziierte aktuelle abnorme \\ psychosoziale Umstände \\ Ätiologie \\ Auditiv \\ Cortex \\ Kinästhesie \\ Kognitiv \\ Komorbid/Komorbidität \\ Magnetresonanztomographie \\ Neurobiologie \\ abnorme psychosoziale Situationen, die \\ für die Verursachung der psychischen \\ Störung relevant sein können \\ Wissenschaft von den Ursachen von \\ Krankheiten \\ Sinneswahrnehmung von Tönen, Sprach- \\ reizen etc. \\ Wesentliche Gehirnregionen der visuellen \\ und auditiven Wahrnehmung, des Ge- \\ dächtnisses und der Aufmerksamkeits- \\ steuerung \\ Wahrnehmung von Bewegungen, Körper- \\ verlagerungen und des Raum-Lage- \\ Verhältnisses \\ Bezeichnet Funktionen, die mit Wahr- \\ nehmung, Lernen, Erinnern und Denken \\ in Zusammenhang stehen \\ Nebeneinander von unterschiedlich dia- \\ gnostizierbaren und eigenständigen \\ Krankheitsbildern bei einem Patienten, \\ die ursächlich mit einer Grunderkrankung \\ zusammenhängen können \\ Bildgebendes Verfahren, das in der medi- \\ zinischen Diagnostik zur Darstellung von \\ Struktur und Funktion der Gewebe und \\ Organe eingesetzt wird. \\ Sammelbegriff für biologische, medizini- \\ sche, physikalische und psychologische \\ Wissenschaften, die den Aufbau und die
}




\section{Occipitaler Cortex \\ Organisch-endogen \\ Pathogenese \\ Pathologischer Befund \\ Phonem \\ Phonologie}

Phonologische Bewusstheit

Positronen-Emissionen-

Tomographie

Psychopathologischer Befund

Psychosozial

Standardabweichung

Temporaler Cortex

Temporo-parietaler Bereich

Visuelle Wahrnehmung

Zentral auditive Wahrnehmung

Zentralnervös

Zentralnervensystem
Funktion des Nervensystems untersuchen Hinterhauptslappen

aus inneren (organischen) Ursachen entstehend

Entstehung von Krankheiten

Befund, dass eine Krankheit vorliegt

Sprachlaut - kleinste Einheit der Sprache

Analyse lautsprachlicher Einheiten als

Voraussetzung für die Unterscheidung von Wörtern

Fähigkeit, die Aufmerksamkeit auf die formalen Eigenschaften der gesprochenen Sprache zu lenken, z.B. auf den Klang der Wörter beim Reimen, auf Wörter als Teile von Sätzen, auf Silben als Teile von Wörtern und auf die einzelnen Laute der gesprochenen Wörter.

Methode zur Darstellung zentralnervöser Prozesse mittels radioaktiv markierter Substanzen

Ergebnis einer systematischen psychiatrischen Untersuchung hinsichtlich des Denkens, Erlebens und Empfindens

Soziale Gegebenheiten, die die Psyche beeinflussen

Statistisches Maß für die Streuung der Werte einer Zufallsvariablen um ihren Mittelwert

Temporallappen

Temporal- und Parietallappen betreffend Fähigkeit, visuelle Reize zu erkennen, zu unterscheiden und zu verarbeiten Aufnahme, Speicherung, Selektion, Differenzierung, Analyse, Synthese, Ergänzung, Integration von akustischen Reizen

Das Zentralnervensystem betreffend Gehirn und Rückenmark 


\section{Anhang 2: \\ Beschlüsse und Empfehlungen der Kultusministerkonferenz}

Grundsätze zur Förderung von Schülerinnen und Schülern mit besonderen Schwierigkeiten im Lesen und Rechtschreiben oder im Rechnen

Beschluss der Kultusministerkonferenz vom 04.12.2003 i.d.F. vom 15.11.2007 http://www.kmk.org/fileadmin/veroeffentlichungen_beschluesse/2003/2003_ 12_04-Lese-Rechtschreibschwaeche.pdf (= KMK-Lesen/Rechtschreiben/Rechnen)

Inklusive Bildung von Kindern und Jugendlichen mit Behinderungen in Schulen, Beschluss der Kultusministerkonferenz v. 20.10.2011

http://www.kmk.org/fileadmin/veroeffentlichungen_beschluesse/2011/2011_ 10_20-Inklusive-Bildung.pdf

(= KMK-Inklusive Bildung)

Vereinbarung über die Schularten und Bildungsgänge im Sekundarbereich I, Beschluss der Kultusministerkonferenz vom 03.12.1993 i.d.F. vom 04.10.2012 http://www.kmk.org/fileadmin/veroeffentlichungen_beschluesse/1993/1993 12_03-VB-Sek-I.pdf (= KMK-Sekl)

Vereinbarung zur Gestaltung der gymnasialen Oberstufe in der Sekundarstufe II, Beschluss der Kultusministerkonferenz v. 07.07.1972 i.d.F. v. 09.02.2012 http://www.kmk.org/fileadmin/veroeffentlichungen_beschluesse/1972/1972_ 07_07-Vereinbarung-Gestaltung-Sek2.pdf (= KMK-SekII)

Vereinbarung über die Abiturprüfung der gymnasialen Oberstufe in der Sekundarstufe II. Gemeinsame Vereinbarung der Kultusministerkonferenz vom 07.07.1972 i.d.F. vom 24.10.2008 http://www.kmk.org/fileadmin/veroef fentlichungen_beschluesse/2008/2008_10_24-Abitur-Gymn-Oberstufe.pdf (= KMK-Abitur-Gym-Oberstufe)

Empfehlungen zur Sonderpädagogischen Förderung in den Schulen der Bundesrepublik Deutschland, Beschluss der Kultusministerkonferenz v. 6.5.1994 http://www.kmk.org/fileadmin/veroeffentlichungen_beschluesse/1994/1994_ 05_06-Empfehl-Sonderpaedagogische-Foerderung.pdf $(=\bar{K} M K-S P)$

Hochschulzugang für beruflich qualifizierte Bewerber ohne schulische Hochschulzugangsberechtigung, Beschluss der Kultusministerkonferenz v. 6.3.2009 
http://www.kmk.org/fileadmin/veroeffentlichungen_beschluesse/2009/2009_ 03_06-Hochschulzugang-erful-qualifizierte-Bewerber.pdf

(= KMK Hochschulzugang beruflich qualifizierte Bewerber)

Beschlüsse der KMK zu Bildungsstandards:

Übersicht über alle Beschlüsse:

http://www.kmk.org/dokumentation/veroeffentlichungen-

beschluesse/bildung-schule/allgemeine-bildung.html\#c7347

\section{Hauptschule:}

Vereinbarung über Bildungsstandards für den Hauptschulabschluss (Jahrgangsstufe 9) (Beschluss der KMK vom 15.10.2004);

http://www.kmk.org/fileadmin/veroeffentlichungen_beschluesse/2004/2004_ 10_15-Bildungsstandards-Haupt.pdf;

Bildungsstandards im Fach Deutsch für den Hauptschulabschluss (Jahrgangsstufe 9) (Beschluss der KMK vom 15.10.2004);

http://www.kmk.org/fileadmin/veroeffentlichungen_beschluesse/2004/2004_ 10_15-Bildungsstandards-Deutsch-Haupt.pdf;

Bildungsstandards für die erste Fremdsprache (Englisch/ Französisch) für den Hauptschulabschluss (Jahrgangsstufe 9) (Beschluss der KMK vom 15.10.2004); http://www.kmk.org/fileadmin/veroeffentlichungen_beschluesse/2004/2004_ 10_15-Bildungsstandards-ersteFS-Haupt.pdf;

Bildungsstandards im Fach Mathematik für den Hauptschulabschluss (Jahrgangsstufe 9) (Beschluss der KMK vom 15.10.2004);

http://www.kmk.org/fileadmin/veroeffentlichungen_beschluesse/2004/2004_ 10_15-Bildungsstandards-Mathe-Haupt.pdf

\section{Mittlerer Bildungsabschluss:}

Vereinbarung über Bildungsstandards für den Mittleren Schulabschluss (Jahrgangsstufe 10) (Beschluss der KMK vom 04.12.2003);

http://www.kmk.org/fileadmin/veroeffentlichungen_beschluesse/2003/2003_ 12_04-Vereinbarung-Bildungsstandards-MS.pdf;

Bildungsstandards im Fach Deutsch für den Mittleren Schulabschluss (Jahrgangsstufe 10) (Beschluss der KMK vom 04.12.2003);

http://www.kmk.org/fileadmin/veroeffentlichungen_beschluesse/2003/2003_ 12_04-BS-Deutsch-MS.pdf;

Bildungsstandards im Fach Mathematik für den Mittleren Schulabschluss (Jahrgangsstufe 10) (Beschluss der KMK vom 04.12.2003);

http://www.kmk.org/fileadmin/veroeffentlichungen_beschluesse/2003/2003_ 12_04-Bildungsstandards-Mathe-Mittleren-SA.pdf; 
Bildungsstandards für die erste Fremdsprache (Englisch/Französisch) für den Mittleren Schulabschluss (Jahrgangsstufe 10) (Beschluss der KMK vom

04.12 .2003

http://www.kmk.org/fileadmin/veroeffentlichungen_beschluesse/2003/2003

12_04-BS-erste-Fremdsprache.pdf

\section{Abitur}

Grundlage: Vereinbarung über Einheitliche Prüfungsanforderungen in der Abiturprüfung;

Beschluss der Kultusministerkonferenz vom 01.06.1979 i.d.F. vom 24.10.2008 http://www.kmk.org/fileadmin/veroeffentlichungen_beschluesse/2008/2008 10_24-VB-EPA.pdf

$(=\bar{E} P A)$

Liste der EPA für die einzelnen Fächer:

http://www.kmk.org/dokumentation/veroeffentlichungen-

beschluesse/bildung-schule/allgemeine-bildung.html\#c7348. 



\section{Anhang 3: \\ Schulrechtliche Vorschriften der Bundesländer (außer Gesetze)}

Eine Synopse aller maßgeblichen schulrechtlichen Rechte aus den Landesverfassungen, dem Schulrecht und der Regelungen zum Benachteiligungsverbot, allerdings nicht bezüglich Legasthenie und Dyskalkulie, mit dem Stand August 2010 bietet die Arbeitsgemeinschaft Gemeinsam leben - gemeinsam lernen e.V. an: http://www.gemeinsamleben-gemeinsamlernen.de/themen/inklusivebildung/70-regelungen-zur-sonderpaedagogischen-foerderung-in-deutschland

\section{Baden-Württemberg (BW)}

Kinder und Jugendliche mit besonderem Förderbedarf und Behinderungen, Verwaltungsvorschrift vom 8. März 1999, Az.: IV/1-6500.333/61, Fundstelle: K. u. U. 1999, S. 45, zuletzt geändert durch Verwaltungsvorschrift vom 22.08.2008 (K. u. U. 2008, S. 149 ber. S. 179), http://www.landesrecht-bw.de/jportal/?quelle=jlink\&query=VVBW-2205-1KM-19990308-SF\&psml=bsbawueprod.psml\&max=true (= BW VV Besonderer Förderbedarf).

Datenschutz an öffentlichen Schulen, Erlass vom 25,11,2009, Az.: 11-0551.0/38 http://www.landesrecht-bw.de/jportal/?quelle=jlink\&query=VVBW-KM20091125-01-SF\&psml=bsbawueprod.psml\&max=true\#ivz6 (BW- Erlass Datenschutz).

\section{Bayern}

Schulordnung für die Gymnasien in Bayern (Gymnasialschulordnung - GSO) vom 23. Januar 2007, zuletzt geändert am 29.7.2010, http://www.gesetze-bayern.de/jportal/portal/page/bsbayprod.psml?doc.id=jlrGymSchulOBY2007rahmen\&showdoccase=1\&paramfrom HL=true\#focuspoint

Förderung von Schülern mit besonderen Schwierigkeiten beim Erlernen des Lesens und des Rechtschreibens,

Bekanntmachung des Bayerischen Staatsministeriums für Unterricht und Kultus vom 16. November 1999, Amtsblatt - KWMBI. I S. 379, geändert am 11. August 2000, KWMBI I S. 403)

Veröffentlichung der KMBek und aller weiteren Schreiben zur KMBek unter http://www.schulberatung.bayern.de/schulberatung/index_05163.asp (= BY KMBek Legasthenie) 
Erläuternde Hinweise für die Schulen zum Vollzug des Bayerischen Datenschutzgesetzes,

Bekanntmachung des Bayerischen Staatsministeriums für Unterricht und Kultus vom 19. April 2001 Nr. III/4 - III/1 - L 0572 - 1/38 570 zuletzt geändert durch

KMBek vom 10. Oktober 2002 Nr. III/4 - III/1 - L 0572 - 1/101 407 http://byds.juris.de/byds/044_6.4_DSchGErlHinSchul_BY_rahmen.html (= BY KMBek Datenschutz)

Ausgleich von Prüfungsnachteilen an Berufsschulen, Berufsfachschulen, Wirtschaftsschulen, Fachschulen, Fachakademien, Fachoberschulen und Berufsoberschulen aufgrund dauernder Behinderung,

Bekanntmachung des Bayerischen Staatsministeriums für Unterricht und Kultus Vom 17. 3. 2011 Nr. VII.8-5 S 9500-6-7.3 363, KWMBI Nr. 10/2011 http://www.schulberatung.bayern.de/imperia/md/content/schulberatung/pdf/ nachteilsausgl_bs_5_11.pdf

(= BY KMBek Berufsschulen Behinderung)

\section{Berlin (BE)}

Verordnung über den Bildungsgang der Grundschule (Grundschulverordnung GsVO) vom 19. Januar 2005 (GVBI. S. 16) in der Fassung vom 9. September 2010 (GVBI. S. 440)

Insbesondere $\S \S 14$ und 16

http://www.berlin.de/imperia/md/content/sen-bildung/rechtsvorschriften/ grundschulverordnung. pdf?start\&ts=1265788173\&file=grundschulverordnung. pdf

(= BE GsVO)

Verordnung über die Schularten und Bildungsgänge der Sekundarstufe I (Sekundarstufe I - Verordnung - Sek I-VO) vom 19. Januar 2005 (GVBI. S. 28), geändert durch Artikel II der Verordnung vom 11. Dezember 2007 (GVBI. S. 677), zuletzt geändert durch Artikel I der Verordnung vom 11. Februar 2010 (GVBI. S. 82) http://www.berlin.de/imperia/md/content/sen-bildung/ rechtsvorschriften/vo_sek_i.pdf?start\&ts=1270040962\&file=vo_sek_i.pdf (= BE Sek I-VO)

Verordnung über die gymnasiale Oberstufe (VO-GO) vom 18. April 2007 (GVBI. S. 156), zuletzt geändert durch Artikel III der Verordnung vom 11. Februar 2010 (GVBI. S. 82) und Verordnung v. 18. August 2010, http://www.berlin.de/imperia/md/content/sen-bildung/ rechtsvorschriften/vo_go.pdf?start\&ts=1266916205\&file=vo_go.pdf (= BE VO-GO)

Verordnung über die sonderpädagogische Förderung (Sonderpädagogikverordnung - SopädVO)

Vom 19. Januar 2005 (GVBI. S. 57) in der Fassung v. 23. Juni 2009 (GVBI. S. 309) 
http://www.berlin.de/imperia/md/content/sen-bildung/rechtsvorschriften/ sopaed_vo.pdf?start\&ts=1266916205\&file=sopaed_vo.pdf (= BE SopädVO)

\section{Brandenburg (BB)}

Verwaltungsvorschriften über die Förderung von Schülerinnen und Schülern mit einer besonderen Schwierigkeit im Lesen und Rechtschreiben oder mit einer besonderen Schwierigkeit im Rechnen (VV-LRS) vom 6. Juni 2011 http://www.bravors.brandenburg.de/sixcms/detail.php?gsid=land_bb_bravors 01.c.50280.de (=BB VV-LRSR)

Regelungen zu den zentralen schriftlichen Abiturprüfungen 2011, Rundschreiben 7/09 vom 20.07.2009 http://www.bravors.brandenburg.de/sixcms/detail.php?gsid=land_bb_bravors 01.c.48260.de

(= BB Leistungsbewertung Abitur 2011)

\section{Bremen (HB)}

Richtlinien zur Förderung von Schülerinnen und Schülern mit besonderen Schwierigkeiten im Lesen, Schreiben und Rechnen (LRS-Erlass in der Fassung vom 01.02.2010) http://www.bildung.bremen.de/sixcms/media.php/41/e_02-2010_a.pdf (=HB LRS-Erlass)

Richtlinien zur Durchführung des Persönlichen Assistenzprogramms für körperbehinderte Schülerinnen und Schüler in den allgemeinen Schulen der Stadtgemeinde Bremen vom 1. 10.2000

http://712.joomla.schule.bremen.de/gesetze/html/341_02.htm (= HB RL Assistenz)

Richtlinien über Nachteilsausgleich für Schülerinnen und Schüler mit körperlichen Behinderungen und mit Sinnesbehinderungen bei Leistungsanforderungen und Leistungskontrollen vom 20. September 1998

http://712.joomla.schule.bremen.de/gesetze/html/241_02.htm (= HB RL Nachteilsausgleich)

Verordnung über die Aufnahme von Schülerinnen und Schülern in öffentliche allgemeinbildende Schulen vom 13. November 2009(Brem.GBI. S. 520), zuletzt geändert durch Nr. 2.1 i.V.m. Anl. 1 ÄndBek vom 24. 1. 2012 (Brem.GBI. S. 24) http://bremen.beck.de/?bcid=Y-100-G-BrAufnVOOeffSch (= HB-AufnahmeVO)

Verordnung über die Abiturprüfung im Lande Bremen (AP-V) Vom 1. Dezember 2005 (Brem.GBI. S. 585)Sa BremR 223-a-10, zuletzt geändert durch Nr. 2.1 i.V.m. Anl. 1 ÄndBek vom 24. 1. 2012 (Brem.GBI. S. 24) 
http://beck-online.beck.de/?bcid=Y-100-G-BrAPV

$(=$ HB AP-V)

Lernstandsbeschreibungen zum Übergang vom Primar- in den Sekundarbereich Die Senatorin für Bildung und Wissenschaft, 28.05.2009, Vorlage Nr. L 82/17 für die Sitzung der Deputation für Bildung am 11.06.2009 http://www2.bildung.bremen.de/sfb/behoerde/deputation/depu/l82v_17.pdf

\section{Hamburg (HH)}

Richtlinie zur Förderung von Schülerinnen und Schülern mit besonderen Schwierigkeiten im Lesen, Rechtschreiben oder Rechnen

Vom 1.11.2006, MBISchul 2006 Seite 110 http://www.hamburg.de/contentblob/69558/data/bbs-vo-richtl-foerderunglesen-rechnen-11-06.pdf (=HH Förder-Richtlinie)

Ausbildungs- und Prüfungsordnung für die Grundschule und die Jahrgangsstufen 5 bis 10 der Stadtteilschule und des Gymnasiums vom 22. Juli 2011 (HmbGVBI. S. 325) http://www.landesrecht.hamburg.de (= HH APO-GrundStGy)

Ausbildungs- und Prüfungsordnung für die Klassen 1 bis 10 der allgemeinbildenden Schulen (APO-AS) vom 22. Juli 2003, zuletzt geändert durch Artikel 1 der Verordnung vom 2. Februar 2010 (HmbGVBI. S. 118) http://www.landesrecht.hamburg.de (= HH APO-AS)

Ausbildungs- und Prüfungsordnung zum Erwerb der allgemeinen Hochschulreife (APO-AH)

Vom 25. März 2008 (HmbGVBI. S. 137), zuletzt geändert am 18. März 2009 (HmbGVBI. S. 66)

http://www.landesrecht.hamburg.de (= HH APO-AH)

\section{Hessen (HE)}

Verordnung zur Gestaltung des Schulverhältnisses vom 19. August 2011 (Gült. Verz. Nr. 721) http://www.kultusministerium.hessen.de (= HE VO-GestSchulVerh)

\section{Mecklenburg-Vorpommern (MV)}

Förderung von Schülerinnen und Schülern mit besonderen Schwierigkeiten im Lesen, Rechtschreiben oder Rechnen Verwaltungsvorschrift des Ministeriums für Bildung, Wissenschaft und Kultur 
vom 3. Mai 2011, 200H-3211-05/590, Mittl.bl. BM M-V 5/2011, S. 242 ff http://www.regierung-mv.de/cms2/Regierungsportal_prod/Regierungsportal/ $\mathrm{de} / \mathrm{bm} /$ Rechtsvorschriften/Schule/index.jsp?\&downloads=1 (=MV VV-LRR)

Die Arbeit des zentralen Diagnostischen Dienstes zur Feststellung von sonderpädagogischem Förderbedarf an allgemein bildenden Schulen in MecklenburgVorpommern

Runderlass des Ministeriums für Bildung, Wissenschaft und Kultur v. 13.8.2010 http://www.bildung-mv.de/export/sites/lisa/de/startseitennachrichten/ download/schuljahresbeginn_2010/Runderlass_DD-_E_12.08.2010.pdf (= MV Diagnostischer Dienst)

Verordnung über die Versetzung, Kurseinstufung und den Wechsel des Bildungsganges an den allgemein bildenden Schulen (Versetzungs-, Kurseinstufungs- und Durchlässigkeitsverordnung - VKDVO M-V)

vom 10. April 2007, GVOBI. M-V 2007, S. 143, Mittl.bl. BM M-V 2007, S. 250 http://mv.juris.de/mv/gesamt/SchulVersetzV_MV_2007.htm\#SchulVersetzV_M V_2007_rahmen (= MV-VKDVO)

\section{Niedersachsen (NI)}

Erlass zur Förderung von Schülerinnen und Schülern mit besonderen Schwierigkeiten im Lesen, Rechtschreiben oder Rechnen

RdErl. d. MK vom 04.10.2005 - 26 - 81631-05 VORIS 22410

http://www.mk.niedersachsen.de/live/live.php?navigation_id=1891\&article_id $=6411 \&$ \&psmand $=8$

(=NI LRR-Erlass)

Verordnung über die Durchlässigkeit sowie über Versetzungen und Überweisungen an den allgemein bildenden Schulen vom 19. Juni 1995 (Nds. GVBI S. 184), zuletzt geändert am 17.5.2010, Nds. GVBI. Nr. 14/2010 S. $227 \mathrm{ff}$ (Durchlässigkeits- und Versetzungsverordnung)

http://www.schure.de/

(= NI DVO Versetzung)

Ergänzende Bestimmungen zur Verordnung über die Abschlüsse in der gymnasialen Oberstufe, im Fachgymnasium, im Abendgymnasium und im Kolleg (EBAVO-GOFAK)

RdErl. d. MK v. 19.5.2005 - 33-83213 (SVBI. 7/2005 S. 361), geändert durch RdErl. vom 17.7.2006 (Nds.MBI. Nr. 25/2006 S. 694), zuletzt geändert am 7.6.2011, (Nds.MBI. Nr. 12/2011, S. 224)

http://www.schure.de/

(= NI EB-AVO-GOFAK) 
Die Arbeit in den Schuljahrgängen 5 bis 10 des Gymnasiums

Erl. des MK vom 3.2.04 - 303-81011, zuletzt geändert durch RdErl. v. 5.3.2009 http://www.schure.de/

(= NI Erlass „Arbeit Sek I Gymnasium“)

Die Arbeit in der Grundschule

Erlass des MK vom 3.2.2004 - 301.2-31020 - zuletzt geändert durch RdErl. v. 20.7.2005

http://www.schure.de/

(= NI Erlass „Arbeit in der Grundschule“)

\section{Nordrhein-Westfalen (NRW)}

Förderung von Schülerinnen und Schülern bei besonderen Schwierigkeiten im Erlernen des Lesens und Rechtschreibens (LRS)

RdErl. d. Kultusministeriums v. 19.7.1991 (GABI. NW. I S. 174/BASS 14-01 Nr. 1) http://www.schulministerium.nrw.de/BP/Schulrecht/Erlasse/LRS-Erlass.pdf (=NRW LRS-Erlass)

Verordnung über die Ausbildung und die Abschlussprüfungen in der Sekundarstufe I

(Ausbildungs- und Prüfungsordnung Sekundarstufe I-APO-S I)

Vom 29. April 2005 zuletzt geändert durch Verordnung vom 10. Juli 2011 (SGV. NRW. 223)

http://www.schulministerium.nrw.de/BP/Schulrecht/APOen/APO_SI.pdf (= NRW APO-S-I)

\section{Rheinland-Pfalz (RP)}

Förderung von Schülerinnen und Schülern mit besonderen Schwierigkeiten im Lesen und Rechtschreiben

Verwaltungsvorschrift des Ministeriums für Bildung, Wissenschaft, Jugend und Kultur vom 28. August 2007 (9321 - Tgb. Nr. 2308/07)

http://sonderpaedagogik.bildungrp.de/uploads/media/VV_Lernschwierigkeiten_Sek_I.pdf (=RP VV-LRS)

Abiturprüfungsordnung vom 14. Juli 1999, gültig bis 31.7.2011

GVBI 1999, S. 175; zuletzt geändert durch Verordnung v 12.1.2006 (GVBI. S. 25) http://landesrecht.rlp.de (= RP-AbiturVO)

Abiturprüfungsordnung vom 21. Juli 2010

http://gymnasium.bildung-rp.de/fileadmin/user_upload/gymnasium.bildungrp.de/mss/AbiPrO2010.pdf

(= RP AbiturVO 2010) 


\section{Saarland (SL)}

Richtlinien zur Förderung von Schülerinnen und Schülern mit besonderen Schwierigkeiten beim Erlernen des Lesens

Vom 15. November 2009, Az.: A4/B 5 - 3.7.2.0, ABI. des Saarlandes vom 3. Dezember 2009, Seite 1814

http://www.vorschriften.saarland.de/verwaltungsvorschriften/vorschriften/03_ 1853_dez_09.pdf

(=SL VV-LRS)

\section{Sachsen (SN)}

Verwaltungsvorschrift des Sächsischen Staatsministeriums für Kultus zur Förderung von Schülern mit Lese-Rechtschreib-Schwäche (VV LRS-Förderung) vom 29. Juni 2006, Az.: 34-6504.20/237, geändert durch VV vom 23. Januar 2008 (MBI.SMK S. 284) mit Wirkung vom 6. Juni 2008 http://www.revosax.sachsen.de/Details.do?sid=6379112051856\&jlink=ef\&jabs $=1$

(=SN VV-LRS)

Verordnung des Sächsischen Staatsministeriums für Kultus über Mittelschulen im Freistaat Sachsen und deren Abschlussprüfungen (Schulordnung Mittelschulen Abschlussprüfungen - SOMIAP) vom 3. August 2004, rechtsbereinigt mit Stand vom 6. März 2009 http://www.revosax.sachsen.de/Details.do?sid=3469712836453 (= SN SOMIAP)

Verwaltungsvorschrift des Sächsischen Staatsministeriums für Kultus über den Datenschutz beim Umgang mit personenbezogenen Daten an Schulen (VwV Schuldatenschutz)

Az.: 12-0278.30-40/12/2 vom 1. Februar 2007

http://www.revosax.sachsen.de/GetXHTML.do?sid=6363110685463

(= SN VwV Schuldatenschutz)

\section{Sachsen-Anhalt (ST)}

Verordnung über die sonderpädagogische Förderung vom 2.8.2005 GVBI. LSA, 2231.97, S. 482

insbesondere $\S 7$ Nachteilsausgleich http://www.mk-intern.bildung-Isa.de/Bildung/ve-sonderpaedagogische_ foerderung.pdf (=ST VO Sonderpäd. Förderung)

Unterrichtsorganisation in der Grundschule RdErl. des MK vom 3. 5. 2005 - 32-84003 http://www.mk-intern.bildung-Isa.de/Bildung/er- 
unterrichtsorganisation_gs.pdf

(=ST Grundschulunterichts-Erlass)

Leistungsbewertung in der Grundschule

RdErl. des MK vom 2. 7. 2003-31-83200 (SVBI. LSA S. 205) zuletzt geändert

durch RdErl. des MK vom 30.06.2008

insbesondere $\mathrm{Nr}$. 4. Besondere Bestimmungen

http://www.mk-intern.bildung-lsa.de/Bildung/er-

leistungbeurteilunggrundschulen.pdf

(=ST Erlass Grundschulbewertung)

Leistungsbewertung und Beurteilung an allgemein bildenden Schulen und

Schulen des Zweiten Bildungsweges der Sekundarstufen I und II

RdErl. des MK vom 1. 7. 2003 (SVBI. LSA S. 195), geändert durch RdErl. des MK vom 1. 7. 2004 (SVBI. LSA S. 129)

insbesondere Nr. 5. Besondere Bestimmung zur Leistungsbewertung http://www.mk-intern.bildung-lsa.de/Bildung/er-

leistungbeurteilungzwbildungsweg.pdf

(=ST Sekundarstufenbewertungs-Erlass)

Verordnung über Abschlüsse in Sek I v. 20. 7. 2004

GVBI. LSA, 2231.9, S. 476, zuletzt geändert durch 2. Ä-VO vom 10.9.2010, GVBL LSA, S. 503

insbes. § 20 Schüler und Schülerinnen mit diagnostizierten Lernstörungen

http://www.mk-intern.bildung-lsa.de/Bildung/ve-

abschlussverordnungsek1_2004.pdf

(=ST VO Abschlüsse)

Verordnung über die Übergänge zwischen den Schulformen in der Sekundarstufe I

(Sek I-Üg-VO) vom 1. April 2004.

http://www.mk-intern.bildung-lsa.de/Bildung/ve-uebergangsek1.pdf (= ST Sek I-Üg-VO)

\section{Schleswig-Holstein (SH)}

Förderung von Schülerinnen und Schülern mit Lese-Rechtschreib-Schwäche (Legasthenie)

Erlass des Ministeriums für Bildung und Frauen vom 27. Juni 2008 - III 316 321.01-20 (NBI. MBF. Schl.-H. 2008 S. 226)

http://www.schooloffice-sh.de/texte/l/legasthenie.htm (=SH Legasthenie-Erlass)

FAQs zum Erlass „Förderung von Schülerinnen und Schülern mit LeseRechtscheib-Schwäche (Legasthenie)" vom 27. Juni 2008 - Stand März 2009 http://www.schooloffice-sh.de/texte/l/legasthenie.htm\# 
Ministerium_für_Bildung_und_Frauen_des_Landes_Schleswig-Holstein (= SH Legasthenie FAQ)

Förderung von Schülerinnen und Schülern mit besonderen Schwierigkeiten im Rechnen (Rechenschwäche)

Erlass des Ministeriums für Bildung und Kultur v. 19.3.2012, III 225

http://www.schleswig-holstein.de

/= SH Rechenschwäche-Erlass)

LRS-Erlass: Verwahrung der Unterlagen

Erlass des Ministeriums für Bildung und Frauen vom 18. März 2009

http://www.schooloffice-sh.de/texte/I/legasthenie.htm\#

Ministerium_für_Bildung_und_Frauen_des_Landes_Schleswig-Holstein (= SH Legasthenie Datenschutz)

Landesverordnung über die Erteilung von Zeugnissen, Noten und anderen ergänzenden Angaben in Zeugnissen (Zeugnisverordnung - ZVO) v. 29. April 2008 NBI. MBF. Schl.-H. 2008, 146

http://www.schooloffice-sh.de/texte/z/zeugnisordung.htm (=SH ZeugnisVO)

\section{Thüringen (TH)}

Thüringer Schulordnung für die Grundschule, die Regelschule, das Gymnasium und die Gesamtschule - ThürSchulO -

vom 20. Januar 1994 (GVBI. S. 185), zuletzt geändert durch die 11. Änderungsverordnung vom 10. Juni 2009 (GVBI. S. 511)

http://www.thueringen.de/de/tmbwk/bildung/schulwesen/schulordnungen/sc hulordnung

(= TH SchulO)

Fachliche Empfehlung zu Fördermaßnahmen für Kinder und Jugendliche mit besonderen Lernschwierigkeiten in den allgemein bildenden Schulen (außer Förderschule) in Thüringen vom 20. August 2008

Internes Schreiben an die Schulen und Schulämter.

Eine Veröffentlichung ist nicht erfolgt. Die Fachliche Empfehlung kann daher nur im Internet abgerufen werden.

http://www.thueringen.de/imperia/md/content/tkm/schule/foerderung/fachli che_empfehlung_lernschwierigkeiten_20_08_2008.pdf

(=TH Fachliche Förderempfehlung)

Erlass TMBWK: Schreiben an die Staatlichen Schulämter zum Nachteilsausgleich nach § 59 ThürSchulO v. 19.7.2011 http://www.thueringen.de/imperia/md/content/tmbwk/bildung/gemeinsamer unterricht/aktuelles/nachteilsausgleich.pdf 



\section{Abkürzungsverzeichnis}

Für die üblichen und juristischen Abkürzungen wird verwiesen auf Kirchner/ Butz, Abkürzungsverzeichnis der Rechtssprache, 6. Auflage 2008

CRPD

Committee on the Rights of Persons with Disabilities

DIMDI

Deutsches Institut für Medizinische Information und Dokumentation

GdB

Grad der Behinderung

$I C D$

International Statistical Classification of Diseases and Relates Health Problems

ICF

International Classification of Functioning, Disability and Health (ICF)

MAS Multiaxiales Klassifikationsschema für psychische Störungen des Kindes- und Jugendalters nach ICD-10 der WHO

$\mathrm{PR}$

Prozentrang 



\section{Zeitschriftenverzeichnis}

Für die deutschen juristischen Zeitschriften wird verwiesen auf Kirchner/Butz, Abkürzungsverzeichnis der Rechtssprache, 6. Auflage 2008

Deutsches Ärzteblatt

Disability Studies Quarterly

E.H.R.L.R.

European Human Rights Law Review

Europäische Zeitschrift zum Anti-

diskriminierungsrecht

European Child and Adolescense

Psychiatry

Fortschritte der Neurologie Psychi-

atrie

Gemeinsam leben - Zeitschrift für

integrative Erziehung

Journal of Applied Psychology

Journal of Learning Disabilities

Lernen und Lernstörungen

Molecular Psychiatry

Neurowissenschaft, Psychologie,

Pädagogik

report psychologie

Scientific Studies of Reading

Sonderpädagogische Förderung

heute

Sozialextra

Zeitschrift für Soziale Arbeit und Sozialpolitik

VHN

Vierteljahreszeitschrift für Heilpädagogik und ihre Nachbargebiete

Z Erziehungswiss

Zeitschrift für Erziehungswissenschaften

Zeitschrift des BVL

Mitgliederzeitschrift des Bundesverbandes Legasthenie und Dyskalkulie e.V.

Zeitschrift für Heilpädagogik

Zeitschrift für integrative Erziehung 
Zeitschrift für Kinder- und Jugendpsychiatrie und Psychotherapie Zschr.klin. Psychol. Psychiatr. Psy- Zeitschrift für Klinische Psychologie, Psychother chiatrie und Psychotherapie 


\section{Literaturverzeichnis}

Aguilar, Yolanda: Neuropsychologische Profile von mexikanischen und deutschen Kindern mit Lese- Rechtschreibstörungen, Diss. Universität Tübingen 2006, http://w210.ub.uni-tuebingen.de/volltexte/2007/3009, zuletzt geprüft am 20.01.2013.

Aichele, Valentin: Stellungnahme der Monitoring-Stelle zur UN-Behindertenrechtskonvention zur UN-Behindertenrechtskonvention zur Stellung der UN-Behindertenrechtskonvention innerhalb der deutschen Rechtsordnung und ihre Bedeutung für behördliche Verfahren und deren gerichtliche Überprüfung, insbesondere ihre Anforderungen im Bereich des Rechts auf inklusive Bildung nach Artikel 24 UN-Behindertenrechtskonvention, http://www.institut-fuer-menschenrechte.de/de/presse/stellungnahmen/ stellungnahme-der-monitoring-stelle-zur-un-behindertenrechtskonvention.html, zuletzt geprüft am 20.01.2013, zit.: Stellungnahme DIMR 2010.

Aichele, Valentin: Die UN-Behindertenrechtskonvention und ihr Fakultativprotokoll, http://www.institut-fuer-menschenrechte.de/uploads/tx commerce/policy_paper_9_die_un_behindertenrechtskonvention_und _ihr_fakultativprotokoll.pdf, zuletzt geprüft am 20.01.2013, zit.: UN-BRK und Fakultativprotokoll.

Aichele, Valentin: Die UN-Behindertenrechtskonvention in der gerichtlichen Praxis; Eine Aufgabe für die Anwaltschaft: Die Rezeption menschenrechtlicher Normen durchsetzen, Anwaltsblatt 2011, S. 727-730.

Aichele, Valentin: Deutsches Institut für Menschenrechte: Stellungnahme der Monitoring-Stelle (31. März 2011) - Eckpunkte zur Verwirklichung eines inklusiven Bildungssystems (Primarstufe und Sekundarstufen I und II), http://www.institut-fuer-menschenrechte.de/uploads/tx_commerce/ stellungnahme_der_monitoring_stelle_eckpunkte_z_verwirklichung_eines inklusiven_bildungssystems_31_03_2011.pdf, zuletzt geprüft am 20.01.2013.

Aichele, Valentin: Barrieren im Einzelfall überwinden: Angemessene Vorkehrungen gesetzlich verankern, 2012, http://www.institut-fuer-menschenrechte.de/uploads/tx_commerce/positionen_nr_5_barrieren_im_einzelfall _ueberwinden.pdf zit.: Angemessene Vorkehrungen.

Aichele, Valentin/Althoff, Nina: Nicht-Diskriminierung und angemessene Vorkehrungen in der UN-Behindertenrechtskonvention, in: Welke, UNBehindertenrechtskonvention mit rechtlichen Erläuterungen, 2012, zit.: Kommentar zur UN-BRK, Nicht-Diskriminierung.

Alexy, Robert: Theorie der Grundrechte, 1994, zit.: Theorie der Grundrechte. 
Althoff, Nina: Diskriminierungsschutz aus den Menschenrechten; Chance und Verantwortung für die Anwaltschaft, Anwaltsblatt 2011, S. 482-484.

Althoff, Nina: Das Individualbeschwerdeverfahren zu den UN-

Fachausschüssen, Anwaltsblatt 2012, S. 52-53.

Amelow, Kathrin: Lese- und Rechtschreibschwierigkeiten; Möglichkeiten schulischer Früherkennung und Förderung, 2007, zit.: Lese- und Rechtschreibschwierigkeiten.

Amorosa, Hedwig/Noterdaeme, Michèle: Therapie schwerer Lese-Rechtschreib-Störungen, in: Suchodoletz/Amorosa, Therapie der Lese-Rechtschreib-Störung (LRS), 2003, S. $79 \mathrm{ff}$.

Anderson, Peggy L./Meier-Hedde, Regine: International case studies of dyslexia, 2011, zit.: International Case Studies.

Ansari, Daniel: Was haben wir durch die kognitive Neurowissenschaft über die Dyskalkulie gelernt? in: Schulte-Körne, Legasthenie und Dyskalkulie: Stärken erkennen - Stärken fördern, 2011, S. 235-244.

Arnade, Sigrid: Die Gesellschaft der Behinderer; Das Buch zur Aktion Grundgesetz, 1997, zit.: Gesellschaft der Behinderer.

Arnade, Sigrid: Übereinkommen über die Rechte von Menschen mit Behinderungen, http://www.netzwerk-artikel-3.de/dokum/schattenuebersetzungendgs.pdf, zuletzt geprüft am 20.01.2013.

Arnold, Karl-Heinz/Graumann, Olga/Rakhhochkine, Anatoli: Handbuch Förderung; Grundlagen, Bereiche und Methoden der individuellen Förderung von Schülern, 2008, zit.: Hdb. Förderung.

Aßmann: Art. 19 GG, in: Epping/Hillgruber, Beck'scher Online-Kommentar, 2009, zit.: Aßmann in BeckOK GG.

Aster, Michael v./Lorenz, Jens Holger: Rechenstörungen bei Kindern; Neurowissenschaft, Psychologie, Pädagogik, 2005, zit.: Rechenstörungen bei Kindern.

Avenarius, Hermann/Heckel, Hans: Schulrechtskunde, 7. Aufl., 2000, zit.: Schulrecht.

Bader, Johann/Funke-Kaiser, Michael/Kuntze, Stefan/von Albedyll, Jörg: Verwaltungsgerichtsordnung; Kommentar anhand der höchstrichterlichen Rechtsprechung, 4. Aufl., 2007, zit.: VwGO.

Badura, Peter: Art. 7, in: Maunz/Dürig/Herzog, Kommentar zum Grundgesetz, 55. Aufl., 2009, zit.: Badura in Maunz/Dürig/Herzog GG.

Badura, Peter/Merten, Detlef/Papier, Hans-Jürgen: Handbuch der Grundrechte in Deutschland und Europa, 2004, 2006, 2009, zit.: Hdb. Grundrechte Band, §.

Banafsche, Minou: Anmerkung zu den Urteilen des BVerwG vom 3. März 2011, Az.: 5 C 15.10 und 5 C 16.10, br 2011, S. 180-182. 
Banafsche, Minou: Die UN-Behindertenrechtskonvention und das deutsche Sozialrecht; eine Vereinbarkeitsanalyse anhand ausgewählter Beispiele, SGb 2012, S. 373-379, 440-444.

Bastian-Becker, Barbara: Entgleisende Beziehungen in deutschen Schulen - Zur malignen Dynamik zwischen Kindern, Lehrern und Eltern aus psychoanalytischer Sicht, in: Overwien/Prengel, Recht auf Bildung, 2007, S. 224-236.

Bauer, Jobst-Hubertus/Göpfert, Burkard/Krieger, Steffen: Allgemeines Gleichbehandlungsgesetz; Kommentar, 2. Aufl., 2008, zit.: AGG.

Baumbach, Adolf/Lauterbach, Wolfgang/Albers, Jan/Hartmann, Peter: Zivilprozessordnung, 67. Aufl., 2009, zit.: ZPO.

Beaucamp, Guy: Das Behindertengrundrecht (Art. 3 Abs. 3 Satz 2 GG) im System der Grundrechtsdogmatik, DVBI 2002, S. 997-1004.

Beaucamp, Guy: Das Verbot der Diskriminierung Behinderter (Art. 3 III 2 GG), JA 2001, S. 36-41.

Behrens, Ulrike: Förderung von Schülerinnen und Schülern mit besonderen Schwierigkeiten im Lesen, Rechtschreiben oder Rechnen, Nds. SVBI 2006, S. 189-191.

Behrens, Ulrike/Wachtel, Peter: Nachteilsausgleich in der Schule, Nds. SVBI 2008, S. 144-146.

Benda, Ernst/Maihofer, Werner/Vogel, Hans-Jochen: Handbuch des Verfassungsrechts, 2. Aufl., 1994, zit.: Hdb. des Verfassungsrechts.

Berlit, Uwe: Rechtspolitik zur Gleichstellung behinderter Menschen, RdJB 1996, S. 145-153.

Bernstorff, Jochen von: Anmerkungen zur innerstaatlichen Anwendbarkeit ratifizierter Menschenrechtsverträge: Welche Rechtswirkungen erzeugt das Menschenrecht auf inklusive Schulbildung aus der UN-Behindertenrechtskonvention im deutschen Sozial- und Bildungsrecht, RdJB 2011, S. 203-217.

Berufsverband für Kinder-und Jugendpsychiatrie, Psychosomatik und Psychotherapie Deutschland (BKJPP) in: Statistische Daten über die Versorgung mit Kinder- und Jugendpsychiatern in Deutschland, http://www.bkjpp.de/files/ situation_kjpp2007.pdf, zuletzt geprüft am 20.01.2013.

Beyerlin, Ulrich: Schulische Integration und der Handlungsauftrag des Staates aus Art. 3 Abs. 3 Satz 2 GG, RdJB 1999, S. 157-169.

Beyer, Regina-Maria: Kognitive und psychosoziale Faktoren bei Kindern mit Aufmerksamkeitsdefizit-/ Hyperaktivitätsstörung (ADHS) und Lese-Rechtschreibstörung. Univ. Diss. Würzburg 2006, http://www.opus-bayern.de/ uni-wuerzburg/volltexte/2006/1893/, zit.: Kognitive und psychosoziale Faktoren. 
Bickel, Heike/Marwege, Gabriele/Zier, Johanna: Ratgeber zur Finanzierung außerschulischer Therapien bei Legasthenie und Dyskalkulie; Broschüre des Bundesverbandes Legasthenie und Dyskalkulie e.V., 2007, zit.: Ratgeber Finanzierung außerschulische Therapien.

Biermann, Adrienne/Goetze, Herbert: Sonderpädagogik; Eine Einführung, 2005, zit.: Sonderpädagogik.

Birnbaum, Christian: Die Rügepflicht des Prüflings, NVwZ 2006, S. 286-296.

BMBF: Zur Entwicklung nationaler Bildungsstandards, Bildungsforschung Band 1; http://www.bmbf.de/pub/zur_entwicklung_nationaler_ bildungsstandards.pdf, zuletzt geprüft am 20.01.2013.

$B M B F$ : Begabte Kinder finden und fördern, Ein Ratgeber für Eltern und Lehrer; www.bmbf.de/pub/b_Kinder.pdf, zuletzt geprüft am 20.01.2013.

Bogdanowicz, Marta/Sayles, H. Alan: Rights of Dyslexic Children in Europe, 2004, zit.: Rights of Dyslexic Children in Europe.

Bohl, Johannes: Der „ewige Kampf“ des Rechtsanwalts um Akteneinsicht, NVwZ 2005, S. 133-140.

Böhm, Thomas: Elternrechte in der Schule; So machen Sie sich stark für Ihr Kind, 2007, zit.: Elternrechte in der Schule.

Bonk, Heinz Joachim/Kallerhoff, Dieter: § 29, in: Stelkens/Bonk/Sachs, Verwaltungsverfahrensgesetz, 7. Aufl., 2008.

Bos, Wilfried: IGLU 2006: Lesekompetenzen von Grundschulkindern in Deutschland im internationalen Vergleich, 2007, zit.: Bos, IGLU 2006.

Boysen, Sigrid: Art. 3 GG, in: von Münch/Kunig, Grundgesetz, 6. Aufl., 2012.

Brockhaus: Enzyklopädie, 17. Aufl., 1970, zit.: Enzyklopädie.

Brockmann, Jürgen/Littmann, Klaus-Uwe/Schippmann, Thomas: Niedersächsisches Schulgesetz; (NSchG); Kommentar mit Ausführungsbestimmungen, 2002, zit.: Nds. SchulG.

Brüggen, Georg: Kurzgutachten zu den verfassungsrechtlichen, planungsrechtlichen und vergaberechtlichen Gesichtspunkten des Projekts Waldschlößchenbrücke in Dresden, http://www.brueggen-ra.de/pdf/Weltkulturerbe/ GA_UNESCO_Weltkulturerbe_Elbtal_06-08-12.pdf, zuletzt geprüft am 20.01.2013.

Bryde, Brun-Otto: Programmatik und Normativität der Grundrechte, in: Badura/Merten/Papier, Handbuch der Grundrechte in Deutschland und Europa, Bd. I, 2004, § 17.

Buch, Michael: Das Grundrecht der Behinderten (Art. 3 Abs. 3 S. 2 GG), 2001, zit.: Grundrecht der Behinderten. 
Büchner, Britta/Kortländer, Michael/Werner, Birgit/Robering, Nicole/Schönweiss, Friedrich: „Legasthenie - eine Krankheit, eine Behinderung, eine Störung?", http://www.legakids.net/fileadmin/user_upload/Downloads/Info/ Wissenschaft/Krankheit_Artikel.pdf, zuletzt geprüft am 20.01.2013.

Bundesregierung: Gutachten betreffend die innerstaatliche Bindungswirkung des UNESCO-Übereinkommens zum Schutz des Natur- und Kulturerbes der Welt, archiv.welterbe-erhalten.de/pdf/080116_Gutachten_UNESCO.pdf, zuletzt geprüft am 20.01.2013.

Bundesregierung: Unser Weg in eine inklusive Gesellschaft, Der Nationale Aktionsplan der Bundesregierung zur Umsetzung der UN-Behindertenrechtskonvention, September 2011, http://www.bmas.de/SharedDocs/ Downloads/DE/PDF-Publikationen/a740-nationaler-aktionsplanbarrierefrei.pdf?_blob=publicationFile, zuletzt geprüft am 20.1.2013, zit.: Bundesregierung, Nationaler Aktionsplan zur UN-BRK.

Bundesverband Deutscher Augenärzte: Legasthenie und Sehvermögen, http://www.augeninfo.de/patinfo/lega.pdf, zuletzt geprüft am 20.01.2013.

Bundeszentrale für gesundheitliche Aufklärung (BZgA), 51109 Köln: Chronische Erkrankungen als Problem und Thema in Schule und Unterricht, http://www.bzga.de/pdf.php?id=b50e75b047e2c2bd22e3d2e6042e3499, zuletzt geprüft am 20.01.2013.

Bundschuh, Konrad/Heimlich, Ulrich/Krawitz, Rudi: Wörterbuch Heilpädagogik; Ein Nachschlagewerk für Studium und pädagogische Praxis, 3. Aufl., 2007, zit.: Wörterbuch Heilpädagogik.

Burghart, Axel: Die Pflicht zum guten Gesetz, 1996, zit.: Pflicht zum guten Gesetz.

Caspar, Johannes: Das Diskriminierungsverbot behinderter Personen nach Art. 3 Abs. 3 S. 2 GG und seine Bedeutung in der aktuellen Rechtsprechung, EuGRZ 2000, S. 135-144.

Castendiek, Jan: Behinderung und freie Schulartwahl, in: Erbguth/Müller/Neumann, Rechtstheorie und Rechtsdogmatik im Austausch, 1999, S. 337-354.

Cloerkes, Günther/Felkendorff, Kai/Markowetz, Reinhard: Soziologie der Behinderten; Eine Einführung, 3. Aufl., 2007, zit.: Soziologie der Behinderten.

Coester-Waltjen, Dagmar: Art. 6, in: Münch, von/Kunig, GrundgesetzKommentar, 5. Aufl., 2001, zit.: Coester-Waltjen in Münch GG.

Colella, Adrienne/DeNisi, Angelo/Varma, Arup: The Impact of Ratee's Disability on Performance Judgements and Choice as Partner: The Role of Disabilityfit Stereotypes and Interdependence of Rewards, Journal of Applied Psychology 83, 1998, S. 102-111. 
Daniel, Stephanie S./Walsh, Adam K./Goldston, David B./Arnold, Elisabeth M./ Reboussin, Beth A./Wood, Frank B.: Suicidality, school dropout, and reading problems among adolescents, Journal of Learning Disabilities 2006,

S. 507-514.

Däubler, Wolfgang/Bertzbach, Martin/Ambrosius, Barbara: Allgemeines

Gleichbehandlungsgesetz; Handkommentar, 2. Aufl., 2008, zit.: AGG.

Dederich, Markus: Menschen mit Behinderung zwischen Ausschluss und Anerkennung, 2001, zit.: Zwischen Ausschluss und Anerkennung.

Degener, Theresia: Verfassungsrechtliche Probleme mit der Behindertendiskriminierung in Deutschland, Kritische Justiz 2000, S. 425-433.

Degener, Theresia: Antidiskriminierungsrechte für Behinderte; Ein globaler Überblick, ZaöRV 2005, S. 887-935.

Degener, Theresia: The Definition of Disability in German and Foreign Discrimination Law, Disability Studies Quarterly 2006.

Degener, Theresia: Antidiskriminierungsrecht; Handbuch für Lehre und Beratungspraxis; mit Lösungsbeispielen für typische Fallgestaltungen, 2008, zit.: Antidiskriminierungsrecht.

Degener, Theresia: Rechtswissenschaftliche Grundlagen, in: Degener, Antidiskriminierungsrecht, 2008, S. 68-114.

Degener, Theresia: Die UN-Behindertenrechtskonvention als Inklusionsmotor, RdJB 2009, S. 200-219.

Degener, Theresia: Welche legislativen Herausforderungen bestehen in Bezug auf die nationale Implementierung der UN-Behindertenrechtskonvention in Bund und Ländern, br 2009, S. 34-51.

Deinert, Olaf/Neumann, Volker: Rehabilitation und Teilhabe behinderter Menschen; Handbuch SGB IX, 2. Aufl., 2009, zit.: Handbuch SGB IX.

Denninger, Erhard/Hoffmann-Riem, Wolfgang/Schneider, Hans-Peter/Stein, Ekkehard: Kommentar zum GG; Alternativkommentar, 3. Aufl., 2002, zit.: AK-GG.

Deutsch, Erwin/Spickhoff, Andreas: Medizinrecht; Arztrecht, Arzneimittelrecht, Medizinprodukterecht und Transfusionsrecht, 6. Aufl., 2008, zit.: Medizinrecht.

Dirnaicher, Udo: Bayerisches Schulrecht: Keine Benachteiligung Behinderter, BayVBI 1997, S. 545-552.

Dolzer, Rudolf/Waldhoff, Christian/Graßhof, Karin: Bonner Kommentar zum Grundgesetz, Stand April 2010, zit.: Bonner Kommentar GG.

Dreier, Horst: Grundgesetz Kommentar, 2. Aufl., 2004, zit.: Dreier GG. 
Dür, Wolfgang/Fürth, Kristina/Griebler, Robert: Strategien der Schule zur Kompensation importierter und Reduktion intern erzeugter gesundheitlicher Unterschiede bei Kindern und Jugendlichen, in: Richter/Hurrelmann, Gesundheitliche Ungleichheit, 2006, S. 405-421.

Eckertz-Höfer: Art. 3, in: Denninger/Hoffmann-Riem/Schneider/Stein, Kommentar zum GG, 3. Aufl., 2002, zit.: Eckertz-Höfer in AK-GG

Eggers, Christian/Fegert, Jörg M./Resch, Franz: Psychiatrie und Psychotherapie des Kindes- und Jugendalters, 2004, zit.: Psychiatrie.

Ehlert, Antje/Schroeders, Ulrich/Fritz-Stratmann, Annemarie: Kritik am Diskrepanzkriterium in der Diagnostik von Legasthenie und Dyskalkulie, Lernen und Lernstörungen 1, 2012, S. 169-184.

Ehrich, Andreas: Specialisterne - Die Spezialisten, Impulse, Fachzeitschrift für unterstützte Beschäftigung 4, 2008, S. 34-40; http://www.bag-ub.de/ impulse/download/impulse48-web.pdf, zuletzt geprüft am 20.01.2013.

Eichholz, Reinald: Paradigmenwechsel im Schulwesen?; Zum Vorrang des Kindeswohls nach Art. 3 der UN-Kinderrechtekonvention, R\&B 2007, S. 3-10.

Eichholz, Reinald: „Gibt es ein Recht auf eine Schule für alle?", http://www.mllev.de/uploads/media/Vortrag_Dr_Reinald_Eichholz_Schrift fassung.pdf, zuletzt geprüft am 20.01.2013.

Eid, Michael/Petermann, Franz: Handbuch der Psychologie; Handbuch der Psychologischen Diagnostik, 2006, zit.: Hdb. Psychologie.

Engelken, Klaas: Nochmals: Der Diskriminierungsschutz Behinderter im Grundgesetz; Sonderschulzuweisung als unzulässig Benachteiligung?, DVBI 1997, S. 762-763.

Engelmann, Ruth/Plaum, Ernst: Sind bei Lese- und Rechtschreibschwierigkeiten "Störung" und "Schwäche" als sinnvolle Untergruppen definierbar? report psychologie 2008, S. 16-29.

Enkhardt, Dieter: Zoff mit der Schule; Konfliktgespräche mit Lehrern führen, 1. Aufl., 2002, zit.: Zoff mit der Schule.

Ennuschat, Jörg: Völker-, europa- und verfassungsrechtliche Rahmenbedingungen der Schulpflicht, RdJB 2007, S. 271-291.

Ennuschat, Jörg: Chancengleichheit für Schülerinnen und Schüler mit Legasthenie und Dyskalkulie, br 2008, S. 93-99.

Ennuschat, Jörg: Die Rechte stotternder Menschen in Schule, Ausbildung und Studium; erstattet der Bundesvereinigung Stotterer-Selbsthilfe e.V., 2. Aufl., 2010, zit.: Die Rechte stotternder Menschen in Schule, Ausbildung und Studium.

Ennuschat, Jörg: Die UN-Behindertenrechtskonvention und die Chancengleichheit für Schülerinnen und Schüler mit Behinderung, in: Sachs/Siekmann, Der grundrechtsgeprägte Verfassungsstaat, 2012, S. 711-725. 
Ennuschat, Jörg/Volino, Angela: Art. 3 Abs. 3 S. 2 GG als Grundlage für Notenschutz zugunsten von Schülern und Schülerinnen mit Behinderungen?, br 2009, S. 166-170.

Epping/Hillgruber: Beck'scher Online-Kommentar, 2009, zit.: BeckOK GG.

Epping, Volker: Grundrechte, 4. Aufl., 2010, zit.: Grundrechte.

Erbguth, Wilfried/Müller, Friedrich/Neumann, Volker: Rechtstheorie und Rechtsdogmatik im Austausch; Gedächtnisschrift für Bernd Jean d'Heur, 1999, zit.: GS Jean d'Heur.

Erler, Thomas: Gesprächskreis Legasthenie Albert-Einstein-Schule-Laatzen, http://www.aes-laatzen.de/news/disundleg/leg.pdf, zuletzt geprüft am 20.01.2013.

Esser, Günter/Schmidt, Martin H.: Die langfristige Entwicklung von Kindern mit Lese-Rechtschreibschwäche, Zschr. klin. Psychol. Psychiatr. Psychother 1993, S. 100-116.

Esser, Günter/Wyschkon, Anne/Schmidt, Martin H.: Was wird auch Achtjährigen mit einer Lese-Rechtschreibstörung?; Ergebnisse im Alter von 25 Jahren, Zschr. klin. Psychol. Psychiatr. Psychother 31, 2002, S. 235-242.

Europarat: Common European Framework of Reference for Languages, http://www.coe.int/t/dg4/linguistic/Source/Framework_EN.pdf, zuletzt geprüft am 20.01.2013.

Faber, Angela/Roth, Verena: Die Umsetzung der UN-Behindertenrechtskonvention durch die Schulgesetzgeber der Länder, DVBI 2010, S. 1193-1204.

Fastenrath, Ulrich: Rechtsgutachterliche Stellungnahme zur Zulässigkeit des Baus der Waldschlösschenbrücke, http://www.google.de/url?sa=t\&rct= $j \& q=\& e s r c=s \& f r m=1 \&$ source $=$ web\& $c d=3 \&$ cad $=$ rja\&ved $=0 C C O Q F j A C \& u r l=$ http\%3A\%2F\%2Ftu-dresden.de\%2Fdie_tu_dresden\%2Ffakultaeten\%2 Fjuristische_fakultaet\%2Fjfoeffl3\%2Fnews\%2FWelterbestatus.pdf\&ei= eSCQUMzWHKuK4gThulHABQ\&usg=AFQjCNH_F6Qz9APeOfFNZwv8Vel DLsbrMg, zuletzt geprüft am 20.01.2013.

Fegert, Jörg: Führt der Weg zum Jugendamt der Zukunft über § 35 a SGB VIII als Einstieg zur „großen Lösung“?, JAmt 2010, S. 267-274.

Fegert, Jörg/Streeck-Fischer, Annette/Freyberger, Harald: Adoleszenzpsychiatrie; Psychiatrie und Psychotherapie der Adoleszenz und des jungen Erwachsenenalters, 2009, zit.: Adoleszenzpsychiatrie.

Fehling, Michael/Arnold, Sascha: Verfassungsfragen eines privilegierten Zugangs von Abiturienten mit Migrationshintergrund zum Lehramtsstudium, RdJB 2011, S. 316-335.

Forkel, Hans-Walter: Unterrichtsausfall als Rechtsproblem; Schulpflicht, Teilhaberecht, Chancengleichheit, SächsVBI 2010, S. 282-285. 
Frowein, Jochen Abraham: Rechtsgutachten zu der Frage, inwieweit ein Anspruch auf Aufnahme von Behinderten in allgemeine Schulen besteht, 1996, zit.: Rechtsgutachten.

Fuerst, Anna-Miria: Behinderung zwischen Diskriminierungsschutz und Rehabilitationsrecht; Ein Vergleich zwischen Deutschland und den USA. 2009, zit.: Behinderung zwischen Diskriminierungsschutz und Rehabilitationsrecht.

Füssel, Hans-Peter: „Integrative Beschulung (ist die) verstärkt realisierungswürdige Alternative zur Sonderschule"; Anmerkung zum Beschluss des Bundesverfassungsgerichts vom 8. Oktober 1997, RdJB 1998, S. 250-255.

Gagel, Alexander: Benachteiligung wegen Behinderung, jurisPR-ArbR 9/2009.

Ganser, Bernd/Richter, Wiltrud: Was tun bei Legasthenie in der Sekundarstufe? 2. Aufl., 2005, zit.: Legasthenie.

Gantner, Eva: Fördertipps für schulisches und häusliches Lernen und Korrekturempfehlungen, in: Ganser/Richter, Was tun bei Legasthenie in der Sekundarstufe? 2005, S. 162-187.

Gasteiger-Klicpera, Barbara/Julius, Henri/Klicpera, Christian: Handbuch Sonderpädagogik; Sonderpädagogik der sozialen und emotionalen Entwicklung. Band 3, 2008, zit.: Hdb. Sonderpädagogik.

Geiger, Rudolf: Grundgesetz und Völkerrecht mit Europarecht; Die Bezüge des Staatsrechts zum Völkerrecht und Europarecht, 5. Aufl., 2010, zit.: Völkerrecht.

Geiger, Rudolf/Khan, Daniel-Erasmus/Kotzur, Markus: Kommentar zum EUV/AEUV, 5. Aufl., 2010, zit.: Geiger/Khan/Kotzur EUV/AEUV.

Geist, Alexander: Identifikation, Diagnostik und Förderung von Schüler mit LRS und ADS am Anne-Frank-Gymnasium Erding, in: Schulte-Körne, Legasthenie und Dyskalkulie: Stärken erkennen - Stärken fördern, 2011, S. $165-184$.

Giesen, Thomas: Schutz des Persönlichkeitsrechts im öffentlichen Bereich, http://www.saechsdsb.de/images/stories/sdb_inhalt/oeb/taetigkeitsberic hte/9_TB.pdf, zuletzt geprüft am 20.01.2013.

Gitter, Wolfgang: $\S \S 104$ ff, in: Säcker/Rixecker, Münchener Kommentar zum Bürgerlichen Gesetzbuch, 5. Aufl., 2009, zit.: Gitter in MüKomm BGB.

Glotz, Peter/Faber, Klaus: Richtlinien und Grenzen des Grundgesetzes für das Bildungswesen, in: Benda/Maihofer/Vogel, Handbuch des Verfassungsrechts, 1994, § 28.

Götzinger-Hiebner, Maria: Das Rechtschreibverwirrbuch; oder: Wie man Kinder fertig macht, 3. Aufl., 2002, zit.: Rechtschreibverwirrbuch.

Grimm, Tiemo: Schicksal Legasthenie, http://www.medgenetik.de/2006/ 2006_2_145_grimm.pdf, zuletzt geprüft am 20.01.2013. 
Gronauer, Barbara: Best Practice im Umgang mit legasthenen Schülern, Internationaler Ländervergleich, http://bvl-infothek.de/sites/bvl-infothek.de/ files/Internationaler\%20L\%C3\%A4ndervergleich\%202012.pdf zuletzt geprüft am 20.01.2013.

Gröschner: Art. 7, in: Dreier, Grundgesetz Kommentar, 2. Aufl., 2004, zit.: Gröschner in Dreier GG.

Gros, Daniel/Sagmeister, Sonja: Nachkrisenzeit; Wie die erfolgreichste Denkfabrik Europas unsere Welt für die nächste Generation sieht, http://www.ceps.be/book/nachkrisenzeit-\%E2\%80\%93-und-was-kommtjetzt, zuletzt geprüft am 20.01.2013

Grote, Rainer/Marauhn, Thilo: EMRK/GG; Konkordanzkommentar zum europäischen und deutschen Grundrechtsschutz, 2006, zit.: EMRK/GG.

Gubelt, Manfred: Art. 3, in: Münch, von/Kunig, Grundgesetz-Kommentar, 5. Aufl., 2001, zit.: Gubelt in Münch GG.

Guhl, Peter: Prüfungen im Rechtsstaat; Rechtsstaatliche Anforderungen an Prüfungsverfahren, 1978, zit.: Prüfungen im Rechtsstaat.

Haffner, Johann/Zerahn-Hartung/Pfuller/Parzer/Strehlow/Resch: Auswirkungen und Bedeutung spezifischer Rechtschreibprobleme bei jungen Erwachsenen - empirische Befunde in einer epidemiologischen Stichprobe, Zeitschrift für Kinder- und Jugendpsychiatrie und Psychotherapie 26, 1989, S. 124-135.

Hain, Ulrich: Familiendynamik bei Belastungen durch umschriebene Lern- und Leistungsstörungen; Familiäre Bedingungen der Bewältigung dyskalkulatorischer Entwicklungsstörungen bei Mädchen und Jungen. 2008, zit.: Familiendynamik bei Belastungen durch umschriebene Lern- und Leistungsstörungen.

Haller, Walter: Festschrift für Ulrich Häfelin zum 65. Geburtstag, 1989, zit.: FS Ulrich Häfelin.

Hanert-Möller, Kerstin: Das Konstrukt Legasthenie; Ursache, Diagnose, Förderung aus verschiedenen Perspektiven, 2007, zit.: Konstrukt Legasthenie.

Hanke, Petra/Hein, Anna Katharina: Fördern in der Allgemeinen Schule, in: Arnold/Graumann/Rakhhochkine, Handbuch Förderung, 2008, S. 390-399.

Harnach-Beck, Viola: $\S 35$ a, in: Jans/Happe/Saurbier/Maas, Kinder- und Jugendhilferecht, Stand Mai 2009, zit.: Harnach-Beck in Jans/Happe/Saurbier/Maas KJHG.

Hartmann, Erich: Konzeption und Diagnostik von schriftsprachlichen Lernstörungen im Responsiveness-to-Intervention-Modell: eine kritische Würdigung, VHN 2008, S. 123-137.

Heiden, Hans-Günter: Die Fakten liegen auf dem Tisch, in: Heiden, Niemand darf wegen seiner Behinderung benachteiligt werden, 1996, S. 15-37. 
Heiden, Hans-Günter: Niemand darf wegen seiner Behinderung benachteiligt werden; Grundrecht und Alltag - eine Bestandsaufnahme, 1996, zit.: Benachteiligungsverbot.

Herberger, Maximilian: juris PK BGB, 3. Aufl., 2006, zit.: juris PK BGB.

Herdegen, Matthias: Die Aufnahme besonderer Vorschriften zugunsten behinderter Personen in das Grundgesetz, VSSR 1992, S. 245-264.

Herdegen, Matthias: Der neue Diskriminierungsschutz für Behinderte im Grundgesetz; Entstehung und Tragweite des Benachteiligungsverbots (Art. 3 Abs. 3 Satz 2 GG), 2. Aufl., 1998, zit.: Diskriminierungsschutz für Behinderte im Grundgesetz.

Heun, Werner: Art. 3, in: Dreier, Grundgesetz Kommentar, 2. Aufl., 2004, zit.: Heun in Dreier GG.

Heyden, Kira/von Ungern-Sternberg, Antje: Ein Diskriminierungsverbot ist kein Fördergebot; Wider die neue Rechtsprechung des EGMR zu Art. 14 EMRK, EuGRZ 2009, S. 81-89.

Heyning, Catherine J. Van de: Is it still a Sin to Kill a Mockingbird?; Remedying Factual Inequalities trough Positive Action: What can be Learned from the US Supreme Court and European Court of Human Rights Case Law, E.H.R.L.R. 2008, S. 376-390.

Hirschberg, Marianne: Behinderung; Neues Verständnis nach der Behindertenrechtskonvention, 2011, zit.: DIMR -Behinderung.

Hoffmann, Wolfgang: Nachmittag im Leben eines rechenschwachen Kindes, http://www.ztr-rechenschwaeche.de/files/artikel_nachmittag_im _leben.pdf, zuletzt geprüft am 20.01.2013.

Hofmann, Hans: Art. 7 und Art. 12, in: Schmidt-Bleibtreu/Klein, GG Kommentar zum Grundgesetz, 11. Aufl., 2008, zit.: Hofmann in Schmidt-Bleibtreu/Klein GG.

Hohmann-Dennhardt, Christine: Grundrechte und Solidarität; Durchsetzung und Verfahren; Festschrift für Renate Jaeger, 2011, zit.: FS Renate Jaeger.

Höller, Birgit: Die Beschulung behinderter Kinder und Jugendlicher an öffentlichen Schulen, 2008, zit.: Beschulung behinderter Kinder und Jugendlicher an öffentlichen Schulen.

Huber, Peter M.: Natürliche Personen als Grundrechtsträger, in: Badura/Merten/Papier, Handbuch der Grundrechte in Deutschland und Europa, Band II, 2006, § 49.

Hufen, Friedhelm: Gleichheitssatz und Bildungsplanung; Zum Funktionswandel der Grundrechte im modernen Sozialstaat. 1975, zit.: Gleichheitssatz und Bildungsplanung.

Hufen, Friedhelm: Staatsrecht II; Grundrechte, 2. Aufl., 2009, zit.: Staatsrecht. 
Huster, Stefan/Rux, Johannes: Art. 20 GG in: Epping/Hillgruber, Beck'scher Online-Kommentar GG, 2009, zit.: Huster/Rux in BeckOK GG.

International Federation of Library Associations and Institutions: Richtlinien für das Bibliothekswesen zur Unterstützung von Legasthenikern, http://archive.ifla.org/VII/s9/nd1/iflapr-91.pdf, zuletzt geprüft am 20.01.2013.

Ise, Elena/Engel, Rolf R./Schulte-Körne, Gerd: Was hilft bei Lese-Rechtschreibstörung?; Ergebnisse einer Metaanalyse zur Wirksamkeit deutschsprachiger Förderansätze, Kindheit und Entwicklung 21, 2012, S. 122-136, abrufbar unter http://www.kjp.med.uni-muenchen.de/veroeffentlichungen.php Isensee, Josef/Kirchhof, Paul: Handbuch des Staatsrechts der Bundesrepublik Deutschland, 2. Aufl., 2001, zit.: Hdb. Staatsrecht, Band.

Jacobs, Claus/Petermann, Franz: Rechenstörung, in: Petermann, Lehrbuch der klinischen Kinderpsychologie, 2008, S. 207-222.

Jähnke, Burkhard/Laufhütte, Heinrich Wilhelm/Odersky, Walter: Leipziger Kommentar zum StGB, 11. Aufl., 2005, zit.: Leipziger Kommentar zum StGB.

Jans, Karl-Wilhelm/Happe, Günther/Saurbier, Helmut/Maas, Udo: Kinder- und Jugendhilferecht; Kommentar, Stand Mai 2009, zit.: Jans/Happe/Saurbier/ Maas KJHG.

Jarass, Hans D.: Zum Grundrecht auf Bildung und Ausbildung, DÖV 1995, S. 674-679.

Jarass, Hans D./Pieroth, Bodo: Grundgesetz für die Bundesrepublik Deutschland; Kommentar, 10. Aufl., 2009, zit.: Jarass/Pieroth GG.

Jogschies, Peter: Förderdiagnostik und sonderpädagogische Begutachtung; Ein Rückblick auf 30 Jahre, Zeitschrift für Heilpädagogik 2008, S. 132-142.

Joint Council for Qualifications: Access Arrangements, Reasonable Adjustments and Special Consideration, http://www.jcq.org.uk/exams-office/accessarrangements-and-special-consideration/regulations-and-guidance/ access-arrangements-reasonable-adjustments-and-special-consideration2012-2013, zuletzt geprüft am 20.01.2013.

Joussen, Jacob: Schwerbehinderung, Fragerecht und positive Diskriminierung nach dem AGG, NZA 2007, S. 174-178.

Jürgens, Andreas: Vom Umgang mit Benachteiligungen; Entgegnung auf die Erwiderung von Klaas Engelken, DVBI 1997, S. 764.

Jürgens, Andreas: Der Diskriminierungsschutz im Grundgesetz, DVBI 1997, S. 410-415.

Jürgens, Eiko/Böhm, Thomas/Prüß, Franz: Schulleitung in den Ländern Brandenburg, Mecklenburg-Vorpommern, Sachsen, Sachsen-Anhalt und Thüringen, 1991, zit.: Schulleitung in den Ländern. 
Jürgens, Gunther: Die verfassungsrechtliche Stellung Behinderter nach Änderung des Grundgesetzes, ZfSH/SGB 1995, S. 353-360.

Jürgens, Gunther: Grundrecht für Behinderte, NVwZ 1995, S. 452-453.

Jürgens, Gunther: Gleichstellung nicht erst in der Zukunft, sondern jetzt, in: Heiden, Niemand darf wegen seiner Behinderung benachteiligt werden, 1996, S. 181-192.

Jürgens, Gunther/Römer, Veiko: Aufnahme von Behinderten in allgemeine Schulen, NVwZ 1999, S. 847-850.

Kannengießer, Christoph: Art. 3, in: Schmidt-Bleibtreu/Klein, GG Kommentar zum Grundgesetz, 11. Aufl., 2008, zit.: Kannengießer in Schmidt-Bleibtreu/ Klein GG.

Kany, Werner/Schöler, Hermann: Diagnostik schulischer Lern- und Leistungsschwierigkeiten; Ein Leitfaden mit einer Anleitung zur Gutachtenerstellung, 2009, zit.: Diagnostik.

Kirchhof, Paul: Verfassungsrechtlicher Schutz und internationaler Schutz der Menschenrechte: Konkurrenz oder Ergänzung, EuGRZ 1994, S. 16-44.

Kirchner, Reinhard: Die nicht sichtbare Behinderung, http://www.laghbayern.de/Ab.pdf, zuletzt geprüft am 20.01.2013.

Kischel, Uwe: Art. 3, in: Epping/Hillgruber, Beck'scher Online-Kommentar, 2009, zit.: Kischel in BeckOK GG.

Klasen, Edith: Legasthenie; Umschriebene Lese-Rechtschreib-Störung; Informationen und Ratschläge, 3. Aufl., 1999, zit.: Legasthenie.

Klemm, Klaus: Sonderweg Förderschulen: Hoher Einsatz, wenig Perspektiven, http://www.bertelsmann-stiftung.de/cps/rde/xbcr/SID-149C029F41C39A86/bst/xcms_bst_dms_29959_29960_2.pdf, zuletzt geprüft am 20.01.2013.

Klemm, Klaus/Preuss-Lausitz, Ulf: Gutachten zum Stand und zu den Perspektiven der sonderpädagogischen Förderung in den Schulen der Stadtgemeinde Bremen, http://www.bildung.bremen.de/sixcms/media.php/13/ Sonderp\%E4dagogisches\%20Gutachten.pdf, zuletzt geprüft am 20.01.2013.

Klicpera, Christian/Gasteiger-Klicpera, Barbara: Der Zusammenhang zwischen kognitiver und sozial-emotionaler Entwicklung, in: Gasteiger-Klicpera/Julius/Klicpera, Handbuch Sonderpädagogik, 2008, S. 353-365.

Klicpera, Christian/Heyse, I./Warnke, Andreas/Kutschera, G./Schwaiger, H.: Entstehungsbedingungen von Verhaltensstörungen bei Kindern, Eine Untersuchung an Kindern in stationärer psychiatrischer Behandlung, Fortschritte der Neurologie Psychiatrie 1981, S. 193-199. 
Klicpera, Christian/Schabmann, Alfred/Gasteiger-Klicpera, Barbara: Legasthenie; Modelle, Diagnose, Therapie und Förderung, 3. Aufl., 2010, zit.: Legasthenie.

Knack, Hans Joachim/Henneke, Hans-Günter: Verwaltungsverfahrensgesetz, 9. Aufl., 2010, zit.: VwVfG.

Koch, Sascha/Schemmann, Michael: Neo-Institutionalismus in der Erziehungswissenschaft; Grundlegende Texte und empirische Studien, 2009, zit.: Erziehungswissenschaft.

König, Doris/Peters, Anne: Kapitel 21: Das Diskriminierungsverbot, in: Grote/ Marauhn, EMRK/GG, 2006, zit.: König in Grote/Marauhn EMRK/GG.

Konsortium Bildungsberichterstattung: Bildung in Deutschland, http://www. bildungsbericht.de/daten/gesamtbericht.pdf, zuletzt geprüft am 20.01.2013.

Konsortium Bildungsberichterstattung: Bildung in Deutschland 2008, www.bildungsbericht.de/daten2008/bb_2008.pdf, zuletzt geprüft am 20.01.2013.

Kopp, Ferdinand O/Ramsauer, Ulrich: Verwaltungsverfahrensgesetz, Kommentar, 13. Aufl., 2012, zit.: VwVfG.

Körting, Ehrhart: Grundrechte behinderter Schüler, in: Raabe Fachverlag, Schule in der Rechtsprechung, 1996, Nr. I 1011.1.

Kotzur, Markus/Richter, Clemens: Anmerkung zur Geltung der Verbindlichkeit der Behindertenrechtskonvention im deutschen Recht, in: Welke, UNBehindertenrechtskonvention mit rechtlichen Erläuterungen, 2012, zit.: Kommentar zur UN-BRK, Verbindlichkeit.

Krajewski, Markus: Ein Menschenrecht auf integrativen Schulunterricht; Zur innerstaatlichen Wirkung von Art. 24 der UN-Behindertenrechtskonvention, JZ 2010, S. 120-125.

Krajewski, Markus/Bernhard, Thomas: Inklusive Schule im Freistaat Bayern?; Die Änderung des BayEUG vom 13.7.2011 aus Sicht der UN-Behindertenrechtskonvention, BayVBI 2012, S. 134-139.

Krajewski, Markus/Bernhard, Thomas: Art. 24: Bildung, in: Welke, UN-Behindertenrechtskonvention mit rechtlichen Erläuterungen, 2012, zit.: Kommentar zur UN-BRK, Art. 24.

Krappmann, Lothar/Lüscher, Kurt: Kinderrechte im Generationenverbund; Plädoyer für eine aktuelle Lektüre der Kinderrechtskonvention, RdJB 2009, S. 326-334.

Krug, Heinz/Riehle, Eckart: SGB VIII - Kinder- und Jugendhilfe; Kommentar und Rechtssammlung, Stand 1.8.2007, zit.: SGB VIII.

Kühne, Hans-Heiner: Die Reichweite des ärztlichen Geheimnisschutzes bei kranken Prüfungskandidaten, JA 1999, S. 523-525. 
Kunig, Philip: Art. 1, in: Münch, von/Kunig, Grundgesetz-Kommentar, 5. Aufl., 2001, zit.: Kunig in Münch GG.

Kunkel, Peter-Christian/Binschus, Wolfgang: Sozialgesetzbuch VIII; Kinder- und Jugendhilfe; Lehr- und Praxiskommentar, 3. Aufl., 2006, zit.: Kunkel SGB VIII.

Kupka, Thomas: Verfassungsnominalismus - Hermeneutische Überlegungen zum Problem sprachlicher Benennungen im Recht, Archiv für Rechts- und Sozialphilosophie 2011, S. 44-77.

Lambert, Johannes: Die Entwicklung des Schulrechts in Baden-Württemberg von 1993 bis 1998, RdJB 1998, S. 384 ff.

Lambert, Johannes: Notengebung für Schüler mit Behinderungen, SchulVerwaltung BW 2002, S. 148-151.

Lampe, Mareike: Gerechtere Prüfungsentscheidungen; Möglichkeiten und Grenzen der Herbeiführung materieller Gerechtigkeit durch gerichtliche Kontrolle und Gestaltung des Verwaltungsverfahrens, 1999, zit.: Gerechtere Prüfungsentscheidungen.

Landerl, Karin und das NeuroDys-Konsortium: Neuro-Dys-Dyslexie in sechs europäischen Orthographien, in: Schulte-Körne, Legasthenie und Dyskalkulie: Stärken erkennen - Stärken fördern, 2011, S. 65-75.

Landerl, Karin/Butterworth, Brian: Spezifische Rechenschwierigkeiten / Dyskalkulie: viele Fragen, erste Antworten, in: Schulte-Körne/Amorosa, Legasthenie, 2002, S. 387-394.

Landerl, Karin/Kaufmann, Liane: Dyskalkulie; Modelle, Diagnostik, Intervention, 2008, zit.: Dyskalkulie.

Landerl, Karin/Moll/Kristina: Double Dissociation Between Reading and Spelling Deficits, Scientific Studies of Reading 2009, S. 359-382.

Landesverband Bayerischer Schulpsychologen: LBSP-Stellungnahme zur KMBek Legasthenie, http://archiv.lbsp.de/Beratung/Stoerungsbilder/Legasthenie/ files/Stellungnahme\%20Erlass\%20LBSP.pdf, zuletzt geprüft am 20.01.2013.

Langenfeld, Christine: Integration und kulturelle Identität zugewanderter Minderheiten, 2001, zit.: Integration und kulturelle Identität zugewanderter Minderheiten.

Langenfeld, Christine: Kap. 23: Das Recht auf Bildung, in: Grote/Marauhn, EMRK/GG, 2006, zit.: Langenfeld in Grote/Marauhn EMRK/GG.

Langenfeld, Christine: Das Recht auf Bildung in der Europäischen Menschenrechtskonvention, RdJB 2007, S. 412-429.

Langenfeld, Christine: Maßnahmen des Nachteilsausgleichs und des besonderen Schutzes für Schüler und Schülerinnen mit Legasthenie an allgemeinbildenden Schulen, RdJB 2007, S. 211-227. 
Langfeldt, Hans-Peter: Behindertendiagnostik, in: Eid/Petermann, Handbuch der Psychologie, 2006, S. 626-632.

Larenz, Karl: Methodenlehre der Rechtswissenschaft, 6. Aufl., 1991, zit.: Methodenlehre.

Latham/Watkins: Völkerrechtliche Fragen des inklusiven Unterrichts in Deutschland im Zusammenhang mit dem Inkrafttreten des Übereinkommens der Vereinten Nationen über die Rechte von Menschen mit Behinderungen; Gutachten erstellt für die Bundesarbeitsgemeinschaft ,Gemeinsam leben - gemeinsam lernen' e.V., April 2009, zit.: Gutachten zur UN-BRK.

Lawson, Anna: Angemessene Vorkehrungen und Zugänglichkeit: Ist ein einheitlicheres EU-Konzept möglich? Europäische Zeitschrift zum Antidiskriminierungsrecht 2011, S. 11-24.

Leder, Tobias: Das Diskriminierungsverbot wegen einer Behinderung, 2006, zit.: Diskriminierungsverbot wegen einer Behinderung.

Legakids: Legakids.net: Pressemitteilung zur Elternumfrage 2009 v. 23.3.2010, http://www.legakids.net/eltern-lehrer/info-ueber-Irs-co/neues/meldung/ article/3/Pressemitteilung-zur-Elternumfrage-2009/, zuletzt geprüft am 20.01.2013.

Leibholz, Gerhard/Rinck, Hans-Justus/Hesselberger, Dieter/Burghart, Axel: Grundgesetz für die Bundesrepublik Deutschland, Stand 2006, zit.: Leibholz/Rinck, GG.

Leitherer, Stephan: Kasseler Kommentar Sozialversicherungsrecht, zit.: Kasseler Kommentar Sozialversicherungsrecht.

Leitlinien: Leitlinien zu Diagnostik und Therapie von psychischen Störungen im Säuglings-, Kindes- und Jugendalter, http://www.awmf.org/uploads/tx szleitlinien/028-017_S1_Umschriebene_Entwicklungsstoerungen_schüli scher_Fertigkeiten_F81_11-2006_11-2011_01.pdf, zuletzt geprüft am 23.01.2013.

Lenhard, Wolfgang/Küspert, Petra/Becker, Heike: Legasthenie und Lese-Rechtschreibschwierigkeiten; Fallgeschichten aus der Praxis, 2009, zit.: Legasthenie.

Leonhardt, Annette/Wember, Franz B.: Grundfragen der Sonderpädagogik; Bildung - Erziehung -Behinderung, 1. Aufl., 2003, zit.: Grundfragen Sonderpädagogik.

Lerche, Peter: Vorbehalt des Gesetzes und Wesentlichkeitsgarantie, in: Badura/ Merten/Papier, Handbuch der Grundrechte in Deutschland und Europa, Band III, 2009, § 62

Ligges, Carolin: Die Bedeutung der Phonologie für die Lese-Rechtschreibstörung, in: Schulte-Körne, Legasthenie und Dyskalkulie, 2007, S. 237-245. 
Ligges, Marc: fMRI-Untersuchung von Sprachverarbeitungsprozessen bei der Lese-Rechtschreibstörung, Diss. Uni Jena 2002, http://nbn-resolving.de/ urn:nbn:de:gbv:27-dbt-000821-5, zuletzt geprüft am 20.01.2013.

Lindner, Josef Franz: Die Prägung des Prüfungsrechts durch den Grundsatz der Chancengleichheit - ein vielschichtiges Phänomen, BayVBI 1999, S. 100-107.

Lindner, Josef Franz: Theorie der Grundrechtsdogmatik, 2004, zit.: Theorie der Grundrechtsdogmatik.

Lindner, Josef Franz: Konsequente Zweckverfolgung als Verfassungspflicht des Gesetzgebers; Eine dogmatische Analyse des „Sportwetten-Urteils" des BVerfG, ZG 2007, S. 188-203.

Löffler, Ilona/Meyer-Schepers, Ursula/Lischeid, Thomas: Rechtschreibschwäche im Fokus der Kompetenzdiagnostik, in: Schulte-Körne, Legasthenie und Dyskalkulie, 2007, S. 267-275.

Lohrenscheit, Claudia: Die UN-Sonderberichterstattung zum Recht auf Bildung und ihre Grundlegung durch Katarina Tomasevski, in: Overwien/Prengel, Recht auf Bildung, 2007, S. 34-50.

Lorz, Ralph Alexander: Der Vorrang des Kindeswohls nach Art. 3 der UNKinderrechtskonvention in der deutschen Rechtsordnung, 2003, zit.: Vorrang Art. 3 UN-KRK.

Lorz, Ralph Alexander: Nach der Rücknahme der deutschen Vorbehaltserklärung: Was bedeutet die uneingeschränkte Verwirklichung des Kindeswohlvorrangs nach der UN-Kinderrechtskonvention im deutschen Recht? 2010, zit.: Rücknahme Vorbehaltserklärung.

Luthe: Gesetzesziele und Begriff der Behinderung, in: Luthe/Bieritz-Harder, Rehabilitationsrecht, 2009, S. $105 \mathrm{ff}$.

Luthe, E.W: Bildungsrecht: Leitfaden für Ausbildung, Administration und Management, 2003, zit.: Bildungsrecht.

Luthe, Ernst-Wilhelm: Optimierende Sozialgestaltung; Bedarf - Wirtschaftlichkeit - Abwägung, 2001, zit.: Optimierende Sozialgestaltung.

Luthe, Ernst-Wilhem: Verfassungsrecht, in: Luthe/Bieritz-Harder, Rehabilitationsrecht, 2009, S. 48 ff, zit.: Rehabilitationsrecht-Verfassungsrecht.

Luthe, Ernst-Wilhem/Bieritz-Harder, Renate: Rehabilitationsrecht, 2009, zit.: Rehabilitationsrecht.

LVL Hessen: Empfehlungen zum individuellen Nachteilsausgleich für Schülerinnen und Schüler mit besonderen Schwierigkeiten beim Lesen, Rechtschreiben oder Rechnen und Legasthenie oder Dyskalkulie, http://www.seb-ess.de/infos/Empfehlung_Nachteilsausgleich.pdf, zuletzt geprüft am 20.01.2013. 
Mahler, Claudia/Weiß, Norman: Der Einfluss der internationalen Menschenrechtsverträge auf die deutsche Bildungsrechtsordnung, RdJB 2007, S. 430-445.

Mangoldt, Hermann von/Klein, Dietrich/Starck, Christian: Kommentar zum Grundgesetz, 5. Aufl., 2005, zit.: Mangoldt/Klein/Starck GG.

Manssen, Gerrit: Art. 12, in: Mangoldt/Klein/Starck, Kommentar zum Grundgesetz, 5. Aufl., 2005.

Martis, Rüdiger/Winkhart-Martis, Martina: Arzthaftungsrecht; Fallgruppenkommentar, 3. Aufl., 2010, zit.: Arzthaftungsrecht.

Marwege, Gabriele: Welche Stellung hat der Schulpsychologe/die Schulpsychologin im Verfahren um die Anerkennung der Legasthenie in der Schule? Zeitschrift des BVL, 2007, S. 36-39.

Marwege, Gabriele: Anmerkung zum Beschluss VG Köln v. 26.9.2008, DVBI 2009, S. 538-540.

Marwege, Gabriele: Die Berücksichtigung von Behinderungen in der Schule und in Schulprüfungen; Eine Rechtsprechungsübersicht, RdJB 2009, S. 229-241.

Masuch, Peter: Die UN-Behindertenrechtskonvention anwenden! in: Hohmann-Dennhardt, Grundrechte und Solidarität, 2011, S. 245-263.

Mathe, Thomas: Von der Segregation zur Inklusion behinderter Menschen; Ein Paradigmenwechsel aus sozialwissenschaftlicher Sicht, br 2011, S. 34-40.

Maunz, Theodor/Dürig, Günter/Herzog, Roman: Kommentar zum Grundgesetz, 55. Aufl., 2009, zit.: Maunz/Dürig/Herzog GG.

Maurer, Hartmut: Das akzeptierte Grundgesetz; Festschrift für Günter Dürig zum 70. Geburtstag, 1990, zit.: FS Günter Dürig.

Maurer, Urs: Erforschung von Hirnfunktionen bei Lesen und Dyslexie mittels bildgebender Verfahren, Zeitschrift des BVL, 2009, S. 15-18.

Maydell, Bernd, von/Ruland, Franz/Becker, Ulrich: Sozialrechtshandbuch, 4. Aufl., 2008.

Merten, Detlef: Grundrechtsverzicht, in: Badura/Merten/Papier, Handbuch der Grundrechte in Deutschland und Europa, Band III, 2009, § 73.

Meyer-Ladewig, Jens/Keller, Wolfgang/Leitherer, Stephan: Sozialgerichtsgesetz, 9. Aufl., 2008, zit.: SGG.

Meysen, Thomas: Die Kinder- und Jugendhilfe als Ausfallbürge bei schwerer Legasthenie und/oder Dyskalkulie, JAmt 2003, S. 53-62.

Meysen, Thomas: $\S 35$ a, in: Münder/Meysen/Trenczek, Frankfurter Kommentar zum SGB VIII: Kinder- und Jugendhilfe, 6. Aufl., 2009.

Michael, Lothar/Morlok, Martin: Grundrechte, 2. Aufl., 2010, zit.: Grundrechte. 
Mierau, Johannes: Legasthenie und Recht - ein Überblick, in: Schulte-Körne, Legasthenie und Dyskalkulie: Stärken erkennen - Stärken fördern, 2011, S. 33-42.

Mokatef, Mona: Das Menschenrecht auf Bildung und Schutz vor Diskriminierung; Exklusionsrisiken und Inklusionschancen, 2006, zit.: Menschenrecht auf Bildung und Schutz vor Diskriminierung.

Mrozynski, Peter: SGB VIII Kinder- und Jugendhilfe; Kommentar, 5. Aufl., 2009, zit.: SGB VIII.

Mrozynski, Peter: Sozialgesetzbuch - allgemeiner Teil (SGB I); Kommentar, 4. Aufl., 2010, zit.: SGB I.

Münch, Ingo, von: Chancengleichheit im Prüfungsrecht; Besprechung des Urteils des OVG Koblenz v. 16.1.1980, Jura 1980, S. 448.

Münch, Ingo, von: Vorb. Art. 1 - 19, in: Münch, von/Kunig, GrundgesetzKommentar, 5. Aufl., 2001.

Münch, Ingo, von/Bryde, Brun-Otto/Kunig, Philip: Grundgesetz-Kommentar, 5. Aufl., 2001, zit.: Münch GG.

Münch, Ingo, von/Bryde, Brun-Otto/Kunig, Philip: Grundgesetz-Kommentar, 6. Aufl., 2012, zit.: Münch GG 2012.

Münder, Johannes/Baltz, Jochem/Kreft, Dieter: Frankfurter Kommentar zum SGB VIII: Kinder- und Jugendhilfe, 5. Aufl., 2006, zit.: Frankfurter Kommen$\operatorname{tar}$ SGB VIII.

Münder, Johannes/Meysen, Thomas/Trenczek, Thomas: Frankfurter Kommentar zum SGB VIII: Kinder- und Jugendhilfe, 6. Aufl., 2009, zit.: Frankfurter Kommentar SGB VIII.

Muñoz, Vernon: Report of the Special Rapporteuer on the right to education, http://www.institut-fuer-menschenrechte.de/fileadmin/user_upload/PDFDateien/UN-Dokumente/Sonderberichterstatter/report_Munoz_mission_ Germany_2006.pdf, zuletzt geprüft am 20.01.2013.

Muñoz, Vernon: Bildung ist ein Recht und keine Ware - Für eine Bildung gleich hoher Qualität für alle, http://munoz.uri-text.de/VernorMunoz7teJuni09 OL_deutscheUebersetzung.pdf, zuletzt geprüft am 20.1.2013.

Murswiek, Dietrich: Grundrechte als Teilhaberechte, soziale Grundrechte, in: Isensee/Kirchhof, Handbuch des Staatsrechts der Bundesrepublik Deutschland, 2. Aufl., 2000, Band V Allgemeine Grundrechtslehren, § 112.

Neumann, Volker: Der verfassungsrechtliche Begriff der Behinderung, NVwZ 2003, S. 897-900.

Neumann, Volker: Verfassungsrecht, in: Deinert/Neumann, Rehabilitation und Teilhabe behinderter Menschen, 2009, § 2, S. 13 ff. 
Neumärker, Klaus Jürgen/Bzufka, Michael Werner: Diagnostik und Klinik der Rechenstörung, in: Aster/Lorenz, Rechenstörungen bei Kindern, 2005, S. $73 \mathrm{ff}$.

Niehues, Norbert: Schul- und Prüfungsrecht; Schulrecht, 3. Aufl., 2000, zit.: Schul- und Prüfungsrecht.

Niehues, Norbert: Schul- und Prüfungsrecht, 4. Aufl., 2004, zit.: Prüfungsrecht.

Niehues, Norbert/Rux, Johannes: Schul- und Prüfungsrecht; Schulrecht, 4. Aufl., 2006, zit.: Schulrecht.

Oelkers, Jürgen: Chancengleichheit, Integration und Schule: Ein Essay, RdJB 2009, S. 190-199.

Oppermann, Thomas: Schule und berufliche Ausbildung, in: Isensee/Kirchhof, Handbuch des Staatsrechts der Bundesrepublik Deutschland, 2. Aufl., 2001, Band VI Freiheitsrechte, § 135.

Orth, Johannes: Verfassungsrechtliche Anforderungen an die Schulstruktur, NVwZ 2011, S. 14-19.

Orthmann Bless, Dagmar: Das schulsystemische Paradigma, in: Walter/Wember/Borchert/Goetze, Sonderpädagogik des Lernens, 2007, S. 93-103.

Ossenbühl, Fritz: Rechtliche Grundfragen der Erteilung von Schulzeugnissen, 1978, zit.: Schulzeugnisse.

Ossenbühl, Fritz: Grundsätze der Grundrechtsinterpretation, in: Badura/Merten/Papier, Handbuch der Grundrechte in Deutschland und Europa, Band I, 2004, § 15.

Osterloh, Lerke: Art. 3, in: Sachs, Kommentar zum Grundgesetz, 5. Aufl., 2009, zit.: Osterloh in Sachs GG.

Overwien, Bernd/Prengel, Annedore: Recht auf Bildung; Zum Besuch des Sonderberichterstatters der Vereinten Nationen in Deutschland, 2007, zit.: Recht auf Bildung.

Papier, Hans-Jürgen: § 839 BGB, in: Säcker/Rixecker, Münchener Kommentar zum Bürgerlichen Gesetzbuch, 5. Aufl., 2009.

Peine, Franz-Joseph: Grundrechtsbeschränkungen in Sonderstatusverhältnissen, in: Badura/Merten/Papier, Handbuch der Grundrechte in Deutschland und Europa, Band III (2009), § 65.

Petermann, Franz: Lehrbuch der klinischen Kinderpsychologie, 6. Aufl., 2008, zit.: Lehrbuch der klinischen Kinderpsychologie.

Pfahl, Lisa/Powell, Justin J.W.: Die Exklusion von Schüler/innen mit sonderpädagogischem Förderbedarf; Ein Beitrag zur Debatte um nationale Bildungsstandards und die Schule für alle, Gemeinsam leben - Zeitschrift für integrative Erziehung 2005, S. 68-78.

Pieroth, Bodo/Schlink, Bernhard: Grundrechte; Staatsrecht II, 23. Aufl., 2007, zit.: Grundrechte. 
Pietzcker, Jost: Verfassungsrechtliche Anforderungen an die Ausgestaltung staatlicher Prüfungen, 1975, zit.: Verfassungsrechtliche Anforderungen an staatliche Prüfungen.

Pixner, Silvia/Kaufmann, Liane: Förderung numerisch-rechnerischer Fertigkeiten: in: Schulte-Körne, Legasthenie und Dyskalkulie: Stärken erkennen Stärken fördern, 2011, S. 199-214.

Plate, Elisabeth: Betrachtungen „inklusiver" Schulentwicklungen in England aus einer internationalen Perspektive, Sonderpädagogische Förderung heute 53, 2008, S. 399-427.

Plume, Ellen/Warnke, Andreas: Legasthenie, in: Steinhausen, Schule und psychische Störungen, 2006, S. 196-204.

Poelmans, G./Buitelaar, J.K./Pauls, D./Franke, B.: A theoretical molecular network for dyslexia: integrating available genetic findings, Molecular Psychiatry 2011, S. 365-382.

Poscher, Ralf/Langer, Thomas/Rux, Johannes: Gutachten zu den völkerrechtlichen und innerstaatlichen Verpflichtungen aus dem Recht auf Bildung nach Art. 24 des UN-Abkommens über die Rechte von Menschen mit Behinderungen und zur Vereinbarkeit des deutschen Schulrechts mit den Vorgaben des Übereinkommens; erstellt im Auftrag der Max Troeger Stiftung, 2008, zit.: Gutachten zu Art. 24 BRK.

Poscher, Ralf/Rux, Johannes/Langer, Thomas: Das Recht auf Bildung; Völkerrechtliche Grundlagen und innerstaatliche Umsetzung, 1. Aufl., 2009, zit.: Recht auf Bildung.

Powell, Justin J.W.: Von schulischer Exklusion zur Inklusion? Eine neoinstitutionalistische Analyse sonderpädagogischer Fördersysteme in Deutschland und den USA, in: Koch/Schemmann, Neo-Institutionalismus in der Erziehungswissenschaft, 2009, S. 213-232.

Prengel, Annedore: Diagnostik muss machtsensibel sein, Sozialextra 2006, S. 15-17.

Proelss, Alexander: Der Grundsatz der völkerrechtsfreundlichen Auslegung im Lichte der Rechtsprechung des BVerfG, in: Rensen/Brink, Linien der Rechtsprechung des Bundesverfassungsgerichts, 2009, S. 553-584.

Raabe Fachverlag: Schule in der Rechtsprechung; Rechtssetzung und Richtersprüche praxisnah kommentiert, 1996, zit.: Schule in der Rechtsprechung.

Rahn, Manfred: Legasthenie bei Erwachsenen, 1981, zit.: Legasthenie bei Erwachsenen.

Reichelt, Helmut: Trotz Schule: Analphabeten in Deutschland, http://www.legakids.net/fileadmin/user_upload/Downloads/Info/Wissens chaft/Analphabetismus_Reichelt.pdf, zuletzt geprüft am 20.01.2013. 
Reichenbach, Peter: Der Anspruch behinderter Schülerinnen und Schüler auf Unterricht in der Regelschule; Zugleich ein Beitrag zur Interpretation des Art. 3 Abs. 3 Satz 2 GG, 2001, zit.: Anspruch behinderter Schülerinnen und Schüler auf Unterricht in der Regelschule.

Reimann, Johannes: Die Sicherstellung des Schulbesuchs behinderter Kinder mit Mitteln des Schul- und Sozialrechts; Eine Untersuchung am Beispiel der Länder Hamburg und Schleswig-Holstein, 2007, zit.: Sicherstellung des Schulbesuchs behinderter Kinder.

Remschmidt, Helmut/Mattejat, Fritz/Warnke, Andreas: Therapie psychischer Störungen bei Kindern und Jugendlichen; Ein integratives Lehrbuch für die Praxis, 2008, zit.: Therapie psychischer Störungen.

Rensen, Hartmut/Brink, Stefan: Linien der Rechtsprechung des Bundesverfassungsgerichts; Erörtert von den wissenschaftlichen Mitarbeitern, 2009, zit.: Rechtsprechung BVerfG.

Reuter-Liehr, Carola: Das Konzept der „Lautgetreuen Lese-Rechtschreibförderung", in: Schulte-Körne, Legasthenie und Dyskalkulie, 2007, S. 107-133.

Reuter, Lutz R.: Gesetzesvorbehalt und Migration: Anforderungen an schulrechtliche Standards für zugewanderte Kinder und Jugendliche, RdJB 2003, S. 23-36.

Richter, Ingo: Recht im Bildungssystem; Eine Einführung, 2006, zit.: Recht im Bildungssystem.

Richter, Matthias/Hurrelmann, Klaus: Gesundheitliche Ungleichheit; Grundlagen, Probleme, Konzepte, 2006, zit.: Gesundheitliche Ungleichheit.

Richter, Wiltrud: Tipps für Eltern und Lehrer, in: Ganser/Richter, Was tun bei Legasthenie in der Sekundarstufe? 2005, S. 72-77.

Riedel, Eibe: Gutachten zur Wirkung der internationalen Konvention über die Rechte von Menschen mit Behinderung und ihres Fakultativprotokolls auf das deutsche Schulwesen, http://gemeinsam-leben-nrw.de/sites/default/ files/gutachten_riedel.pdf, zuletzt geprüft am 20.01.2013, zit.: Gutachten UN-Behindertenrechtskonvention

Riedel, Eibe; Arend, Jan-Michael, Im Zweifel Inklusion: Zuweisung an eine Förderschule nach Inkrafttreten der BRK, NVwZ 2010, S. 1346-1349.

Riedel, Eibe/Söllner, Sven: Studiengebühren im Lichte des UN-Sozialpakts, JZ 2006, S. 270-277.

Riehle, Eckart: § 35 a, in: Krug/Riehle, SGB VIII - Kinder- und Jugendhilfe, Stand 1.8.2007, zit.: Riehle in Krug/Riehle SGB VIII.

Ritgen, Klaus: § 29, in: Knack/Henneke, Verwaltungsverfahrensgesetz, 9. Aufl., 2010, zit.: Ritgen in Knack/Henneke, VwVfG. 
Robbers, Gerhard: Art. 6 und Art. 7, in: Mangoldt/Klein/Starck, Kommentar zum Grundgesetz, 5. Aufl., 2005, zit.: Robbers in Mangoldt /Klein/Starck GG.

Roetteken, Torsten v.: Nichtigkeit der Regelung über den Versorgungsabschlag für ehemals teilzeitbeschäftigte Beamte, jurisPR-ArbR 17/2009 Anm. 6, zuletzt geprüft am 20.01.2013.

Roetteken, Torsten v.: Bleiben diskriminierende Verfahrensfehler sanktionslos? jurisPR-ArbR 41/2009 Anm. 5, zuletzt geprüft am 20.01.2013.

Roos, Jeanette/Schöler, Hermann: Entwicklung des Schriftspracherwerbs in der Grundschule, http://dx.doi.org/10.1007/978-3-531-91574-6, zuletzt geprüft am 20.01.2013.

Rose, Jim: Rose-Review, http://dera.ioe.ac.uk/14790/1/00659-2009DOMEN.pdf, zuletzt geprüft am 20.01.2013.

Rothfritz, Lauri Philipp: Die Konvention der Vereinten Nationen zum Schutz der Rechte von Menschen mit Behinderungen, 2010, zit.: UN-BRK.

Ruffert, Matthias: Art. 12, in: Epping/Hillgruber, Beck'scher Online-Kommentar, 2009, zit.: Ruffert in BeckOK GG.

Rüfner: Art. 3, in: Dolzer/Waldhoff/Graßhof, Bonner Kommentar zum Grundgesetz, Oktober 1996.

Ruß, Hans-Jürgen: Legasthenie und Hochbegabung; Kritische Analyse der Theorien, Erlasse und Rechtsprechung zur LRS. 1992, zit.: Legasthenie und Hochbegabung.

Rust, Ursula/Falke, Josef/Bertelsmann, Klaus: AGG; Allgemeines Gleichbehandlungsgesetz mit weiterführenden Vorschriften; Kommentar, 2007, zit.: AGG.

Rux, Johannes: Die pädagogische Freiheit des Lehrers; Eine Untersuchung zur Reichweite und zu den Grenzen der Fachaufsicht im demokratischen Rechtsstaat, 2002, zit.: Pädagogische Freiheit des Lehrers.

Rux, Johannes: Kein Handlungsbedarf oder Anlass für eine bildungspolitische Revolution? Zur innerstaatlichen Umsetzung der Behindertenrechtskonvention der Vereinten Nationen, RdJB 2009, S. 220-228.

Sachs, Michael: Kommentar zum Grundgesetz, 5. Aufl., 2009, zit.: Sachs GG.

Sachs, Michael: Das Grundrecht der Behinderten aus Art. 3 Abs. 3 Satz 2 GG, RdJB 1996, S. 154-174.

Sachs, Michael: Legasthenie und Zulassung zum Gymnasium, JuS 1998, S. 263.

Sachs, Michael: Verbot der Benachteiligung Behinderter, JuS 1998, S. 553-555.

Sachs, Michael: Besondere Gleichheitsgarantien, in: Isensee/Kirchhof, Handbuch des Staatsrechts der Bundesrepublik Deutschland, 3. Aufl., 2012, Band VIII, § 182, S. 839-1011. 
Sachs, Michael/Siekmann, Helmut: Der grundrechtsgeprägte Verfassungsstaat; Festschrift für Klaus Stern zum 80. Geburtstag, 2012, zit.: FS Klaus Stern.

Säcker, Franz Jürgen/Rixecker, Roland: Münchener Kommentar zum Bürgerlichen Gesetzbuch, 5. Aufl., 2009, zit.: MüKomm BGB.

Sacksofsky, Ute: Art. 3, in: Umbach/Clemens, Grundgesetz, 2002, zit.:

Sacksofsky in Umbach GG.

Scerri, Thomas S./Schulte-Körne, Gerd: Genetics of development of dyslexia, European Child and Adolescense Psychiatry 19, 2010, S. 179-197.

Schenke, Wolf-Rüdiger: Justizgewähr und Grundrechtsschutz, in: Badura/Merten/Papier, Handbuch der Grundrechte in Deutschland und Europa, Band III, 2009, § 78.

Schiek, Dagmar: Gleichbehandlungsrichtlinien der EU; Umsetzung im deutschen Arbeitsrecht, NZA 2004, S. $873 \mathrm{ff}$.

Schiek, Dagmar/Waddington, Lisa/Bell, Mark: Cases, Materials and Text on National, Supranational and International Non-Discrimination Law, 2007, zit.: Non-Discrimination Law.

Schlee, Jörg: 30 Jahre „Förderdiagnostik“ - eine kritische Bilanz, Zeitschrift für Heilpädagogik 2008, S. 122-131.

Schlee, Jörg: Legasthenieforschung am Ende?, 1. Aufl., 1976, zit.: Legasthenieforschung am Ende?

Schlegel, Rainer/Engelmann, Klaus/Noelzke, Thomas: Juris Praxiskommentar SGB V; Sozialgesetzbuch fünftes Buch; gesetzliche Krankenversicherung, 2008, zit.: JurisPK SGB V.

Schleider, Karin: Lese- und Rechtschreibstörungen, 2009, zit.: Lese- und Rechtschreibstörungen.

Schlichte-Hiersemenzel, Barbara: Zu Entwicklungsschwierigkeiten hochbegabter Kinder und Jugendlicher in Wechselwirkung mit ihrer Umwelt, http://www.begabtenzentrum.at/wcms/picture/upload/File/Handreichung en/03_broschuere_entwicklungsschwier_2006.pdf, zuletzt geprüft am 20.01.2013.

Schmidt-Aßmann, Eberhard: Art. 19, in: Maunz/Dürig/Herzog, Kommentar zum Grundgesetz, 55. Aufl., 2009, Schmidt-Aßmann in Maunz/Dürig/Herzog GG.

Schmidt-Bleibtreu, Bruno/Klein, Franz: GG Kommentar zum Grundgesetz, 11. Aufl., 2008, zit.: Schmidt-Bleibtreu/Klein GG.

Schmitt-Kammler, Arnulf/Coelln, Christian von: Art. 6, in: Sachs, Kommentar zum Grundgesetz, 5. Aufl., 2009, zit.: Schmitt-Kammler/Coelln in Sachs GG.

Schrader, Peter/Schubert, Jens: § 3, in: Däubler/Bertzbach, Allgemeines Gleichbehandlungsgesetz, 2. Aufl., 2008, zit.: Schrader/Schubert in Däubler/ Bertzbach AGG. 
Schulte, Bernd: Die Behindertenrechtskonvention der Vereinten Nationen; Von der Integration zur Inklusion: Änderungsbedarf im deutschen Behindertenrecht, br 2011, S. 41-49.

Schulte-Körne, Gerd: Legasthenie und Dyskalkulie; Aktuelle Entwicklungen in Wissenschaft und Schule, 2007, zit.: Legasthenie und Dyskalkulie 2007.

Schulte-Körne, Gerd: Elternratgeber Legasthenie, 2009, zit.: Elternratgeber Legasthenie.

Schulte-Körne, Gerd: Diagnostik und Therapie der Lese-Rechtschreib-Störung, Deutsches Ärzteblatt 107, 2010, S. 718-727, abrufbar unter http://www.kjp.med.uni-muenchen.de/veroeffentlichungen.php

Schulte-Körne, Gerd: Legasthenie und Dyskalkulie: Stärken erkennen - Stärken fördern; Mit 15 Tabellen, 2011, zit.: Legasthenie und Dyskalkulie 2011.

Schulte-Körne, Gerd u.a.: Further evidence for DYX1C1 as a susceptibility factor for dyslexia, Psychiatric Genetics 2008, abrufbar unter http://www.kjp.med.uni-muenchen.de/veroeffentlichungen.php

Schulte-Körne, Gerd/Amorosa, Hedwig: Legasthenie; Zum aktuellen Stand der Ursachenforschung, der diagnostischen Methoden und der Förderkonzepte, 2002, zit.: Legasthenie 2002.

Schulte-Körne, Gerd/Stieglitz, Rolf-Dieter: Lese-Rechtschreibstörung, in: Fegert/ Streeck-Fischer/Freyberger, Adoleszenzpsychiatrie, 2009, S. 476-487.

Schulte-Körne, Gerd/Deimel, Wolfgang/Remschmidt, Helmut: Zur Diagnostik der Lese-Rechtschreibstörung, Kinder- und Jugendpsychiatrie und Psychotherapie 29, 2001, S. 113-116, abrufbar unter http://www.kjp.med.unimuenchen.de/veroeffentlichungen.php

Schulte-Körne, Gerd/Deimel, Wolfgang/Jungermann, Marc/Remschmidt, Helmut: Nachuntersuchung einer Stichprobe von lese-rechtschreibgestörten Kindern im Erwachsenenalter, Zeitschrift für Kinder- und Jugendpsychiatrie und Psychotherapie 31, 2003, S. 267-276, abrufbar unter http://www.kjp.med.uni-muenchen.de/veroeffentlichungen.php

Schulte-Körne, Gerd/Deimel, Wolfgang/Remschmidt, Helmut: Rechtschreibtraining in schulischen Fördergruppen - Ergebnisse einer Evaluationsstudie in der Primarstufe, Zeitschrift für Kinder- und Jugendpsychiatrie und Psychotherapie 31, 2003, S. 85-98, abrufbar unter http://www.kjp.med.unimuenchen.de/veroeffentlichungen.php

Schumann, Brigitte: „Ich schäme mich ja so!“; Die Sonderschule für Lernbehinderte als "Schonraumfalle“. 2007, zit.: "Ich schäme mich ja so!“.

Schünemann, Bernd: § 203, in: Jähnke/Laufhütte/Odersky, Leipziger Kommentar zum StGB, 11. Aufl., 2005. 
Schweizer, Kerstin: Der Gleichberechtigungssatz - neue Form, alter Inhalt? Untersuchung zu Gehalt und Bedeutung des neugefaßten Art. 3 Abs. 2 GG unter Einbeziehung Europäischen Gemeinschaftsrechts, 1998, zit.: Gleichberechtigungssatz.

Schwengers, Clarita: Eingliederungshilfe für seelisch behinderte Kinder und Jugendliche nach $\S 35$ a SGB VIII im Verhältnis zu konkurrierenden Leistungen nach dem (Sozial-)Leistungsrecht; zugleich ein Beitrag zu öffentlichrechtlichen Ausgleichsansprüchen bei Doppelzuständigkeiten von Leistungsträgern. 2007, zit.: Eingliederungshilfe $\S 35$ a SGB VIII.

Sellin, Katrin: Legasthenie und Fremdsprachen, in: Ganser/Richter, Was tun bei Legasthenie in der Sekundarstufe? 2005, S. 141-161.

Sellin, Katrin: Wenn Kinder mit Legasthenie Fremdsprachen lernen, 2. Aufl., 2008, zit.: Wenn Kinder mit Legasthenie Fremdsprachen lernen.

Sidortschuk, Klaus: Zum Verhältnis von Jugendhilfeträger und Schule bei vorliegender Teilleistungsstörung: Kostenerstattung für erbrachte Leistungen im Rahmen der Eingliederungshilfe nach Anspruchsüberleitung des Jugendhilfeträgers gegenüber der Schulverwaltung nach § 95 SGB VIII, JAmt 2005, S. 552-553.

Sodan, Helge/Ziekow, Jan: Grundkurs Öffentliches Recht; Staats- und Verwaltungsrecht, 3. Aufl., 2008, zit.: Öffentliches Recht.

Spickhoff, Andreas: Medizinrecht; [AMG, ApoG, BGB, GenTG, KHG, MBO, MPG, SGB V, SGB XI, StGB, TFG, TPG], 2011, zit.: Medizinrecht.

Spranger, Tade Matthias: Umfassender Schutz aller Behinderten über Art. 3 Abs. 3 S. 2 GG, br 1998, S. 92-93.

Starck, Christian: Art. 3, in: Mangoldt/Klein/Starck, Kommentar zum Grundgesetz, 5. Aufl., 2005, zit.: Starck in Mangoldt/Klein/Starck GG.

Stefek, Helle: Das Recht auf Bildung in der Europäischen Gemeinschaft; Möglichkeit und Notwendigkeit gemeinschaftsrechtlicher Vorgaben zur (Grund-)Bildung in Europa. 2006, zit.: Recht auf Bildung in der EG.

Steinbrück, Hans-Joachim: Die Bedeutung des Übereinkommens der Vereinten Nationen über die Rechte von Menschen mit Behinderungen für die Bildungspolitik in Deutschland, 29.1.2009, zit.: Die Bedeutung des Übereinkommens der Vereinten Nationen über die Rechte von Menschen mit Behinderungen für die Bildungspolitik in Deutschland.

Steinhausen, Hans-Christoph: Psychische Störungen bei Kindern und Jugendlichen; Lehrbuch der Kinder- und Jugendpsychiatrie und -psychotherapie; mit 159 Tabellen sowie 65 aktuellen Original-Fragebögen und Skalen, 6. Aufl., 2006, zit.: Psychische Störungen bei Kindern und Jugendlichen.

Steinhausen, Hans-Christoph: Schule und psychische Störungen, 1. Aufl., 2006, zit.: Schule und psychische Störungen. 
Stelkens, Paul/Bonk, Heinz Joachim/Sachs, Michael: Verwaltungsverfahrensgesetz; Kommentar, 7. Aufl., 2008, zit.: Stelkens/Bonk/Sachs VwVfG.

Stelkens, Ulrich: § 36, in: Stelkens/Bonk/Sachs, Verwaltungsverfahrensgesetz, 7. Aufl., 2008, Stelkens in Stelkens/Bonk/Sachs VwVfG.

Stern, Klaus: Das Gebot zur Ungleichbehandlung, in: Maurer, Das akzeptierte Grundgesetz, 1990, S. 207-219.

Stern, Klaus: Das Staatsrecht der Bundesrepublik Deutschland, 1. Aufl., 1994, zit.: Staatsrecht.

Straßmair, Stefan M.: Der besondere Gleichheitssatz aus Art. 3 Abs. 3 Satz 2 GG; Eine Untersuchung zu Gehalt und Struktur des Diskriminierungsverbotes sowie seiner Bedeutung für die verfassungsrechtliche Stellung und soziale Gleichstellung von Menschen mit Behinderungen. 2002, zit.: Der besondere Gleichheitssatz aus Art. 3 Abs. 3 Satz 2 GG.

Suchodoletz, Waldemar von: Alternative Therapieangebote im Überblick, in: Suchodoletz/Amorosa, Therapie der Lese-Rechtschreib-Störung (LRS), 2003, S. 161-256.

Suchodoletz, Waldemar von: Therapie der Lese-Rechtschreib-Störung (LRS); traditionelle und alternative Behandlungsmethoden im Überblick, 2. Aufl., 2006, zit.: Therapie der Lese-Rechtschreib-Störung.

Suchodoletz, Waldemar v./Amorosa, Hedwig: Therapie der Lese-RechtschreibStörung (LRS); Traditionelle und alternative Behandlungsmethoden im Überblick, 1. Aufl., 2003, zit.: Therapie Lese-Rechtschreib-Störung.

Tacke, Gerd: Die Wirksamkeit von Trainingsprogrammen und Übungen zur Förderung der Rechtschreibung: wissenschaftliche Studien und praktische Erfahrungen, in: Schulte-Körne, Legasthenie und Dyskalkulie, 2007, S. 135-152.

Theben, Bettina: Blinder Bariton, gehörlose Geigerin, rollender Romeo - nicht an deutschen Hochschulen, in: Arnade, Die Gesellschaft der Behinderer, 1997, S. 79-83.

Thiel, Markus: Der Erziehungsauftrag des Staates in der Schule; Grundlagen und Grenzen staatlicher Erziehungstätigkeit im öffentlichen Schulwesen. 2000, zit.: Erziehungsauftrag des Staates in der Schule.

Thiel, Oliver: Rechenschwache Grundschüler - Ursachen, Diagnose, Förderung, https://www.familienhandbuch.de/schule/schulprobleme/rechenschwach e-grundschuler-ursachen-diagnose-forderung, zuletzt geprüft am 20.01.2013.

Thomé, Günther/Eichler, Wolfgang: Lese-Rechtschreib-Schwierigkeiten (LRS) und Legasthenie; Eine grundlegende Einführung, 2. Aufl., 2004, zit.: LRS und Legasthenie. 
Tiedemann, Joachim/Billmann-Mahecha, Elfriede: Wie erfolgreich sind Gymnasiasten ohne Gymnasialempfehlung?, Die Kluft zwischen Schullaufbahnempfehlung und Schulformwahl der Eltern, Z Erziehungswiss 2010, S. 649-660.

Tiemann, Burkard: Zur Rechtsstellung Behinderter im Prüfungsverfahren, BayVBI 1976, S. 650-652.

Tobler, Christa: Indirect discrimination: a case study into the development of the legal concept of indirect discrimination under EC law, 2005.

Tulzer, Friedrich: Deutsch am Gymnasium; Beiträge zu einer Kritik des Deutschunterrichts an höheren Schulen mit einem Erfahrungsbericht und Fallstudien aus Wien und Oberösterreich, 2008, zit.: Deutsch am Gymnasium.

Umbach, Dieter: Art. 12, in: Umbach/Clemens, Grundgesetz, 2002, zit.: Umbach GG, zit.: Umbach in Umbach GG.

Umbach, Dieter/Clemens, Thomas: Grundgesetz; Mitarbeiterkommentar und Handbuch, 2002, zit.: Umbach GG.

United Nations: From Exclusion to Equality, http://www.un.org/disabilities/ documents/toolaction/ipuhb.pdf, zuletzt geprüft am 20.01.2013.

Valtin, Renate: Das Konstrukt Legasthenie - Wem schadet es? Wem nützt es? in: Thomé/Eichler, Lese-Rechtschreib-Schwierigkeiten (LRS) und Legasthenie, 2004, S. 56-63.

Valtin, Renate: Brauchen wir die Legasthenie? http://www.legakids.net/ fileadmin/user_upload/Downloads/Info/Wissenschaft/ValtinStellungnahme-Legasthenie-Bayern.pdf, zuletzt geprüft am 20.01.2013.

Valtin, Renate/Hornberg, Sabine/Buddeberg, Magdalena/Voss, Andreas/ Kowoll, Magdalena E./Potthoff, Britta: Schüler und Schülerinnen mit Leseproblemen: eine ökosytemische Betrachtungsweise, in: Bos, IGLU 2006: Lesekompetenzen von Grundschulkindern in Deutschland im internationalen Vergleich, 2007, S. 109-160.

Vanselow, Kurt/Dummer-Smoch, Lisa: Die Vernachlässigung individueller Begabungsstrukturen im deutschen Bildungssystem; Ergebnisse einer experimentellen Untersuchung, 2002, zit.: Vernachlässigung individueller Begabungsstrukturen.

Verband Dyslexie Schweiz: Richtlinien für die Produktion von Lernmaterialien, http://www.verband-dyslexie.ch/images/stories/pdf/gut_zu_wissen/ Richtlinien_fuer_die_Produktion_von_Lernmaterialien.pdf, zuletzt geprüft am 20.01.2013.

Vondung, Ute: $\S 35$ a, in: Kunkel, Sozialgesetzbuch VIII, 3. Aufl., 2006, zit.: Vondung in Kunkel SGB VIII.

Voßkuhle, Andreas: Der europäische Verfassungsverbund, NVwZ 2010, S. 1-8. 
Waddington, Lisa: Reasonable Accommodations, in: Schiek/Waddington/Bell, Cases, Materials and Text on National, Supranational and International Non-Discrimination Law, 2007, S. 629-758, zit: Waddington in: NonDiscrimination Law.

Walter, Jürgen/Wember, Franz B./Borchert, Johann/Goetze, Herbert: Sonderpädagogik des Lernens, 2007, zit.: Sonderpädagogik des Lernens.

Wansing, Gudrun: Der Inklusionsbegriff in der Behindertenrechtskonvention, in: Welke, UN-Behindertenrechtskonvention mit rechtlichen Erläuterungen, 2012, zit.: Wansing, Kommentar zur UN-BRK, Inklusionsbegriff.

Warnke, Andreas/Hemminger, Uwe/Plume, Ellen: Lese-Rechtschreibstörungen, 2004, zit.: Lese-Rechtschreibstörungen.

Warnke, Andreas/Hemminger, Uwe/Roth, Ellen/Schneck, Stefanie: Legasthenie - Leitfaden für die Praxis, 2002, zit.: Legasthenie - Leitfaden für die Praxis.

Warnke, Andreas/Plume, Ellen/Oehler, Claudia: Rechenstörungen, in: Remschmidt/Mattejat/Warnke, Therapie psychischer Störungen bei Kindern und Jugendlichen, 2008, S. 162-167.

Warnke, Andreas/Schulte-Körne, Gerd: Legasthenie, in: Remschmidt/Mattejat/ Warnke, Therapie psychischer Störungen bei Kindern und Jugendlichen, 2008, S. 153-161.

Warnke, Andreas/Wewetzer, Christoph: Teilleistungsschwächen - seelische Behinderung?! Berufsverband der Ärzte für Kinder- und Jugendpsychiatrie und Psychotherapie Deutschland e.V. 1997, S. 61-78.

Weber-Dürler, Beatrice: Chancengleichheit und Rechtsgleichheit, in: Haller, Festschrift für Ulrich Häfelin zum 65. Geburtstag, 1989, S. 205-222.

Wehrmann, Michael: Qualitative Diagnostik der Rechenschwäche, in: SchulteKörne, Legasthenie und Dyskalkulie, 2007, S. 333-337.

Wehrmann, Michael: Prävention von Dyskalkulie, in: Schulte-Körne, Legasthenie und Dyskalkulie: Stärken erkennen - Stärken fördern, 2011, S. 245-260.

Weichert, Thilo: Schulleitungsaufgaben, schulische Handlungsfelder und Datenschutz, in: Jürgens/Böhm/Prüß, Schulleitung in den Ländern Brandenburg, Mecklenburg-Vorpommern, Sachsen, Sachsen-Anhalt und Thüringen, 1991, Abschnitt 11.1.

Welke, Antje: UN-Behindertenrechtskonvention mit rechtlichen Erläuterungen, 2012, zit.: Kommentar zur UN-BRK.

Welti, Felix: Behinderung und Rehabilitation im sozialen Rechtsstaat; Freiheit, Gleichheit und Teilhabe behinderter Menschen. Habilitationsschrift Kiel, 2005, zit.: Behinderung und Rehabilitation im sozialen Rechtsstaat.

Welti, Felix: § 2, § 12, in: Welti/Lachwitz/Schellhorn, Handkommentar zum Sozialgesetzbuch IX, 3. Aufl., 2009, zit.: HK-SGB IX. 
Welti, Felix: Rechtliche Voraussetzungen von Barrierefreiheit, NVwZ 2012, S. 725-730.

Welti, Felix/Lachwitz, Klaus/Schellhorn, Helmut: Handkommentar zum Sozialgesetzbuch IX (HK-SGB IX); Rehabilitation und Teilhabe behinderter Menschen, 3. Aufl., 2009, zit.: HK-SGB IX.

Wendeling-Schröder, Ulrike/Stein, Axel: Allgemeines Gleichbehandlungsgesetz; Kommentar, 2008, zit.: AGG.

Weuster, Arnulf: Personalauswahl; Anforderungsprofil, Bewerbersuche, Vorauswahl und Vorstellungsgespräch, 2. Aufl., 2008, zit.: Personalauswahl.

Wieland, Joachim: Art. 12, in: Dreier, Grundgesetz Kommentar, 2. Aufl., 2004, zit.: Wieland in Dreier GG.

Wieneke, Rudolf: Symptomorientierter Kriterienkatalog - Rechenschwäche, http://www.ztr-rechenschwaeche.de/files/lehrer_symptomkatalog.pdf, zuletzt geprüft am 20.01.2013.

Wiesner, Reinhard: SGB VIII; Kinder- und Jugendhilfe, Kommentar, 3. Aufl., 2006, zit.: SGB VIII.

Will, Günther: Legastheniebedingte Probleme beim Erlernen der englischen Sprache, in: Ganser/Richter, Was tun bei Legasthenie in der Sekundarstufe? 2005, S. 188-189.

Wimmer, Raimund: Ein halbes Jahrhundert Gesetzesvorbehalt im Schulwesen, RdJB 1997, S. 15-18.

Windthorst, Kay: Verfassungsrecht I; Grundlagen, 1994.

Wirth, G./Ptok, M.: Sprachstörungen, Sprechstörungen, kindliche Hörstörungen: Lehrbuch für Ärzte, Logopäden und Sprachheilpädagogen, 2000, zit.: Sprachstörungen.

Wocken, Hans: Andere Länder, andere Schüler? Vergleichende Untersuchungen von Förderschülern in den Bundesländern Brandenburg, Hamburg und Niedersachen (Forschungsbericht), Mai 2005, http://bidok.uibk.ac.at/down load/wocken-forschungsbericht.pdf, zuletzt geprüft am 20.01.2013.

Wölms, Barbara: Aspekte der schulischen LRS-Förderung im Fach Englisch in der Sekundarstufe I, in: Schulte-Körne, Legasthenie und Dyskalkulie, 2007, S. 153-161.

Wolter, Marlene: Änderungen in der Versetzungsordnung und im Zeugniserlass für die Grundschule, Nds. SVBI 2009, S. 271-272.

Wößmann, Ludger/Piopiunik, Marc: Was unzureichende Bildung kostet; Eine Berechnung der Folgekosten durch entgangenes Wirtschaftswachstum; http://www.bertelsmann-stiftung.de/cps/rde/xbcr/SID-F648D22795AAE8ED/bst/xcms_bst_dms_30242_31113_2.pdf, zuletzt geprüft am 20.01.2013. 
Zier, Johanna: Recht für Diplom-Psychologen; Eine Einführung, 1. Aufl., 2002, zit.: Recht für Diplom-Psychologen.

Zimmerling, Wolfgang: Prüfungsunfähigkeit und (amts-)ärztliches Attest, MedR 2001, S. $634 \mathrm{ff}$.

Zimmerling, Wolfgang: Vorläufiger Rechtsschutz im Prüfungsrecht, NVwZ 2004, S. $651 \mathrm{ff}$.

Zimmerling, Wolfgang: § 839 BGB, in: Herberger, juris PK BGB, 3. Aufl, 2006.

Zimmerling, Wolfgang: Prüfungsrecht aus anwaltlicher Sicht, Vortrag vor der Deutschen Richterakademie am 18.11.2008; http://www.zimmerling.de/ veroeffentlichungen/volltext/richterakademie-2008.htm, zuletzt geprüft am 20.01.2013.

Zimmerling, Wolfgang/Brehm, Robert G.: Prüfungsrecht, 3. Aufl., 2007, zit.: Prüfungsrecht.

Zinsmeister, Julia: Mehrdimensionale Diskriminierung; Das Recht behinderter Frauen auf Gleichberechtigung und seine Gewährleistung durch Art. 3 GG und das einfache Recht. 2007, zit.: Mehrdimensionale Diskriminierung.

Zinsmeister, Julia: Diskriminierungen wegen einer Behinderung, in: Degener, Antidiskriminierungsrecht, 2008, S. 167-178. 
Schüler und Schülerinnen mit Legasthenie und/oder Dyskalkulie haben in der Schule erhebliche Probleme und erreichen häufig nur niedrige Bildungsabschlüsse, weil sie aufgrund ihrer Beeinträchtigung nicht im geforderten Maß lesen, rechtschreiben und/oder rechnen können. Ob und inwieweit Legasthenie und Dyskalkulie in der Schule berücksichtigt werden müssen, ist pädagogisch und schulrechtlich sehr umstritten. Die Arbeit untersucht auf der Grundlage des Verfassungsrechts und der UNBehindertenrechtskonvention, welche Rechte Schüler und Schülerinnen mit Legasthenie/Dyskalkulie haben. Sie kommt zu dem Ergebnis, dass Legasthenie und Dyskalkulie Behinderungen im Sinne des Art. 3 III GG und der UN-BRK sind. Daraus ergibt sich für die betroffenen Schüler und Schülerinnen ein umfassender Anspruch auf Nachteilsausgleich, sowohl bei den Prüfungsbedingungen als auch bei den Prüfungsinhalten. Im umfangreichen Verfahrensteil werden praktische Fragestellungen geprüft, wie z.B. das Verfahren zur Anerkennung der Behinderung in der Schule, die Ausgestaltung von Nachteilsausgleich, der Umfang des Datenschutzes und die Zulässigkeit von Zeugnisbemerkungen. 